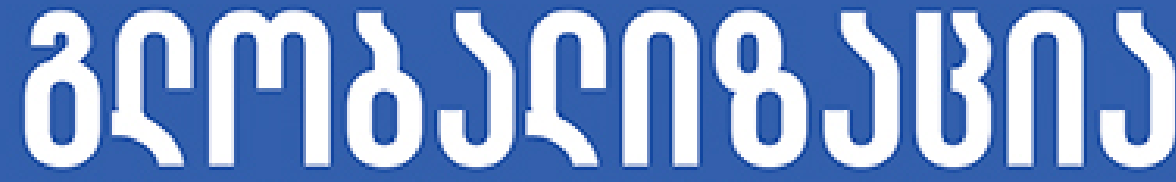

№7

\section{is dก86Jull}

3

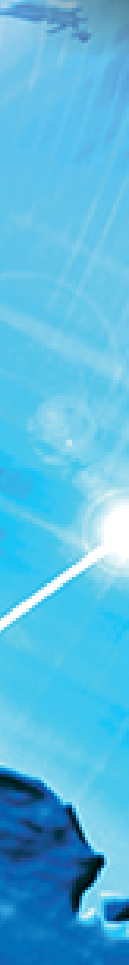

OLOBALIZATION

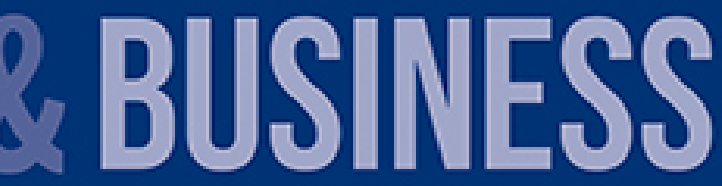




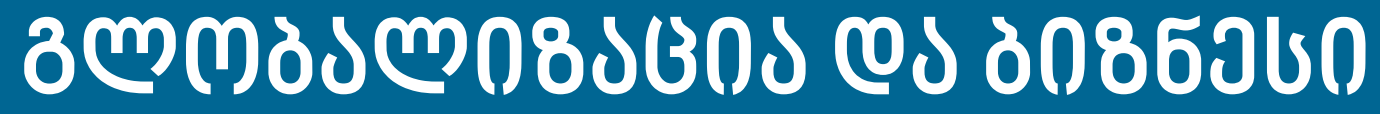

\section{GLOBALIZATION AND BUSINESS}

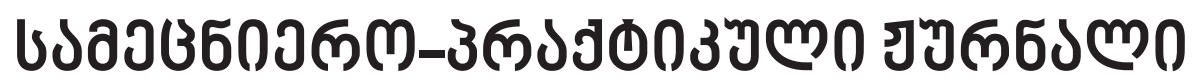

aзmбmanz

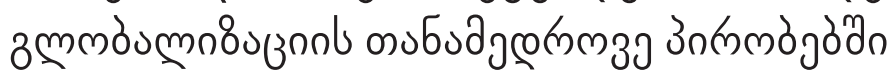

ogmkno gos उnofonzo

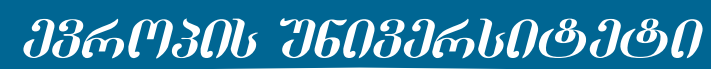
EUROPEAN UNIVERSITY

\section{SCIENTIFIC-PRACTICAL JOURNAL}

Actual Problems of Economy and Business in Modern Globalization

Theory and Practice 


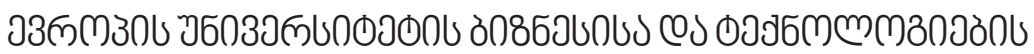

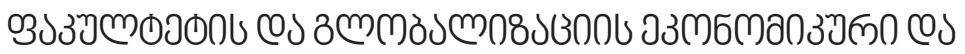

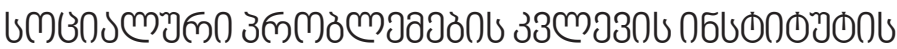

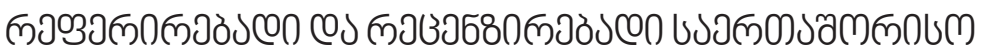

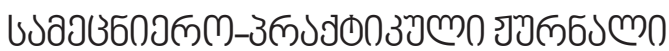

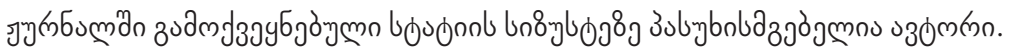

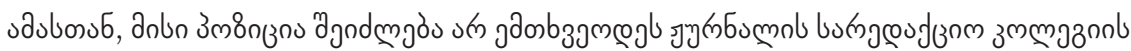
anbusmgàjoll.

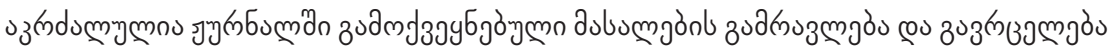

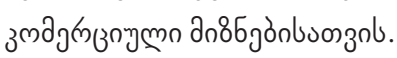

REFEREED AND PEER-REVIEWED INTERNATIONAL SCIENTIFIC-PRACTICAL JOURNAL OF THE FACULTY OF BUSINESS AND TECHNOLOGY AND THE INSTITUTE OF RESEARCH OF ECONOMIC AND SOCIAL PROBLEMS OF GLOBALIZATION OF THE EUROPEAN UNIVERSITY

Each author is responsible for the accuracy of the article in this journal. His/her position may not coincide with the opinions of the editorial board.

Reproduction on distribution of the materials published in this journal for commercial purposes is strictly prohibited.

ISSN 2449-2396 


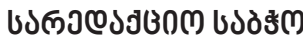

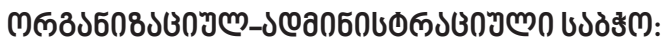

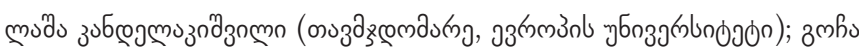

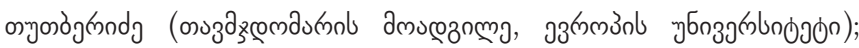

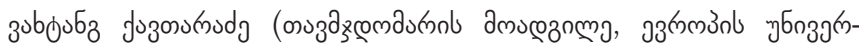

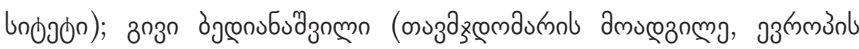

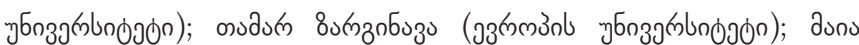

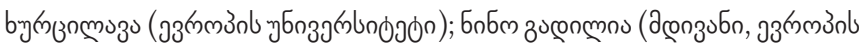

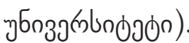

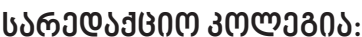

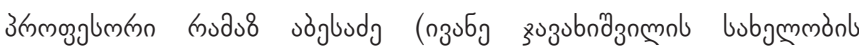

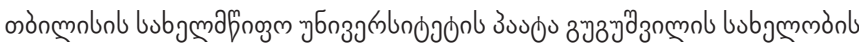

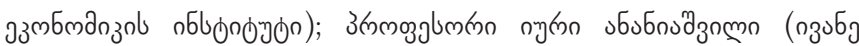

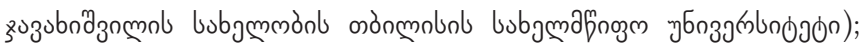

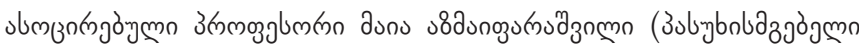

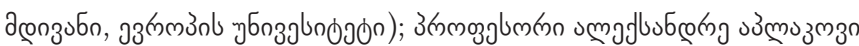

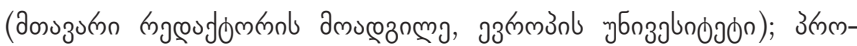

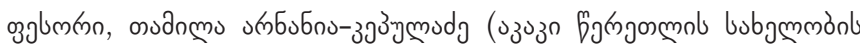

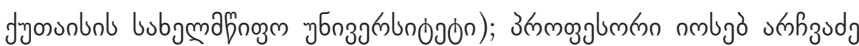

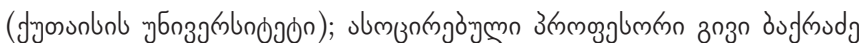

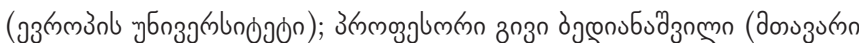

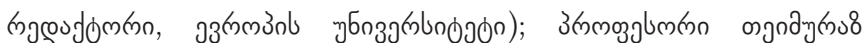

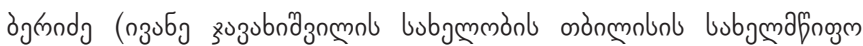

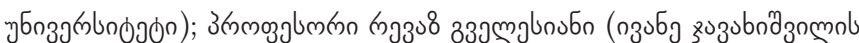

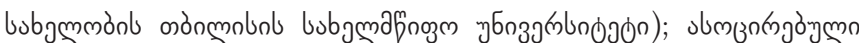

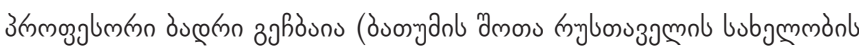

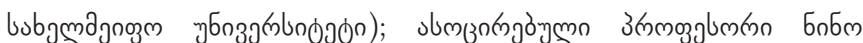

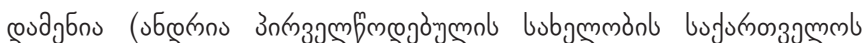

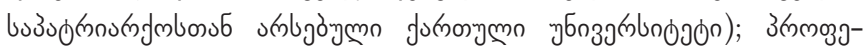

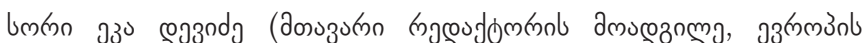
चб

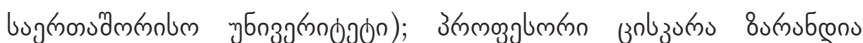

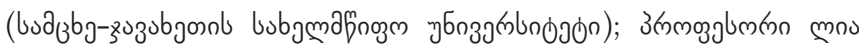

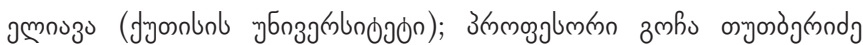

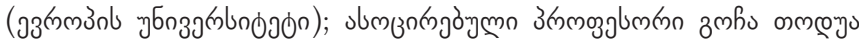
(эз

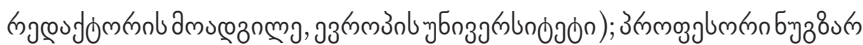

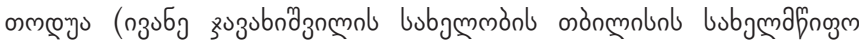

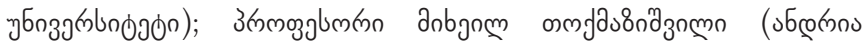

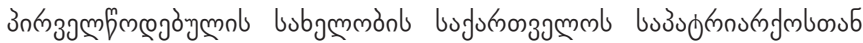

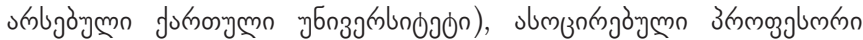

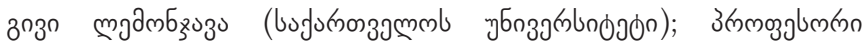

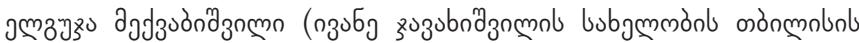

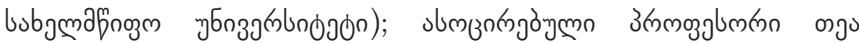

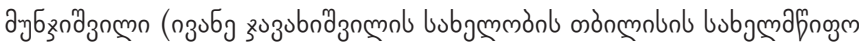

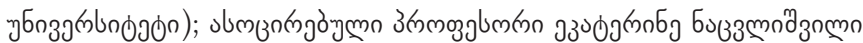

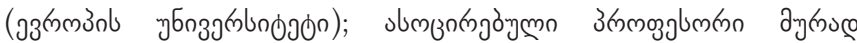

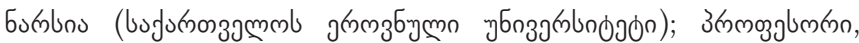

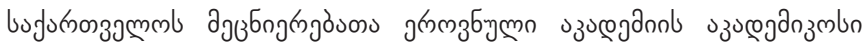

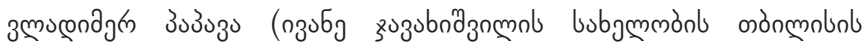

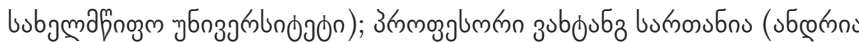

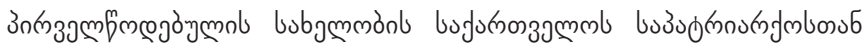

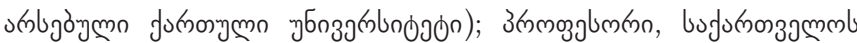

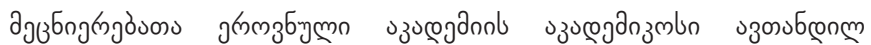

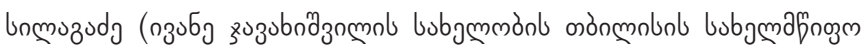

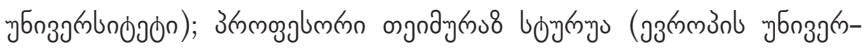

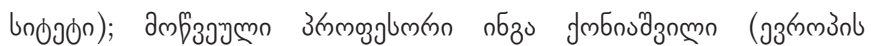

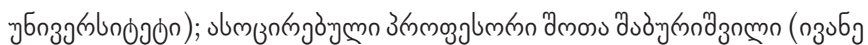

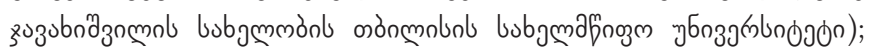

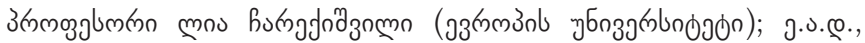

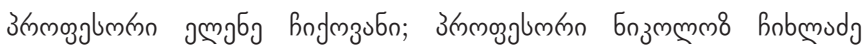

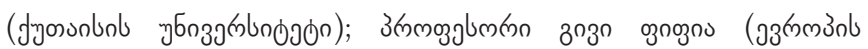

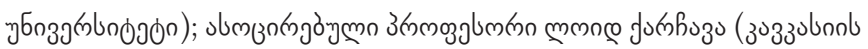

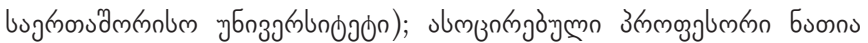

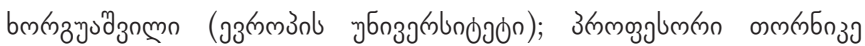

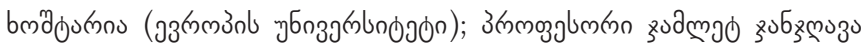

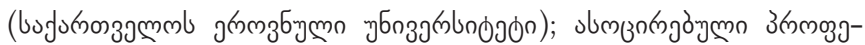

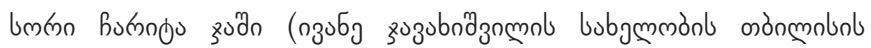

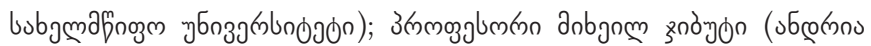

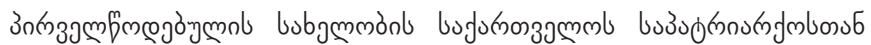
د

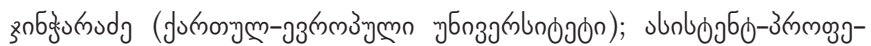

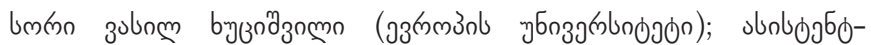

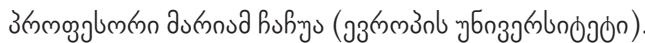

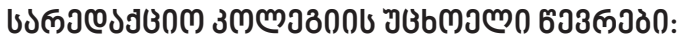

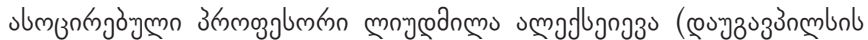
चб

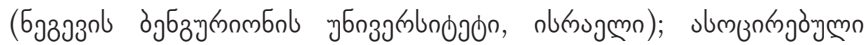

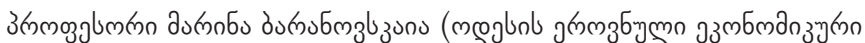
चб

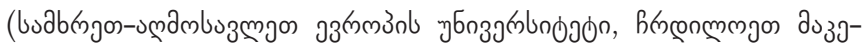

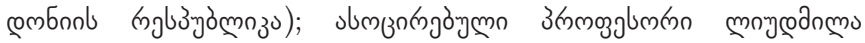

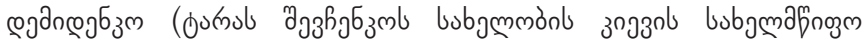

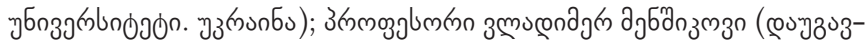

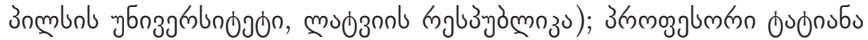

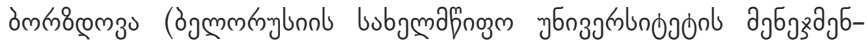

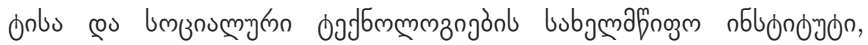

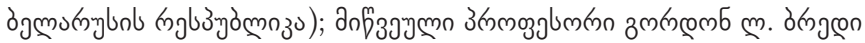

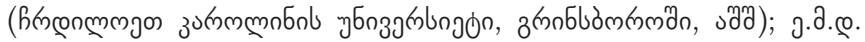

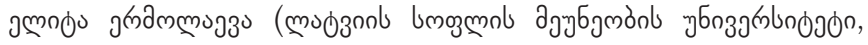

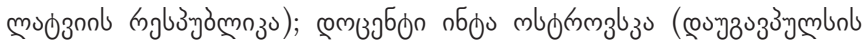

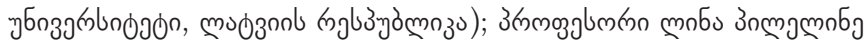

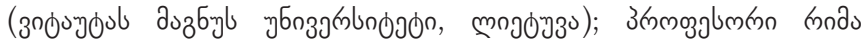

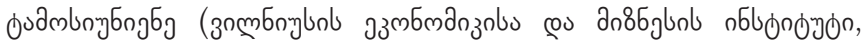

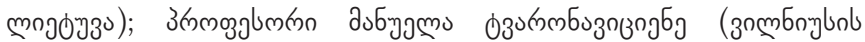

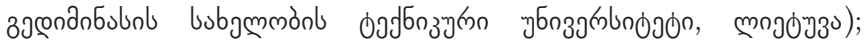

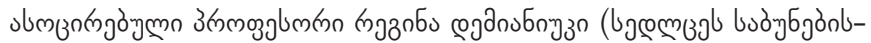

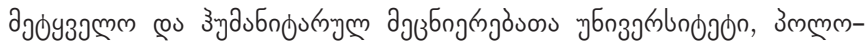

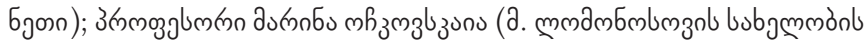

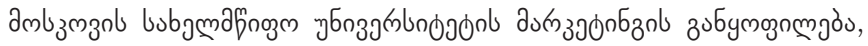

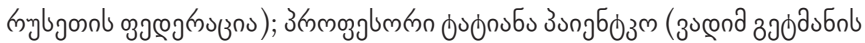

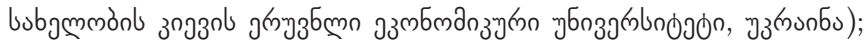

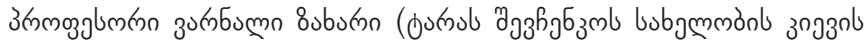

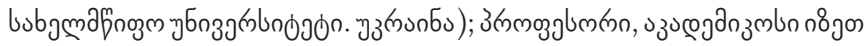

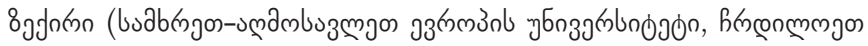

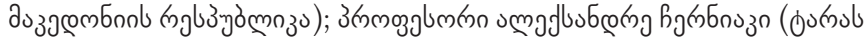

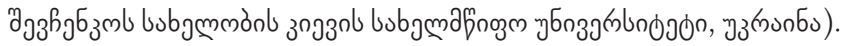




\section{EDITORIAL BOARD}

\section{ORGANIZATIONAL-ADMINISTRATIVE BOARD:}

Lasha Kandelakishvili (Chairman, European University); Gocha Tutberidze (Deputy Chairman, European University); Vakhtang Kavtaradze (Deputy Chairman, European University); Givi Bedianashvili (Deputy Chairman, European University); Tamar Zarginava (European University); Maia Khurtsilava (European University); Nino Gadilia (secretary, European University).

\section{EDITORIAL BOARD:}

Ramaz Abesadze, Professor (Paata Gugushvili Institute of Economics of Ivane Javakhishvili Tbilisi State University); Iuri Ananiashvili, Professor (Ivane Javakhishvili Tbilisi State University); Maia Azmaiparashvili, Associated Professor (Responsible Secretary, European University); Alexander Aplakov, Professor, (Deputy Chief Editor, European University); Ioseb Archvadze, Professor (Kutaisi University); Tamila ArnaniaKepuladze, Professor (Akaki Tsereteli Kutaisi State University); Givi Bakradze, Associated Professor (European University); Givi Bedianashvili, Professor (Chief editor, European University); Teimuraz Beridze, Professor (Ivane Javakhishvili Tbilisi State University); Revaz Gvelesiani, Professor (Ivane Javakhishvili Tbilisi State University); Badri Gechbaia, Associated Professor (Batumi Shota Rustaveli State University); Nino Damenia, Associated Professor (St. Andrew the First-Called Georgian University of the Patriarchate of Georgia); Eka Devidze, Professor (Deputy Chief Editor, European University); Natela Dogonadze - professor (International Black Sea University); Tsiskara Zarandia, Professor (Samtskhe-Javakheti State University), Lia Eliava, Professor (Kutaisi University); Charita Jashi - Associated Professor (Ivane Javakhishvili Tbilisi State University); Gocha Tutberidze, Professor (European University); Gocha Todua, Associated Professor (European University); Thea Todua, Professor (Deputy chief editor, European University); Nugzar Todua, Professor (Ivane Javakhishvili Tbilisi State University); Mikheil Tokmazishvili, Professor (St. Andrew the First-Called Georgian University of the Patriarchate of Georgia); Inga Koniashvili, (European University); Givi Lemonjava, Associated Professor (Georgian University); Elguja Mekvabishvili, Professor (Ivane Javakhishvili Tbilisi State University); Tea Munjishvili, Associated Professor (Ivane Javakhishvili Tbilisi State University); Ekaterine Natsvlishvili, Associated Professor (European University); Murad Narsia, Associated Professor (Georgian National University); Vladimer Papava, Professor, Academician of the National Academy of Sciences of Georgia (Ivane Javakhishvili Tbilisi State University), Vakhtang Sartania, Professor (St. Andrew the First-Called Georgian University of the Patriarchate of Georgia); Avtandil Silagadze, Professor, Academician of the National Academy of Sciences of Georgia (Ivane Javakhishvili Tbilisi State University); Teimuraz Sturua, Professor (European University); Shota Shaburishvili, Associated Professor (Ivane Javakhishvili Tbilisi State University); Lia Charekishvili, Professor (European University); Nikoloz Chikhladze, Professor (Kutaisi University); Givi Pipia, Professor, (European University); Loid Karchava, Associated Professor, (Caucasus International University); Natia Khorguashvili, Associated Professor (European University); Tornike Khoshtaria, Professor (European University); Jamlet Janjghava, Professor (Georgian National University); Mikheil Jibuti, Professor (St. Andrew the First-Called Georgian University of the Patriarchate of Georgia); Khatuna Jintcharadze, assistant-professor (Georgian European University); Vasil Khutsishvili, Assistant professor, (European University); Mariam Chachua, Assistant-Professor (European University).

\section{FOREIGN PART OF EDITORIAL BOARD MEMBERS:}

Ludmila Aleksejeva, Associated Professor (Daugavpils University, Republic of Latvia); Moshe Barak, Professor (Ben-Gurion University of the Negev, Israel); Marina Baranovskaya, Associated professor (Odessa National Economics University, Ukraine); Tatiana Borzdova, Professor (Belarusian State Institute of Management and Social Technologies of State University, Republic of Belarus); Gordon L. Brady, Visiting professor (University of North Carolina at Greensboro, USA); Oleksandr Chernyak, Professor (Taras Shevchenko National University of Kyiv, Ukraine); Regina Demianiuk, Associated professor (Siedlce University of Natural Sciences and Humanities, Republic of Poland); Fitim Deari, Associated Professor (South East European University, Tetovo, Republic of North Macedonia); Liudmila Demydenko, Associated Professor (Taras Shevchenko National University of Kyiv, Ukraine); Elita Jermolajeva, Doctor of Economics (Latvia University of Agriculture, Republic of Latvia); Vladimir Menshikov, professor (Daugavpils University, Republic of Latvia); Marina Ochkovskaya, Professor (Moscow M. Lomonosov State University Marketing Department, Russian Federation); Inta Ostrovska, docent (Daugavpils University, Republic of Latvia); Tatiana Paientko, Professor (Kyiv national economic university named after Vadym Hetman, Ukraine); Lina Pilelienè, Professor (Vytautas Magnus University, Republic of Lithuania); Rima Tamosiuniene, Professor, (Vilnius Institute of Economics and Business, Republic of Lithuania); Manuela Tvaronaviciene, Professor (Vilnius Gediminas Technical University, Republic of Lithuania); Varnalii Zakharii, professor (Taras Shevchenko National University of Kyiv, Ukraine); Izet Zeqiri, professor, Academician (South East European University, Tetovo, Republic of North Macedonia). 


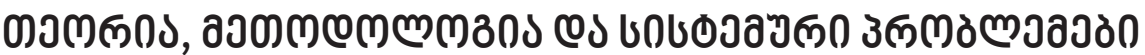

\section{THEORY, METHODOLOGY AND SYSTEMIC PROBLEMS}

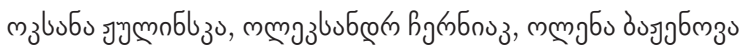

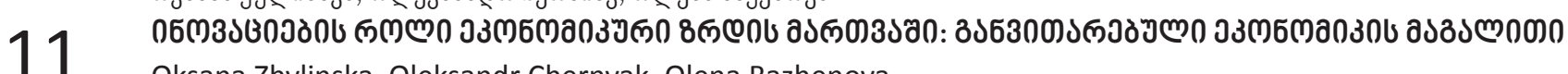

Oksana Zhylinska, Oleksandr Chernyak, Olena Bazhenova

THE ROLE OF INNOVATIONS IN DRIVING ECONOMIC GROWTH: CASE OF ADVANCED ECONOMIES

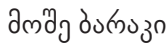

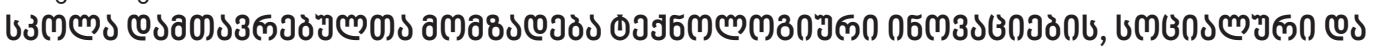

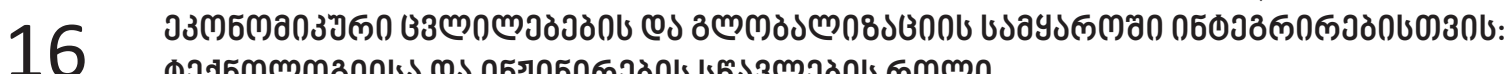

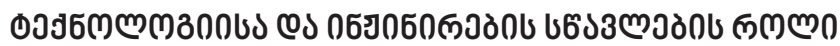

Moshe Barak

PREPARING SCHOOL GRADUATES TO INTEGRATE INTO THE WORLD OF TECHNOLOGICAL INNOVATION, SOCIAL AND ECONOMIC CHANGES, AND GLOBALIZATION: THE ROLE OF TEACHING TECHNOLOGY AND ENGINEERING

zommga nuguenodg

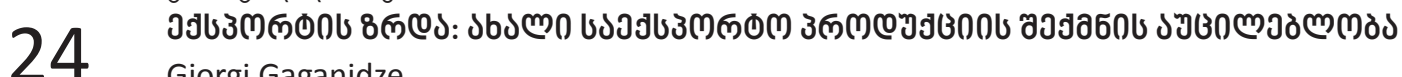

Giorgi Gaganidze

GROWTH OF EXPORT: THE NEED TO CREATE NEW EXPORT PRODUCTS

ńm3ง8 33gmglouton

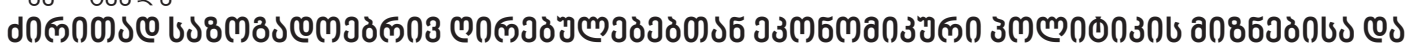

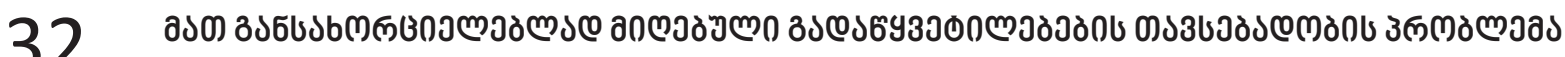

Revaz Gvelesiani

COMPATIBILITY PROBLEM OF BASIC PUBLIC VALUES WITH ECONOMIC POLICY GOALS AND

DECISIONS FOR THEIR IMPLEMENTATION

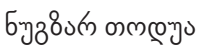

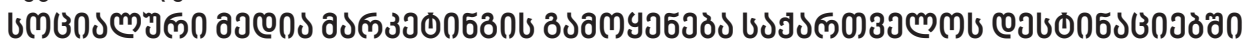

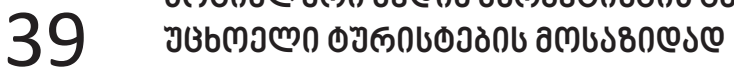

Nugzar Todua

USING SOCIAL MEDIA MARKETING FOR ATTRACTING FOREIGN TOURISTS TO GEORGIAN DESTINATIONS

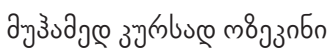

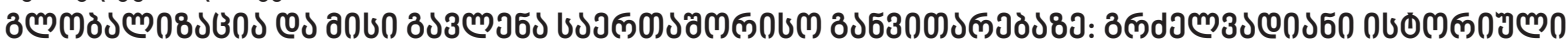

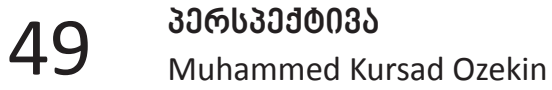

GLOBALISATION AND ITS IMPACTS ON INTERNATIONAL DEVELOPMENT:

A LONG-TERM HISTORICAL PERSPECTIVE

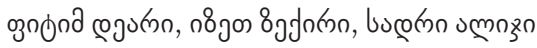

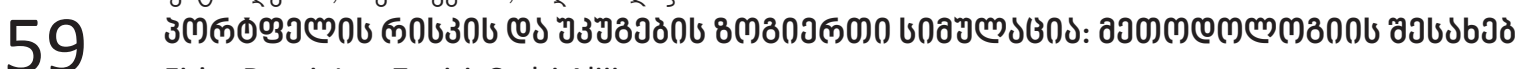

Fitim Deari, Izet Zeqiri, Sadri Aliji

SOME SIMULATIONS ON THE PORTFOLIO RISK AND RETURN: A METHODOLOGICAL NOTE

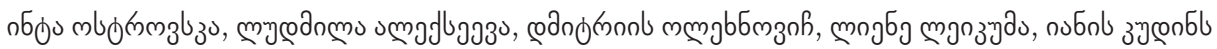

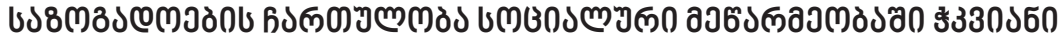

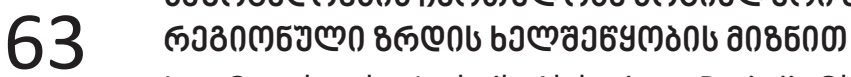

Inta Ostrokovska, Ludmila Aleksejeva, Dmitrijs Olehnovics, Liene Leikuma, Janis Kudins

SOCIETY'S INVOLVEMENT IN SOCIAL ENTREPRENEURSHIP TO PROMOTE SMART REGIONAL GROWTH 


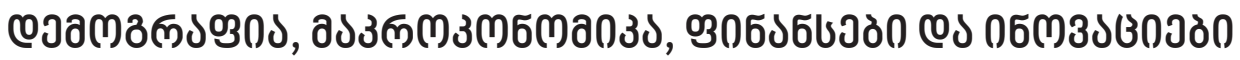

\section{DEMOGRAPHY, MACROECONOMICS, FINANCES AND INNOVATIONS}

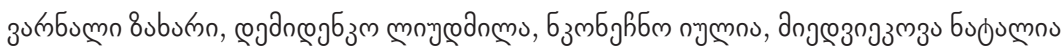

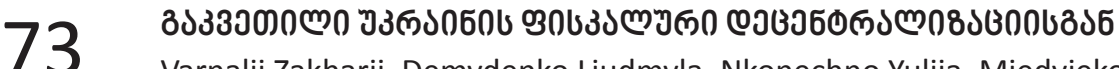

Varnalii Zakharii, Demydenko Liudmyla, Nkonechno Yuliia, Miedviekova Nataliia

LESSONS FROM FISCAL DECENTRALIZATION IN UKRAINE

nmbgo sinfzodo

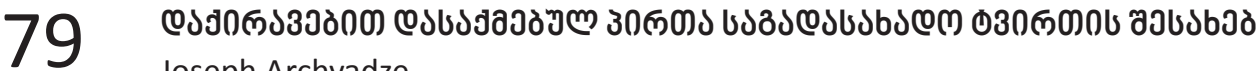

Joseph Archvadze

TAX BURDEN OF EMPLOYED PERSONS

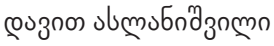

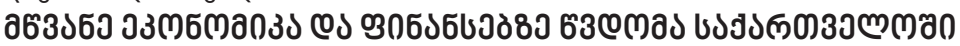

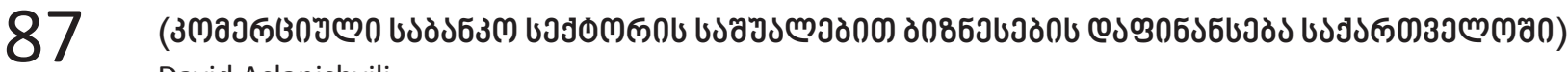

David Aslanishvili

GREEN ECONOMY AND ACCESS TO FINANCE IN GEORGIA

(GOING BEYOND THE COMMERCIAL BANKING SECTOR TO FINANCE BUSINESSES IN GEORGIA)

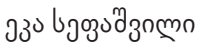

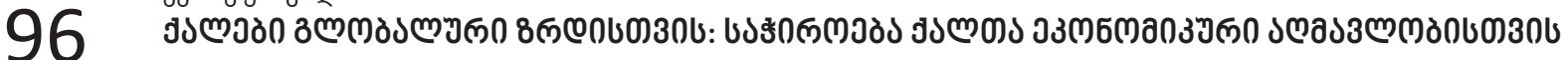

Eka Sepashvili

WOMEN FOR GLOBAL GROWTH: NEED FOR WOMEN ECONOMIC EMPOWERMENT

oknozmo costgmos

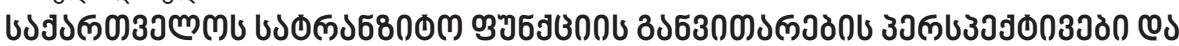

104

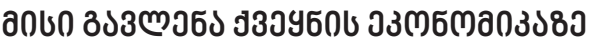

Irakli Danelia

PROSPECTS OF DEVELOPMENT OF GEORGIA'S TRANSIT FUNCTION AND

ITS IMPACT ON THE COUNTRY'S ECONOMY

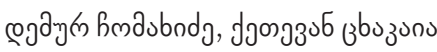

117

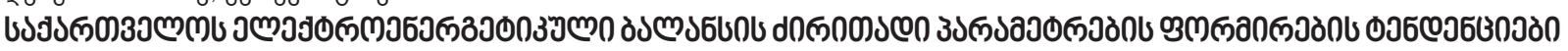

Demur Chomakhidze, Ketevan Tskhakaia

THE TRENDS OF DRAWING UP THE ELECTRICITY BALANCE OF GEORGIA

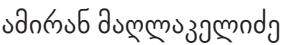

123

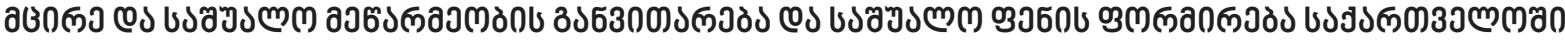

Amirani Maglakelidze

DEVELOPMENT OF SMALL AND MEDIUM ENTREPRENEURSHIP AND

FORMATION OF A MIDDLE CLASS IN GEORGIA

ปgoง 335 oुnogno

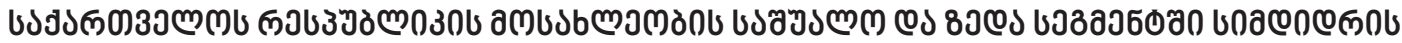

130

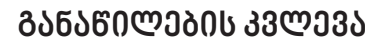

Qetevan Pipia

STUDY OF THE DISTRIBUTION OF WEALTH IN THE MIDDLE AND

TOP SEGMENTS OF THE POPULATION OF GEORGIA REPUBLIC 


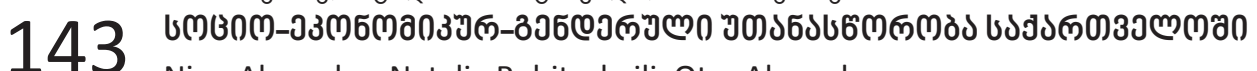

Nino Abesadze, Natalia Robitashvili, Otar Abesadze SOCIO-ECONOMIC-GENDER DISBALANCES IN GEORGIA

$\checkmark 50$ frommndg

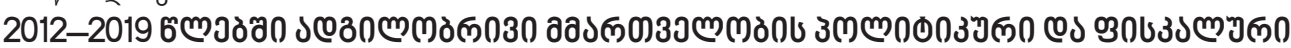

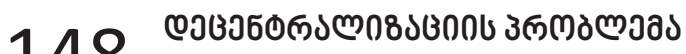

Ana Tsitlidze

THE PROBLEM OF THE POLITICAL AND FISCAL DECENTRALIZATION OF

THE LOCAL GOVERNMENT IN 2012-2019

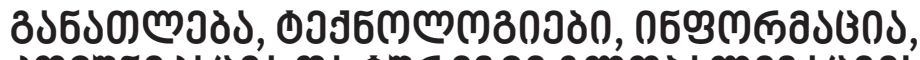

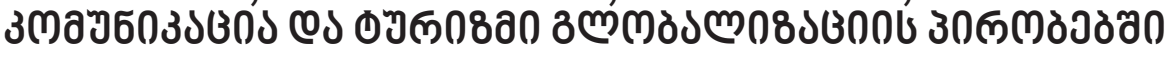

\section{EDUCATION, TECHNOLOGIES, INFORMATION, COMMUNICATION AND TOURISM IN TERMS OF GLOBALIZATION}

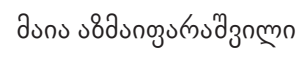

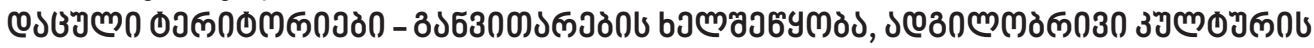

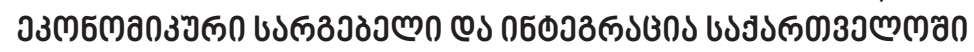

Maia Azmaiparashvili

PROTECTED AREAS - DEVELOPMENT SUSTAINABILITY, ECONOMIC BENEFIT AND

INTEGRATION OF LOCAL CULTURE IN GEORGIA

n.

163

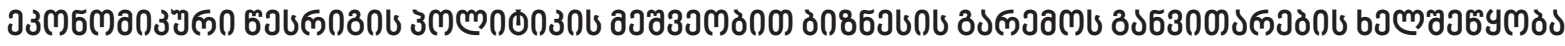

Irakli Aslanishvili

SUPPORTING THE DEVELOPMENT OF BUSINESS ENVIRONMENT THROUGH ECONOMIC ORDER POLICY

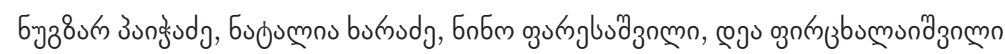

168

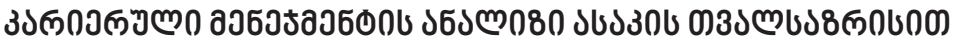

Nugzar Paichadze, Natalia Kharadze, Nino Paresashvili, Dea Pirtskhalaishvili

CAREER MANAGEMENT ANALYSIS IN TERMS OF AGE

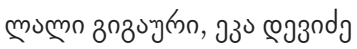

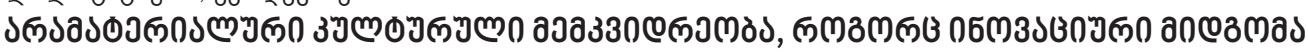

176

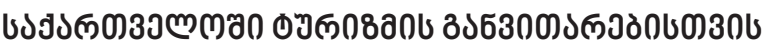

Lali Gigauri, Eka Devidze

INTANGIBLE CULTURAL HERITAGE AS AN INNOVATIVE APPROACH FOR TOURISM

DEVELOPMENT IN GEORGIA

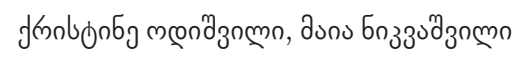

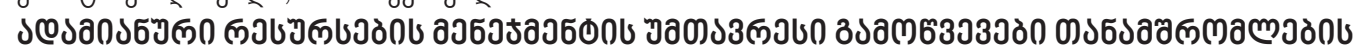

182

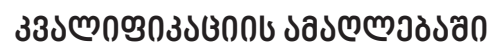

Kristine Odishvili, Maia Nikvashvili

MAIN CHALLENGES OF HUMAN RESOURCES MANAGEMENT IN EMPLOYEE CAREER DEVELOPMENT

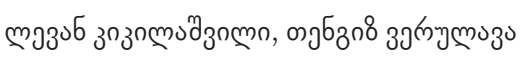

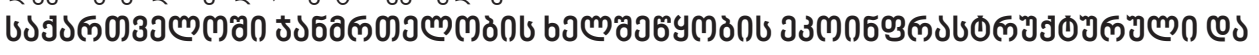

187

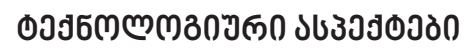

Levan Kikilashvili, Tengiz Verulava

HEALTH PROMOTION IN GEORGIA ECO INFRASTRUCTURE DEVELOPMENT AND TECHNOLOGICAL ASPECTS 


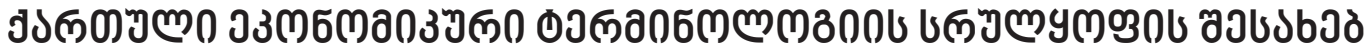
ON THE IMPROVEMENT OF GEORGIAN ECONOMIC TERMINOLOGY

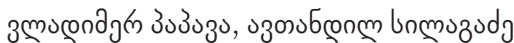

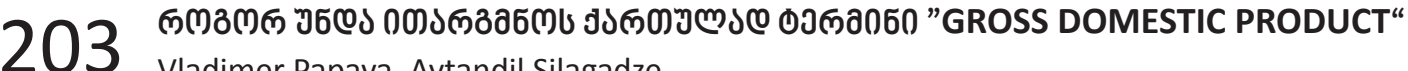

Vladimer Papava, Avtandil Silagadze

“HOW THE TERM THE “GROSS DOMESTIC PRODUCT" SHOULD BE TRANSLATED INTO GEORGIAN."

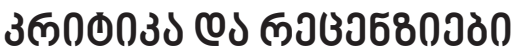

\section{CRITICISM AND REVIEWS}

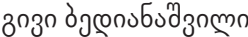

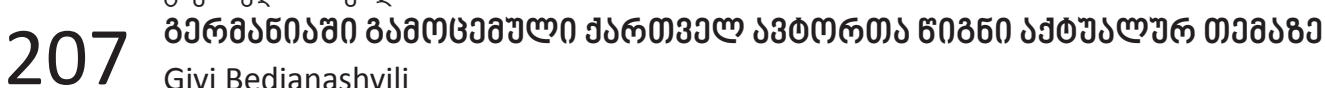

Givi Bedianashvili

BOOK ABOUT THE KEY CHALLENGING ISSUES BY THE GEORGIAN AUTHORS THAT IS PUBLISHED IN GERMANY 


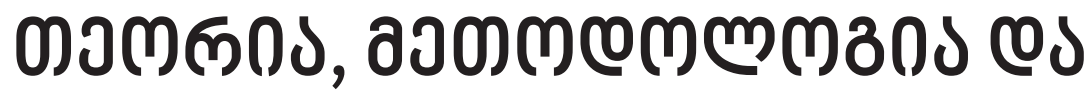

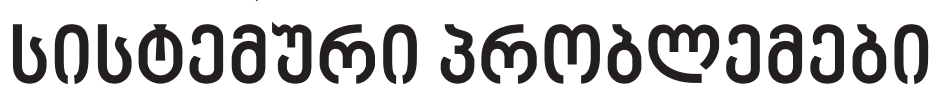

THEORY, METHODOLOGY AND SYSTEMIC PROBLEMS 



\title{
THE ROLE OF INNOVATIONS IN DRIVING ECONOMIC GROWTH: CASE OF ADVANCED ECONOMIES
}

\section{OKSANA ZHYLINSKA}

\author{
D. Sc., Professor, \\ Taras Shevchenko National University of Kyiv, Ukraine \\ zhylinska@ukr.net
}

\section{OLEKSANDR CHERNYAK}

\author{
D. Sc., Professor, \\ Taras Shevchenko National University of Kyiv, Ukraine \\ chernyak@univ.kiev.ua
}

\section{OLENA BAZHENOVA}

\section{Sc., Associate Professor,}

Taras Shevchenko National University of Kyiv, Ukraine olenabazhenova@univ.net.ua

KEYWORDS: ECONOMIC GROWTH, INNOVATION, ECONOMETRIC MODELLING, PANEL DATA MODELS.

For citation: Zhylinska O., Chernyak O., Bazhenova O. (2019), The Role Of Innovations In Driving Economic Growth: Case Of Advanced Economies, Globalization And Business, №7, pp. 11-15. https://doi.org/10.35945/gb.2019.07.001

\section{INTRODUCTION}

Issues of economic growth have been paid attention even in ancient times. For instance, Mesopotamian brick plates contained inscriptions devoted to the calculation of the barley surplus product as the difference between the total output (gross collection of barley) per year in the condition of "normal harvest" and expenditures related to the provision of seed fund, consumption of labor and other costs measured in barley units, It has been considered as a primitive system of social accounting (Kurz, Salvadori).

During last two millennia the world's GDP has increased 497 times, GDP per capita - 17 times, which corresponded to 29 times population growth according to Angus Maddison (Maddison, 2001). Thus, income gap between the poorest and richest regions of the world, which at the beginning of the second millennium was 1.1: 1 , at the beginning of the third millennium comprised 18: 1 (Maddison, 2005).
Throughout the history of world economic development, the periods of rapid economic growth have been replaced by periods of recession. Thus, the countries that demonstrated high growth rates over the long term, in the future had worsened welfare due to wars, natural disasters etc.

Although it should be noted that in developed countries, the growth rate is characterized by greater stability over the long term than in the poorer ones, which are characterized by sudden unexpected changes in economic growth, mainly due to political instability or military action (Lucas, 1988).

Due to up-to-date economic growth models, it is mainly determined by scientific and technological factors.

Thus, this paper is devoted to the research of innovations influence on economic growth in advanced economies. The level of innovations is likely to be determined by quantity of researchers in R\&D (per million people) and research and development expenditure (\% of GDP). 


\section{ANALYSIS OF RECENT RESEARCH AND PUBLICATIONS}

Nowadays economic growth is considered to be determined mainly by the scientific and technological factors, among which there are innovations, accumulation of physical and human capital, external effects etc.

For instance, in Grossman and Helpman's model (Grossman, Helpman, 1991, 1994) the rate of economic growth is determined by the rate of innovation and the index of specialization in intermediate consumption goods sector (Guarini, 2009).

Agion and Howitt included uncertainty into the endogenous growth model (Aghion, Howitt, 1992). The main factor of economic growth in model is considered to be technological progress, which is stimulus for firms engaged in research activities that produce innovations to compete. The model includes vertical innovation when new inventions result in the aging of existing ones. For example, innovations represents inventions of intermediate goods and inventions that promote the productivity of intermediate goods in the production of final goods.

Model of Lukas focuses on the relationship between the rate of growth of the economy and the productivity of training its individuals (Lucas, 1988). On the one hand, higher productivity of teaching contributes to increasing the marginal product of labor and wages and, accordingly, increasing incentives to study. On the other hand, the less individuals will prefer current consumption in comparison with the future, the more time they will be ready to devote to training, which will lead to acceleration of economic growth.

\section{RESEARCH METHOD}

For testing the innovations influence on economic growth in advanced economies we constructed panel vector model of autoregression.

In the reduced form the developed panel vector model of autoregression has the following form:

$$
Y_{i t}=\Gamma_{0}+\sum_{j=1}^{k} \Gamma_{j} Y_{i t-j}+\varepsilon_{i t},
$$

where $Y_{i t}$ - vector of endogenous variables,

$\Gamma_{0}$ - vector of intercepts, $\Gamma_{j}$ - matrix of

coefficients $(j=\overline{1, k}), \varepsilon_{i t}$ - disturbances.

Given the shock to system, variables of model deviate from the equilibrium state and should return to it (Chernyak, 2014). The trajectory of returning to equilibrium state is an impulse response.

The analysis of the decomposition of the prediction errors variances of modelled variables allows determining the sources of their volatility.

\section{RESULTS}

In the paper we have examined the hypothesis of impact of research and development factors on economic growth of advanced economies.

For this purpose it has been taken such indicators as GDP per capita, PPP (constant 2011 international \$), researchers in R\&D (per million people) for advanced countries in period 1996-2017. This group of economies due to International Monetary Fund includes such countries as Australia, Austria, Belgium, Canada, Cyprus, Czech Republic, Denmark, Estonia, Finland, France, Germany, Greece, Hong Kong SAR, Iceland, Ireland, Israel, Italy, Japan, Korea, Latvia, Lithuania, Luxembourg, Macao SAR, Malta, Netherlands, New Zealand, Norway, Portugal, Puerto Rico, Singapore, Slovak Republic, Slovenia, Spain, Sweden, United States and United Kingdom.

The analyzed indicators are presented in Table 1.

Table 1.

Main characteristics of GDP per capita, PPP (constant 2011 international \$) and researchers in R\&D (per million people) for advanced countries

\begin{tabular}{|l|c|c|c|c|}
\hline & \multicolumn{2}{|c|}{$\begin{array}{c}|c| \\
\end{array}$} & $\begin{array}{c}\text { GDP per capita, PPP } \\
\text { (constant 2011 inter- } \\
\text { national \$) }\end{array}$ & $\begin{array}{c}\text { Researchers in R\&D } \\
\text { (per million people) }\end{array}$ \\
\cline { 2 - 5 } & Mean & St. Dev. & Mean & St. Dev. \\
\hline Australia & 39148,56 & 4165.669 & 3889.61 & 471.22 \\
\hline Austria & 39532.86 & 3337.00 & 4049.44 & 828.86 \\
\hline Belgium & 37587.70 & 2788.83 & 3444.99 & 682.01 \\
\hline Canada & 37822.47 & 3287.65 & 4050.43 & 608.60 \\
\hline Cyprus & 29988.62 & 2673.71 & 842.97 & 262.67 \\
\hline Czech Republic & 24303.53 & 4130.70 & 2293.32 & 867.88 \\
\hline Denmark & 41771.84 & 2376.00 & 5648.53 & 1516.54 \\
\hline Estonia & 21377.14 & 5428.78 & 2736.36 & 545.32 \\
\hline Finland & 35317.69 & 3821.42 & 7377.34 & 383.78 \\
\hline France & 34781.53 & 2035.02 & 3416.62 & 565.22 \\
\hline Germany & 38007.48 & 3475.26 & 3663.02 & 3475.26 \\
\hline Greece & 25258.18 & 3177.62 & 1972.14 & 638.37 \\
\hline $\begin{array}{l}\text { Hong Kong SAR, } \\
\text { China }\end{array}$ & 40198.96 & 8521.25 & 2420.11 & 792.12 \\
\hline Iceland & 38446.10 & 4857.55 & 6572.40 & 987.83 \\
\hline Ireland & 40295.16 & 9569.33 & 3019.13 & 1042.42 \\
\hline Israel & 26958.92 & 2790.17 & 7770.74 & 678.44 \\
\hline Italy & 35102.25 & 1536.67 & 1527.42 & 345.74 \\
\hline Japan & 23993.42 & 5941.84 & 4280.84 & 1793.77 \\
\hline Korea, Rep. & & & & \\
\hline
\end{tabular}

Source: World Development Indicators and authors' calculations 


\begin{tabular}{|l|c|c|c|c|}
\hline Latvia & 16819.27 & 5218.50 & 1582.86 & 301.47 \\
\hline Lithuania & 18903.10 & 6191.45 & 2471.76 & 332.23 \\
\hline Luxembourg & 81523.17 & 9020.67 & 4610.90 & 386.75 \\
\hline Macao SAR, China & 70280.47 & 31926.85 & 733.53 & 584.25 \\
\hline Malta & 25350.42 & 4238.74 & 1425.42 & 445.96 \\
\hline Netherlands & 41408.54 & 3371.27 & 3271.16 & 816.96 \\
\hline New Zealand & 29472.67 & 3233.41 & 3223.25 & 668.29 \\
\hline Norway & 57851.16 & 3595.61 & 5085.82 & 650.26 \\
\hline Portugal & 25062.92 & 1313.04 & 2666.92 & 1082.30 \\
\hline Puerto Rico & 31248.74 & 2770.57 & 290.45 & 42.71 \\
\hline Singapore & 58926.28 & 13354.97 & 5005.43 & 1389.40 \\
\hline Slovak Republic & 20307.19 & 5354.11 & 2228.88 & 419.07 \\
\hline Slovenia & 26019.72 & 3675.19 & 3026.83 & 883.25 \\
\hline Spain & 29848.00 & 2437.98 & 2306.93 & 538.02 \\
\hline Sweden & 38383.43 & 4538.05 & 5615.74 & 864.86 \\
\hline Switzerland & 51991.71 & 3631.58 & 3871.80 & 821.60 \\
\hline United States & 46251.26 & 3703.33 & 3776.60 & 327.01 \\
\hline United Kingdom & 33980.52 & 2925.96 & 3667.30 & 647.58 \\
\hline & & & & \\
\hline
\end{tabular}

The number of lags in panel VAR model has been chosen based on values of information criteria such as Akaike, Schwarz, Hannan-Quinn information criteria (chosen number of lags is 3).

Commenting the results of estimation, it should be noted that increasing in number of researches in R\&D sector causes the rising gross domestic product per capita beginning in the first period (figure 1).

Fig. 1. Impulse response function for gross domestic product per capita

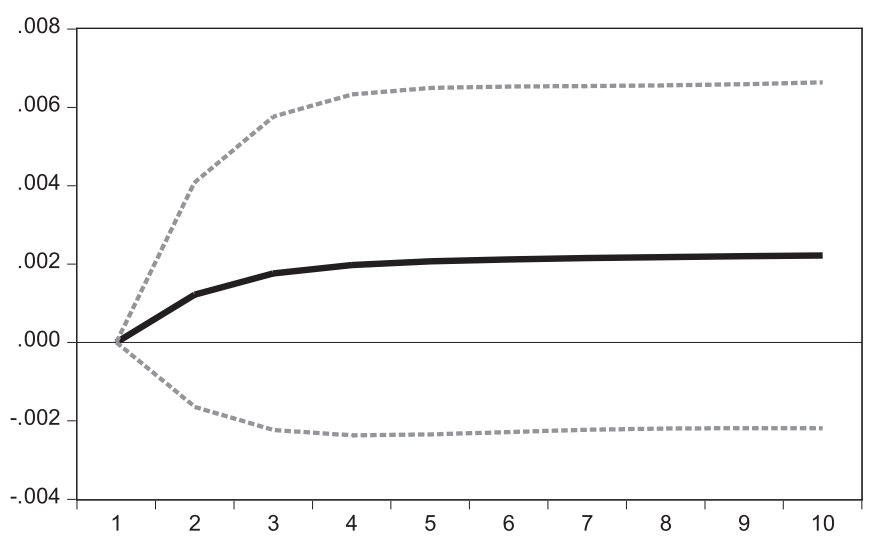

Source: authors' calculations

According to World Development Indicators data and authors' calculations, the mean of researchers in R\&D (per million people) is more than 5000 in such countries as Denmark, Finland, Iceland, Israel, Japan, Norway, Singapore and Sweden. Moreover, for example, in Finland this indicator is maximal (7377.34). Although the number of researches in Finland now is declining. Puerto Rico, Macao, Cyprus have minimal values of mean of researchers in R\&D despite high values of their GDP per capita. For instance, Puerto Rico has the lowest level of this indicator (290.45) with not so small mean of GDP per capita (31248.74).

In Denmark, Germany, Ireland, Korea, Portugal and Singapore the number of researches engaged in R\&D sector is more volatile (more than 1000). For instance, in Denmark this indicator has increased by more than $236 \%$, in Germany - by $73 \%$.

The variables have been tested for a unit root using tests for panel data models such as Lewin, Lina, and Chu and Breitung criteria for common process of a unit root and Ima, Pesaran, and Tina criteria, criteria based on the use of ADF and PP statistics that include individual processes of unit root.

Testing for the presence of a unit root has indicated that almost all variables are first order integrated. Thus, in the panel VAR model the first differences of variables have been considered (there is no cointegration between modelled variables).

Moreover, obtained results have shown the significant impact of researchers in R\&D sector on GDP per capita in advanced economies.

\section{CONCLUSIONS}

Summing up the results of the research, we should note that innovations production really has significant impact on GDP per capita in advanced economies. Moreover, innovations really determines the economic growth in advanced economies. Besides that, investments in human capital create a reliable basis for economic development in the future. Ignoring these facts may lead to the loss of economy's potential in the aspect of its economic development.

Constructed panel data model for estimating the effect of number of researchers in R\&D sector on GDP per capita in advanced economies has shown that increasing in number of researches in R\&D sector causes the rising gross domestic product per capita beginning in the first period.

Future direction of research is considered to be the examining of analyzed relationship for developing countries, comparing the results with advanced ones and paving the way for converging of their economies. 


\section{REFERENCES:}

1. Aghion, P., Howitt, P. A (1992). Model of Growth through Creative Destruction. Econometrica. №60. P. $323-351$.

2. Chernyak, O., Stavytskyy, A., Bazhenova, O., Shebanina, (2014). O. Econometrics. Mykolayiv: MNAU, (In Ukrainian).

3. Grossman, G.M. Helpman, E. (1994). Endogenous Innovations in the Theory of Growth. The Journal of Economic Perspectives. Vol.8, No.1. pp. 23-44.

4. Grossman, G.M. Helpman, E. (1991). Innovation and Growth in the Global Economy. Cambridge, MA: MIT Press.

5. Grossman, G.M. Helpman, E., Oberfield, E., Sampson, T. (2017). Balanced Growth Despite Uzawa. https://www.princeton.edu/ grossman/Uzawa.pdf.

6. Guarini, G. (2009). Innovation and growth in the Grossman-Helpman's model with increasing returns: a note. Munich Personal RePEc Archive. URL: http://mpra.ub.uni-muenchen.de/19612

7. International Monetary Fund. World Economic Outlook Database. April (2019). URL: https://www.imf.org/external/pubs/ $\mathrm{ft} /$ weo/2019/01/weodata/index.aspx

8. Kurz, H.D., Salvadori, N. (2003). Theories of Economic Growth - Old and New URL: https://www.researchgate.net/publication/252313772_theories_of_economic_growth_-_old_and_new

9. Lucas, R. (1988). On the Mechanics of Economic Development. Journal of Monetary Economics. № 22. P. 3-42.

10. Maddison, A. (2001). The World Economy: a Millennial Perspective. Organization for Economic Cooperation and Development. 383 p.

11. Maddison, A. (2005). Growth and Interaction in the World Economy. URL: http://www.ggdc.net/maddison/other_books/ growth_and_interaction_in_the_world_economy.pdf

12. World Development Indicators. The World Bank. http://data-bank.worldbank.org/data/reports.aspx?source=wdi-database-archives-\%28beta\%29\# 


\section{THE ROLE OF INNOVATIONS IN DRIVING ECONOMIC GROWTH: CASE OF ADVANCED ECONOMIES}

\section{OKSANA ZHYLINSKA}

D. Sc., Professor,

Taras Shevchenko National University of Kyiv, Ukraine

zhylinska@ukr.net

\section{OLEKSANDR CHERNYAK}

\section{Sc., Professor,}

Taras Shevchenko National University of Kyiv, Ukraine chernyak@univ.kiev.ua

\section{OLENA BAZHENOVA}

\section{Sc., Associate Professor,}

Taras Shevchenko National University of Kyiv, Ukraine olenabazhenova@univ.net.ua

KEYWORDS: ECONOMIC GROWTH, INNOVATION, ECONOMETRIC MODELLING, PANEL DATA MODELS.

For citation: Zhylinska O., Chernyak O., Bazhenova O. (2019), The Role Of Innovations In Driving Economic Growth: Case Of Advanced Economies, Globalization And Business, №7, pp 11-15. https://doi.org/10.35945/gb.2019.07.001

\section{SUMMARY}

The paper explores the impact of innovations on the cross-country income differences among advanced economies based on the economic and mathematical models construction. As indicator that defines level of innovations production in the country, we have used number of researchers in R\&D sector (per million people). Therefore, it has been con- structed panel data model to estimate the effect of number of researchers in R\&D sector on GDP per capita in advanced economies. Results of estimation have shown the significant impact of researchers in R\&D sector on GDP per capita for the group of advanced economies testifying that innovation really matters in driving economic growth. 


\section{PREPARING SCHOOL GRADUATES TO INTEGRATE INTO THE WORLD OF TECHNOLOGICAL INNOVATION, SOCIAL AND ECONOMIC CHANGES, AND GLOBALIZATION: THE ROLE OF TEACHING TECHNOLOGY AND ENGINEERING}

\section{MOSHE BARAK}

D. Sc., Professor

Ben-Gurion University of the Negev, Israel

mbarak@bgu.ac.il

KEYWORDS: COMPUTATIONAL THINKING, PROJECT-BASED LEARNING, TECHNOLOGY AND ENGINEERING EDUCATION.

For citation: Barak M. (2019), Preparing School Graduates To Integrate Into The World Of Technological Innovation, Social And Economic Changes, And Globalization: The Role Of Teaching Technology And Engineering, Globalization And Business, №7, pp. 16-23. https://doi.org/10.35945/gb.2019.07.002

\section{INTRODUCTION}

Technological innovation, social and economic changes, and accelerating globalization are constraining the education system to prepare school graduates to integrate successfully into the rapidly changing world. The OECD (2018) report entitled The Future of Education and Skills 2030 states that the future is uncertain and we cannot predict it; but we need to be open and ready for it. Children entering the education system today will be young adults by 2030. Schools have to prepare them for jobs that have not yet been created, for technologies that have not yet been invented, and to solve problems that have not yet been anticipated. To navigate through such uncertainty, students will need to develop curiosity, imagination, resilience and self-regulation. They will need to respect and appreciate the ideas, perspectives and values of others, cope with failure and rejection, and move forward in the face of adversity.

Heinrich, Bhattacharya, and Rayudu, (2007) emphasize that rapid technological change, increasing globalization and a changing world of employment with multiple roles during oness professional life are necessitating a change from knowledge to learning societies. Full participation requires lifelong learning skills, meaning the ability to solve problems, work both independently and in a team, communicate effectively in different formats, and selfdirect oness learning and professional development needs.
What tools do schools have in order to deal with this challenge? In the past, technology education had often been associated with crafts or vocational education for low-achieving students. However, in recent years, leading countries at the forefront of science and technology around the world have been making efforts to include the teaching of innovative subjects in technology and engineering as an integral part of the curriculum for all students in K-12 education. Along with teaching basic subjects such as the humanities, languages, mathematics and science, schools are expected to expose all students to new technologies, impart knowledge and skills to them to use these technologies effectively, and foster their motivation to integrate into the world of research and innovation in technology and engineering.

The present paper aims at highlighting some directions for technology and engineering education in K-12 schooling intended to meet the challenges mentioned above based partially on the experience obtained in schools in Israel and in other countries.

\section{INTERDISCIPLINARY VIEW OF TECHNOLOGY AND ENGINEERING EDUCATION}

Educators today are increasingly aware of the importance of imparting an interdisciplinary view of 
technology and engineering to students, as illustrated in Figure 1.

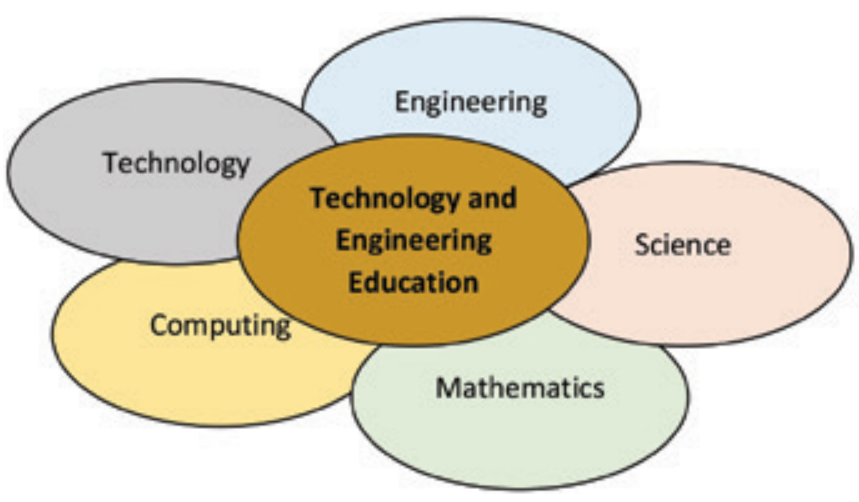

Figure 1: Interdisciplinary view of technology and engineering.

The five aspects illustrated in Figure 1 are not discrete but overlap one another to a certain extent. For example, technology is often described as the field of knowledge and experience through which people change the environment to meet their needs and desires. Engineering is the division of technology that uses science and mathematics for design and problem solving. Computing is a central aspect of developing new technological artefacts and services. These five components are often 'hidden' in projects that the students do, and it is necessary to guide the learners to address these aspects directly in their project design, construction, testing and documentation, as demonstrated in the following examples.

Example 1: addressing aspects of mathematics, physics and computational thinking in robotics

Robotics is one of the most common learning environments in technology and engineering. Barak and Assal (2018) present a case in which students explore the velocity of a robot by marking the distance the robot moves on the floor every five seconds. They use a spreadsheet to draw a graph of the distance $X(\mathrm{Cm})$ depending on the Time $(\mathrm{sec})$ and calculate the robot's average velocity $v(\mathrm{~m} / \mathrm{s})$, as illustrated in Figure 2.

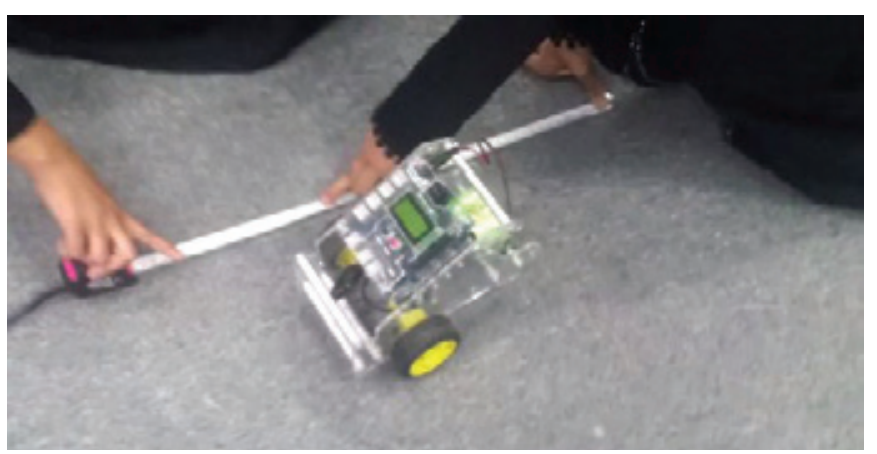

Figure 2: Robotics, STEM and computational thinking: students explore a robot's velocity by measuring the distance $X(\mathrm{Cm})$ the robot moves on the floor over Time (sec).

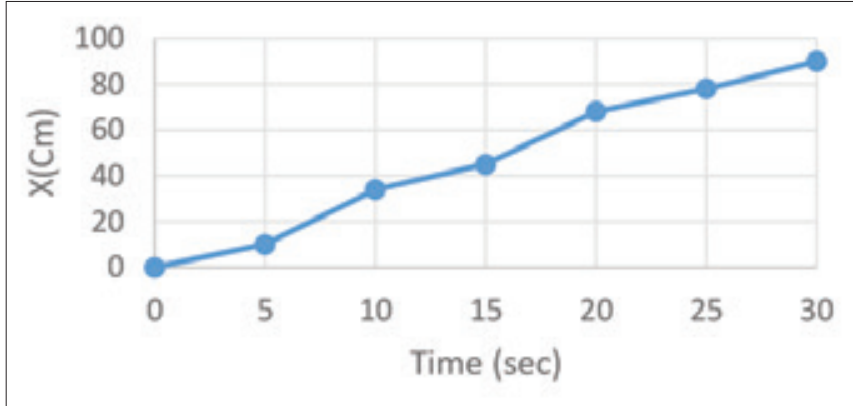

In the example described in Figure 2, the students deal with concepts of technology, physics, mathematics and computational thinking, including programming the robot and using the computer for data presentation and analysis. In the study from which this example was taken (Barak \& Assal, 2018), the students liked this activity because it related to what they had learned in other subjects in school.

Technological aspects of projects in robotics include the concepts of feedback and control, sensing, amplification and optimization. According to Yadav, Hong and Stephenson (2016), computational thinking (CT) refers to exposing students to computing ideas and principles within the context of the subject areas they are already learning, for example, mathematics, science, technology or the humanities. Wing (2006) defined computational thinking as "solving problems, designing systems, and understanding human behavior by drawing on concepts fundamental to computer sciences." It is important to note that the concept of CT is not just restricted to the teaching of computer science or programming, but also to the use of principles or tools from the computer world for investigation, planning or problem solving. For example, in a project on temperature control in a greenhouse, students can use a temperature sensor connected to a computer to store information on temperature changes in the greenhouse over 24 hours and analyze these data to design an adequate airconditioning system. Let us see two additional examples of teaching engineering and technology focused on fostering computational thinking

\section{Example 2: teaching computer-controlled systems}

Hacker (2017) asserts that a sound pedagogical approach for fostering computational thinking would guide students in revisiting computer science (CS) and computational thinking (CT) concepts in both physical world contexts, for example, robotics and computer control, and virtual world contexts, for example, game design. In the past decade, we have witnessed the rapid development of advanced, easy-to-use inexpensive microcontrollers such as the Arduino, together with sensors and devices for measuring and controlling physical variables such as temperature, humidity, light and sound. This has made it possible for teachers and students to design sophisticated control systems with only basic electronics or programming knowledge. For example, Barak (2018) presented the case 
of two Israeli high school students who developed a system to prevent forgetting a baby in a car. The system uses an ultrasonic sensor and Arduino microcontroller to identify whether there is a baby in the baby's car seat. In case of emergency, the microcontroller: (1) opens the car windows automatically; (2) sounds an alarm; and (3) sends a signal to the car owner via a GSM cellular card.

This example demonstrates remarkable options of experiencing problem solving through project work in a computerized environment, from choosing the conceptual solution to the problem, to troubleshooting specific problems in the system's components or software. Students working on non-computerized electronics projects in contrast are more likely to progress along a linear path: planning, constructing, troubleshooting and improving.

\section{Example 3: learning with Lego Mindstorms robots}

A useful platform for fostering computational thinking in problem solving is robotics. Among the most popular educational robots is the Lego EV3 robot, which comes with a rich set of sensors, including a touch sensor, a light color sensor, an ultrasonic sensor and a gyro, as illustrated in Figure 3.

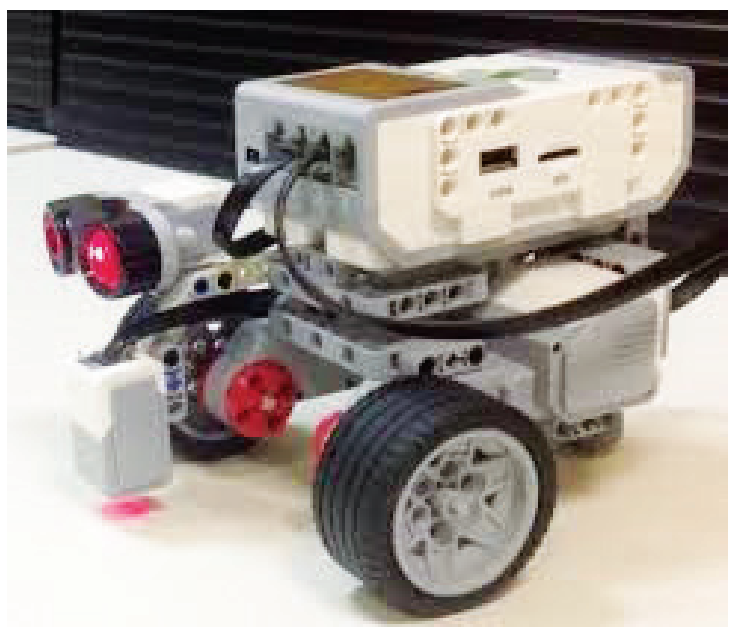

Figure 3: Lego Mindstorms EV3 robot, including a light color sensor and an ultrasonic sensor.

The programming of the EV3 Logo robot consists of:

- Action blocks that control motor operation;

- Flow blocks, for example, loops and conditions;

- Sensor blocks;

- Data operation blocks.

Figure 4 shows a typical EV3 robot program that includes two motor blocks, a sensor block and a loop. This is one of the most sophisticated and popular educational robotic educational systems, although it is quite expensive for schools.

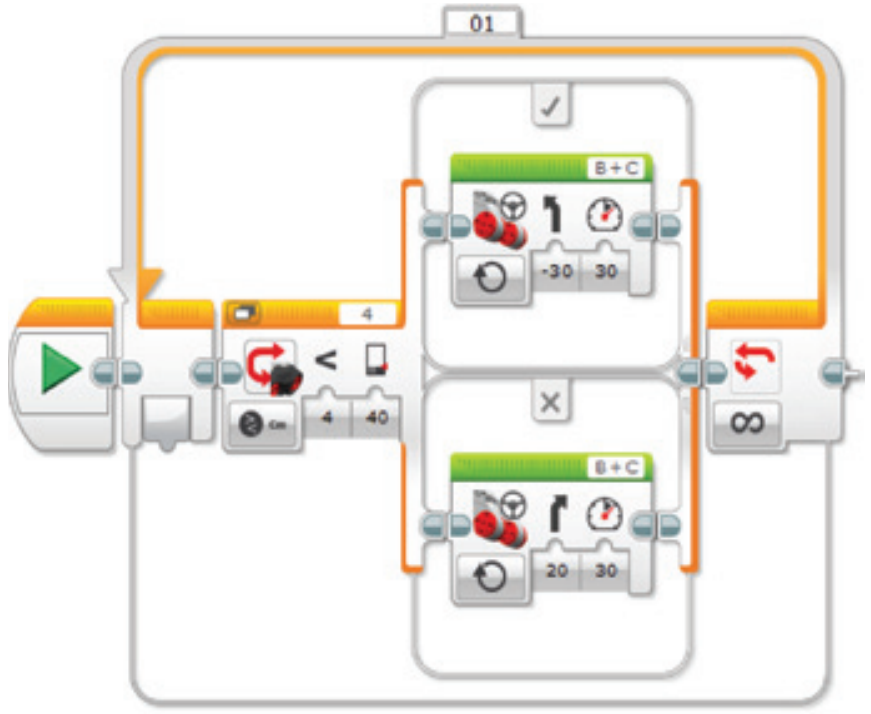

Figure 4: A typical Lego EV3 robot program.

Example 4: learning programming and control concepts with Scratch

A common environment for teaching computational thinking and problem solving is the free Scratch educational programming language, which was developed at MIT and released in 2013. Scratch has been translated into 70+ languages and is used in homes, schools and after-school clubs in almost every country around the world. In its basic version, Scratch is oriented at controlling the movement of a virtual spirit, for example, a cat, on a working surface. There are many programming blocs, such as action, flow, control, sensing and mathematical operations, which are similar to the EV3 programming method. Recently, Scratch has been used increasingly to control objectives in the physical world, for instance, activating motors, reading data from sensors, controlling the Arduino microcontroller or the Lego EV3 robot, as seen in Figure 5.

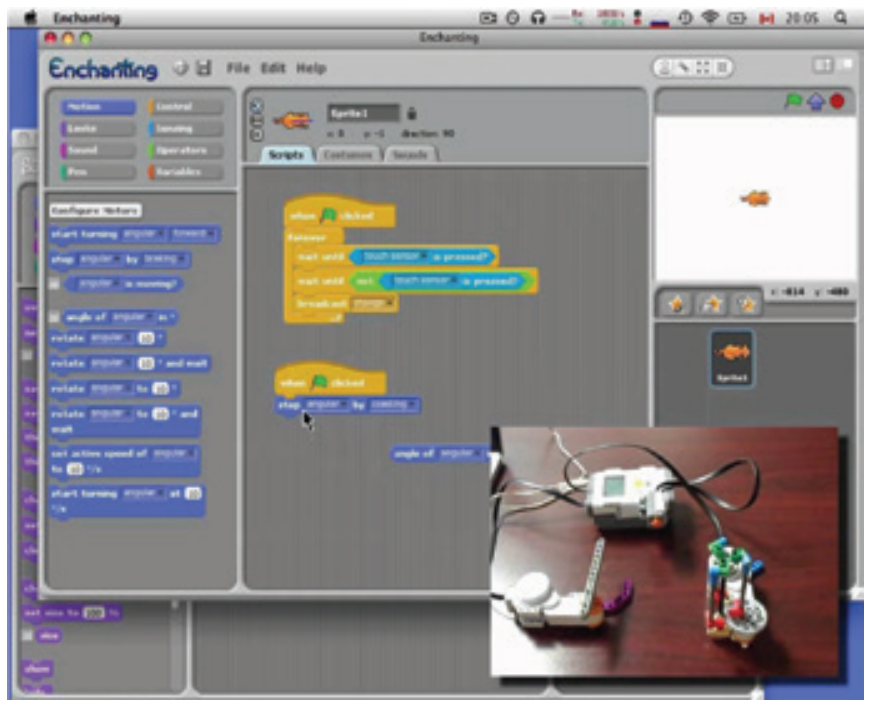

Figure 5: Using Scratch to control a Lego NXT robot. 


\section{Example 5: robotic control by Python}

Molins-Ruano, Gonzalez-Scaristan and Gracia-Saura (2018) point out that although Scratch is a great tool for new young learners, the design and default spirit of Scratch might be considered childish and could discourage high school or college students from using it. These researchers developed a sophisticated robot called Tortoise, shown in Figure 6.

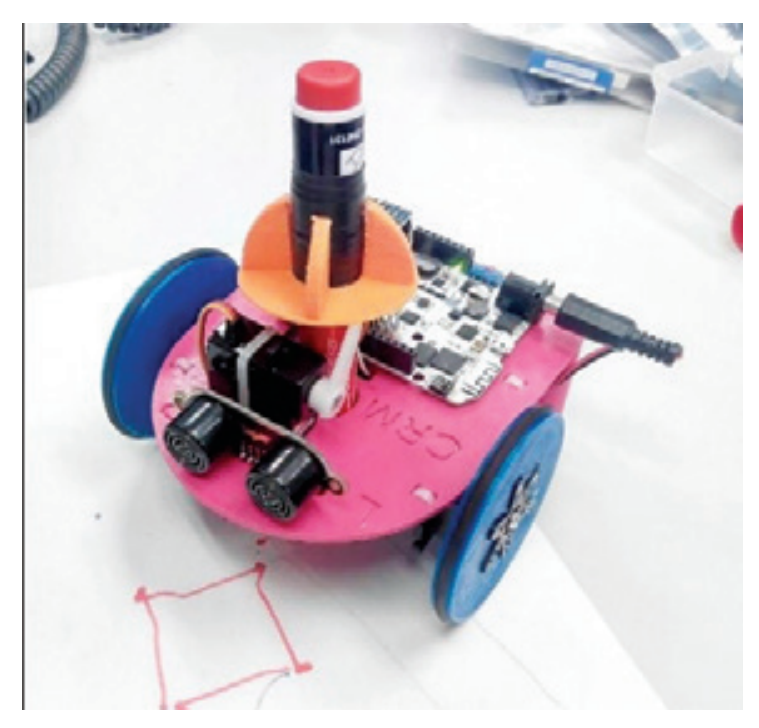

Figure 6: The Tortoise robot.

According to the authors, Tortoise is based on the Arduino microcontroller and simple mechanical components that schools can produce themselves using a 3D printer. The total cost of Tortoise, including a set of sensors, is less than $\$ 100$. The developers call this robotic system PHOGO and show that it can be controlled by the Python programming language, which was ranked the fourth most popular language after Java, $\mathrm{C}$ and $\mathrm{C}++$.

Programming the robot by Python is text-based, and the functions available to the students are similar to the ones in the historical Logo programming environment developed at MIT in the 1970s. For example:

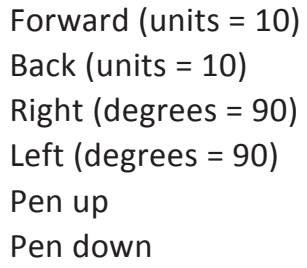

The students can start learning to program the robot by writing a simple code in Python and gradually advance to coping with more sophisticated tasks. While working with Scratch or programming, the LEGO robots can be suitable for students from the age of 10; programming the PHOGO robot using Python may be suitable for older students, for example, those aged 14.

Example 6: teaching concepts of digital image processing

Image processing is one of the most fascinating subjects in the field of computer science and technological applications. Although professional image processing uses advanced mathematics and programming, we can teach basic concepts of image processing to young children (for example, junior high school students), as was observed in a course developed at Ben-Gurion University of the Negev (Barak \& Asad, 2012).

Figure 7 shows that in presenting a black-and-white image, which is actually composed of gray levels, we can use a format of 8-bit digital data that distinguishes between 256 brightening levels from 010 (full black) to 25510 (full white).

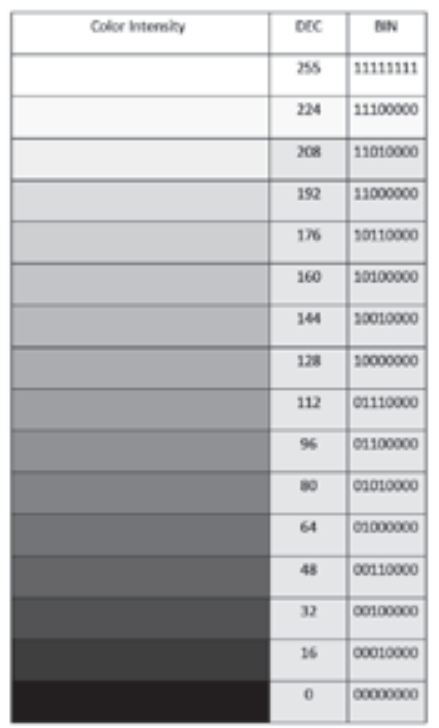

Figure 7: Representation of 256 brightness levels from black (010) to white (25510).

Figure 8 shows an example of 'digitizing' a picture to $12 \times 16$ pixels, assigning a number (brightness level) to each pixel, and finally presenting the entire picture in a matrix of $12 \times 16$ numbers. In other words - the picture is represented by numbers! It is worth mentioning that in real life, for example, on a computer screen or a digital camera, we often use much higher resolution, such as 1440 x 1080 pixels.
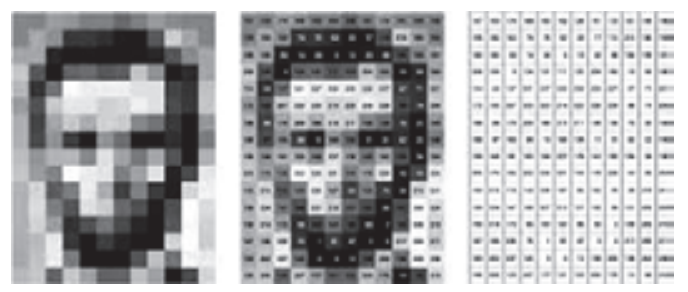

Figure 8: Image representation by $12 \times 16$ pixels at brightness levels 0-255. 
So far we have seen the method of digitizing a blackand-white image. How is a color picture digitized? In a color picture, each pixel gets a different color. From physics, we know that any color could be created by a composition of the three basic colors of red, green and blue (RGB). Figure 9 illustrates an example of creating an orange pixel through the composition of red=255, green $=178$ and blue $=102$. A colored picture of $\mathrm{n} \times \mathrm{m}$ pixels is created by a composition of three matrixes of the same dimension representing the $\mathrm{R}, \mathrm{G}$ and $\mathrm{B}$ colors.
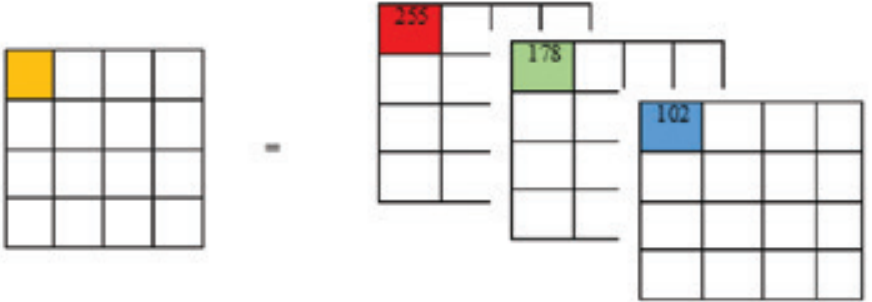

Figure 9: An orange pixel created by the red, green and blue (RGB) colors.

In summary, when we take a picture with our digital camera, we are actually saving a file of numbers, and computers can handle numbers very well. For example, if we want to increase the brightness of an entire image, we will add a number, for example, 20, to the brightness level of each pixel.

\section{FACIAL RECOGNITION}

Facial recognition is one of the most interesting image processing applications (Midrak, 2018). However, how can we use a digital image to identify a person's face? Following is a simple method for facial recognition that was learned successfully by junior high school students (Barak \& Asad, 2012). Figure 10 shows that for facial recognition, we can measure eight parameters from the picture of a face:
be - distance between eyes
bn - distance between nostrils
mw - mouth width
en - distance between eyes and nose
$\mathrm{nm}$ - distance between nose and mouth
mh - mouth width
w - total width
$\mathrm{h}$ - total height

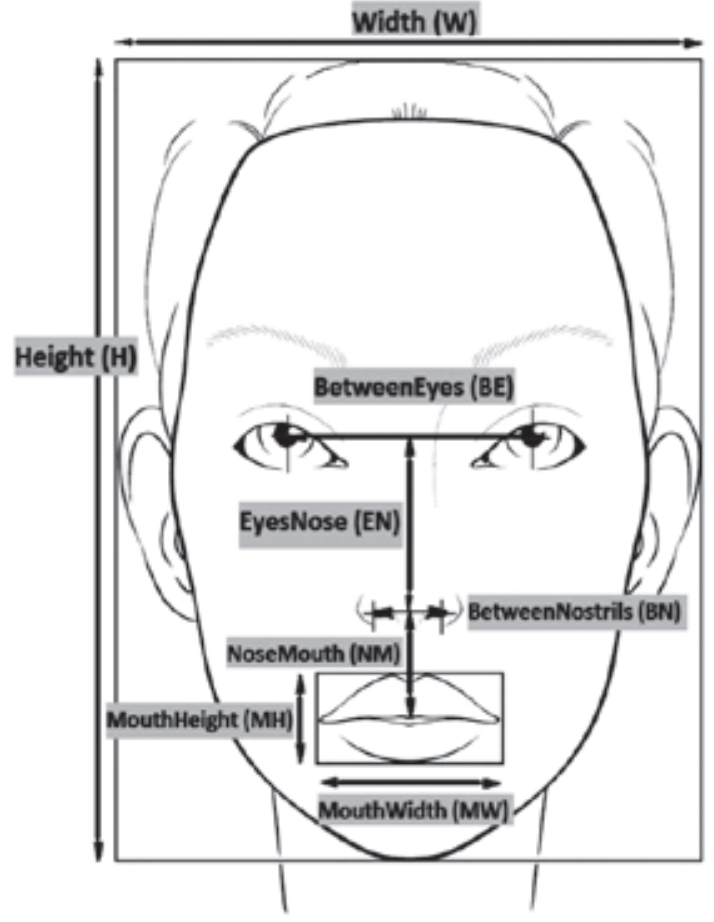

Figure 10: Eight parameters used for facial recognition.

Let us assume that we have values of the eight parameters for two faces:

Face $1=\{\mathrm{be} 1, \mathrm{bn} 1, \mathrm{mw} 1, \mathrm{en} 1, \mathrm{~nm} 1, \mathrm{mh} 1, \mathrm{w} 1, \mathrm{~h} 1\}$

Face $2=\{\mathrm{be} 2, \mathrm{bn} 2, \mathrm{mw} 2, \mathrm{en} 2, \mathrm{~nm} 2, \mathrm{mh} 2, \mathrm{w} 2, \mathrm{~h} 2\}$

Since the faces could be of different sizes, we normalize the six parameters $\mathrm{b} 1$ to $\mathrm{mh}$ by dividing each one by total width $w$ or total height $h$, respectively. We get six normalized parameters $a, b, c, d$, e and f for each picture, as seen in Equations 3 and 4.

$$
\begin{aligned}
& a 1=\frac{b e 1}{w 1} b 1=\frac{b n 1}{w 1} c 1=\frac{m w 1}{w 1} d 1=\frac{n m 1}{h 1} e 1=\frac{m h 1}{h 1} f 1=\frac{e n 1}{h 1} \\
& a 2=\frac{b e 2}{w 2} b 2=\frac{b n 2}{w 2} c 2=\frac{m w 2}{w 2} d 2=\frac{n m 2}{h 2} \text { e2 }=\frac{m h 1}{h 2} f 2=\frac{e n 2}{h 2}
\end{aligned}
$$

In this process, a vector of parameters a-f represents each facial picture.

The last step is calculating the difference $D$ between the vectors of two faces by the formula presented in Equation 5.

$D=\sqrt{(a 1-a 2)^{2}+(b 1-b 2)^{2}+(c 1-c 2)^{2}+(d 1-d 2)^{2}+(e 1-e 2)^{2}+(f 1-f 2)^{2}}$

Equation 5 shows the calculation of the 'Euclidean distance' between two vectors of six dimensions each that can be explained as an extension of the Pythagoras theorem for calculating the diagonal in a straight triangle. Students can perform the process described above using a spreadsheet. 
In class, the students worked in pairs, took photos of themselves, and manually measured the eight parameters be, bn, mw, en, $\mathrm{nm}, \mathrm{mh}, \mathrm{w}$, h of their photos, as illustrated in Figure 11. They entered the data into a simple computer program that created a database of vectors for all students' faces in the class. To identify a student, the program calculates the difference $D$ between the 'unknown' picture and all the pictures stored in the class database. The case of the smallest $D$ identifies the desired image.

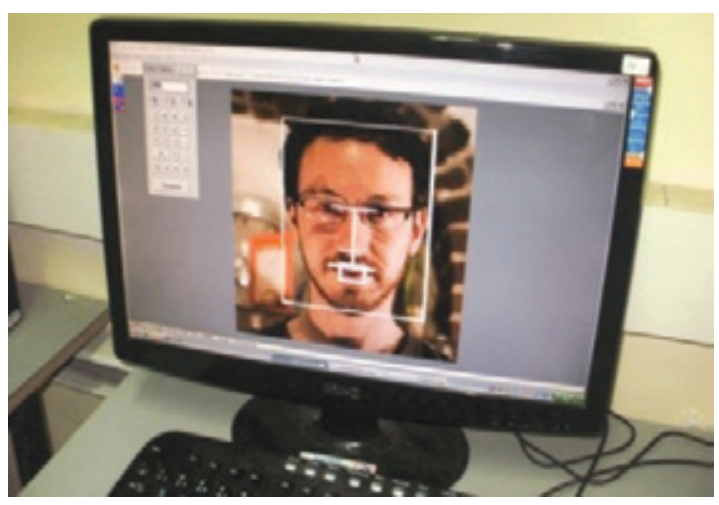

Figure 11: Measuring the eight facial parameters.

In an era when children are growing up with mobile phones, tablets and computers, technological education must be integrated into the digital environment and realize the inherent potential of computerized technologies to develop students> problem-solving and creative thinking abilities.

\section{APPLYING PROJECT-BASED LEARNING IN SCHOOL: THE P3 TASK TAXONOMY}

In technology education, educators often use the term project-based learning, which implies that at the end of the learning process, students build a product or technological system that meets the needs and aspirations of human beings. Project-based learning (PBL) is a constructivist approach that requires a small student group to take responsibility for their learning. This instructional method aims at engaging students in solving reality-based problems, encouraging them to become active, independent and collaborative learners (Mioduser \& Betzer, 2008; Blumenfeld et al., 1991; Savery, 2006). Project-based learning develops students' critical thinking and problem-solving skills, and gives them experience in applying these skills to realworld situations. Project-based learning is one of the tools education has in order to prepare students to integrate into a world in which the vast majority of business organizations are not bureaucratic but rely instead on work teams, shared decision making and a great deal of risk-taking in the effort to compete in the global market (Nordgren, 2002). The digital revolution that has been affecting all aspects of our lives, including education, has pushed forward efforts to introduce project-based learning into traditional schooling because students today have access to tremendous resources and tools on the network for investigating scientific and technological issues, suggesting solutions to problems and designing innovative technological systems.

However, despite the wide consensus in the literature about the advantages of PBL over traditional schooling, educators are increasingly aware of the limitations of applying these methods within the regular school context. Kirschner, Sweller and Clark (2006) write about the failure of constructivist-oriented instructional methods such as discovery, problem-based and inquiry-based learning because the notion of minimal guidance during learning does not work. Minimal guided instruction is less effective and efficient than instructional approaches, which place strong emphasis on guiding the student learning process (Hushman \& Marely, 2015). Some supporters of PBL (Hmelo-Silver, 2004; Hmelo-Silver et al., 2007; Savery, 2006) address the limitations of this method, and mention that it is important to tailor the scope and complexity level of assignments to students' prior knowledge and skills, and provide instruction and support in order to reduce the cognitive load and enable students to learn in a complex domain. Dolman et al. (2005) also writes that PBL curricula should consist more of tutor guidance at the beginning through shared guidance of both the students and the tutor, and move to more student guidance at the end.

To address this issue, we developed the P3 Task Taxonomy (Barak \& Assal, 2018), which distinguishes between three levels of student assignments:

- Practice: exercises and closed-ended tasks in which learners know the final solution in advance and can check if they arrived at the correct answer;

- Problem solving: small-scale, open-ended tasks in which students might use different solution methods and arrive at different answers;

- Projects: challenging open-ended tasks in which the students take part in defining the problem, setting objectives, identifying constraints and choosing the solution method;

\section{CONCLUSIONS}

The OECD (2018) report entitled The Future of Education and Skills 2030, mentioned in the Introduction to the present paper, raises two far-reaching questions countries will have to find answers to in order to prepare students to the rapidly changing world characterized by scientific and technological innovation and accelerating globalization: 
- What knowledge, skills, attitudes and values will today's students need to thrive and shape their world?

- How can instructional systems develop knowledge, skills and attitudes effectively?

Throughout this article, we have seen several examples of teaching technology and engineering that could provide partial answers to these questions. One common feature of the examples we have seen is the interdisciplinary approach in teaching science and technology, which combines the learning of concepts in technology, engineering, science, mathematics and computing. A second common feature of these examples is the transition from the traditional chalk-and-board teaching approach to the constructivistoriented project-based learning approach. The challenge for educators is how to gradually make these changes in the subjects we teach and in the instruction method, adapting to the students previous knowledge and their ability to cope with learning challenging interdisciplinary topics independently.

\section{REFERENCES:}

1. Barak, M. \& Asad, K. (2012). Teaching image processing concepts in junior high school: boys' and girls' achievements and attitudes towards technology. Research in Science and Technological Education, 30(1), 81-105.

2. Barak, M. (2018). Teaching electronics: from building circuits to system thinking and programming. In: M. De Vries (Ed.), Handbook of Technology Education, Cham, Switzerland: Springer, 337-360.

3. Barak, M., \& Assal, M. (2018). Robotics and STEM learning: students' achievements in assignments according to the P3 Task Taxonomy - Practice, Problem solving, and Projects. International Journal of Technology and Design Education, 28(1), 121-144, doi:10.1007/s10798-016-9385-9.

4. Blumenfeld, P. C., Soloway, E., Marx, R. W., Krajcik, J. S., Guzdial, M., \& Palinscar, A. (1991). Motivating project-based learning: sustaining the doing, supporting the learning. Educational Psychologist, 26(3 \& 4), 369-398.

5. Dolmans, D. M., de Grave, W., Wolfhagen, I. P., \& van der Vleuten, C. M. (2005). Current perspectives: problem-based learning: future challenges for educational practice and research. Medical Education, 39(7), 732-741.

6. Hacker, M. (2017). Integrating computational thinking into technology and engineering education. Technology and Engineering Teacher, 77(4), 8-14.

7. Heinrich, E., Bhattacharya, M., \& Rayudu, R. (2007). Preparation for lifelong learning using ePortfolios. European Journal of Engineering Education, 32(6), 653-663. https://doi-org.ezproxy.bgu.ac.il/10.1080/03043790701520602

8. Hmelo-Silver C. E., Duncan, R. G., \& Chinn, C. A. (2007). Scaffolding and achievement in problem-based and inquiry learning: a response to Kirschner, Sweller, and Clark 2006. Educational Psychologist, 42(2), 99-107.

9. Hmelo-Silver, C. E. (2004). Problem-based learning: what and how do students learn? Educational Psychology Review, 16(3), 235-266.

10. Hushman C. J., \& Marley S. C. (2015). Guided instruction improves elementary student learning and self-efficacy in science. Journal of Educational Research, 108(5), 371-381.

11. Kirschner, P. A., Sweller, J., \& Clark, R. E. (2006). Why minimal guidance during instruction does not work: an analysis of the failure of constructivist, discovery, problem-based, experiential, and inquiry-based teaching. Educational Psychologist, 41(2), 75-86.

12. Midrak, R. L. (2018). What is facial recognition? Lifewire, September 3, https://www.lifewire.com/how-does-acomputer-recognize-your-face-4154178

13. Mioduser, D., \& Betzer, N. (2008). The contribution of project-based-learning to high-achievers' acquisition of technological knowledge and skills. International Journal of Technology \& Design Education, 18(1), 59-77. https://doiorg.ezproxy.bgu.ac.il/10.1007/s10798-006-9010-4.

14. Molins-Ruano, P., Gonzalez-Sacristan, C., \& Garcia-Saura, C. (2018). Phogo: A low-cost, free and "maker" revisit to Logo. Computers in Human Behavior, 80, 428-440.

15. Nordgren, R. D. (2002). Globalization and education: what students will need to know and be able to do in the global village. Phi Delta Kappan, 84(4), 318-321.

16. Organization for Economic Co-operation and Development (OECD) (2018). The Future of Education and Skills Education 2030 https://www.oecd.org/education/2030/E2030\%20Position\%20Paper\%20(05.04.2018).pdf

17. Savery J. R. (2006). Overview of problem-based learning: definitions and distinctions. Interdisciplinary Journal of Problem-Based Learning, 1(1):9-20.

18. Wing, J. M. (2006). Computational thinking. Communications of the ACM, 49(3), 33-35.

19. Yadav, A., Hong, H., \& Stephenson, C. (2016). Computational thinking for all: pedagogical approaches to embedding 21st century problem solving in K-12 classrooms. Tech Trends, 60(6), 565-568. doi:10.1007/s11528-016-0087-7. 


\section{PREPARING SCHOOL GRADUATES TO INTEGRATE INTO THE WORLD OF TECHNOLOGICAL INNOVATION, SOCIAL AND ECONOMIC CHANGES, AND GLOBALIZATION: THE ROLE OF TEACHING TECHNOLOGY AND ENGINEERING}

\section{MOSHE BARAK}

\section{Sc., Professor}

Ben-Gurion University of the Negev, Israel

mbarak@bgu.ac.il

KEYWORDS: COMPUTATIONAL THINKING, PROJECT-BASED LEARNING, TECHNOLOGY AND ENGINEERING EDUCATION.

For citation: Barak M. (2019), Preparing School Graduates To Integrate Into The World Of Technological Innovation, Social And Economic Changes, And Globalization: The Role Of Teaching Technology And Engineering, Globalization And Business, №7, pp. 16-23. https://doi.org/10.35945/gb.2019.07.002

\section{ABSTRACT}

Technological innovation, social and economic changes, and globalization are forcing the education system to prepare school graduates to integrate successfully into the rapidly changing world. Along with teaching basic subjects such as the humanities, languages, mathematics and science, schools should expose all students to new technologies, impart knowledge and skills to them to use these technologies effectively, and foster their motivation to integrate into the world of research and innovation in technology and engineering. Schools need also to prepare students to integrate into a world in which the vast majority of business organizations are not bureaucratic but rely instead on work teams, shared decision making and a great deal of risk-taking in the effort to compete in the global market. One of the main tools available to schools to achieve these goals is the teaching of subjects in technology and engineering as an integral part of the curriculum from kindergarten to high school. One challenge for technological education worldwide is to change what we have been teaching in the technological class. While in the past, technology education dealt with teaching subjects such as crafts, auto-mechanics or electronics, technology studies today have to focus more on developing students' computational thinking, for example, in the context of computerized control systems and robotics. A second challenge to technological education today is to change how we teach in the technological classroom. We must move from the traditional chalk-and-board teaching method to teaching that develops students' learning skills, for example, project-based learning. In this article, I will elaborate on these issues and present examples of the implementation of these ideas in the Israeli education system. 


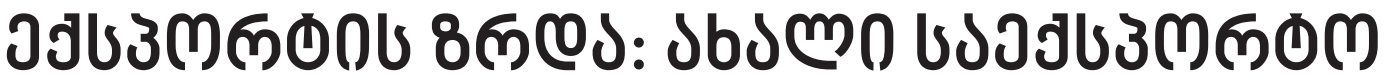

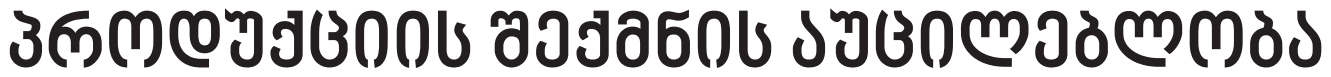

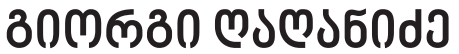

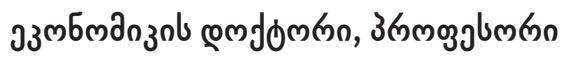

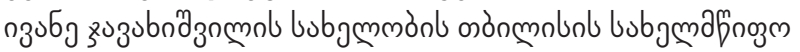

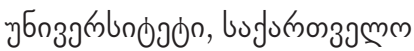

giorgi.gaganidze@tsu.ge

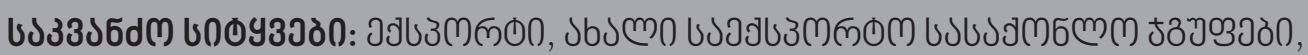

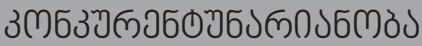

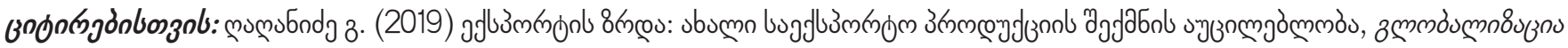
sou do86jlon, №7, 33. 24-31. https://doi.org/10.35945/gb.2019.07.003

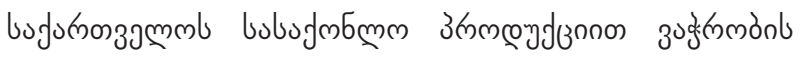

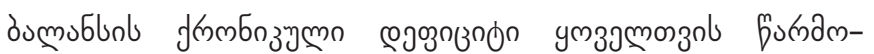

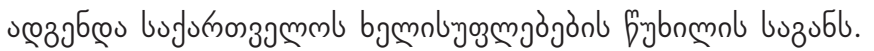

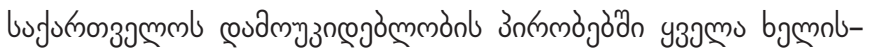

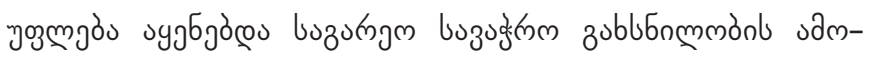

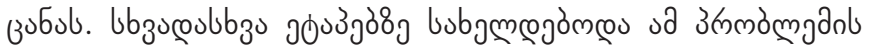

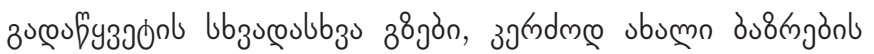

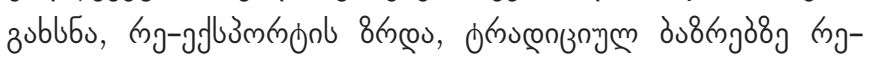

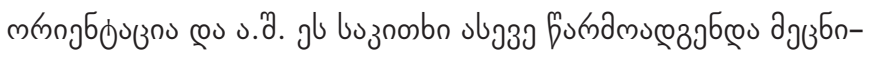

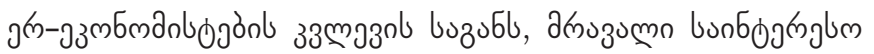

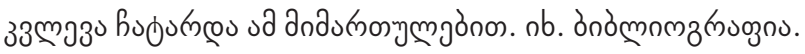

uмб yзэмs

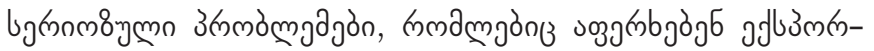

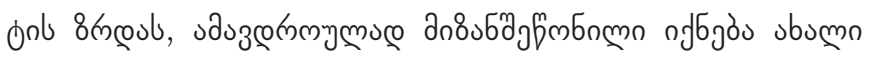

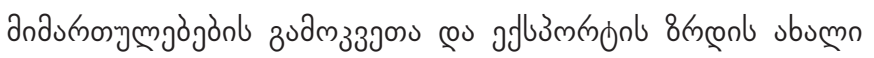

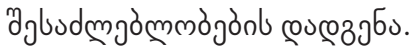

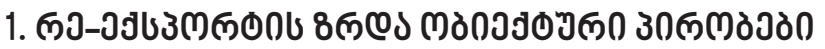

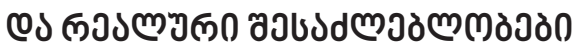

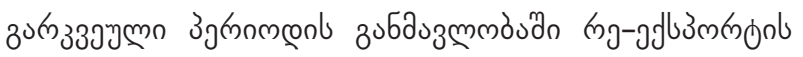

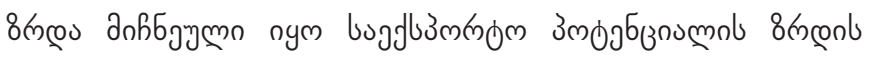

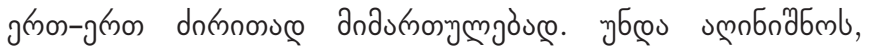

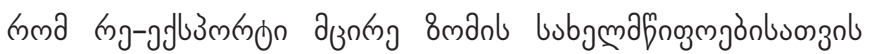

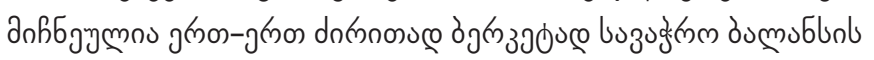

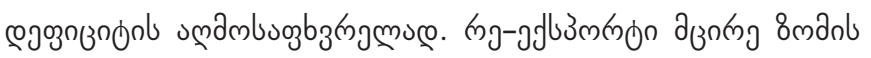

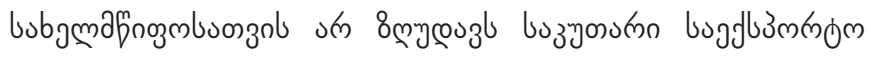

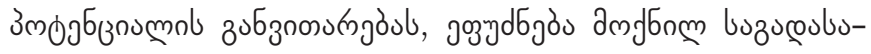

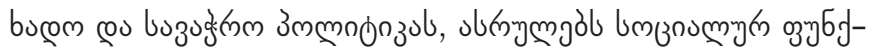

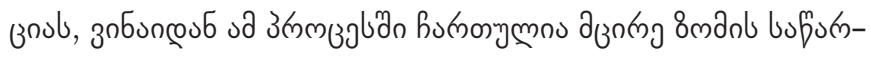

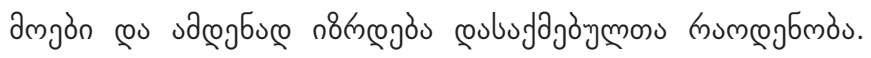

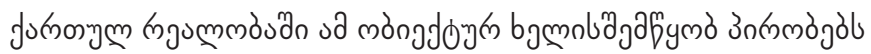

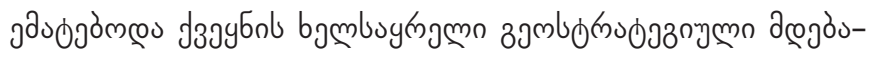

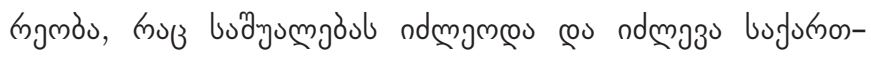

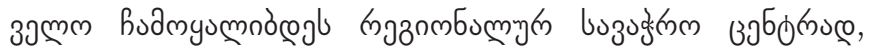

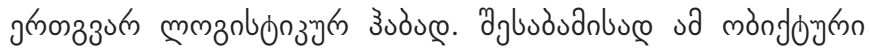

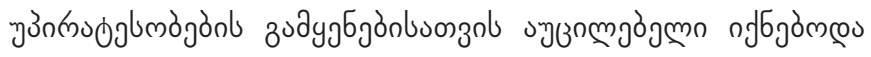

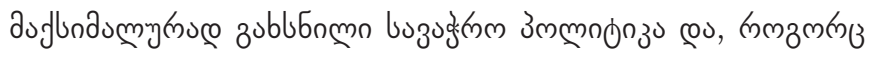

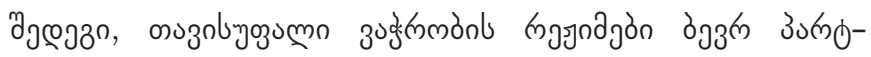

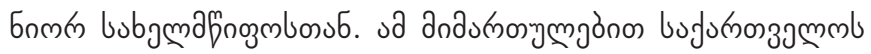

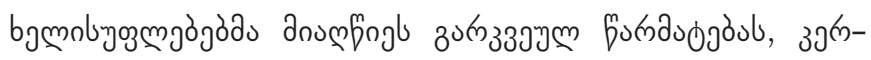

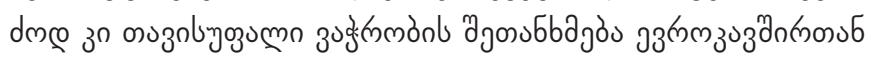

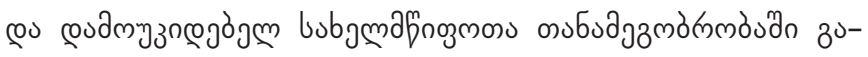

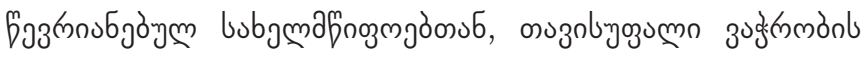

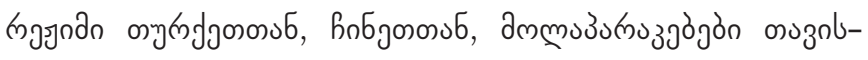

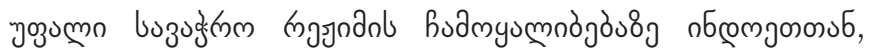

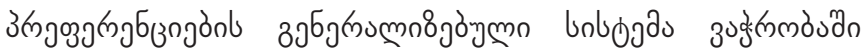

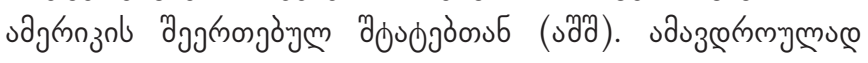

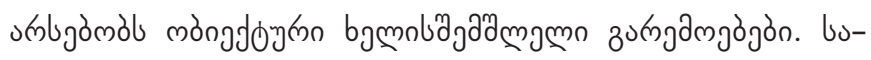

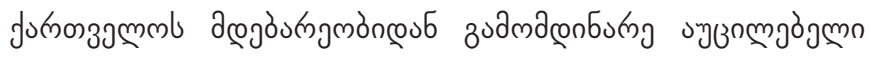

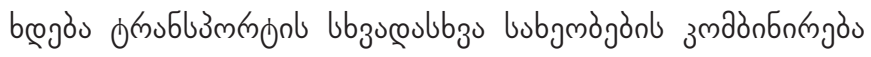

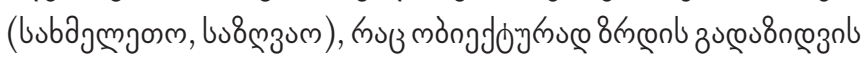

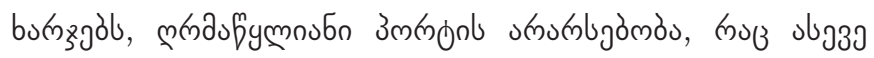




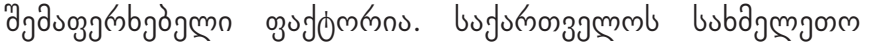

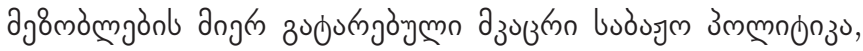

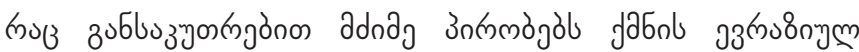

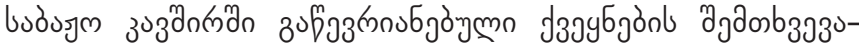

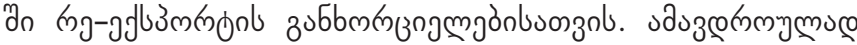

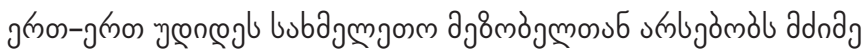

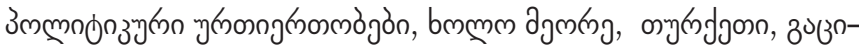

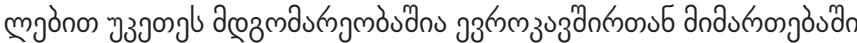

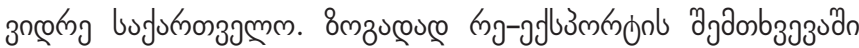

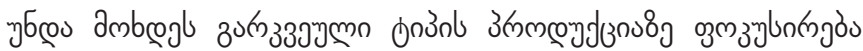

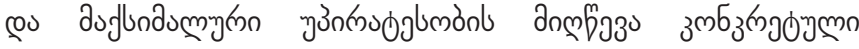

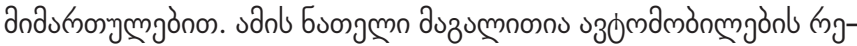

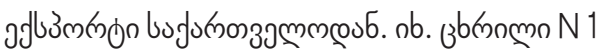

उb)

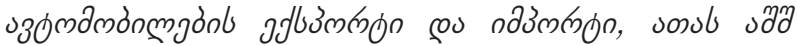
compungidon

\begin{tabular}{|c|c|c|c|c|c|c|c|c|c|c|}
\hline fagmo & 2009 & 2010 & 2011 & 2012 & 2013 & 2014 & 2015 & 2016 & 2017 & 2018 \\
\hline 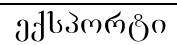 & 78,462 & 227,360 & 450,297 & 587,296 & 703,817 & 517,787 & 179,646 & 166,634 & 234,885 & 278 \\
\hline одзмп & 304,364 & 401,980 & 510,679 & 662,599 & 710,493 & 715,051 & 468,136 & 475,899 & 474,360 & 1,596 \\
\hline
\end{tabular}

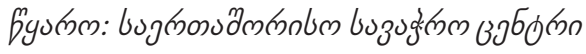

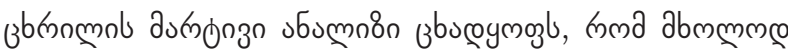

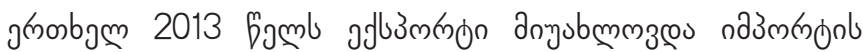

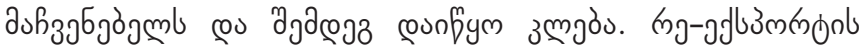

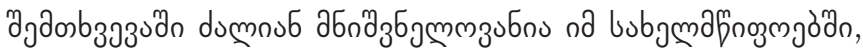

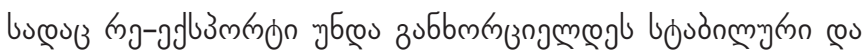

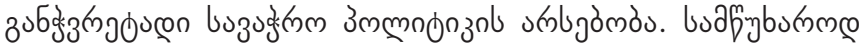

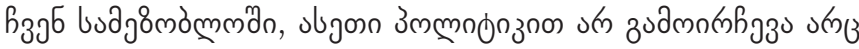
grnon bubjgmafpogn.

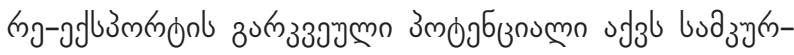

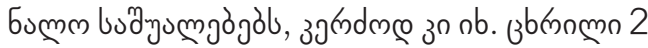

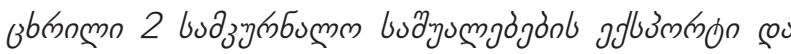

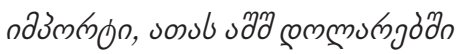

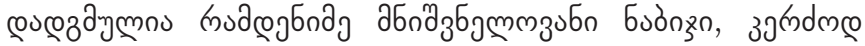

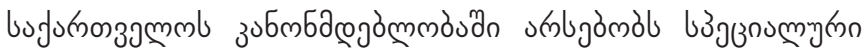

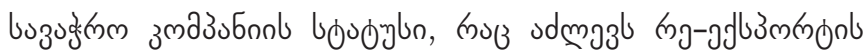

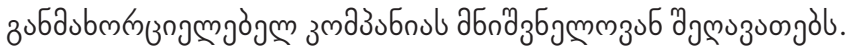

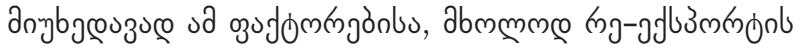

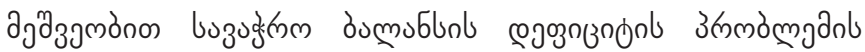

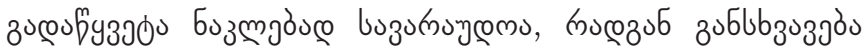

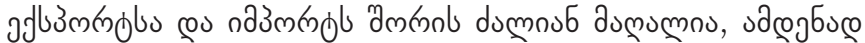

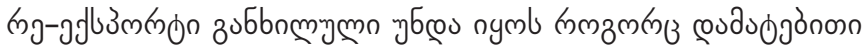
bymäa

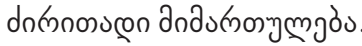

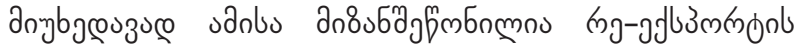

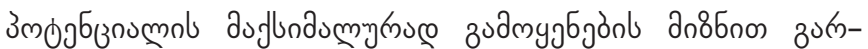

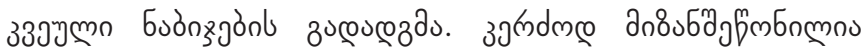

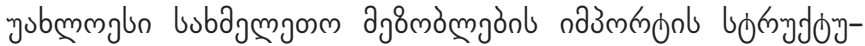

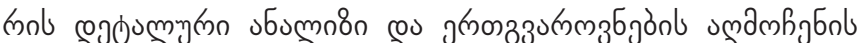

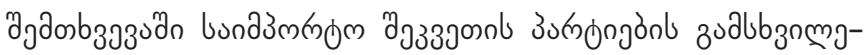

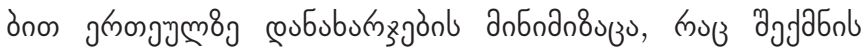

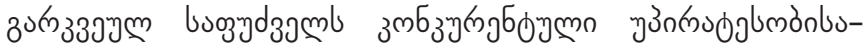

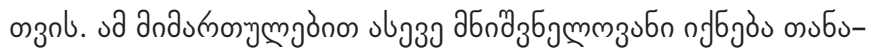

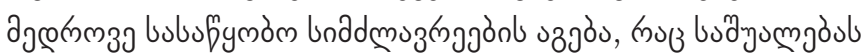

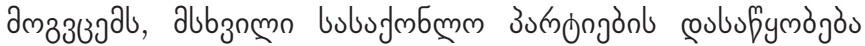

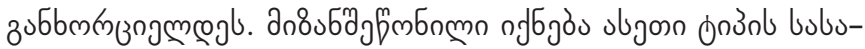

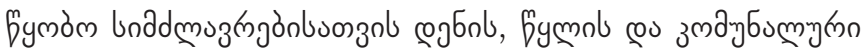

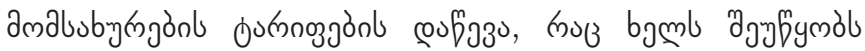

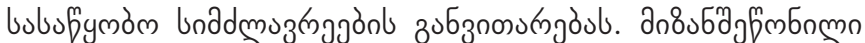

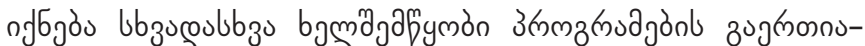

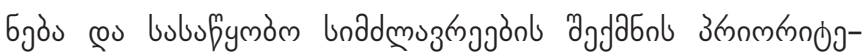

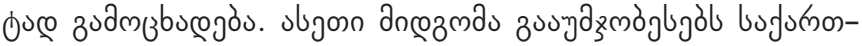

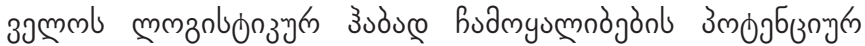
நjbudmgàmmòjal.

\begin{tabular}{|c|c|c|c|c|c|c|c|c|c|c|}
\hline Famo & 2009 & 2010 & 2011 & 2012 & 2013 & 2014 & 2015 & 2016 & 2017 & 2018 \\
\hline adb3 & 5,38 & 720 & 44,1 & 55,249 & 59,000 & 104,703 & 154,389 & 118,798 & 152,437 & 112 \\
\hline 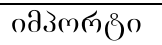 & 199,425 & 21,043 & 28,481 & 264,556 & 318,269 & 364,797 & 790,728 & 314,165 & 392,42 & 285,028 \\
\hline
\end{tabular}

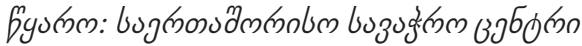

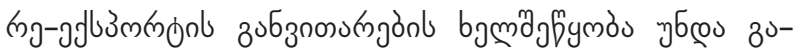

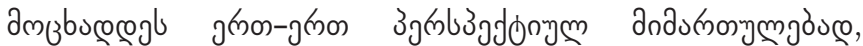

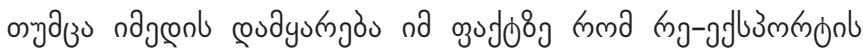

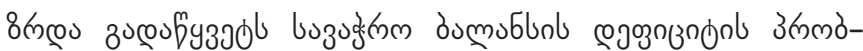

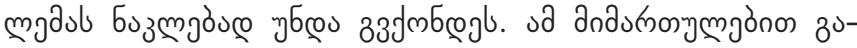

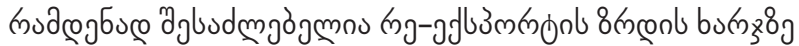

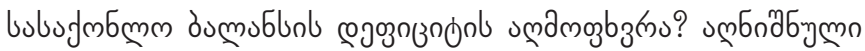

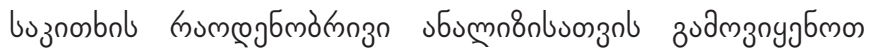

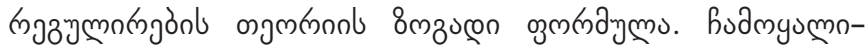

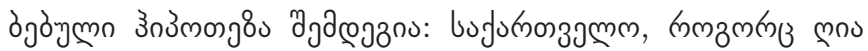

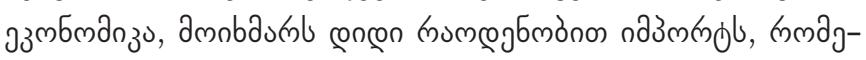

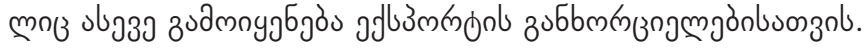




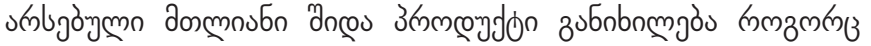

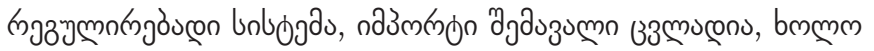

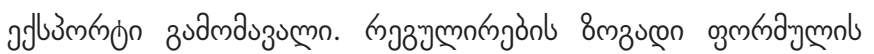

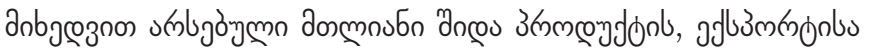

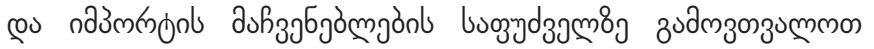

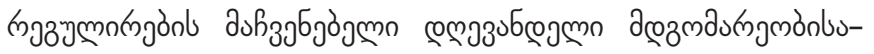

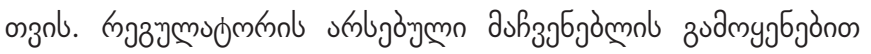

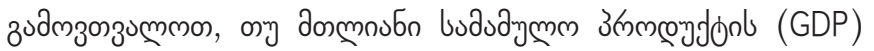

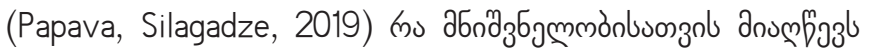

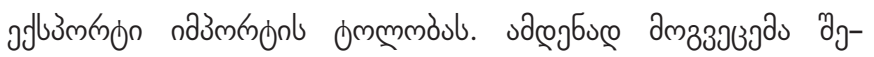

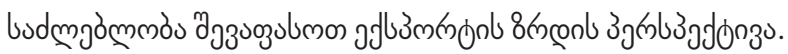

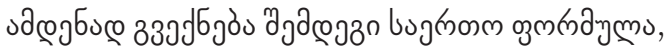 \\ $\mathrm{Y}=\mathrm{S} /(1-\mathrm{SXR}) \mathrm{X}$, \\ $Y$ jلjb3mtron \\ $X$ oдзminon

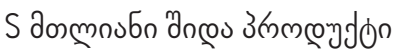

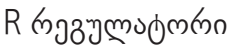

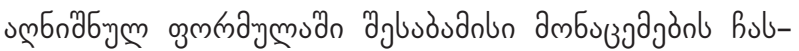

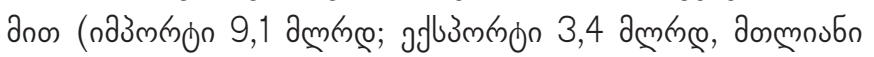

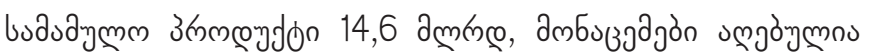

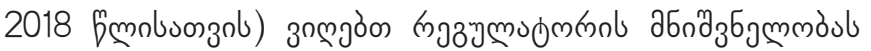

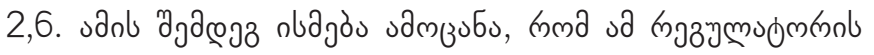

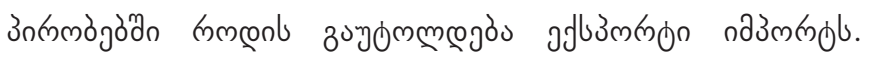

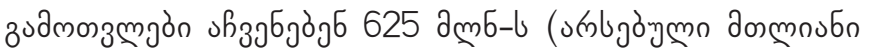

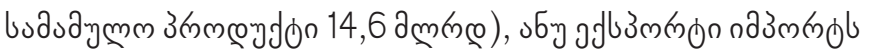

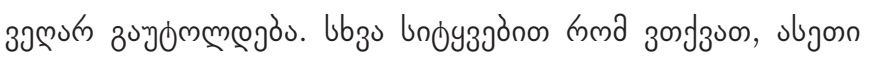

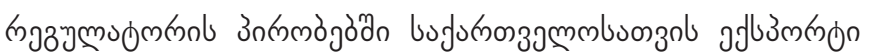

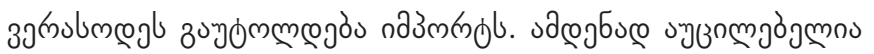

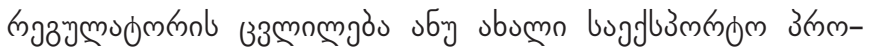

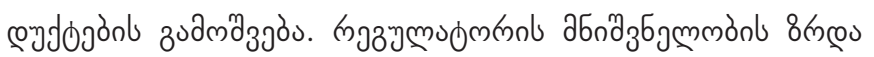

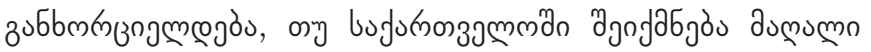

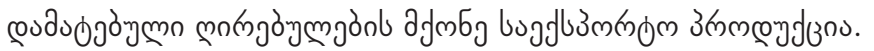

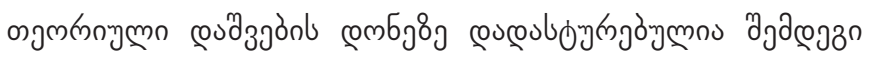

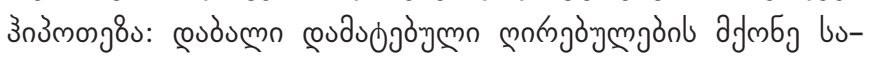

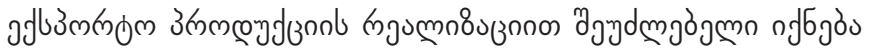

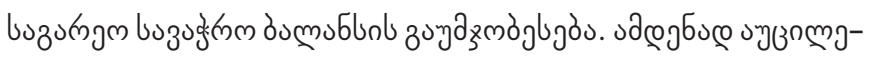

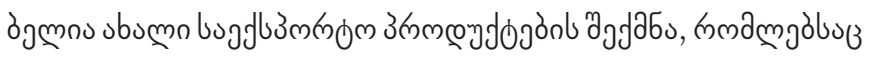

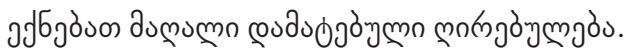

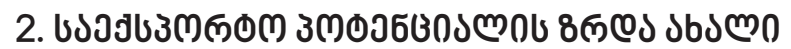

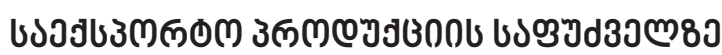

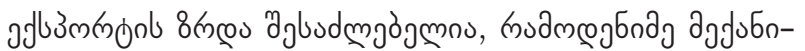
8anl aj

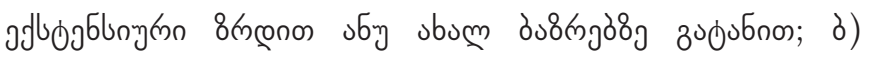

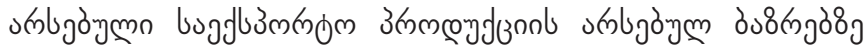

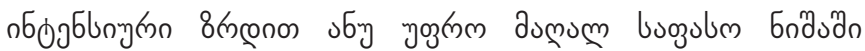

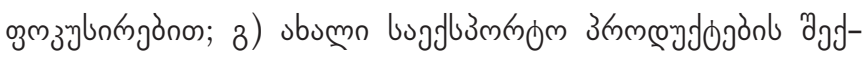

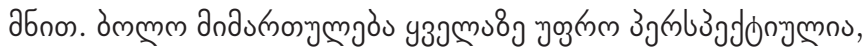

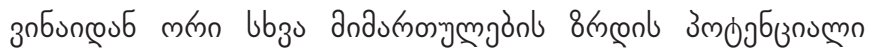

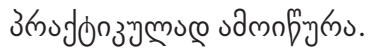

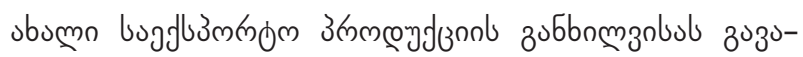

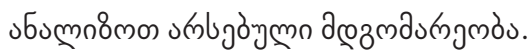

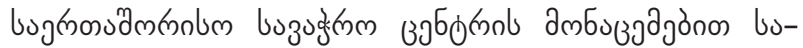

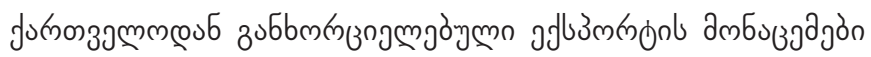

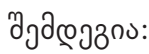

\begin{tabular}{|c|c|c|c|}
\hline & 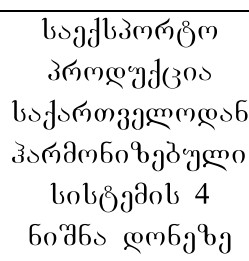 & 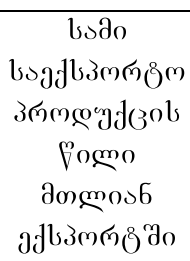 & 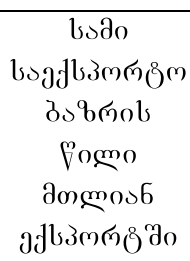 \\
\hline $\begin{array}{l}2016 \\
\text { fagmo }\end{array}$ & 1735 & 30,9 & 26,0 \\
\hline
\end{tabular}

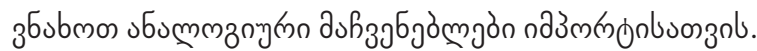

\begin{tabular}{|c|c|c|c|}
\hline & 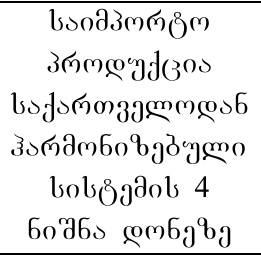 & 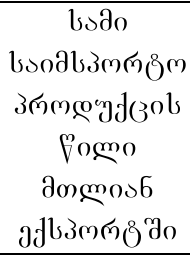 & 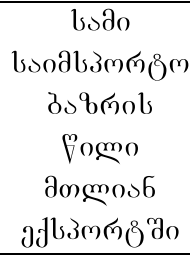 \\
\hline 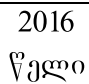 & 3801 & 12,9 & 35,6 \\
\hline
\end{tabular}

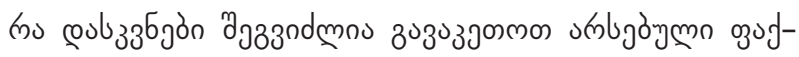

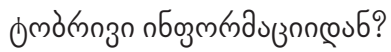

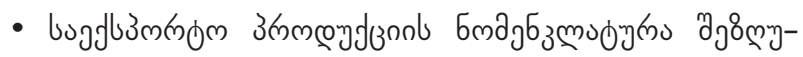
eymas;

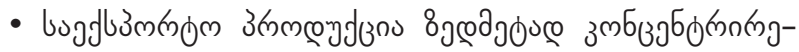

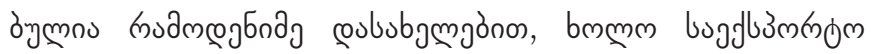

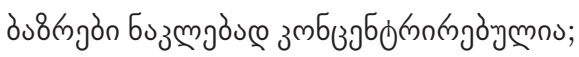

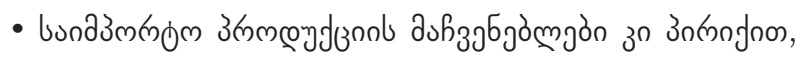

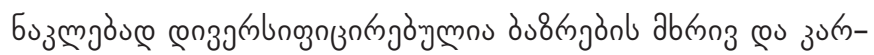

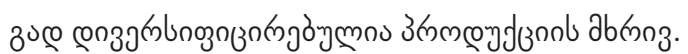

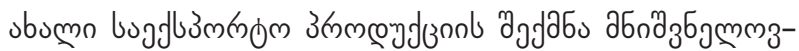

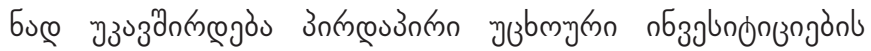

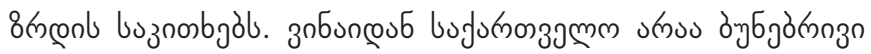

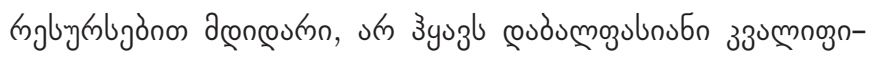

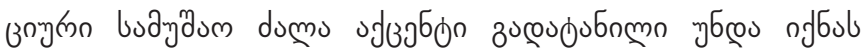

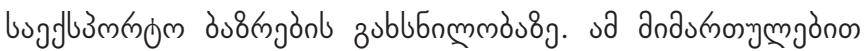

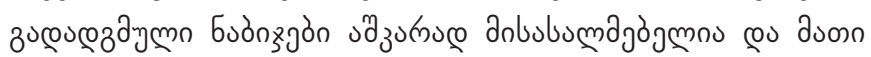

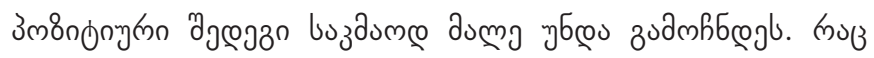




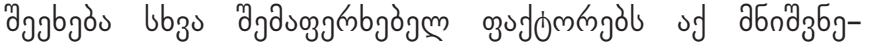

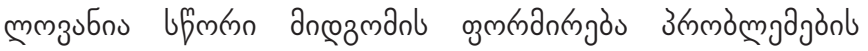

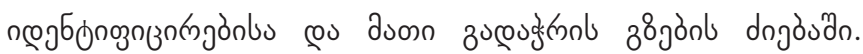

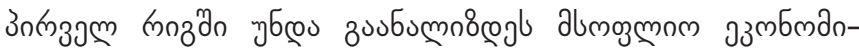

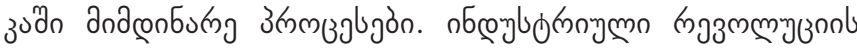

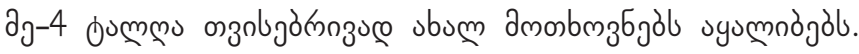

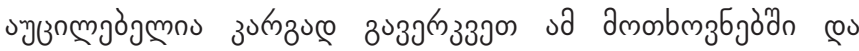

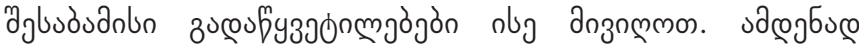

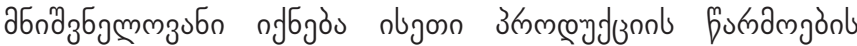

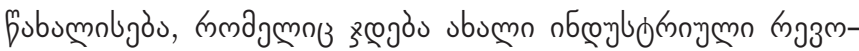
minzonb humbngiòn.

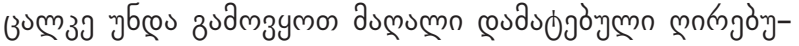

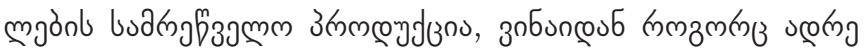

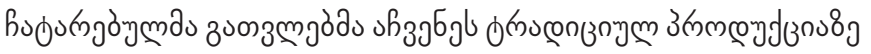

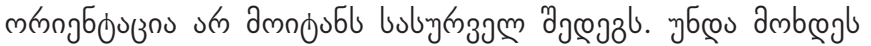

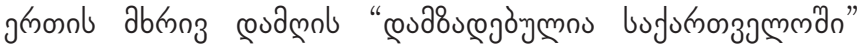

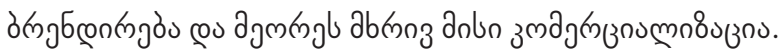

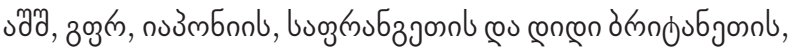

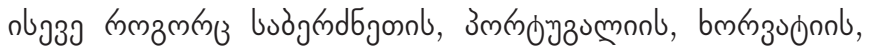

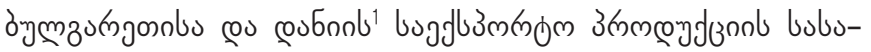

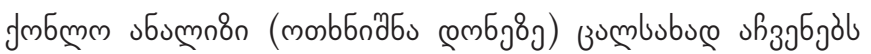

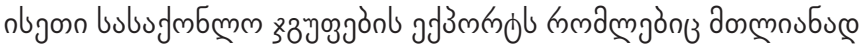

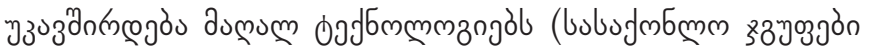

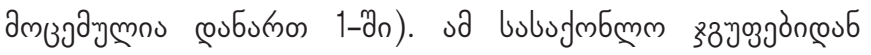

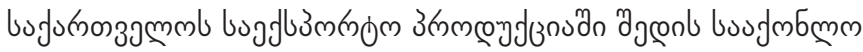

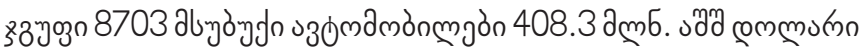

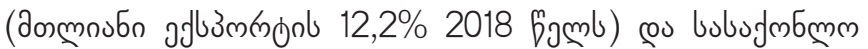

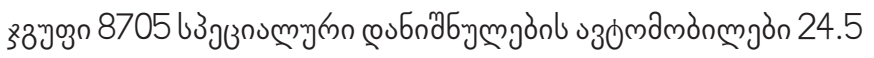

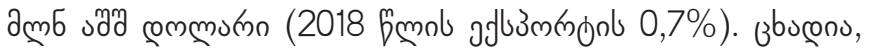

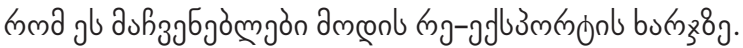

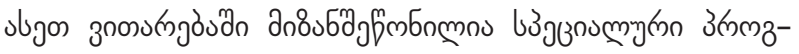

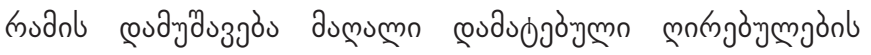

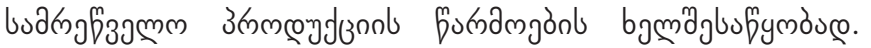

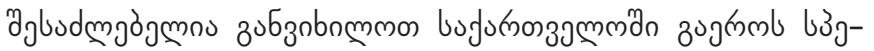

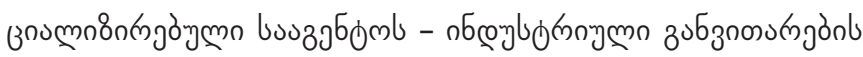

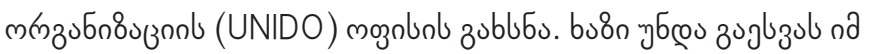

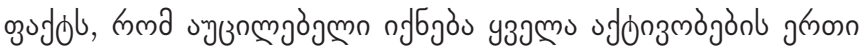

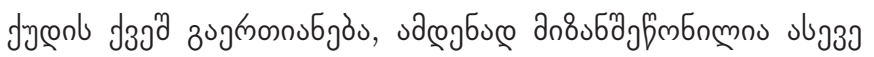

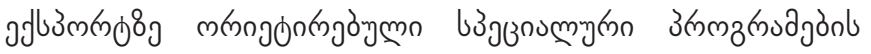

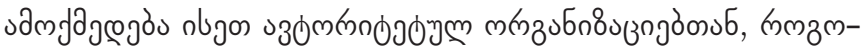

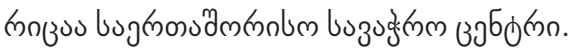

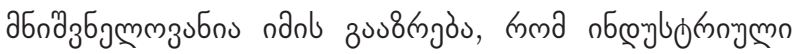

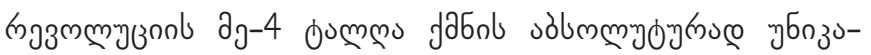

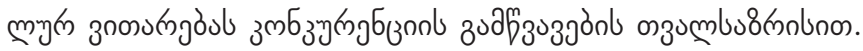

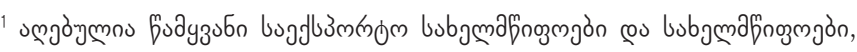

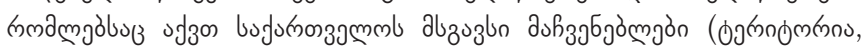

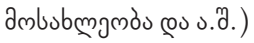

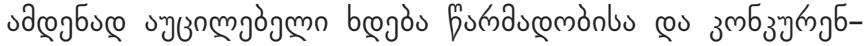

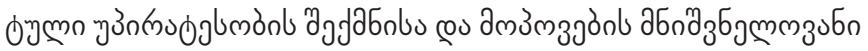

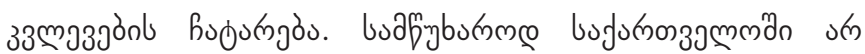

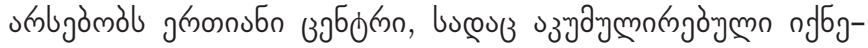

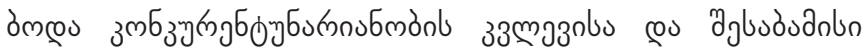

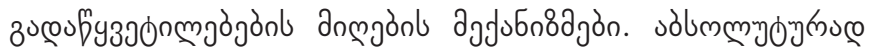

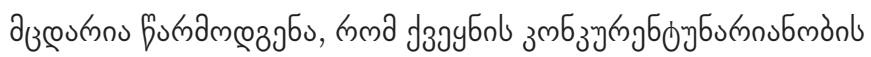

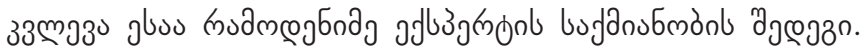

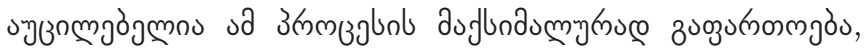

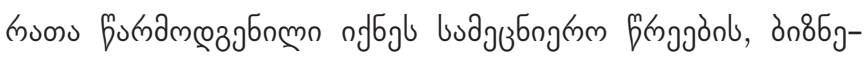

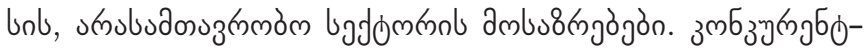

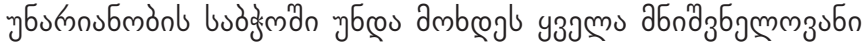

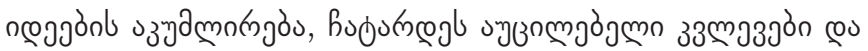

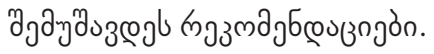

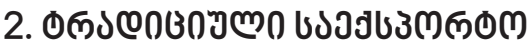

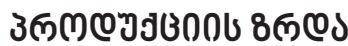

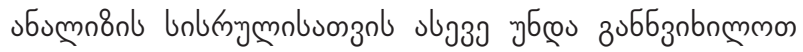

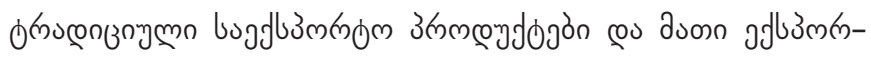

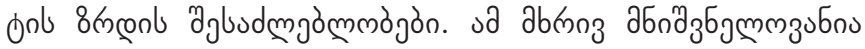

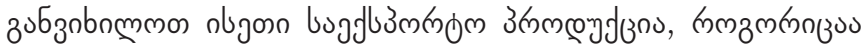

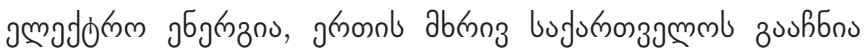

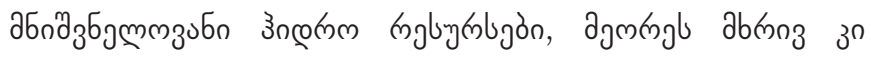

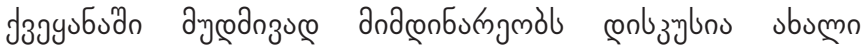

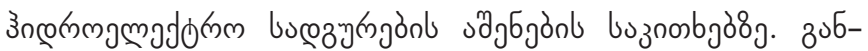

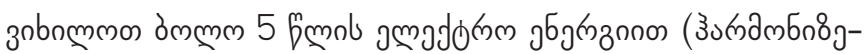

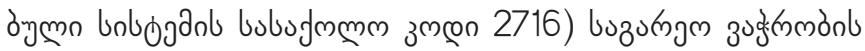
oumublon. zbrnnmo 3.

\section{6hongm 3}

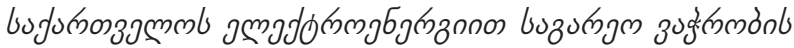

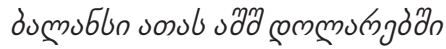

\begin{tabular}{|c|c|}
\hline 6ymo & sımsblon \\
\hline 2014 & $-21,562$ \\
\hline 2015 & $-17,756$ \\
\hline 2016 & 683 \\
\hline 2017 & $-44,518$ \\
\hline 2018 & $-56,756$ \\
\hline
\end{tabular}

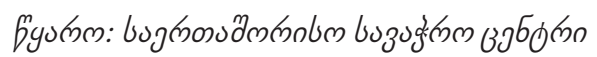

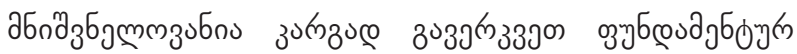

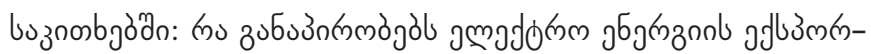

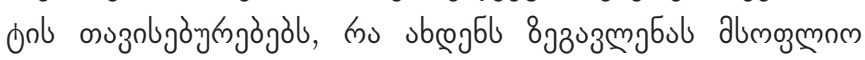

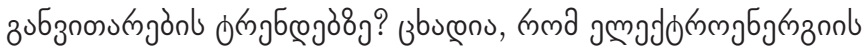




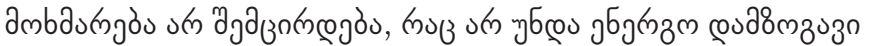

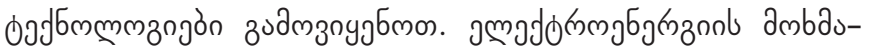

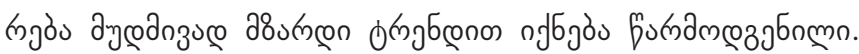

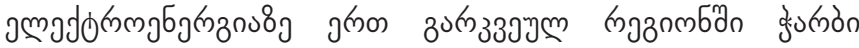

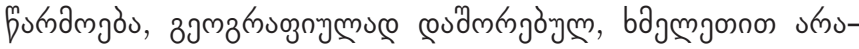

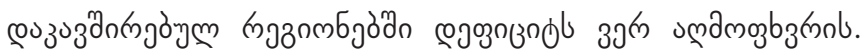

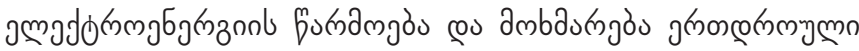

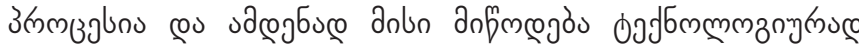

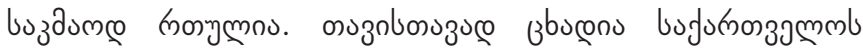

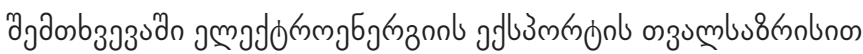

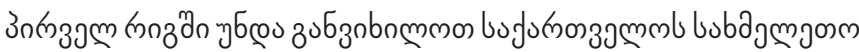

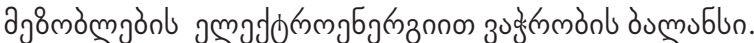

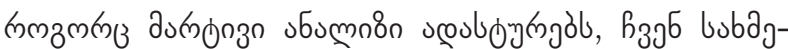

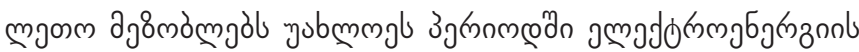

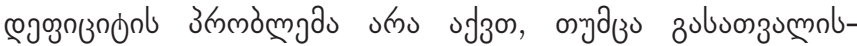

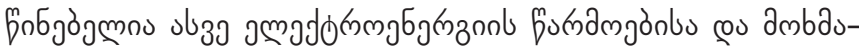

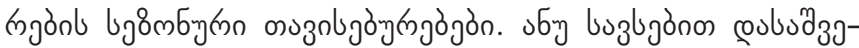

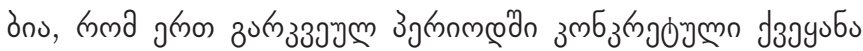

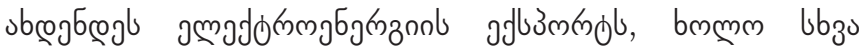

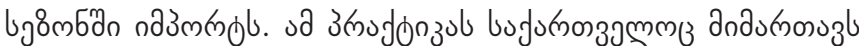

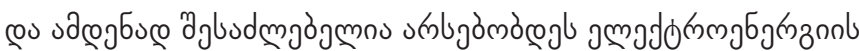

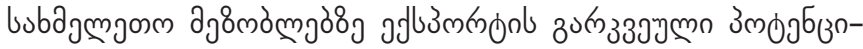

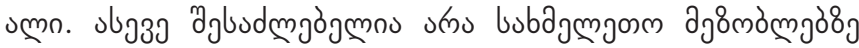

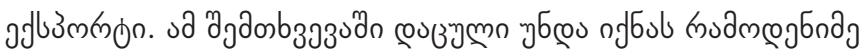

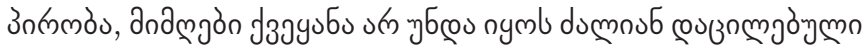

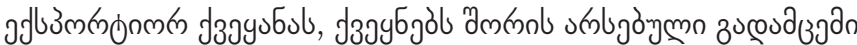

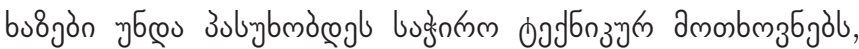

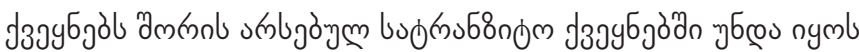

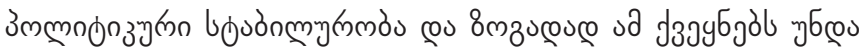

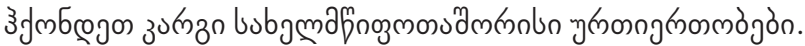

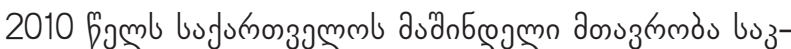

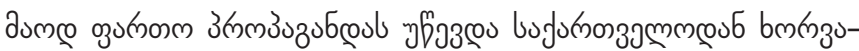
ono⿰氵 jmadd

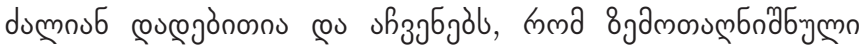

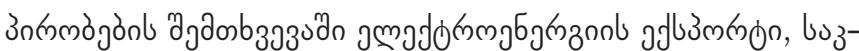

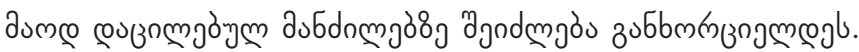

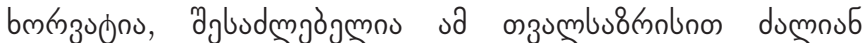

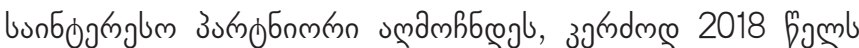

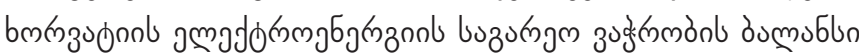

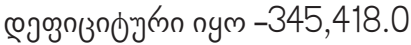

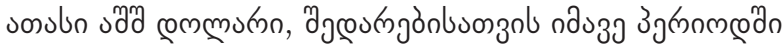

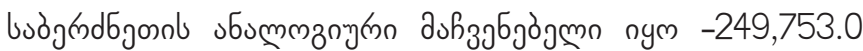
sosubn uag commutron.

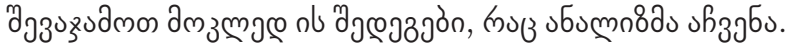

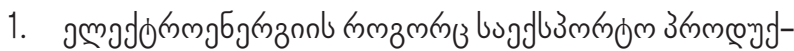

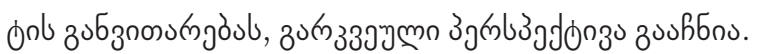

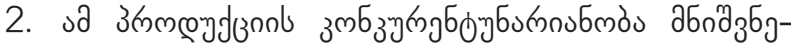

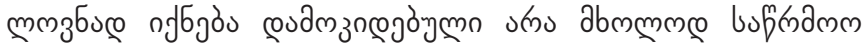

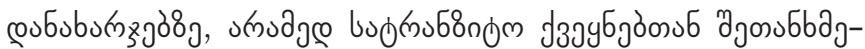
og

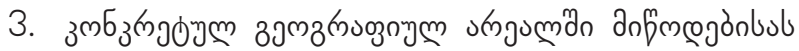

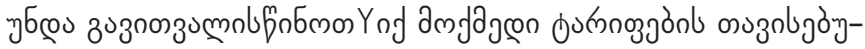

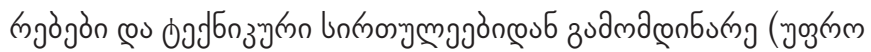

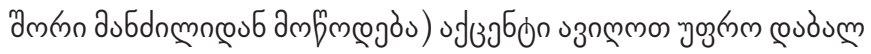

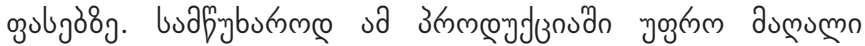
bumnlubnon zmb

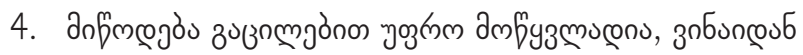

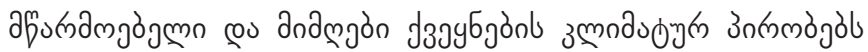

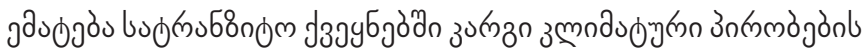

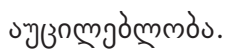

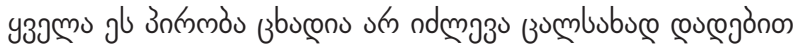

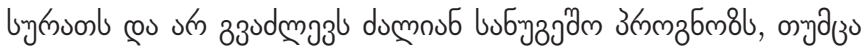

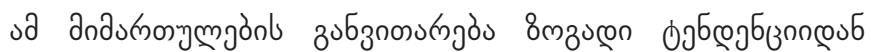

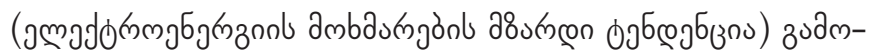

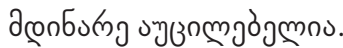

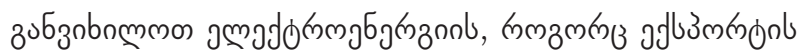

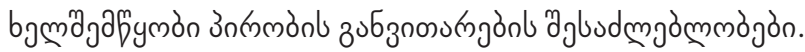

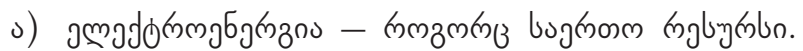

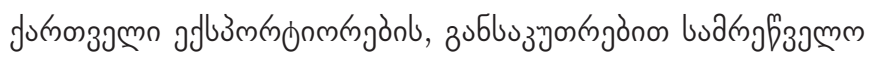

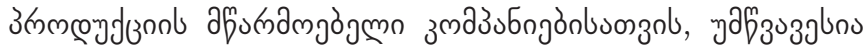

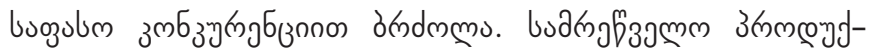

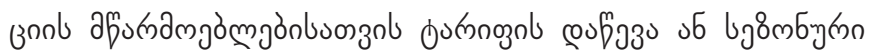

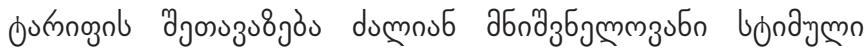

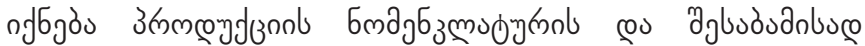

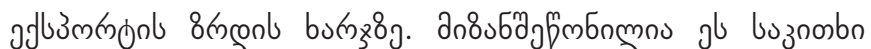

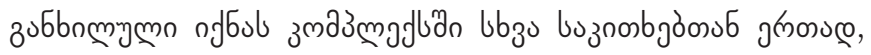

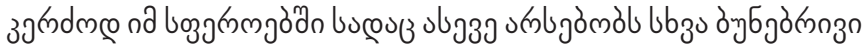

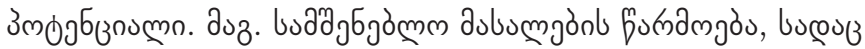

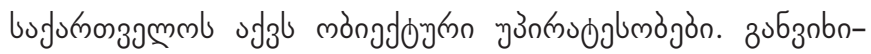

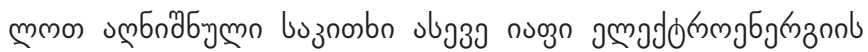

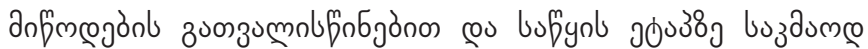

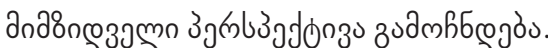

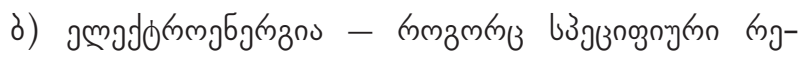

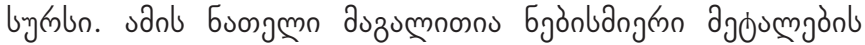

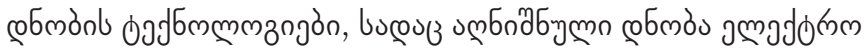

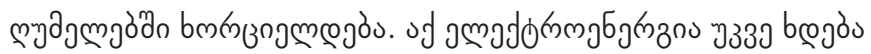

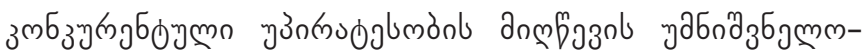

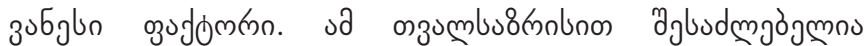

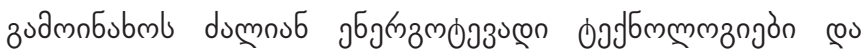

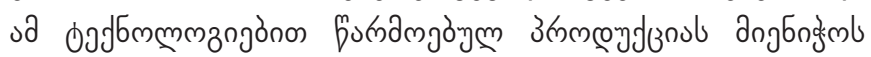

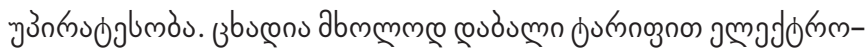




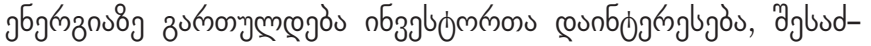

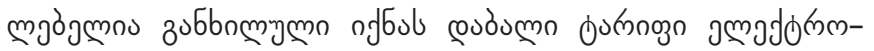

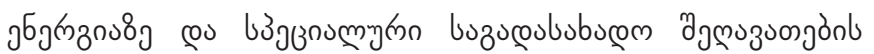

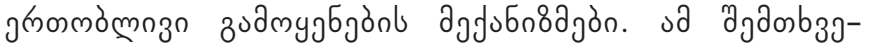

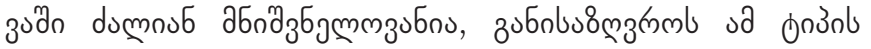

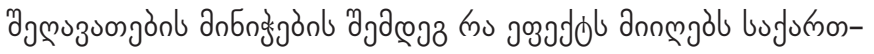

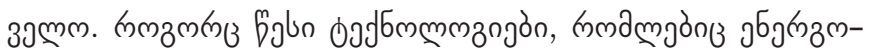

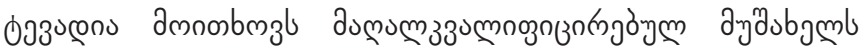

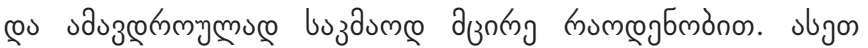

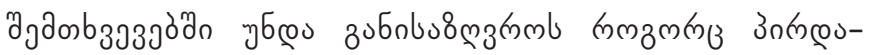

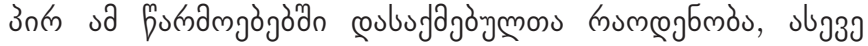

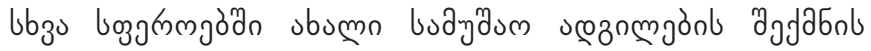

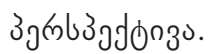

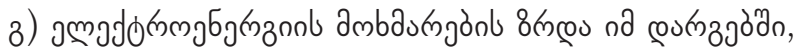

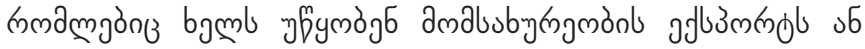

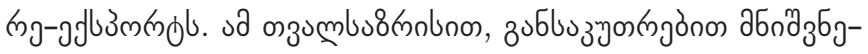

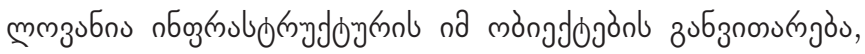

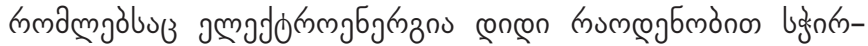

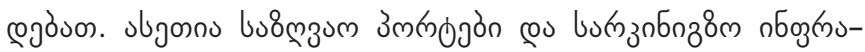

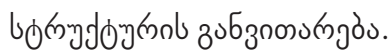

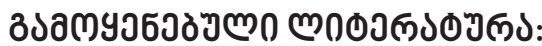

1. O'Cass, Aron and Craig Juliann (2003), "Examining Firm and Environmental Influences on Export Marketing Mix Strategy and Export Performance of Australian Exporters". European Journal of Marketing, 37 9314) 366-84

2. Papava, Vladimer, Retroeconomics - Moving from Dying to Brisk Economy, Journal of Reviews on Global economics, 2017, 6, pp 455-462

3. Papava, V. (2013). Economic Reforms in Post-Communist Georgia: Twenty Years After. New York: Nova Science Publishers.

4. Papava V., Silagadze A., 2019. "How the Term the "Gross Domestic Product" should be translated into Georgian." Globalization and Business, No. 7, pp. 203-204. (In Georgian.)

5. Gaganidze, G. (2016). Georgian Export Potential Utilization on the EU Market, Journal of International Management Studies, Volume16, Number 1.

6. Gaganidze, G. (2015). Export Potential and Competitive Advantage, scientific and practical journal, Economics and Business, N3.

7. Gaganidze,G. (2015). Export Potential of Georgian Agricultural Products on the EU Market (Based on Competitive Advantages and Market Entry Modes), European Journal of Business Research, Volume 15, Number 2, ISSN326:1945-2977.

8. Gaganidze,G.(2014) Competitive advantages of Georgian non-agricultural products on the EU Market, International Academic Conference, Business and Globalization, Dubai, 2-3rd February, ABRM, ISBN 2047-2854

9. Gaganidze,G.(2014). Competitive Advantage of Georgian Agricultural Products on the EU Market, International Academic Conference on Economics, Management and Marketing in Prague, (IAC-EMM 2014) 7-8, August, Conference proceedings, ISBN 978-80-905442-6-0

10. Gaganidze,G. (2014). Systematic Approach to Research the Competitive Advantage, TSU, scientific and practical journal, Economics and Business, N4, 2014

11. Gaganidze,G. (2014). Determining the production export potential index, TSU, scientific and practical journal, Economics and Business, N1, January-February.

12. Gaganidze,G.(2013). Competitiveness of Georgian agro products and creation of export strategies; II International Scientific-Practical Conference, Bioeconomy and Sustainable Development of Agriculture, October, Tbilisi, Proceedings 13. Gaganidze,G.(2013). New Challenges in Managing International Companies, Global Business Conference Proceedings, ISSN 1848-2252;p.73.(on English)

14. Gaganidze,G. (2013).Competitiveness of Georgian Agro products and creation of the export strategies on the EU market, TSU, scientific and practical journal, Economics and Business, N6November-December.

15.Silagadze, A., Zubiashvili, T. (2015). Parameters of the European Union and the Post-Soviet Georgia's Economy. Refereed International Journal of Business and Management Studies (IJBMS), pp. 441-448.

16.Silagadze, A., Atanelishvili,T. (2014). The main economic indicators of the EU and Georgia "Topical problems of the development of economy and economic science." Collection of scholarly works of Paata Gugushvili Institute of Economics TSU, pp.50-52. 


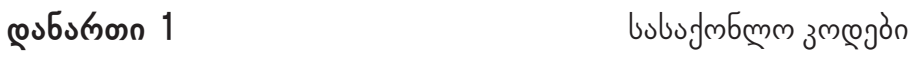

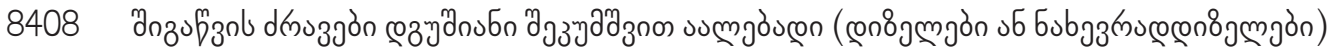

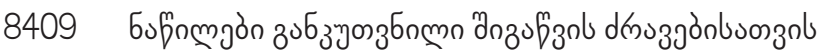

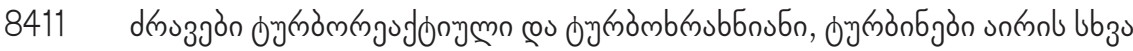

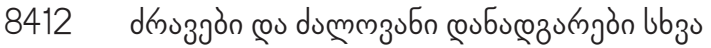

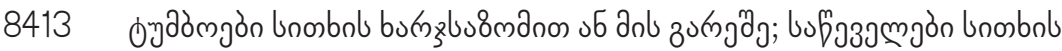

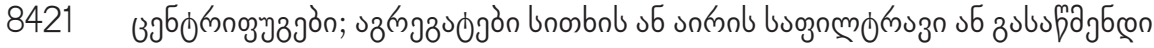

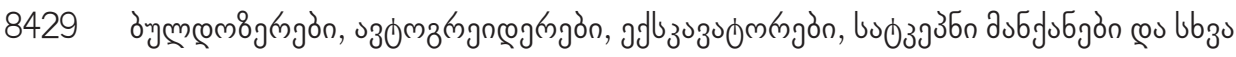

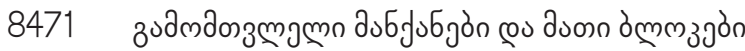

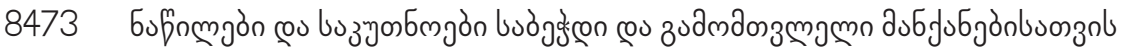

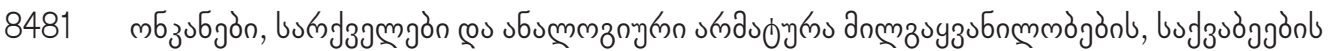

8535 د3دmod

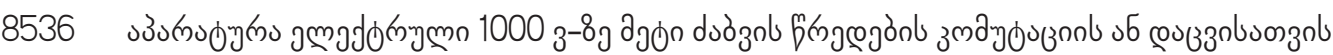

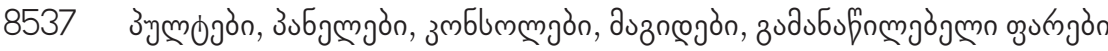

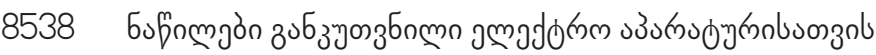

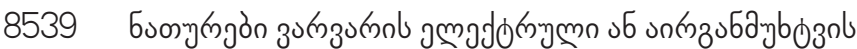

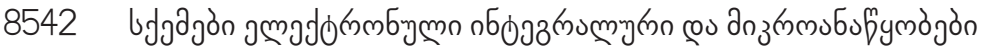

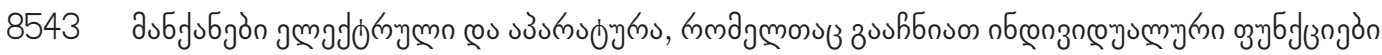

8544 aszongmgòn n8mmnn

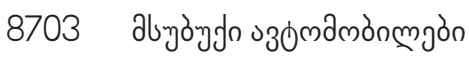

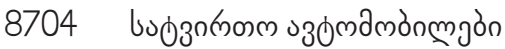

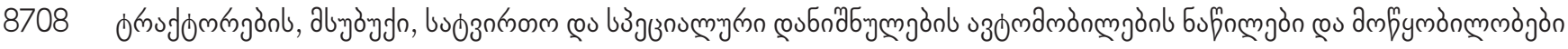

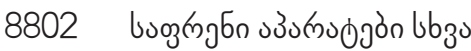

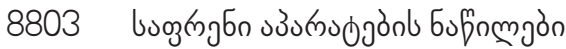




\section{GROWTH OF EXPORT: THE NEED TO CREATE NEW EXPORT PRODUCTS}

\section{GIORGI GAGANIDZE}

\section{Doctor of Economics, Professor}

Ivane Javakhishvili Tbilisi State University, Georgia

giorgi.gaganidze@tsu.ge

\section{KEYWORDS: EXPORT; NEW EXPORT COMMODITY GROUPS; COMPETITIVENESS.}

For citation: Gaganidze G. (2019), Growth Of Export: The Need To Create New Export Products, Globalization And Business, №7, pp. 24-31. (In Georgian). https://doi.org/10.35945/gb.2019.07.003

\section{SUMMARY}

In this article, the author tries to prove the advantages of exporting the export price. It is proposed to evaluate export growth based on the common formula of regulation.

The chronic shortage of balance of trade in commodity products of Georgia was always the subject of concerns of Georgian authorities. Under the independence of Georgia all governments have set a goal of foreign trade openness. At different stages, different ways of solving this problem, name- ly opening new markets, increasing re-export, re-orientation in traditional markets, etc. This issue was also a subject of research for scientists and researchers, and many interesting researches were conducted in this direction. See Bibliography.

All these directions are distinguished in this study and are considered to be the most serious problems that impede export growth, at the same time it is expedient to identify new directions and establish new opportunities for export growth. 


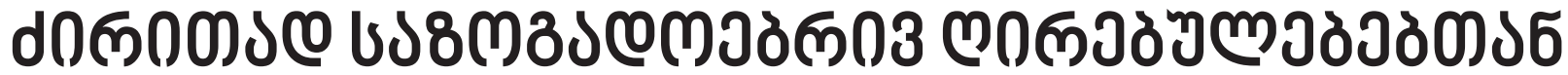

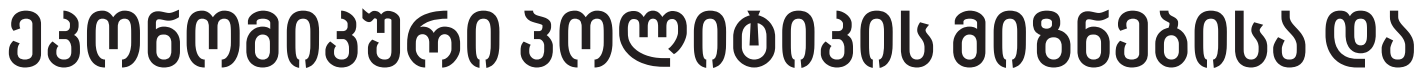

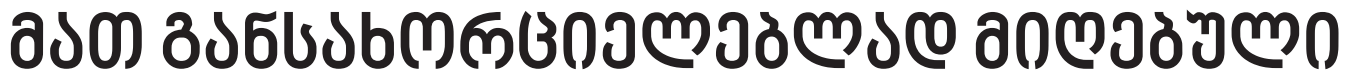

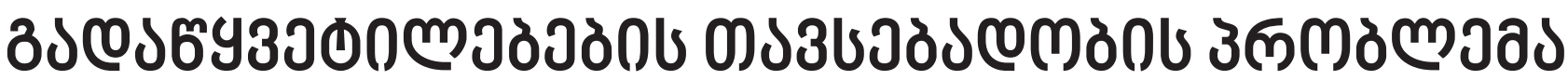

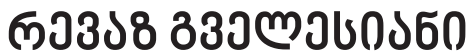

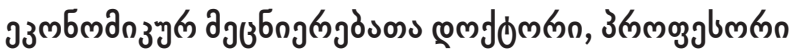

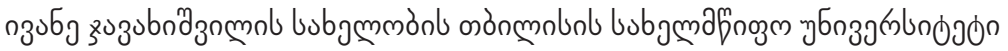

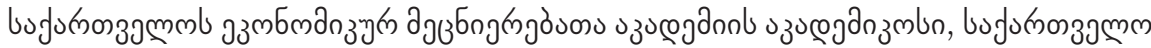

revaz.gvelesiani@tsu.ge

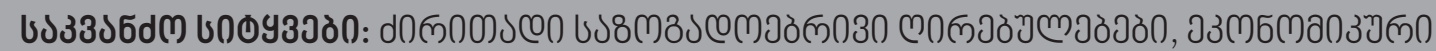

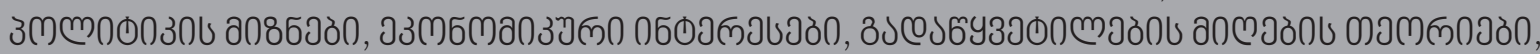

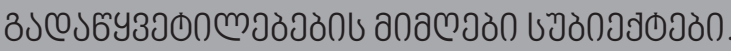

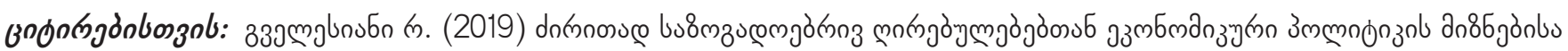

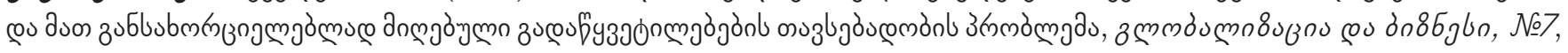
33. 32-38. https://doi.org/10.35945/gb.2019.07.004

әอเ১з১ल0

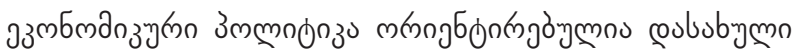

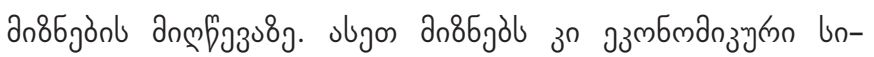

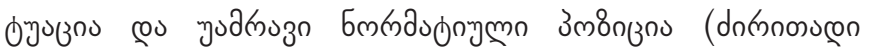

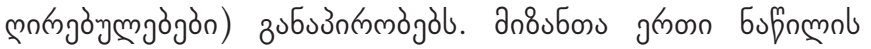

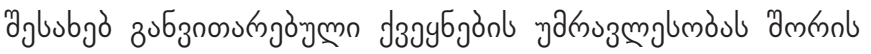

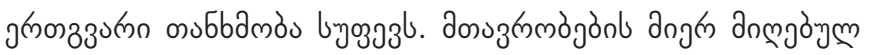

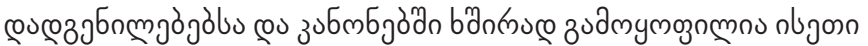

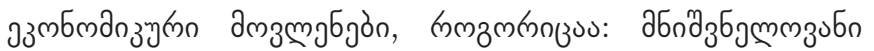

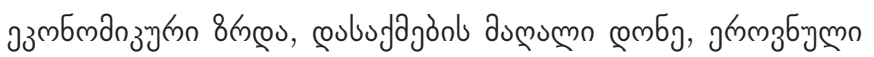

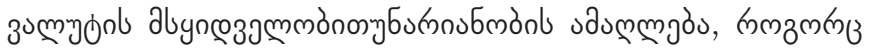

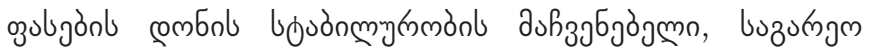

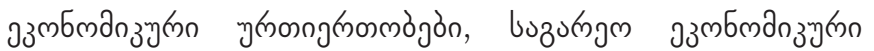

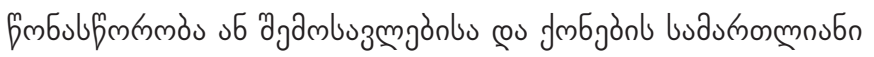

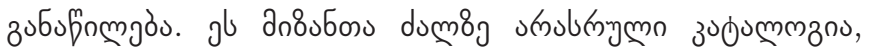

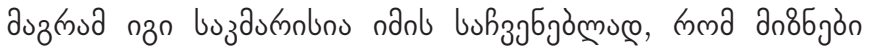

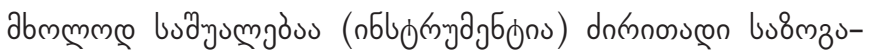

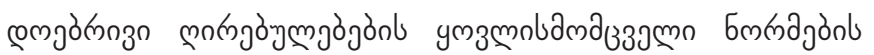

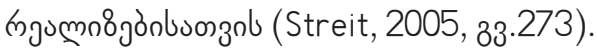

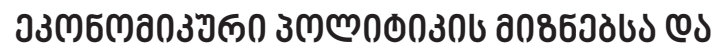

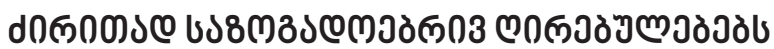

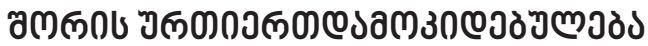

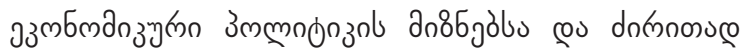

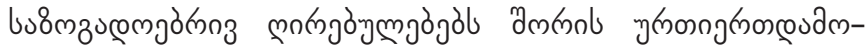

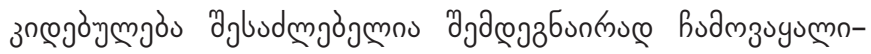

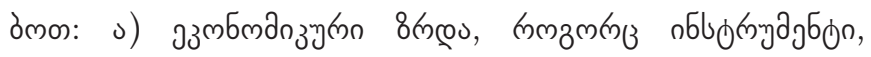

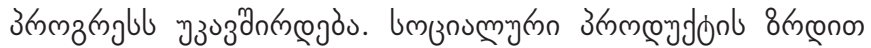

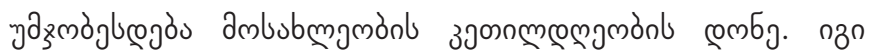

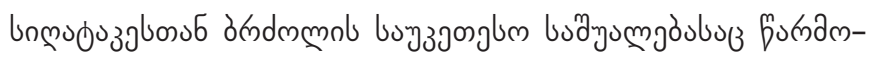

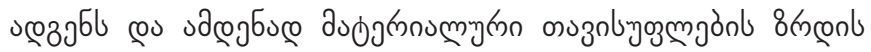

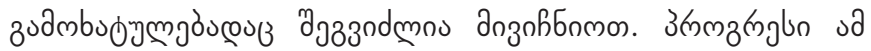

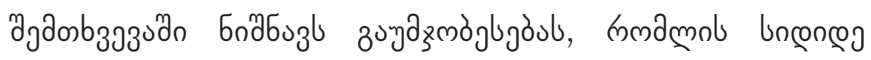

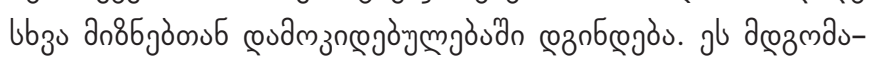

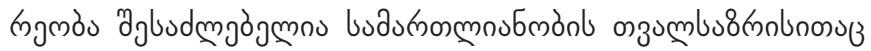

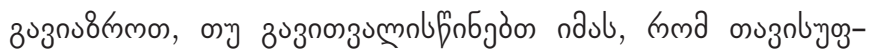

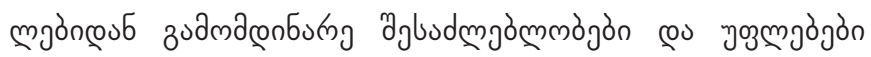

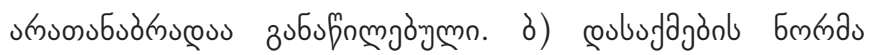

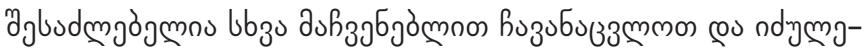




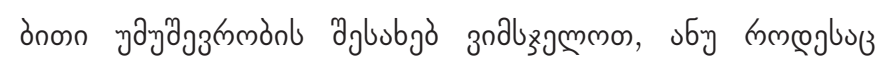

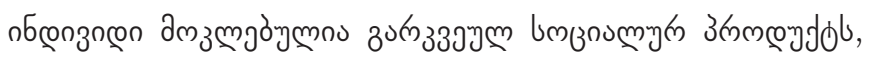

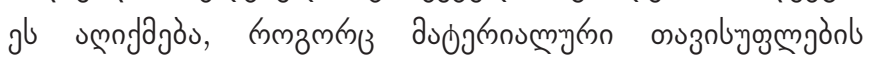

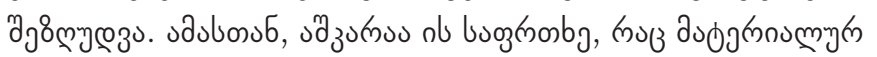

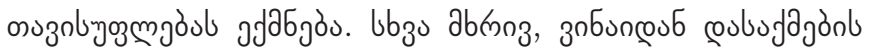

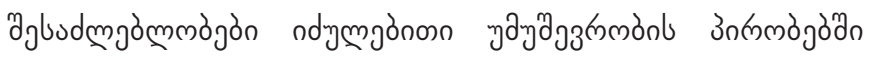

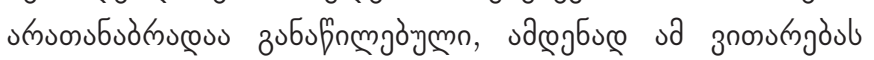

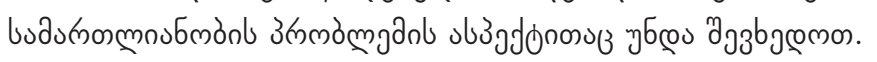

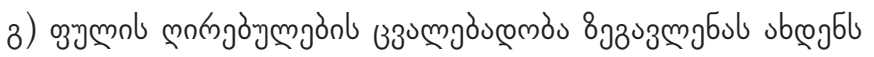

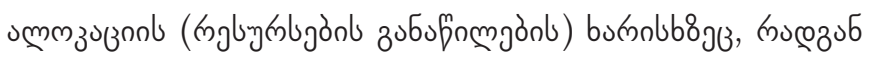

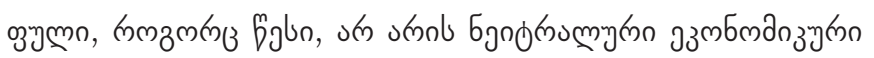

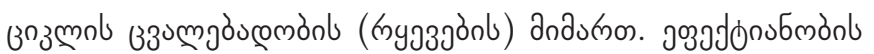

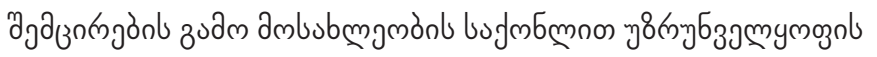

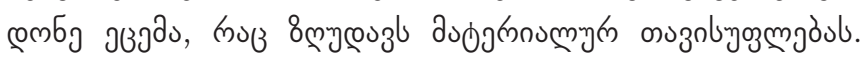

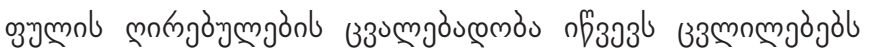

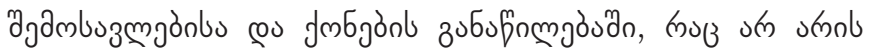

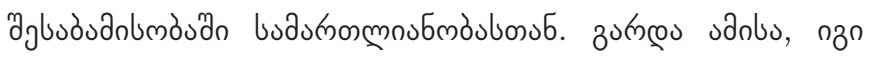

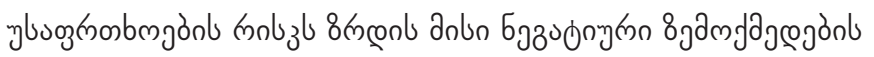

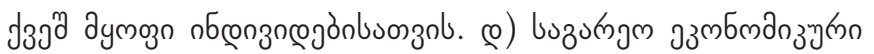

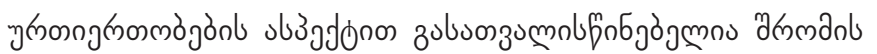

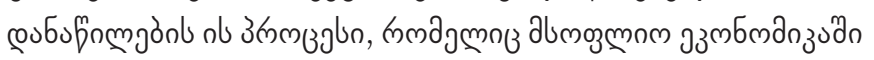

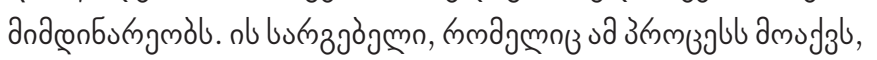

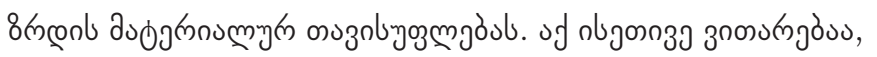

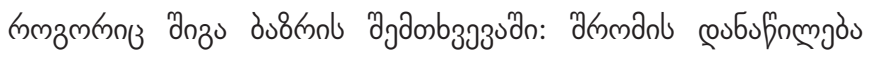

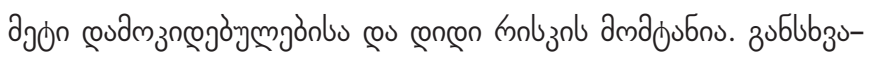

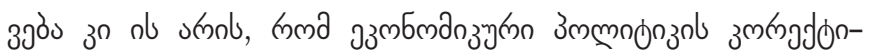

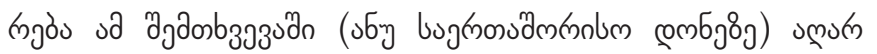

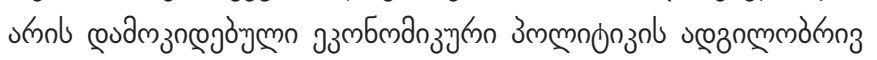

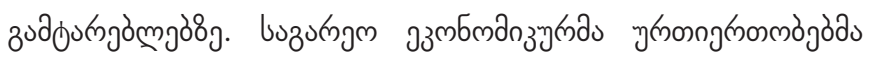

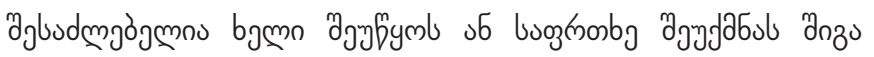

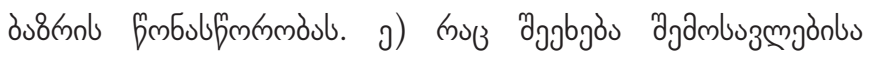

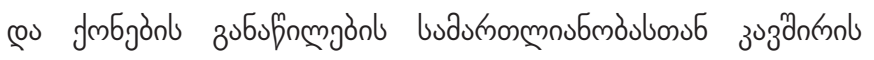

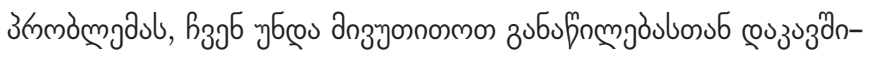

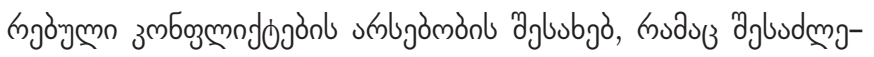

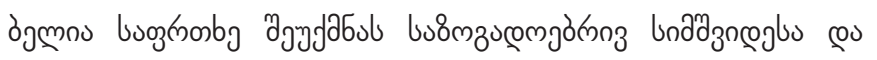

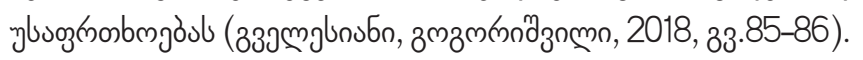

oुn fun

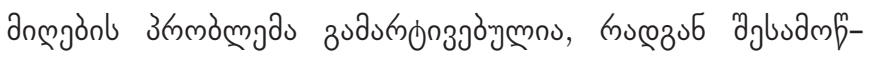

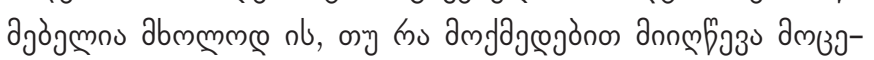

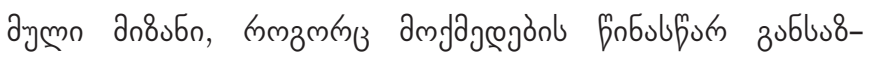

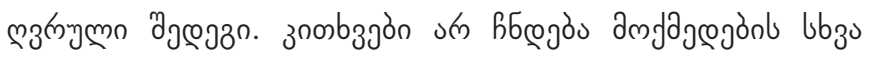

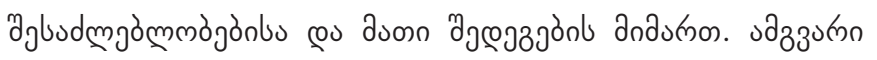

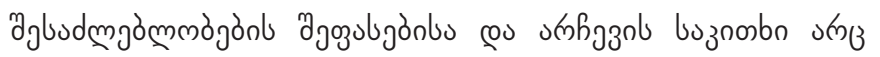

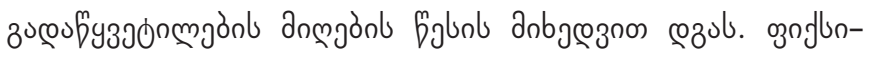

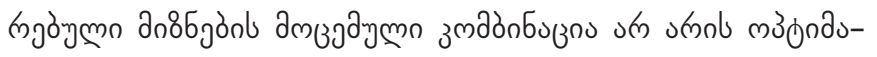

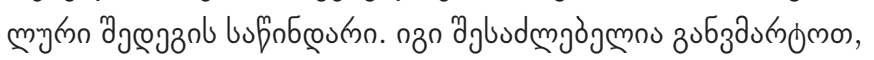

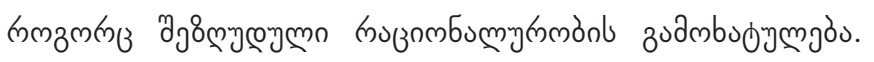

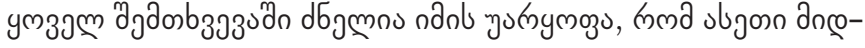

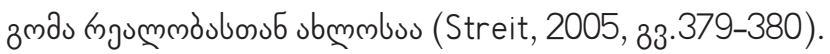

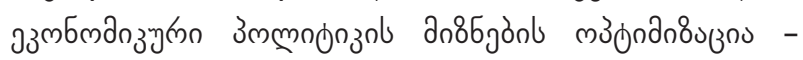

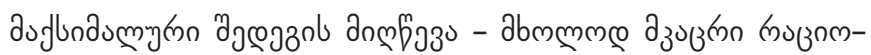

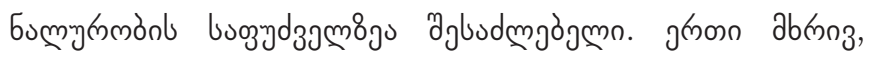

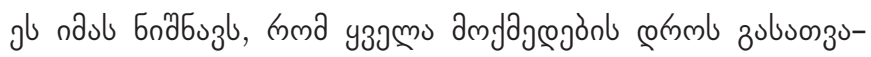

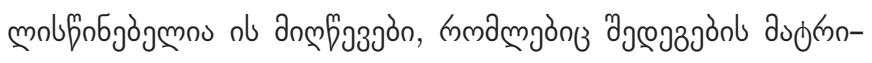

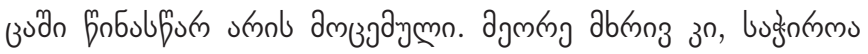

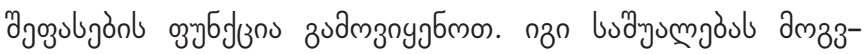

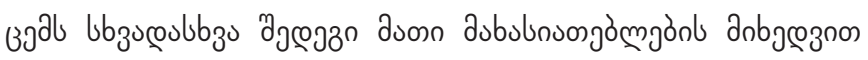

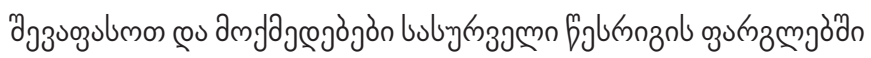

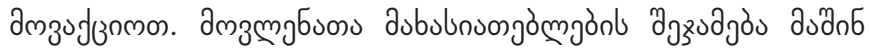

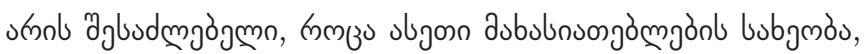

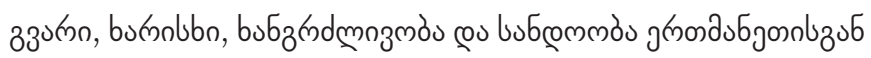

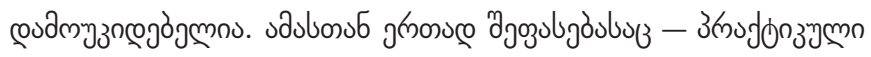

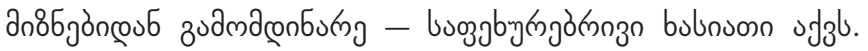

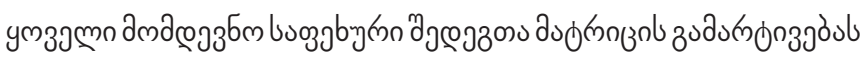

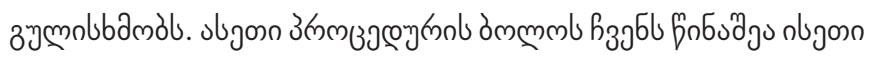

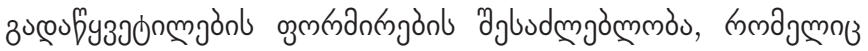

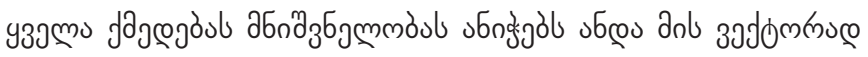

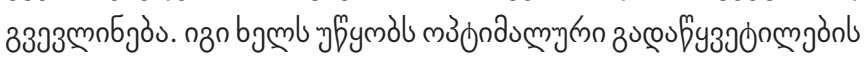
dnģobou (Streit, 2005, 33.380).

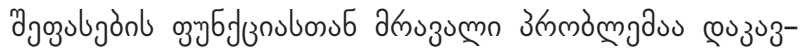

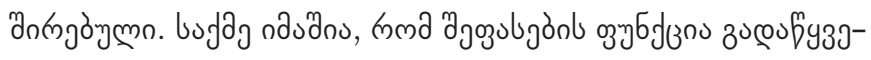

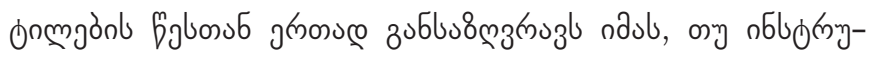

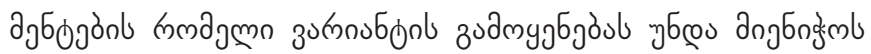

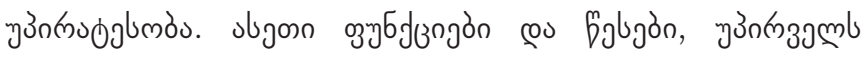

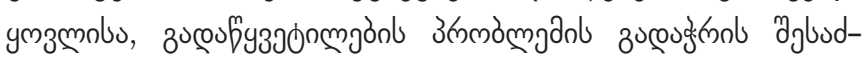

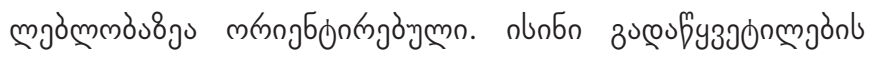

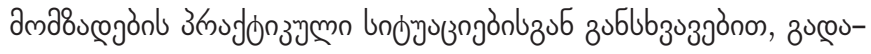

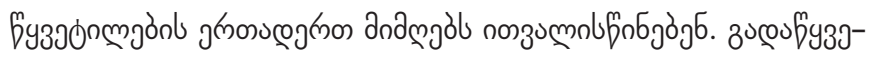

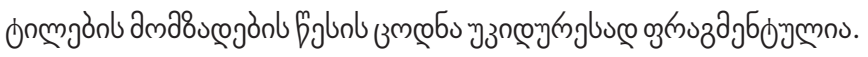

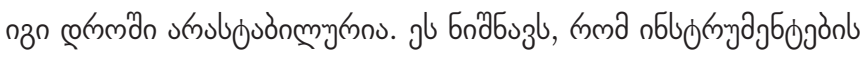

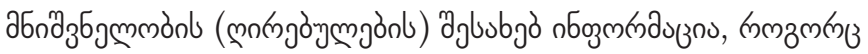

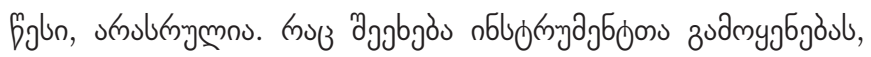

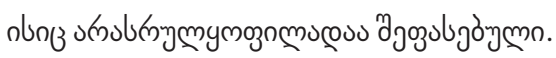

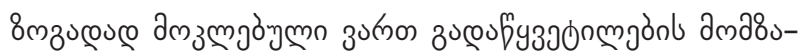

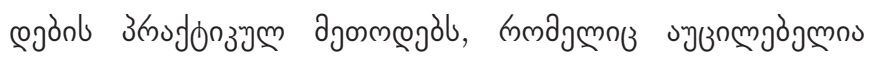

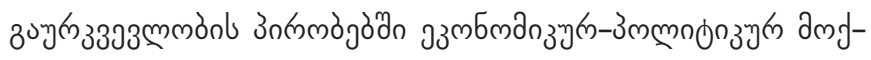

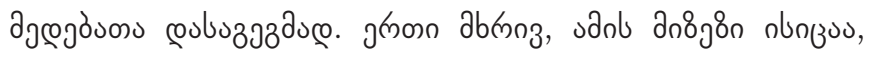

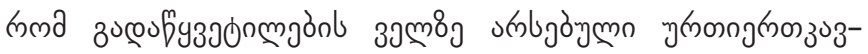

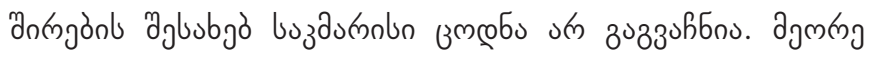

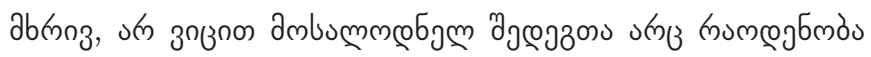

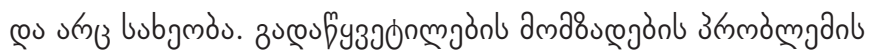
змазмm

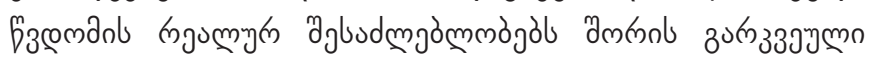

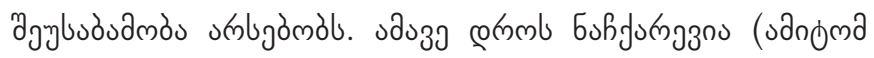




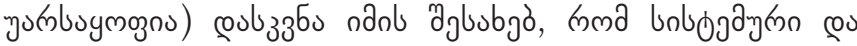

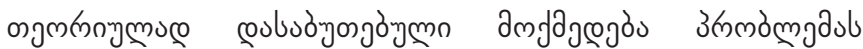

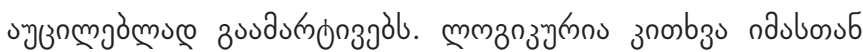

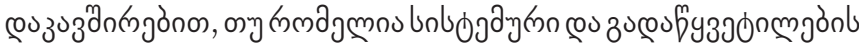

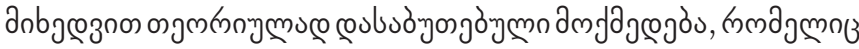

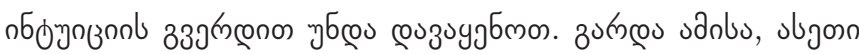

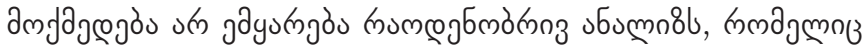

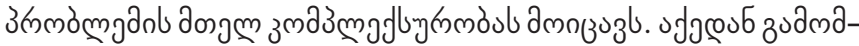

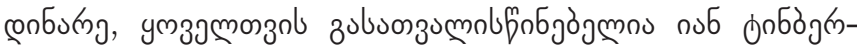

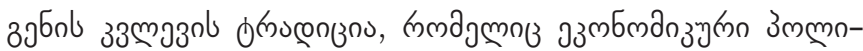

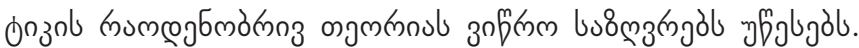

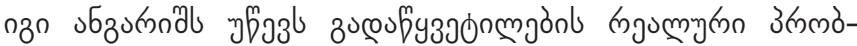

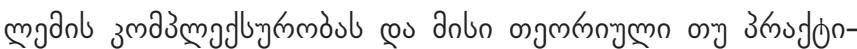

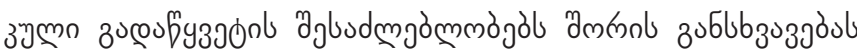

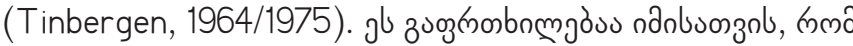

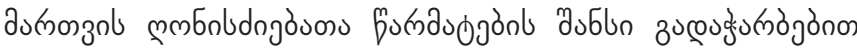

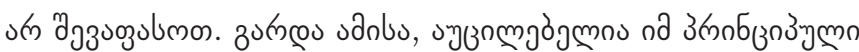

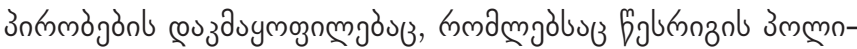

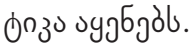

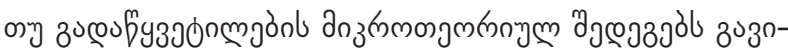

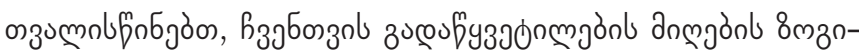

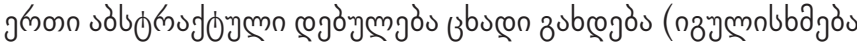

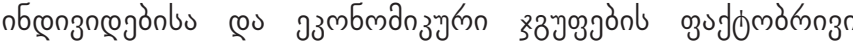

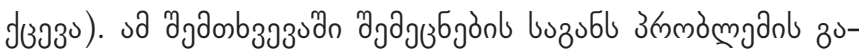

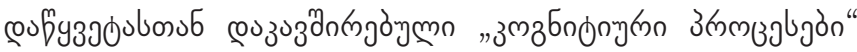

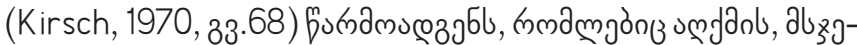

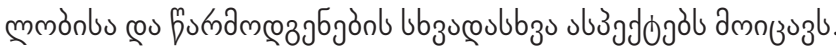

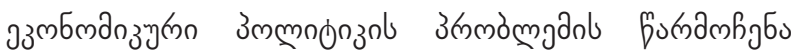

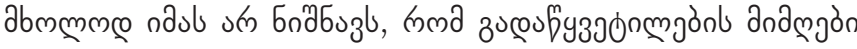

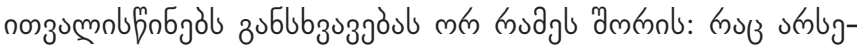

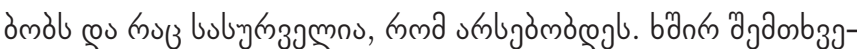

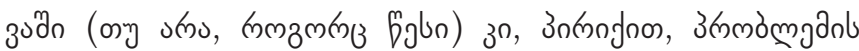

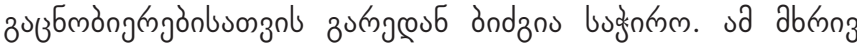

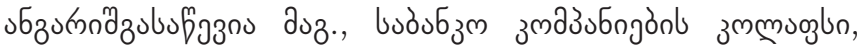

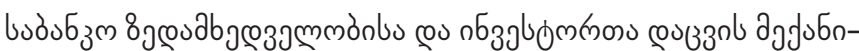
8ajön s s.

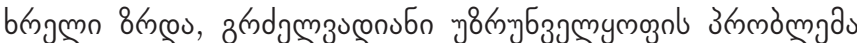

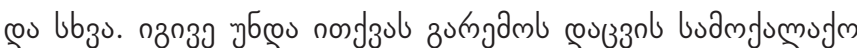

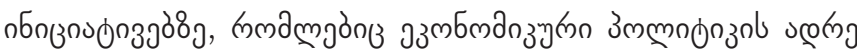

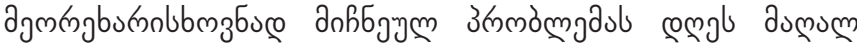

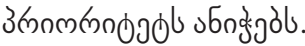

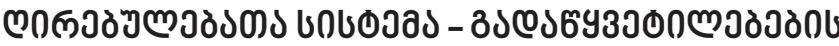

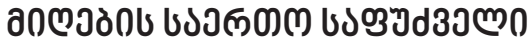

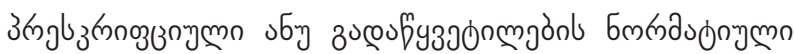

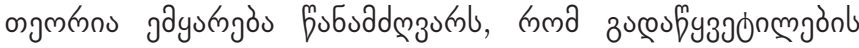

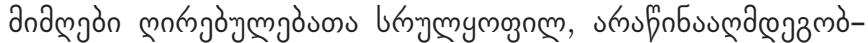

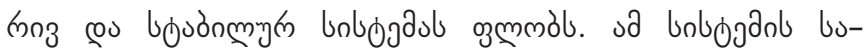

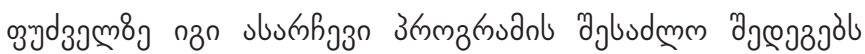

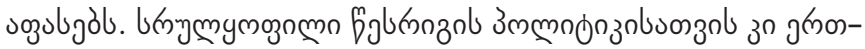

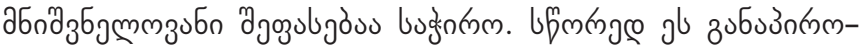

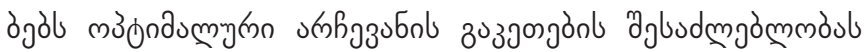

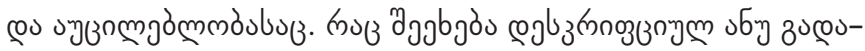

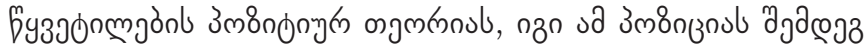

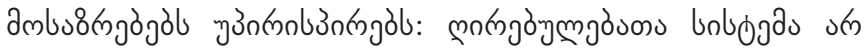

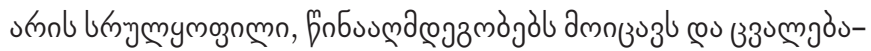

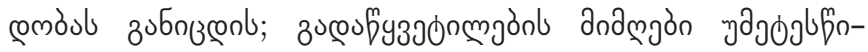

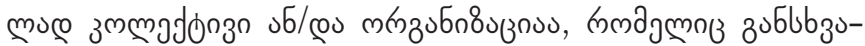

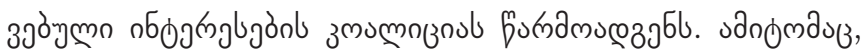

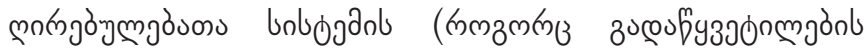

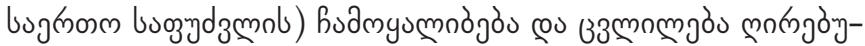

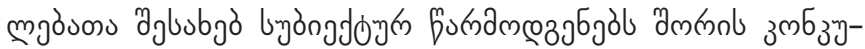

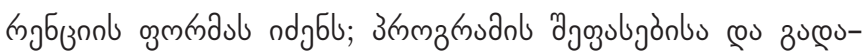

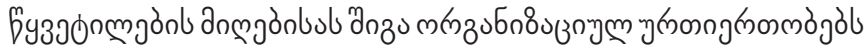

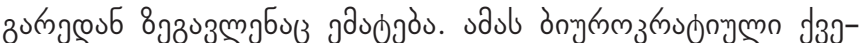

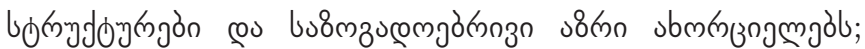

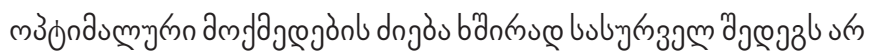

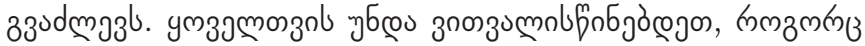

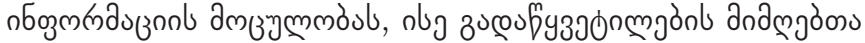

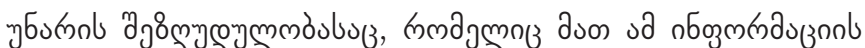

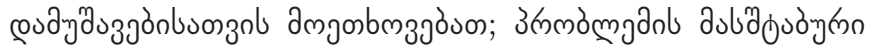

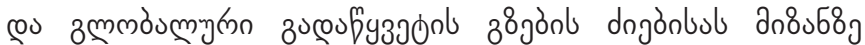

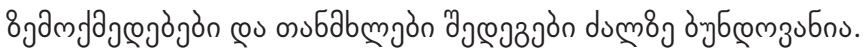

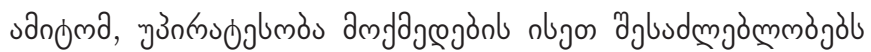

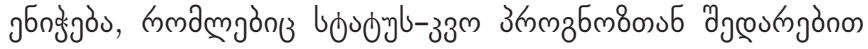

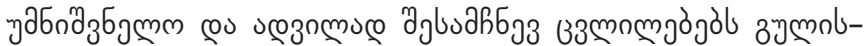
bamoll (Lindblom, 1958).

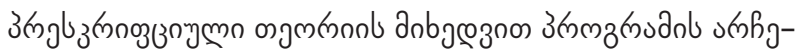

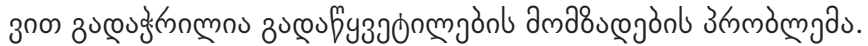

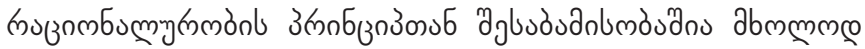

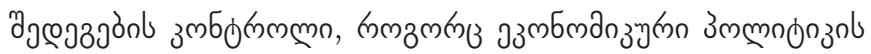

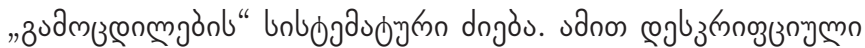

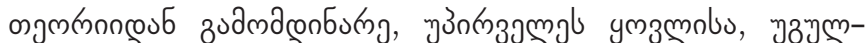

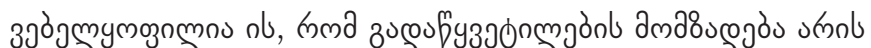

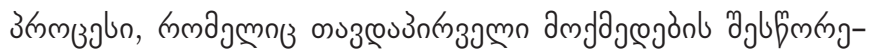

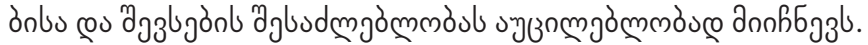

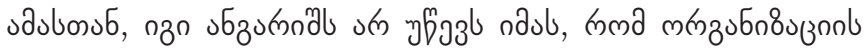

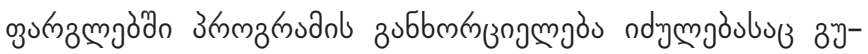

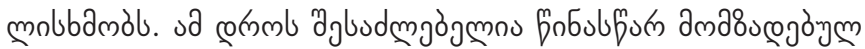

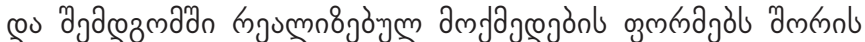

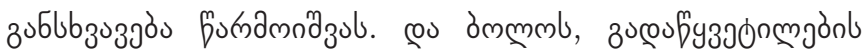

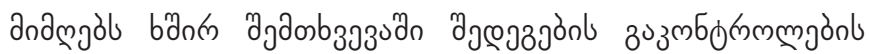

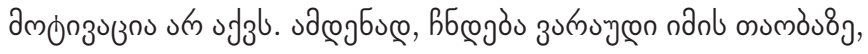




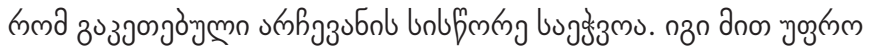

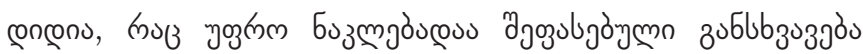

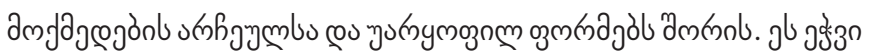
zuмouf

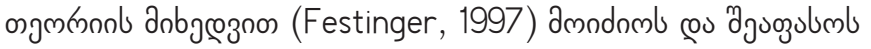

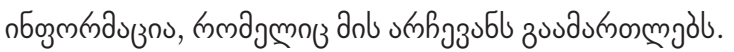

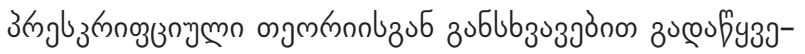

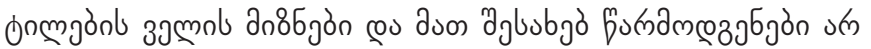

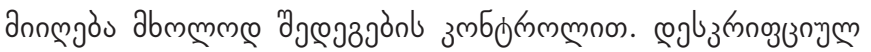

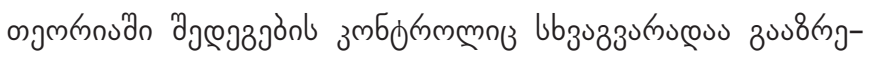

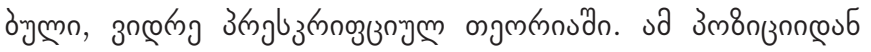

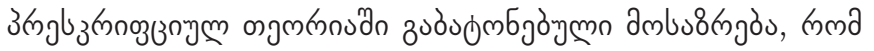

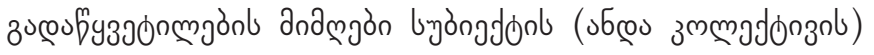

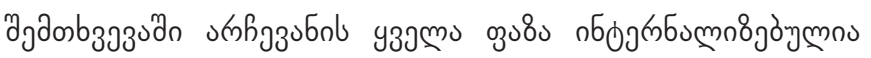

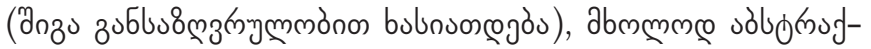

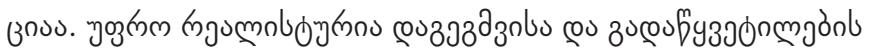

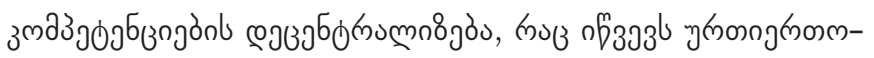

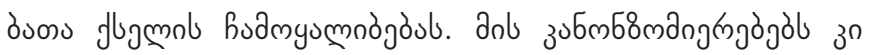
s «h

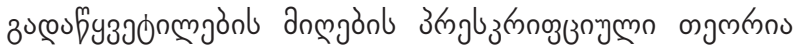

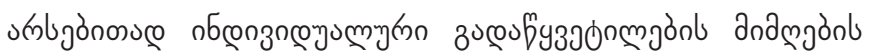

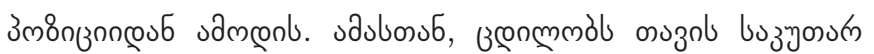
пбоgng

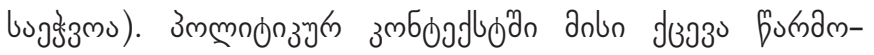

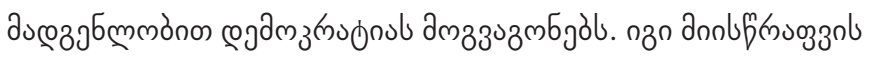

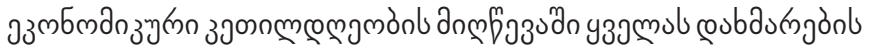

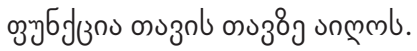

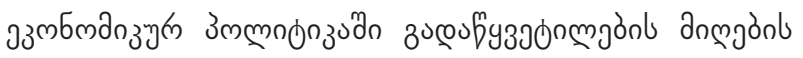

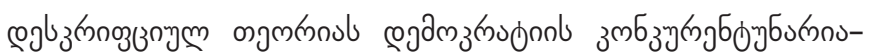

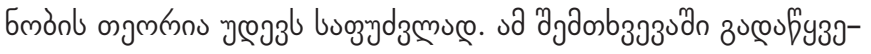

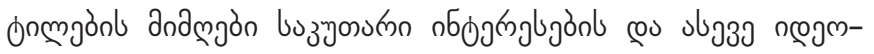

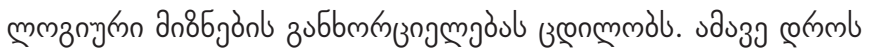

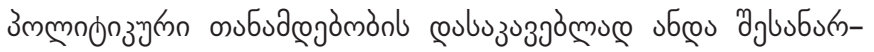

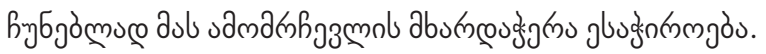

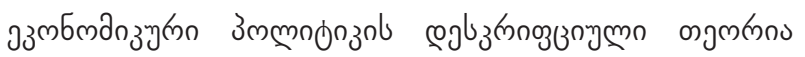

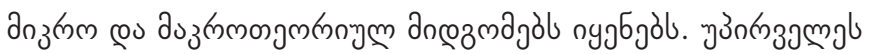

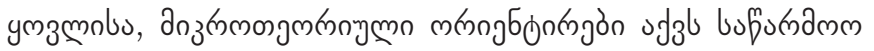

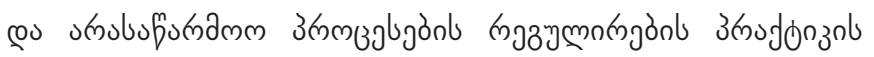
sbumn8ls [Stigler, 1975; Peltzman, 1976]. sas3a conmb,

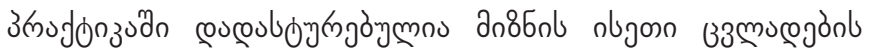

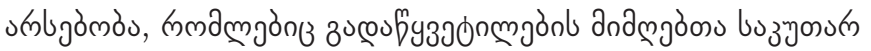

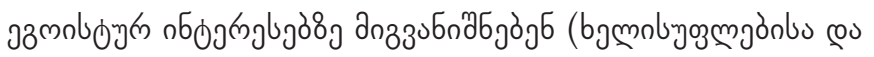

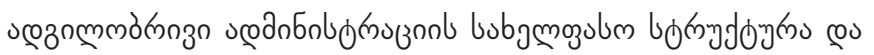

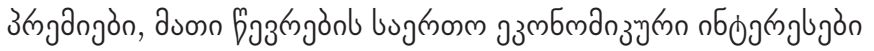

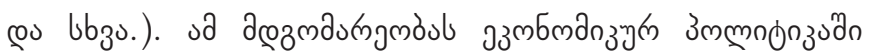

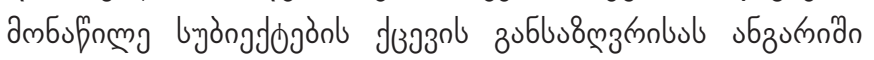

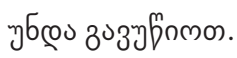

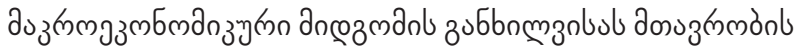

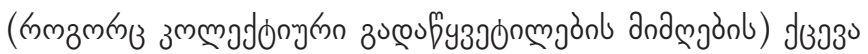

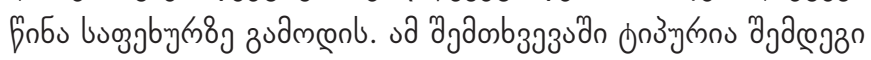

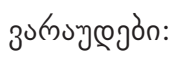

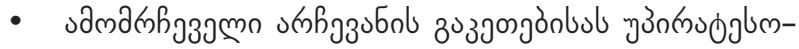

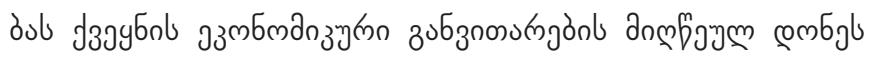

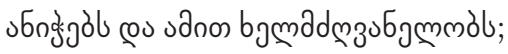

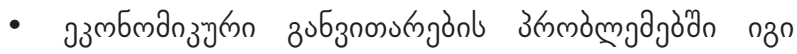

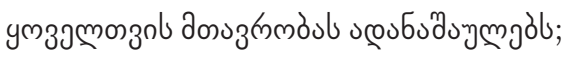

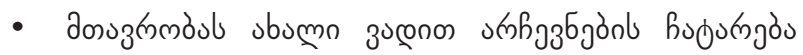

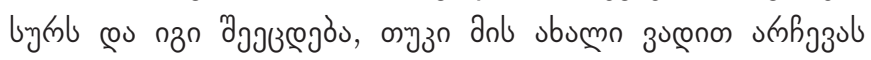

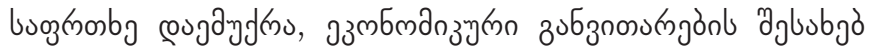

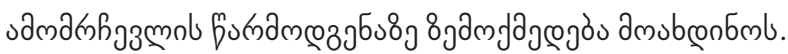

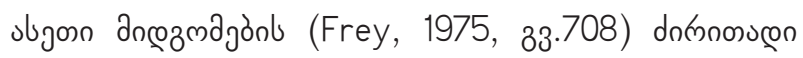

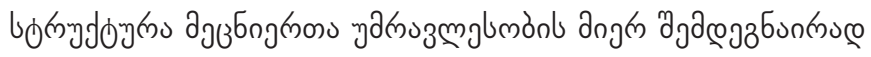
smol fornamezjбомmo:

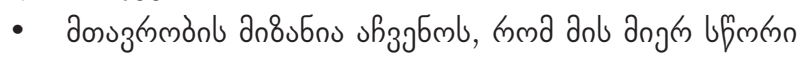

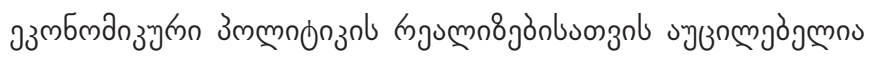

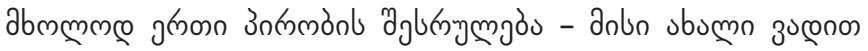

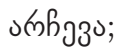

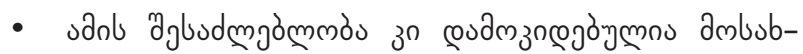

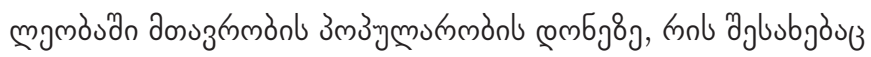

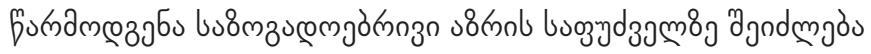
gaz3oda6sb;

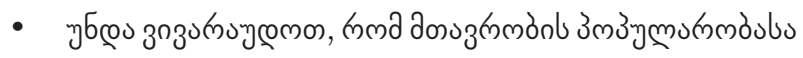

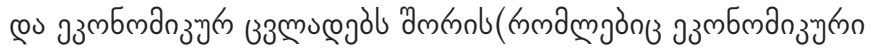

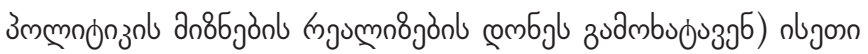

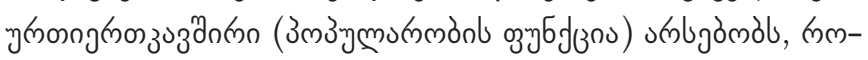

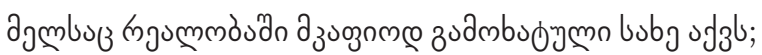

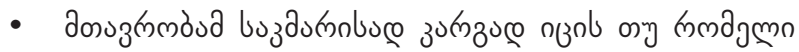

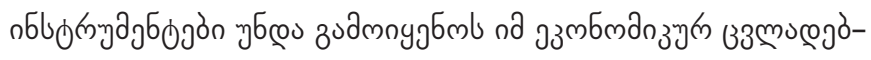

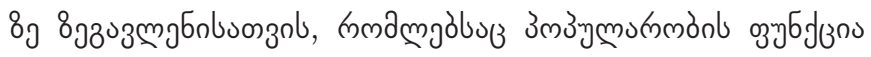
zush6noo;

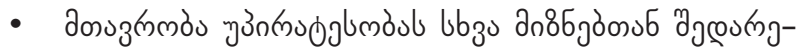

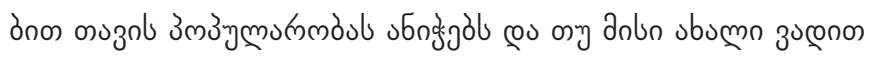

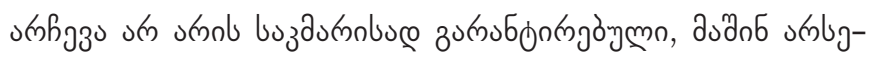

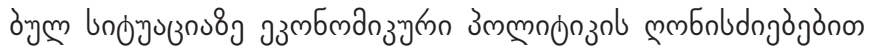

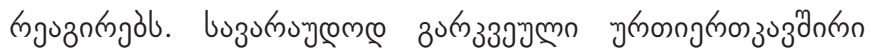

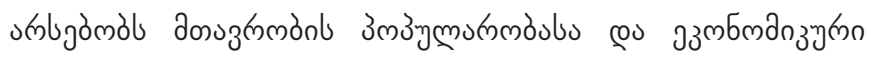

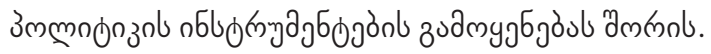

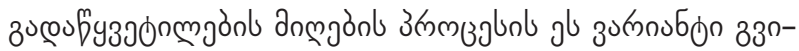

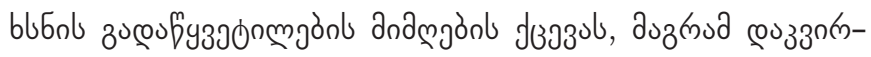

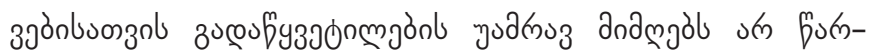

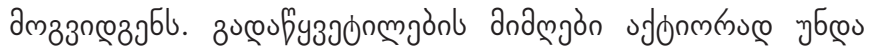

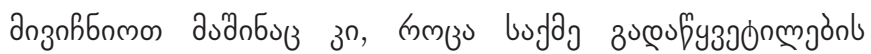

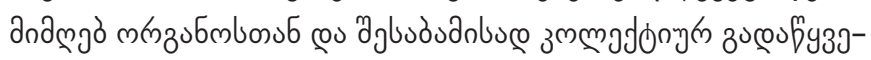

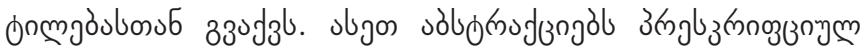




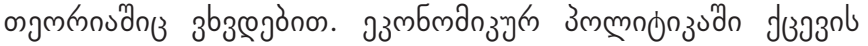
3ublu8 8 zुm

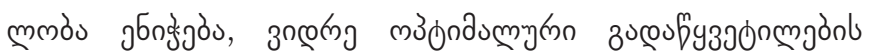

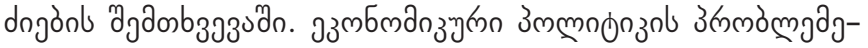

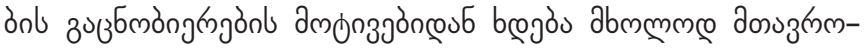

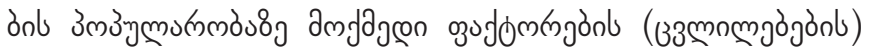

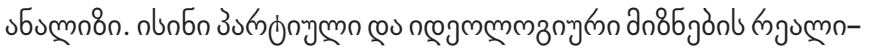

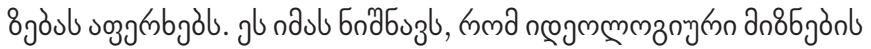

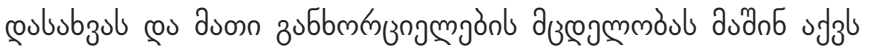

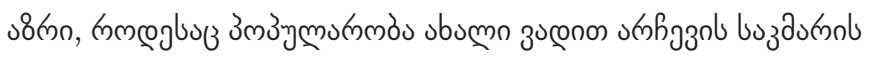

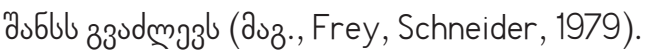

\section{esu335s}

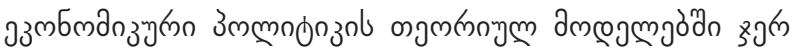

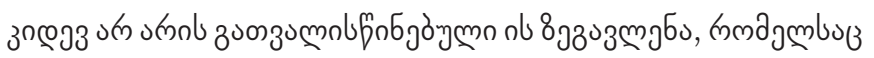

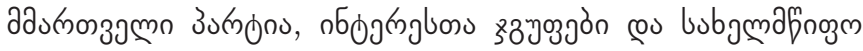

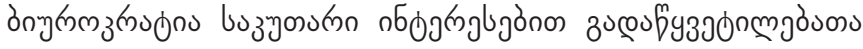

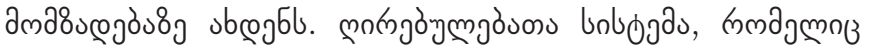

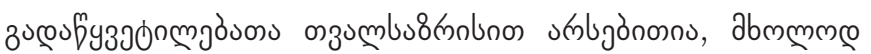

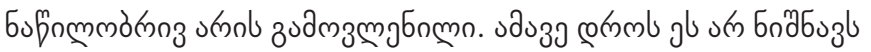

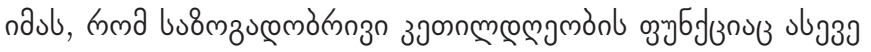

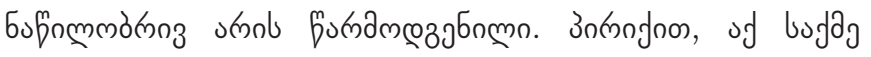

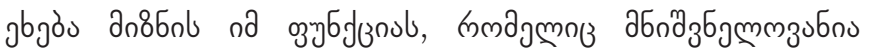

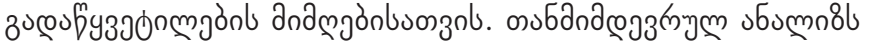

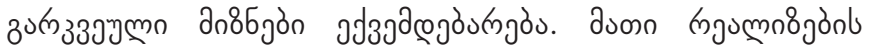

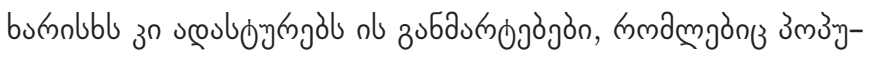

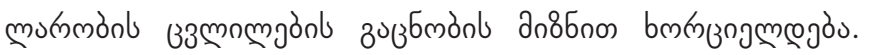

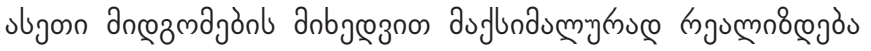

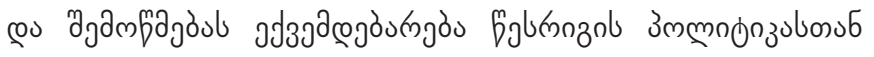

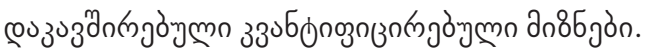

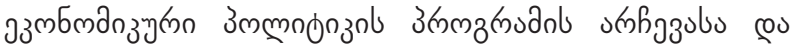

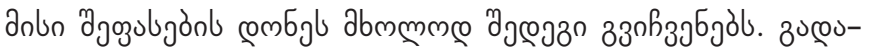

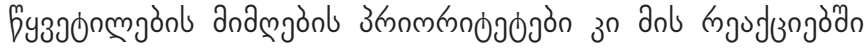

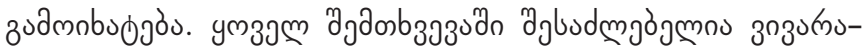

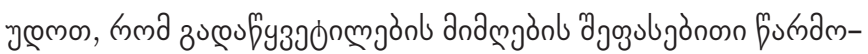

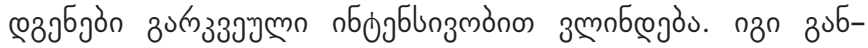

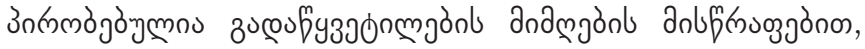

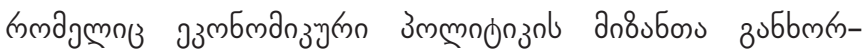

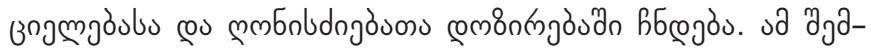

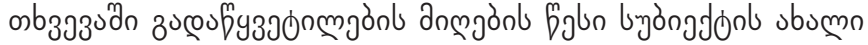

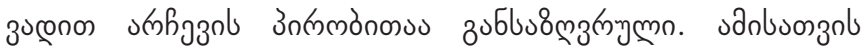

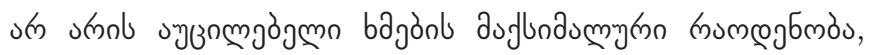

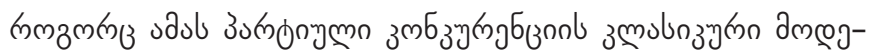

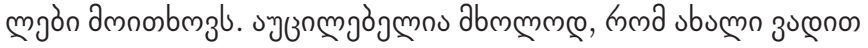

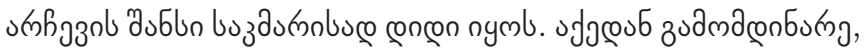

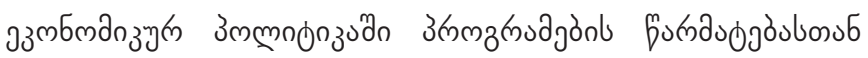

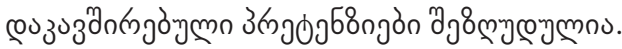

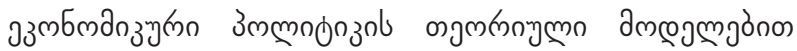

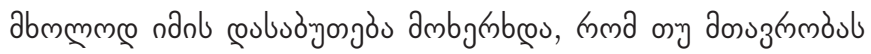

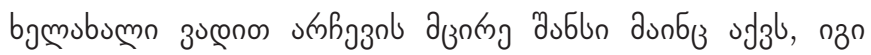

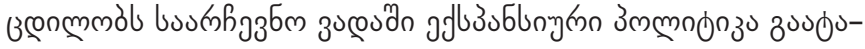

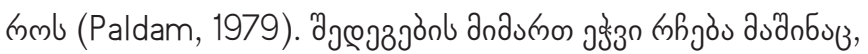

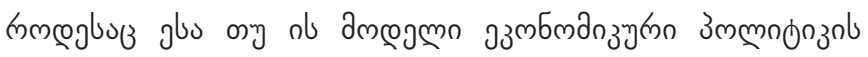

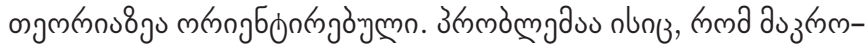

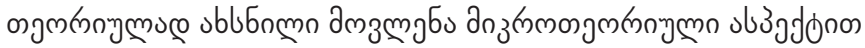

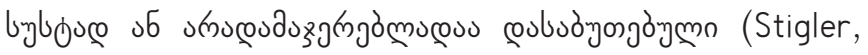

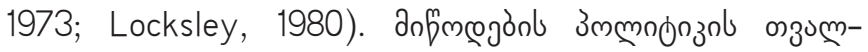

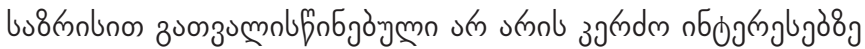

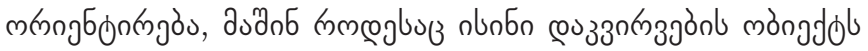

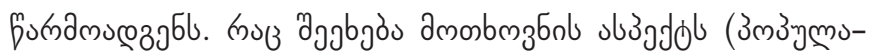

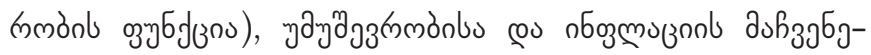

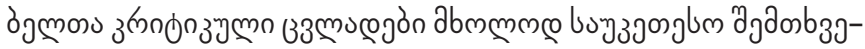

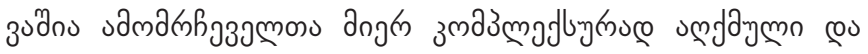

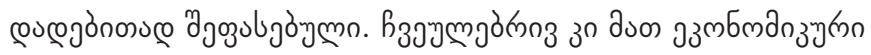

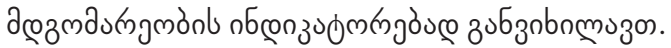




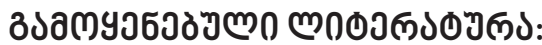

1. Gvelesiani R. (2017). The problem of considering possible consequences of the consistent reforms in the economic-political decisions, Review of Business research (RBR), Volume 17, N2, ISSN: 1546-2609, University of Sassari, Italy, Editor: University of Osijek, Croatia, p.19-24.

2. Gvelesiani R. (2018). The Problem of Compatibility of Market Liberty With Social Equality, european journal of business researchtm (EJBR), Volume 18, N1, ISSN: 1945-2977, Institute for Social Science Research (ISF), Munich, editor-in-chief: Professor Christine Duller, Johannes Kepler University Linz, Austria, p.39-44. www.iabe.org

3. Festinger, L. (1957). A Theory of Cognitive Dissonance, Neudruck, Stanford 1997.

4. Frey, B.S. (1975). Eine Einschaetzung der Neuen Politischen Oekonomie der siebziger Jahre. Zeitschrift fuer die gesamte Staatswissenschaft 131, S.697-718.

5. Frey, B.S. und F. Schneider (1979). An Economeric Model with an Endrogenous Government Sector. Public Choice 34, S.29-43. 6. Kirsch, W. (1970). entscheidungsprozesse (Bd. 1: Verhaltenswissenschaftliche Ansaetze der Entscheidungstheorie), Wiesbaden, S.68.

7. Lindblom, CH. E. (1958). Policy Analysis. American Economic Review 48, S.298-312.

8. Locksley, G. (1980). The Political Business Cycle: Alternative Interpretations: inp. Whiteley (Hrsg.): Models of Political Economy, London, Beverly Hills, S.177-198.

9. Paldam, M. (1979). Is there an Electional Cycle? - A Comparative Study of National Accounts. Scandinavian Journal of Economics 81, S.323-342.

10. Peltzman, S. (1976). Toward a More General Theory of Regulation. Journal of Law and Economics 19, S.211-240.

11. Stigler, G.J. (1973). General Economic Conditions and National Elections American Economic Review 63, Papers and Proceedings, S.160-167.

12. Stigler, G. J. (1975). The Citizen and the State - Essays on Regulation, Chicago und London.

13. Streit, M. E. (2005). Theorie der Wirtschaftspolitik, 6. Auflage, Stuttgart, S.273; 379-380.

14. Tinbergen, J. (1964), Economic Policy - Principles and Design, 4 th rev. ed., reprint, Amsterdam 1975.

15. ззэ 33.85-86. [Gvelesiani R., Gogorishvili I. (2018). Decision-making Technology in Economic Policy. Tbilisi, pp.85-86. In Georgian]. 16. з3лmgl

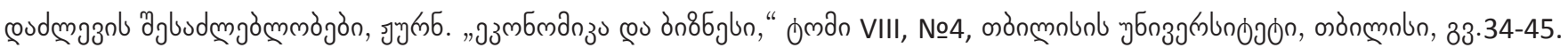
[Gvelesiani R., Gogorishvili I. (2015). Problems of Economic-Political Decision-Making and Opportunities to Overcome, Journal. «Economics and Business» Volume VIII, No. 4, Tbilisi University, Tbilisi, pp. 34-45. In Georgian].

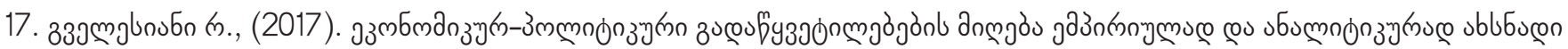

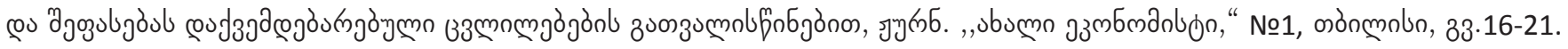
[Gvelesiani R., (2017). Taking into account economic and political decisions empirically and analytically, and taking into account the changes subordinated to the assessment. Journal «Akhali Ekonomisti» №1, Tbilisi, pp. 16-21. In Georgian].

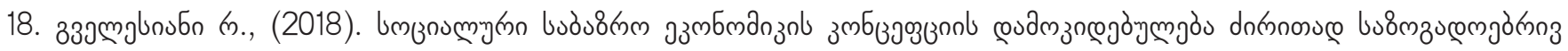

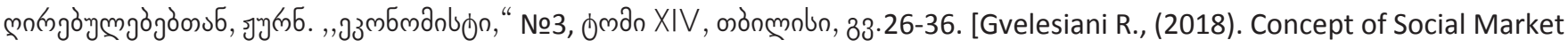
Economy Concept with Basic Public Values, Journal. «Ekonomisti» No. 3, Volume XIV, Tbilisi, pp.26-36. In Georgian]. 


\section{COMPATIBILITY PROBLEM OF BASIC PUBLIC VALUES WITH ECONOMIC POLICY GOALS AND DECISIONS FOR THEIR IMPLEMENTATION}

\section{REVAZ GVELESIANI}

\section{Sc., Professor,}

Ivane Javakhishvili Tbilisi State University,

Academician of the Academy of Economic Sciences of Georgia, Georgia

revaz.gvelesiani@tsu.ge

\section{KEYWORDS: BASIC PUBLIC VALUES, ECONOMIC POLICY GOALS, ECONOMIC INTEREST,} DECISION-MAKING THEORIES, DECISION-MAKERS.

For citation: Gvelesiani R. (2019), Compatibility Problem Of Basic Public Values With Economic Policy Goals And Decisions For Their Implementation, Globalization And Business, №7, pp. 32-38. (In Georgian). https://doi.org/10.35945/gb.2019.07.004

\section{SUMMARY}

Economic policy related decisions are very complex in their logical structure because of the multilateral interdependence of the Social-economic events. Every decision includes some kind of vagueness. It is either clear or vague whether economic-political situation and results of discussed actions are analyzed well. The logical structure of decisions is the objective of the theory of the decision making. This theory helps to assess and overcome the problems in economic policy related decision making.

Economic policy is oriented on achieving the predefined goals. These goals are determined by economic situation and many normative attitudes (basic values). There is an agreement on one part of the goals among the majority of developed countries. The laws and resolutions issued by different governments often include economic indicators, including: significant economic growth, high level of employment, appreciating the currency as an indicator of stability of prices, foreign economic relations, foreign economic equilibrium or fair distribution of the income and the property. This is the partial list of the goals, but it is enough to demonstrate that goals are the only means (instruments) for realizing all-inclusive norms of basic public values. 


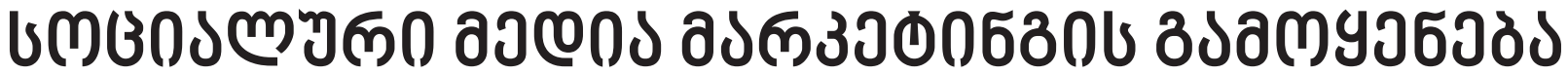

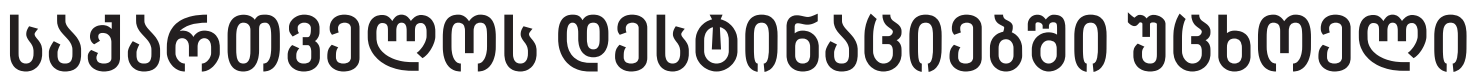

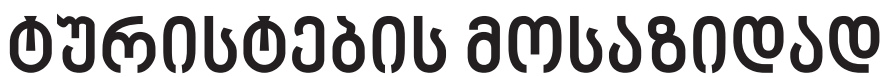

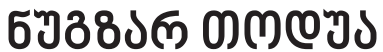

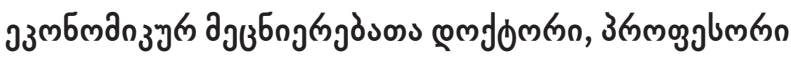

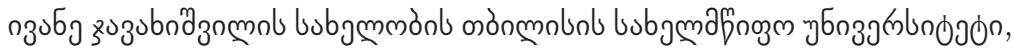

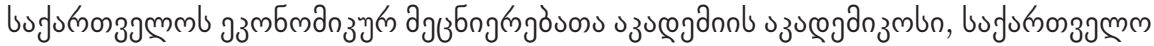

Nugzar.todua@tsu.ge

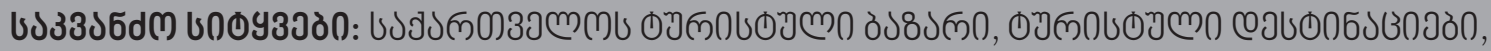

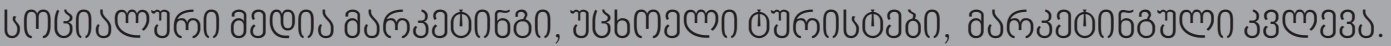

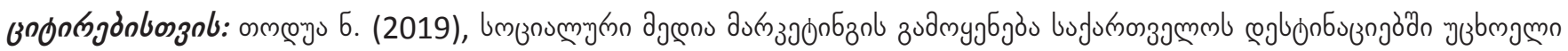

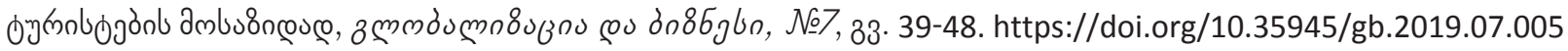

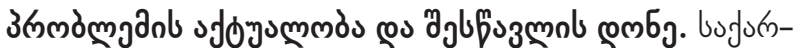

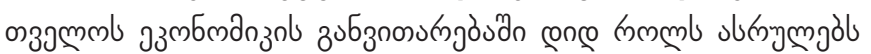

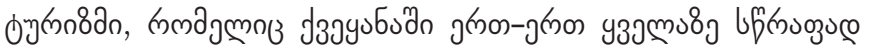

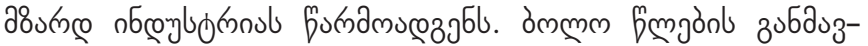

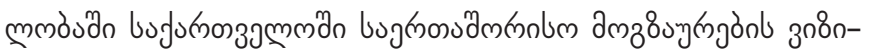

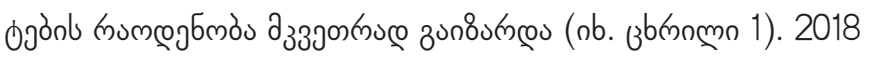

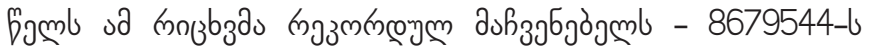

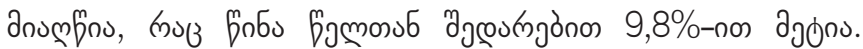

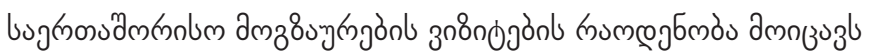

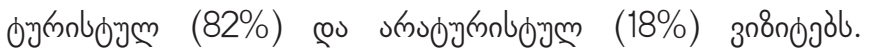

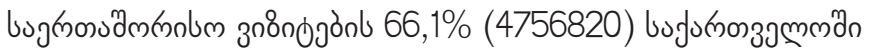

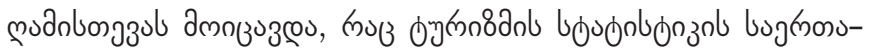

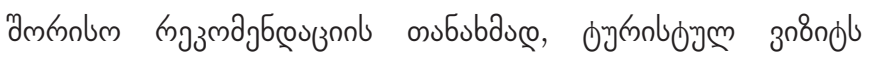

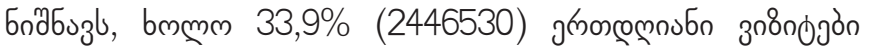

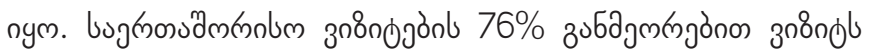

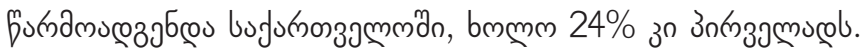

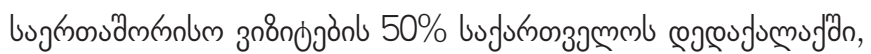

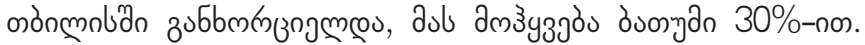

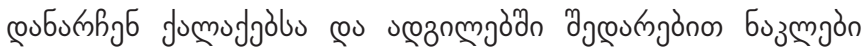

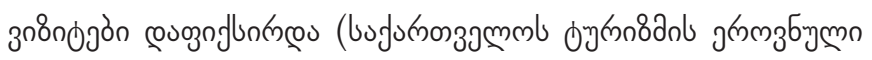

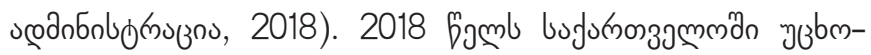

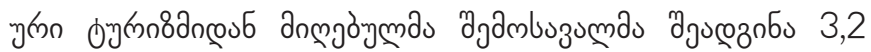

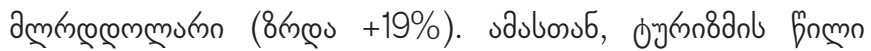

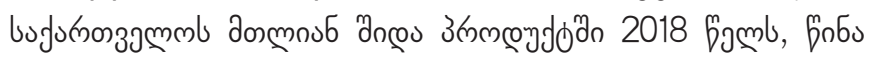

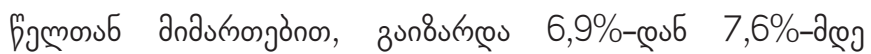

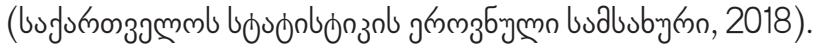

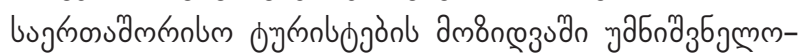

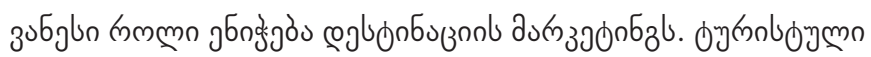

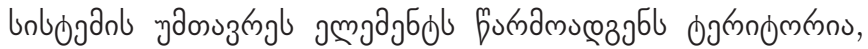

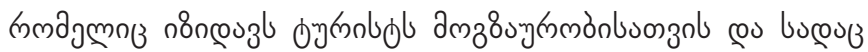

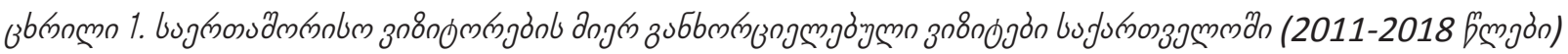

\begin{tabular}{|c|c|c|c|c|c|c|c|c|}
\hline 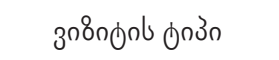 & 2011 bुตฺn & 2012 bुวmก & 2013 бృตรก & 2014 bృmก & 2015 ด̆อмก & 2016 fృตฺn & 2017 Øึวตn & 2018 бวตूก \\
\hline 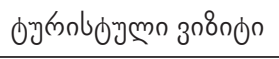 & 1832788 & 2459794 & 2884295 & 2938892 & 3011663 & 3297275 & 4069354 & 4756820 \\
\hline 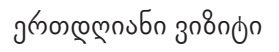 & 878909 & 1646080 & 2070153 & 2065439 & 2244336 & 2095541 & 2413476 & 2446530 \\
\hline $\operatorname{ligm}$ & 2711697 & 4105874 & 4954448 & 5004331 & 5255999 & 5392816 & 6482830 & 7203350 \\
\hline
\end{tabular}

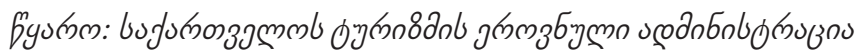




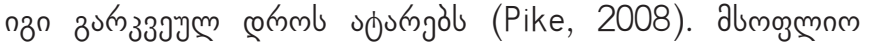

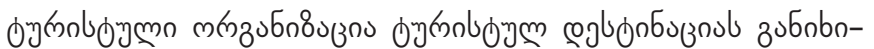

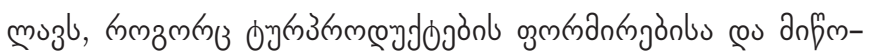

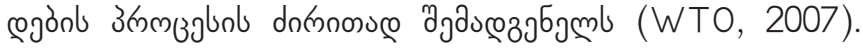

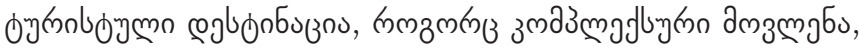

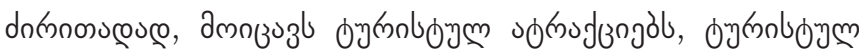

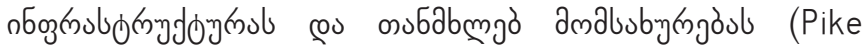

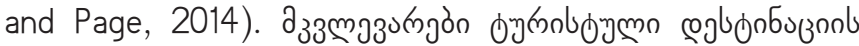

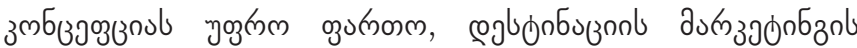

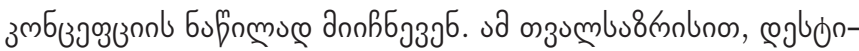

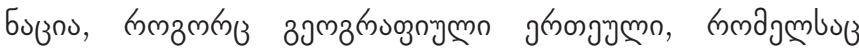

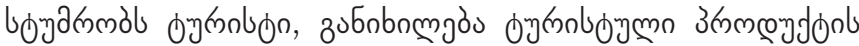

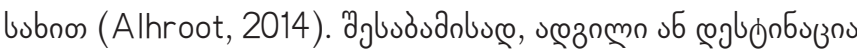

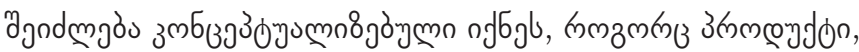
mmajmmn dmnbaurngos (Hausteinova, 2013; Nguyen,

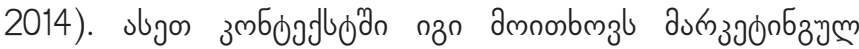

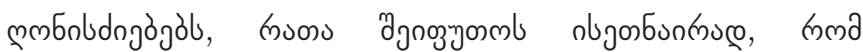

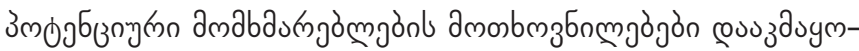
oुnmolb (Benckendorff, and Black, 2005).

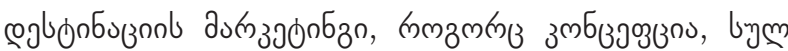

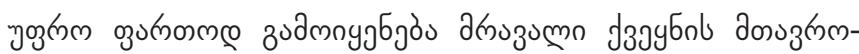

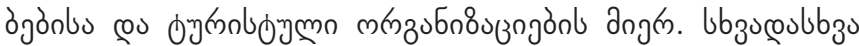

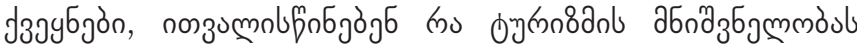

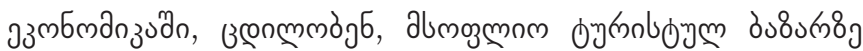

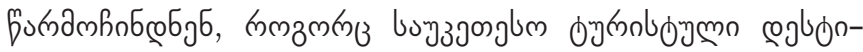

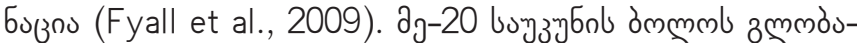

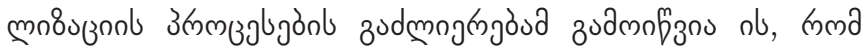

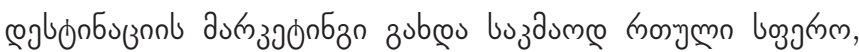

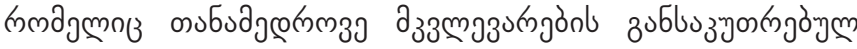

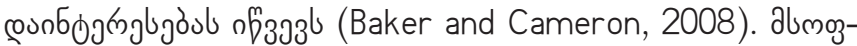

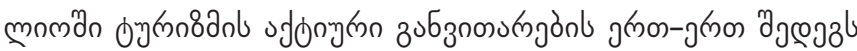

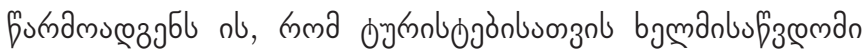

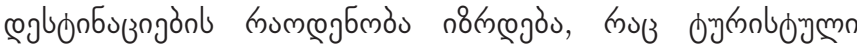

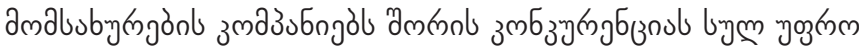

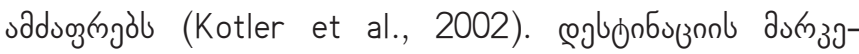

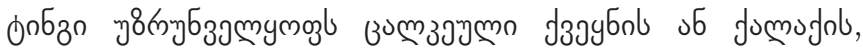

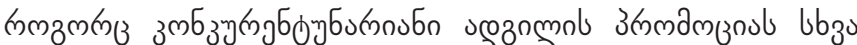

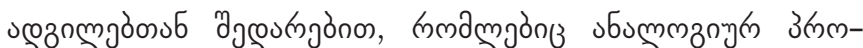

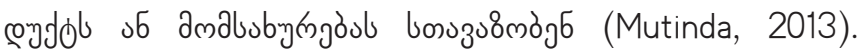

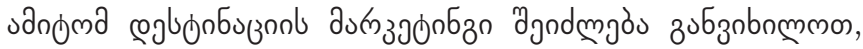

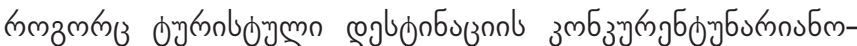

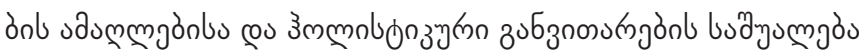
(Wanjala, 2015).

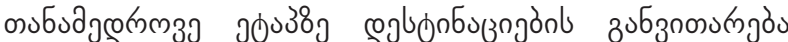

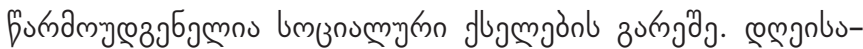

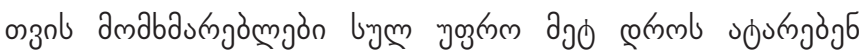

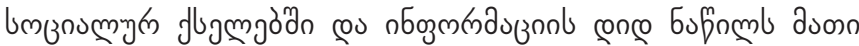

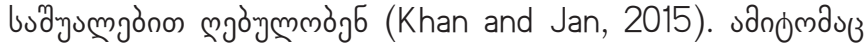

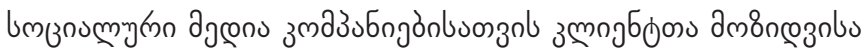

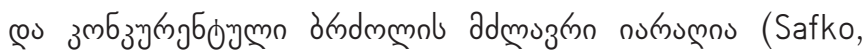

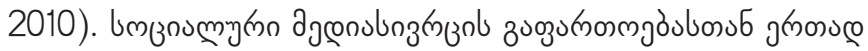

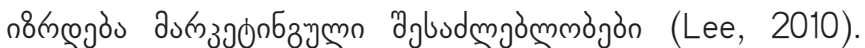

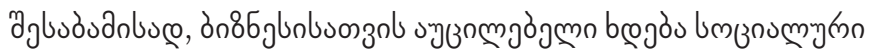

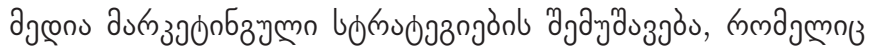

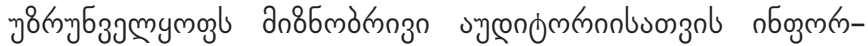

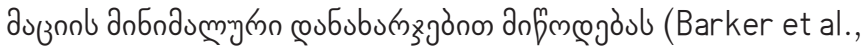

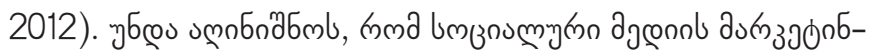

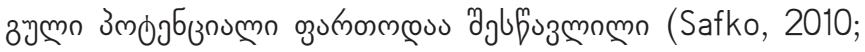

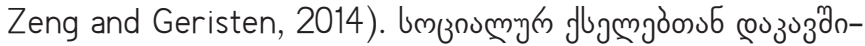

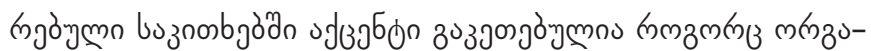

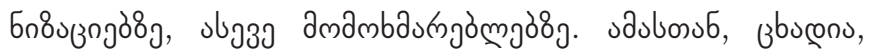

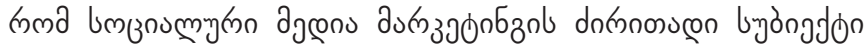

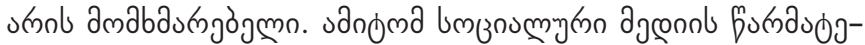

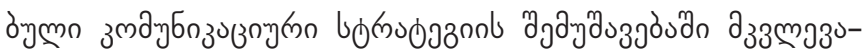

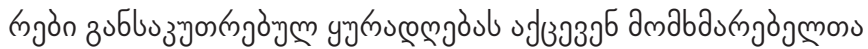

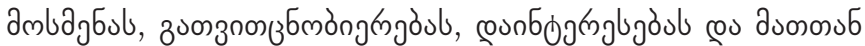
yrnongrnomobul (Sweeney and Craig, 2011).

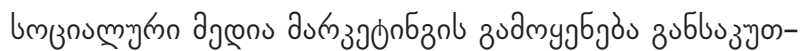

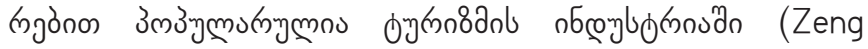
and Geristen, 2014; Evangelos, 2012). Jubmoknnzo dgenols

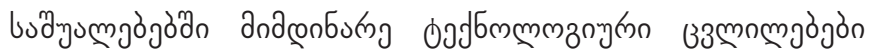

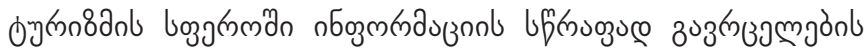

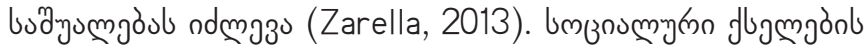

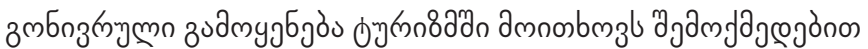

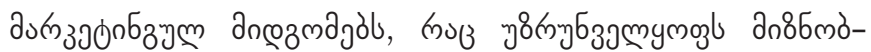

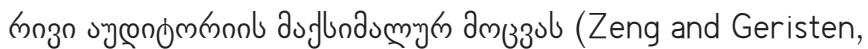

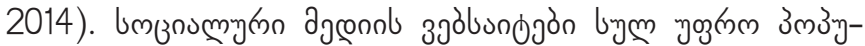

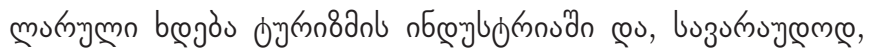

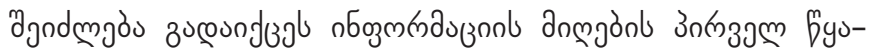

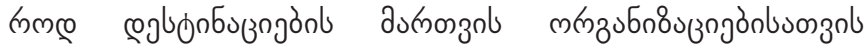

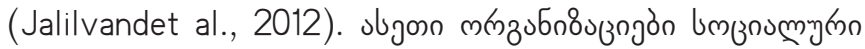

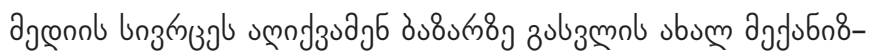

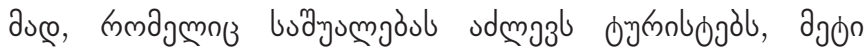

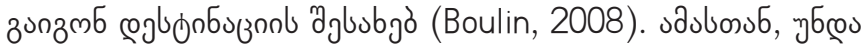

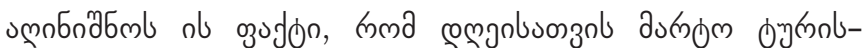

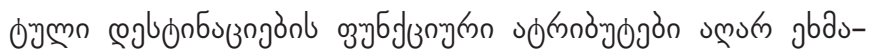

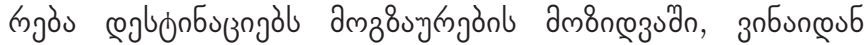

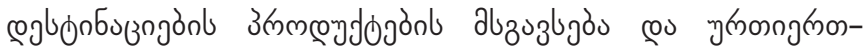

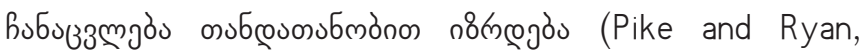

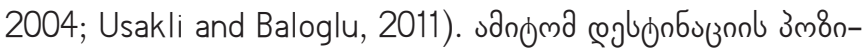

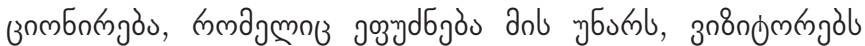

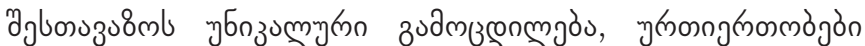

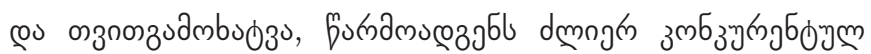

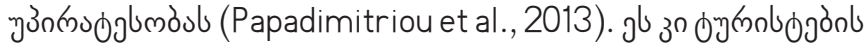




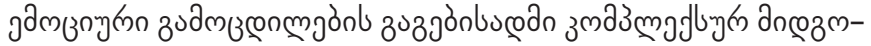
aub amnosmasl (Garcia et al., 2012; Blain et al., 2005).

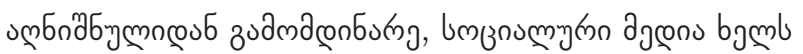

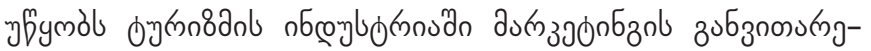

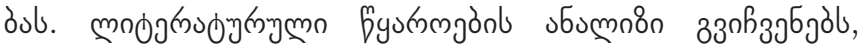

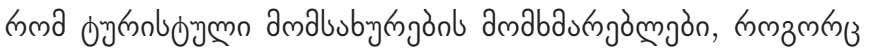

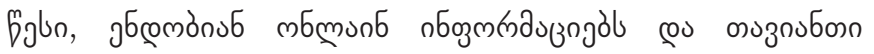

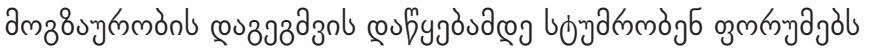

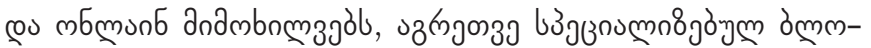
3

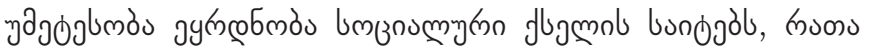

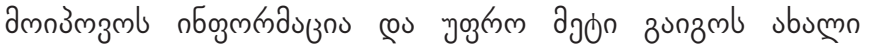

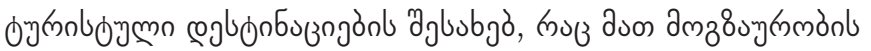

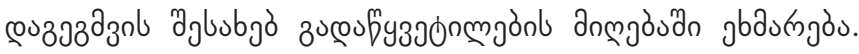

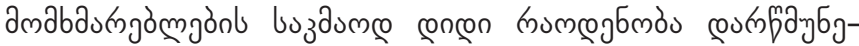

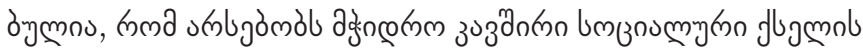

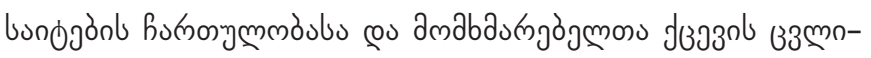

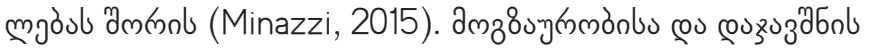

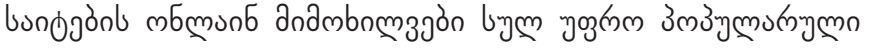

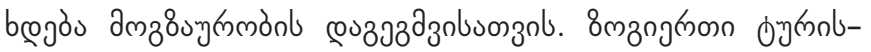

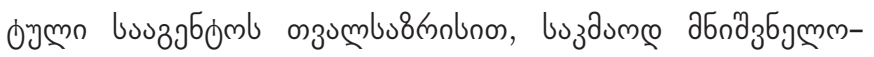

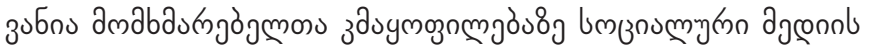

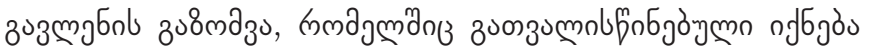

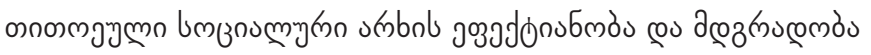
(Schaefer, 2014).

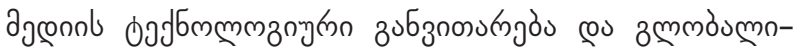

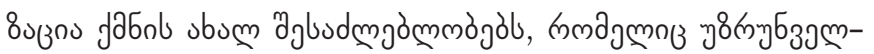

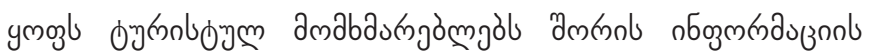

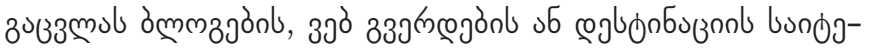

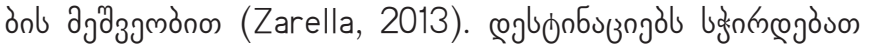

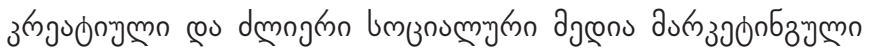

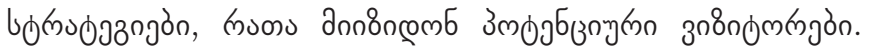

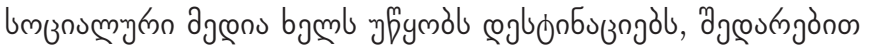

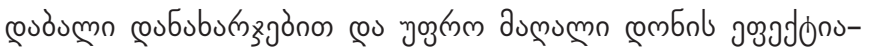

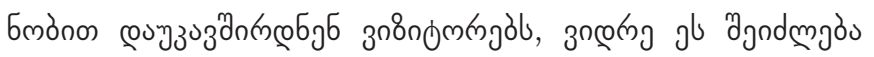

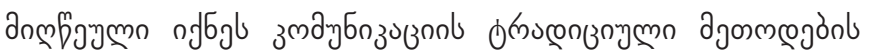

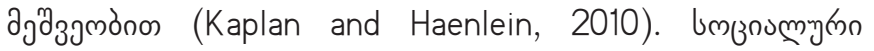

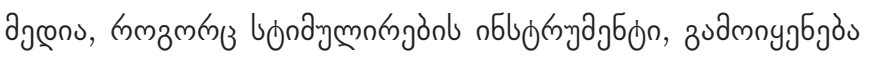

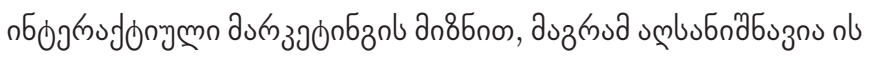

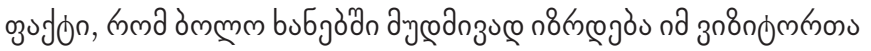

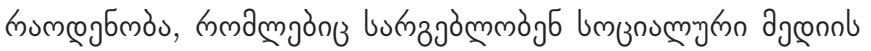

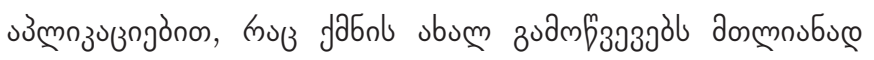

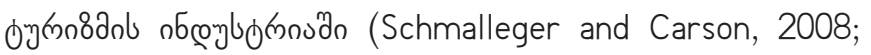
Heinonen, 2011; Carrand Hayes, 2015).

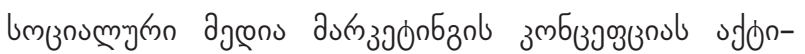

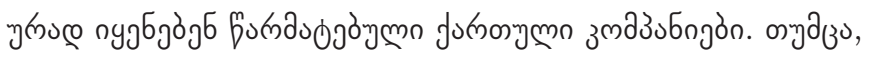

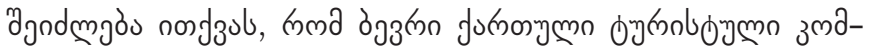

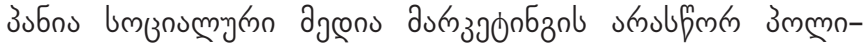

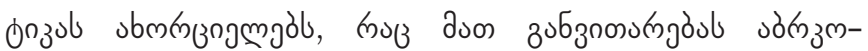

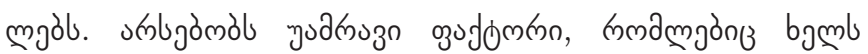

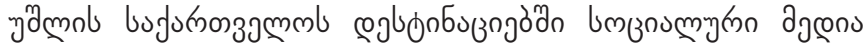

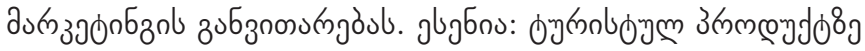

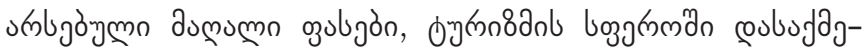

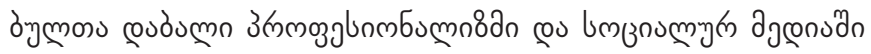

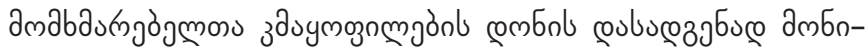

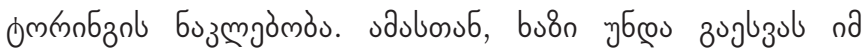

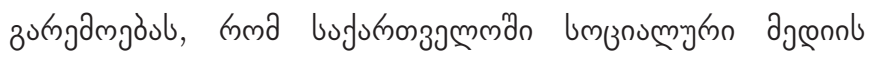

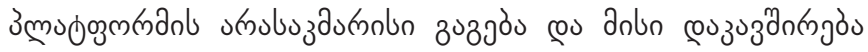

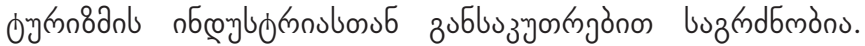

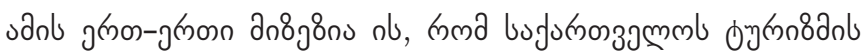

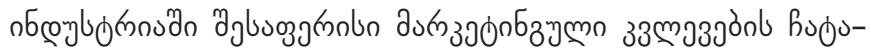

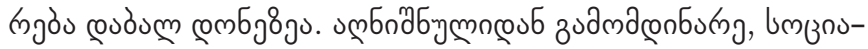

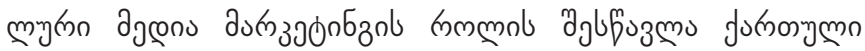

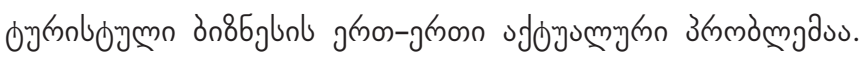

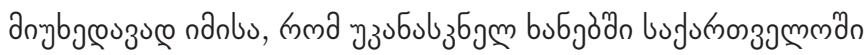

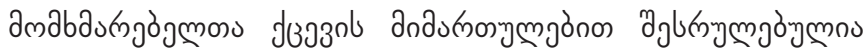

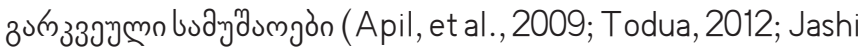
and Todua, 2013; Todua et al., 2013; Todua and Dotchviri, 2015 a; Todua and Dotchviri, 2015 b; Todua and Jashi, 2015; Todua et al., 2015; Todua and Jashi, 2016; Todua et al., 2016; Mghebrishvili and Urotadze, 2016; Todua et al., 2017; Todua and Gogitidze, 2017; Todua, 2017 a; Todua, 2017 b; Seturi

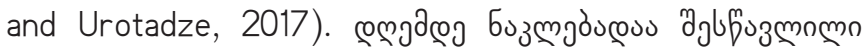

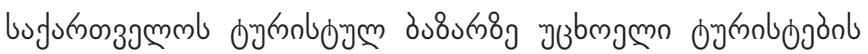

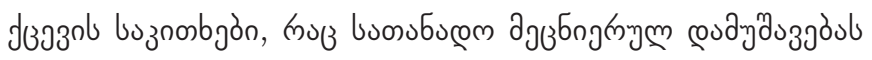

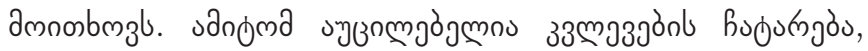

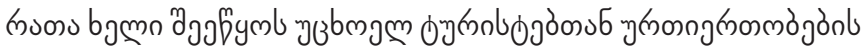

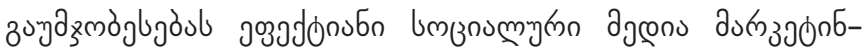

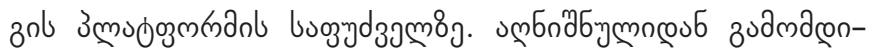

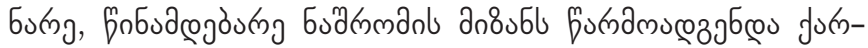

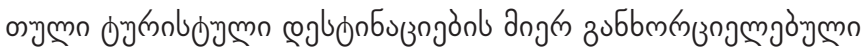

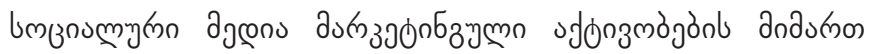

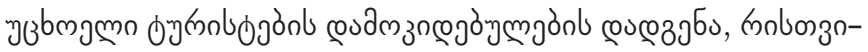

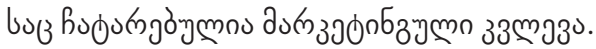

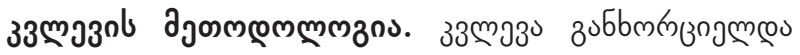

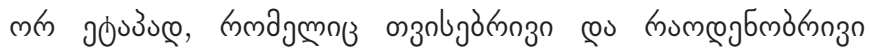

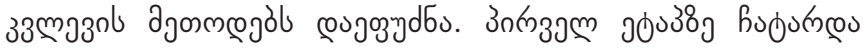

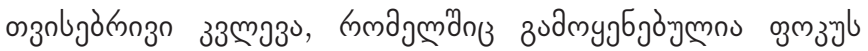

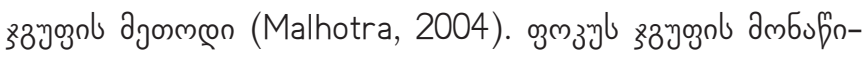

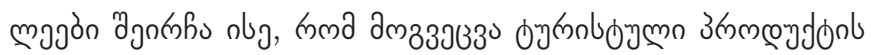

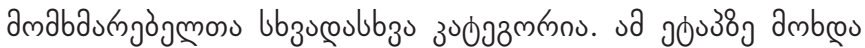

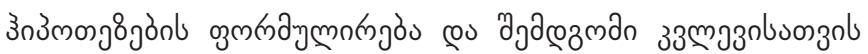

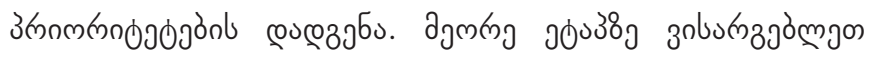

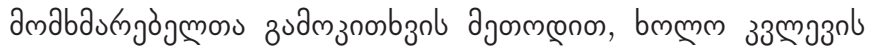




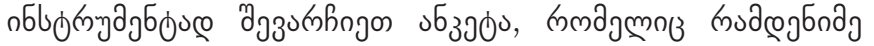

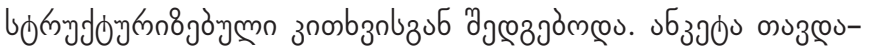

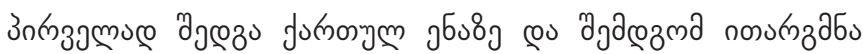

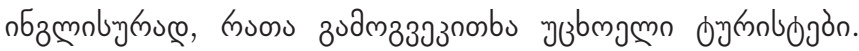

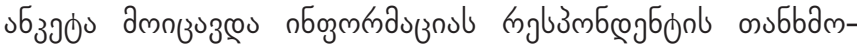

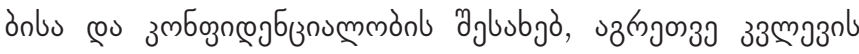

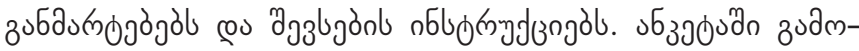

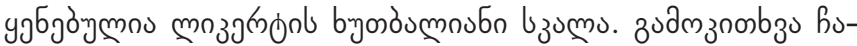

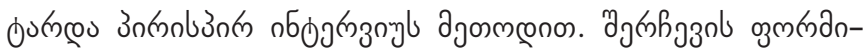

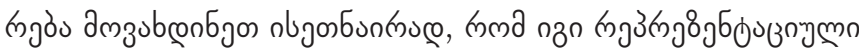

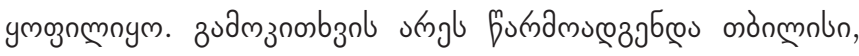

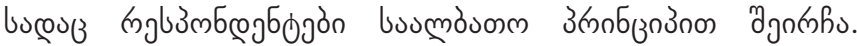

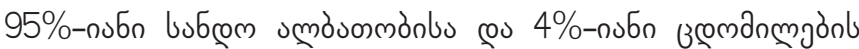
zunzummolf

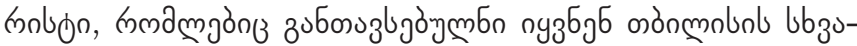

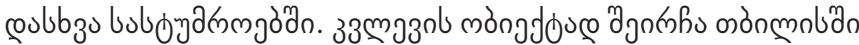

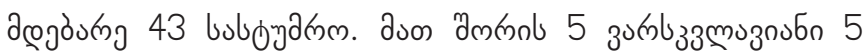

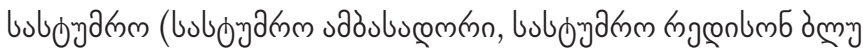

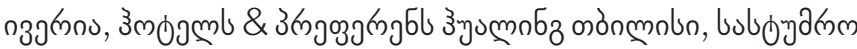

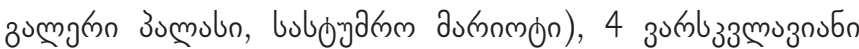

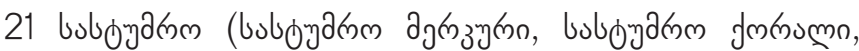

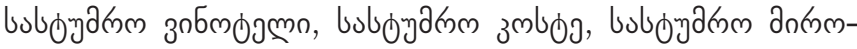

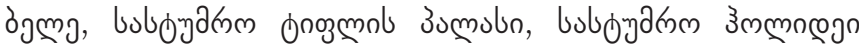

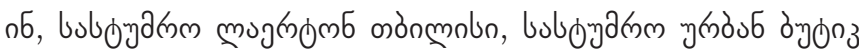

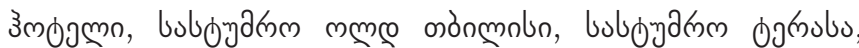

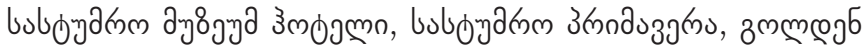

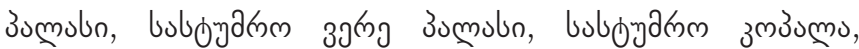

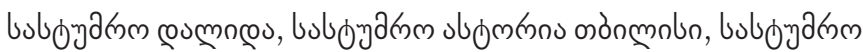

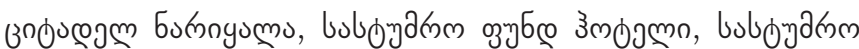

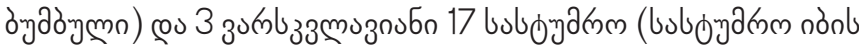

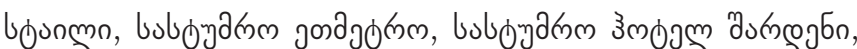

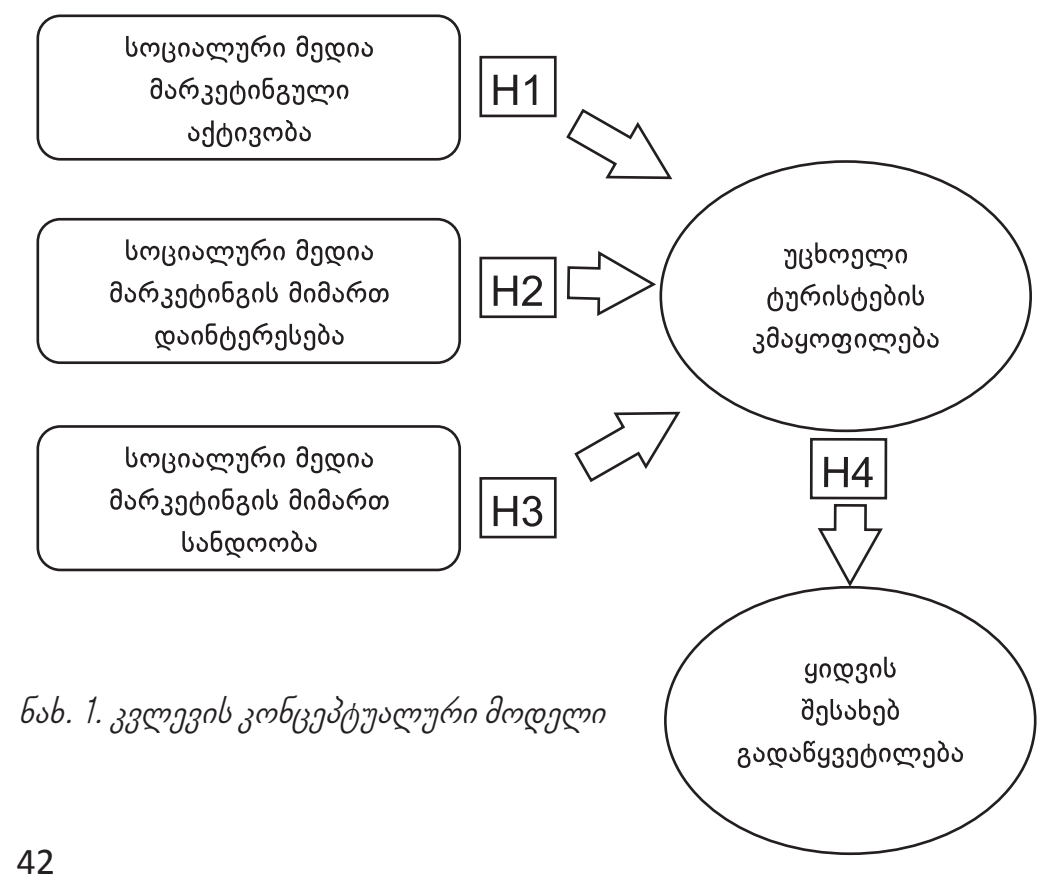

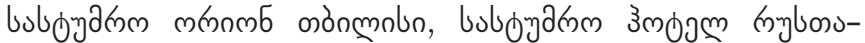

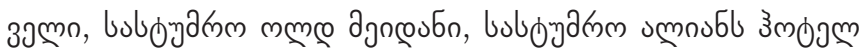

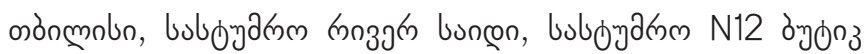

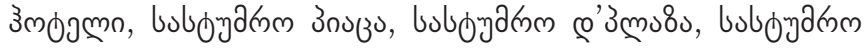

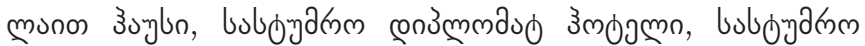

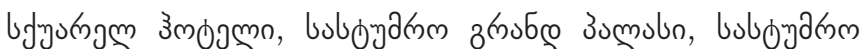

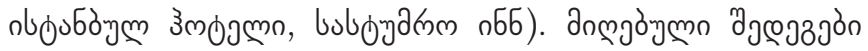

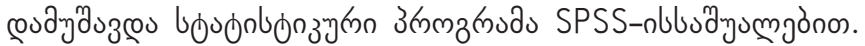

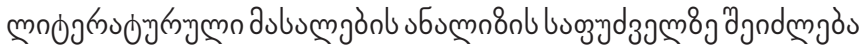

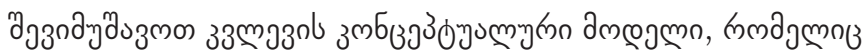

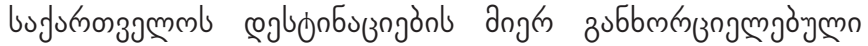

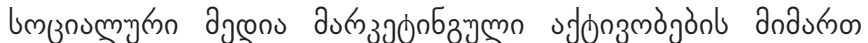

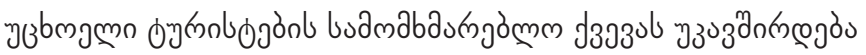
(ob. Eob. 1).

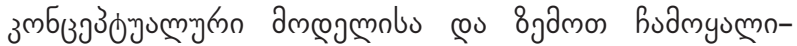

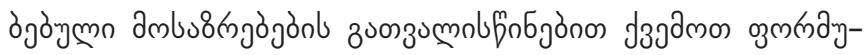

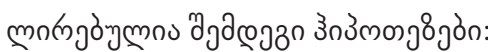

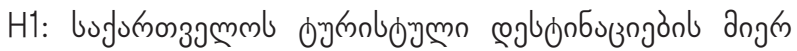
зubbmkrzojm

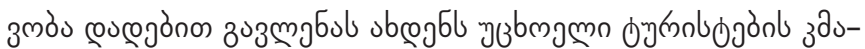

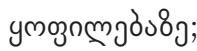

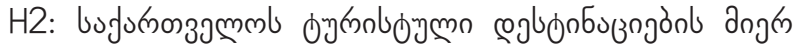

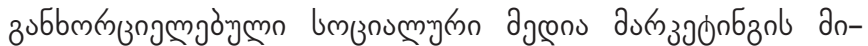

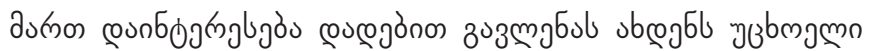

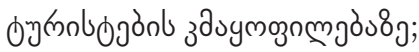

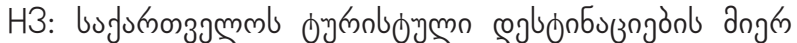

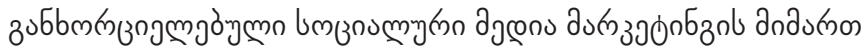

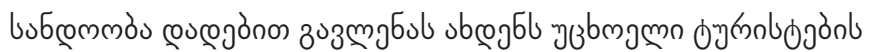
здаумоुпм

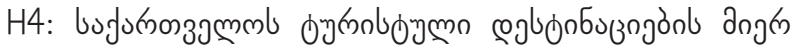

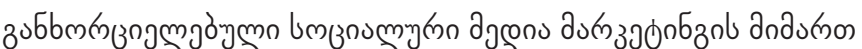

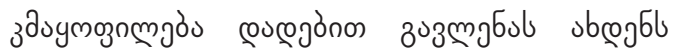

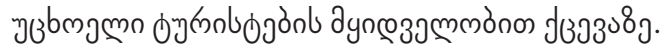

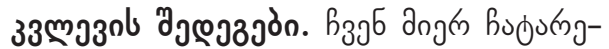

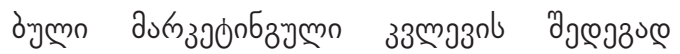

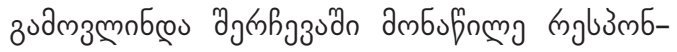

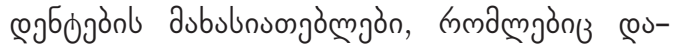

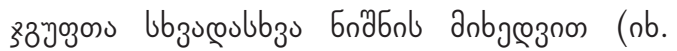

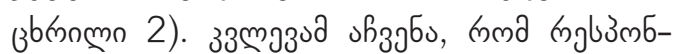

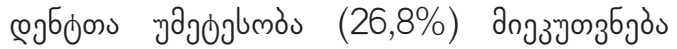

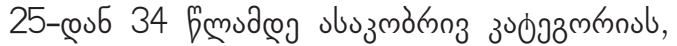

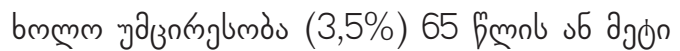

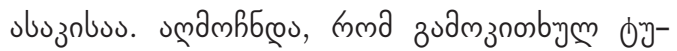

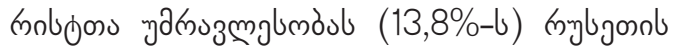

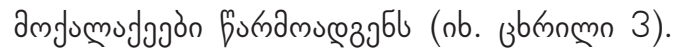

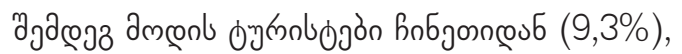

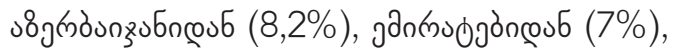




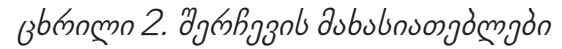

\begin{tabular}{|c|c|c|c|}
\hline aububnuongòmgòn & $\%$ & asbulunuogjąmgòn & $\%$ \\
\hline nozozn & & coubufajos & \\
\hline 18-24 & 16,7 & $\begin{array}{l}\text { agfondgl } \\
\text { agmmmógmn }\end{array}$ & 13,4 \\
\hline $25-34$ & 26,8 & 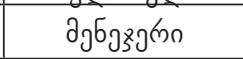 & 27,8 \\
\hline $35-44$ & 22,3 & b3joznumalbon & 39,4 \\
\hline $45-54$ & 18,7 & 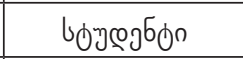 & 12,7 \\
\hline $55-64$ & 12,0 & $6 b_{3}$ & 6,7 \\
\hline 65 bुmº̀ ajon & 3,5 & 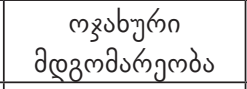 & \\
\hline bjglon & & durnombjms & 41,4 \\
\hline dumn & 56,8 & cumz̧ubjàyman & 58,6 \\
\hline 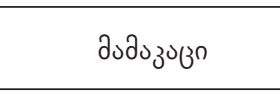 & 43,2 & 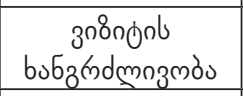 & \\
\hline subsongyoso & & $1-3 \cos 0$ & 29.8 \\
\hline bodyumm & 12,0 & 4-5 gем0 & 34,6 \\
\hline 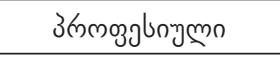 & 21,5 & 1330 mo & 21,2 \\
\hline dozumuzmo & 29,3 & 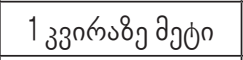 & 14,4 \\
\hline duzollogn & 34,1 & 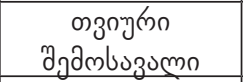 & \\
\hline smdommo & 3,1 & $\begin{array}{c}1000 \text { ady } \\
\text { commumagrog }\end{array}$ & 5,6 \\
\hline 3nonon & & $\begin{array}{c}1001-2000 \text { s.d } \\
\text { cmmomn }\end{array}$ & 8,7 \\
\hline 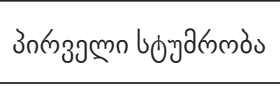 & 51 & 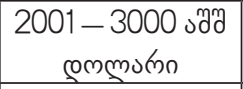 & 23,8 \\
\hline 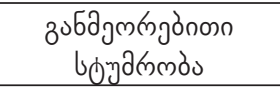 & 49 & $\begin{array}{c}3001-4000 \text { sdy } \\
\text { emmumn }\end{array}$ & 17,4 \\
\hline 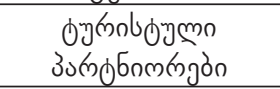 & & $\begin{array}{c}4001-5000 \text { sd } \\
\text { cmmumn }\end{array}$ & 13,9 \\
\hline dumom & 19,5 & 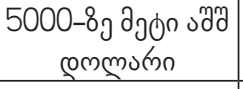 & 12,1 \\
\hline аээкмл & 34,8 & yు & 18,5 \\
\hline mz̧obn & 5,7 & & \\
\hline ajzmòmgòn & 26,7 & & \\
\hline змm & 13,3 & & \\
\hline
\end{tabular}

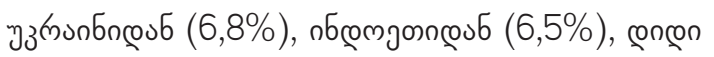

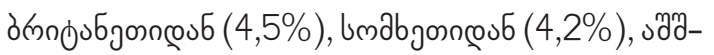

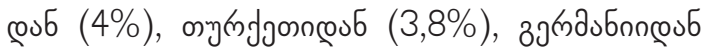

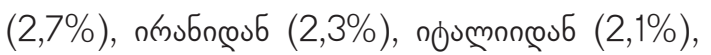

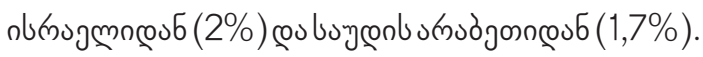

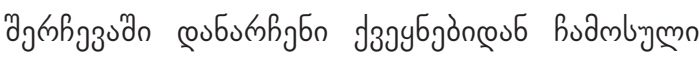

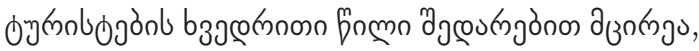

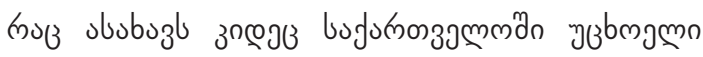

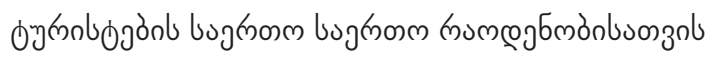

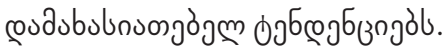

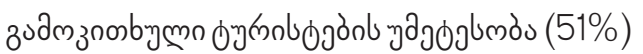

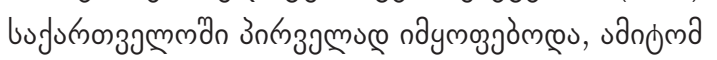

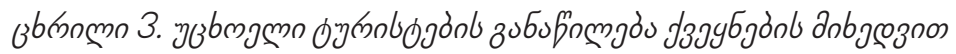

\begin{tabular}{|c|c|c|c|c|c|c|c|}
\hline \multirow[t]{2}{*}{$\mathrm{N}$} & \multirow{2}{*}{ dзэys 60} & \multicolumn{2}{|c|}{ 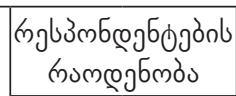 } & \multirow[t]{2}{*}{$N$} & \multirow{2}{*}{ dзаgsбo } & \multicolumn{2}{|c|}{ 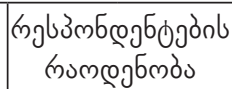 } \\
\hline & & bnbzomg & $\%$ & & & 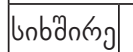 & $\%$ \\
\hline 1 & fribgon & 83 & 13,8 & 26 & fabjon & 4 & 0,7 \\
\hline 2 & hoбjon & 56 & 9.3 & 27 & nuउmbno & 4 & 0,7 \\
\hline 3 & 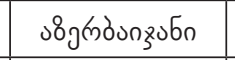 & 49 & 8,1 & 28 & 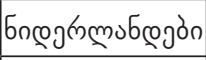 & 4 & 0,7 \\
\hline 4 & 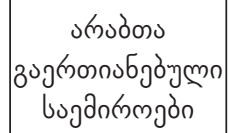 & 42 & 7,0 & 29 & $\begin{array}{c}\text { sbummo } \\
\text { 8gmubons }\end{array}$ & 4 & 0,7 \\
\hline 5 & ग3 & 41 & 6,8 & 30 & उmrnotyzumos & 4 & 0,7 \\
\hline 6 & nбқолдоп & 39 & 6,5 & 31 & jb306jon & 4 & 0,7 \\
\hline 7 & 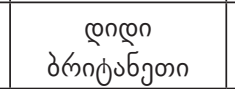 & 27 & 4,5 & 32 & coubno & 3 & 0,5 \\
\hline 8 & umabjon & 25 & 4,1 & 33 & 5mtrossonos & 3 & 0,5 \\
\hline 9 & د & 24 & 4,0 & 34 & gmomsutons & 3 & 0,5 \\
\hline 10 & oyjuggon & 23 & 3,8 & 35 & bghòjon & 3 & 0,5 \\
\hline 11 & 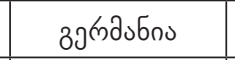 & 16 & 2,7 & 36 & 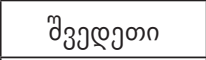 & 3 & 0,5 \\
\hline 12 & omsobn & 14 & 2,3 & 37 & dumunonos & 2 & 0,3 \\
\hline 13 & nougnos & 13 & 2,2 & 38 & дnuбaumn & 2 & 0,3 \\
\hline 14 & nbmogmn & 12 & 2,0 & 39 & mдेбn & 2 & 0,3 \\
\hline 15 & 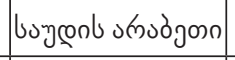 & 10 & 1,7 & 40 & 303060060 & 2 & 0,3 \\
\hline 16 & ogmoknglon & 9 & 1,5 & 41 & 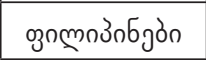 & 2 & 0,3 \\
\hline 17 & 383ก300 & 8 & 1,3 & 42 & bodms & 2 & 0,3 \\
\hline 18 & s3bomos & 7 & 1,2 & 43 & 788ठ03900ก & 2 & 0,3 \\
\hline 19 & bsogh & 7 & 1,2 & 44 & 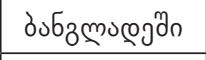 & 1 & 0,2 \\
\hline 20 & 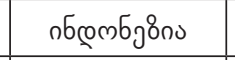 & 7 & 1,2 & 45 & उ०50000 & 1 & 0,2 \\
\hline 21 & $\partial_{3 \partial n \text { gotmos }}$ & 7 & 1,2 & 46 & nodunzo & 1 & 0,2 \\
\hline 22 & mกjठju & 6 & 1,0 & 47 & з"च3อกסо & 1 & 0,2 \\
\hline 23 & उмल्мmБјол & 6 & 1,0 & 48 & dungmuten & 1 & 0,2 \\
\hline 24 & nmmencobno & 5 & 0,8 & 49 & 8sabos & 1 & 0,2 \\
\hline 25 & 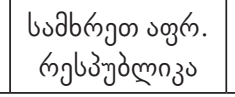 & 5 & 0,8 & & bygm & 600 & 100 \\
\hline
\end{tabular}

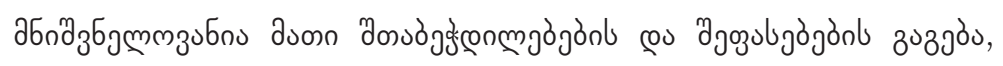

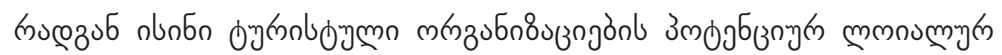

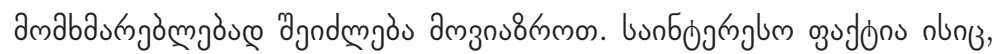
има пु

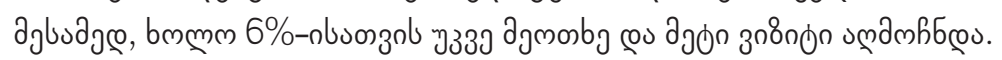

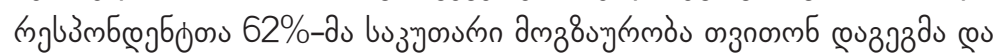

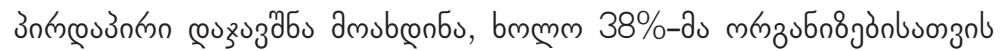

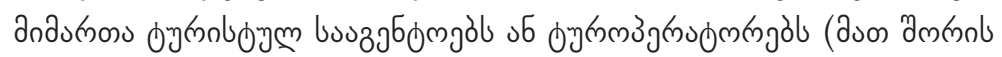

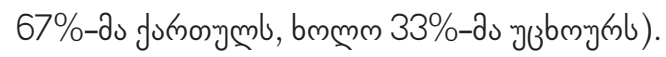

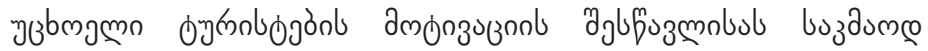

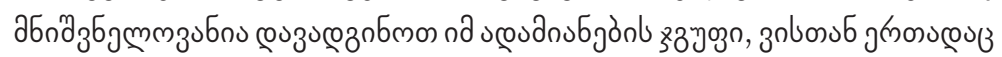

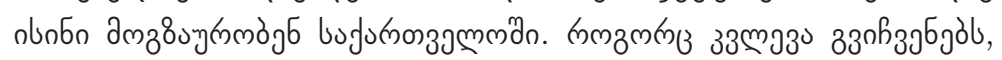




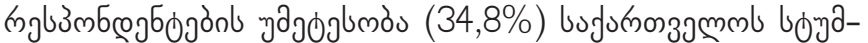

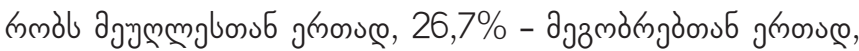

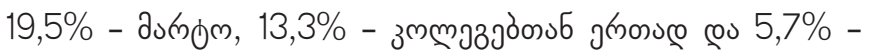

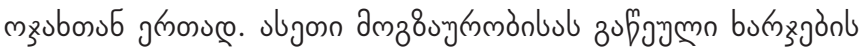

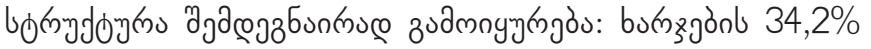

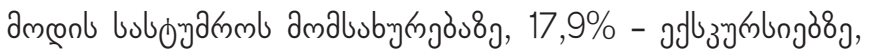

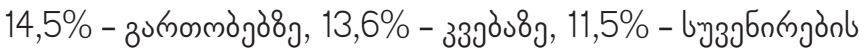

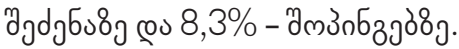

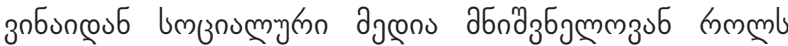

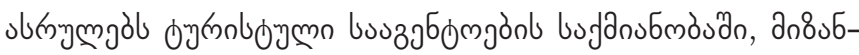

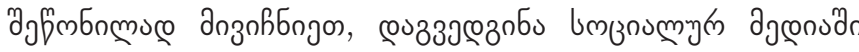

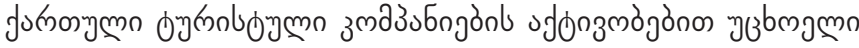

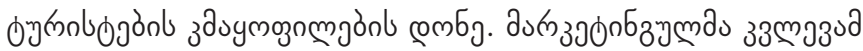

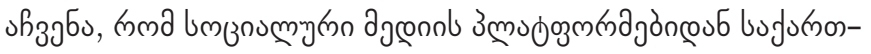

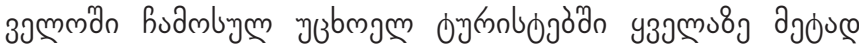

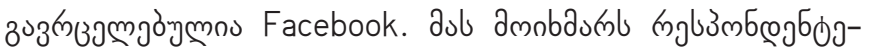

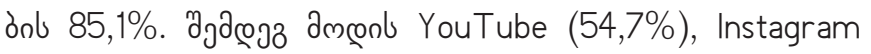
(32,4\%), Google+ (31,1\%), Linkedln (29,9\%), Twitter

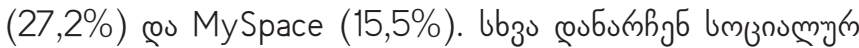
Jlugmgdb (Pinterest, Tumblr, Flickr, Reddit, Ask.fm, Vkontakte, Odnoklassniki) dmnbaukr zudmznorbymons 14\%.

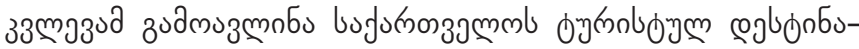

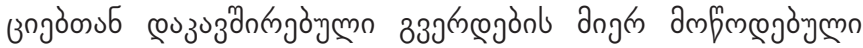

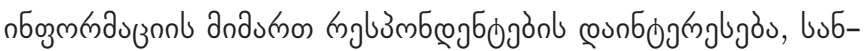
एмm

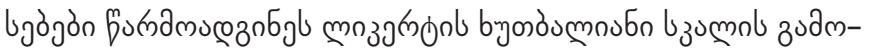

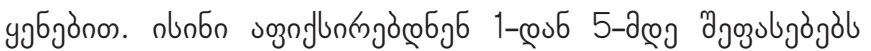

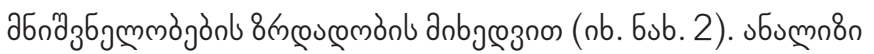

\section{6ub. 2}

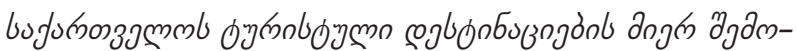

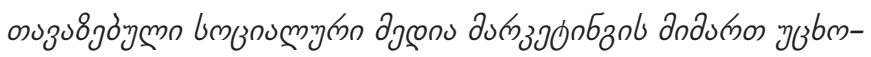

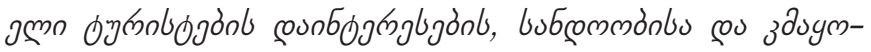
gुnmgonb gem6jgón

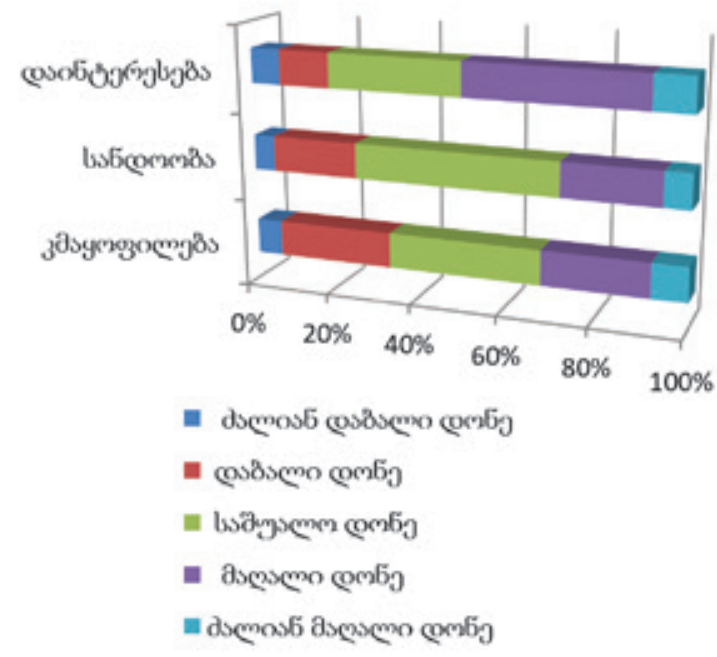

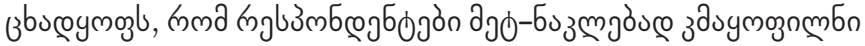

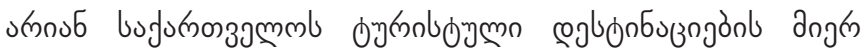

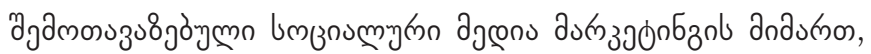

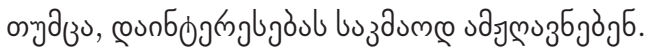

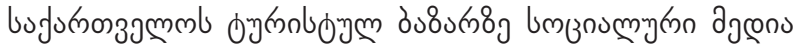

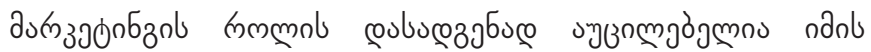

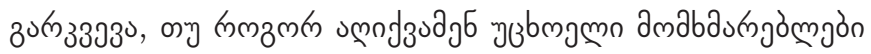

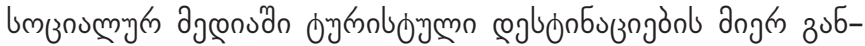

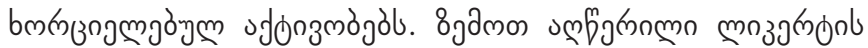

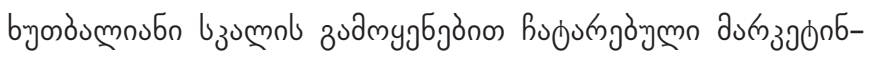

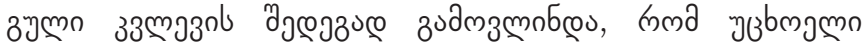

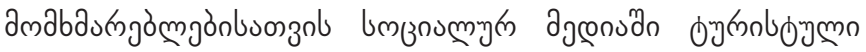

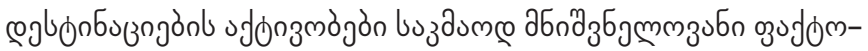

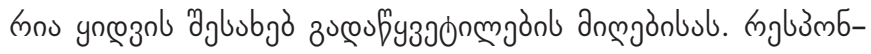

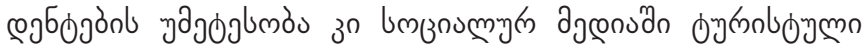

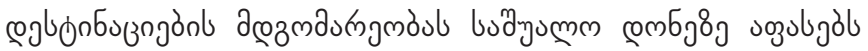
(nb. 5ub. 3).

6ub. 3

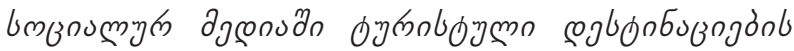

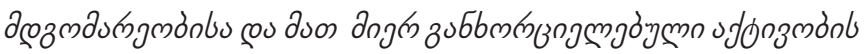

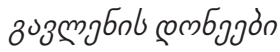

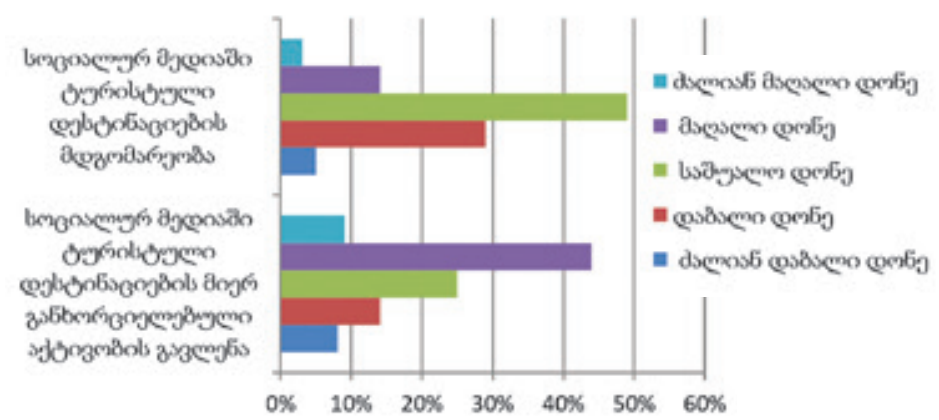

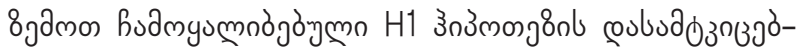

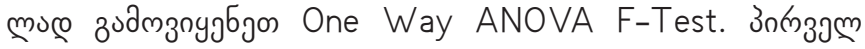

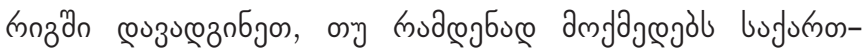

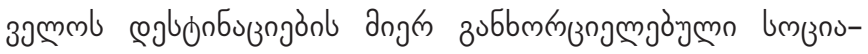

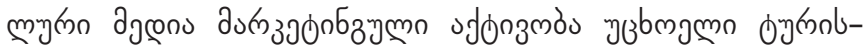

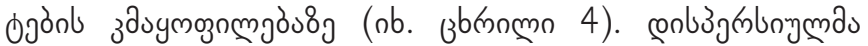

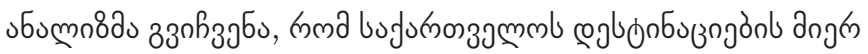

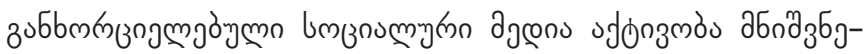

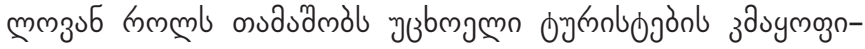
mgaेव̊g ( $F=1,930 ; P=0,007)$.

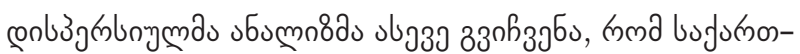

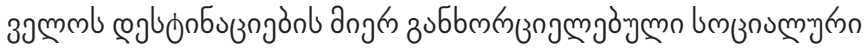

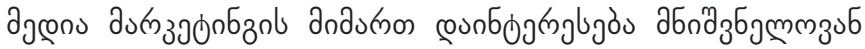

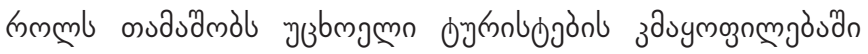

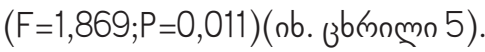




\section{3brongmo 4}

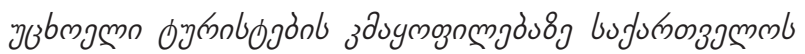

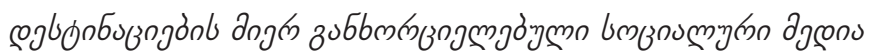

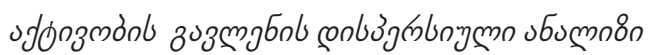

\begin{tabular}{|c|c|c|c|c|c|}
\hline \multicolumn{6}{|c|}{ 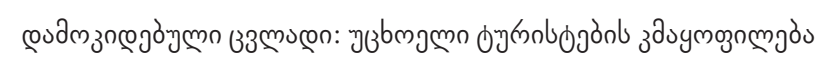 } \\
\hline & 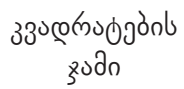 & df & $\begin{array}{l}\text { bud yumm } \\
\text { 33ugenofon }\end{array}$ & $\mathrm{F}$ & $p$ \\
\hline 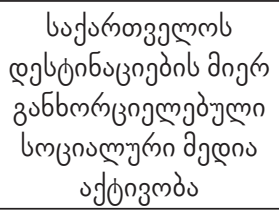 & 6,155 & 3 & 2,052 & 1,930 & 007 \\
\hline 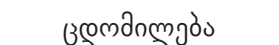 & 868,343 & 817 & 1,063 & & \\
\hline
\end{tabular}

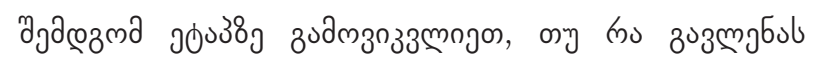

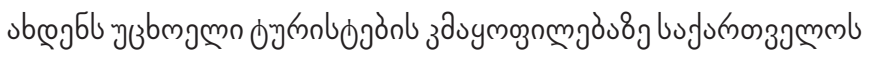
œ

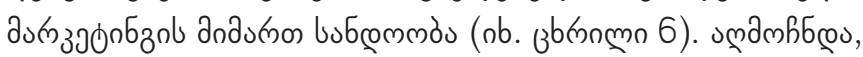

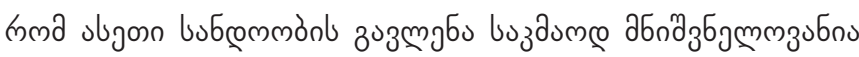
( $F=1,316 ; P=0,008)$.

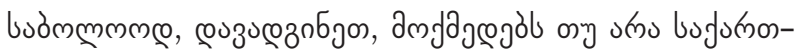

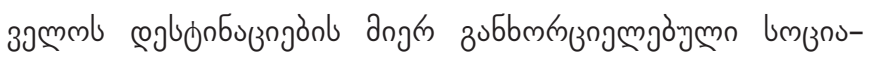

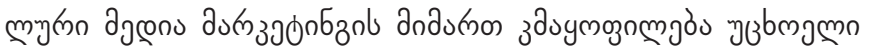

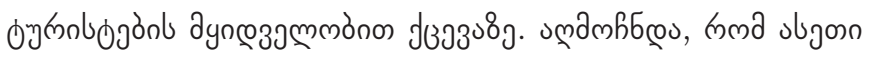

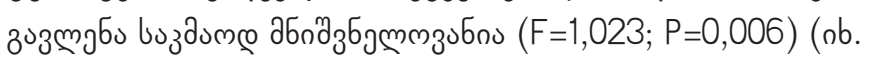
(z) manno 7).

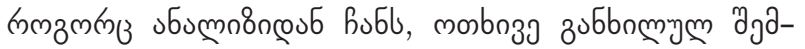

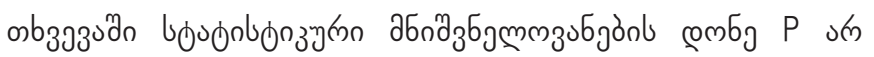

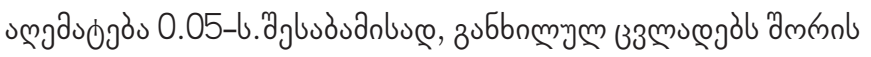

\section{3brongm 5}

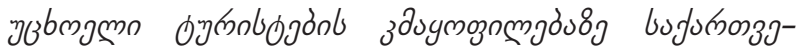

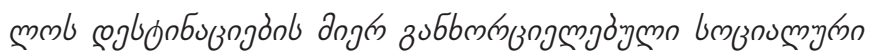

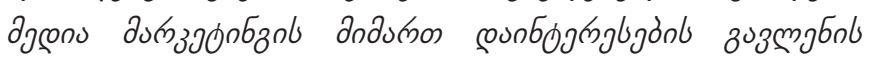
conbughbringmo v6umoso

\begin{tabular}{|c|c|c|c|c|c|}
\hline \multicolumn{6}{|c|}{ 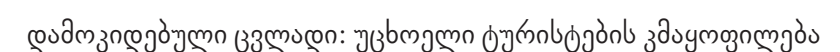 } \\
\hline & 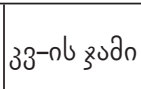 & $d f$ & $\begin{array}{l}\text { bodyumm } \\
\text { 33uconuodn }\end{array}$ & $\mathrm{F}$ & $p$ \\
\hline 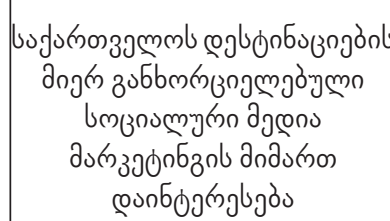 & 9,930 & 5 & 1,986 & 1,869 & 011 \\
\hline 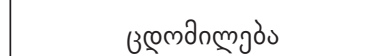 & 868,343 & 817 & 1,063 & & \\
\hline
\end{tabular}

36trongon 5

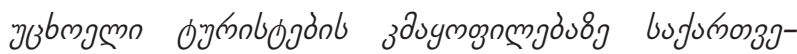

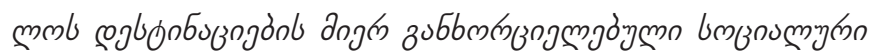

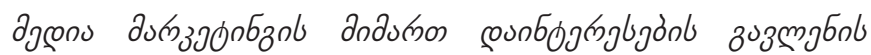
sonbughtongmo ubumnon

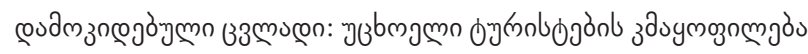

\begin{tabular}{|c|c|c|c|c|c|}
\hline & $33-n$ s $30 \partial n$ & $d f$ & 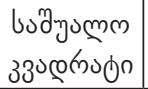 & $\mathrm{F}$ & p \\
\hline 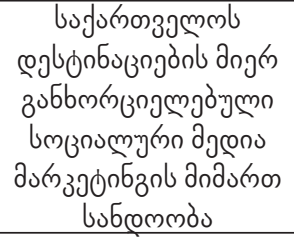 & 8,166 & 5 & 1,633 & 1,316 & 008 \\
\hline 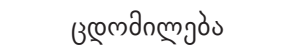 & 1014,087 & 817 & 1,241 & & \\
\hline
\end{tabular}

3b6rngno 6

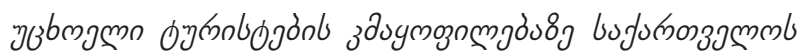

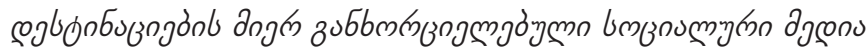

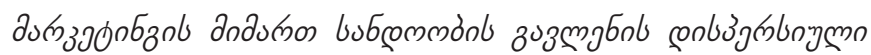
ง6uलmo8n

\begin{tabular}{|c|c|c|c|c|c|}
\hline \multicolumn{6}{|c|}{ 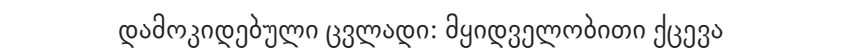 } \\
\hline & $33^{-n}$ b zuan & $d f$ & 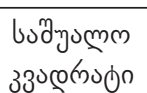 & $\mathrm{F}$ & p \\
\hline 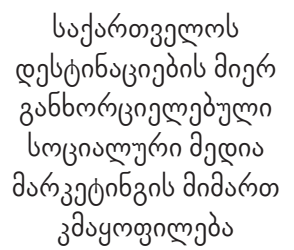 & 6,160 & 5 & 1,232 & 1,023 & 006 \\
\hline зомдnмmgos & 983,439 & 817 & 1,204 & & \\
\hline
\end{tabular}

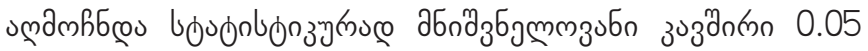

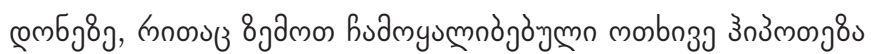
cosadosizes.

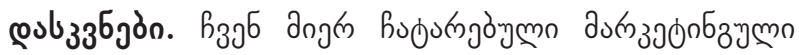

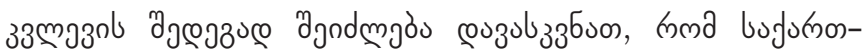

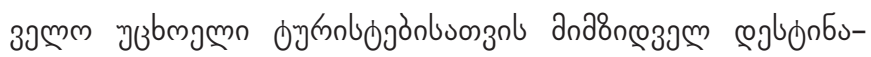
znol fuknamuœ

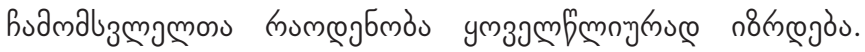

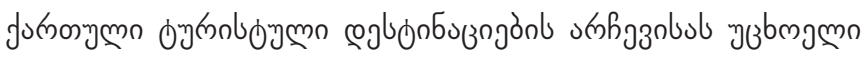

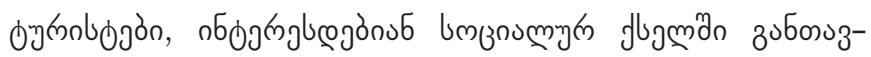

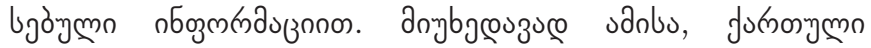

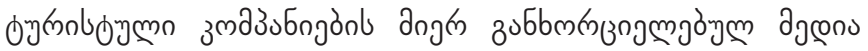

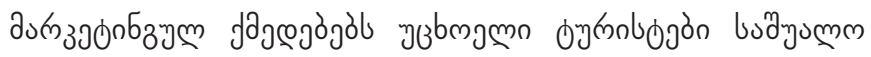
кемб

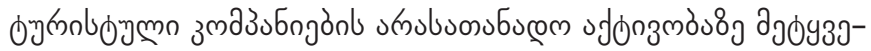




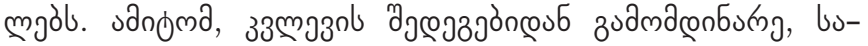

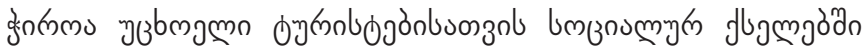

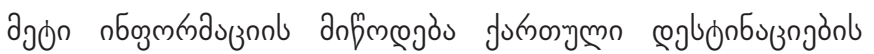

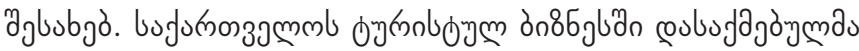

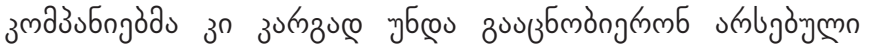

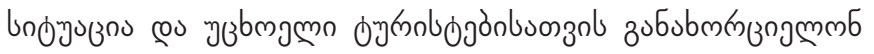

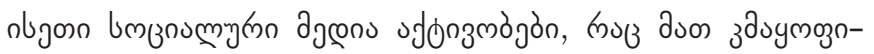

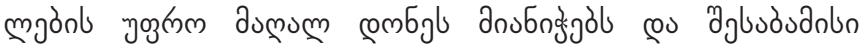

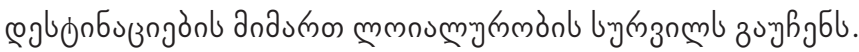

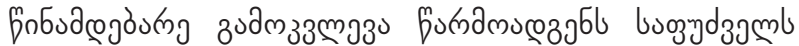

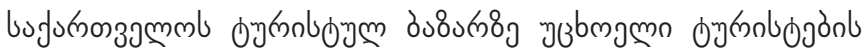

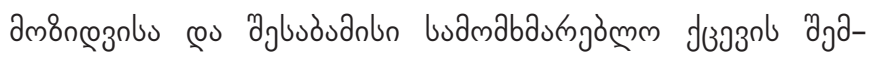

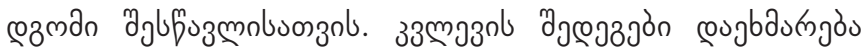

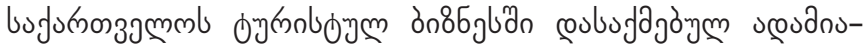

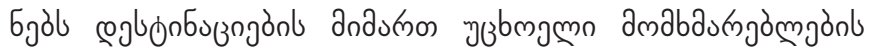

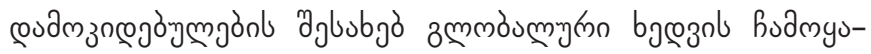

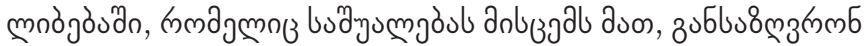

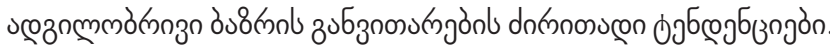

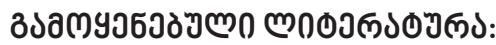

1. Alhroot, J. (2014). Marketing of A Destination; A Case Study of Jordan.International Journal of Tourism Research, Vol.1, No. 2, pp.45-50.

2. Apil, A. R., Kaynak, E., and Todua, N. (2009). Product Preference Differences of High and Low Ethnocentric Consumers in Georgia. In Proceedings of the 18th World Business Congress "Management Challenges in an Environment of Increasing Regional and Global Concerns". International Management Development Association, pp. 531-539.

3. Baker, M. J. and Cameron, E. (2008).Critical Success Factors in Destination Marketing. Tourism \& Hospitality Research, Vol.8, No. 2, pp. 79-97.

4. Barker, M., Barker, D., Bormann, N., and Neher, K. (2012). Social mediamarketing - A strategic approach. NV: SouthWestern Publishing/Cengage Learning.

5. Benckendorff, P. and Black, N. L. (2005). Destination Marketing on the Internet: A Case Study ofAustralian Authorities. IOSR Journal of Business and Management, Vol.11, No.1, pp.11-21.

6. Blain, C., Levy, S. E., and Ritchie, N. (2005). Destination branding; Insights and practices fromdestination management organizations. Journal of Travel Research, Vol. 43 No.4 pp. 328-338

7. Boulin, J. L. (2008). Institutional tourism does not elude Web 2.0. Tourisme \& Loisirs, Vol. 265, pp. 30-34

8. Carr, C. T., and Hayes, R. A. (2015). Social media: Defining, developing, and divining. Atlantic Journal of Communication, Vol. 23, No.1, pp. 46-65.

9. Evangelos, Chr. (2012). Social Media in Travel, Tourism and Hospitality: Theory, Practice and Cases. Abingdon: Routledge

10. Fyall, A., Kozak, M., Andreu, L., Gnoth, J. and Lebe, S. S. (2009). Marketing innovations for sustainable destinations, Goodfellow Publishers, Oxford.

11. Garcia, J. A., Gomez, M., and Molina, A. (2012). A destination-branding model; An empiricalanalysis based on stakeholders. Tourism Management, Vol. 33, No. 3, pp. 646-661.

12. Hausteinova, A. (2013). Web Site Evaluation of National Destination Marketing Organization. International Journal of Contemporary Hospitality, Vol.2, No.1, pp.25-30.

13. Heinonen, K. (2011). Consumer activity in social media: Managerial approaches to consumers' social media behavior. Journal of Consumer Behavior, Vol. 10, No. 6, pp. 356-364.

14. https://gnta.ge/ge/

15. https://www.geostat.ge

16. Jalilvand, M., Samiei, N., Dini, B., and Manzari, P. (2012). Examining the structural relationships ofelectronic word of mouth, destination image, tourist attitude toward destination and travelintention; An integrated approach. Journal of Destination Marketing and Management, Vol. 1, pp. 134-143.

17. Jashi, C., and Todua, N. (2013). Behavior Changing through of Social marketing (Georgian Case).Proceedings of third World Social Marketing Conference. Toronto (Canada). pp. 95-97.

18. Kaplan, A. M., and Haenlein, M. (2010). Users of the world, unite! The challenges and opportunities of Social Media. Business Horizons, Vol.53, pp. 59-68.

19. Khan, M. F., and Jan, A. (2015). Social Media and Social Media Marketing: A Literature Review. IOSR Journal of Business and Management, Vol.17, No. 11, pp.12-15.

20. Kotler, P., Haider, D. and Rein, I. (2002). Marketing places, Free press, New York.

21. Lee, D.H. (2010). Growingpopularity of social mediaand business strategy. SERIQuarterly, Vol. 3, No. 4, pp. $112-117$.

22. Malhotra, N. (2004). Marketing Research: an Applied Orientation. 4th ed., Upper Saddle River, NJ: Prentice Hall. 
23. Mghebrishvili, B., and Urotadze, E. (2016). Functions of Retailing. In Proceedings of 23rd International Academic Conference, Venice, Italy, pp. 356-360.

24. Minazzi, R. (2015). Social Media Marketing in Tourism and Hospitality.Springer International Publishing, New York.

25. Mutinda, R. (2013). Perspectives of Hotel Investors on Kenya's Competitiveness as a Tourismlnvestment Destination. Journal of Hospitality and Management, Vol.2, No. 4, pp.25-30.

26. Nguyen, P. (2014). Joint Destination Marketing in the South Central Coast Region of Vietnam. Journal of Tourism, Vol.1, No. 1, pp.74-80.

27. Papadimitriou, D., Apostolopoulou, A., and Kaplanidou, K. (2013). Destination personality, affectiveimage, and behavioral intentions in domestic urban tourism. Journal of Travel Research,Vol. 54, No. 3, pp. 1-14.

28. Pike S. (2008).Destination marketing: An integrated marketing communication approach. Elsevier, Oxford.

29. Pike, S. and Page, S. (2014). Destination Marketing Organizations and destination marketing: A narrative analysis of the literature. Tourism Management, Vol. 41, No. 1, pp.202-227.

30. Pike, S., and Ryan, C. (2004). Destination positioning analysis through a comparison of cognitive, affective, and conative perceptions. Journal of Travel Research, Vol. 42, No. 2, pp. 333-342.

31. Safko, Lon. (2010). The social media bible: Tactics, tools, and strategies for business success. John Wiley \& Sons.

32. Schaefer, M. W. (2014). Social Media Explained: Untangling the World ıs Most Misunderstood Business Trend. Mark W. Schaefer, PU.

33. Schmalleger, D., and Carson, D. (2008). Blogs in tourism: Changing approaches to information exchange.Journal of Vacation Marketing, Vol. 14, No. 2, pp. 99-110.

34. Seturi, M., and Urotadze, E. (2017). About Marketing Process Model and Relationship Marketing. In Proceedings of International Workshop „Model-Based Governance for Smart Organizational Future“, Sapienza University of Rome, Italy, pp. $169-171$.

35. Sweeney, S., and Craig, R. (2011). Social Media for Business: 101 Ways to Grow Your Business Without Wasting Your Time.Maximum Press, Toronto.

36. Todua, N. (2017). Influence of Food Labeling Awereness on healthy behavior of Georgian Consumers. Ecoforum Journal, 6(2).

37. Todua, N. (2017). Social Media Marketing for Promoting Tourism Industry in Georgia. In Proceedings of 22nd International Conference on Corporate and Marketing Communications: Challenges of Marketing Communications in a Globalized World. Zaragoza, Spain, pp. 168-171.

38. Todua, N. (2012). Marketing Research of Consumer Behavior in Georgian Market.Publishing House Universal, Tbilisi.

39. Todua, N., and Gogitidze, T. (2017). Marketing Research of Attitudes Towards Genetically Modified Crops by Georgian Farmers. Annals of Constantin Brancusi University of Targu-Jiu. Economy Series. Issue 1, pp. 69-76.

40. Todua, N., and Dotchviri, T. (2015). Anova in Marketing Research of Consumer Behavior of Different Categories in Georgian Market. Annals of Constantin Brancusi University of Targu-Jiu. Economy Series, Issue 1, pp. 183-190.

41. Todua, N., and Dotchviri, T. (2015). On the Marketing Research of consumer prices and inflation process. British Journal of Marketing Studies. Vol. 3, No. 2, pp.48-57.

42. Todua, N., and Jashi, Ch. (2015). Some Aspects of Social Media Marketing (Georgian Case).International Journal of Social, Behavioral, Educational, Economic and Management Engineering, Vol.9, No. 4, pp. 1160-1163.

43. Todua, N., and Jashi, Ch. (2016). Main Aspects of Service Quality in the Hotel Industry of Georgia. In Proceedings of 4th International Conference on Contemporary Marketing Issues (ICCMI). Heraklion, Greece, pp. 431-435.

44. Todua, N., Babilua, P., and Dochviri, T. (2013). On the Multiple Linear Regression in Marketing Research. Bulletin of the Georgian National Academy of Sciences, Vol. 7, No. 3, pp. 135-139.

45. Todua, N., Gogitidze, T., and Phutkaradze, J. (2015). Georgian consumer attitudes towards genetically modified products. International Journal of Management and Economics, Vol. 46, No. 1, pp. 120-136.

46. Todua, N., Gogitidze, T., and Phutkaradze, B. (2017). Georgian Farmers Attitudes towards Genetically Modified Crops. Economics World, Vol. 5, No. 4, pp. 362-369.

47. Todua, N., Mghebrishvili, B., and Urotadze, E. (2016). Main aspects of formation of legal environment on the Georgian food market. In Proceedings of International Scientific-Practical Conference "Challenges of Globalization in Economics and Business". Universal, Tbilisi, pp. 250-256.

48. Usakli, A., and Baloglu, S. (2011). Brand personality of tourist destination; An application of selfcongruitytheory. Tourism Management, Vol. 32, pp. 114-127.

49. Wanjala, J. (2015). Cultural Factors Influencing Consumer Choice for Holiday Destination AmongLocal Tourists in Kenya. Journal of Hospitality and Tourism Research, Vol.2, No. 3, pp.17-24.

50. WTO. (2007).A practical guide to tourism destination management. World Tourism Organization, Madrid.

51. Zarella, D. (2013). The Science of Marketing: When to Tweet, What to Post, How to Blog, and Other Proven Strategies. Hoboken: John Wiley \& Songs Inc.

52. Zeng, B. , and Geristen, R. (2014). What do We know about Social media in Tourism. Tourism Management Perspectives. Vol.10, pp. 27-36. 


\section{USING SOCIAL MEDIA MARKETING FOR ATTRACTING FOREIGN TOURISTS TO GEORGIAN DESTINATIONS}

\section{NUGZAR TODUA}

D.Sc. Professor,

Ivane Javakhishvili Tbilisi State University,

Academician of the Academy of Economic Sciences of Georgia, Georgia

Nugzar.todua@tsu.ge

KEYWORDS: GEORGIAN TOURISM MARKET, TOURIST DESTINATIONS, SOCIAL MEDIA MARKETING, FOREIGN TOURISTS, MARKETING RESEARCH.

For citation: Todua N. (2019), Using Social Media Marketing For Attracting Foreign Tourists To Georgian Destinations, Globalization And Business, №7, pp. 39-48. (In Georgian). https://doi.org/10.35945/gb.2019.07.005

SUMMARY

At current stage business development cannot be imagined without social networks. The customers spend more and more time in social networks and get most part of the information from them. Therefore, for the companies, the social media is a powerful tool for attraction of clients and for competition. Usingthe social media marketing is particularly popular in tourism industry. Technological changes in mass media allow immediate distribution of information in the sphere of tourism. Reasonable using of social networks in tourism requires creative marketing approaches ensuring coverage of the target audience to maximal possible extent. Sites of social media became the primary source of information for the destination management organizations. Usually the tourists trust online information and before commencement of travel they visit the forums and online reviews, as well as specialized blogs. Hence, there is a close relationship between involvement of tourists' social network sites and change in their behavior. Technological development and globalization of media creates new opportunities providing sharing the information between tourist consumers through blogs, web sites or destination sites. The destinations need creative and powerful social media marketing strategies to attract potential visitors. Social media helps the destinations to communicate with the visitors at relatively low costs and higher effectiveness than traditional communication methods. Social media, as an instrument for stimulation is used for the purposes of interactive marketing. Though, it is notable that there is a growing number of visitors, who use social media applications, creating new challenges in entire tourism industry.

As social media plays a significant role in the activities of travel agencies, we found reasonable to find out the level of satisfaction of the foreign tourists with the activities of Georgian tourist companies in social media. Market research showed that of social media platforms, among the foreign tourists the most widespread is Facebook. 85.1\% of the respondents use it. It is followed by YouTube (54.7\%), Instagram (32.4\%), Google+ (31.1\%), Linkedin (29.9\%), Twitter (27.2\%) and MySpace (15.5\%). $14 \%$ of the respondents use the other social networks (Pinterest, Tumblr, Flickr, Reddit, Ask.fin, Vkontakte, Odnoklassniki). Study demonstrated interest of the respondents to the information provided by the sites related to tourism, their trust and satisfaction. Analysis shows that the respondents are satisfied with the social media marketing offered by Georgian travel agencies to certain extent.

As a result of our market study we can conclude that Georgia is an attractive destination for the foreign tourists. Therefore, the number of repeated visits to Georgia increases from year to year. In making choice of Georgian travel agencies, the foreign tourists basically rely on the information in social networks and word-of-mouth marketing. Though, interest, trust and satisfaction of the foreign customers to social media marketing offered by Georgian travel companies are at the average level. Therefore, foreign tourists evaluate media marketing activities conducted by Georgian tourist companies as average and this underlines insufficient activity of Georgian tourist companies in social networks.

With the help of marketing research obtained levels of interest, reliability and satisfaction of foreign tourists in relationto social media marketing provided by Georgian destinations.On the basis of analysis of the research results statistically significant values were obtained that show influence of the activity, interest and reliability regarding to social media marketing provided by Georgian destinations on the foreign tourists' satisfaction. In the work statistically significant value is also received reflecting the influence of the social media activity provided by Georgian destinations on buying behavior of foreign tourists' 


\title{
GLOBALIZATION AND ITS IMPACTS ON INTERNATIONAL DEVELOPMENT: A LONG-TERM HISTORICAL PERSPECTIVE
}

\section{MUHAMMED KURSAD OZEKIN}

\author{
(MA (King's College London), DPhil (University of Sussex)) \\ Usak University - Department of Political Science andInternational Relations, Turkey
}

\section{KEYWORDS: GLOBALIZATION, ECONOMIC GROWTH, UNEVEN DEVELOPMENT, CONVERGENCE.}

For citation: Ozekin M. (2019), Globalization And Its Impacts On International Development: A Long-Term Historical Perspective, Globalization And Business, №7, pp. 49-58. https://doi.org/10.35945/gb.2019.07.006

\section{INTRODUCTION}

The impact of globalization on international development is one of the most controversial issues of today's development discourse. Many studies come to different conclusions about this issue depending on how the questions are asked, and which data series are used. Broadly speaking, there are two distinct accounts about whether globalization brings international economic equality or not. The neoliberal school of thought or optimists argue that globalization creates an integrated world market in which prosperity, wealth and power are being diffused around the globe so that the globalization brings international equality in the long run. Particularly the rise of contemporary globalization both as a social process and a political project has brought about a considerable optimism concerning the role of global market forces in promoting growth, reducing poverty, and achieving economic development and prosperity particularly in developing countries. In this respect, the period from the late 1970s to the early 1990s witnessed a marked upsurge of highly optimistic neoliberal discourse both in development theory and practice (Ruckert, 2006).

However, probing into the empirical evidence, a growing intellectual doubt has been cast on the fundamental claim of the optimistic development discourse that globalization and fullscale market liberalization based on the neoclassical principle of comparative advantages leads to successful economic performance and overall growth in the world economy. In contrast so-called 'radical' school or pessimistic view argues that with the impact of globalization, the world is becoming more fragmented that income gap between the developed and developing countries is widening. For the pessimistic accounts, the development that the world economy experienced in the period from the early 1970s to the late 1990s, which could be termed the age of globalization, was considerably lower and unstable compared to the earlier period from the late 1940s to the early 1970s, which has been described as "the golden age of capitalism" (Kiely, 2005 and Nayyar, 2006). Moreover, not only has overall growth been lower, but also the degree of inequality in the world economy has also increased during the same period (see inter alia, UNCTAD, 1997 and Wade, 2001).

Rather than taking sides with either of these two camps, this study embraces a middle ground approach to the matter of globalization and development. Overall the study argues that globalization causes complex patterns of convergence and divergence across regions and between countries that its impacts cannot be observed at the same degree and effect in all ages and economies of the world. In this sense, it is argued that some countries benefit from it whereas some face economic losses, and so despite the relative industrial convergence and economic growth in developing countries inequality in international development is still persistent. In the light of this argument, this study aims to explore the impact of globalization on inequality in international development through two main stages. In the first stage, the study critically discusses the nexus between globalization and development by putting special emphasis on the complex patterns of development in the global economy, Particularly drawing on the works of Milanovic (2003) and Arrighi, Silver and Brewer (2003), the conceptualization of contemporary globalization either as a win-win situation or as a zero-sum game will be questioned based on the uneven, limited 
and asymmetric nature of economic globalization. In the second stage, the impact of globalization on international inequality is later explained through a long-term historical perspective. To this end, the relationship between globalization and development is situated in the wider historical context of the international capitalist system which has been in a phase of farreaching structural transformation for a long time. In doing so, the implications of globalization for development are explored in retrospect, focusing on the questions of uneven development in diverse industrialization processes. Finally, the last section concludes with a review of the arguments and key findings, and provided a broad view on the matter of Globalization and development.

\section{GLOBALIZATION AND INTERNATIONAL DEVELOPMENT: AN ECONOMIC OUTLOOK}

The buzzword globalization has been defined in many ways throughout the years ${ }^{1}$. In economic terms, globalization refers to the expansion and deepening of international trade, technology, capital and information. In other words, economic globalization means the flow of goods, services, investment, production, and technology across nations. This flow is inevitably associated with the liberalization of the markets, and international trade (Global Policy Forum, 2006). Another economic aspect of globalization is the integration of financial and commodity market, internationalization of production and increasing competitiveness. (Callaghan, 2002). In this regard, Globalization cannot be understood separately from the capitalist economic system of the world and should not be regarded as a driving force of international development per se.

Globalization creates an economic interdependence between countries on a global scale by increasing the volume and variety of cross-border transactions in terms of goods, services, capital and technology (Bhagwati, 2004). Within this explanation of globalization, free trade, capital mobility and technology transfer can be regarded as major economic dimensions of globalization which can be found in a greater or lesser degree all over the world. Each dimension has negative or positive economic impacts on both developed and developing countries depending on the case of each country.

\footnotetext{
${ }^{1}$ How to define and measure globalization is a quite controversial issue in development studies literature. As a multifaceted concept, globalization has economic, social, political aspects that move beyond basic indicators such as FDI movements and trade openness. Thus, given its multifaceted character, several indices have been developed to measure divergent aspects of globalization; for example, the CSGR Globalization Index (Lockwood and Redoano, 2005), the Maastrict Globalization Index (Martens and Raza, 2009), the KOF Index, (Dreher et al., 2008). Among them, the most often used index is the KOF Globalization index which see globalization as a process of creating networks through a variety of flows including capital, goods, people, information, ideas and etc.
}

According to neo-liberal point of view, international trade, as a one of the major economic dimensions of globalization, is more beneficial for poor economies because of three main reasons. First of all, free trade brings technology transfer which enables lower income economies to grow faster than higher income economies. (Weeks Gerschenkron 1962) Secondly, open trade policies have a bigger effect on the terms of trade of the countries integrating world economy than on countries already integrated (Lindert and Williamson, 2003). The third line of argument is that trade liberalization can facilitate the diffusion of knowledge and increase the steady-state income of lower income economies to a higher level.

In contrast, the globalization process and free trade have not shown their impacts at the same degree in all economies of the world. Contrary to what the international trade theory emphasizes, income distribution in developing countries is not improved with the rise in the volume of international trade. Depending on data derived from household surveys, Milanovic (2003) asserts that in poor countries upper income groups in social stratification are better off with open trade policies whereas lower income groups do not benefit from it adequately which leads to unequal income distribution within country. As the income level of poor countries increases, lower and middle-income groups begin to benefit from international trade. Therefore, initial income level of countries is a decisive factor in understanding to what extend will a country and social class benefit from open trade policies. In other words, with open trade policies, income distribution within poor countries could worsen, eventually leading to losses in overall income level of country. Therefore, it seems that countries having relatively abundant factors of production may benefit from free trade, whereas those having scarce factors may encounter problems in their economies.

The Capital mobility is another important dimension of globalization process. Both developed and developing countries benefit from foreign direct investment (FDI) and portfolio investments. FDI is a long term of investment in physical assets that creates employment opportunities whereas portfolio investments are short-term investments in financial assets which is more prone to create speculative impacts. Especially capital mobility in the form of portfolio investment might trigger the financial crises in vulnerable developing economies which do not have the stable financial market and well-established capital accumulation to handle potential crises. In this context, Woepking (2009) underlines that increasing liberalization of capital flow in developing countries makes their financial markets more liable to volatility and the crisis that brings along an increase in foreign debts and credits. By the same token, as Fischer (2003) further puts forward, integration of developing economies to global markets should be materialized gradually and with the establishment of necessary infrastructures. Otherwise their economies would be open to capital flow volatility, which might lead to an economic crisis, loss of output and unemployment. 
Over the past four decades, with the liberalization of international trade and capital flow, and the accompanying technological transfer, there has been a widespread convergence in the degree of industrialisation between rich and poor countries. However, over the same forty years there was virtually no income convergence between these states so that historical inequality in international development seems persistent. In fact, there is no doubt that as two of the most widely accepted aspects of the global economy, the restructuring of production and trade on a global scale has reshaped international divisions of labour to a considerable extent. Contrary to the post-war expansion of world capitalism in the 1950s and 1960s, the dismantling of production processes accompanied by dynamic growth in world trade has narrowed the gap between core and peripheral countries in terms of industrialization. The global spread of manufacturing capabilities to developing economies makes dichotomous denominations such as 'industrialized' and 'non-industrialized' increasingly untenable. Moreover, production is increasingly taking place in the framework of global value chains through which capital-, technology- and knowledge-intensive processes are dispersed to a greater number of countries, including developing ones. All these structural shifts become more obvious as time goes by. However, the question still remains: whether all these changes have brought economic and technological convergence between peripheral and core countries, and diminished the income gap between them in socio-spatial terms.

In response to this question, Arrighi et al. (2003, pp.1216) suggest that despite widespread convergence in levels of industrialization, the development gap and income disparity between the global south and global north have not been diminished but reproduced. Their findings reveal that the south as a whole converged with and even in some cases overtook the north in terms of industrialization levels. While the north's manufacturing sector comprised $28.9 \%$ of GDP in 1960 , it later incrementally descended to $24.5 \%$ by 1980 and to $19.8 \%$ by 1999 . On the other hand, the percentage of manufacturing in the south's GDP exhibits an opposite trend, ascending from $21.6 \%$ in 1960 to $24.3 \%$ in 1980 , with a slight fall to $23.3 \%$ in $1999^{2}$. Thus, the global south's percentage of GDP in manufacturing as a percentage of the global north's rose from $74.6 \%$ in 1960 to $99.4 \%$ and $118 \%$ in 1980 and 1998, respectively. However, Arrighi et al. empirically prove that the convergence in industrialization levels has not been accompanied by convergence in levels of income. As a proportion of the north's GNP per capita, GNP per capita in the global south has remained almost stagnant, with very slight changes from $4.5 \%$ in 1960 to $4.3 \%$ and $4.6 \%$ in 1980 and 1998 , respectively (Arrighi et al., 2003, pp.12-16).

Thus it is fair to discuss that the persistence of northsouth income disparity, despite apparent industrial convergence, reveals that the polarizing tendency of the world capitalist system is still at work and continuing to reproduce economic disparities between different sociospatial entities. Despite the bifurcation within the global south - due to conspicuous cases such as South Korea, Taiwan and more recently China - geographical asymmetries in income levels remain among the premier issues informing today`s global inequalities. In fact, the industrial convergence between rich and poor states has not been followed by convergence in the levels of income. The industrialization in Third World countries after 1980 increased the disparity of development levels and unevenness within the region. In this period, Sub-Saharan African and Latin American countries experienced major deterioration whereas East

2 The United Nations Industrial Development Organization's report reveals very similar figures regarding the period between 1980 and 2000. See UNIDO (2004, p.137).
Regions are separated as follows: 1. Asia, 2. Sub-Saharan Africa, 3. Middle East and North Africa, 4. Latin America, 5. Developing world, 6. Developing world, excluding China and India, 7. China and India, 8. Eastern Europe, 9. Non-industrialized world, 10.Industrialized world, 11. World. The developing world is the world excluding the industrialized world and Eastern Europe, and the non-industrialized world is the world excluding the developed (industrialized) world.

Source: World Bank, World Development Indicators. CD-ROMs, 2001.
Figure 1

Average per capita daily incomes of regions, 1960, 1980, and 2000 (1993 PPP dollars)

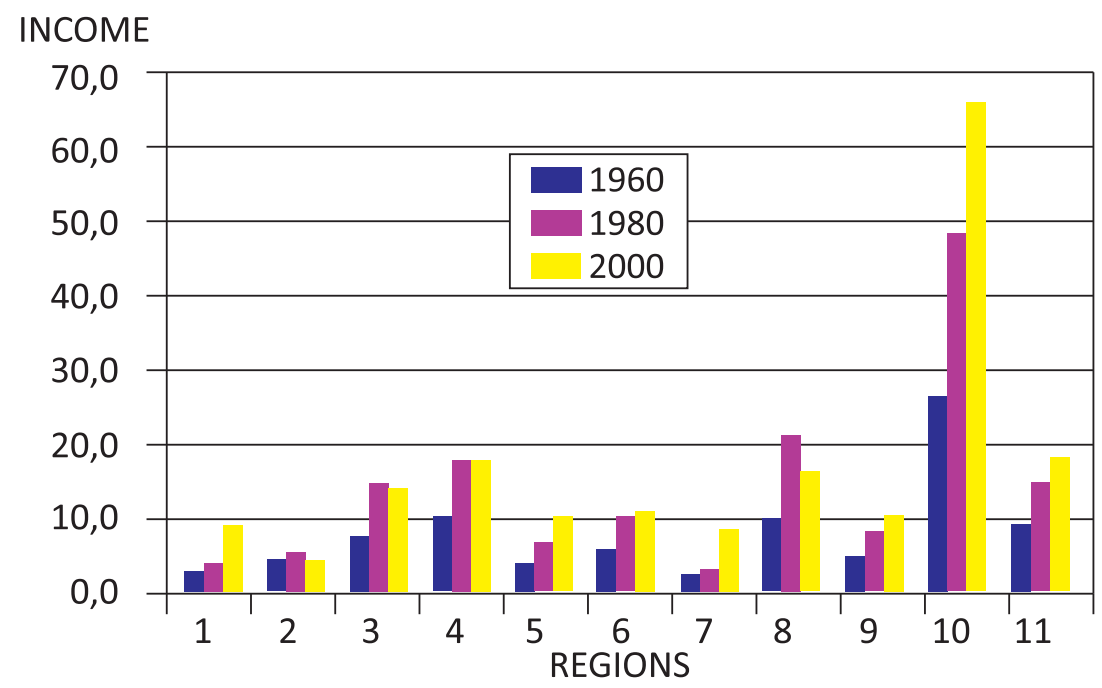


Asian ones developed considerably. Development disparities and rising unevenness created a bifurcation within Third World countries which creates a need to redefine historical North-South division. Nevertheless, despite the industrial convergence in Third World the structural North-South division maintains its importance as being a significant phenomenon in international development of global world.

The income divergence between developed and developing world can be retrospectively observed by assessing the average per capita daily incomes of regions between the years 1960 and 2000. As can be seen in World Bank global indicator of average per capita daily incomes, people of the industrialized countries earn the highest level of daily income in the world by comparison with the rest of the world. Although, Eastern Europe, Latin America, the Middle East, and North Africa have experienced a relative increase in their incomes, there is an ongoing income gap between developed and developing countries. Moreover, the income level of the industrialized world in 1960 still exceeds the other regions income levels in 2000.

In contrast the ongoing income gap, by giving weight to between country inequality rather than within country inequality in their measurement, Firebaugh and Goesling (2004) states that with the spread of industrialization throughout populous poor regions, globalization has decreased the inequality of income in the world. Their findings indicate that rapidly developing economies of China and South Asia are the major equalizing force in decreasing income inequality. However, Milanovic's (2002) study depending on household surveys indicated an increase in income inequality throughout the world. He stated (2005a) that rapidly developing economies of China and India may lead to misleading conclusions in measuring the effect of globalization

Figure 2

Global Output Growth Rate between 1951 and 2015 on the world income inequality. To Milanovic the between and within income inequality of several countries should be taken into consideration in order to improve the reliability of results about the impact of globalization on world income inequality. Moreover, the impact of globalization and open economic policies implemented by developing countries naturally generate different outcomes in each case. Some countries which have been able to govern globalization can benefit from it and others can face economic losses (Stiglitz, 2006).

Thus, the overall economic and social gains derived from the current wave of globalization have not presented simpler pattern for generalisations. First of all, with the exception of the 2000s, global growth rate shows a downward trend since the late 1960s (See, Figure 2). In fact, it is quite hard to reach a common view why this slowdown has occurred in the global economy, but it is fair to say that it has mostly originated from the overall economic slowdown in the developed economies. Probing into further empirical evidence reveals that over the last three decades, the slowdown of economic growth in developed world has been accompanied by an upward trend of growth in the developing world (See Table, 1). ${ }^{3}$ Thus, considering the overall growth trends both in the developed and developing economies, one might easily jump into the conclusion that the current phase of globalization has led to global convergence not only in terms of industrialisation but also in terms of global income. However, taking a closer look to the regional and country level indicators can help to gain further insight into the matter. In fact, looking into the recent data of the last three and half decades, two main feature stands out from the Table 1: first, there are varying

3 The 1970 s could also be considered as a period of convergence as the growth in the developed economies considerably decelerated due to the series of financial turmoil in global economy.

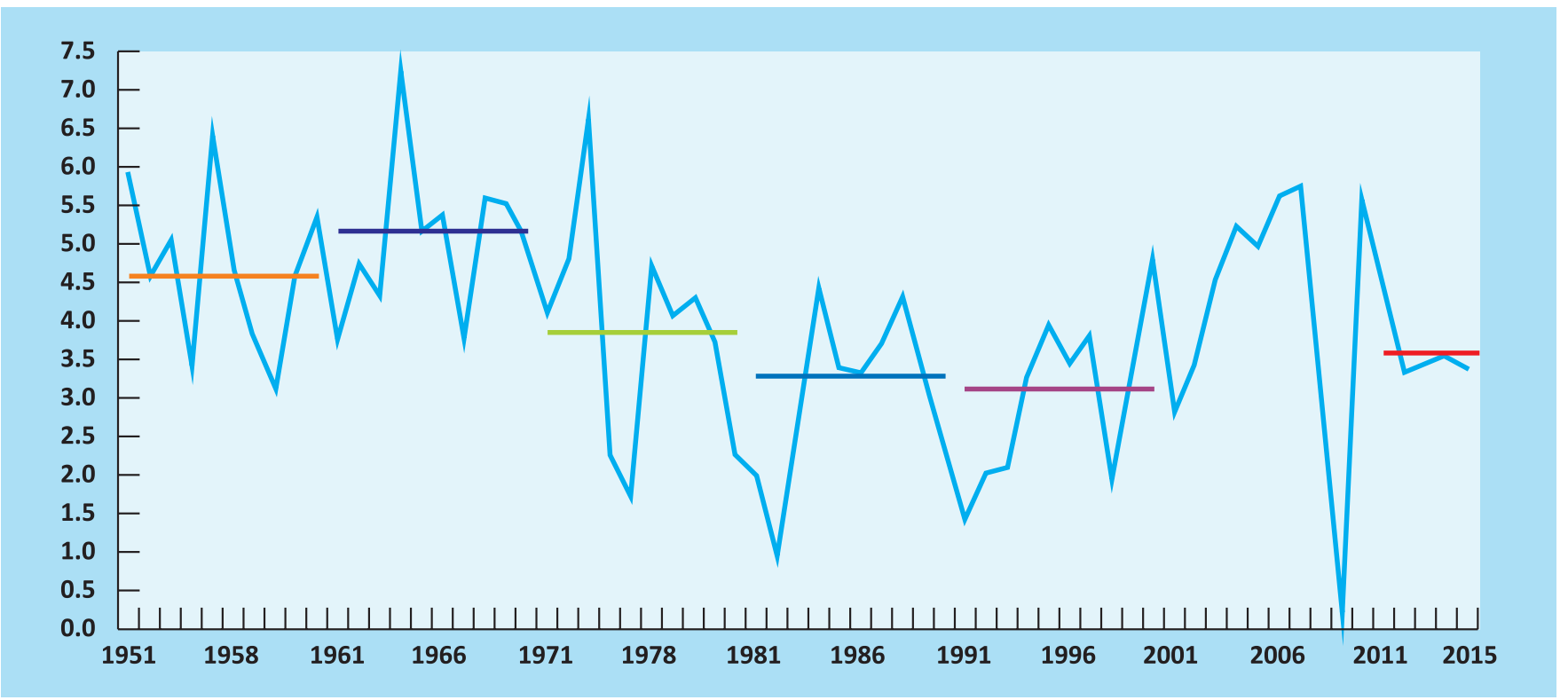

Source: UNCTAD (2016a) secretariat calculations, based on The Conference Board, Total Economy Database. 
periods of convergence and divergence between developed and developing economies and second, there is a growing bifurcation and disparity within the developing world as some regions and countries has grown much faster than others. As the Table 1 reveals, the first decade of the 2000s distinguished itself as a period of rapid and widespread growth in almost all developing world. However, a quick look to the current decade rather reveal that this might have been something anomaly since average annual growth rates in many parts of the developing world dropped down to the growth rates of the 1960 s and the 1970 s or even lower. More importantly, considering the period 1981-2018 in its entirety, developing regions and countries have presented divergent trajectories. Whereas East Asian economies, particularly China, exhibits a steady pattern of convergence, many parts of the developing world, namely West Asia and Latin America, have fallen
At this juncture, as most commonly used indicator of economic growth and welfare, GDP per capita could help us to assess the expansion of economies across and within regions but it remained limited when it comes to account for the well-being of nations and the quality of life of the world population. Thus, despite the relative improvements in overall growth indicators there remains in fact more work to be done in order to ensure more inclusive economic growth globally. Although extreme poverty has been declining globally thanks to the achievements in China and India, as the most populous countries in the world, poverty reduction has been quite unequal across regions and countries. Today, more than 830 million people live below the extreme poverty line of $\$ 1.90$ a day. When the poverty line is adjusted to the moderate poverty threshold from $\$ 1.90$ to $\$ 2.50$ a day, 1.8 million people- around 25 percent of the world population- fall below

Table 1

Growth of Real GDP Per Capita for Selected Regions and Countries, 1951-2018, (Average annual growth, per cent at PPP)

\begin{tabular}{|l|l|l|l|l|l|l|l|l|l|}
\hline & $\begin{array}{l}1951- \\
1980\end{array}$ & $\begin{array}{l}1981- \\
2018\end{array}$ & $\begin{array}{l}1951- \\
1960\end{array}$ & $\begin{array}{l}1961- \\
1970\end{array}$ & $\begin{array}{l}1971- \\
1980\end{array}$ & $\begin{array}{l}1981- \\
1990\end{array}$ & $\begin{array}{l}1991- \\
2000\end{array}$ & $\begin{array}{l}2001- \\
2010\end{array}$ & $\begin{array}{l}2011- \\
2018\end{array}$ \\
\hline Developed Countries & 3.5 & 1.7 & 3.1 & 4.2 & 2.6 & 2.5 & 2.1 & 1.2 & 1.3 \\
\hline USA & 2.3 & 1.9 & 1.3 & 3.4 & 2.2 & 2.6 & 2.4 & 0.9 & 1.6 \\
\hline Developing Countries & 2.7 & 3.9 & 2.7 & 2.6 & 3.0 & 2.1 & 3.2 & 5.8 & 4.1 \\
\hline Africa & 1.8 & 1.3 & 1.5 & 1.9 & 1.2 & -0.4 & 0.7 & 3.0 & 1.9 \\
\hline Latin America & 2.6 & 1.3 & 2.4 & 2.4 & 3.0 & -0.4 & 1.6 & 2.4 & 1.2 \\
\hline Asia & 2.8 & 5.1 & 2.8 & 2.7 & 3.3 & 3.6 & 4.2 & 7.0 & 5.0 \\
\hline East Asia & 3.0 & 7.2 & 4.2 & 3.4 & 4.1 & 6.7 & 5.8 & 9.6 & 6.6 \\
\hline China & 2.3 & 7.7 & 4.1 & 2.7 & 3.1 & 6.5 & 6.2 & 11.1 & 7.3 \\
\hline South East Asia & 2.6 & 3.4 & 2.3 & 1.6 & 4.0 & 2.6 & 3.0 & 4.2 & 4.0 \\
\hline West Asia & 4.4 & 1.3 & 3.2 & 4.9 & 3.4 & -1.6 & 1.6 & 3.3 & 0.1 \\
\hline $\begin{array}{l}\text { Developing countries, } \\
\text { excl. China }\end{array}$ & 2.7 & 2.5 & 2.4 & 2.5 & 2.9 & 1.1 & 2.3 & 3.6 & 2.4 \\
\hline $\begin{array}{l}\text { Developing countries, } \\
\text { excl. East Asia and } \\
\text { South-East-Asia }\end{array}$ & 2.8 & 1.1 & 2.4 & 2.8 & 2.7 & -0.8 & 1.2 & 2.5 & 0.6 \\
\hline
\end{tabular}

Source: Authors calculation, based on the UNCTAD (2018) database.

behind in terms of GDP per capita growth. Thus, looking into the overall data, it is fair to argue that only certain part of the developing world - mostly confined to the East and South East Asian countries- has made noticeable strides in terms of narrowing the income gap with the developed economies. the poverty line (UNCTAD, 2016b, p.4). Again when two most populous countries in the developing world, namely China and India, are excluded, the extreme poverty still pose a global problem and strikes many parts of developing world including Asia, the Caribbean and in particular Sub-Saharan Africa. It is in

Figure 3 Extreme Poverty Around the world (Percentage of population) 2016

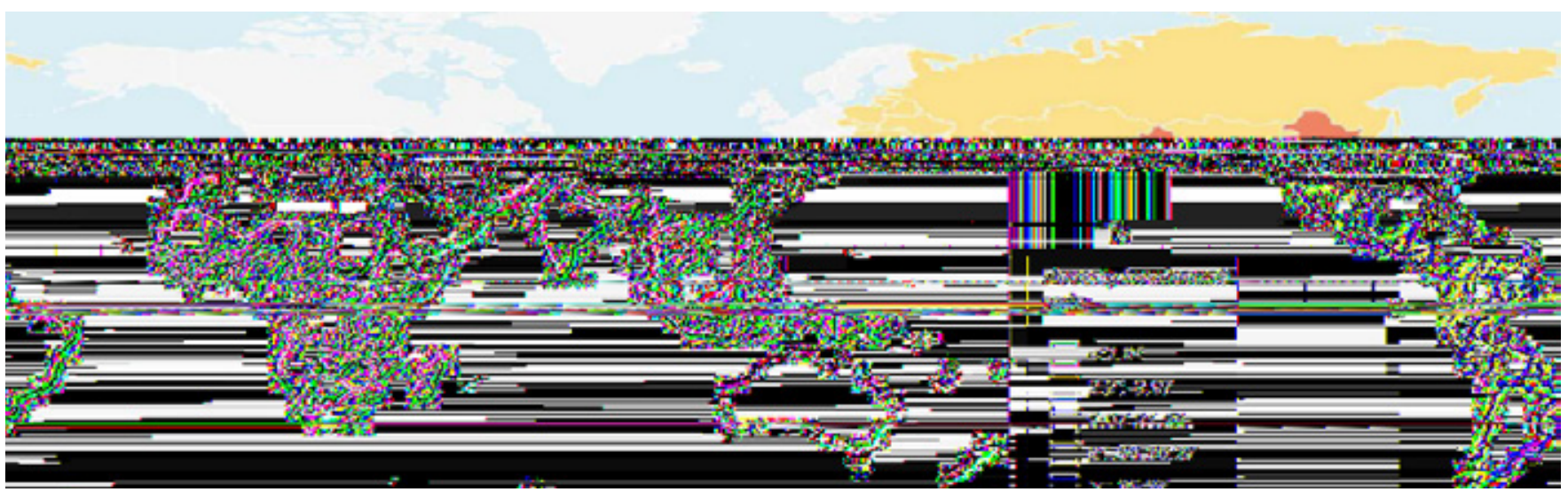

Sources: Derived from World Bank, Poverty and Equity Database. 
Table 2

Levels of GDP per capita and Interregional Spreads, 1000-1998

\begin{tabular}{|c|c|c|c|c|c|c|c|c|}
\hline \multicolumn{9}{|c|}{$\begin{array}{c}\text { Table } 2 \\
\text { Levels of GDP per capita and Interregional } S\end{array}$} \\
\hline & 1000 & 1500 & 1820 & 1870 & 1913 & 1950 & 1973 & 1998 \\
\hline Western Europe & 400 & 774 & 1232 & 1974 & 3473 & 4594 & 11534 & 17921 \\
\hline Western offshoots & 400 & 400 & 1201 & 2431 & 5257 & 9288 & 16172 & 26146 \\
\hline Japan & 425 & 500 & 669 & 737 & 1387 & 1926 & 11439 & 20413 \\
\hline Asia (excluding Japan) & 450 & 572 & 575 & 543 & 640 & 635 & 1231 & 2936 \\
\hline Latin America & 400 & 416 & 665 & 698 & 1511 & 2554 & 4531 & 5795 \\
\hline Eastern Europe \& former USSR & 400 & 483 & 667 & 917 & 1501 & 2601 & 5729 & 4354 \\
\hline Africa & 416 & 400 & 418 & 444 & 585 & 852 & 1365 & 1368 \\
\hline World & 435 & 565 & 667 & 867 & 1510 & 2114 & 4104 & 5709 \\
\hline $\begin{array}{l}\text { Inter-regional spreads- } \\
\text { Richest/poorest }\end{array}$ & 1.1:1 & $2: 1$ & $3: 1$ & $5: 1$ & $9: 1$ & $15: 1$ & 13:1 & 19:1 \\
\hline
\end{tabular}

Source: Maddison, The World Economy. A Millennial Perspective. Development Centre Studies, OECD, (2001).

fact hard to get up-to-date data covering all these regions, but according to the latest available, extreme poverty remains as a dire issue in Southern Asia and sub-Saharan Africa, with 80 per cent of population in these regions live less than $\$ 1.25$ a day. When examining the developing world as a whole, extreme poverty is not exclusively African and South-Asian problem: in the rest of the developing world such as Madagascar, Haiti and the Micronesian Island, the people living in extreme poverty constitutes the majority of the population (see, Figure 3 )

\section{GLOBALIZATION AND INTERNATIONAL DEVELOPMENT: A HISTORICAL OUTLOOK}

Globalization is not a new phenomenon in world history. Historical origins of modern globalization can be traced back to the second half of the 19th century. Therefore, the effect of globalization on international development should be considered with the historical perspective in order to comprehend the ongoing disparities in international development. Broadly, globalization process can be divided into three main historical periods.

The first wave of globalization or liberal era covers the period between 1870 and 1913.It was characterized by increasing international trade, unrestricted capital mobility under the gold Standard and international migration between Europe and the New World. The second period (19141950), covering two world wars, can be denominated as deglobalization era because of increasing restrictive practices toward international trade and capital with a widespread inflation and instability in Europe. The last period of 19502000 covers the golden age of capitalism (1950-1973) and the second wave of globalization or neo-liberal era (1973-2000 and onwards) which is characterized by more open trade regimes, increasing international financial flow, and more marketoriented economic policies in both industrialized, post-socialist and developing countries.
Since 1870 , the globalization process has caused complex patterns of economic convergence and divergence across regions of the world. For example, Maddison (2001) indicates that interregional disparities of GDP per capita between the Western Europe and the poor regions increased in the first wave of globalization period. As can be seen in Table 1, interregional disparities of GDP per capita between richest and poorest region increased from 5:1 in 1870 to 9:1 in 1913. The economic convergence of the first wave of globalization in fact occurred between the countries of Western Europe and the New World, but peripheral regions such as East Europe, Asia, and Latin America were left behind this process. (O'Rourke and Williamson, 2000). Therefore, the first period of modern globalization witnessed a convergence of GDP per capita and a decrease in international inequality across nations within the Atlantic Economy which underpinned the historical NorthSouth division of world economy.

The following period of "de-globalization" (1913-1950), characterized by two world wars, high inflation, instability and restrictive policies, reverted the trend of convergence between Atlantic Economy that had observed during the first wave of globalization. In this period global disparity between the richest and the poorest regions widened by the negative effects of the two world wars and the turbulence of the interwar period. (Solimano 2001) (also see table 2)

The next period of 1950-1973 which is labelled as the golden age of capitalism brought rapid growth, relative stability and declining inequality based on the regulated economy. Post world war economy was characterized by the balance of payment adjustment mechanism and Keynesian policies. During this period GDP in per capita gap between the poorest and the richest regions fell from 15:1 in 1950 to 13:1 in 1973 (see Table 2). Post world war period also witnessed a considerable convergence among western European Economies. Japan became a member of advanced capitalist economies. Some Asian countries such as Taiwan, South Korea, Hong Kong, Singapore experienced rapid growth rate in terms of GDP per capita (Salimano 2001). 
Hey day of golden age of capitalism was shaken by the collapse of the Bretton Woods system and the two international oil shocks of the 1970s which was followed by a slowdown in growth rates and an acceleration in inflation in the OECD countries. The second wave of globalization (19732000 and onwards) is characterized by pro-market economic policies and an increase in global financial flows. These policies were accompanied by a rise in global inequalities and regional disparities (Madison 2001). However, this period witnessed a convergence in Asia due to the high growth rate in Taiwan, Hong Kong, China and India (Salimano 2001). It can be also claimed that in the second wave of globalization period, weighted international inequality (weighted Gini coefficient for world) has decreased considerably with the rapid economic growth in China and India. However, it seems misleading to calculate international inequality in this way because the equalizing impact of rapid growth in these two countries, which possess the $45 \%$ of world population, do not enable us to understand the international inequalities rightly.

Milanovic (2001) has computed the weighted international inequality (1950-1998) with and without taking these two countries into account. As can be seen in Figure 4 since the 1960 's there has been a decline in the weighted international Gini coefficient (a 10\% decline, from 55.6 in 1965 to 50.1 in 1998).

Figure 4

Weighted International Inequality 1950-1998

Source: Milanovic, B. World Income Inequality in the Second Half of the Twenty Century mimeo, World Bank, (2001).

Figure 5

Weighted International Inequality without China and India 1950-1998

Source: Milanovic, B. World Income Inequality in the Second Half of the Twenty Century mimeo, World Bank, (2001).
However, the decline in the Gini reverses if China and India are excluded from the calculation. (see figure 5) With the exclusion of India and China, the weighted international Gini coefficient shows an increase since the 1980's which means that international inequality has been rising since the 1980's. Therefore, the rapid growth in Chinese and Indian economies which are faster than the growth rate of both world economy and economies of rich countries may mislead us. In fact, in the second wave of globalization period world witnessed a rapid economic growth in Asia which was accompanied by reduction in poverty. However, inequality has increased in Latin America, post-socialist Eastern Europe and former Soviet Union and the importance of these regions in world economy has relatively declined. (Salimano 2001)

To sum up when we consider the overall globalization process from 1870 to 2000 , it can be claimed that globalization brings remarkable improvements in living conditions and human development (IMF, 2000). However, it has also caused complex patterns of economic convergence and divergence across regions of the world. It has generated different outcomes for each country that some countries have benefited from it and some faced economic losses. Since 1870, the world has witnessed unprecedented increase in material wealth, growth and experienced dramatic technical development. However, this was also a period of large disparities in income per head
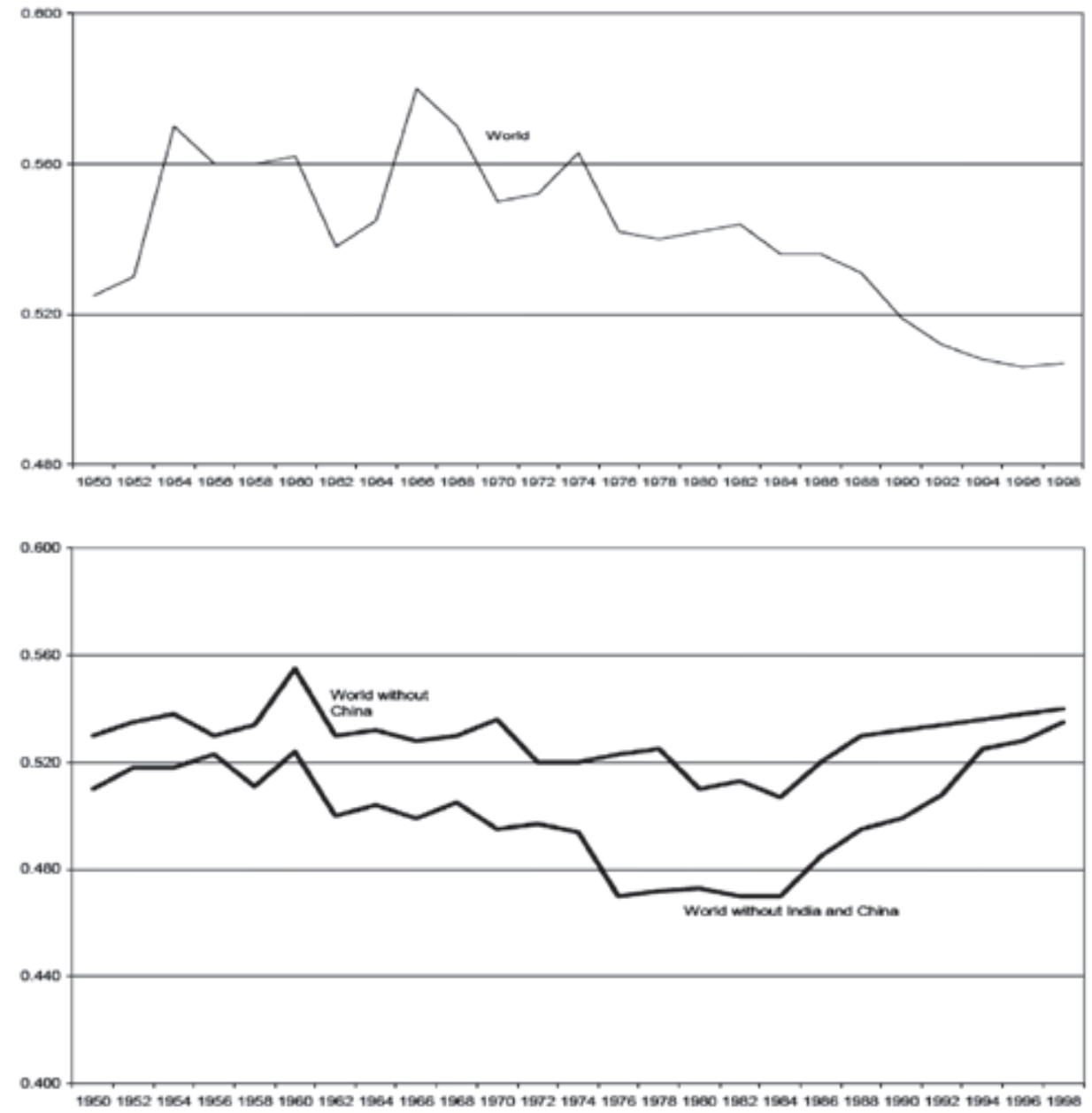
and living standards across countries and regions of the world and despite the economic growth in the developing regions of the world, inequality in international development is still persistent that today one billion people living in developed countries earn 80 percent of the world domestic product whereas the remaining 20 percent is distributed between five billion people who live in developing countries (Rizvi, 2005, p. 2)

\section{CONCLUSION}

Overall globalization process (second stage) and its impact on international development (first stage) shows that globalization causes complex patterns of convergence and divergence across regions and between countries that its impacts cannot be observed at the same degree in all economies of the world. In the first wave of globalization, economic convergence occurred within Atlantic Economy (Europe and the New World) and it did not extend to Central, Eastern European, Asian and Latin American countries.
After the rapid growth in golden age of capitalism, regional convergence was observed within Asia due to the economic growth in South Korea, Taiwan, Hong Kong, India and China. Despite the claims of narrowing international income gap with the equalizing effect of two rapidly growing Asian giant economies India and China weighted international Gini coefficient indicates an ongoing increase since the 1980's.

Moreover, despite the industrialization of Third World with market friendly economic policies, industrial convergence between developed and developing countries has not been followed by an income convergence (first stage). Therefore, expected positive impacts of open economic policies and globalization on international inequalities did not materialize adequately on a global scale. Whereas East Asian countries experienced high growth rates Sub-Sahara African and Latin American countries suffered from economic deterioration. Despite the growth and the improvement of living conditions in developing countries, uneven development and ongoing international economic inequality continues to be a persistent phenomenon of international development.

\section{REFERENCES:}

1. Arrighi G., Silver B. J., and Brewer B. D., (2003). Industrial Convergence, Globalization, and the Persistence of the North-South Divide. Studies in Comparative International Development, 38 (1), pp. 3-31.

2. Bhagwati, J., (2004). In Defense of Globalization. Oxford: Oxford University Press.

3. Callaghan, J., (2002). Social Democracy and Globalization: The Limits of Social Democracy in Historical Perspective. British Journal of Politics and International Relations, 4(3), pp. 429-451.

4. Dreher, A., N. Gaston and P. Martens, (2008). Measuring Globalization - Gauging Its Consequences. Berlin: Springer.

5. Firebaugh, G. and Goesling, B., (2004). Accounting for the Recent Decline in Global Income Inequality. American Journal of Sociology, 110(2), pp. 283-312

6. Fischer, S., (2003). Globalization and Its Challenges. The American Economic Review, 93 (2), pp.1-30.

7. Kiely R., 2005. Empire in the Age of Globalization: US Hegemony and Neo-liberal Disorder. London: Pluto Press.

8. Lockwood, B. and M. Redoano, (2005). The CSGR Globalization Index: An Introductory Guide', Working Paper 155, Warwick: Centre for the Study of Globalization and Regionalisation.

9. Maddison, A., (2001). The World Economy. A Millennial Perspective. Development Centre Studies, OECD.

10. Martens, P. and M. Raza, (2009). Globalization in the 21st Century: Measuring Regional Changes in Multiple Domains. Integrated Assessment, 9(1), pp.1-18.

11. Milanovic, B., (2001). World Income Inequality in the Second Half of the Twenty Century . Mimeo, World Bank.

12. Milanovic, B., (2003). Can We Discern the Effect of Globalization and Income Distribution? Evidence from Household Budget Surveys. Policy Research Working Paper, No. 2876.

13. Milanovic, B., (2005). Worlds Apart: Measuring International and Global Inequality. Princeton: Princeton University Press. 14. Nayyar, D., (2006). Globalization, History and Development: A Tale of Two Centuries. Cambridge Journal of Economics, 30(1), pp. 137-159.

15. O'Rourke, K.H. and Williamson J.G., (2000). Globalization and History. The Evolution of a Nineteenth Century Atlantic Economy. Massachusetts: The MIT Press.

16. Ruckert, A., (2006). Towards an Inclusive-Neoliberal Regime of Development: From the Washington to the Post- Washington Consensus. Labour, Capital and Society, 39(1), pp.34- 67.

17. Stiglitz, J. E., (2008). Making Globalization Work. Economic and Social Review, 39(3), pp 171-190.

18. Solimano A., (2001). The Evolution of World Income Inequality: Assessing the Impact of Globalization. CEPAL - SERIE:United Nations Publication.

19. UNCTAD, (1997). Trade and Development Report 1997, New York and Geneva: United Nations Publication. 20. UNCTAD, (2016a).Trade and Development Report 2016, New York and Geneva: United Nations Publication. 
21. UNCTAD, (2016b). Development and Globalization Facts and Figures, New York and Geneva: United Nations Publication. 22. Wade, R., (2001). Is Globalization Making World Income Distribution More Equal?. LSEDESTIN Working Paper No 01-01. London: London School of Economics, Development Studies Institute.

23. Weeks J., (1999). The Expansion of Capital and Uneven Development on a World Scale. Centre for Development Policy \& Research (CDPR). SOAS University of London.

24. International Monetary Fund, (2000.) Globalization: Threat or Opportunity? [online] Available at <http://www.imf.org/ external/np/exr/ib/2000/041200to.htm> [Accessed 23 July 2018].

25. Rizvi, H., 2005. Globalization Driving Inequality, UN Warns. [online] Available at: <http://globalpolicy.org/component/ content/article/218-injusticeand-inequality/46548.pdf> [Accessed 23 July 2018].

26. Woepking, J., (2008). International Capital Markets and Their Importance, The University of Iowa Center for International Finance and Development. [online] Available at: <http://www.uiowa.edu/ifdebook/ebook2/contents/part3-II.shtml> [Accessed 23 July 2018].

27. Global Policy Forum, (2006). [online] Available at: <http://www.globalpolicy.org/globalization/globalization-of-theeconomy-2-1/generalanalysis-on-globalization-of-the-economy.html> [Accessed 23 July 2018].

28. World Bank, World Development Indicators. [online] Available at: <http://search.worldbank.org/data?qterm=World+Devel opment+Indicators\&language=EN\&format=html> [Accessed 23 July 2018]. 


\section{GLOBALIZATION AND ITS IMPACTS ON INTERNATIONAL DEVELOPMENT: A LONG-TERM HISTORICAL PERSPECTIVE}

\section{MUHAMMED KURSAD OZEKIN}

(MA (King's College London), DPhil (University of Sussex))

Usak University - Department of Political Science and International Relations, Turkey

\section{KEYWORDS: GLOBALIZATION, ECONOMIC GROWTH, UNEVEN DEVELOPMENT, CONVERGENCE.}

For citation: Ozekin M. (2019), Globalization And Its Impacts On International Development: A Long-Term Historical Perspective, Globalization And Business, №7, pp. 49-58. https://doi.org/10.35945/gb.2019.07.006

\section{SUMMARY}

The nexus between globalization and international development is one of the most debatable issues in today's development literature. Broadly, there are two camps: the diffusionist accounts of neoliberal school of thought or what we might call optimists and the so-called radical school of thought or pessimists. Rather than taking sides with either of these two camps, this study embraces a middle ground approach to the matter of globalization and development. Overall, it argues that globalization causes complex patterns of convergence and divergence across regions and between countries that its impacts cannot be observed at the same degree and effect in all ages and economies of the world. In the light of this argument, this study aims to explore the impact of globalization on the unevenness of international development through two main stages. In the first stage, the study critically discusses the nexus between globalization and development by putting special emphasis on the complex patterns of development in the global economy, To that end, the conceptualization of contemporary globalization either as a win-win situation or as a zero-sum game is questioned based on the uneven and asymmetric nature of economic globalization. Later in the second stage, the relationship between globalization and development is situated in a wider historical context of the international capitalist system which has been in a phase of far-reaching structural transformation for a long time. In doing so, the implications of globalization for development are explored in retrospect, focusing on the question of uneven development throughout the history. 


\title{
SOME SIMULATIONS ON THE PORTFOLIO RISK AND RETURN: A METHODOLOGICAL NOTE
}

\section{FITIM DEARI}

\author{
Associated Professor, \\ South East European University, Republic of North Macedonia \\ f.deari@seeu.edu.mk.
}

\section{IZET ZEQIRI}

\author{
Full Professor, \\ South East European University, Associate Member of the \\ Macedonian Academy of Sciences and Arts, Republic of North Macedonia \\ i.zeqiri@seeu.edu.mk.
}

\section{SADRI ALIJI}

\section{Associated Professor,}

South East European University, Republic of North Macedonia

s.aliji@seeu.edu.mk

For citation: Deari F., Zeqiri I., Aliji S. (2019), Some Simulations On The Portfolio Risk And Return: A Methodological Note, Globalization And Business, №7, pp. 59-62. https://doi.org/10.35945/gb.2019.07.007

\section{INTRODUCTION AND DEFINITION OF THE PROBLEM}

Investing either on short or long-term securities is not an easy decision. Decision makers are in the front of many alternatives in which money can be invested. In the principle, decision making process has to study the relationship between risks and returns. Higher risks should be compensated by higher returns and thus different investors will prefer different investments. While some will prefer a combination of higher risk-return, other will prefer a combination of lower risk-return. But, both face with the same problem: how to distribute investing funds among selected alternatives? With other words, what are investing proportions per each alternative, i.e. security?

Let's consider an investor that has 10,000 Euros available to invest among two alternatives: (1) Agilent Technologies, Inc. (hereafter A) and (2) Barnes Group Inc. (hereafter B). Investor required $10 \%$ return from the overall investment, which is 1,000 Euros. Composition of an optimal portfolio means that $10 \%$ return will be realized on one hand and on other hand risk will be minimized. The problem with which an investor faces in this case is how to share 10,000 Euros: how much to invest on $A$ and $B$. Thus, the study tries to present effects that weight has on overall risk and return (portfolio risk and return). Moreover, the correlation coefficient between $A$ and $B$ should also be considered and lower coefficient provides better effect from diversification.

The rest of this paper is organized as following: 2 . Data and methodology; 3 . Analysis, results and discussion; 4. Conclusions; and 5. References.

\section{DATA AND METHODOLOGY}

Time-series data covers the period from March 2010 to February 2018 downloaded from Yahoo Finance (https:// finance.yahoo.com/). For an experimental purpose Agilent Technologies, Inc. (A) and Barnes Group Inc. (B) are random- 
ly selected. From downloaded data we kept just adjusted prices, whereas other data as: open, high, low, close and volume we are not examined in this study. Data are selected on monthly terms and thus stock's returns are calculated. Data and analysis are performed using Excel. Totally 95 observations are examined.

Stock' return is calculated as: $\quad\left(P_{t}-P_{t-1}\right) / P_{t-1}$

and adjusted prices are used.

Another calculation would be: $\operatorname{Ln}\left[\left(P_{t}+D_{t}\right) / P_{t-1}\right]$

and for more see: Benninga (1997).

Covariance and correlation between stock A and B, and the portfolio variance are calculated as following and for more see: Bundo (2009):

$$
\begin{aligned}
& \operatorname{cov}_{i j}=\frac{1}{T} \sum_{i=1}^{T}\left(R_{i, t}-\bar{R}_{i}\right)\left(R_{j, t}-\overline{R_{j}}\right) \\
& \rho_{i j}=\frac{\operatorname{cov}_{i j}}{\sigma_{i} \sigma_{j}} \\
& \sigma_{p}^{2}=\sum_{i=1}^{N} \sum_{j=1}^{N} w_{i} w_{j} \rho_{i j} \sigma_{i} \sigma_{j}
\end{aligned}
$$

\section{ANALYSIS, RESULTS AND DISCUSSION}

In this section performed analysis and related discussion are presented. Table 1 presents selected summary statistics for both stocks. As it can be noticed stock $B$ offers higher return than stock $A$, but both have almost the same sample variance (0.6\%). On average, per each 100 Euros invested on stock $A$, there is 1.5 Euros return, respectively 1.7 Euros on stock B. Stock $A$ reached higher maximum than stock $B$, whereas almost both have similar minimum points. This makes stock $A$ to have range of 0.412 in comparison with $B$ of 0.381. In other words, based on this measure stock $A$ seems to be more risky than $B$. But, on the other hand stock $B$ has higher variance than stock $A$, i.e. $0.59 \%$ versus $0.55 \%$ (see on Table 1, results are presented as rounded numbers rather than percentages).

\begin{tabular}{|c|c|c|}
\hline Description & Return-Stock A & Return-Stock B \\
\hline Mean & 0.015 & 0.017 \\
\hline Standard Deviation & 0.074 & 0.077 \\
\hline Sample Variance & 0.006 & 0.006 \\
\hline Kurtosis & 0.850 & 0.239 \\
\hline Skewness & 0.298 & 0.158 \\
\hline Range & 0.412 & 0.381 \\
\hline Minimum & -0.175 & -0.172 \\
\hline Maximum & 0.237 & 0.209 \\
\hline Count & 95 & 95 \\
\hline
\end{tabular}

Table 1: Summary statistics

View as trend line, less or more stock A and stock B are moving on the same direction as it's presented on Figure 1.

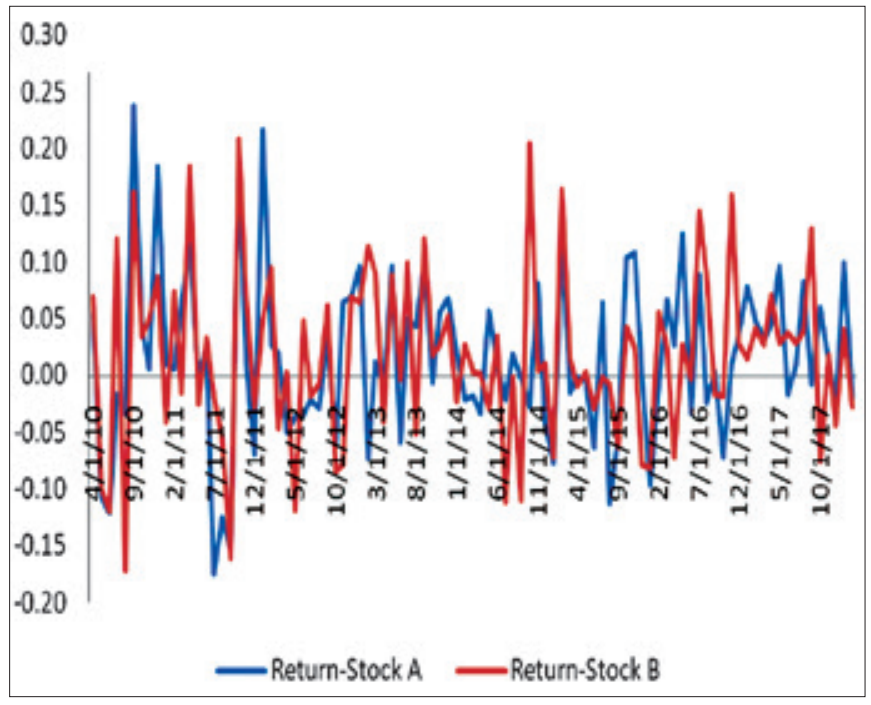

Fig. 1. Historical returns

Table 2 presents correlation between stock $A$ and stock $\mathrm{B}$, whereas Table 3 presents covariance. There is a positive correlation between selected stocks.

\begin{tabular}{|c|c|c|}
\hline & Return-Stock A & Return-Stock B \\
\hline Return-Stock A & 1.000 & \\
\hline Return-Stock B & 0.532 & 1.000 \\
\hline
\end{tabular}

Table 2: Correlation

As usual, investors would chose stocks or securities in general from companies in different business industries due to lower correlation coefficients. Lower return from one business industry is supposed to be compensated with higher return from another one. Thus, the target return or overall return is important, i.e. portfolio return to be achieved under the accepted risk rate.

\begin{tabular}{|c|c|c|}
\hline & Return-Stock A & Return-Stock B \\
\hline Return-Stock A & 0.005 & \\
\hline Return-Stock B & 0.003 & 0.006 \\
\hline
\end{tabular}

\section{Table 3: Covariance}

Moreover, totally five simulations are performed as presented on Table 4. From first to fourth simulation there are presented cases in which arbitrary are given weights, $30 \%$ of funds are invested on stock $A$ and $70 \%$ on stock $B$. In the first simulation, using the actual correlation coefficient of 0.5 , calculated portfolio variance is $0.5 \%$, respectively standard deviation $6.8 \%$. In the second simulation, correlation coefficient is 0.0 (neutral correlation) and thus portfolio standard deviation is $5.8 \%$. In the third simulation, correlation coefficient is 1.0 (perfect positive correlation) and thus portfolio standard deviation is $7.6 \%$. In the fourth simulation, there is a perfect negative correlation, i.e. -1.0 and calculated portfolio standard deviation is $3.1 \%$. 


\begin{tabular}{|c|c|c|}
\hline \multicolumn{3}{|c|}{ Initial data and simulation 1} \\
\hline Description & A & B \\
\hline Weight & 0.300 & 0.700 \\
\hline Correlation coefficient & 0.532 & \\
\hline Portfolio variance & 0.005 & \\
\hline Portfolio standard deviation & 0.068 & \\
\hline \multicolumn{3}{|c|}{ Simulation 2} \\
\hline Weight & 0.300 & 0.700 \\
\hline Correlation coefficient & 0.000 & \\
\hline Portfolio variance & 0.003 & \\
\hline Portfolio standard deviation & 0.058 & \\
\hline \multicolumn{3}{|c|}{ Simulation 3} \\
\hline Weight & 0.300 & 0.700 \\
\hline Correlation coefficient & 1.000 & \\
\hline Portfolio variance & 0.006 & \\
\hline Portfolio standard deviation & 0.076 & \\
\hline \multicolumn{3}{|c|}{ Simulation 4} \\
\hline Weight & 0.300 & 0.700 \\
\hline Correlation coefficient & -1.000 & \\
\hline Portfolio variance & 0.001 & \\
\hline Portfolio standard deviation & 0.031 & \\
\hline \multicolumn{3}{|c|}{ Simulation 5} \\
\hline Optimal weight & 0.536 & 0.464 \\
\hline Correlation coefficient & 0.532 & \\
\hline Portfolio variance & 0.004 & \\
\hline Portfolio standard deviation & 0.066 & \\
\hline
\end{tabular}

Table 4: Simulation of correlation and weight

Results denote clearly that as the correlation coefficient is lower, the portfolio risk is measured by variance and the standard deviation becomes lower too. Theoretically, there is no benefit from the diversification when correlation is +1 as it evidenced here and better result is obtained when correlation is -1 . In the condition of perfect negative correlation, portfolio standard deviation is $3.1 \%$, which is smallest from all other simulations.

Finally, the fifth simulation presents the case that with actual data the best solution is to invest $53.6 \%$ on stock $A$ and $46.4 \%$ on stock $B$ for an investor which attempts to minimize risks. These percentages are optimal weights or proportions how to share funds among both selected stocks. With optimal weights, overall return will be $1.56 \%$, whereas on other cases will be $1.60 \%$. Thus, in this case an investor gets something lower return from portfolio since the risk is slightly decreased comparing with the first simulation. Optimal weights are found using Excel Solver. Otherwise, optimal weight on stock $A$ (then investing on stock $B$ is: $1-w A$ ) can be found using Lagrange function (multiplier) (see for example: Bundo, 2009; Deari, 2015) or other calculation ways (see for example: Bodie, Kane, Marcus, 2002; Roychoudhury, 2007).

Figure 2 presents all simulations performed based on correlation coefficients.

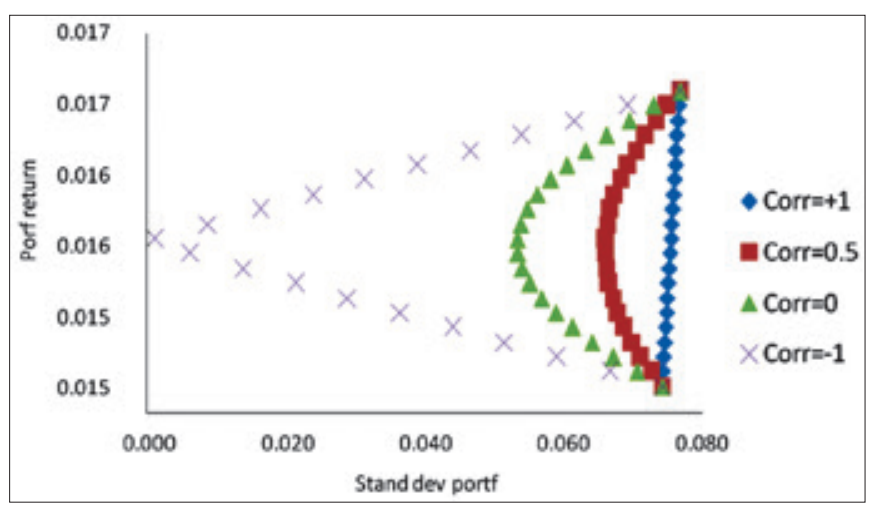

Fig. 2. Portfolio risk and return

\section{CONCLUSION}

This study applied an experimental analysis showed that the risk and the return from a portfolio is influenced by the correlation coefficient and respectively investing weights. Selecting securities with lower correlation coefficients should be an element in the decision making process, as well how to share funds in an optimal way.

\section{REFERENCES:}

1. Benninga, S. (1997). Financial Modeling, Uses Excel. Cambridge: Massachusetts Institute of Technology Press.

2. Bodie, Z., Kane, A., Marcus, J. A. (2002). Investments. Boston: McGraw-Hill Irwin.

3. Bundo, Sh. (2009). Drejtim Portofoli. Tiranë: albPAPER.

4. Deari. F. (2015) “Portfolio Composition: A Methodological Solution Using Lagrange Multiplier", ICESOS'15, Sarajevo.

5. Roychoudhury, S. (2007). "The optimal portfolio and the efficient frontier", National Science Foundation. [Online] 


\section{SOME SIMULATIONS ON THE PORTFOLIO RISK AND RETURN: A METHODOLOGICAL NOTE}

\section{FITIM DEARI}

\section{Associated Professor,}

South East European University, Republic of North Macedonia

f.deari@seeu.edu.mk.

\section{IZET ZEQIRI}

\section{Full Professor,}

South East European University, Associate Member of

the Macedonian Academy of Sciences and Arts, Republic of North Macedonia

i.zeqiri@seeu.edu.mk.

\section{SADRI ALIJI}

\section{Associated Professor,}

South East European University, Republic of North Macedonia

s.aliji@seeu.edu.mk

\section{KEYWORDS: INVESTMENT, CORRELATION, WEIGHT, PORTFOLIO, STOCK.}

For citation: Deari F., Zeqiri I., Aliji S. (2019), Some Simulations On The Portfolio Risk And Return: A Methodological Note, Globalization And Business, №7, pp. 59-62. https://doi.org/10.35945/gb.2019.07.007

\section{SUMMARY}

In this study we try to demonstrate mainly from the methodological and pedagogical perspective how investment weights and the correlation between securities are manifested on the portfolio risk and return. Empirical data are examined from 2010 to 2018 and for experimental purpose two companies are randomly selected. Totally five simulations are performed using Excel.

The fifth simulation presents the case of optimal proportions for an investor which efforts to minimize the portfolio risk. 


\section{SOCIETY'S INVOLVEMENT IN SOCIAL ENTREPRENEURSHIP TO PROMOTE SMART REGIONAL GROWTH}

\section{INTA OSTROVSKA}

Dr.paed., docent Institute of Humanities and Social Sciences

Daugavpils University, Latvia

inta.ostrovska@du.Iv

\section{LUDMILA ALEKSEJEVA}

Dr. oec., docent

Head of the Department of Economics and Sociology, Latvia ludmila.aleksejeva@du.Iv

\section{DMITRIJS OLEHNOVIČS}

Mg.hist, lecturer

The Department of History, Daugavpils University, Latvia

dmitrijs.olehnovics@du.Iv

\section{LIENE LEIKUMA-RIMICĀNE}

\author{
Mg.oec., PhD student \\ Senior Specialist in Cooperation, Issues International and \\ Public relations office Daugavpils University, Latvia \\ liene.rimicane@du.Iv

\section{JĀNIS KUDIṆŠ} \\ Mg.oec., PhD student \\ Deputy rector for development, International and \\ Public relations office \\ Daugavpils University, Latvia \\ janis.kudins@du.Iv
}

KEYWORDS: SOCIAL ENTREPRENEURSHIP, COMMUNITY, ENGAGEMENT, SMART GROWTH, REGION.

For citation: Ostrovska I., Aleksejeva L., Oḷehnovičs D., Leikuma-Rimicāne L., Kudinš J. (2019), Society's Involvement In Social Entrepreneurship To Promote Smart Regional Growth, Globalization And Business, №7, pp. 63-69.

https://doi.org/10.35945/gb.2019.07.008

\section{INTRODUCTION}

The concept of social entrepreneurship, hybrid businesses are relatively new in economic research, but such forms of social benefit-oriented entrepreneurs, associations, philanthropic movements and other market players have long been seen. The development of the social economy sector, which can be described as non-commercial mass business sector. It is based on democratic values and seeks to improve social, economic and environmental conditions of society, often focusing on disadvantaged members of society. It also develops awareness of the third sector in the economy by carrying out its activities between the private sector and the business sector, or between the public sector and government. New organizations are arising, initiatives and research that defined a new type of business- social entrepreneurship.

\section{THEORETICAL INSTITUTIONAL FRAMEWORK FOR SOCIAL ENTREPRENEURSHIP}

This concept appeared for the first time in scientific literature in the 20th century. In the 60's and 70's, more visibility was gained thanks to the active functioning of the "Ashoka" organization and basic social entrepreneurship concepts which were stated by founders such as Mr.Young, Mr.Dreiton, Mr.Durands, Mr.Silbert, Mr.Junus and others. (Alter, Dawans, Miller, 2007)

During various periods of time, young social enterprises developed to deal with socio-economic problems through market mechanisms for the non-governmental sector representatives and entrepreneurs, performing functions that the public or private sector was unable to address. Social enterprises of these different forms are characterized by ethical values and responsibilities, local public interests, social and 
economic opportunities and a primary social and/or environmental objective rather than profit making. In many countries, such social private or partnership companies (hybrid companies) have long been known and have many common features, but in each country, they are called and governed by completely different departments and laws. Hybrid enterprises include the competences of private (economic), national (political) and environmental (ecological) areas, developing new ways of engaging society to meet existing needs (Schieffer and Lessem 2009). These types of companies are mainly developing in countries where legislation has not provided for a clear framework for the implementation of social entrepreneurship. Therefore, through the maximum flexibility of private, non-profit and public sector borders, they form new forms of activity, which seek to maximize the integration and convergence of sectoral boundaries, with a high contribution to social benefits for economic activities. In the same way as social innovation, social entrepreneurship worldwide has raised the need for a common concept in order to promote the smooth development of such businesses across the European Union at the end of 2011, the European Commission approved and made public Social Entrepreneurship initiative that updated the concept of new social enterprises and hybrid businesses. The document clarifies their substance and seeks to establish a common approach in the style of their management and economic activities and sets out measures that will contribute to the development of the sector. This document defines a social enterprise as a member of the social economy, which is a company whose main objective is to exercise social influence and not to create benefits for its owners or partners. It operates in a market, in a business specific and innovative way of producing goods and services; it uses income mostly for social purposes. These undertakings are managed responsibly and transparently, namely by involving its employees, customers and stakeholders in the economic activities of the undertaking. (Social Business Initiative, 2011).

Social welfare can be a catalyst for the smart growth of the area, even though as the theoretical findings indicate, debates on smart development are still young (Steiner and Mossbock, 2014). However, significant changes in paradigms of regional development policy already have occurred (Vanthillo and Verhetsel, 2012). Experts in this field highlight that possible ignorance of regional and sectoral peculiarities (Steiner and Mossbock, 2014), necessity in restructuring of economy (OECD, 2013), and creating new jobs (EPA, 2015a) are the main challenges for smart development. (see more Šipilova, Ostrovska, Aleksejeva et al., 2017)

Rural areas are particularly important for ensuring sustainability and smart development of a state as a whole. The present study reflects the quantitative and qualitative assessment of smart growth challenges in the region of Latgale (Latvia) at the level of 19 districts. This is worked out within the framework of the Latvian National Research Programme EKOSOC-LV. The present study focuses on smart growth as a tool for risk pre- vention and the use of opportunities in regional development within the framework of the concept of smart specialization. The aim is to estimate the risk factors that influence the formation of a smart territory and to analyze the interrelationship of quantitative indicators and expert opinions. By analyzing the theoretical principles, the regional actors' recommendations, taking into account the research group participants' and regional experts' findings, the authors characterize the processes and the risks of smart development, as well as make assumptions about the development of the desired situation. The significant risk in the development of Latgale region is the decline in its population. At the same time, the results of the research show that the population is the most important cornerstone of smart growth of the rural territories in Latgale region. Both the objective data processing (statistical analysis) and the subjective point of view (the results of the expert survey) highlight a number of significant risk factors for promoting smart regional space and the growth of knowledge-based economy: the insufficient development of the population's economic activity particularly in the knowledge-based segment, and the small population size. The assessment of various challenges and risks in the regional development of Latvia, as well as the integrated application of quantitative and qualitative approaches allows for the elaboration of a comprehensive vision of the smart growth processes in Latgale region of Latvia. The quantitative assessment is based on the establishment and testing of the integrated index (Smart Development Index). The qualitative assessment is based on the regional experts' opinions summarized by using the Analytic Hierarchy Process methodology. The research results have both scientific and practical applicability in promoting smart development in rural areas. Firstly, the results of the study offer a possible methodological solution for the assessment of smart development. Secondly, the obtained scientific experience can be used for solving practical problems at the level of the districts of Latgale region. (Aleksejeva, Šipilova et al., 2018)

The greatest challenge in Latgale region is related to the loss of human capital and the sluggish development trends in entrepreneurship (e.g., "Latgale's Region of Planning", 2010a, 2010b). The programme and the strategy of the region's development (e.g., "Latgale's Region of Planning", 2010a, 2010b) envisage a set of measures aimed at mitigating the negative trends. These measures are based on raising efficiency of the use of local resources, on activating the cooperation between the parties involved in the process of development, and in strengthening the business sector (e.g., "'Latgale's Region of Planning", 2010a, 2010b). From an economic perspective, a significant contribution to the development of Latgale region is ensured by the relatively successful operation of the high technology sector of manufacturing, and the use of rich natural resources (e.g., "Latgale's Region of Planning", 2010a, 2010b). However, it should be noted that the potential of rich natural resources of Latgale region, due to the low economic activity, is mostly used with low add- 
ed value (e.g., "Latgale's Region of Planning", 2010a, 2010b). The low growth of labour productivity is obvious not only in Latgale region and it is one of the main reasons for overall worsening of external competitiveness of Latvian economy (e.g., Baldi, Šipilova, 2014). (see more Olehnovičs, Ostrovska, Šipilova et al., 2017) As far as paradigm of smart development is new for Latvia and regions, possibilities of small municipalities are limited not only with their socio-economical capabilities, but also due to the lack of visible example, knowledge and experience in this area. However, such a problem is not topical only for Latvia.

\section{NATIONAL EXPERIENCE IN SOCIAL ENTREPRENEURSHIP}

Social enterprises are not artificially newly created forms of economic activity, nor do they have a future vision of the only right model for the implementation of economic activities, which has been developed over time by companies which consider the promotion of social value and its alignment as an alternative to the profits generated by the company for business owners. Regardless of the above factors and other characteristics, global practice has shown that countries where social entrepreneurship is part of the market are more stable, because during the global economic crises the social usiness sector acts as a stabiliser of the situation, based on activity in local markets and therefore, is less affected by economic fluctuations in the world. Especially in countries where social enterprises are found in all sectors and spheres such as the Netherlands and Belgium.(Leikuma, 2012)

In Georgia, the social entrepreneurship is evolving. The Center for Social Entrepreneurship is a structural unit of The Center for Strategic Research and Development of Georgia. Center's long term goal is establishment of the concept and promoting practical activities linked to the Social Entrepreneurship in Georgia. The Center for Social Entrepreneurship is engaged in the development of Social Entrepreneurship in Georgia. To achieve this goal, Center developed a module of trainings for interested organizations and provides thematic consultations. In order to assist beginning Social Entrepreneurs, Center announces grants competitions and provides periodical consultations to the winner organizations in organizational, financial, marketing and legislative issues. In order to better adapt Social Entrepreneurship with the Georgian reality, Center works with various State agencies on the local and central levels. (The center for social., 2019)

The Russian experience in this field is increasing. Difficulties with self-determination of social entrepreneurs show, that there is a problem with the legislation. The documents and laws from the Ministry of Economic Development of the Russian Federation identify the subject of "social entrepreneurship" as a small and medium-sized business, not NGO. Secondly, due to the lack of significant privileges and encouragement, there is no reason for such identification. Also there is no motivation to unite such people into the professional community. When a professional community starts an activity (for example, on the basis of Center for Innovations in the Social sphere), it causes situations when more enterprises start to identify themselves as social ones. Thirdly, the reluctance to be self-defined as a social entrepreneur arises due to the fact that often "purely business" projects, which have no social component, call themselves "social entrepreneurs". The vast majority of the registered enterprises work in the sphere of socially oriented NGOs (they are social enterprises classifying themselves as NGO). Education, services sector, social services for citizens and health care are popular among entrepreneurs. The brightest projects of social entrepreneurship are successful projects notwithstanding their sphere. In other words, we can infer that it is difficult to speak about existence of certain "niche" where social entrepreneurship would be successful or demanded for sure. So, it is impossible to say that social entrepreneurship needs for some "niche" orientation. In general, common principles of effective business models and niches, could be applied for social entrepreneurship. (Social entrepreneurship in ..., 2018)

\section{SOCIAL ENTREPRENEURSHIP IN LATVIA}

In recent years, there has also been a public debate in Latvia about social enterprises, their meaning, relevance and the need for a regulation of such a type of economic activity, which would ensure equal competitiveness with other companies, as well as enabling them to compete in the market on similar terms by attracting investments and customers, etc. Such initiatives are witnessed by organised social entrepreneurship forums, blogs, media articles and other public initiatives. There are a number of events that could ensure faster development of the social entrepreneurship sector and would be able to combine the pursuit of economic activities and social objectives within the same organisation, by reducing operational costs and red tape, thereby directly contributing to the development of the sector. Support for social enterprises should focus on the following measures: common sectoral components for the implementation of public policy; legislative development governing sector action; promotion of social entrepreneurship support programmes and incentive programmes to attract private investment; use of social enterprises as tools for rural development and integration programmes for vulnerable groups of urban communities; experimental social activities; establishment of companies, analysis and research of the indicators of these companies. The following overall social enterprise activity model is proposed in Latvia. (see Figure 1)

One of the challenges is the sector's weakness in many and different forms of its expression, where synergies between the various elements are needed with a view to strengthen social entrepreneurship and the social economy 
as a whole. One of the necessary measures: promoting visibility, this element would ensure that these companies are identified and that they are aware of their operational objectives and social benefits. Another element to boost the sector's visibility is to promote more and better quality sector research, as well as systematic data collection on the indicators, welfare and quality of life of existing companies and their links to the number of social enterprises. Better sector research would also improve skills and capacity building in social economy organizations, starting with specialized higher education programmes to promote awareness and diverse characteristics of social enterprises, and partly address the third challenge the lack of specialized training, practices and education. Social economy organizations operate under unfair competition compared to traditional forms of business. More research into social enterprises, analysis of laws, methods and generally accepted strategies would increase the advantages of social enterprises competing in the market. Education has a direct impact on advancing new processes and promoting specific initiatives, including the direct impact on the development of the social entrepreneurship sector on education in the development and implementation of sector related research and training programmes. The increase of informative resources would facilitate the formation of social enterprises, as well as support would be given to enthusiasts with rich ideas, driven by social benefits to start a business, the right training and preparation to work in a traditional business-oriented environment, which would improve the sustainability of social enterprises. Such developments would also improve the provision of available support networks and infrastructure for the provision of suitable business development services to third sector players.

On April 1st 2018, the Social Business Act came into force, which aims to create a favourable environment for social entrepreneurship. Representatives of the Latvian Social Business Association also actively participated in the formation of the Law for three years. (Full text of the law is available here: https://likumi.lv/ta/id/294484-social-networking-law)

Important regulations related to Cabinet of Ministers.:

- rules on the procedures for the granting, registration and monitoring of social enterprise status of population groups at risk of social exclusion;

- rules on the Social Business Commission;

- rules on conditions for granting commercial support to social enterprises and procedures for granting aid.

The new law aims to promote the quality of life of the society and to promote the employment of people at risk of social exclusion. The law provides a legal framework for both the criteria for obtaining the status of a social enterprise and the procedures for the state to support this type of business.

The Ministry of Welfare is responsible for the promotion and development of social entrepreneurship. The status of a

Figure 1. Generalised business model of social enterprise in Latvia (Leikuma-Rimicāne, 2013)

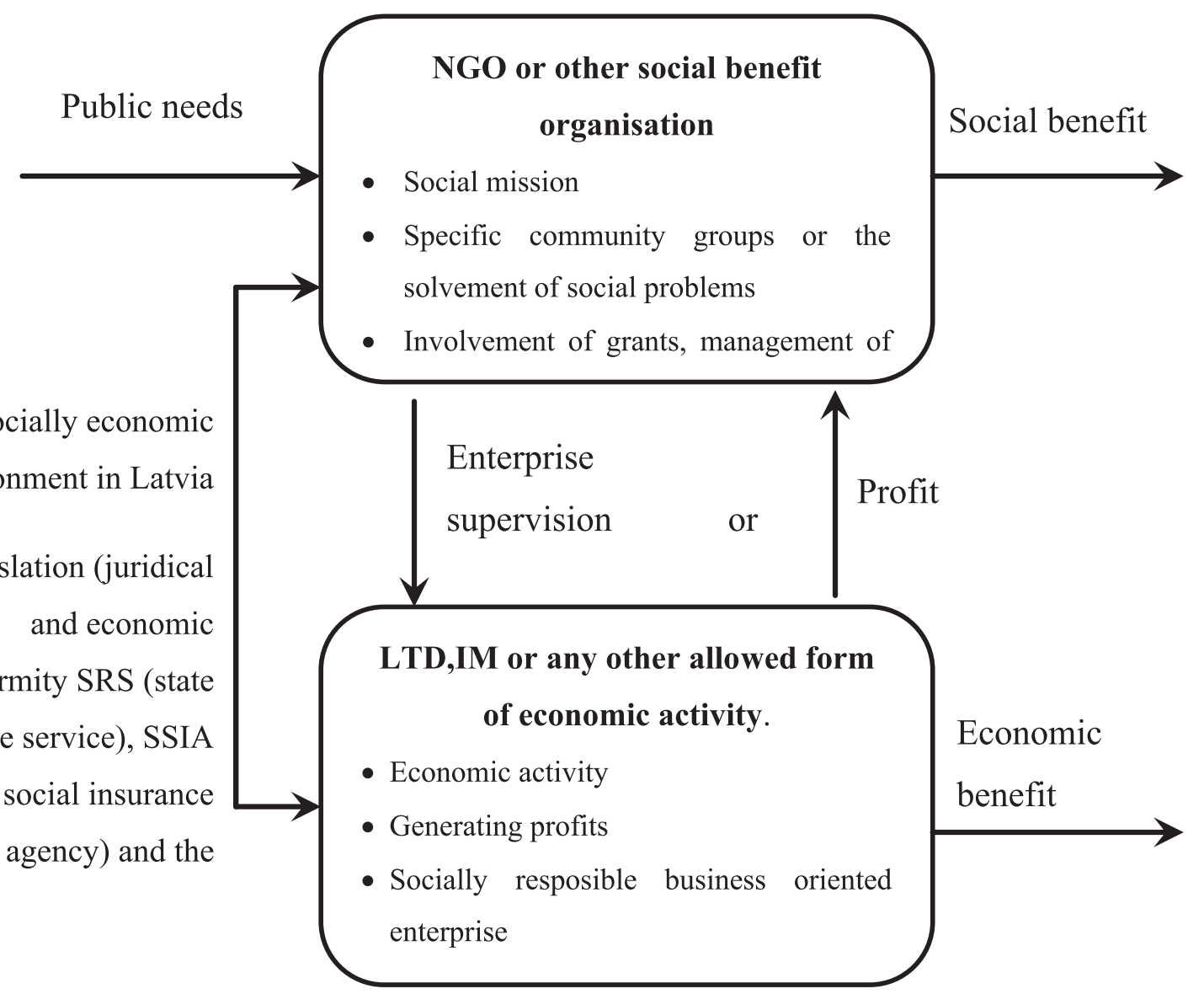


social enterprise is granted by the Ministry of Welfare on the basis of an evaluation of specifically established Social Enterprise Commission. The Ministry also maintains the Register of Social Enterprises by publishing it on their home page.

Latvian Social Entrepreneurship Association is an organization that combines social enterprises and, in various ways, cares about the development and awareness of the social entrepreneurship sector. We participated in the drafting of the law, many meetings with decision makers and municipal representatives, the story of social enterprise work and the benefits that arise if we allow and help social enterprises to work. Also, we travel a lot around Latvia, telling the practical sides of social entrepreneurship: how to start such a company, how to find the right idea of building a business model, what the opportunities to seek financing and investment are. We also maintain the www.socialauznemejdarbiba.Iv homepage, which is the largest resource in Latvian for social entrepreneurship, where there are various kinds of interpretative materials available, which can help to understand the nature and the practical side of social entrepreneurship. (Johansone (Ikstena), 2018)

A fundamental problem is the financial availability. Social purpose-based organizations often find it difficult to access the same funding opportunities as traditional companies because of their specific characteristics, including social objectives, measurability of social benefits, return of profits, efficiency, etc. As a response to the market failure, new opportunities have been found for companies to follow the spotlight in the European Union, where a platform for attracting investment has been launched, which would be open to all social enterprises in the Union. Another potential development event "crowd funding", which takes the form of using small scale capital from many independent individuals to fund new business ideas. Crowd funding is easily available thanks to widely used social networks, which allow the idea to be found in a very broad spectrum and attract their investment to realize the idea. The principle has not yet gained popularity in Latvia, but it shows many features that suggest that this type of financial attraction would work directly in Latvia because of social enterprises and their public friendly objectives, because individuals are prepared to support ideas that are friendly and transparent to society as they are in social companies.

However, more financial attraction tools have been created. For the development of social entrepreneurship, project contests are often important for the development of entrepreneurship. Individual municipalities, for example, with varying degrees of regularity, organize project contests to be followed by the entrepreneurs themselves, for example, twice a year, the call for a "kick-off" grant contest. Also helpful for business development is the Business Ideas Cup.

In the second quarter, "Second Breath" offers an opportunity to participate in a scholarship contest where the winners of the scholarship are selected by the buyers when they are voted in favor. As one of the priorities, the development of social entrepreneurship is precise.
The most important support for the development of social entrepreneurship is the LM and ALTUM support measure, which includes the possibility of obtaining a financial grant for the development of meaningful social entrepreneurship. The funding is available under the measure ranges from $€ 5$ to $€ 200$ thousand, depending on the company's experience and current turnover.

The New Door is an accelerator for social entrepreneurship, helping to practically develop the idea of social entrepreneurship, while Reach for change is a social entrepreneurship incubator that helps develop social entrepreneurship ideas related to improving the quality of life for children and young people. Calls to these organizations are once a year in the autumn.

Services provided by the Latvian Investment and Development Agency are also available to social entrepreneurs. Regional business incubators are particularly suitable for social entrepreneurs, who can help develop the business idea during the pre-accession phase and support the business during the incubation phase. If the idea of social entrepreneurship is innovative, an appropriate programme is also supporting services for innovation vouchers.

\section{SOCIAL ENTREPRENEURSHIP IN LATVIAN REGIONS AND MUNICIPALITIES}

The legal framework also provides the right of municipalities to participate in the creation of a social enterprise. However, municipalities in such companies cannot have the majority of votes, and the purpose of doing so must be the employment of the target group. This provision will be in force three years after the law comes into force (until 2021), but the legislator will have the possibility to extend this period when assessing the functioning of the law if social enterprises without the participation of municipalities do not form a sufficient activity.

Municipalities can be important partners to promote the development of social enterprises. Local governments are announcing grants for business development, and they also tend to apply, for example, a property tax discount. Each municipality operates at its discretion in this respect and grant tenders are mostly published at a given time.

On the other hand, if we look at the regional representation, it is noted (see Figure 2 ) that most social enterprises are registered in Riga and around Riga, which makes it possible to assume that the concept of social entrepreneurship is not yet known to a large part of the society, particularly in those regions (e.g. Latgale), where there are significant and diverse social problems and the tension they create.

Statistical data regarding the regional representation of social enterprises indicate further work, which should be performed by both the Ministry of Welfare as the responsible Ministry for the policy area and the Latvian Association of Social Entrepreneurship - as an organization representing the sector. Only by telling and presenting examples of good practice will the concept of social entrepreneurship be able to reach further corners of Latvia and will find long-term solutions to various 
Figure 2. Social businesses in Latvian regions at the beginning of 2019 (Frīdenberga 2019).

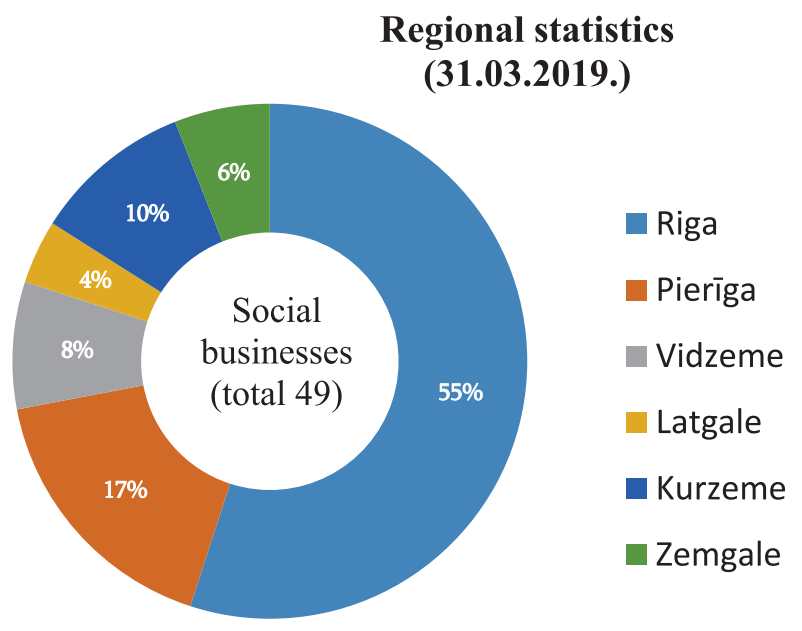

societal challenges -unemployment, education quality issues, environmental challenges and others. https://sua.Iv/pirmaisyear-with-social-awareness-law-secinajumi /

\section{CONCLUSION}

A merchant who conforms to the criteria specified in the Law may obtain the status of a social enterprise in Latvia. State social companies will be given a certain amount of support, but they will be prohibited from distributing profits between the owners of the company. It must be invested in achieving the objectives set out in the articles of association: creating public goods. In addition, certain areas in which a social enterprise is not entitled to operate, such as arms and ammunition, alcoholic beverages, tobacco products, gambling and betting, and financial and insurance sectors.

Developing the increase in the number of social enterprises and hybrid organizations will not only result in a strong economically active sector, but also based on the experience of other countries, will improve the wellbeing of Latvian citizens due to the social objectives of economic activity.

The research is supported by Daugavpils University project "Public involvement in social entrepreneurship in Latgale to enhance smart growth of the region"

\section{REFERENCES:}

1. Aleksejeva L., Šipilova V., Jermolajeva E., Ostrovska I., Oḷehnovičs D. (2018) Regional Risks and Challenges in Smart Growth in Latgale Region (Latvia). Journal of Security and Sustainability Issues ISSN 2029-7017 print/ISSN 2029-7025 online. 2018 June, Volume 7, Number 4, pp. 727-739 http://jssidoi.org/jssi/papers/papers/view/305

2. Alter K., Dawans V., Miller L. (2007) Social Enterprise Typology, [online] [viewed on 12th January 2013]. Available: http:// www.4lenses.org/setypology/history

3. Frīdenberga A., (2019) Pirmais gads ar Sociālā uzṇēmuma likumu - secinājumi [First year with Social Business Law - conclusions] https://sua.Iv/pirmais-gads-ar-sociala-uznemuma-likumu-secinajumi/ [viewed on April 22rd. 2019]

4. Leikuma L., (2012) Development of social entrepreneurship projects in Latvia, Riga, RTU

5. Lešinska A., Litvins G. (2012) Latvija ceḷā uz sociālo uzṇēmējdarbību [Latvia on the way to social entrepreneurship] available: http://providus.lv/article/petijums-latvija-cela-uz-socialo-uznemejdarbibu

6. Johansone (Ikstena) E. (2018) Kādas ir sociālās uzṇēmējdarbības iespējas Latvijā? [What are the opportunities for social entrepreneurship in Latvia?] https://www.Ism.lv/raksts/zinas/ekonomika/kadas-ir-socialas-uznemejdarbibas-iespejas-latvija-intervija-ar-asociacijas-vaditaju.a275216/ [viewed on April 23rd. 2019]

7. Ministry of Welfare. Regulatory framework: Order 24.05.2013. Nr. 35. [online] [viewed on 4th October 2013]. Available: http:// mail.Im.gov.lv/web/ library.nsf/36be6e10494a87fcc2256ade00399621/f3eebcd1a1e5b903c2257b780040f25c?OpenDocument

8. Olehnovičs D., Ostrovska I., Šipilova V., Jermolajeva E., Aleksejeva L. Sustainable development assessment in Latvia: the example of Latgale region. International Scientific-Practical Magazine Globalization and business, 3 / 2017. Pp. 26-31. http:// www.eugb.ge/uploads/content/N3/3-04.pdf

9. Schieffer A, Lessem R (2009) Beyond social and private enterprise: towards the integrated enterprise. Trans Stud Rev 15:713-725 10. Social entrepreneurship initiative: Announcement from the European Commission. 25.10.2011 [online] [viewed on October 23rd 2012.]. Available: http://eur-lex.europa.eu/LexUriServ/LexUriServ.do?uri=COM:2011:0682:FIN:LV:PDF

11. Social entrepreneurship in Russia: ecosystem development. https://sua.lv/wp-content/uploads/2019/04/Mapping_ eng_2018.pdf [viewed on 4th April 2019].

12. Šipilova V., Ostrovska I., Aleksejeva L., Jermolajeva E., Oḷehnovičs D. (2017) A Review of the Literature on Smart Development: Lessons for Small Municipalities. International Journal of Economics and Financial Issues, 2017, 7(1), 460-469. https:// www.econjournals.com/index.php/ijefi

13. The center for social entrepreneurship. http://segeorgia.org/info.php?ID=11\&In=en [online] [viewed on 24th April 2019]. 14. The law of social entrepreneurship. https://likumi.lv/ta/id/294484-sociala-uznemuma-likums [online] [viewed on 24th April 2019]. 


\section{SOCIETY'S INVOLVEMENT IN SOCIAL ENTREPRENEURSHIP TO PROMOTE SMART REGIONAL GROWTH}

\section{INTA OSTROVSKA}

Dr.paed., docent Institute of Humanities and Social Sciences

Daugavpils University, Latvia

inta.ostrovska@du.Iv

\section{LUDMILA ALEKSEJEVA}

\section{Dr. oec., docent}

Head of the Department of Economics and Sociology, Latvia ludmila.aleksejeva@du.Iv

\section{DMITRIJS OLEHNOVIČS}

Mg.hist, lecturer

The Department of History, Daugavpils University, Latvia

dmitrijs.olehnovics@du.Iv

\section{LIENE LEIKUMA-RIMICĀNE}

Mg.oec., PhD student

Senior Specialist in Cooperation, Issues International and Public relations office Daugavpils University, Latvia liene.rimicane@du.Iv

\section{JĀNIS KUDIṆŠ}

Mg.oec., PhD student

Deputy rector for development, International and Public relations office

Daugavpils University, Latvia

janis.kudins@du.Iv

KEYWORDS: SOCIAL ENTREPRENEURSHIP, COMMUNITY, ENGAGEMENT, SMART GROWTH, REGION.

For citation: Ostrovska I., Aleksejeva L., Oḷehnovičs D., Leikuma-Rimicāne L., Kudinš̌ J. (2019), Society's Involvement In Social Entrepreneurship To Promote Smart Regional Growth, Globalization And Business, №7, pp. 63-69.

https://doi.org/10.35945/gb.2019.07.008

\section{SUMMARY}

The essential definition of the promotion of smart regional growth is local resource, potential, exploration of operational mechanisms. That is in order to develop the understanding of local resource feasibility and the community activities. It is important to develop cross-sectoral cooperation, which is focused on non-technological, social and on the ecological innovation processes in connection with entrepreneurship. Social entrepreneurship is an essential mechanism in economic activity balancing, while creating social values that encourage the local community to participate in many areas and reduce socio-economic disparities. The "Traditional" entrepreneur whose main desire is to create a product, find their client and thus, earn money, differs from a social businessman. An entrepreneur who has chosen the social niche, firstly, solves a social problem. In Latvia, social entrepreneurship is becoming more popular. Help and consultation at this point is provided by the Latvian entrepreneurship association (LEA), which has been in operation since the year of 2015. The region of Latgale is also becoming more popular in this field.

In order to evaluate social entrepreneurship feasibility and to determine factors in the region of Latgale, cross-disciplinary research has to be carried out. Moreover, research and development in this area involves the involvement of a wide variety of stakeholders: NGO (non-governmental organisation), state, business, representatives of the public. 



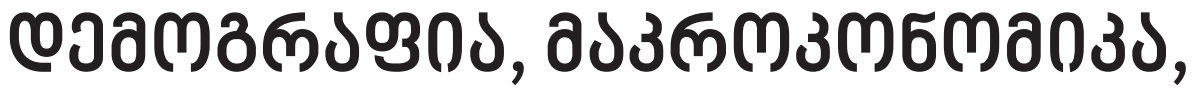

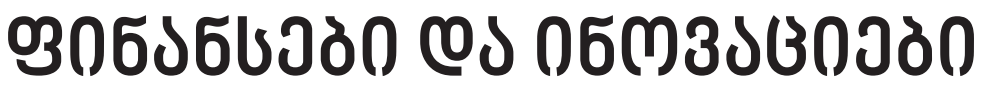

\section{DEMOGRAPHY, MACROECONOMICS, FINANCES AND INNOVATIONS}





\title{
LESSONS FROM FISCAL DECENTRALIZATION IN UKRAINE
}

\author{
VARNALII ZAKHARII \\ Doctor of Economic Sciences, Professor \\ Taras Shevchenko National University of Kyiv, Ukraine, \\ Honored Worker of Science and Technology of Ukraine, \\ Academician of the Academy of Economic Sciences of Ukraine \\ vzs1955@gmail.com
}

\section{DEMYDENKO LIUDMYLA}

PhD in Economics, Associate Professor, Taras Shevchenko National University of Kyiv, Ukraine ipr.demydenko@gmail.com

\section{NAKONECHNA YULIIA}

PhD in Economics, Associate Professor, Taras Shevchenko National University of Kyiv, Ukraine tematika@ukr.net

\section{MIEDVIEDKOVA NATALIIA}

PhD in Economics, Assistant

Taras Shevchenko National University of Kyiv, Ukraine

nsmedvedkova@gmail.com

KEYWORDS: FISCAL DECENTRALIZATION, LOCAL BUDGETS, AMALGAMATED TERRITORIAL COMMUNITY, LOCAL GOVERNMENTS.

For citation: Varnalii Z., Demydenko L., Nakonechna Y., Miedviedkova N. (2019), Lessons From Fiscal Decentralization In Ukraine, Globalization And Business, №7, pp. 73-78. https://doi.org/10.35945/gb.2019.07.009

One of the most sensitive reforms for Ukrainian citizens is fiscal decentralization reform. The results of the activities carried out in this area were the growth of local budgets by 3.4 times over the period 2014-2018 (from UAH 68.6 billion to UAH 231 billion) (Monitoring of the process of decentralization, 2019). The monitoring of the process of decentralization of power and the reform of local selfgovernment as of February 10, 2019 emphasizes that it was created 838 amalgamated territorial communities (hereinafter ATCs), covering 9 million citizens for the period 2015-2018 (Reporting of the State Treasury Service of Ukraine, 2019). Nevertheless, according to international experts, this reform is one of the most successful in Ukraine, many issues remain unresolved.
The object of this study is the process of fiscal decentralization in the context of amalgamated territorial communities.

The subject istheoretical and practical approaches to analysis of reforms in budgeting amalgamated territorial communities and ensuring their transparency under fiscal decentralization in Ukraine.

Theoretical and methodological basis of research isto analyze necessity of fiscal decentralization reform in the context of amalgamated territorial communities and explore advantages and disadvantages of steps provided by the government in this direction; assess the state of transparency of amalgamated territorial communities under fiscal decentralization in Ukraine. 
Statistical analysis - Different statistical data, obtained from an electronic service Open Budget and Monitoring of the process of decentralization of power and the reform of local self-government have been analyzed. The information obtained from the Ministry of Finance of Ukraine,the State Treasury Service of Ukraine, the Ministry of Economic Development and Trade of Ukraine, the Ministry of Regional Development, Building and Housing and Communal Services of Ukraine was analyzed, as well as the dynamics of amendments to Budget and Tax Codes of Ukraine were researched.

The analysis of the official documentation - included the analysis and classification of the official data, documentation, decisions provided by the state departments.

The research is based on the Ukrainian works, the researches carried out and reports prepared by Ukrainian organizations, the official documentation of the state departments, various governmental and legislation documents (Concept of the Reform of Local Self-Government and Territorial Organization of Government, Budget and Tax Codes of Ukraine, laws such as "On Voluntary Association of Territorial Communities", "About the State Budget of Ukraine for 2019", "On Cooperation of Territorial Communities", "On Local Self-Government); as well as the interviews and surveys of the business entities.

The reform of structure of tax revenues of local budgets took place in 2015. It found its legislative recognition in Budget and Tax Codes of Ukraine. Thus, the government introduced tax incentives for the voluntary amalgamation of territorial communities. Such communities began to receive $60 \%$ of personal income taxes paid on territories of communities. Moreover, an additional local retail excise tax on sale of alcoholic beverages and petroleum products was implemented. Regional budgets received $10 \%$ of tax on corporate profits for the first time. Thus, the financial autonomy of local governments began to grow. This trend is continuing in 2019: personal income taxes on lease of land plots to individuals or shares by a tax agent, as well as $5 \%$ of rent on mineral extraction of national significance are charged in addition to local budgets; $13.44 \%$ of excise tax on fuel to local budgets is retained (The Budget Code of Ukraine, 2019; The Tax Code of Ukraine, 2019).

The transfer of significant funds to the local level exacerbates the problem of control over the targeted and efficient use of financial resources. In this context, it is important to comply with the requirements of the Law of Ukraine "About openness of the use of public means» (The Law of Ukraine on September 30, 2015), which requires the publication of information about size and direction of budget spending.

The Ministry of Regional Development, Building and Housing and Communal Services of Ukraine works quite successfully in this context: it accumulates and highlights information on rating of regions in the process of forming
ATCs and publishes monitoring monthly. According to the method of assessing the success of the process, a set of indicators has been determined: the number of ATCs that have amalgamated in the region; coverage of the region by ATCs; amounts of the population of ATCs. In addition, an electronic service Open Budget was created on the portal of the Ministry of Finance of Ukraine and implemented in November 2018 (Open Budget, 2018). It contains open data on tax revenues, transfers of local budgets and directions for spending funds.

The authors analyzed the level of openness of data on the finance of ATC on the availability of information of the following parameters: program of the socio-economic development; draft budget for the current year; public budget (a simplified form of visualization of revenues and expenditures of budget of ATC), minutes of public hearings on the budget; adopted annual budget, annual budget performance report; quarterly budget execution reports, debt policy document; information about borrowing and repaying; information on the status of implementation of investment projects that are being implemented; reports on implementation of budget passports.

Today we can note the insufficient level of disclosure of information on key financial indicators. It has been possible to create only official sites at the level of amalgamated territorial communities that contain a limited amount of information. It means that the sites of most ATCs disclosure only certain documents, and the share of ATCS that have complete information does not exceed $10 \%$. We can state the deep differentiation regarding the publication of information created on the sites of ATC and the importance of coordinating these processes by central authorities in organizing systematic work on ensuring transparency of local budgets.

The Government of Ukraine approved the Concept of the Reform of Local Self-Government and Territorial Organization of Government in April 2014 (Cabinet of Ministers of Ukraine, 2014). The purpose of this document is to change the outdated administrative-territorial structure of the country, inherited from times of the Soviet Union. By the beginning of the reform, the number of territorial communities was about 12 thousand; it means that the average size of the community was significantly less than 5 thousand people. The small average size of territorial communities led to the subsidization of most of them. Therefore, the amalgamation of territorial units was chosen as one of the most important directions of the decentralization policy in Ukraine. The Law of Ukraine "On Voluntary Association of Territorial Communities" created legislative grounds for the consolidation of territorial units. As of February 2019, 878 ATCs were formed and united 4,018 local councils; more than 9 million citizens live in them nowadays (Verkhovna Rada of Ukraine, 2015). The unification process is planned to be completed by the end of 2020. To create a favorable environment for the unification of communities, the Government also allocated funds to 
financially support the development of the infrastructure of the ATCS (UAH 1.94 billion in 2018). It should also be noted that UAH 2.1 billion are provided for the subvention for formation of the infrastructure of ATCs in 2019 (About the State Budget of Ukraine, 2019).

The Law of Ukraine "On Cooperation of Territorial Communities» has created a mechanism for solving general problems of ATCs - garbage collection and processing, the creation of a common infrastructure, etc. As of February 2019, 1262 ATCs used the mechanisms proposed by this law by signing 325 agreements on co-operation.

In parallel with the amalgamation of communities, some changes in their budgets have been made. ATCs have received additional sources of income and new powers to meet the needs of the local population, which allows them to effectively manage local economic processes.

Budget decentralization is the transfer of significant powers and financial resources from state authorities to local self-government bodies, the transfer of new voluntarily amalgamated territorial communities in Ukraine to direct intergovernmental relations with the state budget. The main results of the reform of fiscal decentralization should be the following: improving the quality and accessibility of public services, creating safe and comfortable environment for human life, participation of public in solving local issues.

Fiscal decentralization is the denationalization of power, the expansion of the source base, the transfer of responsibility, rights and resources to the local level. It is accompanied by a complete transformation of the budget system in the process of creating amalgamated territorial communities in Ukraine. The main goal of the budget decentralization reform is to increase the well-being of all members of the territorial community, improve the quality of public services at the local level and reduce the level of corruption and distrust in local authorities.

The main objectives of the reform are to strengthen the legal, organizational and financial viability of the amalgamated territorial communities, to improve the motivation in increase of the revenue base of their budgets. But it is impossible without comprehensive steps in the conduct of decentralization in such areas as: education in rural areas, health care, land relations, provision of administrative services, architectural and construction control (Fig. 1).

Effective changes in these industries are based, on the one hand, on further transfer of large powers to local governments, as such power structures which are closer to people and they know educational, medical and other needs of members of their community and can better meet them. On the other hand, they need state aid - the provision of financial support to communities through the allocation of funds in the form of subventions from the state budget for the formation of appropriate infrastructure, material incentives for rural doctors, teachers and funds from the State Regional Development Fund.

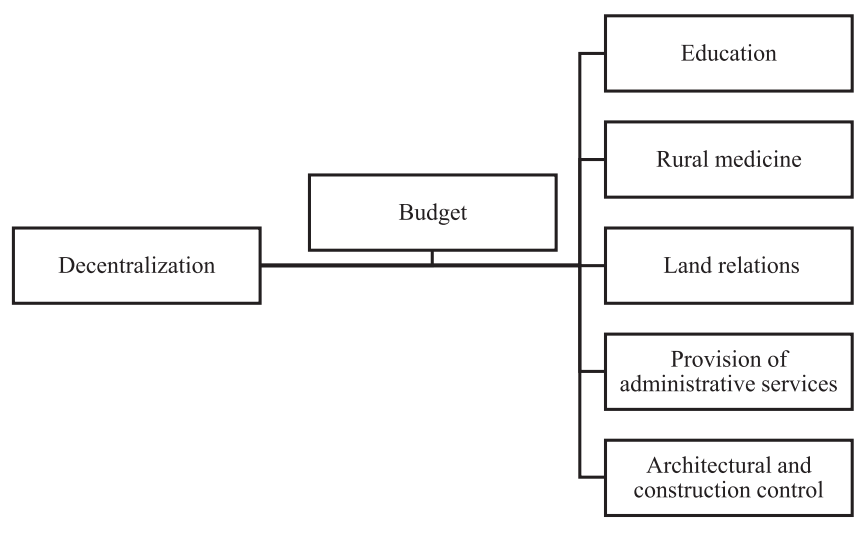

Fig.1. Spheres of decentralization in Ukraine

Since 2014, in accordance with Articles 64, 66 of the Budget Code, income sources of territorial communities have been expanded significantly and consist of $60 \%$ of personal income tax, parts of excise tax, in particular excise tax on retail fuel, alcoholic beverages and tobacco products, ecological tax, rents.

The role of local taxation in formation of capable communities is growing in Ukraine. Budgets of ATCs receive two local taxes, in particular, property tax, which consists of real estate tax other than a land plot, transport tax, land fee and single tax, as well as local fees: parking fee, tourist fee. Local authorities more actively began to use their right to determine, within the limits of legislation, the rates of local taxes and fees.

The openness of budget information of the local government is the key to its effective activities under the control and with the participation of members of territorial administrative unit of the community. The laws of Ukraine clearly indicate the recognition of the basic principles of transparency in Ukraine, in particular publicity, as one of the basic principles of local self-government and publicity and transparency of the budget system. In particular, Art. 7 of the Budget Code of Ukraine noted that "One of the important principles of the functioning of the budget system of Ukraine is informing the public on budget policy, drafting, reviewing, approving, executing the state budget and local budgets, and monitoring the implementation of the state budget and local budgets" [3]. The principle of transparency and openness is also listed among the main conditions for the voluntary association of territorial communities, as stated in the relevant Law of Ukraine.

Funds from local budgets, including financial resources of the ATC, are included in public funds and therefore they are subject to the main provisions of the Law of Ukraine «About openness of the use of public means». It specifies the criteria and standards for informing the public about the subjects and objects of control by the state and the public in the field of public funds. They include the following funds of local budgets: funds of managers and recipients of funds of local budgets, credit resources provided under local guarantees, 
Table 1 shows the system of distribution of tax revenues and the procedure for the formation of incomes of the amalgamated territorial communities in Ukraine.

Table 1. FORMATION OF BUDGET REVENUES OF ATCS IN UKRAINE IN 2019

\begin{tabular}{|c|c|}
\hline STATE TAXES THAT FORM STATE AND LOCAL BUDGETS & REVENUES OF ATCS \\
\hline $\begin{array}{l}\text { Excise tax } \\
\text { Excise tax on retail trade in alcoholic beverages and tobacco products }\end{array}$ & $\begin{array}{l}100 \% \text { (tax rate for beer, alcoholic } \\
\text { beverages, tobacco products, tobacco and } \\
\text { industrial tobacco substitutes sold by } \\
\text { business entities of retail trade of excisable } \\
\text { goods is } 5 \% \text { ) }\end{array}$ \\
\hline $\begin{array}{l}\text { Excise tax on fuel produced in Ukraine and excise tax on goods imported into } \\
\text { the customs territory of Ukraine }\end{array}$ & $\begin{array}{l}13,44 \% \text { of excise tax on fuel produced in } \\
\text { Ukraine and } 13,44 \% \text { of excise tax on goods } \\
\text { imported into the customs territory of } \\
\text { Ukraine are automatically charged to the } \\
\text { budget }\end{array}$ \\
\hline Personal income tax & $60 \%$ \\
\hline \multicolumn{2}{|l|}{ Corporate income tax } \\
\hline Corporate income tax for utility company & $100 \%$ \\
\hline \multicolumn{2}{|l|}{ Ecological tax } \\
\hline Ecological tax (except for the ecological tax on radioactive waste) & $25 \%$ \\
\hline \multicolumn{2}{|l|}{ Rent } \\
\hline Rent on special usage of water & $10 \%$ \\
\hline $\begin{array}{l}\text { Rent on usage of subsoil for the extraction of oil, natural gas and gas } \\
\text { condensate (except for the rent on usage of subsoil within the continental shelf } \\
\text { and /or the exclusive (marine) economic zone of Ukraine) from the extraction of } \\
\text { oil, gas and gas condensate }\end{array}$ & $3 \%$ \\
\hline $\begin{array}{l}\text { Rent on usage of subsoil for the extraction of minerals of local significance; rent } \\
\text { on usage of subsoil for purposes not related to the extraction of minerals; rent } \\
\text { on special usage of water of water objects of local significance; rent on special } \\
\text { usage of forest resources }\end{array}$ & $\begin{array}{l}100 \% \text { (such payments are charged to the } \\
\text { location of the relevant natural resources, } \\
\text { and in relation to water bodies - at the } \\
\text { place of tax registration of the payer of the } \\
\text { rent) }\end{array}$ \\
\hline Local taxes and fees & $100 \%$ \\
\hline $\begin{array}{l}\text { Property tax, which consists of property tax other than a land plot; transport } \\
\text { tax; land fee }\end{array}$ & $100 \%$ \\
\hline Single tax for small businesses & $100 \%$ \\
\hline Fees: parking fee, tourist fee & $100 \%$ \\
\hline
\end{tabular}

Source: Compiled by the authors based on The Budget Code of Ukraine

as well as funds of economic entities of municipal property received by them from their economic activities.

The process of creating the ATC and ensuring its transparency and objectivity is monitored by the Ministry of Finance of Ukraine, the Ministry of Economic Development and Trade of Ukraine, The Ministry of Regional Development, Building and Housing and Communal Services of Ukraine.

The Ministry of Regional Development together withBuilding and Housing and Communal Services of Ukraine is a leader in popularizing the process of creating the ATC and provides the public with qualitative information on the progress of this process. The ministry publishes data on the planned and actual use of funds of ATC for the period 20152019 in the context of all regions of Ukraine on website ${ }^{1}$.

As our analysis shows, nevertheless general information

${ }^{1}$ https://decentralization.gov.ua on the website of the Ministry of Regional Development, Building and Housing and Communal Services on the voluntary association of territorial communities is published quite fully, regularly and constantly updated, the disclosure of information on the functioning of ATCs is characterized by considerable fragmentation and depends on the desire and ability of the management of ATCs. More than half of all ATCs do not provide publicly available information on the revenues and expenditures of the budget, subsidies, subventions and loans. And although sites of ATC have been created and work, they lack financial content. Therefore, any member of the community or ordinary citizen cannot fully obtain financial information or learn about the prospects of the development of a separate ATC.

The achievement of decentralization is the growth of a large number of infrastructure projects where more than 11 million of them have already been implemented. Due to state support 
and local budgets, new schools, kindergartens, dispensaries, stadiums, security centers have appeared in the communities.

Residents of ATCs have the opportunity to actually influence the local authorities, plan the development of their society, receive a new quality of educational, medical, social and administrative services, as a result, significantly reduce corruption.

Due to the state support from the State Regional Development Fund and the constant growth of their own incomes, communities have the opportunity to plan and implement priority projects. If we take the incomes of communities for comparison, we will achieve such results: they amounted to 70 billion UAH in 2014, UAH 234 billion in 2018 and UAH 291 billion was planned for 2019. According to state support, UAH 0.5 billion was allocated to the local level in 2014, UAH 33 billion in 2018. These funds are transferred by state government to the regional level, and at the local levels in accordance with the improved development strategies, priority projects are defined. Thus, we achieve a synergy of the state regional policy and regional development strategies that are adopted locally.

One should note here that some ATCs of Zaporizhzhya region (Veselovskaya and Shirokivska ATCs) are winners in the pilot project "Equal-to-equal», conducted within the working group "Committee of the EU-Ukraine Regions» to support decentralization. It is implemented with the support from the U-LEAD Program with Europe.Under this project Zaporizhzhya region and the communities will cooperate with the European ones.
In September 2018 an international study tour by the representatives from the Shirokovo ATC started as a part of the "Equal-to-equal" initiative in Barleben (Germany). The purpose of the visit is to study the best practices for strengthening the economic and institutional potential of the society. The program of the study visit includes various thematic events and meetings with the representatives of the local government and the business sector.

\section{CONCLUSIONS AND PROSPECTS FOR FURTHER RESEARCH}

To build an effective model of budget management, it is important to ensure continuous and systematic disclosure of information on the accumulation of financial resources of state and local budgets and the direction of their use. Budget transparency provides increased control of community representatives and civil society organizations over the financial and budgetary activities of local governments and contributes to increasing their responsibility for the use of public funds.

The creation of an ATC in Ukraine enhances public attention to the transparency of finances at the level of territorial communities and makes it necessary to make additional efforts by society and researchers on measures and mechanisms that can increase the degree of transparency of local finances, which is not yet sufficient.

\section{REFERENCES:}

1. Monitoring of the process of decentralization of power and the reform of local self-government. URL: https://storage. decentralization.gov.ua/uploads/library/file/378/10.02.2019_ukr.pdf(Accessed 14 March 2019).

2. Reporting of the State Treasury Service of Ukraine. URL: https://www.treasury.gov.ua/ua/file-storage/vikonannyaderzhavnogo-byudzhetu(Accessed 4 March 2019).

3. The Budget Code of Ukraine: Law of Ukraine on July 8, 2010, No. 2456-VI. URL: http: // zakon3.rada.gov.ua/laws/show/275517/ed20150101 (Accessed 4 March 2019).

4. The Tax Code of Ukraine: Law of Ukraine on 02.12.2010, No. 2755-VI. URL: http: // zakon3.rada.gov.ua/laws/show/275517/ed20150101 (Accessed14 March 2019).

5. About openness of the use of public means. The Law of Ukraine on September 30, 2015, No. 183-VIII. URL: https://zakon3. rada.gov.ua/laws/show/183-viii (Accessed 12 March 2019).

6. Open Budget. URL: https://openbudget.gov.ua/local-budget?id = 26400100000 (Accessed 4 March 2019).

7. Cabinet of Ministers of Ukraine (2014), "Concept of the Reform of Local Self-Government and Territorial Organization of Government", available at: https://zakon.rada.gov.ua/laws/show/333-2014-\%D1\%80. (Accessed 21 February 2019).

8. Verkhovna Rada of Ukraine (2015), Law of Ukraine "On Voluntary Association of Territorial Communities", URL: https:// zakon.rada.gov.ua/laws/show/157-19 (Accessed 21 February 2019).

9. Verkhovna Rada of Ukraine (2018), Law of Ukraine "About the State Budget of Ukraine for 2019", URL: https://zakon.rada. gov.ua/laws/show/2629-19(Accessed 1 February 2019).

10. Verkhovna Rada of Ukraine (2014) Law of Ukraine “On Cooperation of Territorial Communities", URL: https://zakon.rada. gov.ua/laws/show/1508-18 (Accessed 21 February 2019).

11. Verkhovna Rada of Ukraine (1997), Law of Ukraine “On Local Self-Government”, URL: https://zakon.rada.gov.ua/laws/ show/280/97-\%D0\%B2\%D1\%80(Accessed 21 March 2019). 


\title{
LESSONS FROM FISCAL DECENTRALIZATION IN UKRAINE
}

\author{
VARNALII ZAKHARII \\ Doctor of Economic Sciences, Professor \\ Taras Shevchenko National University of Kyiv, Ukraine, \\ Honored Worker of Science and Technology of Ukraine, \\ Academician of the Academy of Economic Sciences of Ukraine \\ vzs1955@gmail.com
}

\section{DEMYDENKO LIUDMYLA}

PhD in Economics, Associate Professor, Taras Shevchenko National University of Kyiv, Ukraine ipr.demydenko@gmail.com

\section{NAKONECHNA YULIIA}

PhD in Economics, Associate Professor, Taras Shevchenko National University of Kyiv, Ukraine tematika@ukr.net

\section{MIEDVIEDKOVA NATALIIA}

PhD in Economics, Assistant

Taras Shevchenko National University of Kyiv, Ukraine

nsmedvedkova@gmail.com

KEYWORDS: FISCAL DECENTRALIZATION, LOCAL BUDGETS, AMALGAMATED TERRITORIAL COMMUNITY, LOCAL GOVERNMENTS.

For citation: Varnalii Z., Demydenko L., Nakonechna Y., Miedviedkova N. (2019), Lessons From Fiscal Decentralization In Ukraine, Globalization And Business, №7, pp. 73-78. https://doi.org/10.35945/gb.2019.07.009

\section{SUMMARY}

In order to build an effective model of management of budget funds, it is important to ensure the constant and systematic disclosure of information on the accumulation of financial resources of the state and local budgets and the directions for their use. The first results of the process of fiscal decentralization in Ukraine are considered, which is accompanied by changes in the budget and tax legislation. Specifics of the formation of tax revenues of budgets of joint territorial communities are outlined. The state of disclosure of budget documents by the amalgamated territorial communities is highlighted and the need for coordination of the authorities in this area is indicated.

Understanding citizens) spending processes in the pu- blic sector can significantly increase confidence in state institutions, provide conditions for the implementation of necessary reforms in all-important areas of the country. In the context of the fiscal decentralization reform launched in 2015, the issue of transparency of financial information at the local level in Ukraine is raised, as significant amounts of financial resources are transferred to local budgets. Strengthening the focus on local government action on effective management of budget funds should be a priority for public organizations and political parties. The implementation of the state policy aimed at increasing transparency and openness in the management of financial resources in the public sector will ensure the tangible results in the social and economic spheres that will be obtained by the citizens of the communities. 


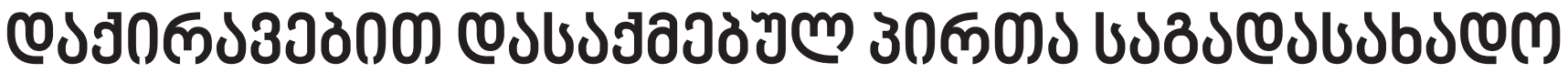

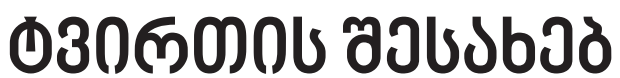

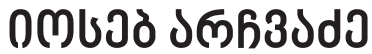

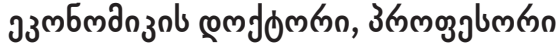

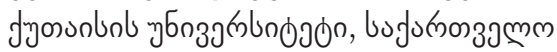 \\ daswreba@yahoo.com
}

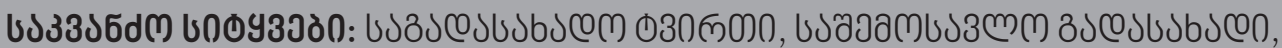

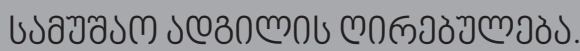

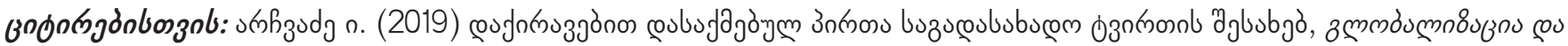
on865lon, №7, 33. 79-86. https://doi.org/10.35945/gb.2019.07.010

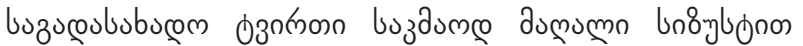

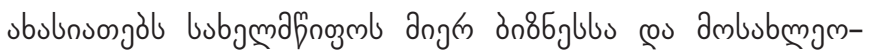

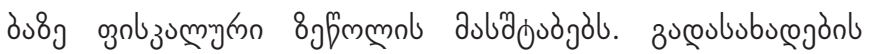

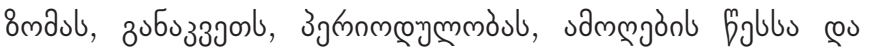

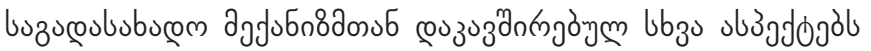

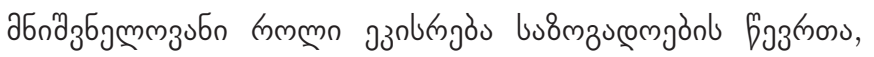

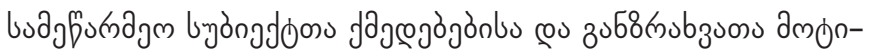

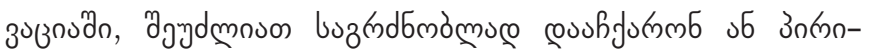

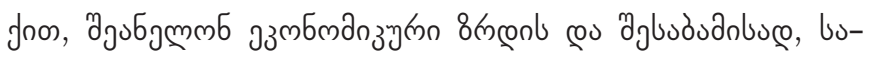

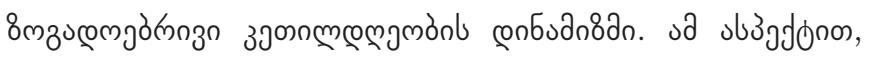

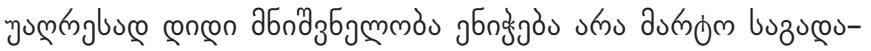

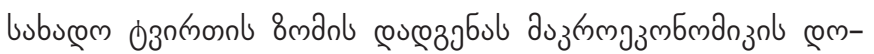

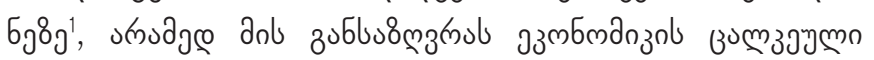

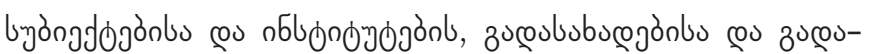

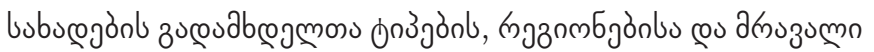

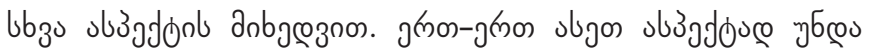

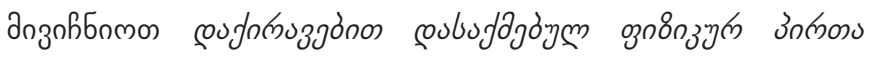

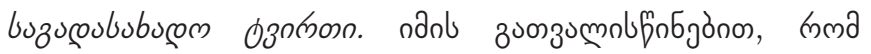

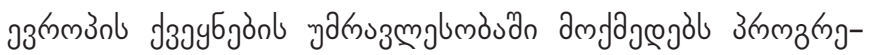

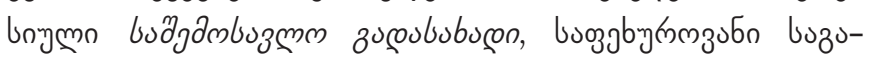

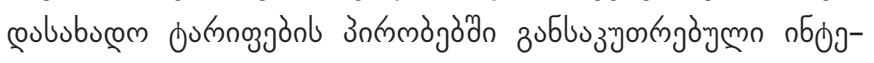

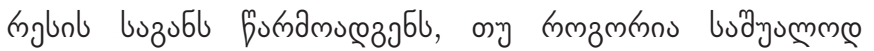

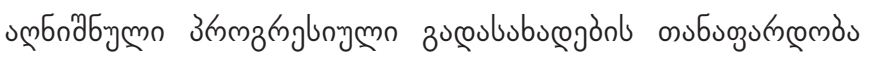

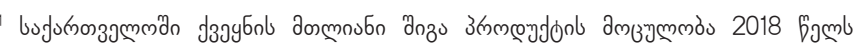

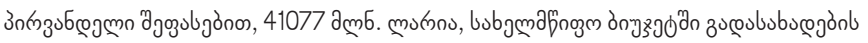
дмззлмm

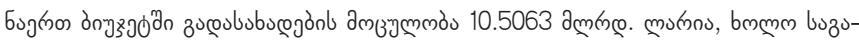

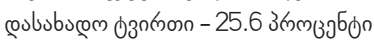

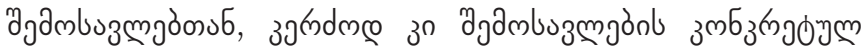

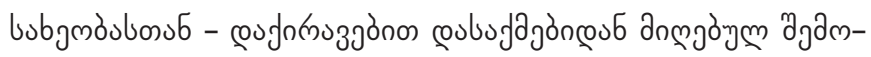

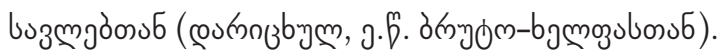

bufunnonzagmel buzucoububucem zmegflun anbjeznon, कुn

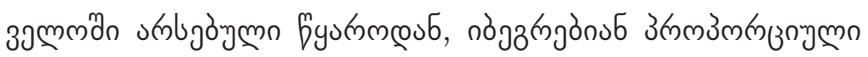

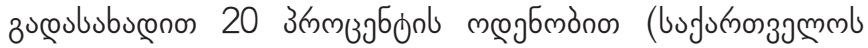

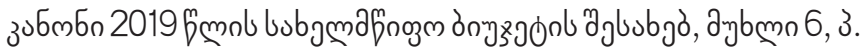

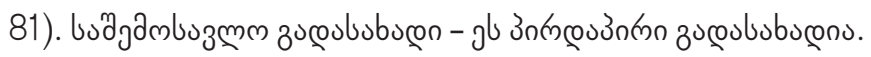

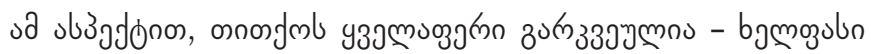

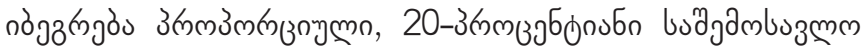

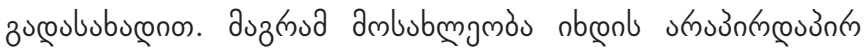

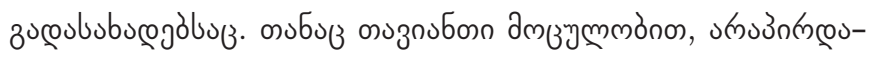

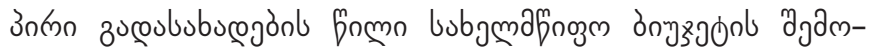

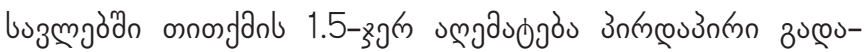

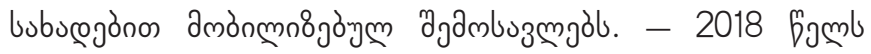

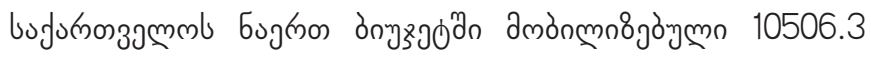

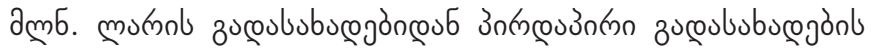

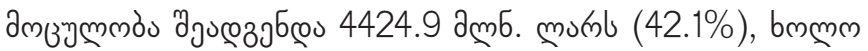

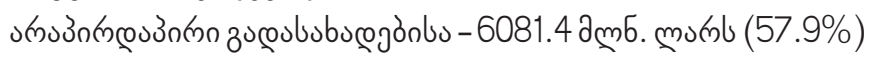

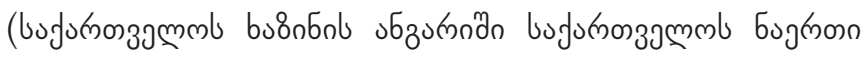

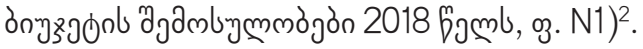

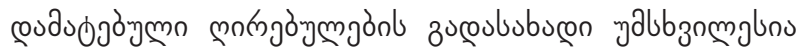

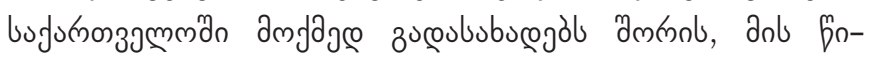

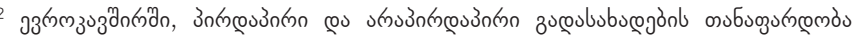

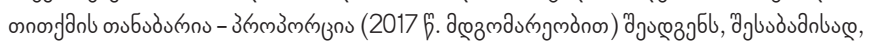
49.1: 50.9-8 [ [6]. 


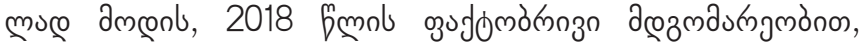

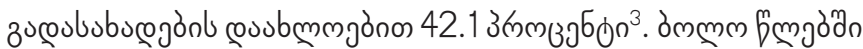

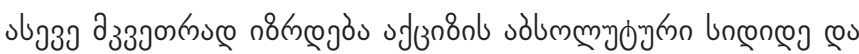

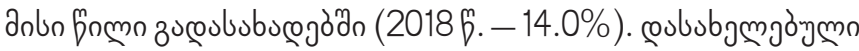

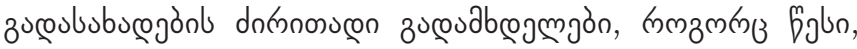

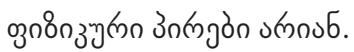

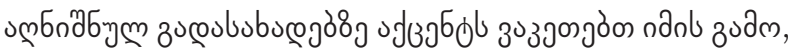

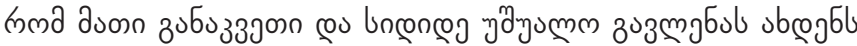

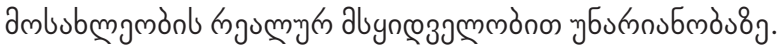

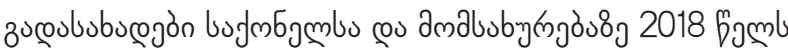

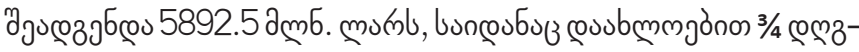

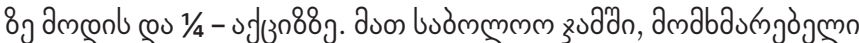

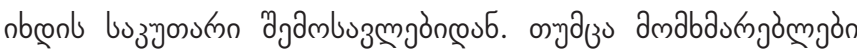

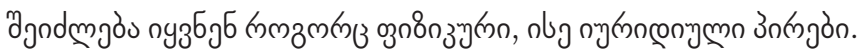

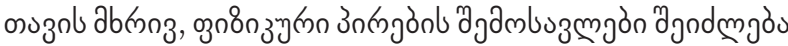

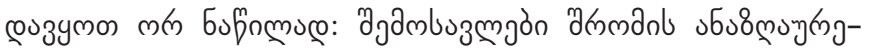

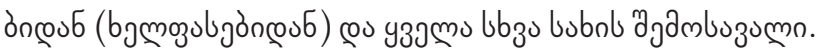

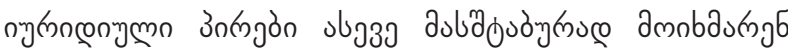

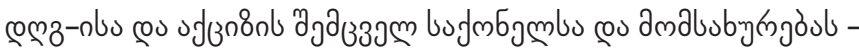

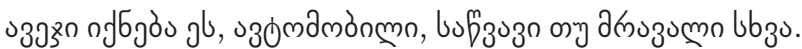

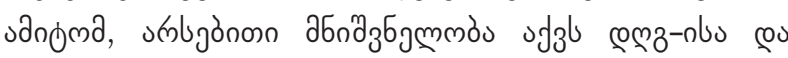

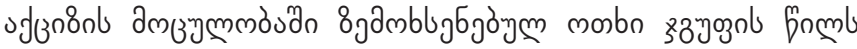

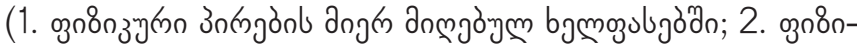

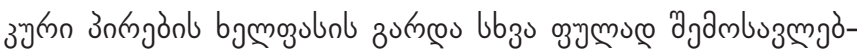

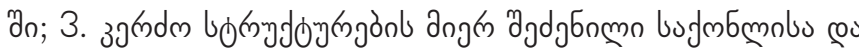

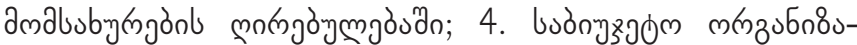

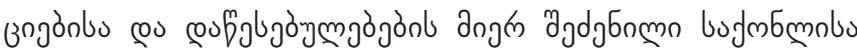

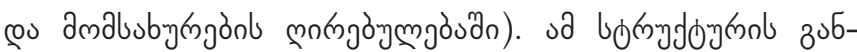

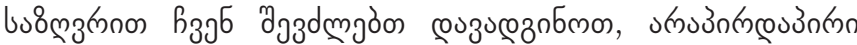

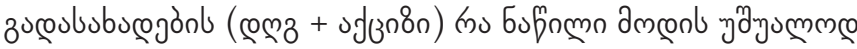

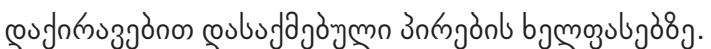

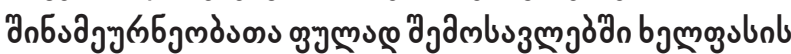
bubnon dng

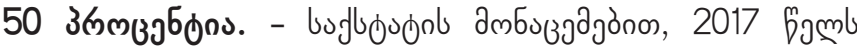

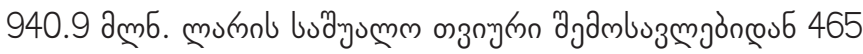

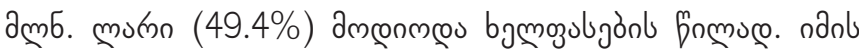

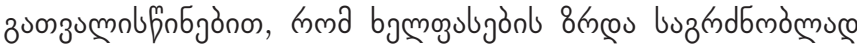

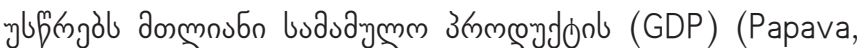

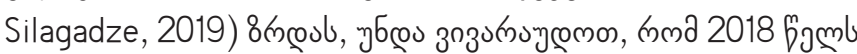

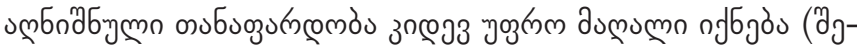

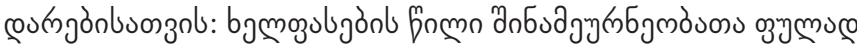

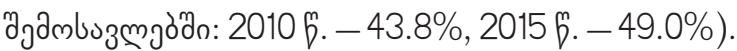

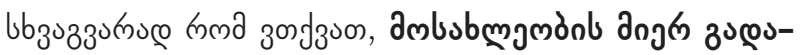

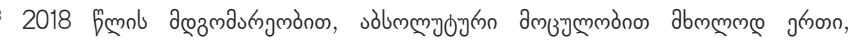

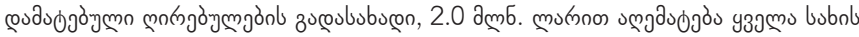

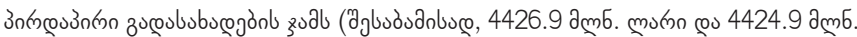
mumn) (f̧yornm:www.mof.ge)
}

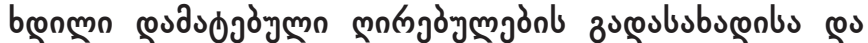

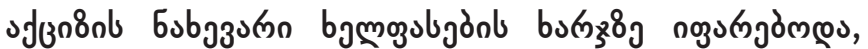

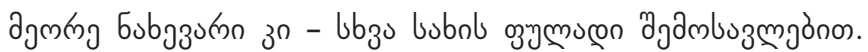

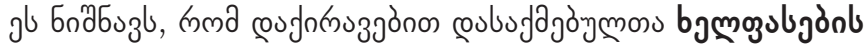

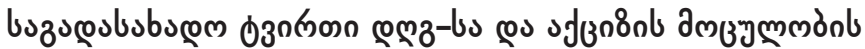

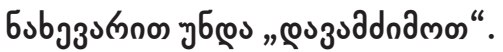

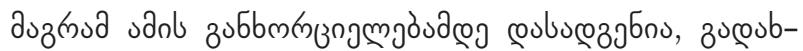

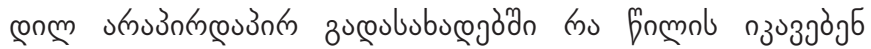

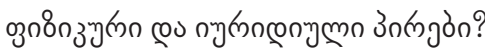

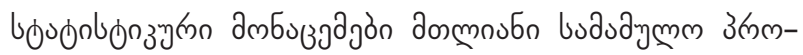

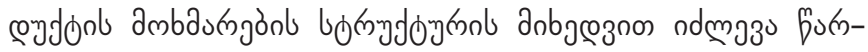

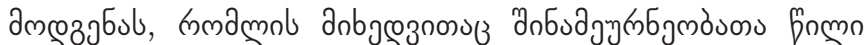

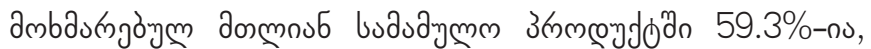

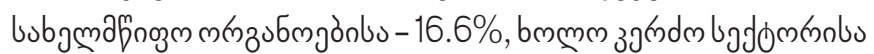

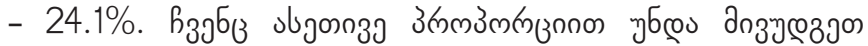

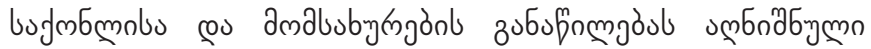

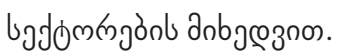
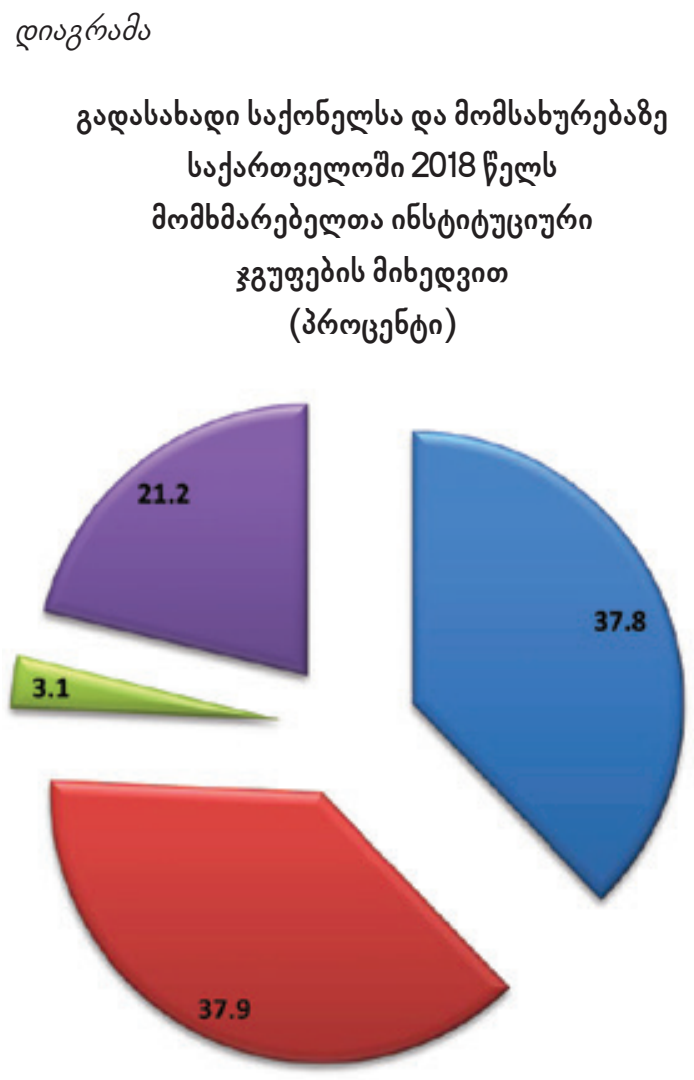

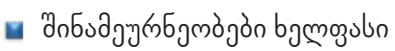

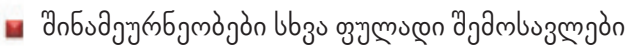

घ әдз

- zgrndm bajom

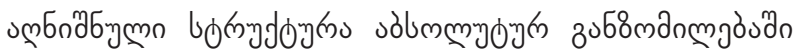

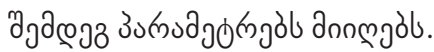


उbrongon 1

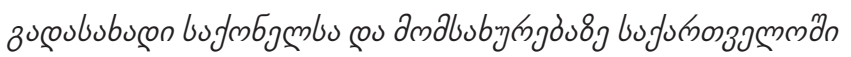

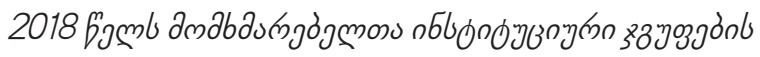

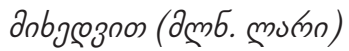

\begin{tabular}{|c|c|c|c|c|c|}
\hline & \multirow{2}{*}{ bym } & \multicolumn{2}{|c|}{ 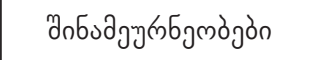 } & \multirow{2}{*}{ дәәзм } & \multirow{2}{*}{$\begin{array}{l}39 \text { mdm } \\
\text { bjJomkn }\end{array}$} \\
\hline & & bymojulon & 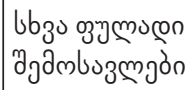 & & \\
\hline 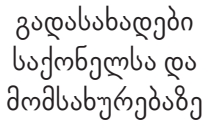 & 5892.5 & 2227.4 & 2233.3 & 182.7 & 1249.2 \\
\hline
\end{tabular}

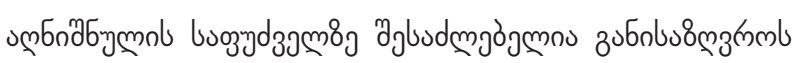

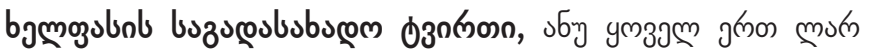

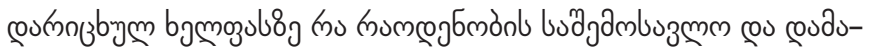

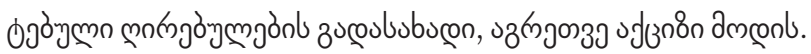

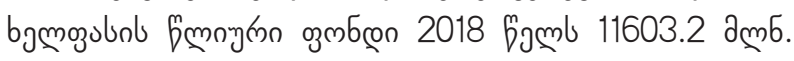

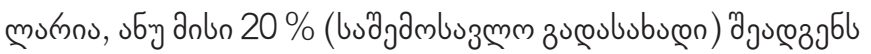
2320.1 ammen. mu们.

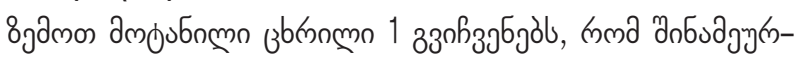

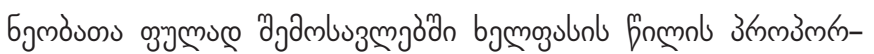

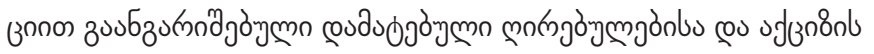

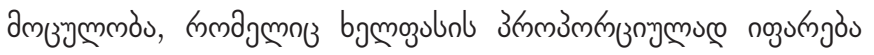

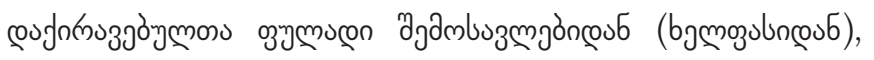

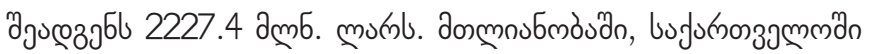

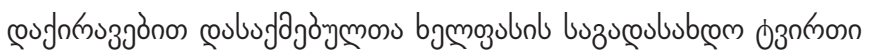
กfбjös: $(2320.1+2227.4): 14471.8=39.2 \%-b$.

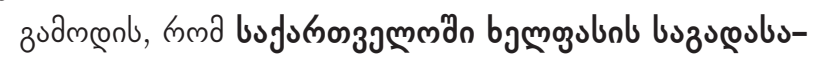

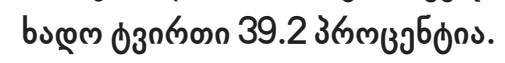

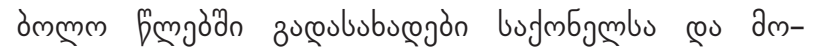

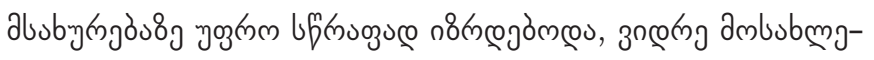

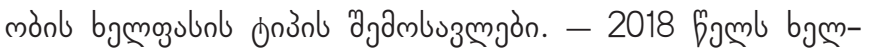

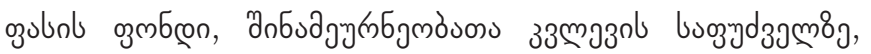

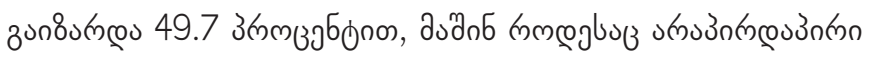

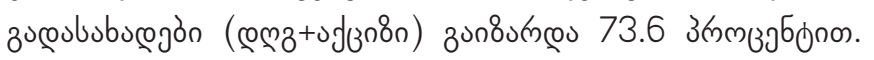

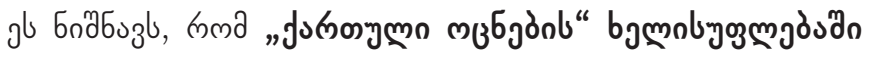

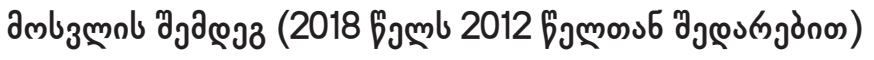

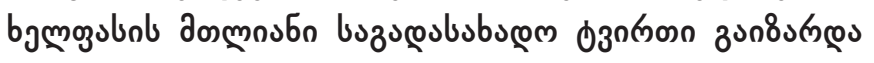

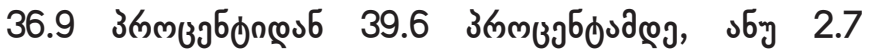

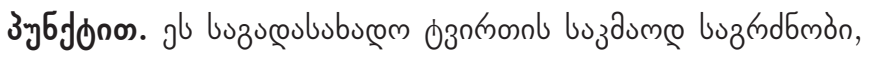

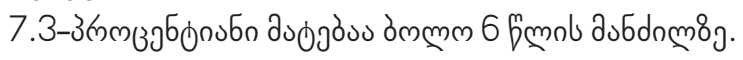

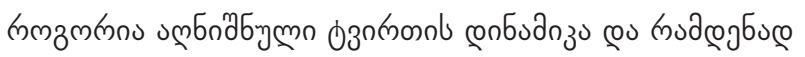

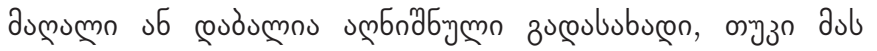

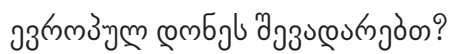

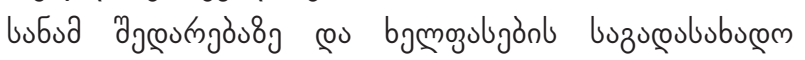

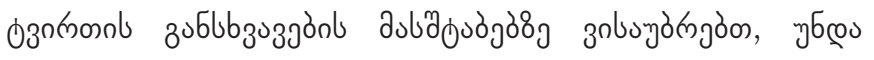

conozinods 2

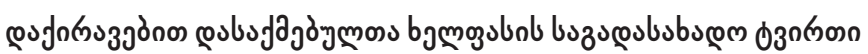

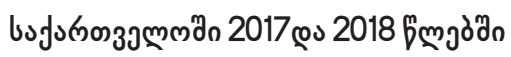

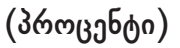

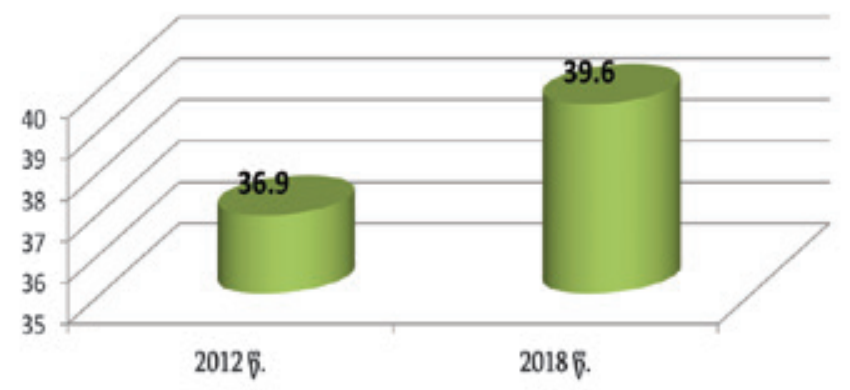

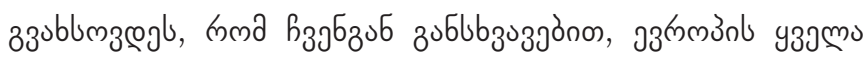

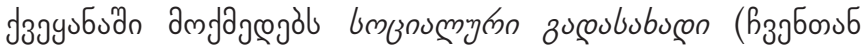

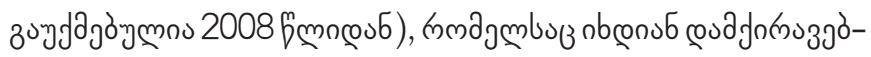

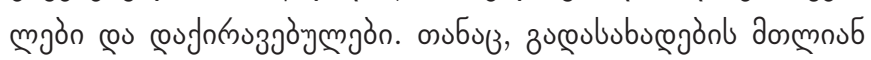

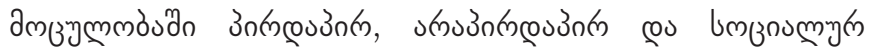

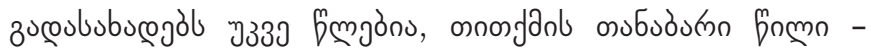

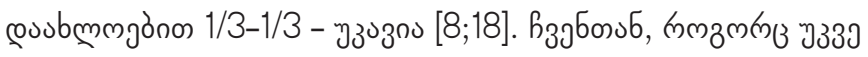

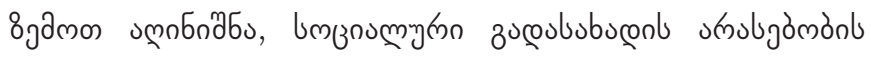

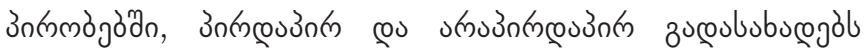

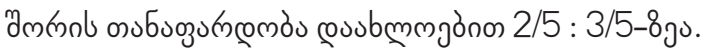

bufunoszamman coubufajöymos agambuzmgònb buzu-

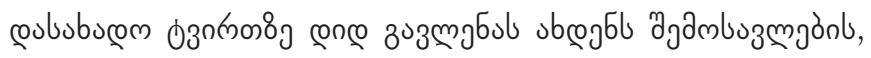

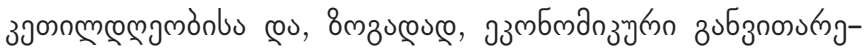

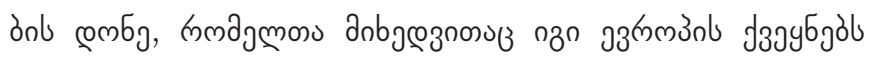

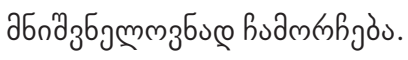

\section{conognodo 3}

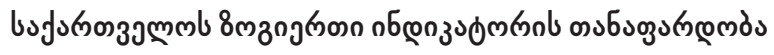
эз пмззง

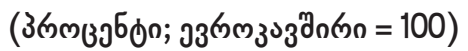

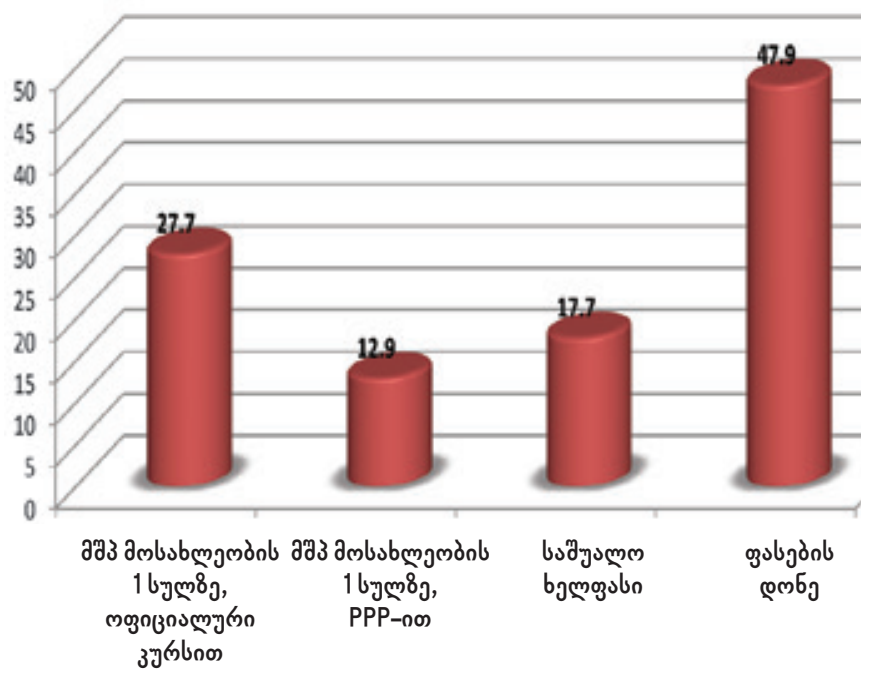




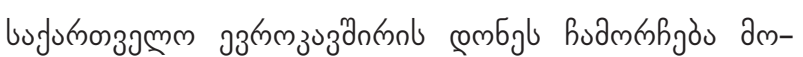

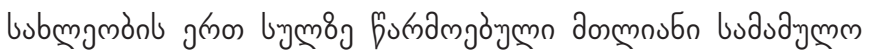

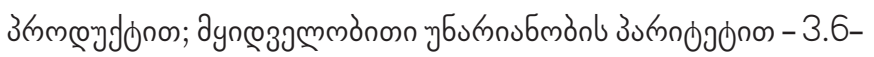

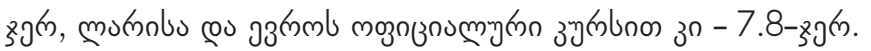

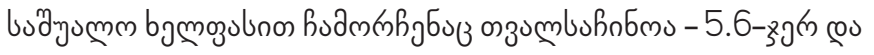
agoue.

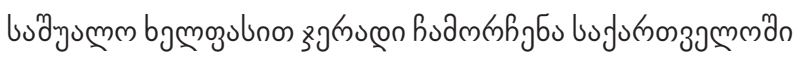

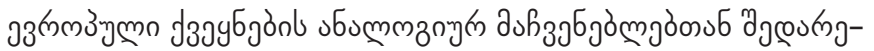

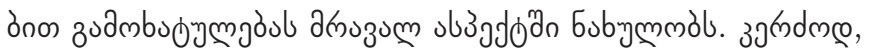

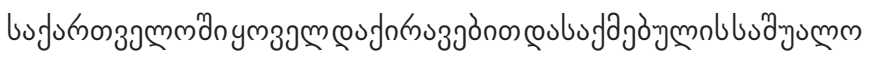

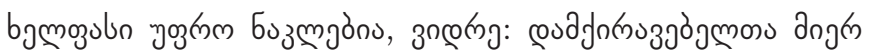

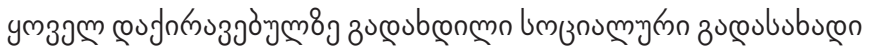

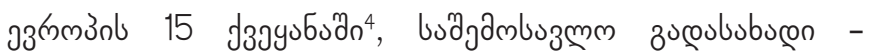

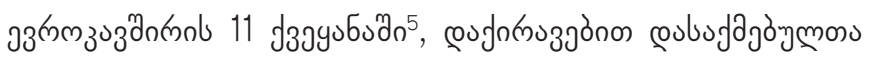

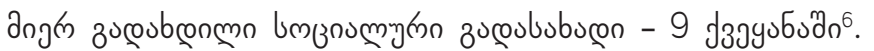
nu bjmgulon a

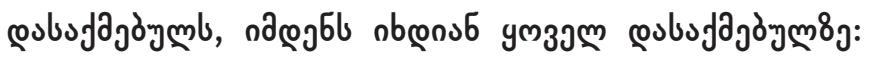

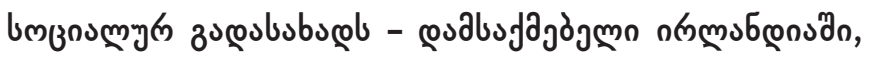
buajambszmm zucoububscol -

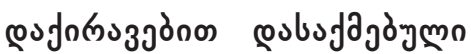

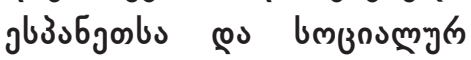

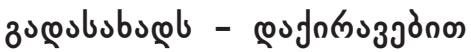

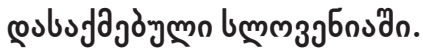

nanl zudm, mma bufuknon-

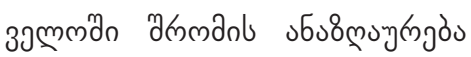

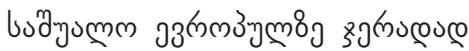
cosoumos co oznon grnosbuntro combnl buzugububugem o3ntronols

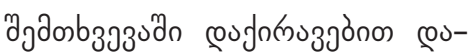
bufagàymgònl bymon counhg-

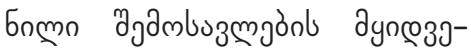

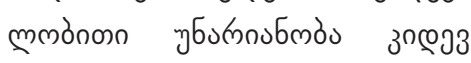

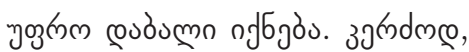
bufuknonzamman 2018 famls

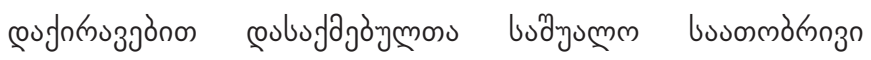

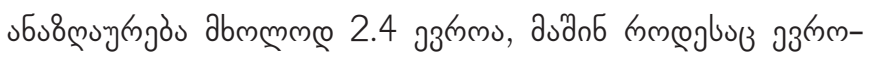

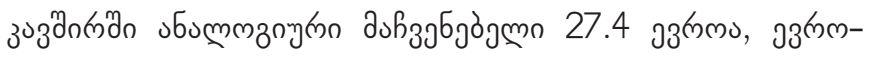

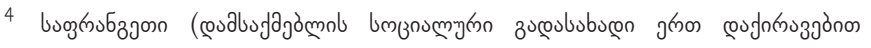

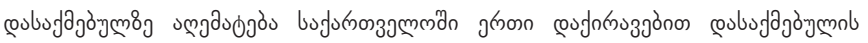

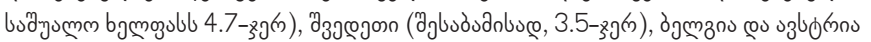

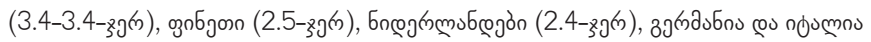

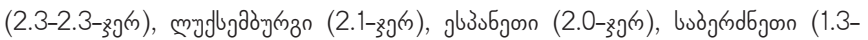

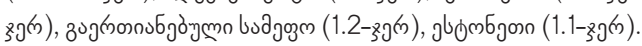

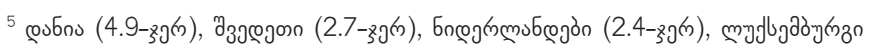

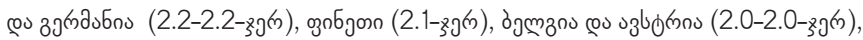

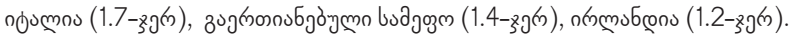

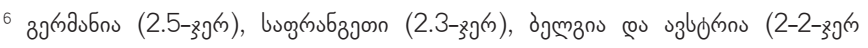

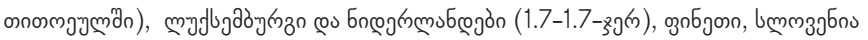

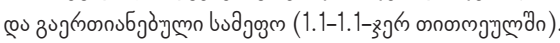

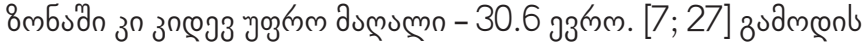

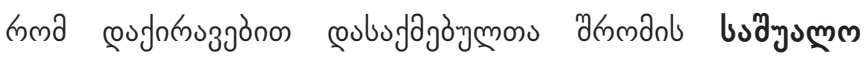

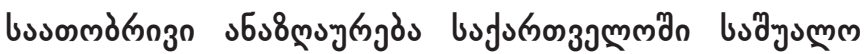

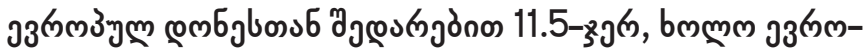

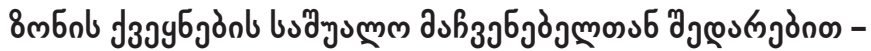

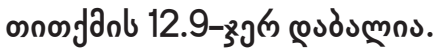

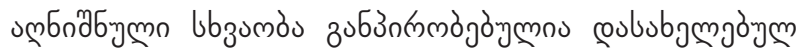

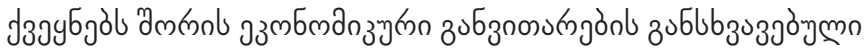

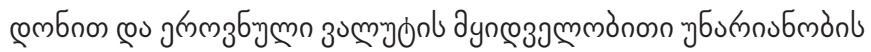

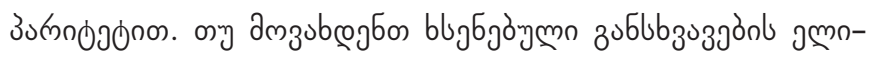

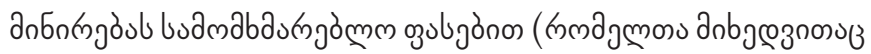

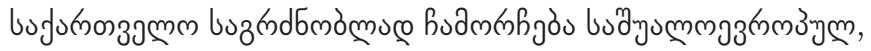

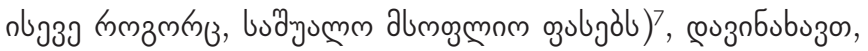

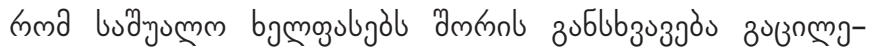

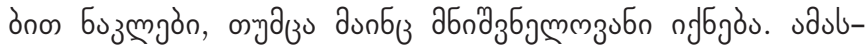

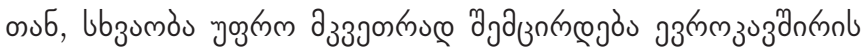

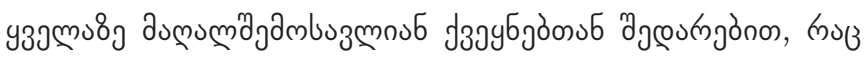

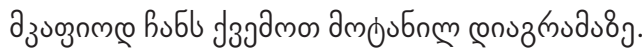

sonoz ro 2004

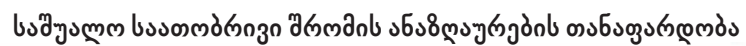

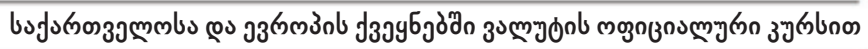

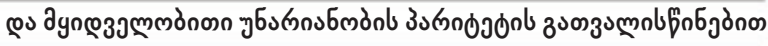

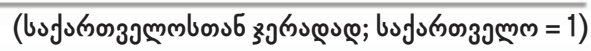

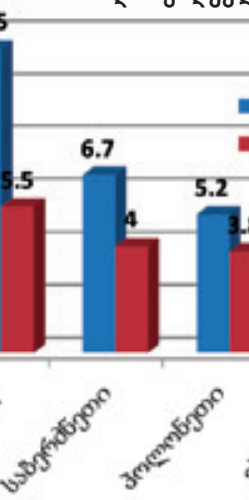

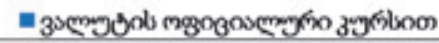

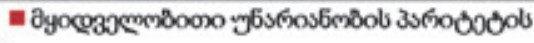

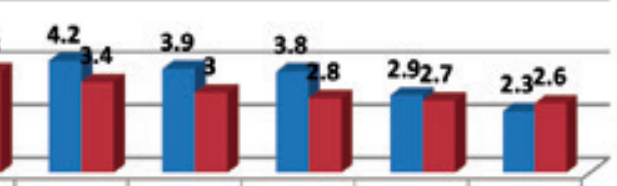

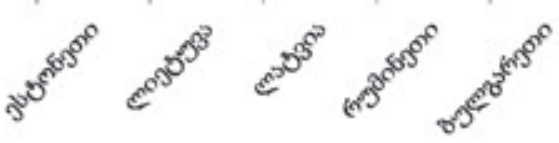

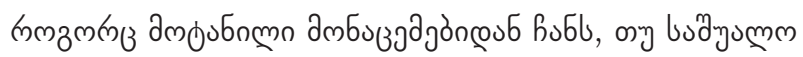

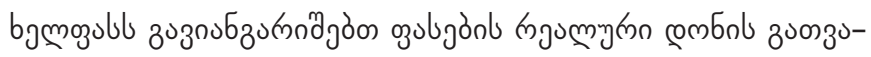

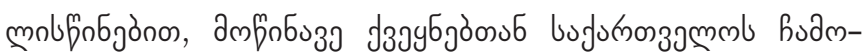

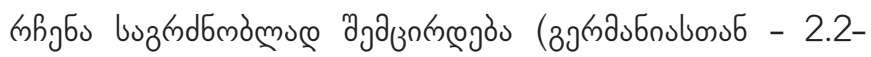

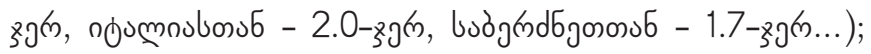

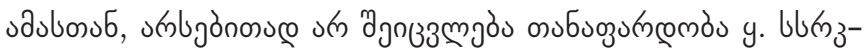

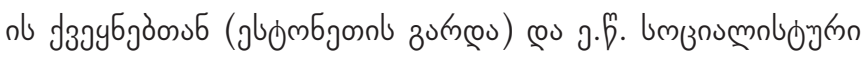

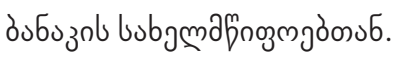

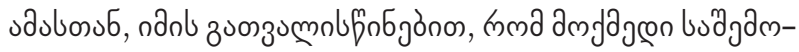

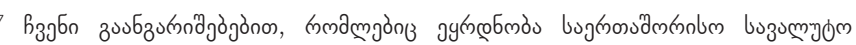

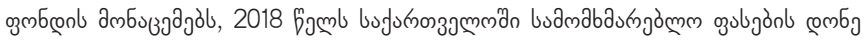

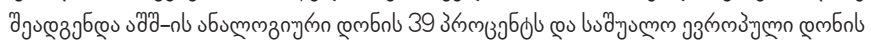

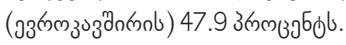




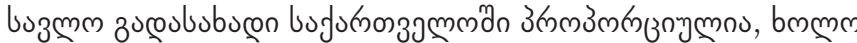

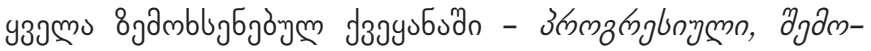

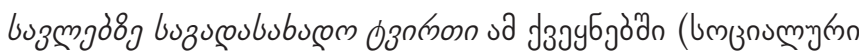

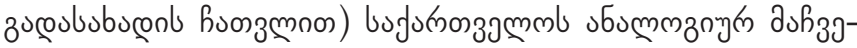

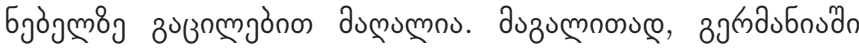

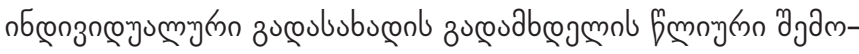

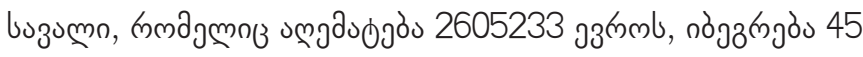

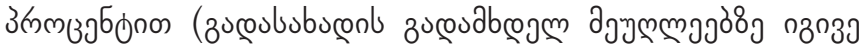

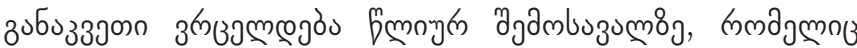

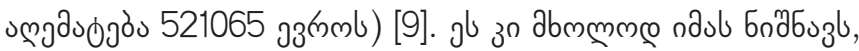

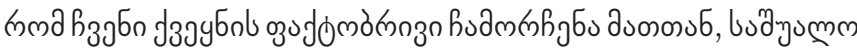

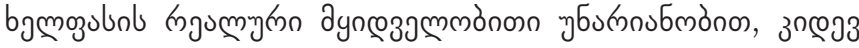

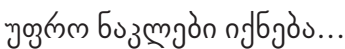

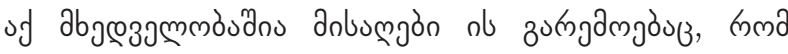

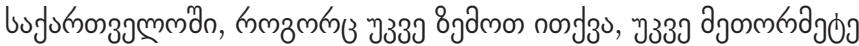

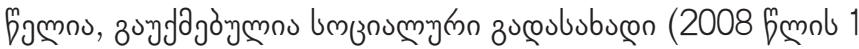

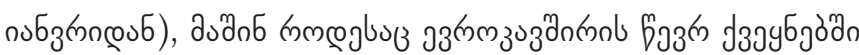

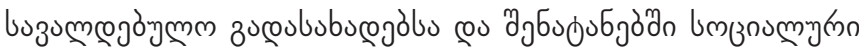

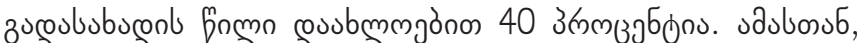

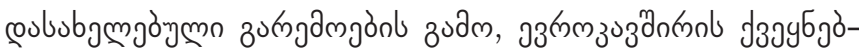

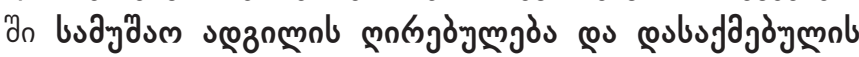

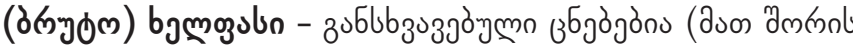

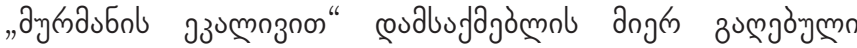

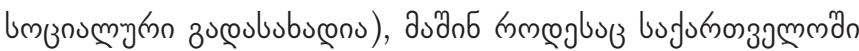

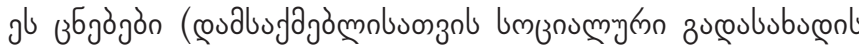

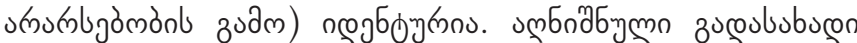

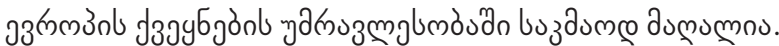

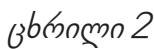

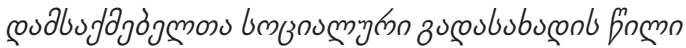

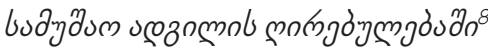

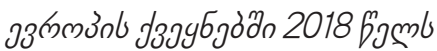

\begin{tabular}{|c|c|c|c|}
\hline & $\%$ & & $\%$ \\
\hline 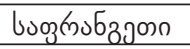 & 33.0 & gुпбјбоп & 18.2 \\
\hline $\operatorname{limm} 30300$ & 26.0 & 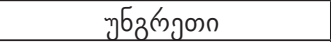 & 17.4 \\
\hline fybjon & 25.4 & 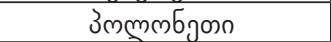 & 17.1 \\
\hline 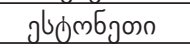 & 24.8 & 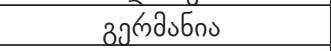 & 16.2 \\
\hline mod 30 & 24.6 & 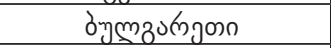 & 16.1 \\
\hline 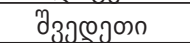 & 23.9 & 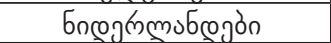 & 15.9 \\
\hline ذ̀manos & 23.3 & bminzodno & 14.7 \\
\hline sदognos & 23.3 & lsmm3jбns & 13.9 \\
\hline jb305gon & 23.0 & 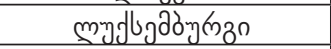 & 13.1 \\
\hline nojomos & 23.0 & 33n3mmbn & 10.3 \\
\hline buoggmdбgon & 20.0 & 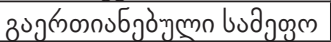 & 10.0 \\
\hline modo30 & 19.4 & ommutronos & 9.7 \\
\hline 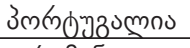 & 19.2 & dugmoo & 9.1 \\
\hline ๓चдобдюn & 19.0 & costons & 0.5 \\
\hline
\end{tabular}

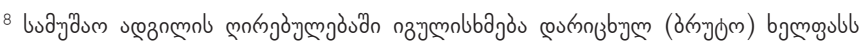

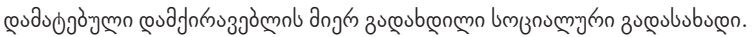

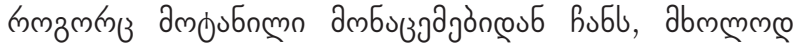

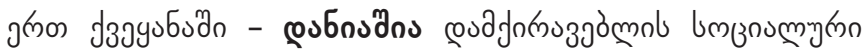

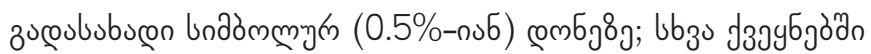

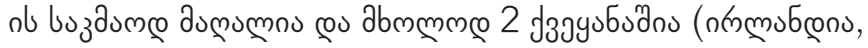

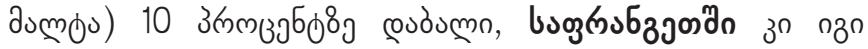

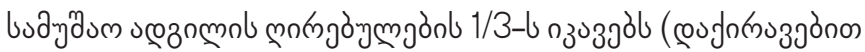

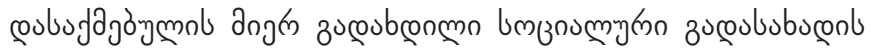

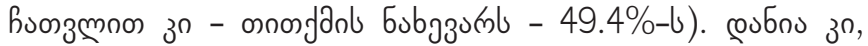

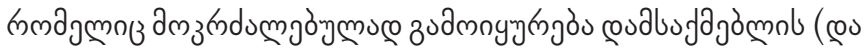

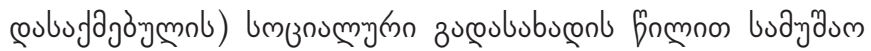

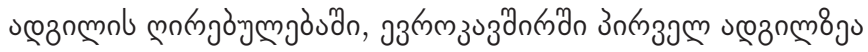

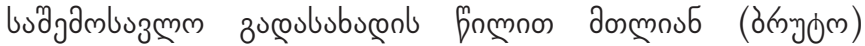

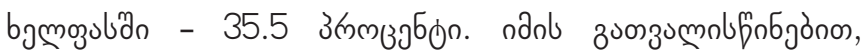

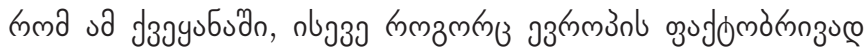

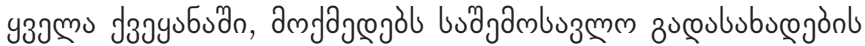

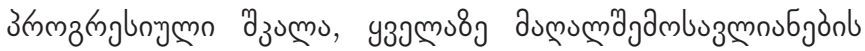

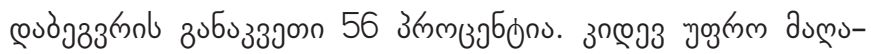

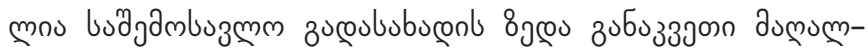

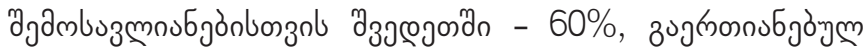

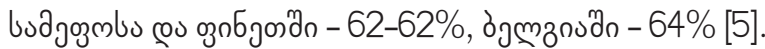

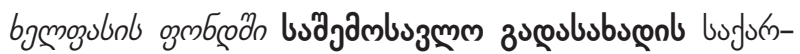
пзэмmml duß

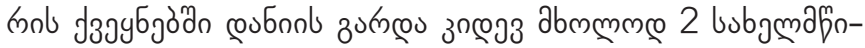

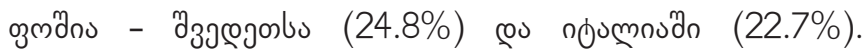

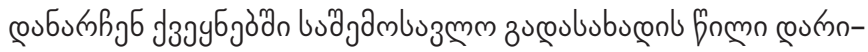

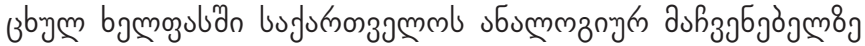

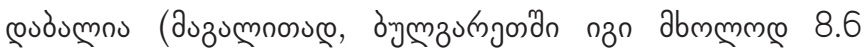

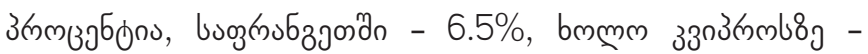

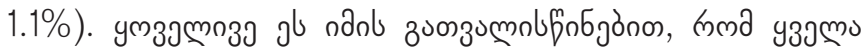

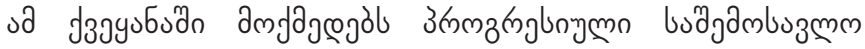

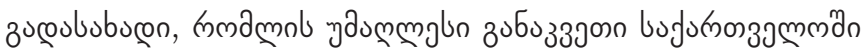

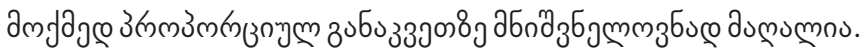

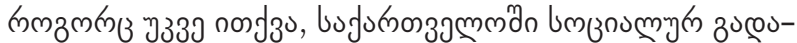

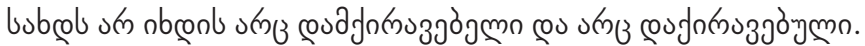
إ

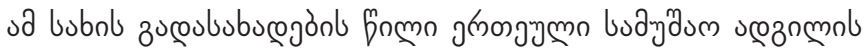

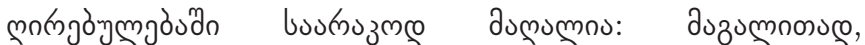

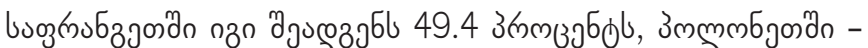

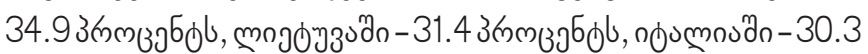

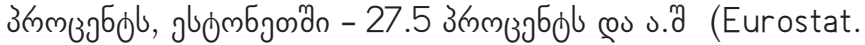
Tax Revenue statistics:7). sanoma bufotronzammobu po

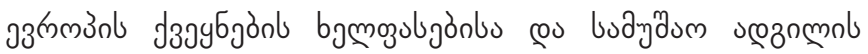

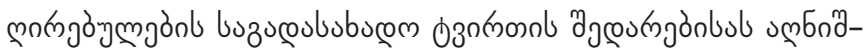

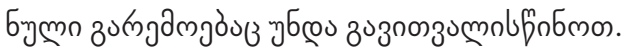

ajon ozummbufon

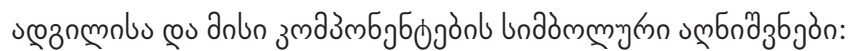


conźs 5

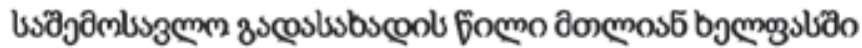

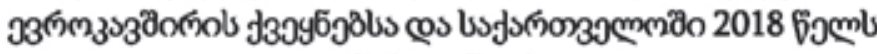

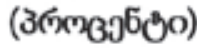

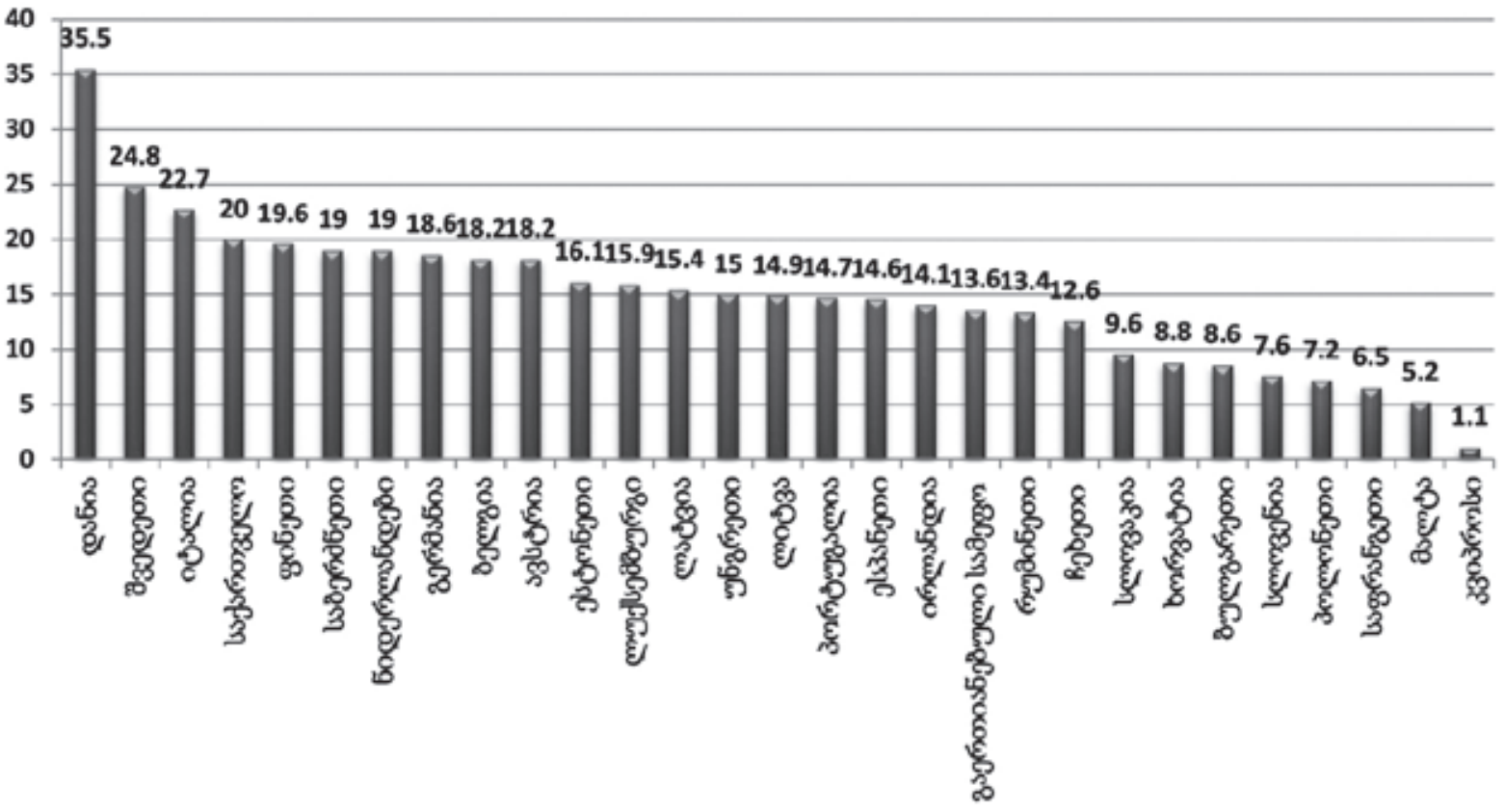

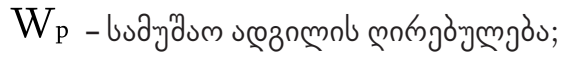

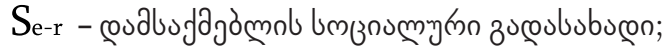

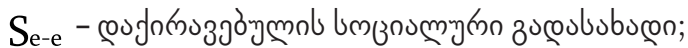

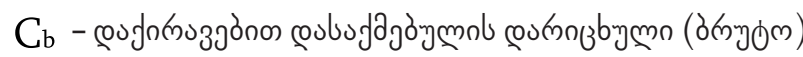
bəmogulo;

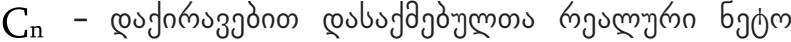
bjmozolo;

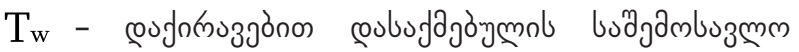
3ocosubsono;

$\mathrm{T}_{\mathrm{i}}$ - cosfntruzjönon coubufajöymol angm zucoubconmo

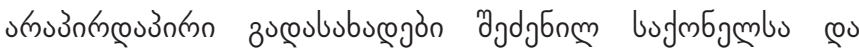

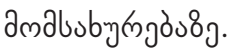

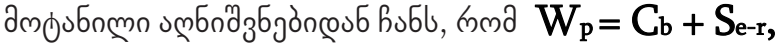
bмmе $\mathrm{Cb}_{\mathrm{b}}=\mathrm{C}_{\mathbf{n}}+\mathrm{S}_{\mathrm{e}-\mathrm{e}}+\mathrm{T}_{\mathbf{w}}+\mathrm{T}_{\mathbf{i}}$

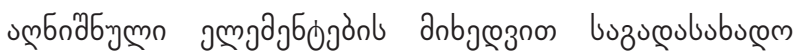

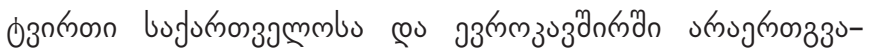

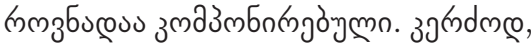

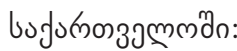

$\left(\mathrm{T}_{\mathrm{w}+} \mathrm{T}_{\mathrm{i}}\right):\left(\mathrm{C}_{\mathrm{n}}+\mathrm{T}_{\mathrm{w}}+\mathrm{T}_{\mathrm{i}}\right)$

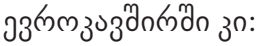

$\left(\mathrm{T}_{\mathrm{w}}+\mathrm{T}_{\mathrm{i}}+\mathrm{S}_{\mathrm{e}-\mathrm{r}}+\mathrm{S}_{\mathrm{e}-\mathrm{e}}\right):\left(\mathrm{C}_{\mathrm{n}}+\mathrm{T}_{\mathrm{w}}+\mathrm{T}_{\mathrm{i}}+\mathrm{S}_{\mathrm{e}-\mathrm{r}}+\mathrm{S}_{\mathrm{e}-\mathrm{e}}\right)$

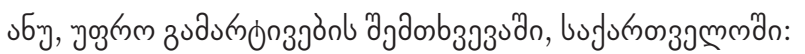

$\left(\mathrm{T}_{\mathrm{w}}+\mathrm{T}_{\mathrm{i}}\right): \mathrm{C}$;

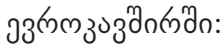

$\left(\mathrm{T}_{\mathrm{w}}+\mathrm{T}_{\mathrm{i}}+\mathrm{S}_{\mathrm{e}-\mathrm{r}}+\mathrm{S}_{\mathrm{e}-\mathrm{e}}\right):\left(\mathrm{C}_{\mathrm{b}}+\mathrm{S}_{\mathrm{e}-\mathrm{r}}\right)$.

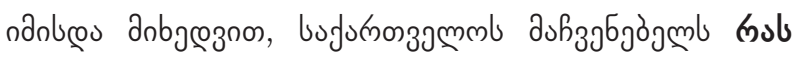

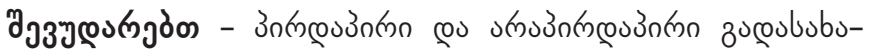

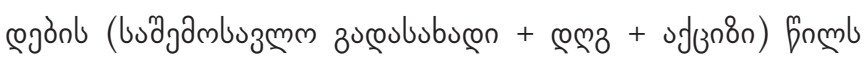
donmoub bjmoguban, on yzamo zucoububuconl fonmb buay-

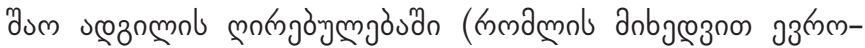

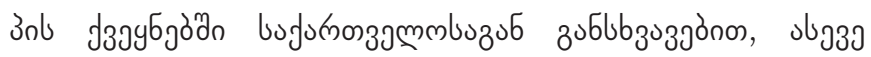

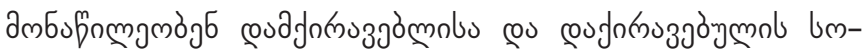

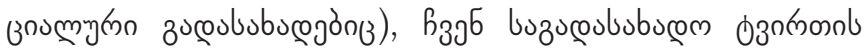

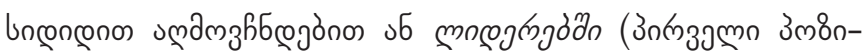

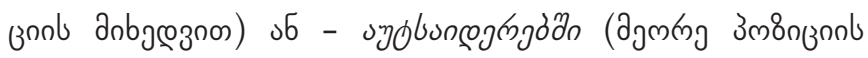

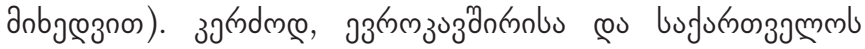

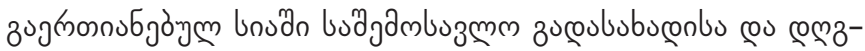

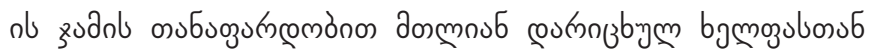

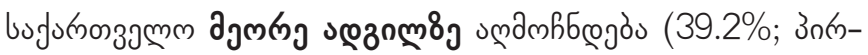

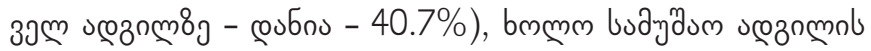

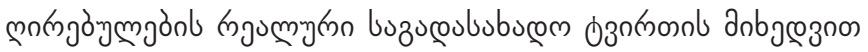

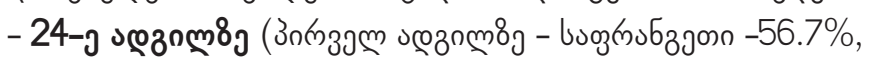

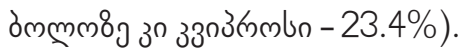

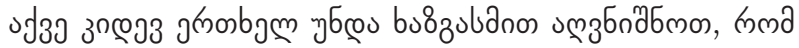

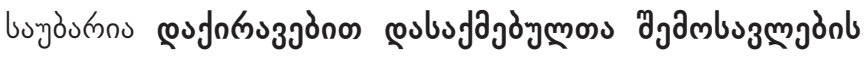

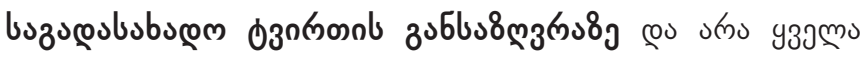

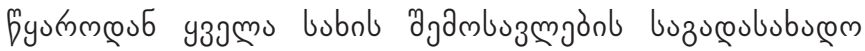

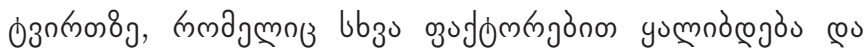
nвmajó.

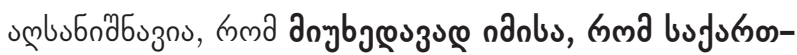
зэмmm

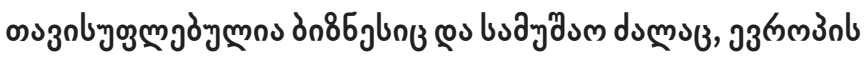

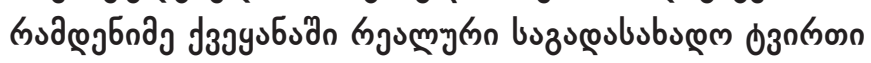

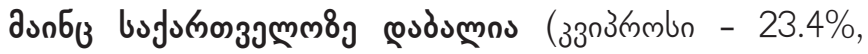




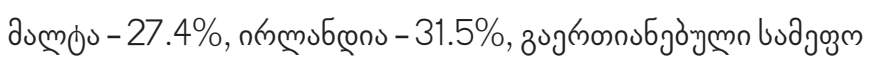
- 35.1\%, ömgüungon - 39.1\%).

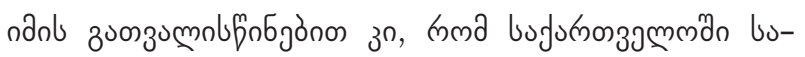

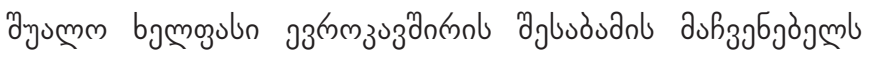

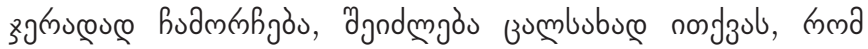

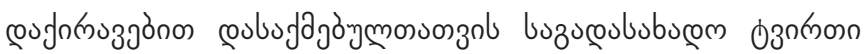

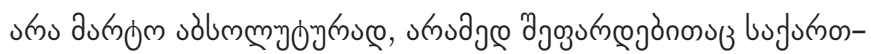

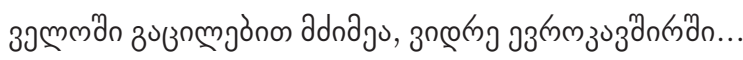

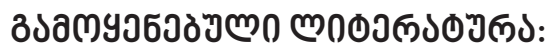

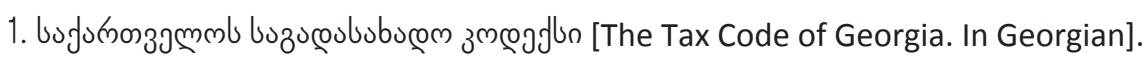

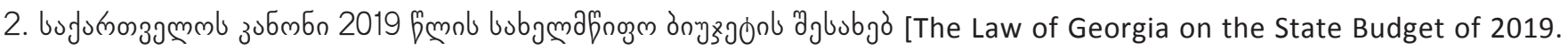
In Georgian].

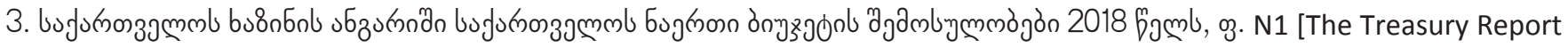
of Georgia Consolidated Budget Receipts in 2018, N1. In Georgian].

4. Papava V., Silagadze A., 2019. "How the Term the "Gross Domestic Product" should be translated into Georgian." Globalization and Business, No. 7, pp. 203-204. (In Georgian.)

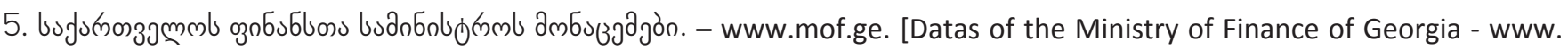
mof.ge. In Georgian].

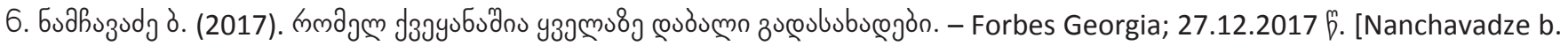
(2017). Which country has the lowest taxes- Forbes Georgia; 27.12.2017. In Georgian].

7. Eurostat. Tax Revenue statistics.

8. James Rogers | Cécile Philippe, The Tax Burden of Typical Workers in the EU 28-2018. Institut Économique Molinari, Paris-Bruxelles;

9. Viktor Trasberg. Dynamics of European Tax Structures (2013). Tartu.

10. Germany Income Taxes and Tax Laws.

https://www.google.com/search?source=hp\&ei=vZvnXNTKB43qrgSsz4fYDQ\&q=Tax+system+in+Germany\&oq=Ta

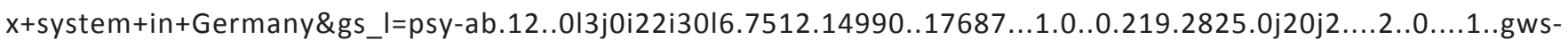
wiz.....6..35i39j0i131j0i67.y2eTQggFZb0

11. Налоговые системы в странах Евросоюза. Минск, 2007 [Tax systems in the EU countries. Minsk, 2007. In Russian].

12. Лыкова, Л. Н., Букина, И. С. (2015). Налоговые системы зарубежных стран. Москва, «Юрайт» [Lykova, L.N., Bukina, I.S. (2015) Tax systems of foreign countries. Moscow, «Yurayt». In Russion].

13. www.geostat.ge

14. www.imf.org 


\section{TAX BURDEN OF EMPLOYED PERSONS}

\section{JOSEPH ARCHVADZE}

Doctor of Economic Science,

Kutaisi University, Georgia

daswreba@yahoo.com

KEYWORDS: TAX, INCOME TAX, SALARY TAX, WORK PLACE VALUE.

For citation: Archvadze J. (2019), Tax Burden Of Employed Persons, Globalization And Business, №7, pp. 79-86. (In Georgian). https://doi.org/10.35945/gb.2019.07.010

\section{SUMMARY}

Tax burden demonstrates very precisely the scope of fiscal pressure on business and population by the state. In Georgia physical persons are taxed as by direct taxes (income tax is $20 \%)$, as indirect taxes. In the end they have much influence on population's purchasing power.

More than population's cash income comes from the salaries, accordingly income tax, VAT and excise could be considered as the tax burden of salaries. According to the calculation by this principle, the salary tax burden in Georgia is $\mathbf{3 9 . 2} \%$. In the last 7 years (In comparison with the year 2012) because of the rapid growth of indirect taxes, the mentioned burden has been increased by 2.7 point.

To compare the salary tax burden with the European Union countries it is complicated, because since 2008 there is annulled social tax in Georgia, which is paid by employee and employer in Europe. There could be made the comparison if according to the European countries we compare the tax burden value with single workplace, which consists of social taxes paid by salary receivers and employers (contributions).

Accordingly, what we will compare with Georgian indexthe share of direct and indirect taxes in whole salaries, or all tax shares in workplace value, Georgia with tax size will be on leader position (according to the first position) or in outsiders (according to the second position). That means, that in joint list of the EU and Georgia, with the sum of income tax and VAT in comparison with accrued salary, Georgia will be on the second place (39.2\%; on the first place is Dania-40.7\%) and by the real tax burden of workplace value - on $\mathbf{2 4}^{\text {th }}$ place (On the first place is France-56.7\%, on the last place is Cypros-23.4\%). 


\title{
GREEN ECONOMY AND ACCESS TO FINANCE IN GEORGIA (GOING BEYOND THE COMMERCIAL BANKING SECTOR TO FINANCE BUSINESSES IN GEORGIA)
}

\section{DAVID ASLANISHVILI}

\author{
PHD, Professor
}

Caucasus University, Georgia

daslanishvili@cu.edu.ge

KEYWORDS: GREEN ECONOMY, ACCESS TO FINANCE IN GEORGIA.

For citation: Aslanishvili D. (2019), Green Economy And Access To Finance In Georgia (Going Beyond The Commercial Banking Sector To Finance Businesses In Georgia), Globalization And Business, №7, pp. 87-95. https://doi.org/10.35945/gb.2019.07.011

\section{GREEN ECONOMY AND ACCESS TO FINANCE IN GEORGIA (GOING BEYOND THE COMMERCIAL BANKING SECTOR TO FINANCE BUSINESSES IN GEORGIA)}

\subsection{Green Bonds - International Practice}

Huge efforts and financial resources are needed to ensure sustainable development and achieve climate and environmental objectives. It is estimated that total global investment needs are around USD 5-7 trillion per year. In particular, support of private finance is needed, with public finance serving to leverage such private capital. (Future we want. 2912. Outcome document of the United Nations Conference)

As a result of the analysis of the World Economic Forum, it is necessary to invest about $\$ 5.7$ trillion in the Green Economy every year, from which 5 trillion funding should be directed directly to green infrastructure and business projects, and the remaining 700 billion will be able to effectively manage these projects.

A large number of financial instruments can also be applied by the government to support the scaling-up of green investments. These include, among others;

- instruments that provide direct financing from the budget (at the national and sub-national level), such as equity, grants, government soft loans;

- instruments that mitigate risks (e.g. guarantees, feed-in tariffs);

- instruments that help raise additional private funds (e.g. green bonds) (Lindenberg, N., 2014).
The concept of green bonds was launched almost 10 years ago, by leading development finance organizations such as the World Bank, the IFC and the EIB, together with pioneering investment banks. In little more than a decade, annual green bond issuance has grown from zero to nearly $\$ 170 \mathrm{bn}$.

- In 2019, global issuance is expected to reach a record $\$ 200 \mathrm{bn}$. That growth is impressive - and a measure of investors' eagerness to address the greatest development challenge of our time.

- Yet green bonds remain a small sliver of the \$100tn global bond market.

- In 2016, the Swedish Pension Fund (AP4) allocated $21.8 \%$ of its global equity portfolio to low-carbon projects.

- AP4 aims to decarbonize its entire global equity portfolio by 2020 .

- Similar initiatives are taking place in other OECD countries as well (e.g. the Portfolio DE carbonization Coalition in the USA embracing 28 institutional investors have pledged to gradually decarbonize a total of USD 600 billion by designing investment portfolios with a smaller climate change impact).

- Currently, Europe is leading the green bond market, with numerous Europe-based mutual funds focusing on green bonds. The EU is in the process of boosting the market for green bonds for infrastructure and SMEs.

- Despite previous experience of some countries in the region with municipal bonds for water supply and sanitation infrastructure (e.g. Ukraine, Kazakhstan) none of the EaP countries and Central Asia seems to have issued green bonds so far to finance low-carbon investments. 
- However, Ukraine is considering the creation of a green bond market and have prepared "Green Bond Guidelines: Roadmap for Ukraine". The introduction of green bonds is also being considered in Kazakhstan.

In the majority of developed countries, many specialized financial institutions that support green investment and support of energy efficient projects in developing countries, including Georgia, have been created to support green projects. One of the examples is the European «Green Growth Fund», which implements energy in the energy industry and reduces greenhouse gas emissions in up to 20 developing economies.

The foundation was founded in Luxembourg in 2009 by the Development Bank of Germany and the European Investment Bank, which has financial support from other leading international financial institutions such as the Austrian Development Bank, the European Bank for Reconstruction and Development Bank, the International Finance Corporation, the Dutch Development Bank, etc.

The EU funded the new multinational program in the context of the Eastern Partnership (EU for Environment), which was implemented in Georgia in April 2019.

The EU4ENVRM program aims at helping EU partner countries to maintain and utilize their natural capital to boost the ecological well-being of the population and use new opportunities for development. For instance, enterprises (particularly small and medium sizes) will receive further assistance in terms of saving energy, water and materials: leading international experts will consult environmental management.

This new program will support Georgiass efforts to improve the challenges in the development of green economy development. For all 6 countries of the Eastern Partnership, this program has a total of 20 million euros. The results of the work carried out by the EU and local institutions are that within the 2019 exhibition Georgian companies have received more than 600000 Euros order from international buyers.

\subsection{Links between economic growth, green growth (e.g. clean energy), high living standards and capital markets "Green" economy}

The Rio Conference in June 2012 reflected a trend that focuses on the economic system. Term "green" economy was first used in 2013. The concept of green economy is transferred in the document of Rio de Janeiro conference dedicated to the sustainable development "future we want". As United Nations Environment Program (UNEP) defines "green" Economy is an economy that provides growth of peopless well-being in the long term and reduces inequalities in order to enable future generations to avoid environmental and its impoverishment risks.

The main focus in the definition on the growth of peopless welfare, because nature conservation, protection and in some cases, improvement by itself serves to increase peopless wellbeing. To be more specific a "green" economy means ecological needs' that have been improving the social and economic situation of people through the rational use of resources, the preservation of the process of nature reproduction, ensuring the safety of living organisms and the growth of production.

"Green" economy goals also serve to provide resources for the future, due to the fact that non-renewable, exhausted resources will be replaced by renewable, environmentally friendly resources. Although the problem of providing resources in the future is much huger and global. The main sectors of the green economy are:

- Renewable energy (solar, wind, geothermal, marine, including waves, biogas and heat energy);

- Green buildings (green modifiers, green products and materials);

- Clean Transport (Alternative Fuel, Public Transport, Hybrid and Electric Vehicle);

- Water management (water and rain treatment systems, internal water landscape, water usage);

- Waste management (utilization, municipal waste, use of materials, soil fertility improvement, cleaning);

- Land management (organic agriculture, urban forests and parks, forest development)

\section{«Green» Economy in Georgia}

«Georgia-EU Association Agreement» focuses on sustainable development and green economy. In particular, Article 301 of the Agreement states that «the Parties will develop and strengthen cooperation on environmental issues, thus contributing to sustainable development and long-term goals of the green economy.

Tbilisi City Hall is particularly active in the direction of a "green economy "that developed the Environmental Strategy on 2015-2020 and Green City Action Plan for 2017-2030.

According to the 2018 Global Green Economy Index (GGEI), Georgia is taking an intermediate position among world countries. On the other hand, according to the Environmental Performance Index (EPI), Georgia meets only in the second half of the world, but with improved indicators. Georgia is rich in natural resources and has the potential for rapid development.

The richness of Georgia has the potential for rapid development of natural resources. There are certain ways for developing countries to achieve global green growth. In many countries the green growth is understood as an inclusive economic development, which envisages the development and maintenance of environmental and social values.

According to the data of 2018, Georgia occupies the 44th place among 130 countries $(0,5183)$. And the first five looks as follows:

- Sweden 0.7608

- Switzerland 0.7594 
- Iceland 0.7129

- Norway 0.7031

- Finland 0.6997

The last places were distributed: Guinea-Bissau, BosniaHerzegovina, Benin, Haiti, Bahrain.

According to the data of 2018, Georgia ranked 94th in the ecological efficiency rating among 180 countries compared to 2016 (111th place), improved by 17 points. In total, Georgia received 55.69 points from 100 points (2018).Among the neighbors is the best situation in Russia - 63.79 points (52nd place), Azerbaijan - 62.33 points (59th place), Armenia - 62,07 points (63rd place). It is noteworthy that in 2016 this situation worsened in Turkey (108) and Ukraine (109th place).

\subsection{Green Tourism As An Important Component Of The Transition To A Green Economy And Economic Growth}

In the mid-2000 the tourism industry accounted for 5\% of global GDP and provided about $8 \%$ of total employment. This industry ranked fourth in world exports (after the fuel, chemical and automotive industries). Since tourism is playing an important role in economy, a green tourism is a considerable component of the transition to a green economy. Thus, all types of tourism should become green and sustainable, namely:

- make optimal use of environmental resources, which are a key element for the development of tourism, support key environmental processes and promote the conservation of natural resources and biodiversity;

- respect the sociocultural identification of local communities, help preserve their cultural heritage and traditional values;

- to ensure sustainable long-term economic activities that provide socioeconomic equitable benefits for all parties involved, including tourist satisfaction, stable employment and income-generating opportunities, and social services to host communities.

\section{Georgian non - traditional funding (Capital Market in Georgia)}

What is the present and the future for the stock and debt capital market in Georgia?

Here we have studied possible financial vehicles that go beyond traditional sources of private capital offered by commercial banks.

\subsection{Georgian Capital Market - On-going trends and development Problem Statement}

The mission of research proposal is to find out the real solution to rapidly develop countries like Georgia based on modern capital market tools. Economic progress and improving living standards of the population depends largely on the provision of high economic growth. That issue itself heavily depends on energy sources and mostly on green sources.

To attract capital into green energy objects, it is obligatory to have reliable and strong market foundation and its tools. One of the real levers, and the most acceptable and practical forms of investment are to invest in equities through the stock market. It is clear for the developed world how important the organized stock market is. This includes Western Europe, USA, Canada, Japan, China and other countries with powerful stock industries such as the New York Stock Exchange; Euronext; NASDAQ OMX; the London Stock Exchange and many others.

The mediation business or brokerage/investment firms include Merrill Lynch; Morgan Stanley; Goldman Sachs; J.P. Morgan; Barclays Capital and many other investment banks.

Georgian corporate bonds eligible in Clearstream from September 2018. Clearstream Banking S.A. started offering settlement, custody and asset servicing for selected Georgian corporate bonds, which is in addition to the securities issued by the Government of Georgia and international financial institutions (the "IFIs"). By offering new services in Georgia, Clearstream further enhances the access to the Georgian capital market for international investors and is the sole international central securities depository (the "ICSD") to offer such services. Non-resident corporate, non-resident individual and resident individual holders of Georgian listed corporate bonds issued prior to 2023 are exempt from capital gain and interest income (withholding) taxes.

MORE THAN 2 BILLION GEL SUCCESSFUL PLACEMENTS OF IPO DONE in Georgia during the last five years:

- FMO (Nederlandse Financierings-Maatschappij voor Ontwikkelingslanden N.V)

- Black Sea Trade and Development Bank

- Bank of Georgia

- Liberty Bank

- M2 Real Estate

- TBC BANK

- EBRD bonds

- ADB bonds

- Georgian Leasing Company

- Nikora

- Zedazeni

- EVEX

- GWP (Georgian Water and Power)

- Lisi Lake

- Teliani Wine

- Silknet

- Microfiance Organisation Crystal

(source: www.gse.ge)

June 28, 2019 Tbilisi, Georgia: TBC Capital has listed the largest Eurobond issue on the Georgian Stock Exchange. TBC 
Bank has successfully priced a debut US\$300 million 5-year $5.75 \%$ ( $6 \%$ yield to maturity) senior unsecured notes issue (the "Notes").

July 12, 2019 - The Asian Development Bank (ADB) raised 60 million GEL (about $\$ 21 \mathrm{~m} / € 18.70 \mathrm{~m}$ ) from two new issues of local currency bonds. The proceeds of the bond issues will be on-lent to Credo Bank to launch new products including home improvement and mortgage loans to lower income households in rural areas and on the outskirts of Georgian capital, Tbilisi.

TEGETA MOTORS LTD - On May 22, 2019, GEL 30 million worth of 3-year bonds issued by the Tegeta Motors LTD have been admitted to the category $A$ listing of the Georgian Stock Exchange.

Research has ascertained that Georgia faces the problem between the two basic means of attracting monetary resources -Commercial Bank lending/loans and the stock market. In financially successful and developed countries, these two mechanisms-bank loans and the stock market-are designed to attract money and create a mutually beneficial synergy. In Georgia, there is only one mechanism to attract money resources -the bank loan.

In general, the Georgian commercial banking system tries its utmost to prevent the use of the second mechanism, the stock market, since it is considered a main competitor. The analysis of the structure of owners of Georgian Stock Exchange (source: securities registrar JSC Kavkasreestri www.kavkasreestri.ge) shows that $58 \%$ of shares of the stock exchange is in hands of its competitor - Georgian commercial banks/holdings. ((D Aslanishvili (2016) Market Foundation...)

There is another stock exchange - Tbilisi Stock Exchange, where owners are just commercial banks/holdings and Georgian Stock Exchange as minority shareholder itself (fully under control of Commercial Banks/holdings).

It means that Georgian commercial banks will not allow the development of their competitor-the stock market--as it threatens their own preferential and successful financial position.

According to this analysis, Commercial banking and Stock Market have one mega regulator - the National Bank of Georgia. It should be noted, that an essential attribute of the stock market - the National Securities Commission of Georgia (NSCG) - was abolished. The power of the NSCG was transferred to the regulator of Georgian commercial banks and lobbyist of their interests - the National Bank of Georgia.

Simultaneously, very tough policies were enacted against non-commercial banking structures - brokerage firms, registrar companies and market participants. As the result-there are practically no non-commercial banking funding ability to attract finance in Georgia.

Fixation rule - unfortunately, there is no public trades and market price on Stock Exchange. Securities admitted to the stock exchange can be transferred by simple inscription on paper, without any auction or trading - it is enough simply to sign the paper document. ( (D Aslanishvili (2016) Market Foundation...)
As the analysis shows, the management of joint stock companies (non-commercial banks) gradually lost any interest in equity trading in an open and transparent environment, once the law didn't require it. As the results - there is no de facto market price on any stock on Georgian Stock Exchange

- BROKERAGE FIRMS

- Galt \& Taggart JSC

- Caucasus Capital Group JSC

- $\quad$ Silk Road Bank JSC

- Heritage Securities JSC

- TBC Capital Ltd

- Cartu Broker LTD

(source: www.gse.ge)

Is there ANY real positive trend? - STOCK MARKET

In 2013, the country's stock exchange turnover amounted to GEL 530,491 or 338-fold less compared to 2007

In the period from January 1, 2014 - July 14, 2019

Number of Trades - 667

Volume - 32,666,230

Total Value (GEL) - 2,770,069.42

(www.gse.ge)

At average per year - 461,678 Georgian Lari turnover Approximately all trades go on Banking stocks and affiliated companies

Is there ANY real positive trend? - BOND MARKET

In the period from January 1, 2014 - July 14, 2019 :

Number of Trades - 140

Volume - 13,243

Total Value (USD) - 13,810,688.40

At average per year - 2,301,781 USD turnover Approximately all trades go on Banking Bonds and affiliated companies

\subsection{So, Where Is The Turnover And Market?}

\section{GRAY MARKET (OTC MARKET)}

In the period from January 1, 2014 - July 14, 2019:

Number of Trades - 2,319

Volume - 6,031,596,962

Total Value (GEL) - 2,323,956,196.85

At average per year - 38,732,603 GEL turnover - Mostly turnover goes on Banking Stocks/Bonds and affiliated companies. 
Presently, Georgia's stock exchange has lost its key function as a foundation for price formation in the stock market. Therefore, any trading in shares or other securities publicly has become senseless.

Fixation of Deals out of Market (gray Market, OTC) represent the only way of funds attraction. As practice shows, Commercial Banks distribute the adopted offer of stocks/ bonds inside the banking/holding structure for own clients and submit the fulfilled IPO to Stock Exchange. Stock Exchange act as Notary, but not as Stock Exchange.

Any suggestion on legislative amendments as a solution in order to save and develop independent from Commercial bank's Georgia's stock exchange had been many times stopped and blocked by Georgian government, National Bank and Commercial Banks, working together to keep the current trend. ((D Aslanishvili (2016) Market Foundation...)

The only way to get finance in Georgia is to be loyal to Commercial Banks and its affiliated structure(s) and show the readiness to give them access to your company share structure and management to receive funding.

\section{3 “THE ROAD TO HELL IS PAVED WITH GOOD INTENTIONS"}

So the reality is simple:

MANY TALKS OF SECURITIES MARKET, ALTERNATIVE SOURCE OF COMMERCIAL BANK LENDING, IPO AND STOCK MARKET DEVELOPMENT, BUT REALITY IS OPPOSITE....

Georgia needs the REAL Glass Steagal/Dodd-Frank Act in action, not in paper.

\section{Situation in Summary}

- Commercial Banks/Holdings fully control non-commercial source of funding - "Georgian Stock Exchange"- 58 percent of Stake of GSE and 100 percent control of "Tbilisi Stock Exchange";

- Supervisory Board and Management of Stock Exchange in hands of Commercial Banks/holdings;

- Commercial Banks and its affiliated structure mostly prefer to have IPO and trades OUT OF GEORGIA and its Stock Market (London Stock Exchange);

- Registrars (Transfer Agents) - the most developed are in hands of Commercial banks/holdings;

- Licensed by NBG and GSE Brokerage Companies almost all in property of Commercial Banks/holdings;

- All emissions and successful placements of companies (Bonds/stocks) are done only by Commercial Banks and for their and affiliated companies;

- THERE IS NO ALTERNATIVE SOURCES OF FUNDING IN GEORGIA EXCEPT COMMERCIAL BANK LOAN DIRECTLY OR INDIRECTLY (via their "green light" to issue bonds/stocks for Banks of affiliated companies).

\section{SOLUTION}

- Georgia needs the REAL Glass Steagal/Dodd-Frank/The Gramm-Leach-Bliley Acts in action, not JUST in paper.

- We need independent from Commercial Bank/Holdings the Supervisor and Stock Market structure

- Commercial Banks/Holding should be prohibited from Stock Market activity.

\section{CONCLUSION}

- Main sources of funding for SME green investments in Georgia are Commercial Banks and affiliated structures;

- Capital market - stock and bond markets in Georgiais not developed as alternative source of funding for any noncommercial banking structure;

- There is a lot of real benefits of non-bank financing, including for clean energy projects and the SME sector (e.g. small hydro, energy efficiency);

- Government can use State Bonds/obligations, its affiliated structures (Partnership fund) to support SME finance in green economy;

- Government of Georgia should intervene and change the "rule of games" on Capital market and to introduce the REAL Glass Steagal/Dodd-Frank/The Gramm-Leach-Bliley Acts in action, not JUST in papers;

- Georgia needs an independent from Commercial Bank/ Holdings and National Bank of Georgia the Supervisor and Stock Market structure - to have the Real alternative source of funding;

- Commercial Banks/Holding should be prohibited from Stock Market activity (depositors risk mitigation - international practice);

- International community (donors, IFIs, international organization) should supervise the REAL implementation of abovementioned steps in Georgian reality to support Georgia's efforts to have the Real developed capital markets, available to finance business.

\section{POSSIBLE INVESTMENTS IN CLEAN ENERGY PROJECTS OF GEORGIA (E.G. SMALL HYDRO, ENERGY EFFICIENCY)}

Georgia has the greatest potential to become an important exporter of electricity received from renewable energy transformation. This gives our country a significant advantage in the entire region. This advantage can be considered as two important aspects:

- Energy independence, which is of paramount importance to maintain political stability in the region,

- Economic benefits, after fully satisfying domestic consumption, export of electricity to neighboring countries.

The first place among the natural riches of Georgia is the 
hydro-electrical. There are 26060 rivers on the territory of Georgia, with total length of approximately 60 thousand km. The common stock of fresh water of Georgia, comprising 96,5 $\mathrm{km} 3$ of glaciers, lakes and reservoirs of water reserves.

From the total number of rivers, there are about 300 rivers with energy value, the annual total potential capacity of 15 thousand megawatts and average annual energy equivalent to 50 billion kWh. Georgia is a wealthy country with renewable energy resources, but it is a poor source of energy sources.

In 2016, 57.6 thousand terrestrial power plants were produced in Georgia, which is 1.4 million tons of oil equivalent. More than half of this energy was on hydropower resources - $58.3 \%$. $28.2 \%$ of the biofuels and waste are still large in the energy produced. The remaining energy sources have a relatively small share of domestic production: coal $-8.7 \%$, crude oil - $2.8 \%$, geothermal $-1.5 \%$, gas $-0.4 \%$

Energy consumed in Georgia is 3.15 times higher than the energy required, which is largely due to demand for nonrenewable energy resources, which is not available in the country, but traditional energy resources are widely used in a number of fields.

As a result, in 2016, almost one-third of the countryss domestic consumption of petroleum products $(33.1 \%)$ and slightly less natural gas (30.5\%).

In the world as well as in Georgia, the role of coal is reduced in the economy (5.8\% of consumption). $20.8 \%$ of the domestic consumption comes from electricity, the source of which is the renewable energy/ unprofitable energy or import of the internal one. Only $0.4 \%$ of domestic internal consumption comes from geothermal energy. Over the next 10 years, new electric power capacity is expected to turn Georgia into an important exporter of electricity.

With realistic calculations, electricity consumption in Georgia will increase by 3.5\% per annum in the following years. As a result, net exports of 9.9 billion kWh electricity will be expected by 2027 , which will be $37.6 \%$ of the total electricity generated.

The list of potential projects is drawn up by the Ministry of Energy of Georgia for the potential investors (Order N125/) on Approving List of Potential Power Plants in Georgia (available to show). For each project, the average annual output is given. Some assumptions are used to calculate the value added by them (assumptions are taken from potential projects).

- Export tariff - 0,080

- Internal supply tariff - 0,048

- Technical losses and their own consumption - 3\%

- Export Share - 80\%

- Share of internal supply - $20 \%$

The following assumptions are used:
- Average Annual Production of Each Project Decreases Technical Losses (0.03\%);

- The derivative output is divided between export and internal supply $(80 \%-20 \%)$;

- The derivatives generated are multiplied by the cost of electric power of Kilowatt and the sum;

- The final added value is divided on macroeconomic indicators to assess its share.

- The macroeconomic indicators used the GDP of 2017 at current prices (15,230 billion USD) and the export indicator of 2017 (2,348 billion USD).

Following the aggregation of the information received at the project level, it is possible to see the general picture, first of all, to project projects according to their status:

- Construction and Licensing Projects (45 projects) After completion of full exploitation, they will generate a total additional cost of $2.5 \%$ of current GDP (2017).

- The second group consists of research projects under construction commitment (22 projects), the potential share of which is $2.2 \%$ of the current GDP. These are the projects for which the terms and obligations of construction are determined, but research works are still under way.

- The next group is the projects at the stage of technical and economic research ( 80 projects). There is an expressed interest in these projects by investors, although only research works are underway, after which the terms of their construction may be determined. The total contribution of these projects to GDP is $4.6 \%$.

- The fourth group is potential projects (99 projects), which are only theoretical and the investoris interest is not expressed. The potential share of potential projects in GDP is $3.2 \%$.

- Renewable energy current and potential projects are determined by several renewable energy sources. As mentioned above, hydropower has a vital role in the Georgian energy sector.

- The total potential contribution of hydro power plants (both ongoing and potential) to the current economy is quite high and $10.2 \%$.

- After hydropower, Georgia is attractive for investors with wind energy, which is $2.0 \%$ for wind power plants.

- Interest in the use of solar energy is scarce $(0.3 \%)$, as well as bio waste recycling.

- Overall, the potential contribution of all four types of projects in the economy is $12.6 \%$, which is quite high.

Obviously the research is based on certain assumptions, and all project implementation will require tens of years, and with the growth of the current economy, their contribution to economic growth in other equal conditions will be reduced. However, potential economic benefits of projects are more visible. 


\section{REFERENCES:}

1. Davit Aslanishvili, (2019). International Conference on: Cooperation, Development, Exchange in Higher Education: Academic practices and achievements from Emerging Europe, Hungary, Miskolc University, May 7, 2019;

2. D Aslanishvili (2016) Market Foundation for Sustainable Economy Growth and Energy Policy (Georgian Case). Modern Economy, volume 7, p. 314 -319Modern Economy, 2016, 7, 314-319 Published Online March 2016 in SciRes. http://www. scirp.org/journal/me, http://dx.doi.org/10.4236/me.2016.73034

3. Aslanishvili, D. and Omadze, K. (2016) Origins and the Reasons of Monetary Crises in Georgia (1995-2016). Modern Economy7, p. 1232-1250.http://dx.doi.org/10.4236/me.2016.711119

4. Aslanishvili, David; Muller, Dietrich; Omadze, Kristine, Analyse of the past Industrial Revolutions and possible outcome of the coming new fourth In-dustrial Revolution (Industry 4.0), Con-ference: International Scientific-Practical Conference Dedicated to the Foundation of the Institute Innova-tive Economy and Problems of Its Formation in Post-Communist Countries. Pages: 100-106, Year: 2016

5. Aslanishvili, David; Omadze, Kristine, Monetary Crises in Georgia - 1995 - 2016 Source: Ekonomisti, Volume: 8, Issue: 1, Pages: 89-108, Published: 2016

6. Aslanishvili, David, Ricardo equivalent, the origin of state debt, its meaning and practical purpose Conference: Modern Tendencies of Development of Economy and Economic Science, Pages: 18-22, Year: 2018

7. Aslanishvili, David; Omadze, Kristine, Georgiass external debt structure and the challenges of the current situation Conference: International Scientific Conference Dedicated to the 65th birth anniversary of Professor George Tsereteli Structural and Innovative Problems of Economic Development, Pages: 25-28, Year: 23-24 June, 2017,

8. Author(s): Aslanishvili, David; Omadze, Kristine, International Discussions on Bank Credit and Economic Growth Relationship (Expanded Summary), Source: Ekonomisti, Issue: 2, Pages: 62-74, Published: 2018

9. Aslanishvili, David, Place and importance of the government securities market in the European Union: recent trends and discussions (Expanded Summary), Source: International Scien-tific-Analytical Journal EKONOMISTI, Issue: 5, Pages: 12-14, Published: 2015

10. United Nations Environment Programme and World Tourism Organization (2012). Tourism in the Green Economy-Background Report, UNWTO, Madrid. [ Electronic resource]: Retrieved from https://www.e-unwto.org/doi/pdf/10.18111/9789284414529 11. World Wide Fund For Nature (WWF, 2019). Over $80 \%$ of marine pollution comes from land-based activities. [Electronic resource]: Retrieved from http://wwf.panda.org/our_work/oceans/problems/pollution/

12. Future we want. 2012. Outcome document of the United Nations Conference on Sustainable. Development Rio de Janeiro, Brazil, 20-22 June 2012 https://sustainabledevelopment.un.org/content/documents/733FutureWeWant.pdf

13. Towards Green Growth. 2011. https://www.oecd.org/greengrowth/48012345.pdf

14. Berger, Roland. 2011. Green Growth, Green Profit How Green Transformation Boosts Business. Green Growth, Green Profit. US, New York: Palgrave Macmillan. https://doi.org/10.1057/9780230303874.

15. Cereceda Rafael, Cuddy Alice. 2018. "9 Out of 10 People Worldwide Breathing Polluted Air." Euro News. https://www. euronews.com/2018/05/02/9-out-of-10-people-breathing-polluted-air-worldwide.

16. Madhipatla, Kishore, S Venkatesh, D J Christopher, Jeffrey D Stanaway, R S Dhaliwal, LalitDandona, Virendra Singh, et al. 2018. "The Impact of Air Pollution on Deaths, Disease Burden, and Life Expectancy across the States of India: The Global Burden of Disease Study 2017." The Lancet PlanetaryHealth 3 (1): e26-39. https://doi.org/10.1016/s2542-5196( 18)30261-4. 17. World Health Organization. 2018. "World Health Organization Releases New Global Air Pollution Data." CCAC Secretariat. http:// ccacoalition.org/en /news/world-health-organization-releases-new-global-air-pollution-data. https://www.energy-community.org/ 18. A strategy for smart, sustainable and inclusive growth" and in 2011 "A resource-efficient Europe - Flagship initiative under the Europe 2020 Strategy"

19. Geoffrey Heal - THE ECONOMICS OF RENEWABLE ENERGY (2009)

20. International Energy Agency - World Energy Outlook 2016

21. Nicholas Apergisa, James E. Payneb - Renewable energy consumption and economic growth: Evi-dence from a panel of OECD countries (2010)

22. OECD (2009), "The Bioeconomy to 2030. Designing a p olicy agenda"; EU (2012), "Innovating for Sustainable Growth: A Bioeconomy for Europe"; U SA (2012), "National Bioeconomy Blueprint

23. TheEuropean Commission's 'Europe 2020' strategy con siders developing a smarter and greener Europe essential

24. Green for Growth Fund. www.ggf.lu

25. United Nations Framework Convention on Climate Change. www.unfccc.int

26. World Resources Institute. http://www.wri.org/our-work/project/climate-finance/climate-finance-and-private-sector

27. https://tradingeconomics.com/switzerland/gdp-per-capita

28. https://tradingeconomics.com/poland/gdp-per-capita

29. https://tradingeconomics.com/hungary/gdp-per-capita

30. https://tradingeconomics.com/georgia/gdp-per-capita

31. www.geostat.ge 


\title{
GREEN ECONOMY AND ACCESS TO FINANCE IN GEORGIA (GOING BEYOND THE COMMERCIAL BANKING SECTOR TO FINANCE BUSINESSES IN GEORGIA)
}

\section{DAVID ASLANISHVILI}

\author{
PHD, Professor
}

Caucasus University, Georgia

daslanishvili@cu.edu.ge

KEYWORDS: GREEN ECONOMY, ACCESS TO FINANCE IN GEORGIA.

For citation: Aslanishvili D. (2019), Green Economy And Access To Finance In Georgia (Going Beyond The Commercial Banking Sector To Finance Businesses In Georgia), Globalization And Business, №7, pp. 87-95. https://doi.org/10.35945/gb.2019.07.011

\section{SUMMARY}

This research will explore other possible financial vehicles that go beyond traditional sources of private capital offered by commercial banks. It will look at international experience and the opportunities to use public support, green bonds to raise green finance as well as the work of energy service companies (ESCOs) to finance green investments.

We have offered our view of what should be done in fact (not in paper in Georgia as it has been in the past 15 years) to change the situation and end the negative and harmful monopoly of the commercial banks and the National Bank of Georgia and to have in place the two independent sources to attract and invest resources in Georgia.

This will increase the capitalization of the country and is a proven way to eradicate the countrys lagging and accelerate economic growth.

Why should we focus on this issue?

1. According to WHOs latest data, over 7 million people die each year because of breathing air with solid particles, and one of its main pollutants is vehicles. (Cereceda Rafael, Cuddy Alice. 2018.....)

2. Georgia's Capital - Tbilisi - is occupying the 3rd place in the light of air pollution,

3. Due to the critical situation, the public demand to live in a clean ecological environment, day by day increases.
In our research the following Questions are discussed and overviewed:

- Is it important to act on the issues of Georgiass position on the global scale?

- What unique components can be used to prolong the average life of people?

- What investors do the country need for building ecoprojects and their realization?

- What type of ecofriendly technologies can be developed for potential customers in Georgia?

In that field we have studied the following:

- The links between economic growth, green growth (e.g. clean energy), high living standards and capital markets;

- Why the Commercial Banks are the main and the only source of finance for green (and not only) investments in Georgia;

- Situation on capital markets of Georgia (stock and bond markets) - as an indicator of economic growth and an alternative source of financing;

- Possible benefits of non-bank financing, including for clean energy projects and the SME sector (e.g. small hydro, energy efficiency);

- The role of government in supporting capital market development;

- The role of international community (donors, IFIs, international organization) to support Georgia's efforts to develop capital markets 


\section{Georgia - Recent level of development}

To illustrate the wide gap between the developed economy and the weak one, let us compare the current level of per capita GDP of Switzerland, Hungary, Poland to Georgian one (source: https://tradingeconomics.com/switzerland/gdpper-capita; https://tradingeconomics.com/poland/gdp-percapita; https://tradingeconomics.com/hungary/gdp-per-capita; https://tradingeconomics.com/georgia/gdp-per-capita);

- The Gross Domestic Product per capita in Switzerland was last recorded at 76667.44 US dollars in 2017. The GDP per Capita in Switzerland is equivalent to 607 percent of the worldıs average.

- The Gross Domestic Product per capita in Hungary was last recorded at 15647.85 US dollars in 2017. The GDP per Capita in Hungary is equivalent to 124 percent of the world ss average.

- The Gross Domestic Product per capita in Poland was last recorded at 15751.23 US dollars in 2017. The GDP per Capita in Poland is equivalent to 125 percent of the world ss average.

- The Gross Domestic Product per capita in Georgia was last recorded at 4290.17 US dollars in 2017). The GDP per Capita in Georgia is equivalent to 34 percent of the worldıs average. 


\section{WOMEN FOR GLOBAL GROWTH: NEED FOR WOMEN ECONOMIC EMPOWERMENT}

\section{EKA SEPASHVILI}

Doctor of Economics

Associate Professor

Ivane Javakhishvili Tbilisi State University, Georgia

Eka.sepashvili@gmail.com

KEYWORDS: GENDER EQUALITY, WOMEN ECONOMIC EMPOWERMENT, GLOBAL GROWTH.

For citation: Sepashvili E. (2019), Women For Global Growth: Need For Women Economic Empowerment, Globalization And Business, №7, pp. 96-103. https://doi.org/10.35945/gb.2019.07.012

\begin{abstract}
"Policy-makers need to take concrete action to help women achieve equal access to opportunities - to education, to productive resources, and to full equality under the law" Christine Lagarde, Managing Director, IMF.
\end{abstract}

\section{INTRODUCTION}

Modern economic development heavily relies on new technologies and unprecedented development of science creating more and more new innovative products and services. The forth industrial revolution fundamentally differs from the previous ones. The new inventions connect digital technologies to almost every product and service and impact the development of all spheres: economic, political, social, cultural, environmental and etc. Modern knowledge-based global economy rises new goals for policy-makers wishing and trying to increase welfare of the country in a way to benefit all citizens of a national state. In such reality the demand for knowledge generation and improvement of resource efficiency is critical.

The equal impact and contribution of women and men in this process of modern transformation, deep economic changes and societal alteration is critical as global economy continuously moves towards the new fifth industrial revolution, where knowledge and cognitive skill have the imperative. More than ever, nations cannot afford to lose the skills, ideas and perspectives of women who are currently under-represented - in order to utilize the promise of a more prosperous and human-centric future that is to be come soon by contemporary innovations and technologies.

New challenges for national economic policies generate new approaches which have to face these modern developments and trends. One of the efficient practices represents women promotion for a very simple reason: women yet are not fully engaged into development and their economic potential has to be unlocked to benefit not only economic goals but also social inclusion and human development to meet upmost goal of every state: inclusive growth and high level of welfare. In this regard, the women promotion and their empowerment are key policy challenges. Nowadays the need for women advancement in all spheres of the society is clear and generates no doubts for reasonability and rationality of such approach to economic growth.

Generally speaking, all types of inequality have economic prerequisite, circumstances and consequences. Therefore, in this paper we mostly focus on the economic side of the discrimination and lack of gender balance between men and women in economic terms. Despite the significant progress mankind has reached in the past 20 century, women still are not equal partners with men in the economy, politics and society: the gap between them remains large. Gender balance in economic, which means equal presentation at economic decision-making, merit-based participation in the labour force, presence in managerial and leadership positions, access to resources and etc., is not inevitably ideal for every nation, society or individual. But creating equal opportunities for both gender and then giving them possibility to choose relying on their own preferences is the key for any state to achieve 
and guarantee higher level of welfare. At the same time, we should not miss the sectors where men are disadvantaged relative to women. Thus, when we speak about transformation and creating incentives for women promotion, we should be conscious not to exaggerate and keep focus on improving parity between men and women.

\section{LITERATURE REVIEW}

The importance of gender balance in economic performance is growing due to the effects of globalization, which deepens the gap between men and women, in terms of access to political-economic power and resources. As Schwab (2017) notes, deepening the globalization may result grave consequences: organizations might be unable to adapt with new environment; governments could fail to employ and regulate new technologies to capture their benefits; inequality may grow and societies' fragmentation continues. As human capital is the key to technology, knowledge and innovations, its gender dimension does matter. Hence, national economies have to devote a great deal of attention to human resource efficiency. Therefore, governments should create appropriate conditions and/or incentives for business actors to focus on innovative products and services (Sepashvili 2018b). In this regard, gender equality represents a key factor for unlocking the economic potential of nation states and for leveraging economic growth (Sepashvili 2019).

Societies with greater gender equality not only offer better socio-economic opportunities for women, but also tend to grow faster and more equitably. There are gains in poverty reduction, environmental sustainability, consumer choice, innovation and decision-making on a wider set of issues when women are supported to engage in economic growth (Abney and González 2018). This kind of development encourages to support the economic empowerment of women and creates incentives to increase investment in female human capital - an investment that has returns not only for women but for men, children and nation as well. Gender equality leads to "smart Economics" (Buvinic \& King, 2007). This approach views gender equality as an integral part of economic development and aims to boost development through investing more efficiently in women and girls. Thus, investing in female human capital is resulting more effective development outcomes (Chant, 2012; Chant and Sweetman 2012). The need for development and facilitation of innovations dictate policy-makers to unlock cognitive potential of women and promote women economically to achieve better participation in decisionmaking and better positions at the top leadership level to influence company profitability at microeconomic level and welfare of population at the macroeconomic level (Sepashvili 2016, Sepashvili 2018a).

Women contribution to the economy is vital and proved by different studies and researches. The World Development
Report 2012 (World Bank, 2011) dedicated to Gender Equality and Development, confirmed the clear positive correlation between the women Economic Participation and the GDP level per capita and overall level of welfare. As world Economic Forum Report (2017) states, many empirical studies demonstrated that improved gender balance generates significant economic benefits, which differ according national characteristics of national economies, national cultural behavior and the challenges they are facing. Notable new estimates displays that economic gender equality could add an additional US\$250 billion to the GDP of the United Kingdom, US\$1,750 billion to the US, US\$550 billion to Japan GDPs, US\$320 billion to France GDP and US\$310 billion to German GDP. Other calculations suggest that China will have US\$2.5 trillion GDP increase if gender balance is realized and that the Global Economy could increase US\$5.3 trillion by 2025 if the gender gap is narrowed in economic participation by $25 \%$. More realistic calculation shows that, if female employment rates in OECD countries reaches the level Sweden experiences, GDP could be boost by over USD 6 trillion (PwC 2018).

The McKinsey Global Institute Report (2015) notes that $\$ 12$ trillion could be added to global GDP by 2025 by advancing women's equality within society by supporting public, private, and social sectors to act in coordination to close gender gaps. Scholars conducting the calculation note, that a "full-potential" scenario in which women participate in the economy is identical to that of men, would add up to $\$ 28$ trillion, or 26 percent, to annual global GDP in 2025 compared with a business-as-usual scenario. Of course reaching absolute parity is unrealistic, it is just noted the volume of the GDP that might be gained. Achieving even slightly better gender balance within society implies changes in cultural behavior, social attitudes, regulations and legislations. And visa-versa: changes in social norms and behavior in favor of women advancement leads to better women involvement in all spheres and especially in decision-making level. At the same time, it concerns personal choice of individuals, both men and women, how to allocate their time between un-paid and paid work, or how to participate in economics or politics.

Globally, Women comprise approximately the half the world's working-age population, but their contribution to the world GDP in only 37\%, the McKinsey Global Institute's Gender Parity Report 2015 says. This global average contribution to GDP covers large variations according the regions. The share of regional output generated by women is only $17 \%$ in India, $18 \%$ in the Middle East and North Africa (MENA), and $24 \%$ in South Asia (excluding India). In North America and Oceania, China, and Eastern Europe and Central Asia, the share is 40 to $41 \%$.

More women involvement leads to economic growth. International Monetary Fund (2018) research proves, that women economic empowerment supports productivity, increases inclusive economic growth and improves income equality alongside with other positive development outcomes. However, studies shows that growth does not inevitably lead 
to a reduction in gender inequality. On the contrary, it is assessed that gender gaps cost the economy some 15 percent of GDP (Cuberes and Teignier, 2016; Ferrant and Kolev, 2016). But just attracting women in labour force solve the problem only partially. Women participation at economic decisionmaking and women presence at top managerial positions is decisive to fully unlock economic potential and cognitive skills women hold.

Gender parity gives additional incentive to business development. Companies significantly benefit by giving possibility to women leadership that leads to increased effectiveness and growth (Sepashvili, 2019). It is assessed that companies with three or more women in senior managerial positions have higher score in all dimensions of performance (McKinsey \& Company, 2018). However, the reality is controversial: women face significant barriers, and often structural discrimination to hold highest leadership positions. According to Cheryl (Cheryl, 2014), only 5\% of Fortune 500 CEOs were women in 2014, and according new 2019 data women hold 24 (4.8\%) of CEO positions at those S\&P 500 companies. This vividly represents the real picture: while in recent years women experienced significant progress in career advancement, men are still majority on leadership positions such as high-level management, board of director seats, CEOs and est. Data from the most recent Global Entrepreneurship Monitor (GEM) suggest (Terjesen, 2012) that women represent one fourth of established business activity in countries and approximately one third of all new business activity around the globe.

\section{INTERNATIONAL RANKING OF COUNTRIES BY GENDER EQUALITY}

The sharpness and importance of barriers and obstacles impeding women promotion, vary by countries and relies on cultural and historical heritage, national mentality, government policy, gender role perception and so on. The difficulties facing women and girls over the globe are a major source of inequality. Women are discriminated against in health, education, political representation, labour market, etc. - with entails negative consequences for development. Despite this significant progress, that is evidenced by the World Bank Report on Development (World Bank, 2011), the situation in terms of gender equality still needs improvement, especially in its economic dimension. Encouraging trends are the higher number of women in the labour market and their progress in securing better education and training, as well as better access to health care and social protection.

The Global Gender Gap Report 2018 proves these significant achievements and notes the new threat of emerging gender misbalance in advanced technologies. In an era of fourth industrial revolution, when cognitive skills are increasingly vital to further technological development, no nation can afford to deprive women's human capital in the innovation development and knowledge generation, where attracting talent is crucial as this aspect of human capital is already scarce over the globe.

Since 2006 the Global Gender Gap Index measures the relative gaps between women and men across four key areas: health, education, economy and politics. Global Gender Gap Index measures gaps between male-female data rather than levels and quality. The Global Gender Gap Index points on the gaps between men and women according four main factors: 1. Economic Participation and Opportunity, 2.Educational Attainment, 3. Health and Survival and 4.Political Empowerment. It provides a comprehensive set of data and shows gaps on critical indicators, helping countries to set priorities within their own economic, political, social and cultural contexts. The Index also reveals those countries that-within their region or income group-are leaders in distributing resources more equitably between women and men, regardless of the overall level of available resources.

We will compare index for selected countries to track the tendency. Thus, for comparison we choose developed countries and countries, which are advanced in terms of gender equality. At the same time we will see the index for some former soviet countries and countries of soviet bloc in Eastern Europe. Soviet legislation had unprecedented high level of women promotion at all level, which was resulted in higher gender equality for former soviet countries, despite the fact that after the collapse of Soviet Union, all of them experienced deep economic crisis. And to completing the picture, some countries with the worst score are presented.

Data shows quite diverse picture according countries, but the tendency is that countries with high gender equality have good results in political empowerment and economic participation. However, direct and clear correlation is not observed as the history of achieving gender equality in these countries is different and greatly depends on social norms, cultural background and law enforcement. Worth to note that in former soviet countries gender equality is lower than the gender balance in economic opportunities. As it was already mentioned above, Soviet gender equality policy helped number of countries to reach better equality in economic terms. The most distinguished example is Belarus, which ranks as $\mathbf{2 8}$ for total score and is 6th in terms of economic participation and opportunity. The same picture is observed for other countries as well. The Global index rank and subindex rank for economic participation significantly differs for Russian Federation, Ukraine, and Kazakhstan: global index ranks are 75, 65 and 60 accordingly for these countries, while sub-index indicating economic opportunities shows a better picture and ranks are 31, 28 and 32, accordingly. Azerbaijan, Armenia and Georgia are considerably lagging back in terms of gender equality: their ranking are as follow: 97, 98 and 99 accordingly. Azerbaijan gained the highest ranking - 47 in terms of economic equality among these countries, while Armenia 
Table 1. Global Gender Gap Index 2018. Rank of selected countries

\begin{tabular}{|c|c|c|c|c|c|c|}
\hline$\#$ & Country & Global Index & $\begin{array}{l}\text { Economic participation } \\
\text { and opportunity }\end{array}$ & $\begin{array}{l}\text { Educational } \\
\text { attainment }\end{array}$ & $\begin{array}{l}\text { Health and } \\
\text { survival }\end{array}$ & $\begin{array}{l}\text { Political } \\
\text { empowerment }\end{array}$ \\
\hline 1 & Iceland & 1 & 16 & 39 & 121 & 1 \\
\hline 2 & Norway & 2 & 11 & 41 & 95 & 3 \\
\hline 3 & Sweden & 3 & 9 & 52 & 115 & 7 \\
\hline 4 & Finland & 4 & 17 & 1 & 60 & 6 \\
\hline 5 & Slovenia & 11 & 15 & 29 & 1 & 22 \\
\hline 6 & France & 12 & 63 & 1 & 78 & 10 \\
\hline 7 & Denmark & 13 & 38 & 1 & 100 & 15 \\
\hline 8 & Canada & 16 & 27 & 1 & 104 & 21 \\
\hline 9 & Latvia & 17 & 10 & 1 & 1 & 42 \\
\hline 10 & Bulgaria & 18 & 50 & 27 & 42 & 45 \\
\hline 11 & Lithuania & 24 & 21 & 53 & 1 & 41 \\
\hline 12 & Belarus & 28 & 6 & 49 & 52 & 74 \\
\hline 13 & Estonia & 33 & 42 & 1 & 42 & 51 \\
\hline 14 & Moldova & 35 & 18 & 69 & 1 & 72 \\
\hline 15 & United States & 51 & 19 & 46 & 71 & 98 \\
\hline 16 & Kazakhstan & 60 & 32 & 30 & 42 & 94 \\
\hline 17 & Ukraine & 65 & 28 & 26 & 56 & 105 \\
\hline 18 & Russian Federation & 75 & 31 & 28 & 1 & 123 \\
\hline 19 & Kyrgyz Republic & 86 & 78 & 79 & 1 & 93 \\
\hline 20 & Azerbaijan & 97 & 47 & 33 & 146 & 137 \\
\hline 21 & Armenia & 98 & 73 & 35 & 148 & 115 \\
\hline 22 & Georgia & 99 & 85 & 60 & 123 & 119 \\
\hline 23 & Iraq & 147 & 149 & 136 & 76 & 90 \\
\hline 24 & Pakistan & 148 & 146 & 139 & 145 & 97 \\
\hline 25 & Yemen & 148 & 147 & 146 & 126 & 149 \\
\hline
\end{tabular}

Source: Global Gender Gap Report (2018), World Economic Forum, 2018

ranks 73 and the worst result in economic sub-index- 85, was confirmed for Georgia. As for other developed countries, like Iceland, Norway, Sweden and Finland, being the best countries in terms of general gender equality, their results in economic sub-index shows worse ranking - 16, 11, 9 and 17 accordingly, but still demonstrating excellent results in comparison with former soviet countries.

Another source estimating gender equality is Human Development Report, which measures Gender Inequality Index (GII) taking into account three main factors of human development. 1. Women reproductive health measured by maternal mortality ratio and adolescent birth rates; 2 . Women empowerment measured by women participation in parliament and women access to education and 3. Economic status estimated by women participation in labour force.

The GIl measures the human development costs of gender inequality. Thus, GII highlights the inequalities between females and males and notes damage to human development in this regard. The Gll gives new picture of the position of women in 160 countries and points on gender gaps in major areas of human development. The component indicators emphasize areas in need of critical political intervention and offers solid food for thoughts. It assists public policy to correctly choose systematic disadvantages of women urgently need to be overcome.

Gender equality index for the same countries are as following: Table 2.

Data on gender equality index for same countries show slightly different picture and ranks, though the clear tendency is that countries with higher index of human development experience higher level of gender equality. However, some countries demonstrate better results in terms of human development (Iceland 6, Norway -1, Canada - 12, United States -13 ,) while their results in gender inequality index in worse (Iceland 9, Norway -5 Canada - 20, United States -41). For majority of countries the gender inequality index is higher in comparison with human development index. Azerbaijan, Armenia and Georgia showed better ranks for gender inequality index, 71, 55 and 78 accordingly, while human development level for these countries is worse: 80, 90 and 70 accordingly.

McKinsey Global Institute's Gender Parity report 2015, assess 15 gender equality indicators for 95 countries. The indicators fall into four categories: equality in work, essential services and enablers of economic opportunity, legal protection and political voice and physical security and autonomy. The scores is set at 1.00 as the ideal gender parity and countries 
Table 2. Gender Inequality Index 2017

\begin{tabular}{|c|c|c|c|c|c|}
\hline & country & $\begin{array}{c}\text { Human } \\
\text { Development index } \\
\text { rank }\end{array}$ & $\begin{array}{l}\text { Gender Inequality } \\
\text { Index rank }\end{array}$ & $\begin{array}{c}\text { Share of seats in } \\
\text { parliament } \\
\text { (\% held by women) }\end{array}$ & $\begin{array}{c}\text { Skilled labour Force } \\
\text { (\% of labor force) } \\
\text { (2011-2017) }\end{array}$ \\
\hline 1 & Iceland & 6 & 9 & 38,1 & 73,4 \\
\hline 2 & Norway & 1 & 5 & 41,4 & 82,4 \\
\hline 3 & Sweden & 7 & 3 & 43,6 & 84,9 \\
\hline 4 & Finland & 15 & 8 & 42,0 & 88,3 \\
\hline 5 & Slovenia & 25 & 7 & 28,7 & 90,6 \\
\hline 6 & France & 24 & 16 & 35,5 & 82,6 \\
\hline 7 & Denmark & 11 & 2 & 37,4 & 76,6 \\
\hline 8 & Canada & 12 & 20 & 30,1 & 90,0 \\
\hline 9 & Latvia & 41 & 42 & 16,0 & 91,2 \\
\hline 10 & Bulgaria & 51 & 46 & 23,8 & 87,6 \\
\hline 11 & Lithuania & 35 & 28 & 21,3 & 95,7 \\
\hline 12 & Belarus & 53 & 31 & 33,1 & 98,6 \\
\hline 13 & Estonia & 30 & 27 & 26,7 & 89,2 \\
\hline 14 & Moldova & 112 & 48 & 22,8 & 60,7 \\
\hline 15 & United States & 13 & 41 & 19,7 & 83,2 \\
\hline 16 & Kazakhstan & 58 & 43 & 22,1 & 75,1 \\
\hline 17 & Ukraine & 88 & 61 & 12,2 & 98,1 \\
\hline 18 & Russian Federation & 49 & 53 & 16,1 & 96,1 \\
\hline 19 & Kyrgyz Republic & 122 & 91 & 19,2 & 92,1 \\
\hline 20 & Azerbaijan & 80 & 71 & 16,8 & 93,0 \\
\hline 21 & Armenia & 83 & 55 & 18,1 & 94,4 \\
\hline 22 & Georgia & 70 & 78 & 16,0 & 94,4 \\
\hline 23 & Iraq & 120 & 123 & 25,3 & 28,4 \\
\hline 24 & Pakistan & 150 & 153 & 20,0 & 28,3 \\
\hline 25 & Yemen & 178 & 160 & 0,5 & 30,9 \\
\hline
\end{tabular}

Source: Human Development Report 2018, United Nations Development Program, http://hdr.undp.org/en/composite/GII

and regions are assessed by their distance each country has to reach absolute gender parity. This index clearly shows a strong link between gender equality in society, beliefs, social norms, cultural behavior and attitudes about the role of women, and gender equality in work. The report concludes that gender equality in work is not achievable without the gender equality in society and social norms. Researchers fail to find a country with high gender equality in society but low gender equality in work. The report concludes that economic development supports countries to narrow gender gaps, but progress in four areas in particular-education level, financial and digital inclusion, legal protection, and unpaid care work-could help to accelerate progress.

McKinsey Global Institute's Gender Parity Report 2015 data indicates the lowest scores 0.44 for South Asia (excluding India), North America and Oceania gained the highest score at 0,74. The Eastern Europe and Central Asia, where former soviet and Eastern European former socialist countries are

\begin{tabular}{|l|l|}
\hline & Very high human development \\
\hline & High human development \\
\hline & Medium human development \\
\hline & Low human development \\
\hline
\end{tabular}

concentered, gained 0.67, a little bit higher as of 0.71 score was given to Western Europe region, where major advanced European countries are located. As for remainder part of the world, the scores were as following for the regions: 0,64 Latin America; 0,62 East and South East Asia, 0,57 Sub-Saharan Africa and 0,48 Middle East and North Africa.

The women high representation was observed in unpaid work, which was accompanied by their lowest involvement in paid work. Globally, $75 \%$ of the world's total unpaid care is carried out by women, such as family care, child and elderly care, cooking, and cleaning. However, this contribution is not calculated in traditional measures of GDP. Report estimates 
Table 3. Women in Business

\begin{tabular}{|c|c|c|c|c|}
\hline & $\begin{array}{c}\text { Percent of firms with } \\
\text { female participation in } \\
\text { ownership }\end{array}$ & $\begin{array}{c}\text { Percent of firms } \\
\text { with majority female } \\
\text { ownership }\end{array}$ & $\begin{array}{l}\text { Percent of firms with a } \\
\text { female top manager }\end{array}$ & $\begin{array}{l}\text { Proportion of permanent } \\
\text { full-time workers that are } \\
\text { female }(\%)\end{array}$ \\
\hline All Countries & 33.6 & 14.6 & 18 & 30.6 \\
\hline East Asia \& Pacific & 46.9 & 28 & 32.6 & 37.6 \\
\hline Europe \& Central Asia & 32.5 & 12.6 & 18.9 & 37.8 \\
\hline Latin America \& Caribbean & 49.9 & 19.9 & 20.1 & 33.8 \\
\hline Middle East \& North Africa & 23.3 & 3.4 & 5.4 & 17.6 \\
\hline South Asia & 18.4 & 9.6 & 11 & 18.3 \\
\hline Sub-Saharan Africa & 29.2 & 13.4 & 15.8 & 26.5 \\
\hline Armenia (2013) & 25.3 & 14.5 & 19.1 & 46.4 \\
\hline Azerbaijan (2013) & 4.5 & 2 & 2.4 & 31.3 \\
\hline Belarus (2013) & 43.6 & 18.6 & 32.7 & 50.9 \\
\hline Bulgaria (2013) & 39 & 12.4 & 23.9 & 41.3 \\
\hline Georgia (2013) & 33.9 & 19.2 & 44.4 & 44 \\
\hline $\operatorname{Iraq}(2011)$ & 6.8 & n.a. & 2.3 & 8.6 \\
\hline Kazakhstan (2013) & 28.3 & 16.4 & 18.9 & 35.5 \\
\hline Kyrgyz Republic (2013) & 49.4 & 16.4 & 28.8 & 46 \\
\hline Latvia (2013) & 36.3 & 22.8 & 31.5 & 44.4 \\
\hline Lithuania (2013) & 35.8 & 16.5 & 21 & 38.4 \\
\hline Moldova (2013) & 47.3 & 28.5 & 25.7 & 42 \\
\hline Pakistan (2013) & 11.8 & 8 & 6 & 7.3 \\
\hline Russian Federation (2012) & 28.5 & n.a. & 20.1 & 43.4 \\
\hline Slovenia (2013) & 34.5 & 13.3 & 18.8 & 33.6 \\
\hline Sweden (2014) & 52.5 & 9.3 & 12.5 & 34.5 \\
\hline Ukraine (2013) & 31.5 & 12.9 & 18.8 & 41.4 \\
\hline Uzbekistan (2013) & 29.2 & 7.9 & 13.4 & 27 \\
\hline
\end{tabular}

Source: Enterprise Surveys 2019, The World Bank, http://www.enterprisesurveys.org

that unpaid work being undertaken by women as much as $\$ 10$ trillion of output per year, approximately equivalent to $13 \%$ of global GDP.

Interesting picture reveals to study women participation in firm ownership, management, and the workforce. The World Bank Enterprise Surveys 2019 give some interesting data on these indicators and provides useful information about women economic participation in the labor force and their entrepreneurship activities.

As data show in the table, women participation in firm ownership is high in countries that experience good level of human development or presented good balance of gender equality. Sweden, Russian, Moldova, Kyrgyz republic, Latvia, Lithuania, Georgia, Ukraine, Slovenia, Belarus, Bulgaria have more than $30 \%$ of women in firm ownership, and just some of them (Belarus, Georgia, Latvia) have more than $30 \%$ women in top management.

As data of different Institutions showed there are no clear correlation of gender equality with some specific factor, though countries with high level of human development an economic development showed better results in terms of gender equality. Despite the varied measuring methodology, the factors affecting gender equality are approximately the same and concern gender balance in decision-making, economic opportunities, access to health care and social protection, education. At the same time, gender equality has clear social character and implies cultural and historical aspects of the countries' development.

\section{CONCLUSION}

Thus, there is no doubt that gender balance in economic terms boost countries' development and hence, welfare. The gender equality means equal opportunities and rights for both men and women in political, economic and social spheres. This kind of approach advocates gender equality and balanced involvement of men and women at macro level and at micro level. These are the areas that policy makers have to address to create national competitive model to increase resource efficiency. Therefore, achieving greater gender equality is becoming one of the most significant factors for modern economic policy which should encourage the factors, features and elements positively contributing country's welfare. The important message to be considered is that gender equality leads to smarts economics and creates sound base for resource efficiency and innovative development which is crucial for reaching higher competitiveness in global markets. 


\section{REFERENCES:}

1. Abney David and González Laya Arancha (2018), This Is Why Women Must Play a Greater Role in the Global Economy, World Economic Forum, January, 2018. Retrieved https://www.weforum.org/agenda/2018/01/this-is-why-women-must-play-a-greaterrole-in-the-global-economy/

2. Chant, S. (2012). «The disappearing of «smart economics»? The World Development Report 2012 on Gender Equality: Some concerns about the preparatory process and the prospects for paradigm change». Global Social Policy. 12 (2): 198-218.

3. Chant, Sylvia; Sweetman, Caroline (2012). «Fixing women or fixing the world? (Smart economics), efficiency approaches, and gender equality in development». Gender \& Development. 20 (3): 517-529..

4. Cheryl. (2014). Women CEOs of the S\&P 500. http://www.catalyst.org/knowledge/women-ceos-sp-500

5. Cuberes, D., \& Teignier, M. (2016). Aggregate Effects of Gender Gaps in the Labor Market: A Quantitative Estimate. Journal of Human Capital, 10(1), 1-32.https://doi.org/10.1086/683847

6. Ferrant, G. and A. Kolev (2016), Does gender discrimination in social institutions matter for long-term growth?: Cross-country evidence. OECD Development Centre Working Papers, No. 330, OECD Publishing, Paris, https://doi.org/10.1787/5jm2hz8dgls6-en

7. Gazzola Patrizia, Sepashvili Eka, Pezzetti Roberta, (2016) Corporate Social Responsibility as a Mean To Promote Gender Equality. Business and Management Sciences International Quarterly Review, Pavia, Giugno 2016, Vol.7 Retrieved from http://riviste. paviauniversitypress.it/index.php/ea/article/viewFile/1788/1859

8. Gazzola Patrizia, Sepashvili Eka, Roberta Pezzetti (2018) How Sustainable Enterprises Can Drive The Sustainable Development, European Scientific Journal February 2018 /SPECIAL/ edition. p 26-36.

9. Global Gender Gap Report (2018), World Economic Forum, 2018, retrieved from: http://www3.weforum.org/docs/WEF_ GGGR_2018.pdf

10. Human Development Report 2018, United Nations Development Program, retrieved from: http://hdr.undp.org/en/composite/GI 11. International Monetary Fund (2018). Pursuing Womenıs Economic Empowerment https://www.imf.org/en/Publications/PolicyPapers/Issues/2018/05/31/pp053118pursuing-womens-economic-empowerment

12. McKinsey \& Company (2018), Women Matter: Time to accelerate. Ten years of insights into gender diversity 2018. Retrieved from: $\quad$ https://www.empowerwomen.org/-/media/files/un\%20women/empowerwomen/resources/hlp\%20briefs/unhlp\%20 full\%20report.pdf?la=en ..[7]

13. PwC, Women in Work Index 2018. Available at: https://www.pwc.co.uk/services/economics-policy/insights/women-in-workindex.html

14. Schwab Klaus (2017) The Fourth Industrial Revolution, World Economic Forum, 2017 retrieved from: https://www.weforum.org/ about/the-fourth-industrial-revolution-by-klaus-schwab

15. Sepashvili Eka (2019) Gender Dimension of Economic Decision-Making: Women on Top Management, Business and Management Sciences International Quarterly Review, Pavia, Giugno 2019, Vol. Retrieved from

16. Sepashvili Eka (2018)a. Innovative Clusters - A Model for Rising International Competitiveness. 5th Business Systems Laboratory International Symposium «Co-creating Responsible Futures in the Digital Age: Exploring new paths towards economic, social and environmental Sustainability». January, 2018; p.2019-22;

17. Sepashvili Eka (2018)b. Innovations and Global Economy. International Scientific-Practical Magazine, Globalization and Business \#5/2018. p. 158-163

18. Sepashvili Eka, (2016). Globalized World Economy, Innovations and National Policies for Economic Growth. Business Systems Laboratory 4th International Symposium, 'Governing Business Systems. Theories and Challenges for Systems. Thinking in Practice. Vilnius, August, 2016, pp. 174-76. Retrieved from: http://bslab-symposium.net/Vilnius.2016/BSLab-Vilnius2016-e-book_of_ Abstracts.pdf

19. Sustainable Development Goals, UN, retrieved from: https://sustainabledevelopment.un.org/?menu=1300;

20. Terjesen Siri, 2012, Women entrepreneurship: A force for growth, Trade forum ASPX, retrieved from: https://www. researchgate.net/profile/Amanda_Elam/publication/285764926_Women_entrepreneurship_A_force_for_growth/ links/56bb7cfc08ae2481ab6ac2c2/Women-entrepreneurship-A-force-for-growth.pdf

21. The Power of Parity: How Advancing Women's Equality Can Add \$12 trillion to Global Growth. (2015), McKinsey Global Institute, September, 2015, retrieved from: https://www.mckinsey.com/ /media/McKinsey/Featured\%20Insights/Employment\%20and\%20 Growth/How\%20advancing\%20womens\%20equality\%20can\%20add\%2012\%20trillion\%20to\%20global\%20growth/MGI\%20 Power\%20of\%20parity_Executive\%20summary_September\%202015.ashx;

22. World Bank (2012). 2012 Development Report. Retrieved from: https://openknowledge.worldbank.org/handle/10986/4391

23. World Bank (2012). Development Report 2012. Retrieved from https://openknowledge.worldbank.org/handle/10986/4391

24. World Economic Forum (2017), Global Gender Gap 2017, retrieved from: http://reports.weforum.org/global-gender-gapreport-2017/results-and-analysis 


\section{WOMEN FOR GLOBAL GROWTH: NEED FOR WOMEN ECONOMIC EMPOWERMENT}

\section{EKA SEPASHVILI}

Doctor of Economics

Associate Professor

Ivane Javakhishvili Tbilisi State University, Georgia

Eka.sepashvili@gmail.com

KEYWORDS: GENDER EQUALITY, WOMEN ECONOMIC EMPOWERMENT, GLOBAL GROWTH.

For citation: Sepashvili E. (2019), Women For Global Growth: Need For Women Economic Empowerment, Globalization And Business, №7, pp. 96-103. https://doi.org/10.35945/gb.2019.07.012

\section{SUMMARY}

Modern global economic development heavily relies on new technologies and unprecedented development of science creating more and more new innovative products and services. In such reality the demand for knowledge generation and improvement of resource efficiency is critical. More than ever, nations cannot afford to lose the skills, ideas and perspectives of women who are currently under-represented - in order to utilize the promise of a more prosperous and human-centric future that is to be come soon by contemporary innovations and technologies.

New challenges for national economic policies generate new approaches which have to face these modern developments and trends. One of the efficient practices represents women promotion for a very simple reason: women yet are not fully engaged into development and their economic potential has to be unlocked to benefit not only economic goals but also social inclusion and human development to meet upmost goal of every state: inclusive growth and high level of welfare. In this regard, women promotion and their empowerment are key policy challenges. Nowadays the need for women advancement in all spheres of the society is clear and generates no doubts for reasonability and rationality of such approach to economic growth.

Women contribution to the economy is vital and proved by different studies and researches. As data of different Institutions showed, there are no clear correlations of gender equality with some specific factors, though countries with high level of human development and economic development showed better results in terms of gender equality. The important message to be considered is that gender equality leads to smarts economics and creates sound base for resource efficiency and innovative development which is crucial for reaching higher competitiveness in global markets. 


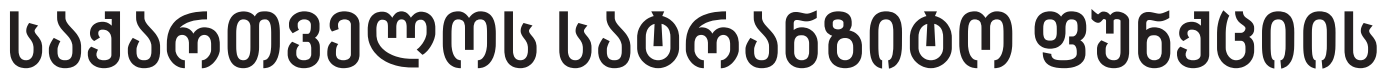

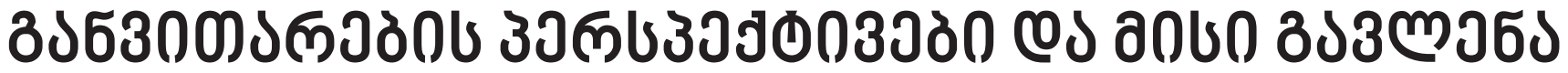

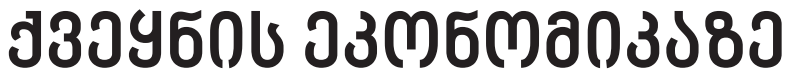

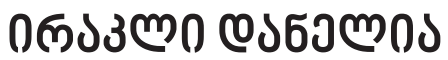

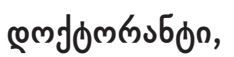

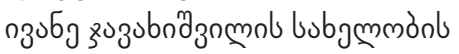

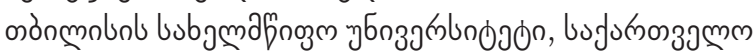

irkli.danelia@tsu.ge

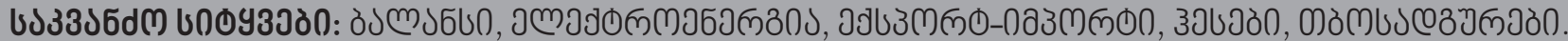

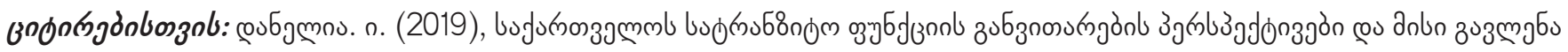

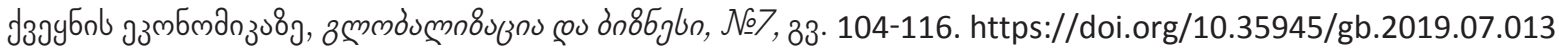

\section{əอง১ว১ตั0}

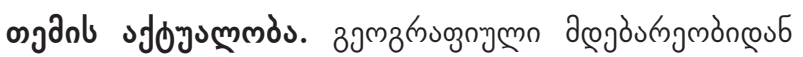

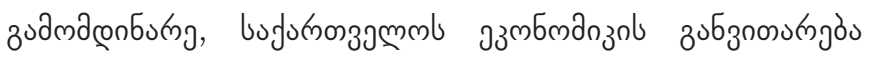

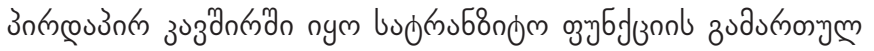

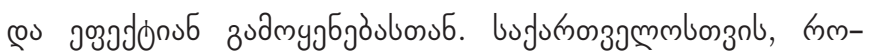

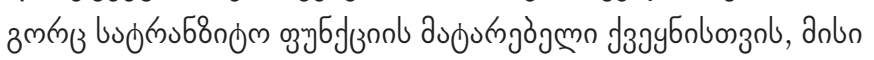

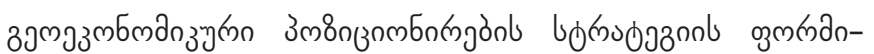

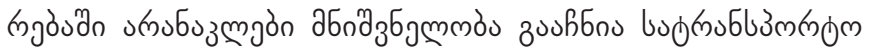

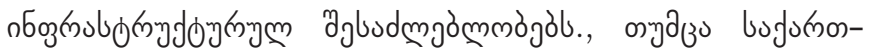

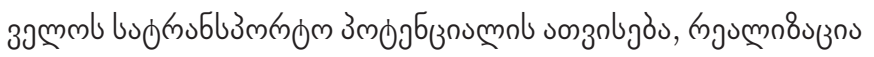

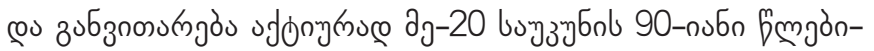

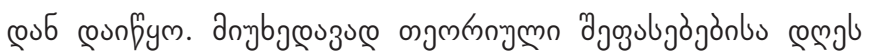

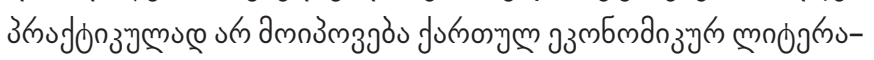

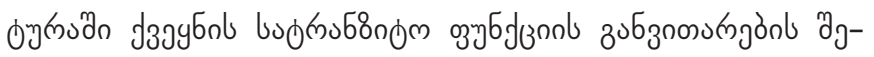

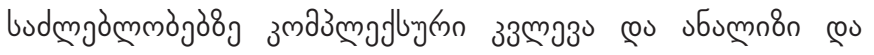

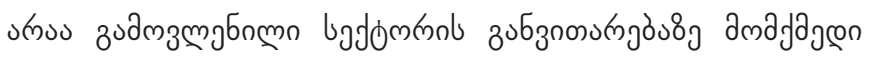

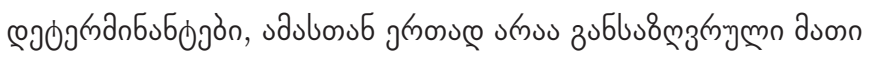

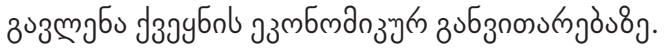

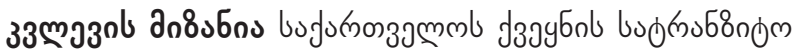

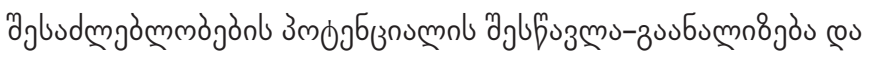

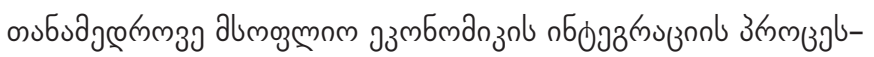

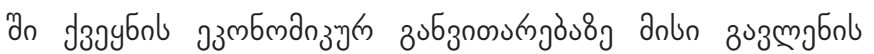

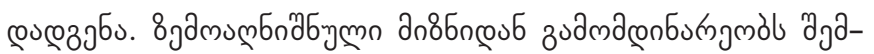

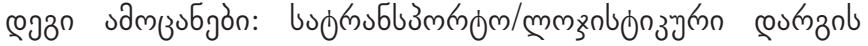

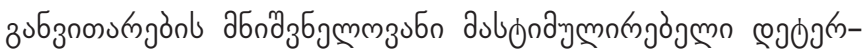

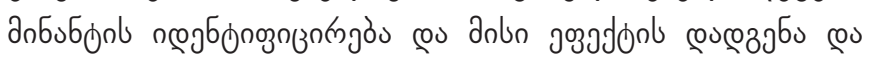

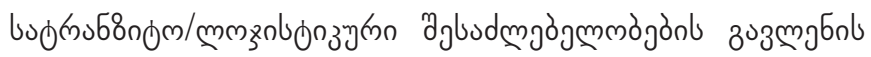

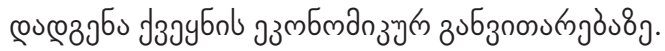

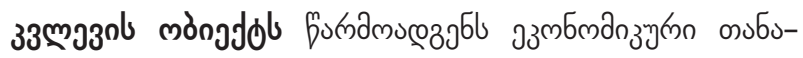

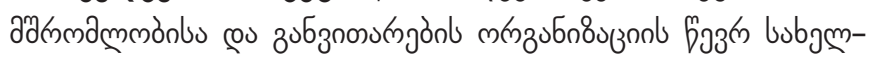

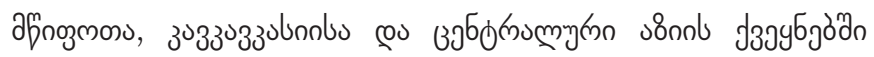

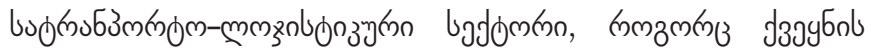

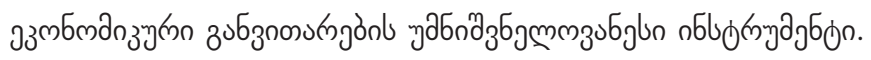

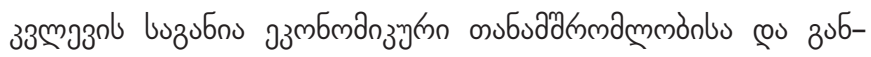

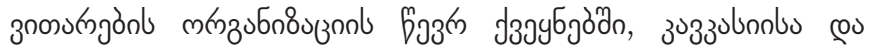

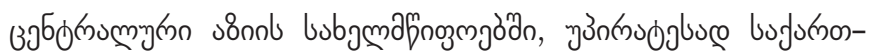

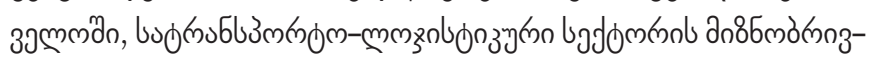

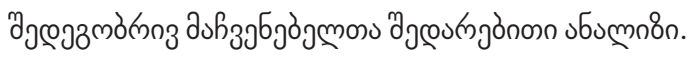

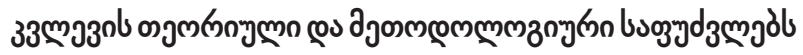

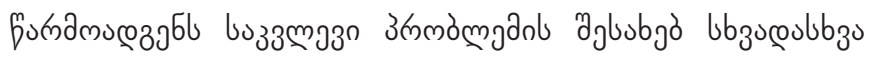

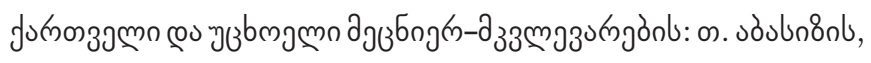

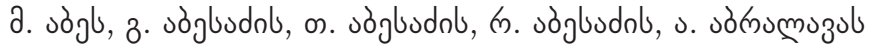

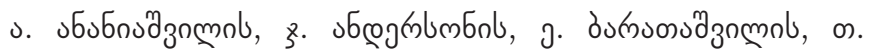

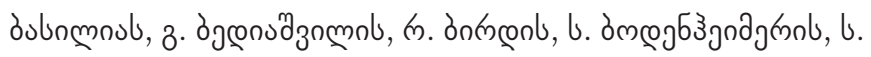

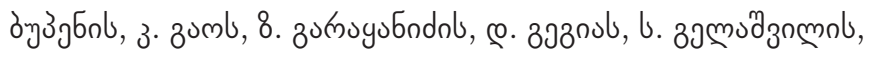

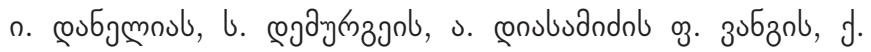

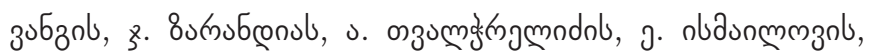




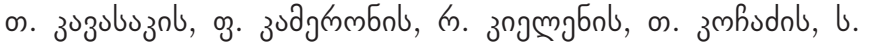

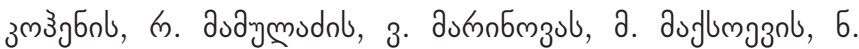

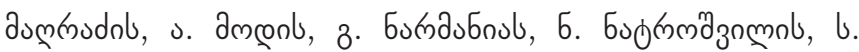

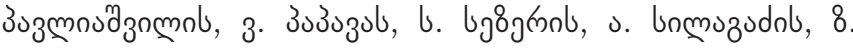

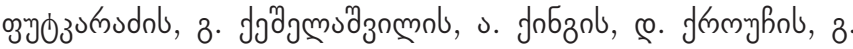

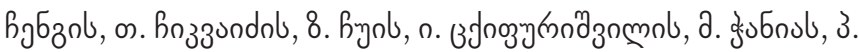

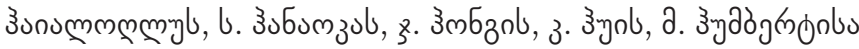
cou lb3. Бs

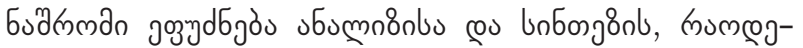

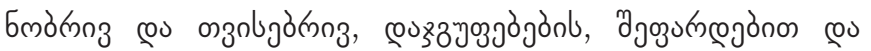

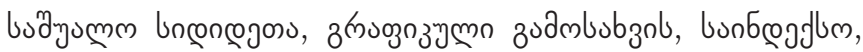

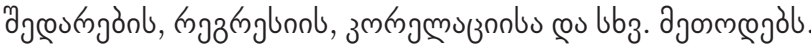

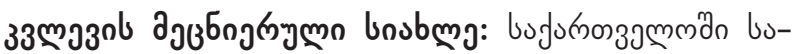

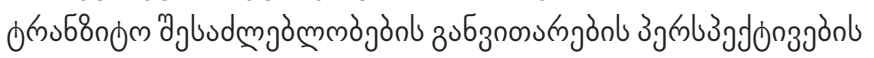

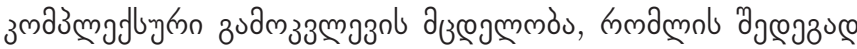

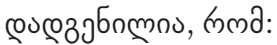

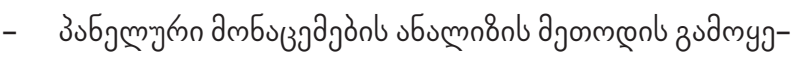

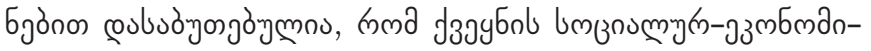

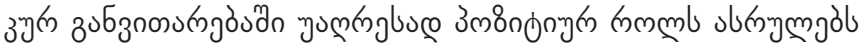

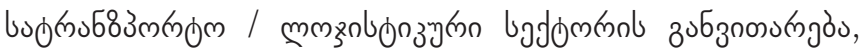

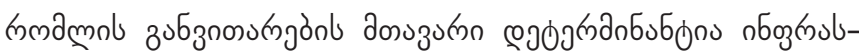

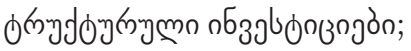

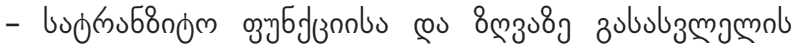

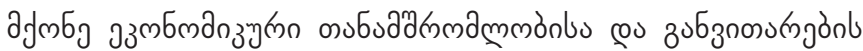

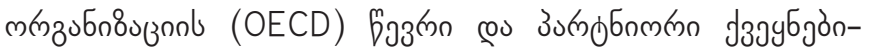

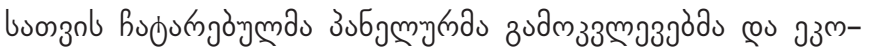

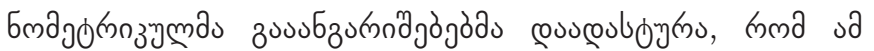

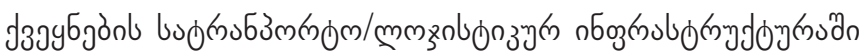

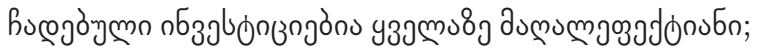

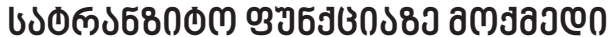

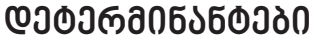

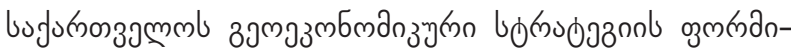

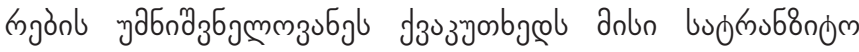
оэचб

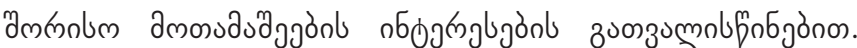

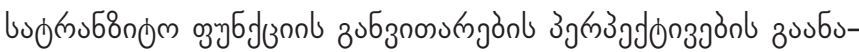

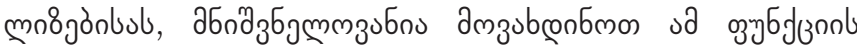

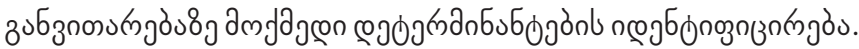

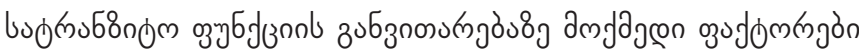

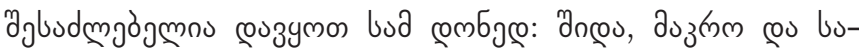

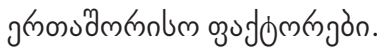

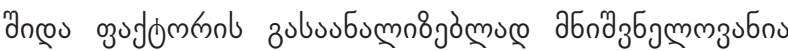

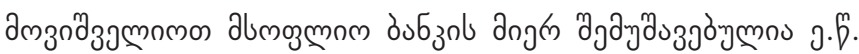

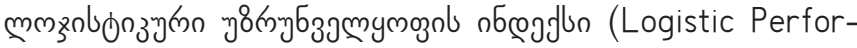

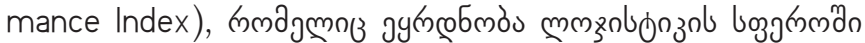

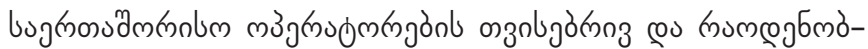

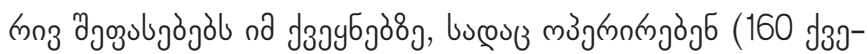
yง

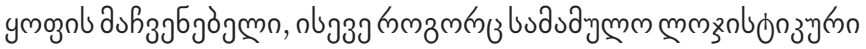

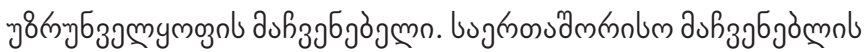

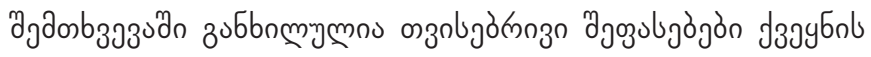

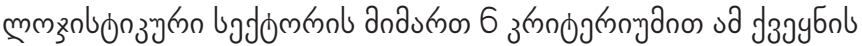

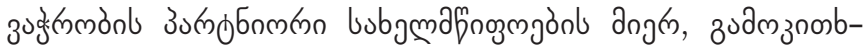

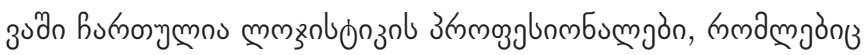

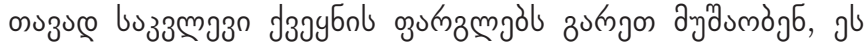

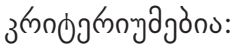

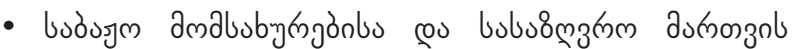

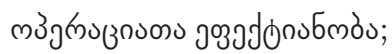

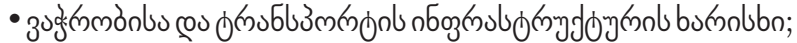

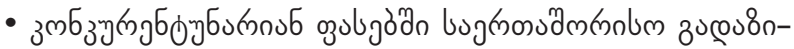

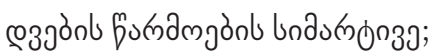

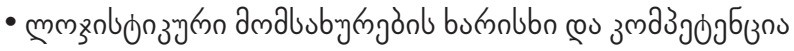
- buszomamónmm

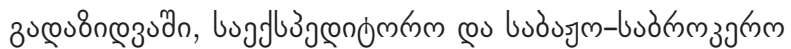
amaluby

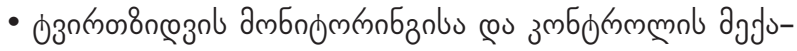

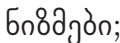

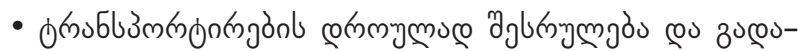

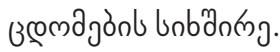

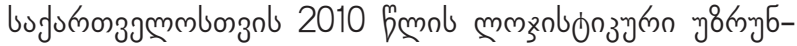

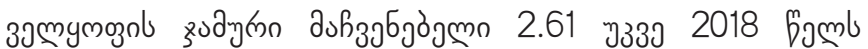

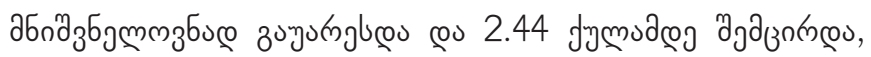

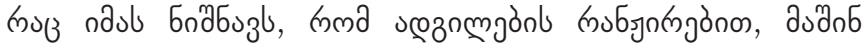

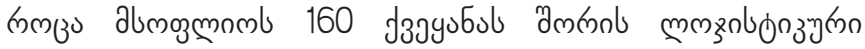

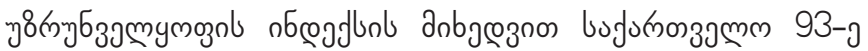

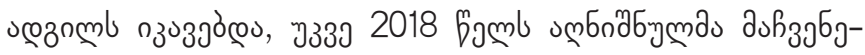

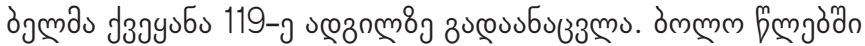

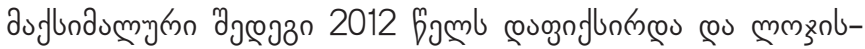

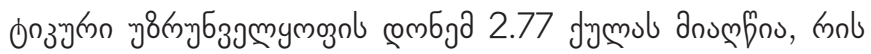

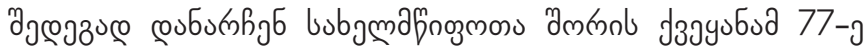

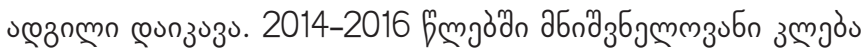

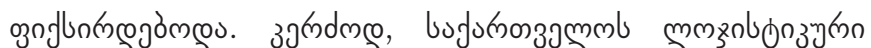

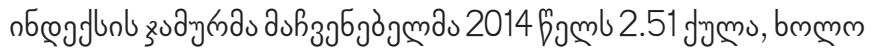

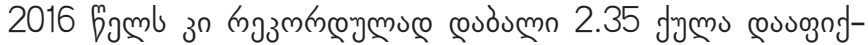

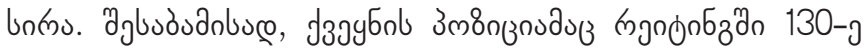

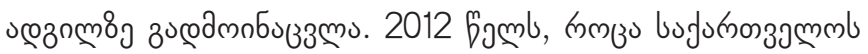

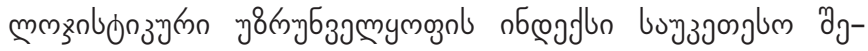

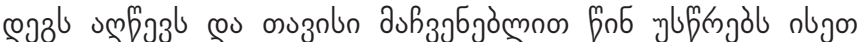

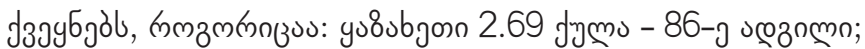

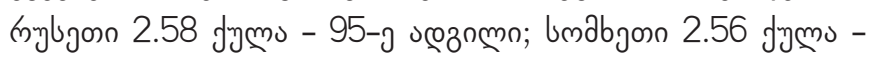

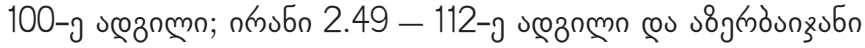




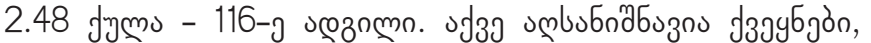

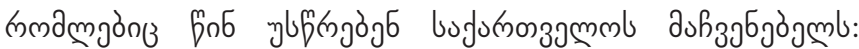

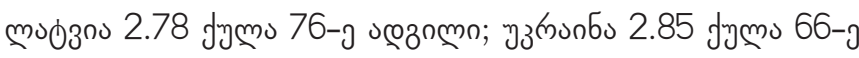

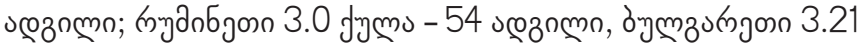

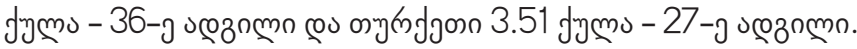

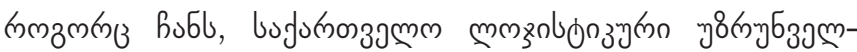

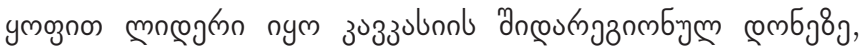

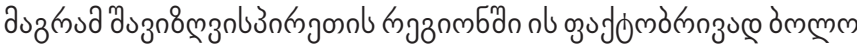

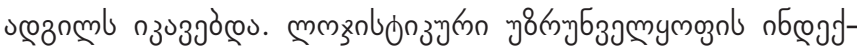

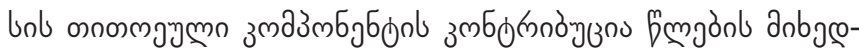

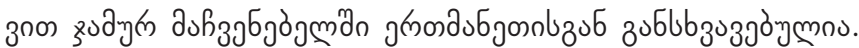

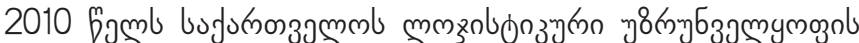

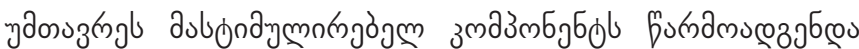
копм चूmm

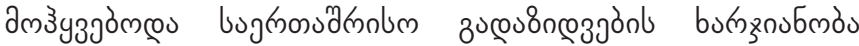

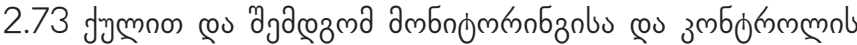

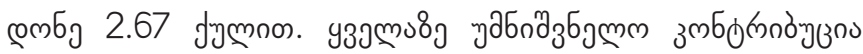

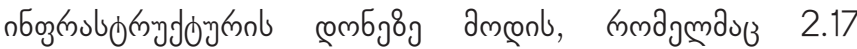

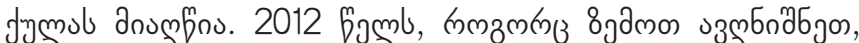

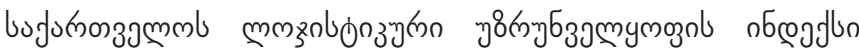

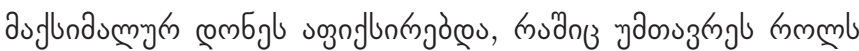

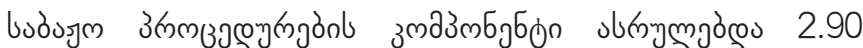
Jymmnon. au bmb a

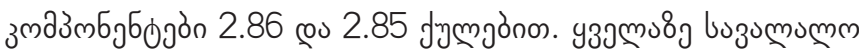

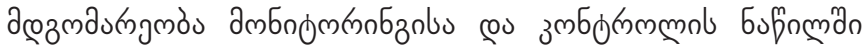

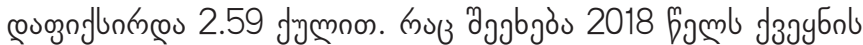

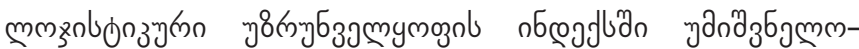

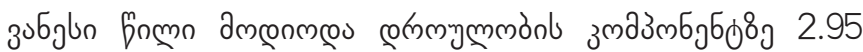

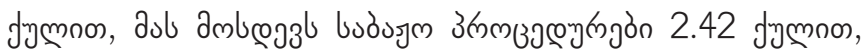

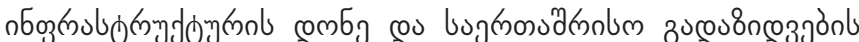

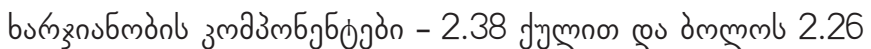

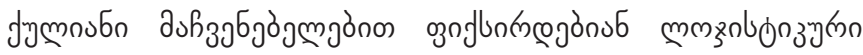

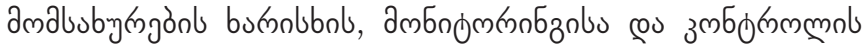

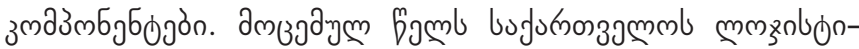

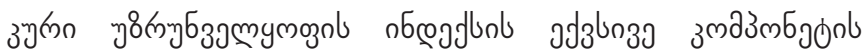

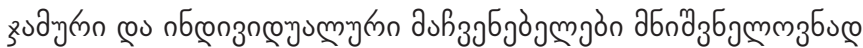

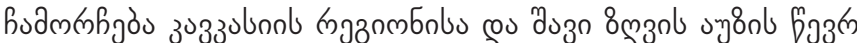

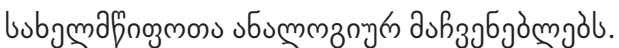

buasaymm mmznlon

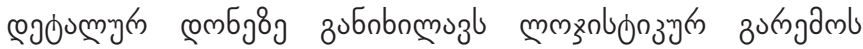

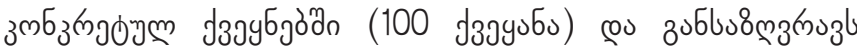

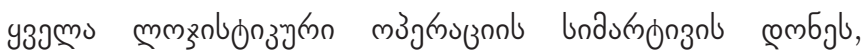

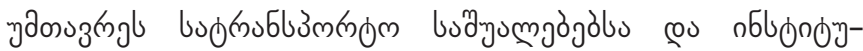

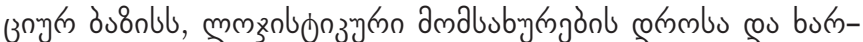

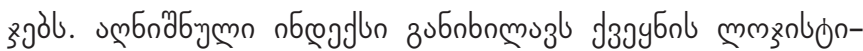

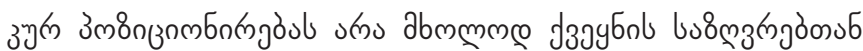

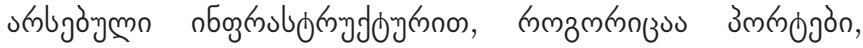

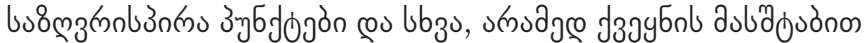

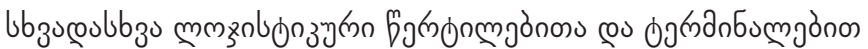

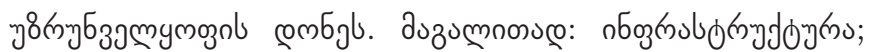

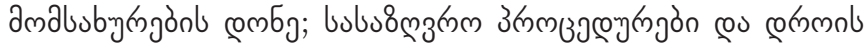

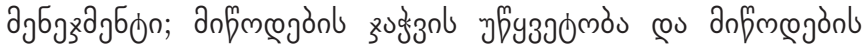
zuäzon bunagcommòs.

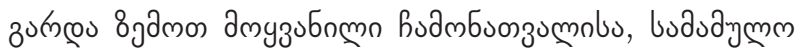

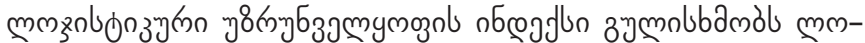

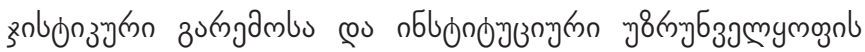

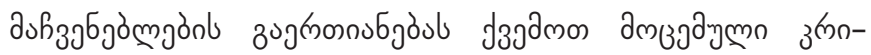

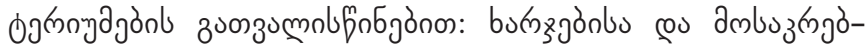

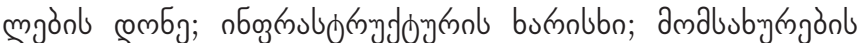

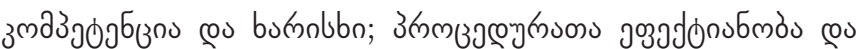
coszznotgönll fyummgòn.

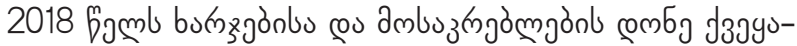

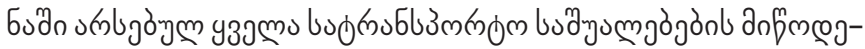

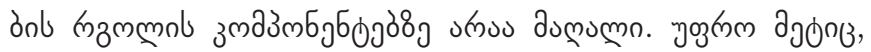

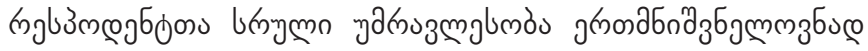

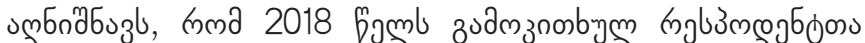

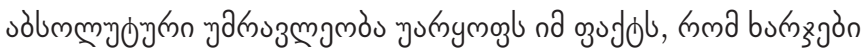

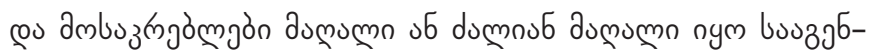

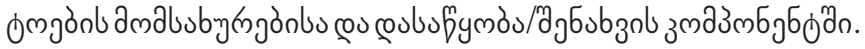

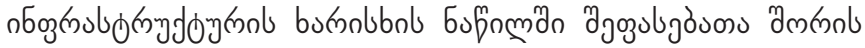

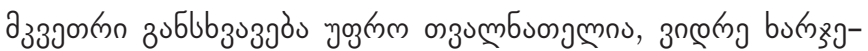

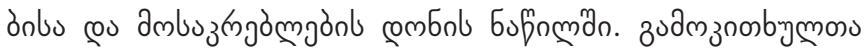

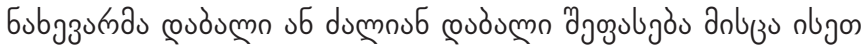

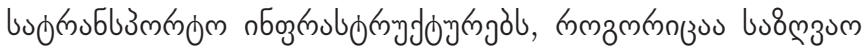

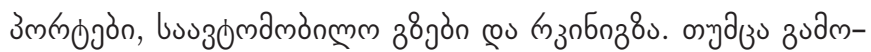
znorbygmosuzut sälmm

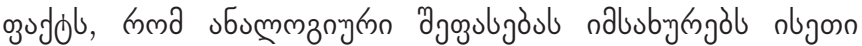

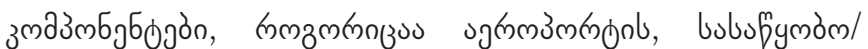

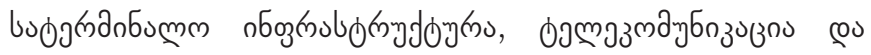

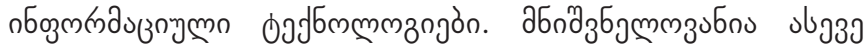

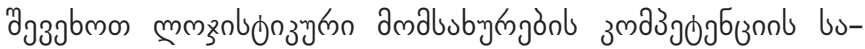

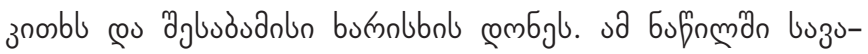

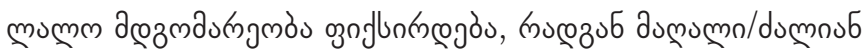

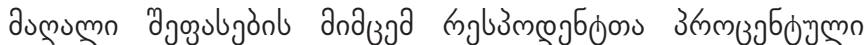

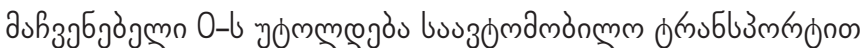

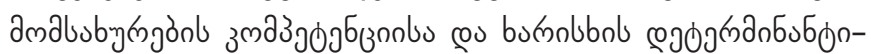

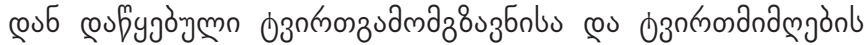

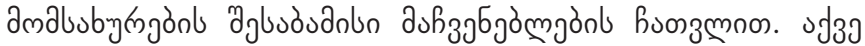

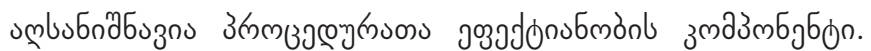

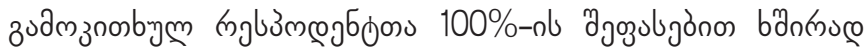

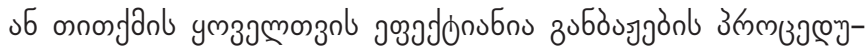
Knl, bubu8

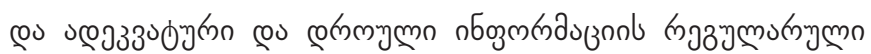

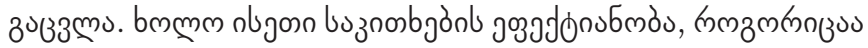




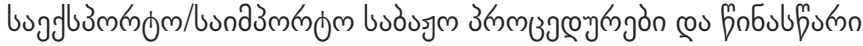

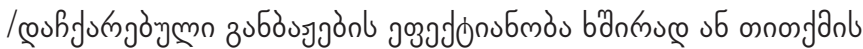

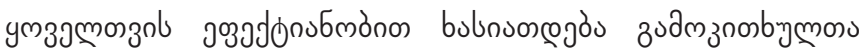

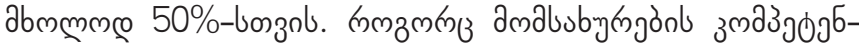

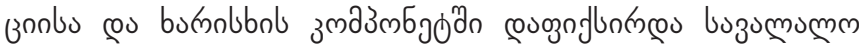

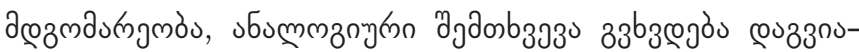

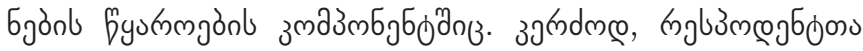

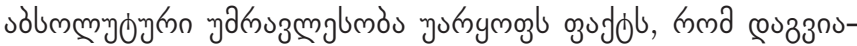

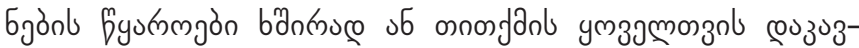

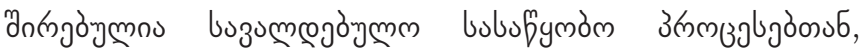
fnб

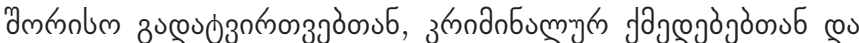

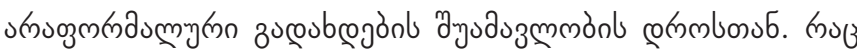

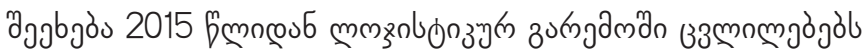

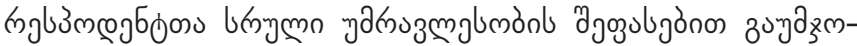

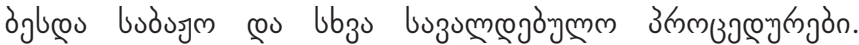

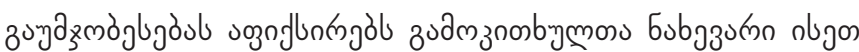

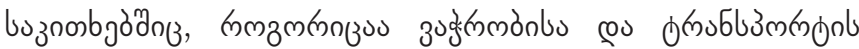
nбoुmulob

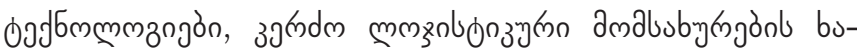

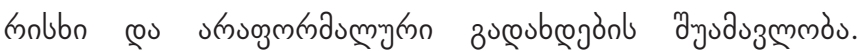

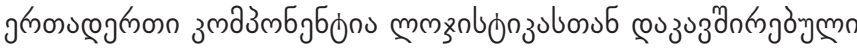

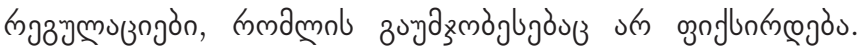

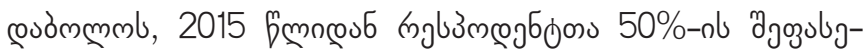

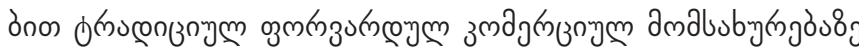

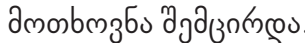

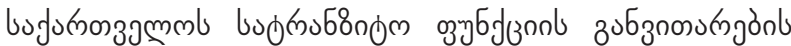

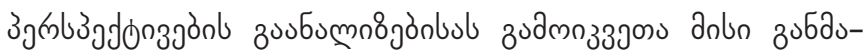

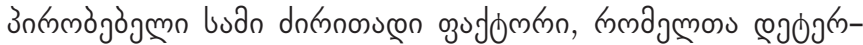

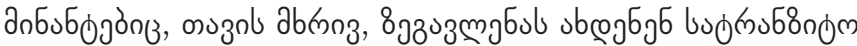

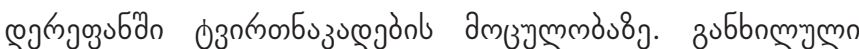

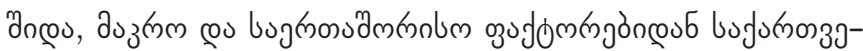

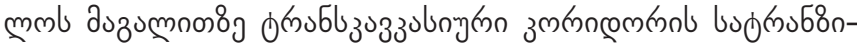

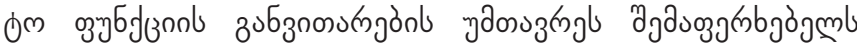

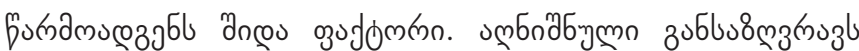

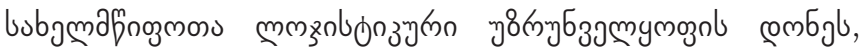

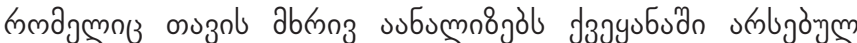

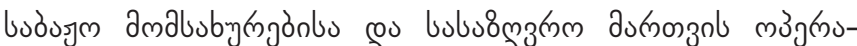

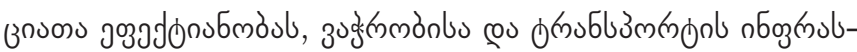

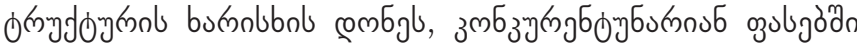

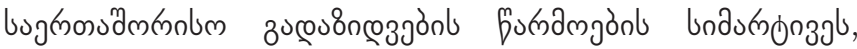

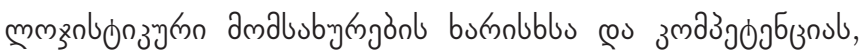

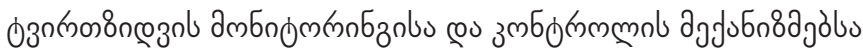

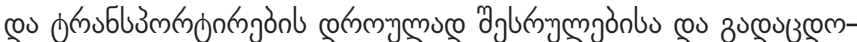
agonol bnbantringl.

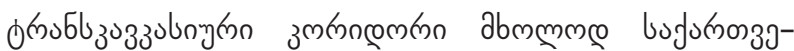

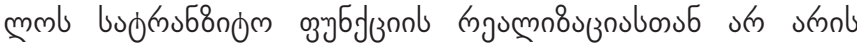

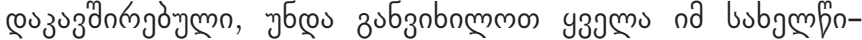

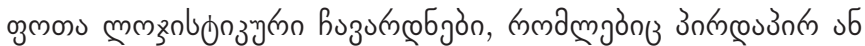

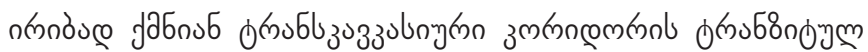

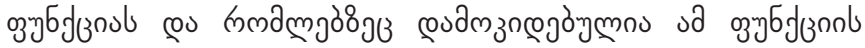

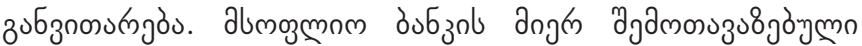

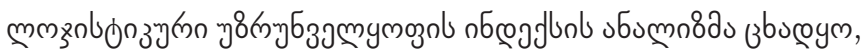

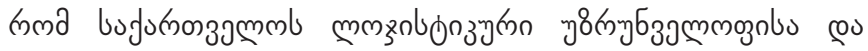

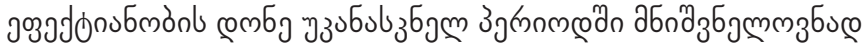

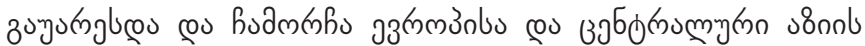

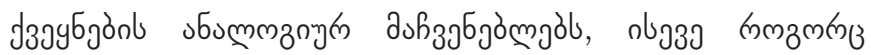

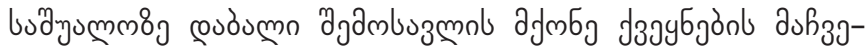

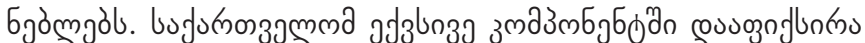

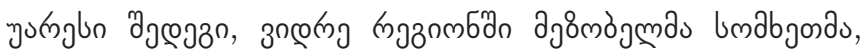

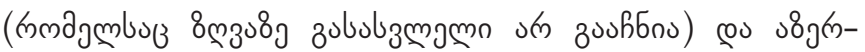

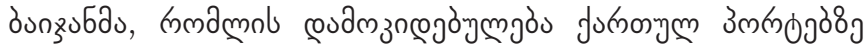

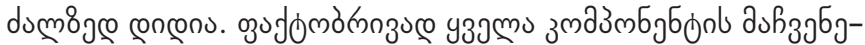

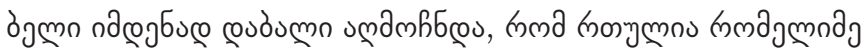

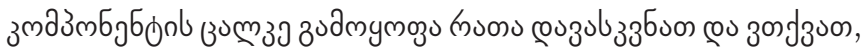

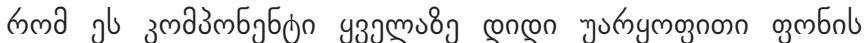

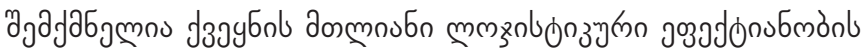

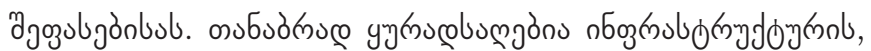

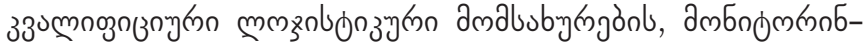

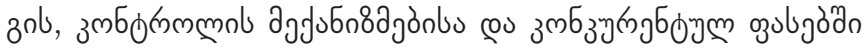

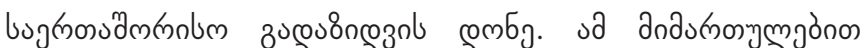
aбпаз

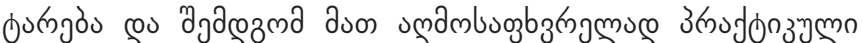

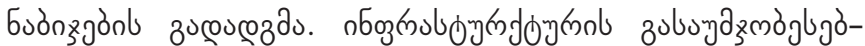

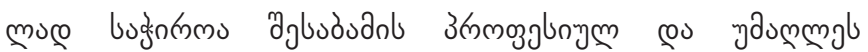

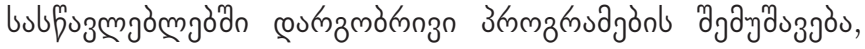

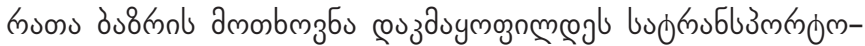

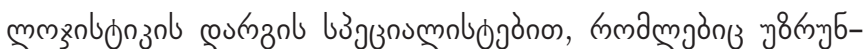

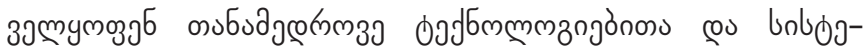

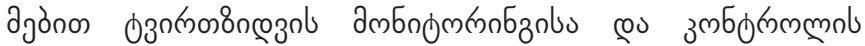

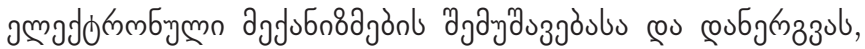

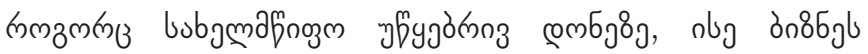

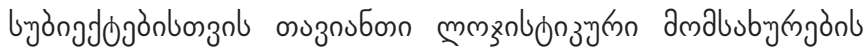

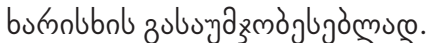

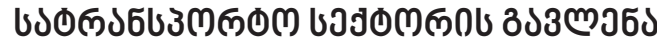

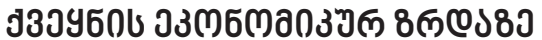

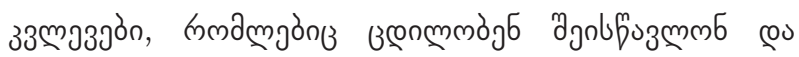

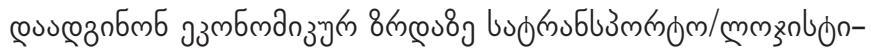

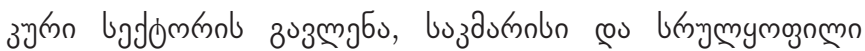

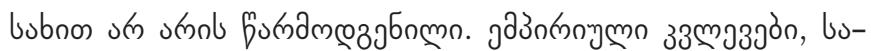

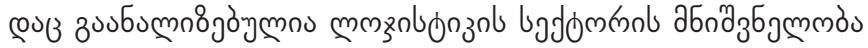




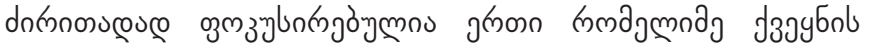

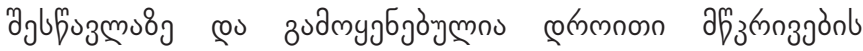

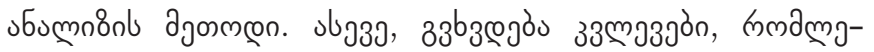

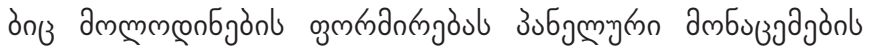

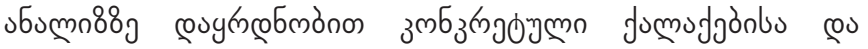

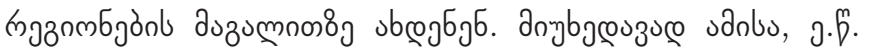

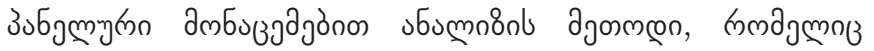

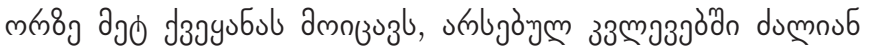

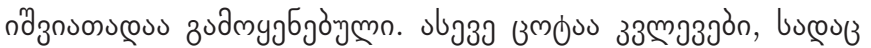

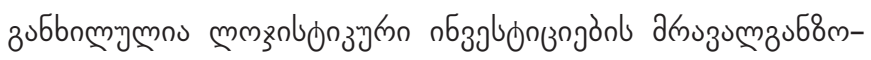

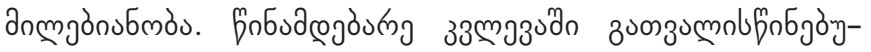

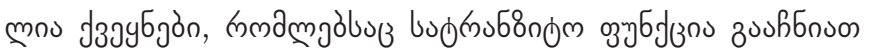

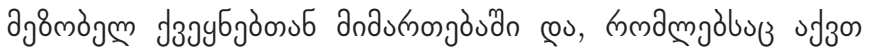

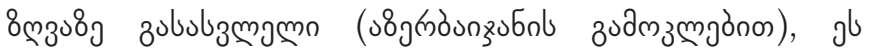

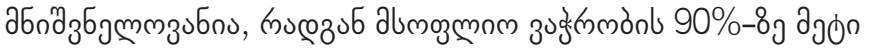

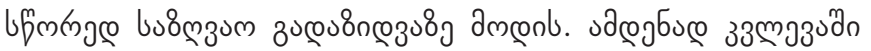

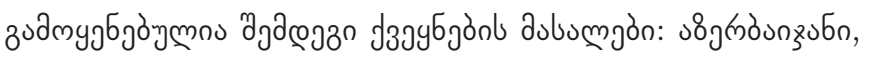

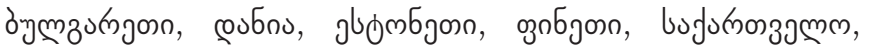

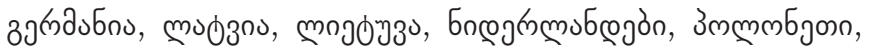

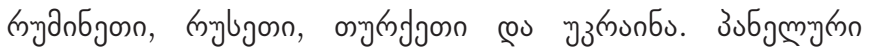

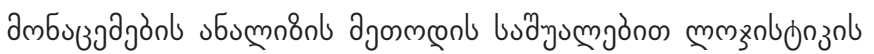

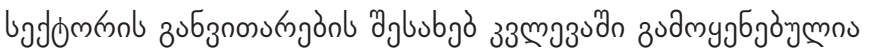

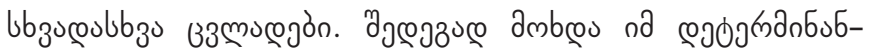

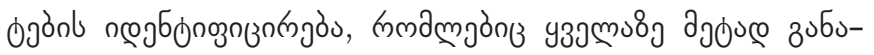

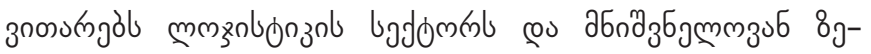

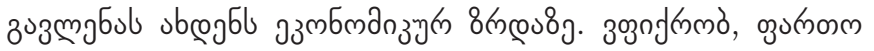

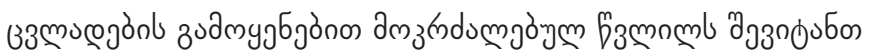

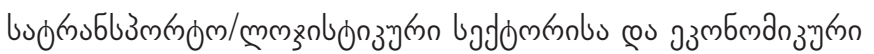

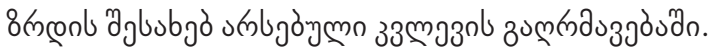

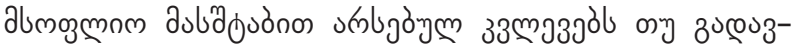

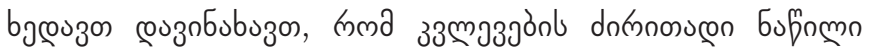

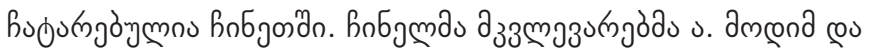

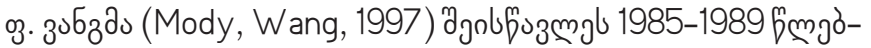

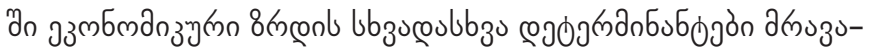

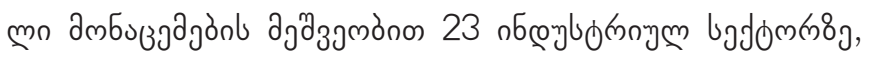

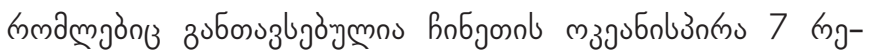

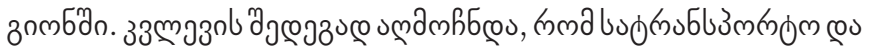

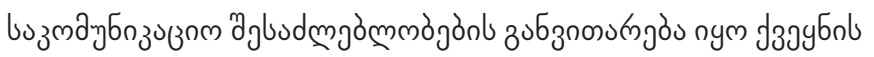

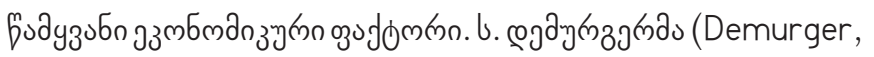

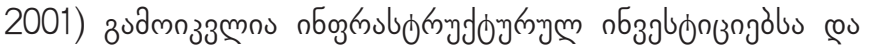

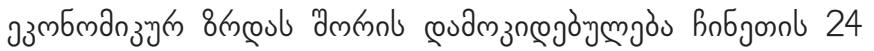

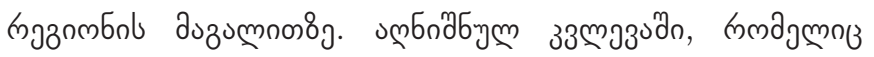

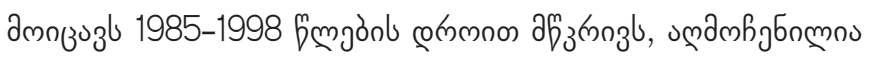

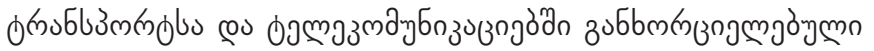

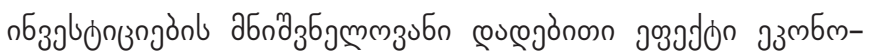

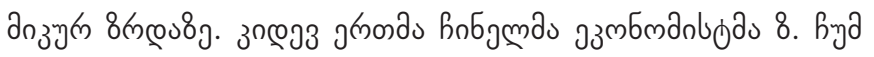

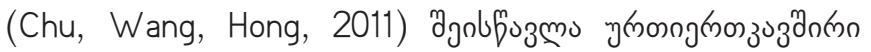

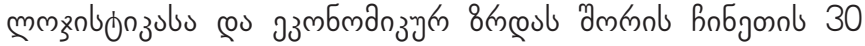

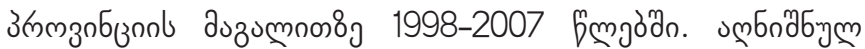

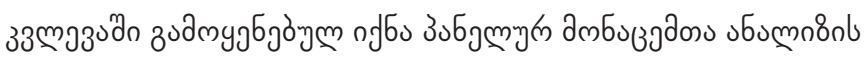

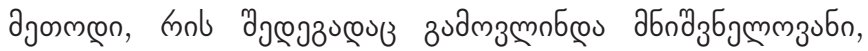

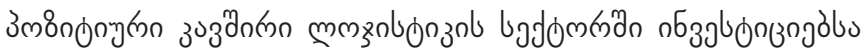

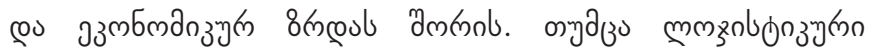

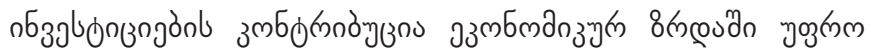

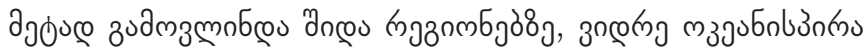

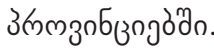

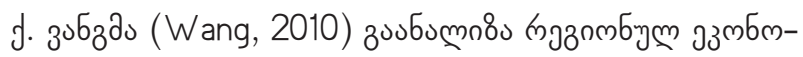

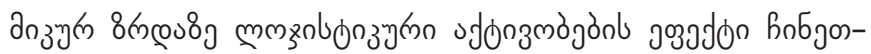

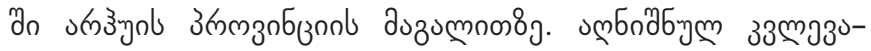

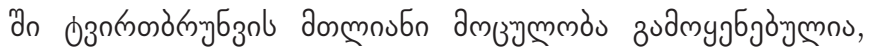

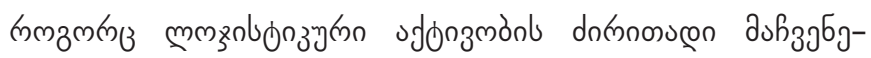

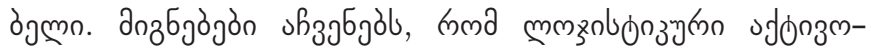

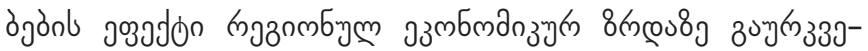

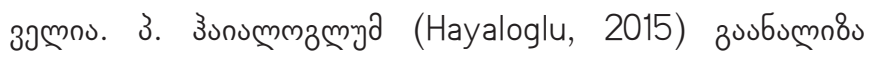

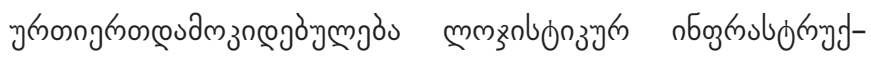

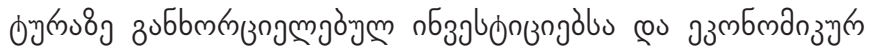

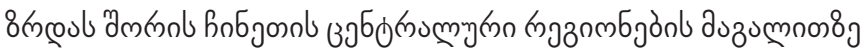

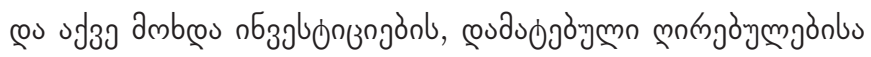

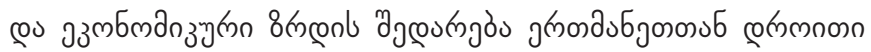

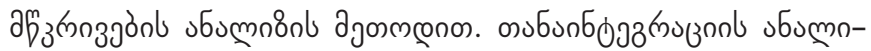

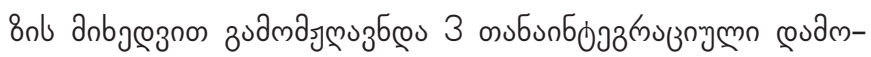

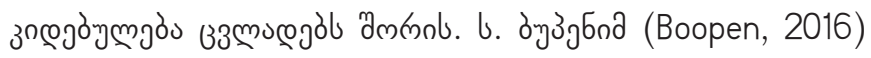

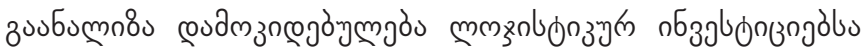

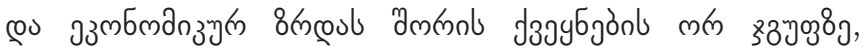

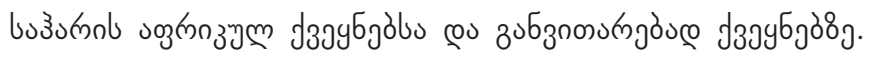

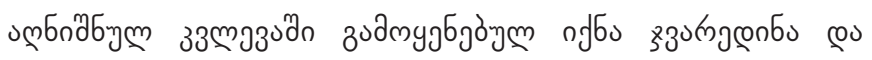

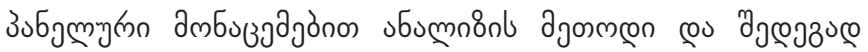

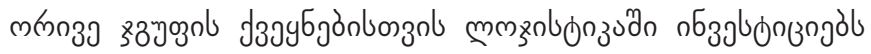

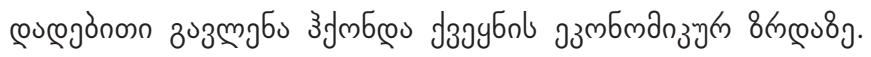

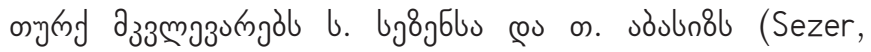

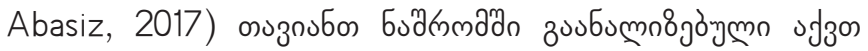

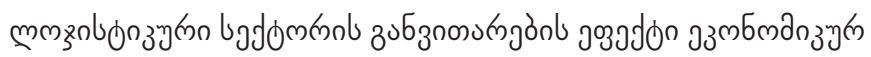

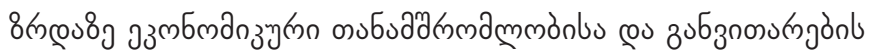

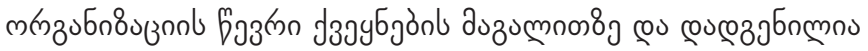

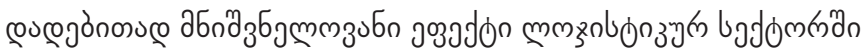

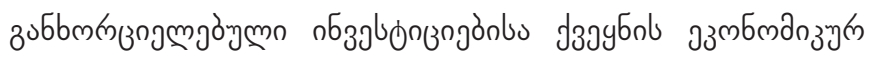

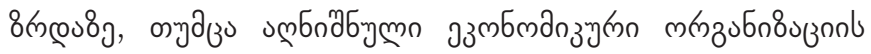

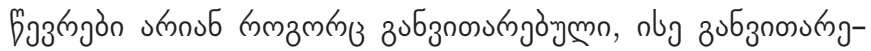

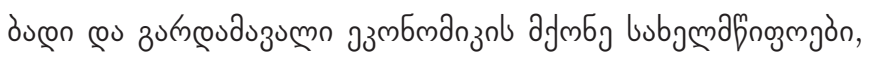

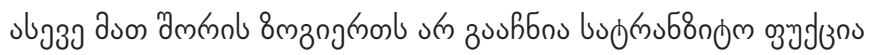

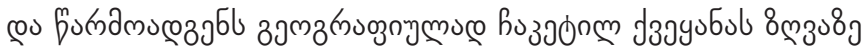

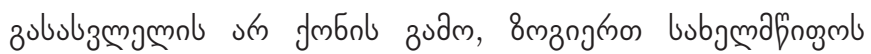

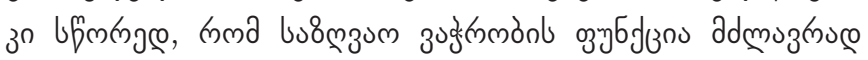

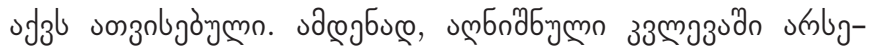




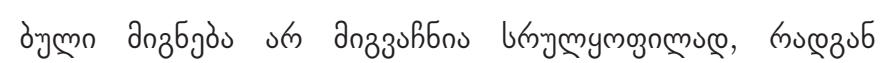
jmoszu

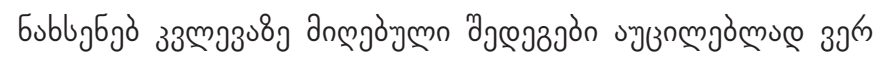

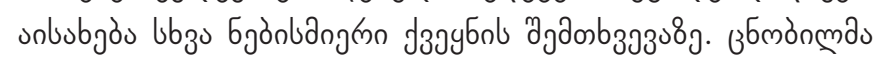

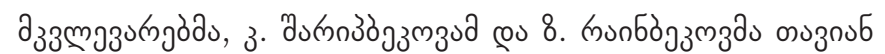

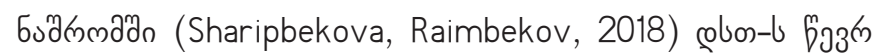

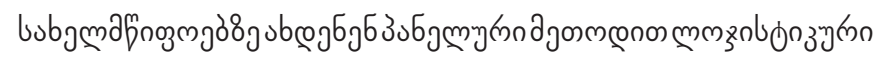

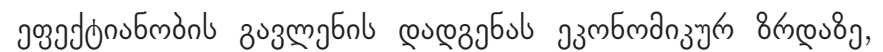

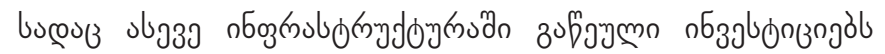

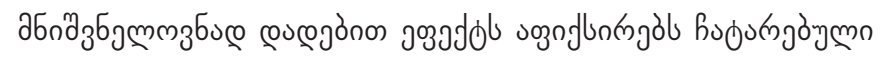

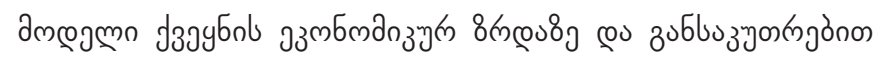

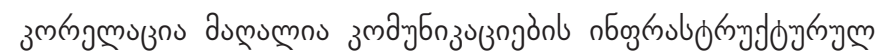

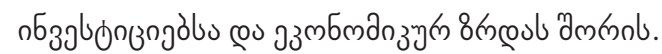

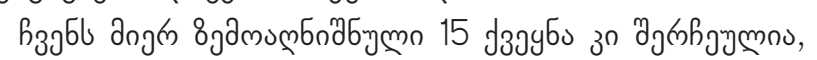

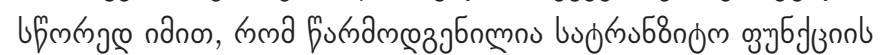

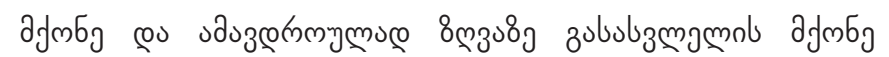

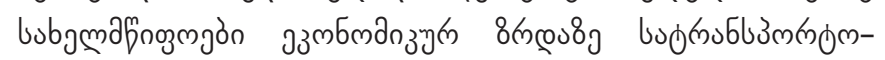

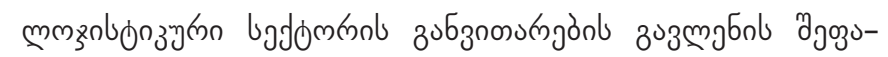

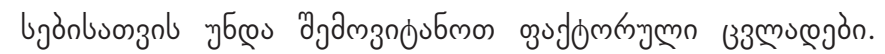

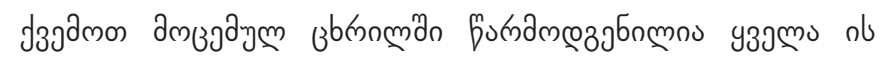

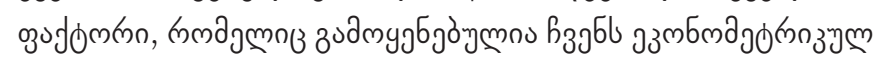

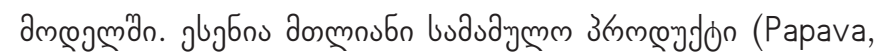

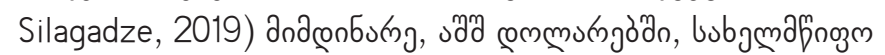

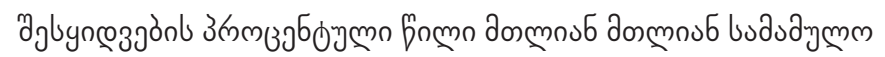

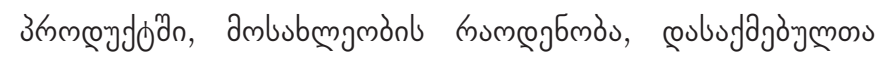

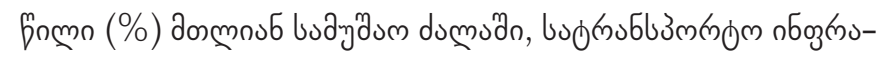

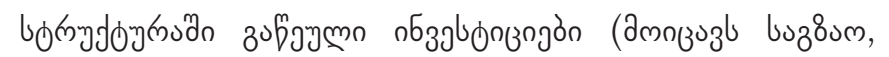

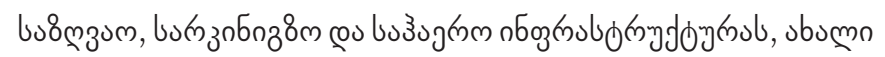

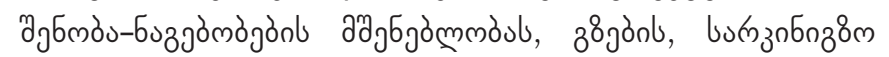

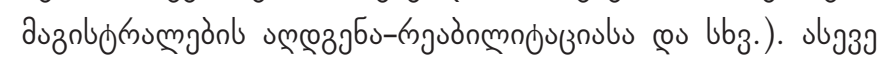

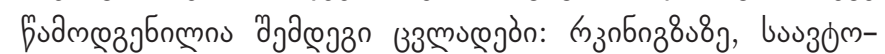

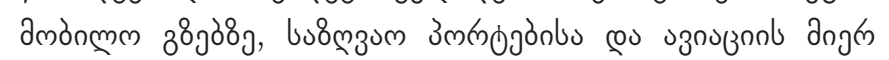
3u cus

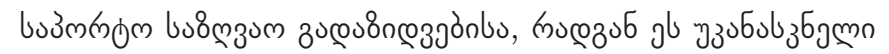

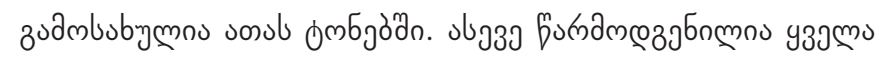

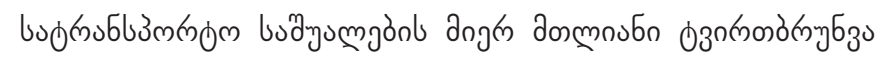

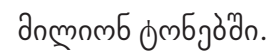

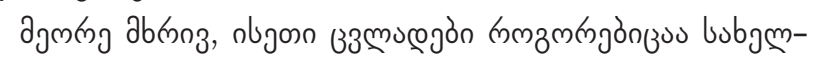

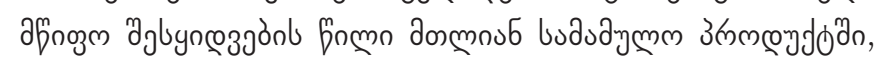

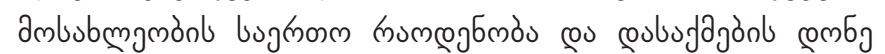

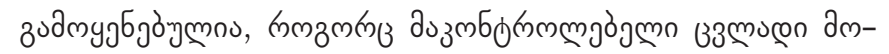

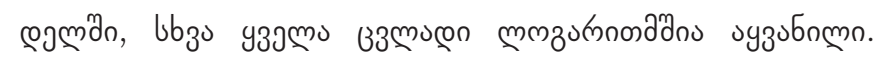

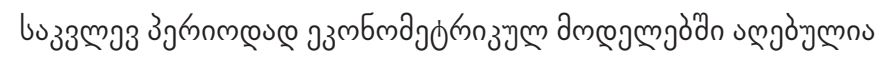

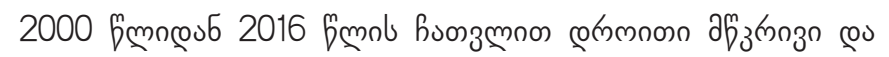

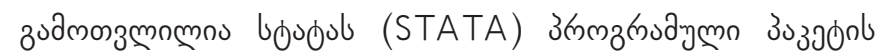
วృ

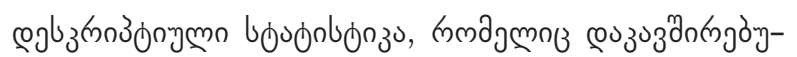

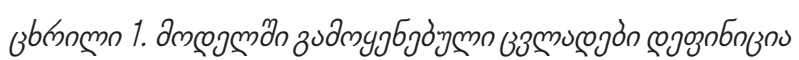

\begin{tabular}{|c|c|c|}
\hline oुufomm no & osudom nnol zublus & fyotrm \\
\hline LGDP & 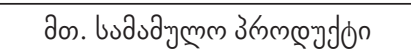 & alımogmom du $\sigma_{3 n}$ \\
\hline GFCE & 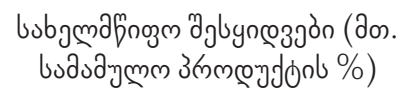 & dbmogmnn du $\sigma_{3}$ \\
\hline POP & 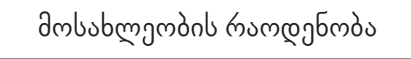 & abmogmmon dub \\
\hline EMP & coubutajàymmos comб (\%) & abmogmnn du $\sigma_{30}$ \\
\hline LTINV & 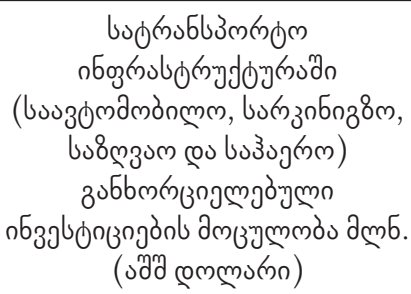 & 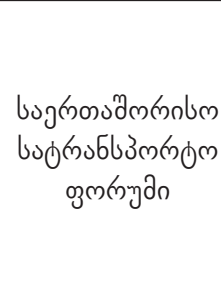 \\
\hline LRAIL & 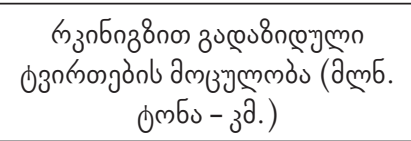 & abmogmnn du $\sigma_{z}$ \\
\hline LROAD & 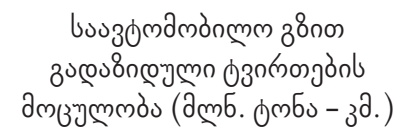 & 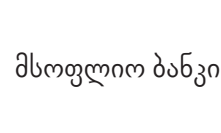 \\
\hline LAIR & 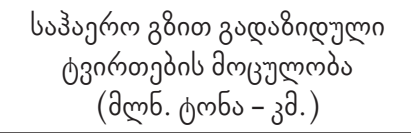 & 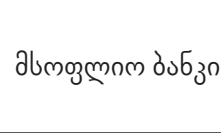 \\
\hline LPORT & 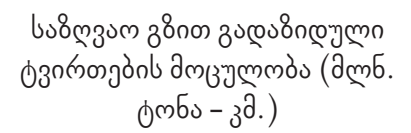 & abmogmnn du $\sigma_{3}$ \\
\hline LTFREIGHT & 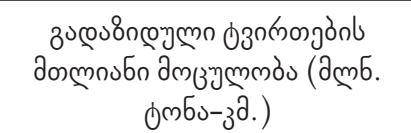 & dbmogmnn du $\sigma_{3}$ \\
\hline
\end{tabular}

fyurnm: alumogmon bubzo www. worldbank.org/; bugnoon-

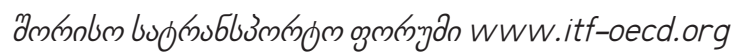

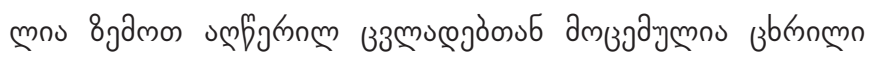

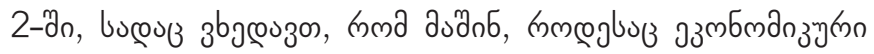

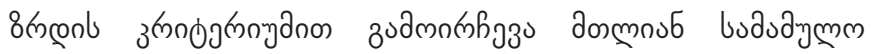

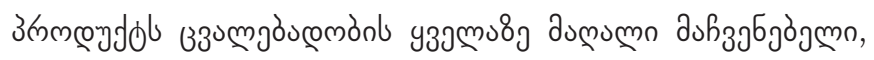

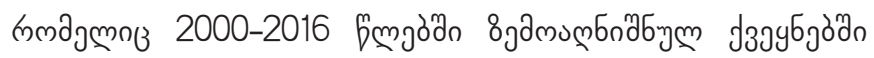

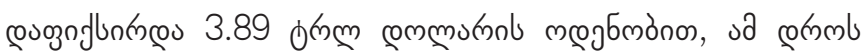

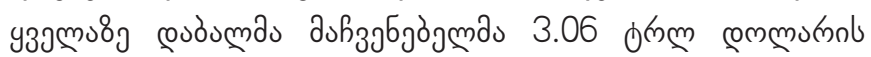

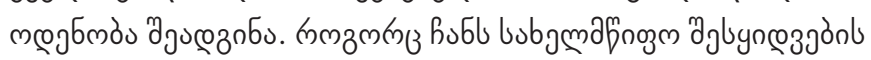

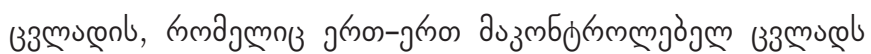

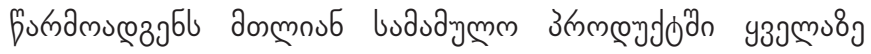

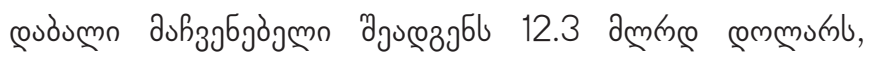

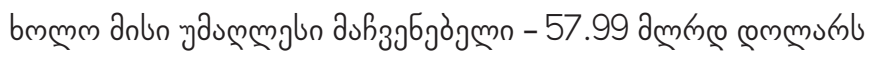

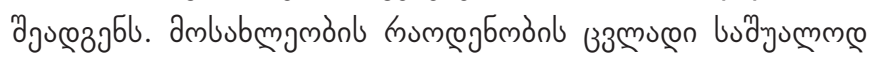

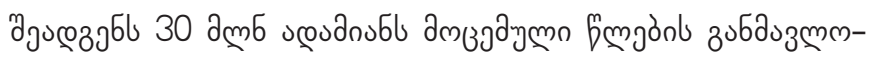

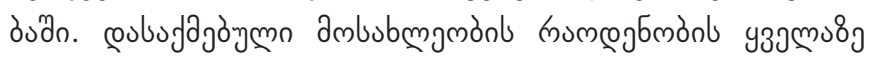

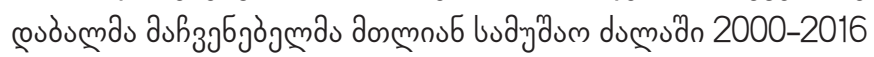




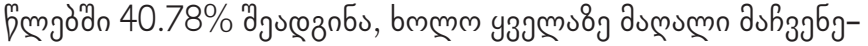

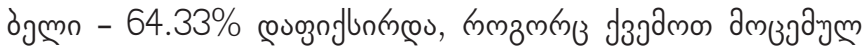

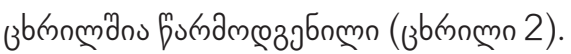

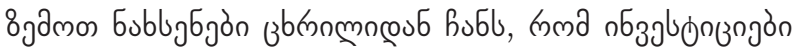

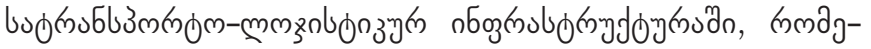

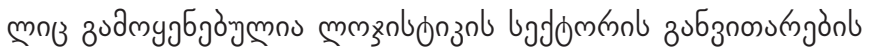

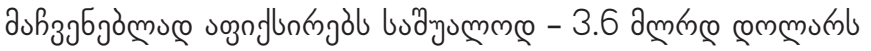

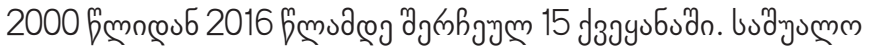

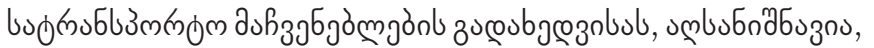

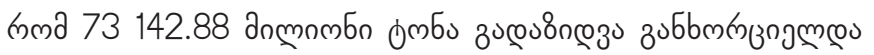

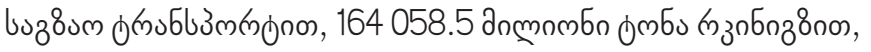

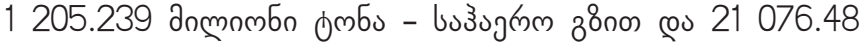

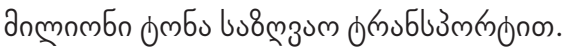

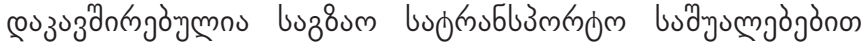

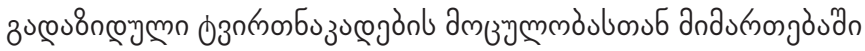

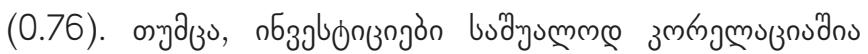

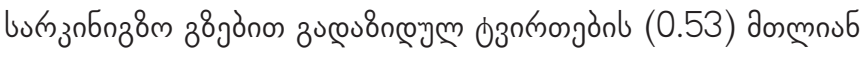

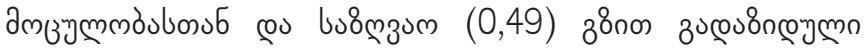

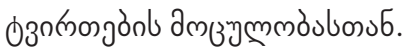

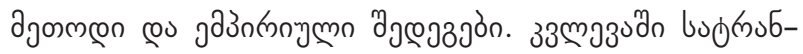

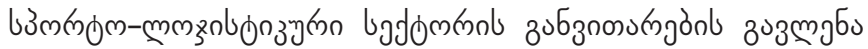

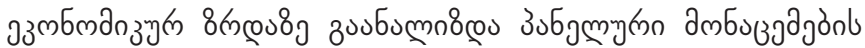

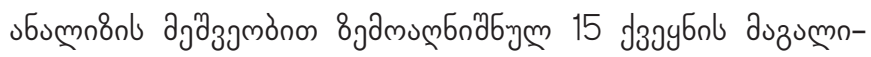

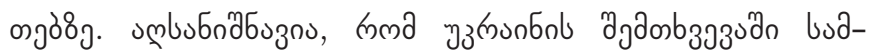

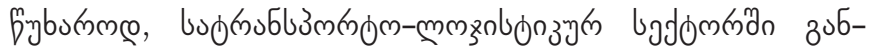

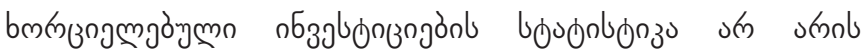

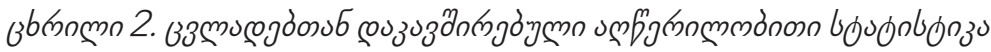

\begin{tabular}{|r|rccrr|}
\hline Variable & Obs & Mean & Std. Dev. & \multicolumn{1}{c|}{ Min } & \multicolumn{1}{c|}{ Max } \\
\hline GDP & 270 & $4.72 \mathrm{e}+11$ & $8.31 \mathrm{e}+11$ & $3.06 \mathrm{e}+09$ & $3.89 \mathrm{e}+12$ \\
GCF & 270 & 24.16702 & 5.662393 & 12.37117 & 57.99043 \\
GGFCE & 270 & 18.40937 & 3.935351 & 8.495112 & 27.93502 \\
POP & 270 & $3.05 \mathrm{e}+07$ & $3.94 \mathrm{e}+07$ & 1314545 & $1.47 \mathrm{e}+08$ \\
EMP & 270 & 53.74861 & 5.535251 & 40.785 & 64.337 \\
\hline INFINVESTeur & 252 & $3.65 \mathrm{e}+09$ & $5.16 \mathrm{e}+09$ & $-7.83 \mathrm{e}+07$ & $2.07 \mathrm{e}+10$ \\
RAILWAY & 270 & 164058.5 & 496995.7 & 1696 & 2493428 \\
Road & 270 & 73142.88 & 98529.01 & 475 & 348559 \\
Port & 270 & 21076.48 & 34684.02 & 2 & 133093 \\
AIR & 270 & 1205.239 & 2313.622 & .003 & 10187.72 \\
\hline TOTAL FREIGHT & 270 & 335006.6 & 833414.1 & 3646 & 4076419 \\
TRADE & 270 & 93.49827 & 29.91048 & 42.00053 & 170.4283 \\
\hline
\end{tabular}

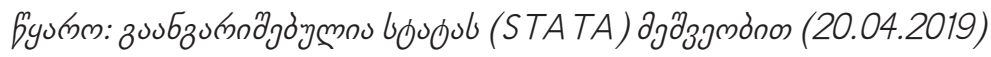

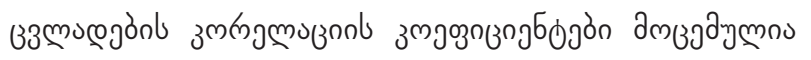

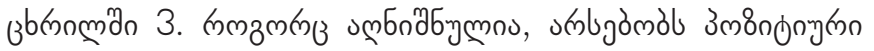

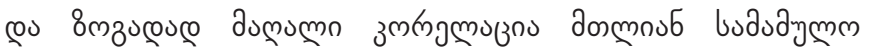

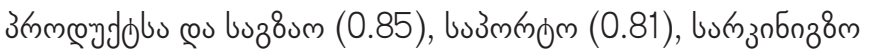

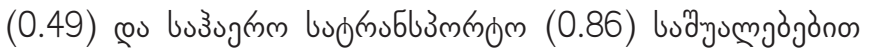

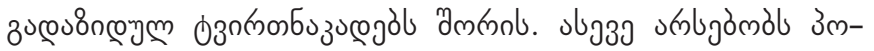

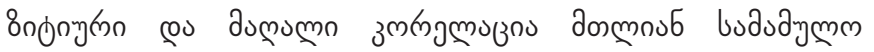

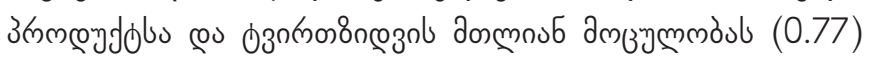

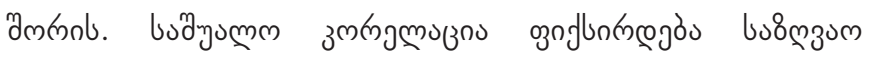

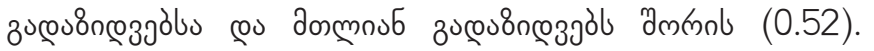

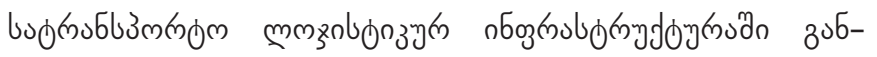

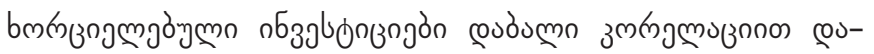

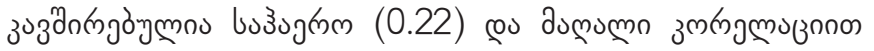

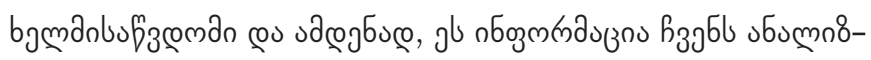

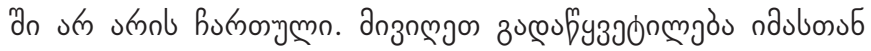

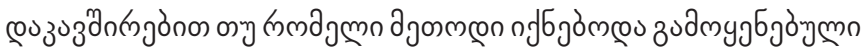

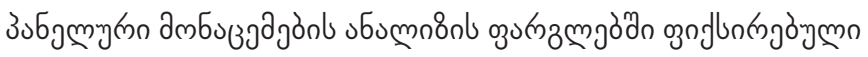

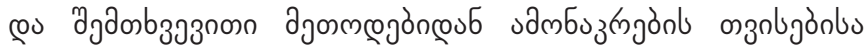

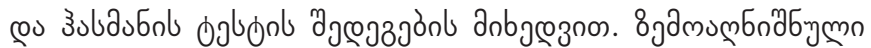

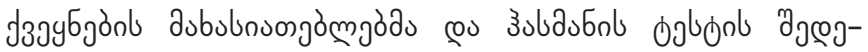

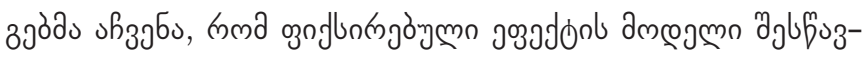

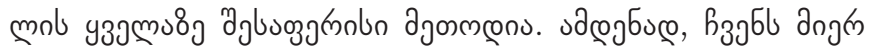

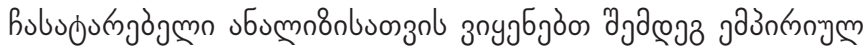
ancogmb:

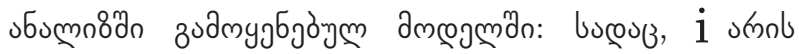

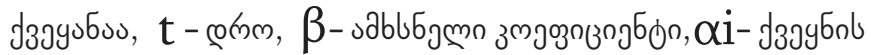

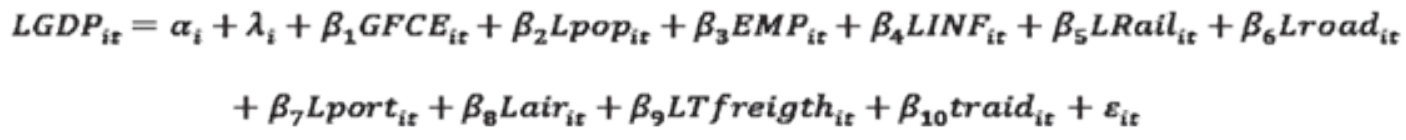




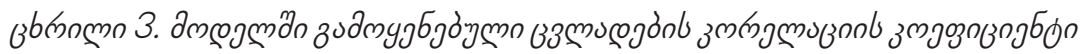

\begin{tabular}{|c|c|c|c|c|c|c|c|c|c|c|c|c|}
\hline & LGDP & GCF & GGFCE & LPOP & EMP & Linfrln & Lrailw & Lroad & Lport & LAIR & LTFREI T & TRADE \\
\hline LGDP & 1.0000 & & & & & & & & & & & \\
\hline GCF & -0.4183 & 1.0000 & & & & & & & & & & \\
\hline GGFCE & 0.2514 & -0.3616 & 1.0000 & & & & & & & & & \\
\hline LPOP & 0.7960 & -0.2526 & -0.1909 & 1.0000 & & & & & & & & \\
\hline EMP & 0.1121 & -0.0312 & 0.3142 & -0.1201 & 1.0000 & & & & & & & \\
\hline Linfrln & 0.8531 & -0.2739 & -0.0301 & 0.8472 & 0.1634 & 1.0000 & & & & & & \\
\hline Lrailw & 0.4957 & -0.1628 & -0.1545 & 0.6794 & 0.0103 & 0.5341 & 1.0000 & & & & & \\
\hline Lroad & 0.8564 & -0.3329 & -0.0448 & 0.8148 & -0.2097 & 0.7633 & 0.6238 & 1.0000 & & & & \\
\hline Lport & 0.8120 & -0.3278 & 0.3870 & 0.5299 & -0.0203 & 0.4988 & 0.3011 & 0.6245 & 1.0000 & & & \\
\hline LAIR & 0.8621 & -0.2965 & 0.2281 & 0.7298 & 0.2448 & 0.7985 & 0.4358 & 0.6942 & 0.6569 & 1.0000 & & \\
\hline LTFREIGHT & 0.7731 & -0.2886 & -0.1682 & 0.8891 & -0.0210 & 0.7614 & 0.8638 & 0.8667 & 0.5216 & 0.7022 & 1.0000 & \\
\hline
\end{tabular}

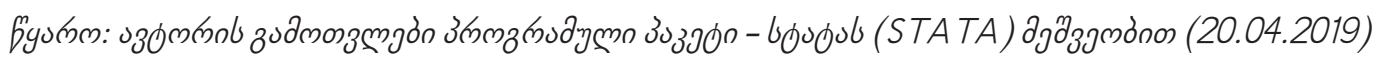

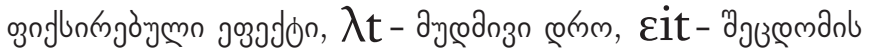

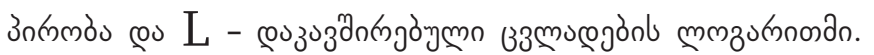

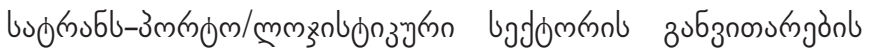
дu

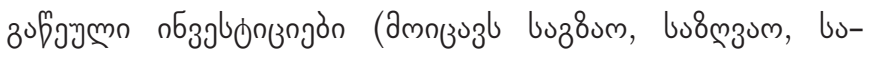

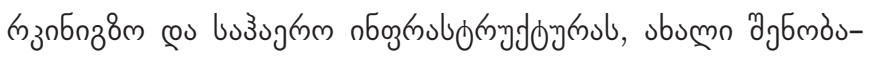

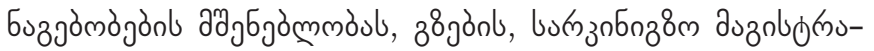
mgàn uल

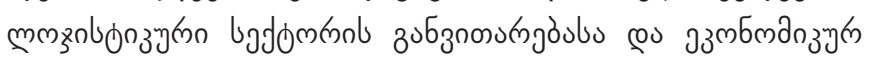

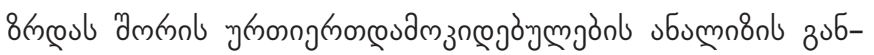

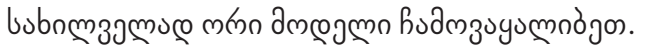

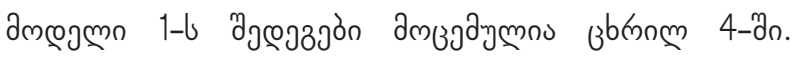

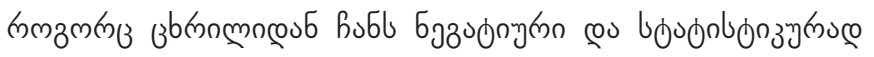

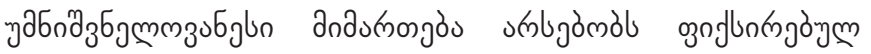

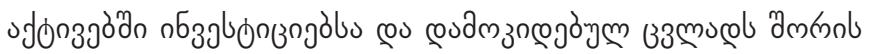

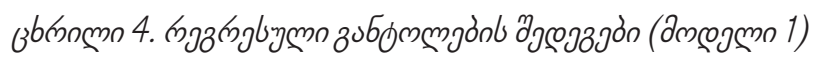

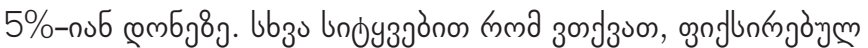

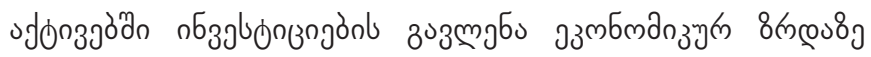

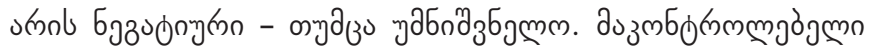

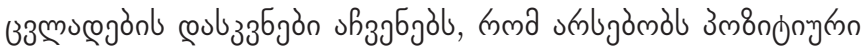

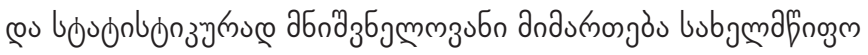

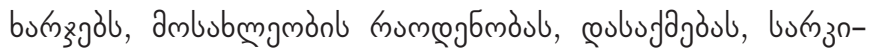

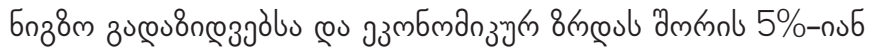

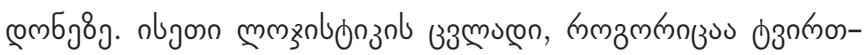

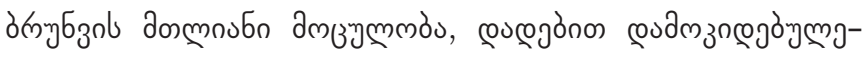

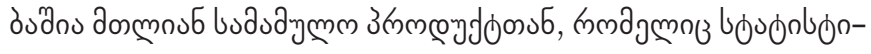

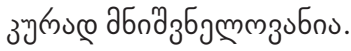

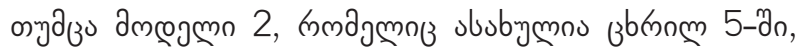

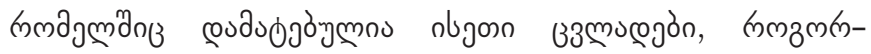

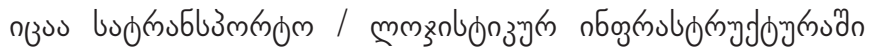

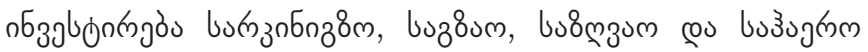

Random-effects GLS regression Group variable: YEAR

$\begin{array}{lll}\text { R-sq: } & \text { within } & =0.7980 \\ \text { between } & =0.4339 \\ \text { overall } & =0.7628\end{array}$

$\operatorname{corr}\left(u_{-} i, x\right)$ $=0$ (assumed)
Number of obs Number of groups

Obs per group:

Wald chi2 (6) Prob $>$ chi2

$\begin{array}{rlr} & = & 270 \\ & = & 18 \\ \min & = & 15 \\ \operatorname{avg} & = & 15.0 \\ \max & = & 15\end{array}$

845.62 0.0000

\begin{tabular}{|r|rrrrrr|}
\hline LGDP & Coef. & Std. Err. & $z$ & $\mathrm{P}>|\mathrm{z}|$ & $(95 \%$ Conf. & Interval) \\
\hline GCF & -.0070415 & .0047323 & -1.49 & 0.137 & -.0163167 & .0022336 \\
GGFCE & .0680004 & .0069597 & 9.77 & 0.000 & .0543595 & .0816412 \\
LPOP & .7635489 & .095969 & 7.96 & 0.000 & .575453 & .9516448 \\
EMP & .0160583 & .0045127 & 3.56 & 0.000 & .0072135 & .024903 \\
LTFREIGHT & .2806713 & .0778936 & 3.60 & 0.000 & .1280026 & .4333401 \\
TRADE & .0000983 & .0010043 & 0.10 & 0.922 & -.0018701 & .0020668 \\
_COnS & 2.352522 & .5772322 & 4.08 & 0.000 & 1.221167 & 3.483876 \\
\hline
\end{tabular}

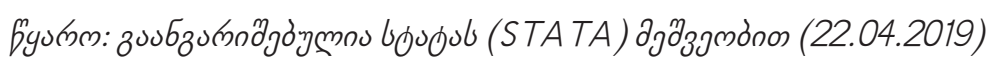




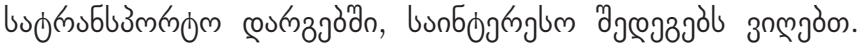
mmznlobnz

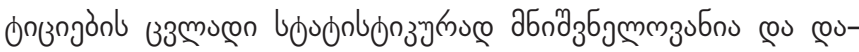

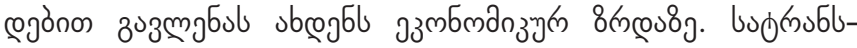

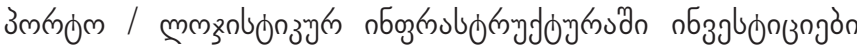

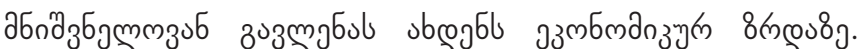

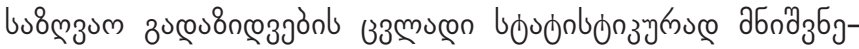

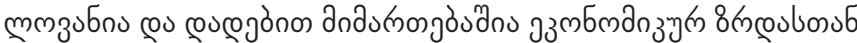

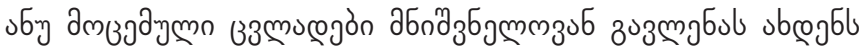

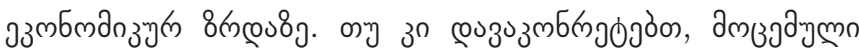

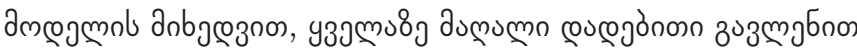

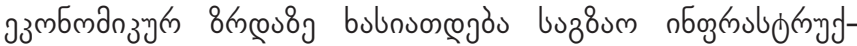

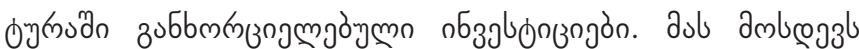

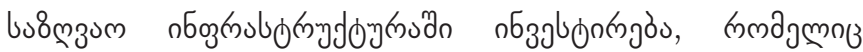

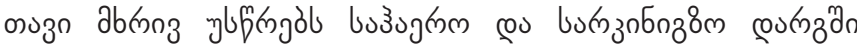

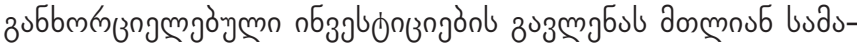

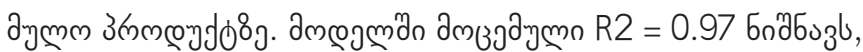

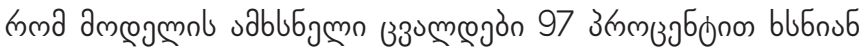
ububl6jom izmugol.

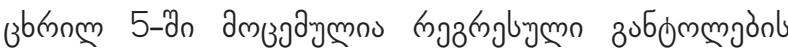

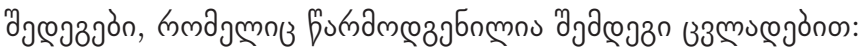

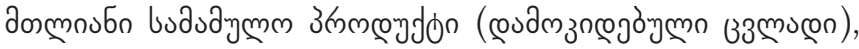

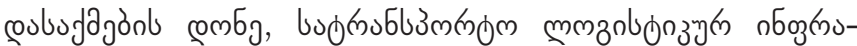

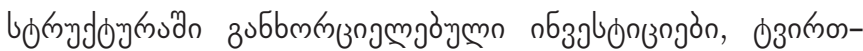

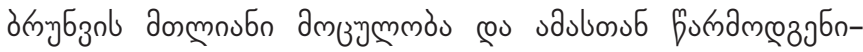

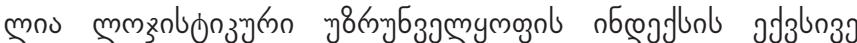
змдзмб бббо.

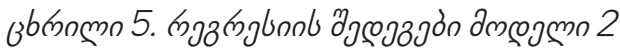

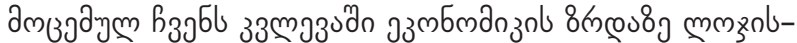

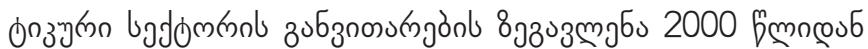

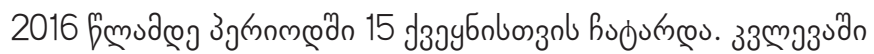

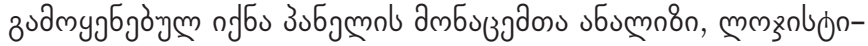

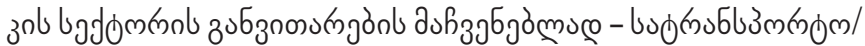

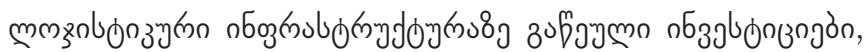

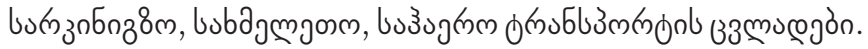

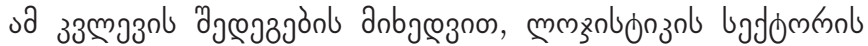

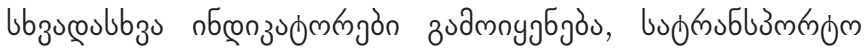

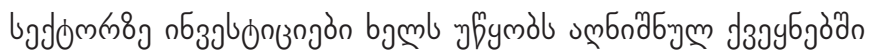

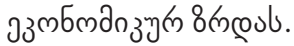

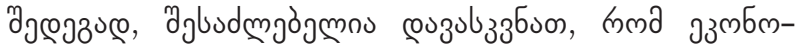

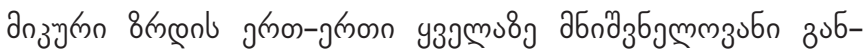

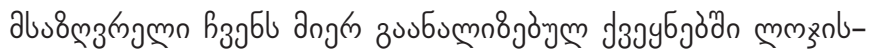

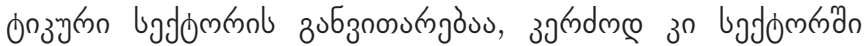

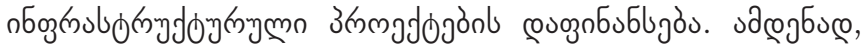

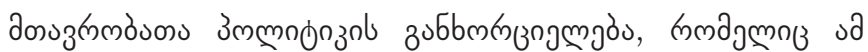
loggrnman nб

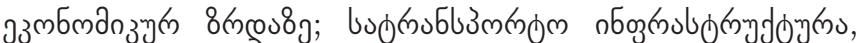

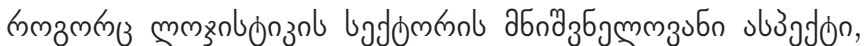

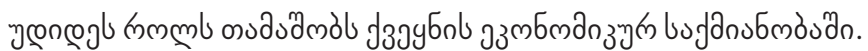

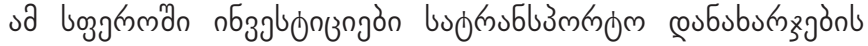

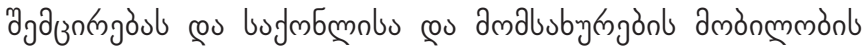

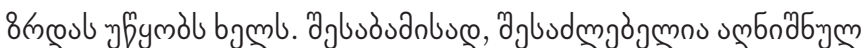

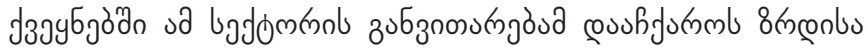

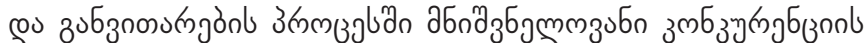

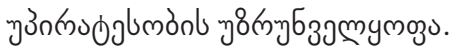

$$
\begin{aligned}
& \text { Random-effects GLS regression } \\
& \text { Group variable: YEAR } \\
& \text { R-sq: } \quad \text { within }=0.9776 \\
& \text { between }=0.9416 \\
& \text { overall }=0.9742
\end{aligned}
$$

\begin{tabular}{|c|c|c|c|c|c|c|}
\hline LGDP & Coef. & Std. Err. & $z$ & $P>|z|$ & (95\% Conf. & Interval) \\
\hline Linfrln & .3030062 & .0323353 & 9.37 & 0.000 & 2396302 & 3663823 \\
\hline LPOP & .1948443 & .0466628 & 4.18 & 0.000 & .1033869 & .2863018 \\
\hline GGFCE & .0271335 & .0031039 & 8.74 & 0.000 & 0210499 & .0332171 \\
\hline Lrailw & -.1435916 & .0322523 & -4.45 & 0.000 & -.206805 & -.0803781 \\
\hline Lroad & .4037676 & .0372555 & 10.84 & 0.000 & .3307482 & .4767871 \\
\hline Lport & .2597323 & .0148955 & 17.44 & 0.000 & .2305375 & .288927 \\
\hline EMP & 0173693 & .0024598 & 7.06 & 0.000 & .0125482 & .0221904 \\
\hline LTFREIGHT & .0944806 & .0690121 & 1.37 & 0.171 & -.0407806 & .22974117 \\
\hline LAIR & .0133481 & .0131114 & 1.02 & 0.309 & -.0123498 & .0390459 \\
\hline cons & .494869 & .4319752 & 1.15 & 0.252 & -.351787 & 1.341525 \\
\hline
\end{tabular}

$\begin{array}{llrr}\text { Number of obs } & & = & 251 \\ \text { Number of groups } & & & 18 \\ & & & \\ \text { Obs per group: } & \min & = & 13 \\ & \text { avg } & = & 13.9 \\ & \text { max } & = & 14 \\ \text { Wald chi2 (9) } & & & \\ \text { Prob }>\text { chi2 } & & = & 9096.49 \\ & & & 0.0000\end{array}$

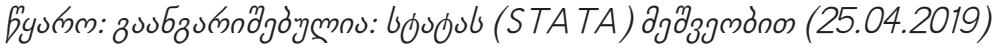




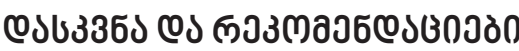

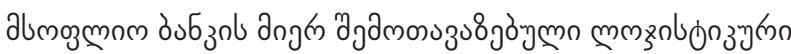

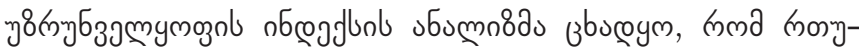

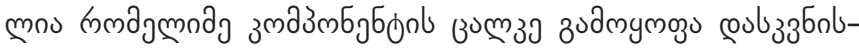

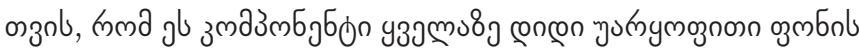

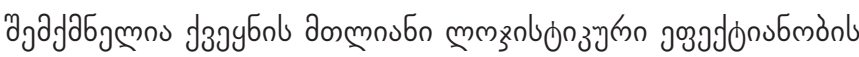

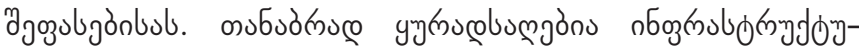

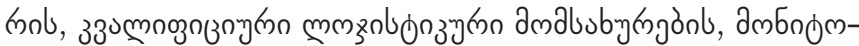

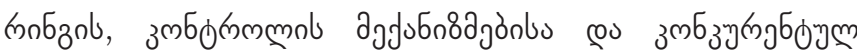

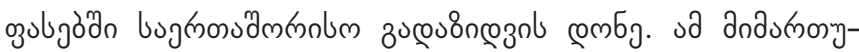

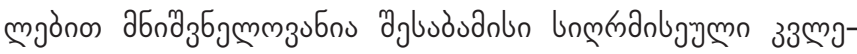

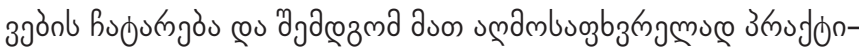

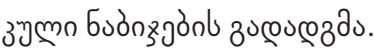

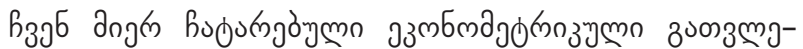

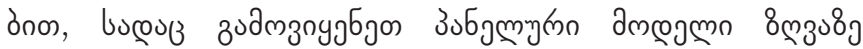

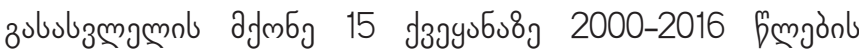

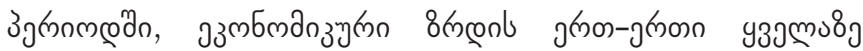

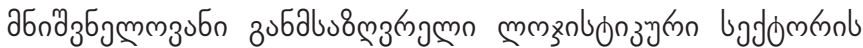

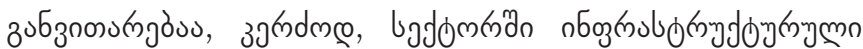

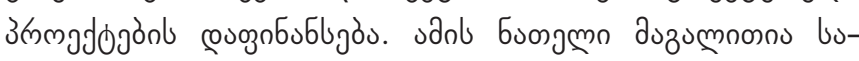

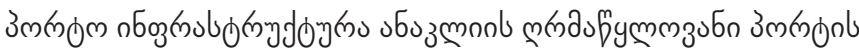

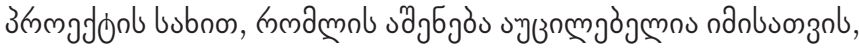

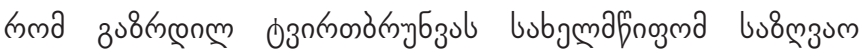

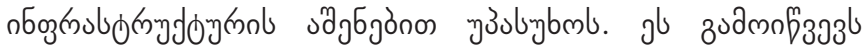

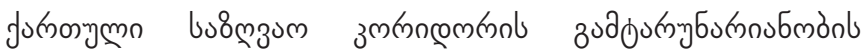

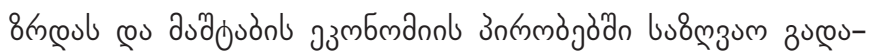

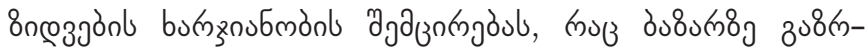

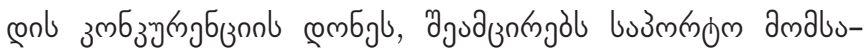

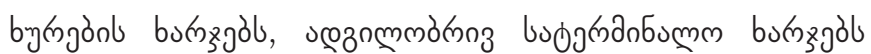

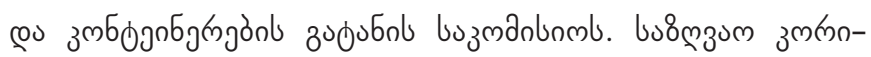

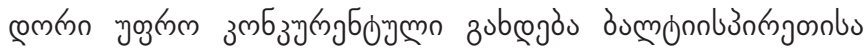

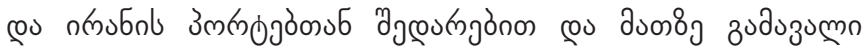

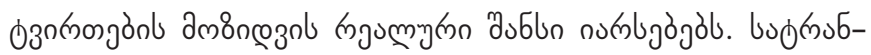

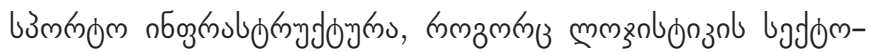

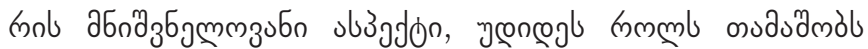

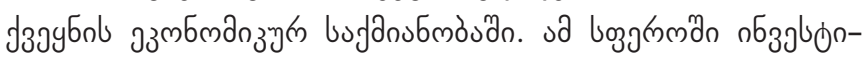

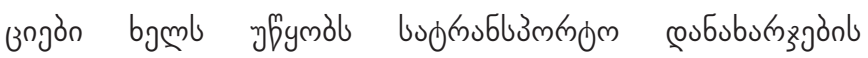

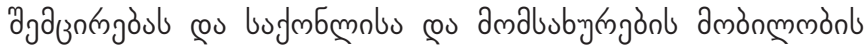

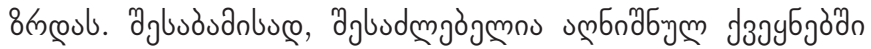

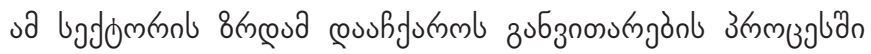

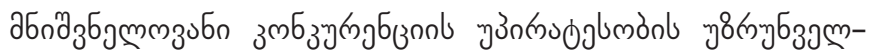

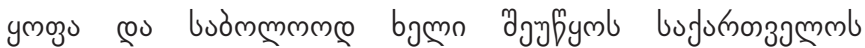

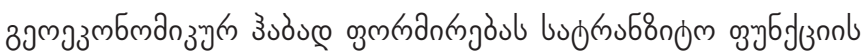

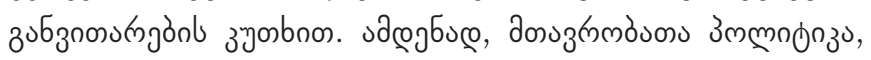

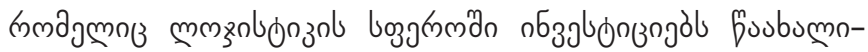

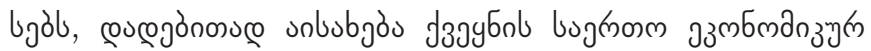

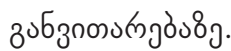

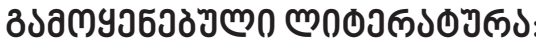

1. Abesadze, G. Abesadze, T. (2011). Globalization and peculiarities of Georgiass transit function. Tbilisi. (In Georgian).

2. Abesadze, R. (2018). Global economic security, international scientific conference, university publishing house. Tbilisi. (In Georgian).

3. Abralava, A. (2005). National economy and globalization. Tbilisi. (In Georgian).

4. Ananiashvili, I. (2018). Econometric analysis of peculiarities of fiscal and monetary instruments on joint demand in Georgian economy, globalization challenges in economy and business. Tbilisi. (In Georgian).

5. Baratashvili, E., Zarandia J., Abalava A. (2009). Regionalism: Theory and Practice. Tbilisi. (In Georgian).

6. Basilia, T., Silagadze, A., Chikvaidze, T. (2001). Post-socialist transformation: the Georgian economy on the eve of the XXI century. Tbilisi. (In Georgian).

7. Gedenidze, m. (2013). Architectural and functional-planning organization of the Silk Road (Traceka) section of Georgia. Methodical guidelines and a project project in urban planning. Technical University Publishing House. Tbilisi. (In Georgian).

8. Gelashvili, S., Maghradze, N. (2018). Geographical statistics of direct foreign investments in Georgia. Collection of TSU. Tbilisi. (In Georgian).

9. Danelia, I. (2019). Peculiarities and possibilities of development of transit corridor of Georgia. Journal: «Economy». Tbilisi. (In Georgian). 10. European Neighborhood Policy (2006). Action Plan, EU-Georgia. (In Georgian). Tbilisi. http://smr.gov.ge/Uploads/ a2dacc8e.pdf

11. Tvalchrelidze, A., Silagadze, A., Keshelashvili, G., Gegia, D. (2011). Georgiass socio-economic development program. International Foundation for Sustainable Development - Georgia. Tbilisi. (In Georgian).

12. Ismailov, E., Papava, V. (2007). Central Caucasus - the essence of geopolitical economy. Search. «Diogene». Tbilisi. (In Georgian). 13. Maksov, M. (1998). Caucasus - complex analysis, ways of political and economic stabilization, issues of geopolitics. Tbilisi University Press. Tbilisi. (In Georgian). 
14. Narmania, G. (2016). Waiting for Silk Road. J. Forbes Georgia. Tbilisi. (In Georgian).http://forbes.ge/news/1099/abreSumisgzis-molodinSi

15. Natroshvili, N. (2018). «APM Terminals» plans to build Mega Port in Poti. (In Georgian). http://www.netgazeti.ge/GE/105/ business/45909/

16. Pavliashvili, S., Garakanidze, Z. (2014). Georgian Transport Corridor - Problems and Prospects. Tbilisi. (In Georgian).

17. Sanikidze, G., Kiguradze, N. (2001). Modern international relations. Tbilisi. (In Georgian).

18. Putkaradze, R. (2011). International markets and main business centers. TSU, Lecture Course: «International Business», Chapter II, «Universal». Tbilisi. (In Georgian).

19. Anderson, J. (2009). 2009 Tax Misery \& Reform Index. J. Forbes. Tbilisi. www.forbes.com/global/2009/0413/034-taxmisery-reform-index.html\#51f9ab 9343b3

20. Bedianashvili, G. (2016). The Global Business Environment, European Integration and the Cultural Potential of Socialeconomic Development of Georgia. Globalization and Business. Tbilisi.

21. Bedianashvili, G. (2018). Formation of Knowledge Economy and Innovative Entrepreneurial Policy: Institutional Aspect. J. Globalization and Business. Tbilisi.

22. Bird, R. M. (2008). Tax Challenges Facing Developing Countries. Institute for International Business Working Paper No. 9. Delhi. http:papers.ssrn.com/sol3/papers. cfm?abstract_id=1114084

23. Boopen, S. (2006). Transport Infrastructure and Economic Growth: Evidence from Africa Using Dynamic Panel Estimates. The Empirical Economics Letters 5(1). (In China).

24. Cameron, F. (2015). The Maritime Silk Road - an EU perspective. World Commerce Review. http://www. worldcommercereview.com/html/cameron-the-maritime-silk-road---an-eu-perspective.html

25. Chania, M., Putkaradze, R. (2018). The Priorities of Georgian Economic Development in Conditions of Globalization.http:// journals.euser.org/files/articles/ejme_v1_i1_18/Maia.pdf

26. Cheng G. P. (2010). The Impact of Developments in the Logistics Sector on Economic Growth. (In China).

27. Chu, Z., Wang, Q., Hong, J. (2011). Transport infrastructure and regional economic growth: Evidence from China. Springer Science+Business Media. (In China). https://www.researchgate.net/publication/225309603_Transport_infrastructure_and_ regional_economic_growth_Evidence_from_China?

28. Cohen, S. (1963). Geography and Politics in a World Divided. Random House. New York.

29. Danelia, I. (2015). The Eurasian Union and the EU: mutually exclusive or mutually complementary economic systems. Journal: "Geoeconomics". http://geoeconomics.ge/en/?p=2987

30. Danelia, I. (2017). Main Determinants of Georgiass Transit Function Development. European Scientific Journal (ESJ). https://eujournal.org/index.php/esj/article/view/10207

31. Danelia, I. (2018). Black Sea Container Market and Georgia's Positioning. European Scientific Journal (ESJ). http://eujournal. org/index.php/esj/article/view/11479

32. Danelia, I. (2019). Georgia's Container Market and The Black Sea Region. Journal: "Economic Alternatives". https://www. unwe.bg/uploads/Alternatives/8_EA_1_2019_en.pdf

33. Danelia, I. (2019). Georgiars Geoeconomic Positioning with Political Concept. European Scientific Journal (ESJ).

34. Danelia, I. (2019). Prospects of Development of Georgiass Transit Function and Its Impact On The Countryss Economy. 4th International Conference - Actual Economic and Social Problems in Modern Globalization. Journal: "Globalization and Business". European University. Tbilisi.

35. Demurger, S. (2001). Infrastructure Development and Economic Growth: An Explanation for Regional Disparities in China? J. Comparative Economics, vol. 29, issue 1. https://www.researchgate.net/publication/222298076_Infrastructure_ Development_and_Economic_Growth_An_Explanation_for_Regional_Disparities_in_China

36. Hanaoka, S., Kawasaki, T. (2010). Survey Report on Freight Transport in Landlocked Countries in Central Asia. Graduate School of Science and Engineering. Tokyo Institute of Technology. Tokyo.

37. Hayaloglu, P. (2015). The Impact of Developments in the Logistics Sector on Economic Growth: The Case of OECD Countries, International Journal of Economics and Financial https://ideas.repec.org/a/ejn/ejssjr/v5y2017i1p11-23.html

38. Hu, K., Wang, X., Gao, K. (2012). Co-integration model of logistics infrastructure investment and regional economic growth in central China. (In China).

39. Humpert, M. (2018). Maersk Container Ship Transits Arctic Ocean With Icebreaker Escort. https://www.highnorthnews. com/en/maersk-container-ship-transits-arctic-ocean-icebreaker-escort

40. Hunter, S. T. (2000). Evolution of the Foreign Policy of the transcaucasian states. In Crossroads and Conflict: Security and Foreign policy in the Caucasus and Central Asia. Rourledge. New York.

41.ImplementTIR-EPDLANS,(16Jun.2016).InternationalRoadTransportUnion.http://ofae.gr/media/documents/2016/06/16/ en-tir-epd-green-lanes-0303.pdf 
42. IRU/World Bank road freight transport service reform. (January 2017). https://www.iru.org/guide-to-road-freighttransport-reform

43. ITF Transport Outlook. (2017). Rail Container Transport. OECD. https://www.ttm.nl/wp-content/uploads/2017/01/itf_study.pdf 44. Kjellen, R. (2001). The State as a Living Organism. Review of International Studies. Berlin.

45. Luttwak, E. N. (1993). The coming Global War for Economic Power. Philadelphia.

46. Marinova, V., Abe, M., Hügel, J., Sceia, A. (2016). E-TIR: Towards Paperless Cross Border Trade. United Nations, ESCAP. https://www.iru.org/sites/default/files/2016-09/eTIR-report-towards-paperless-cross-border-trade-iru-unescap-unece.pdf 47. Mody, A., Wang, F. (1997). Explaining Industrial Growth in Coastal China: Economic Reforms... and What Else? (In China). 48. Papava, V., Silagadze, A. (2019). On the Georgian name of one key economic term "Gross Domestic Product". J. Economics and Business, No. 1, pp.180-182. Tbilisi.

49. Sezer, S., Abasiz, T. (2017). The Impact of Logistics Industry on Economic Growth: An Application in OECD Countries. Istanbul.

50. Transcontinental Infrastructure Needs to 2030/2050 Organization for Economic Co-operation and Development (OECD). (2012). Mumbai Gateway Area Case Study, Opportunities And Challenges, Final Report. https://www.oecd.org/futures/ infrastructureto2030/49996110.pdf

51. Vernon, R., Spar D., Tobin, G. (1991). Iron Triangles and Revolving Doors: Cases in U.S. Foreign Economic Policymaking. Santa Barbara.

52. Wang, X. (2010). Transformation of Growth Pattern and Growth Sustainability in China. (In China).

Internet Resourses:

53. http://lpi.worldbank.org/

54. http://www.economy.ge

55. http://www.geostat.ge

56. http://www.worldbank.org

57. https://fiata.com/home.html 


\section{PROSPECTS OF DEVELOPMENT OF GEORGIA'S TRANSIT FUNCTION AND ITS IMPACT ON THE COUNTRY'S ECONOMY}

\section{IRAKLI DANELIA}

PHd student

Ivane Javakhishvili Tbilisi State University, Georgia

Irakli.danelia@tsu.ge

KEYWORDS: TRANCAUCASUS CORIDOR, TRANSIT, TRANSPORTATION, LOGISTICS, INVESTMENT, ECONOMIC GROWTH.

For citation: Danelia I. (2019), Prospects Of Development Of Georgia`S Transit Function And Its Impact On The Country`S Economy, Globalization And Business, №7, pp. 104-116. (In Georgian). https://doi.org/10.35945/gb.2019.07.013

SUMMARY

Depending on geographical location, the development of Georgian economy was directly related to the efficient use of transit function. For Georgia, as a transit function, the importance of transportation infrastructure is important in the formation of its geo-economic positioning strategy. The acquisition, realization and development of Georgiass transport potential began in the 90s of the 20th century. Nevertheless, complex research and analysis on the possibilities of development of transit function of the country in Georgian economic literature is not practically investigated yet. Morover, there are not identified determinants on the development of the sector, and their impact on the economic development of the country is not defined either.

The study aims to analyze capabilities of Georgiass transit potential and to determine its influence on economic development of the country in the process of integration of modern world economy. The following objectives are derived:

- Identification of significant stimulant determination of development of transport / logistics field and its effectiveness;

- Establishment of transit / logistics potential for economic development of the country.

The objective of the research is the transport and logistics sector in the Member States of the Organization for Economic Cooperation and Development, the Caucasus and Central Asian countries as an important instrument of economic development of the country.

The research is subject to comparative analysis of the targeted and consequential indicators of transport and logistics sector in the member states of the Organization for Economic Cooperation and Development, in the Caucasus and Central Asia.

The theoretical foundation of the research is the works of various Georgian and foreign researchers about the study.

The survey was based on the World Bank, the Organization for Economic Development and Cooperation, Eurostat, National Statistics Office of Georgia, Georgian and Foreign Professionals, Georgian and Foreign Marine Ports, Railway and Motor Operators, as well as the laws of Georgia.

The work is based on analysis and synthesis, quantitative, qualitative, groupings, and medium-size, graphic expression, indexing, comparison, regression and correlation methods.

The scientific innovation of the research is the attempt to conduct a complex examination of transit capabilities in Georgia, as a result of which:

- Using the Panel Data Analysis Method is reasonable as the development of the transport / logistics sector is a very positive role in the socio-economic development of the country, the main determinant of which is the infrastructural investment.

- Panel examinations and econometric calculations for member and partner countries of the Organization for Economic Co-operation and Development (OECD) with transit function and sea exit confirmed that investments in the transport/logistics infrastructure of these countries are the most efficient.

The paper can be used to overcome the challenges of successful transit systems in Georgia and its successful integration into the world economy. Consequently, the findings and recommendations of the research topic are appropriate for the Ministry of Economy and Sustainable Development, the Ministry of Regional Development and Infrastructure, the Parliament of Georgia and other sectoral authorities, non-governmental organizations, scientific organizations and other stakeholders. In the process of teaching geo-economics, «international economy», "world economy» and their neighboring disciplines. 


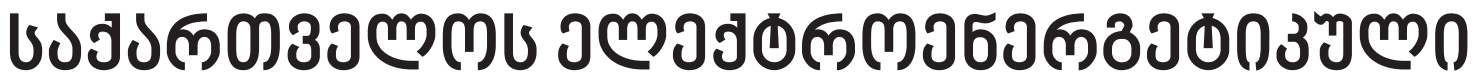

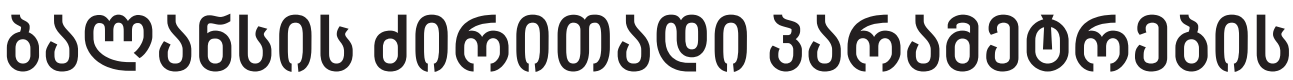

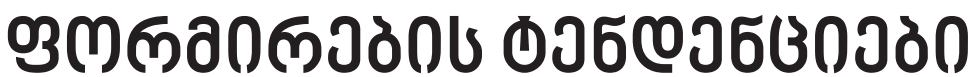

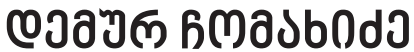

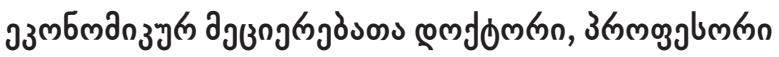 \\ bufuknos $39 \mathrm{mml}$ o oj

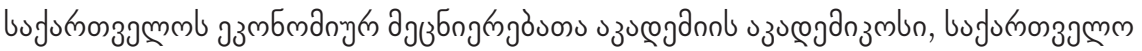 \\ demurchomakhidze@yahoo.com
}

\section{งอ0อ3১ธ Зb১3১0১}

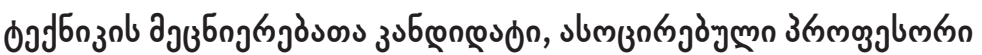

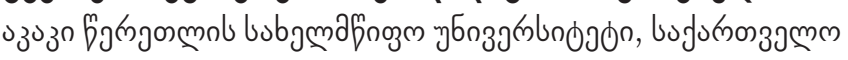

ketevan.tskhakaia@gmail.com

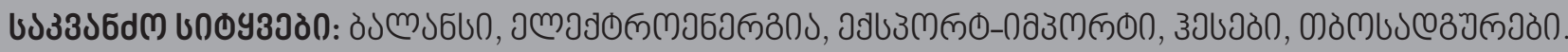

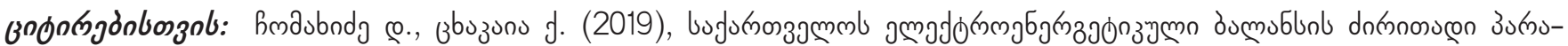
a jom

\section{ฆอง১З১ตั0}

jмaปd

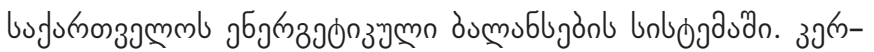

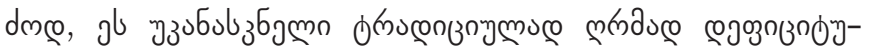

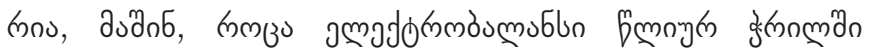

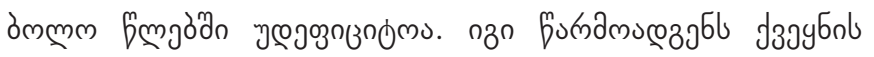

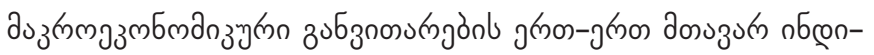

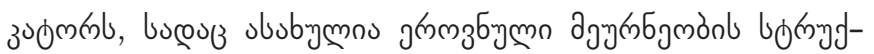

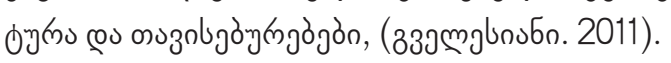

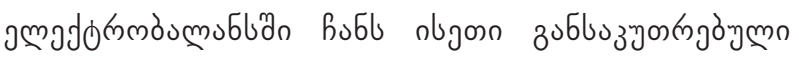

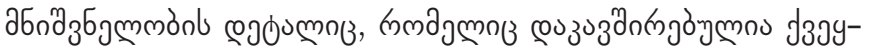

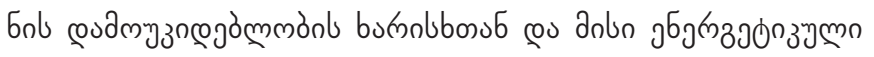

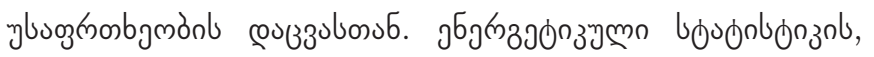

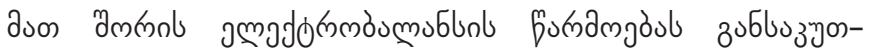

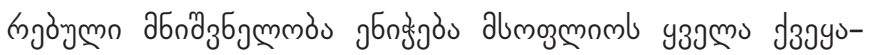
бuдn, anybje

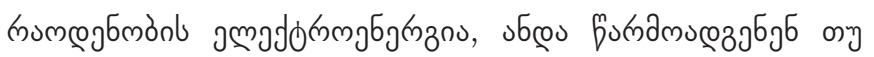

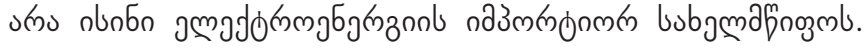

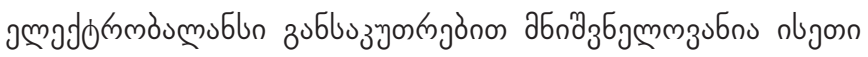

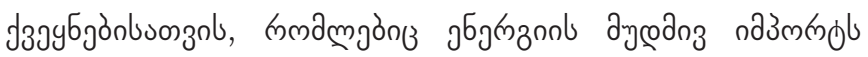

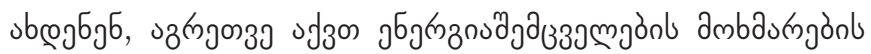

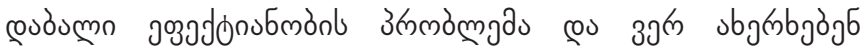

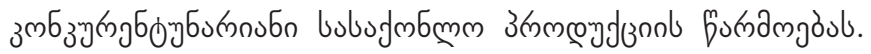
uмб

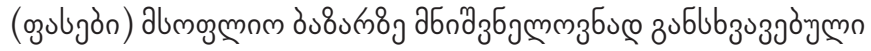

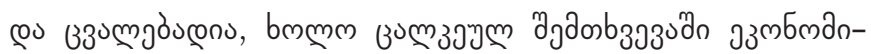

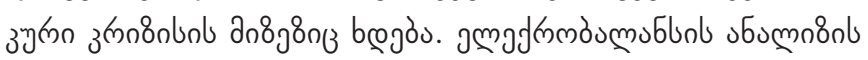

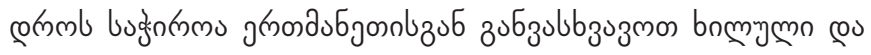

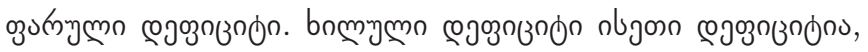

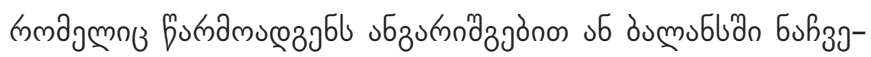

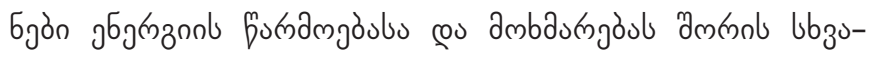

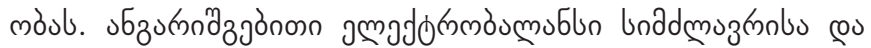

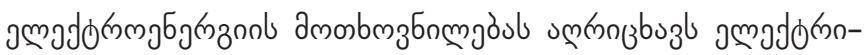

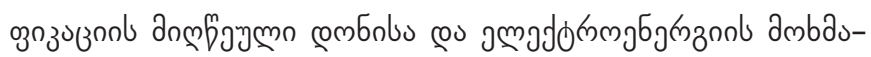

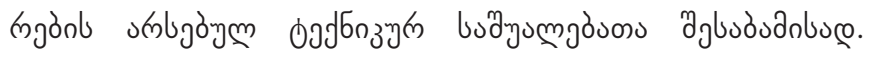




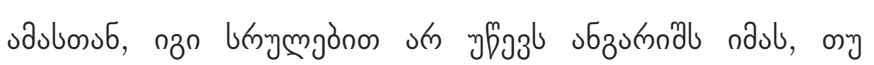

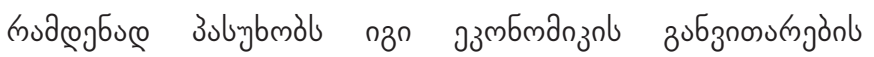

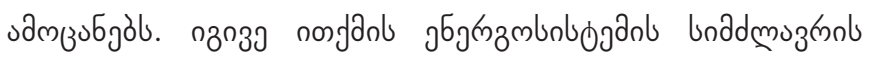

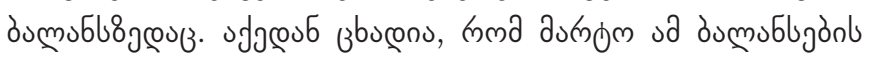

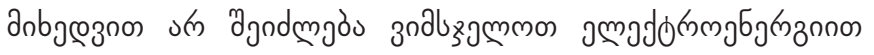

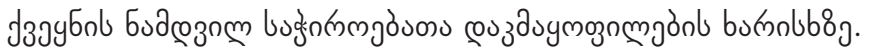

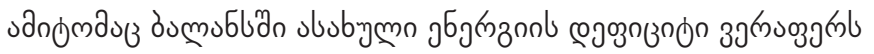

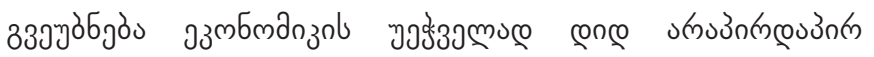

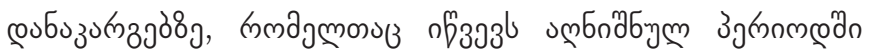

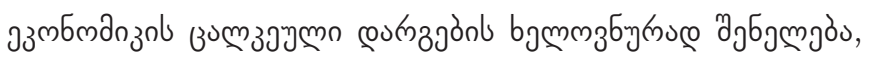

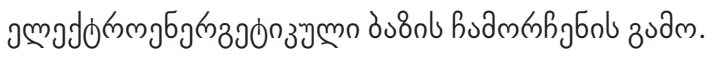

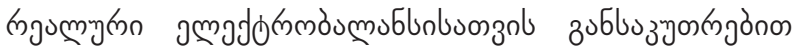

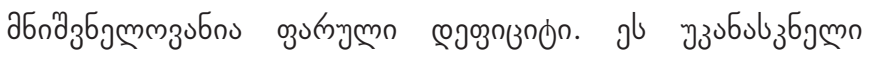

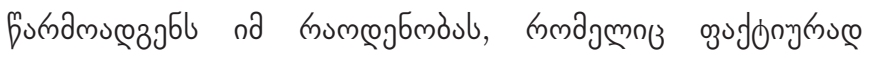

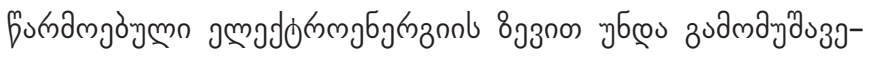

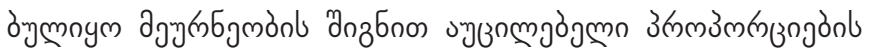

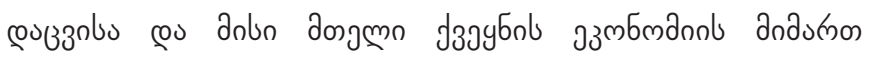

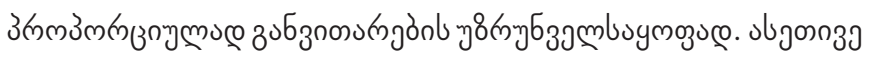

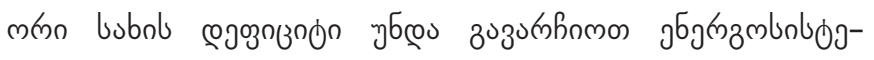

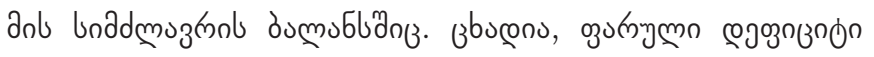

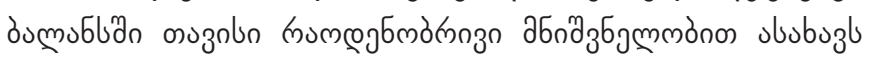

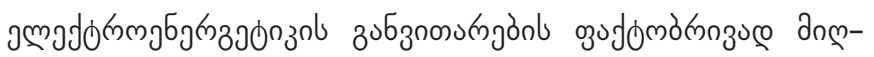

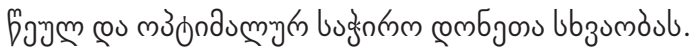

\section{d0m00งео б১60ल0}

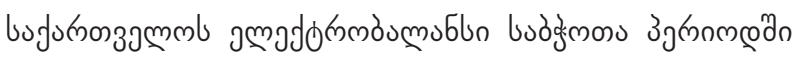

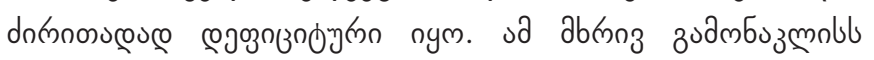

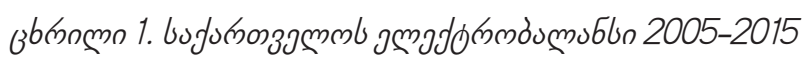

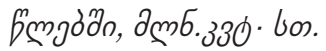

\begin{tabular}{|c|c|c|c|c|c|}
\hline \multirow{2}{*}{ coubsbymgös } & \multicolumn{5}{|c|}{ famn } \\
\hline & 2005 & 2010 & 2012 & 2014 & 2015 \\
\hline 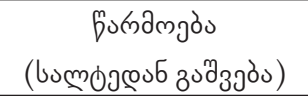 & 6880,8 & 9919,2 & 9471,9 & 10153,7 & 710832,6 \\
\hline àm dmbnt & & & & & \\
\hline 3jon & 5850,2 & 9263,3 & 7122,1 & 8221,1 & 8453,8 \\
\hline का ח & 1030,6 & 655,9 & 2349,8 & 1932,6 & 2378,7 \\
\hline пазмnका & 1398,6 & 222,1 & 614,6 & 851,9 & 699,2 \\
\hline 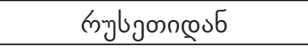 & 615,7 & 211,9 & 517,05 & 665,6 & 511,0 \\
\hline 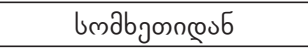 & 252,9 & & & 2,1 & 86,5 \\
\hline 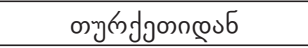 & 9,3 & 0,0001 & 629,0 & - & - \\
\hline 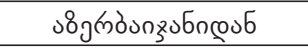 & 20,7 & 4 & 97,54 & 184,2 & 101,7 \\
\hline गd3mகnon & 121,8 & 1524,2 & 528,2 & 603,6 & 659,9 \\
\hline 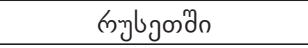 & - & 1117 & 369,43 & 218,6 & 169,6 \\
\hline 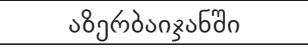 & - & 14,31 & 11,79 & 8,0 & - \\
\hline ๓चங் & 121,5 & 303,4 & 79,0 & 236,5 & 419,4 \\
\hline bmabgoñn & - & 89,5 & 67,9 & 140,5 & 70,9 \\
\hline 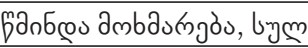 & 7842,2 & 8422,0 & 9379,4 & 10402,0 & 10631,8 \\
\hline dumsoblon $( \pm)$ & $-96,2$ & $+1477,2$ & $+92,5$ & $-248,3$ & $+200,8$ \\
\hline
\end{tabular}

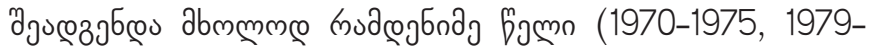

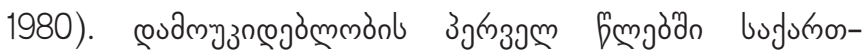

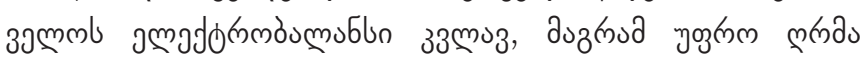

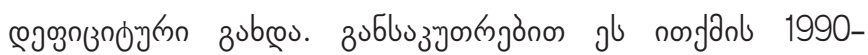

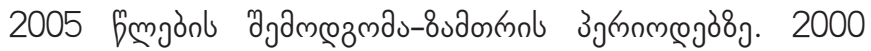

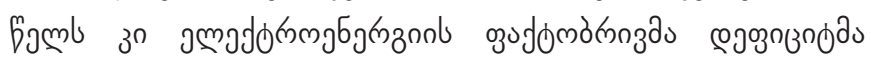

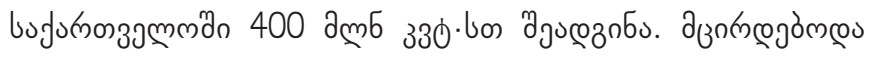

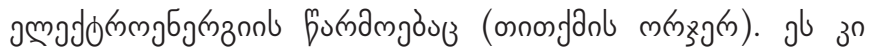

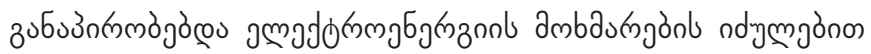

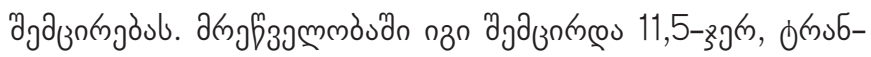

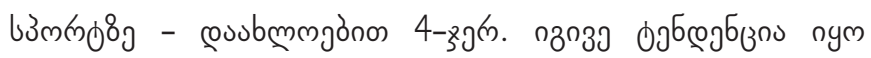

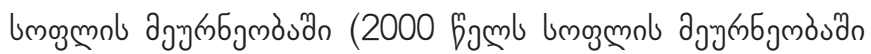

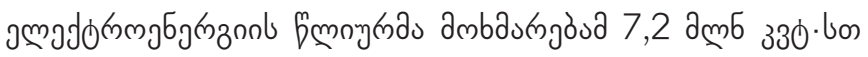

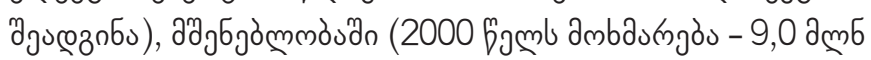

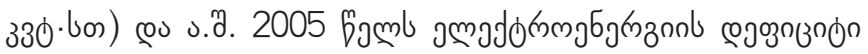

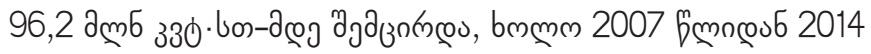

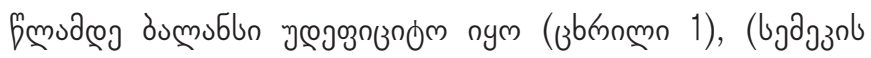

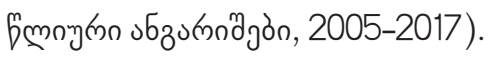

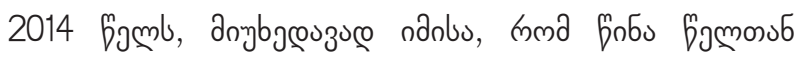

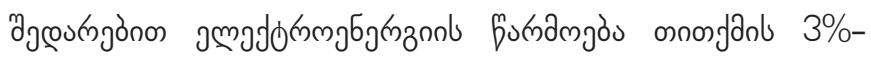

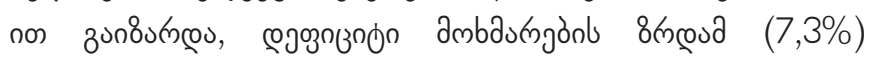

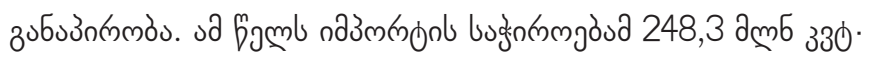

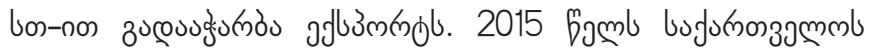

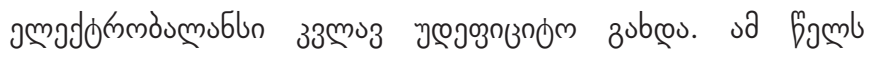

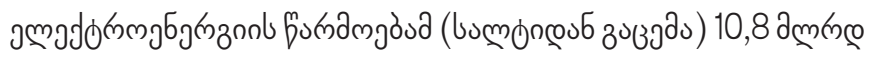

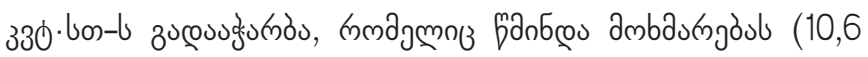
a

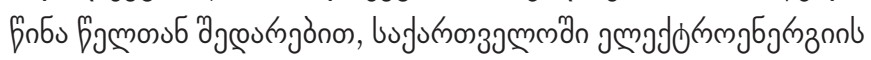

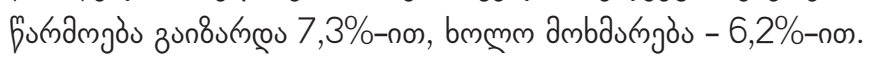

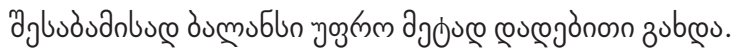

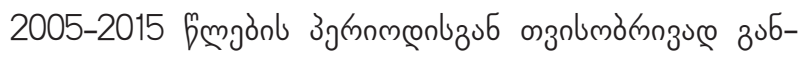

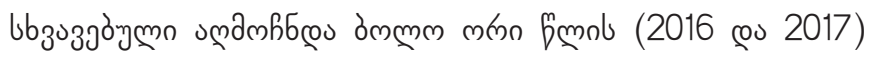

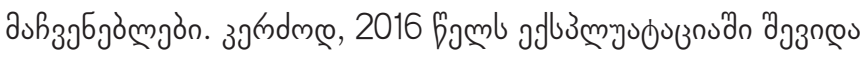

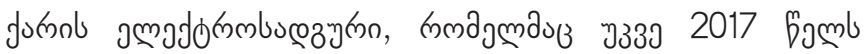

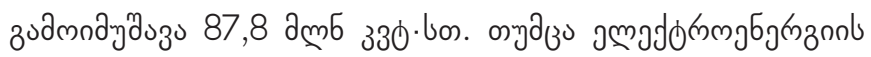

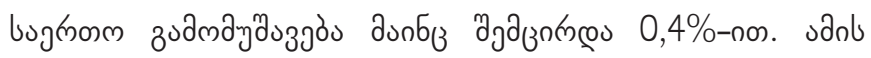

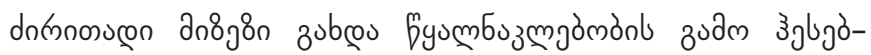

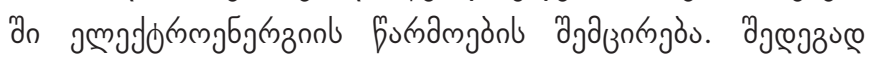

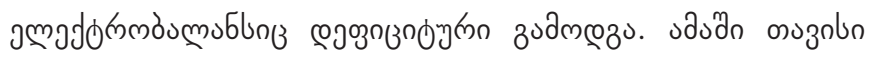

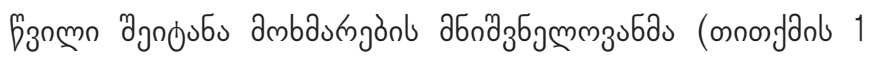

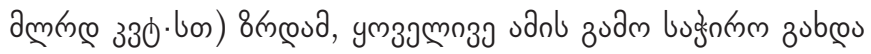

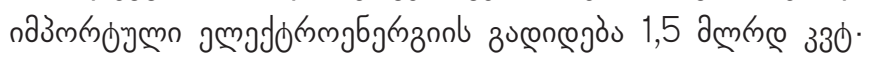

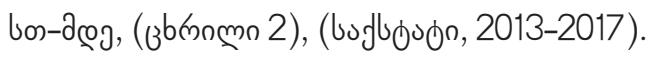

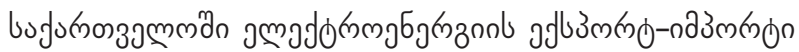

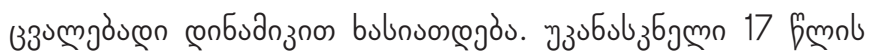

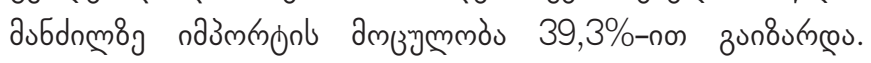

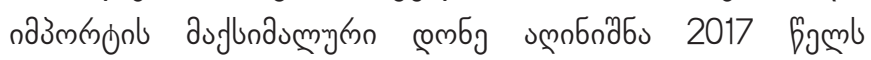




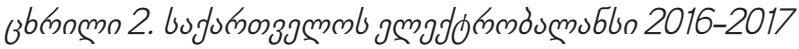

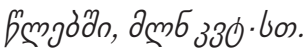

\begin{tabular}{|c|c|c|}
\hline \multirow{2}{*}{ 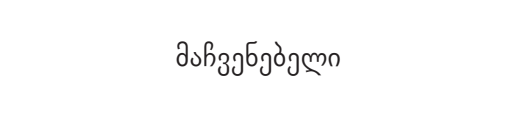 } & \multicolumn{2}{|c|}{ fomo } \\
\hline & 2016 & 2017 \\
\hline 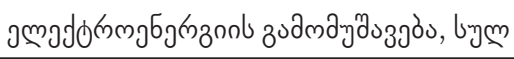 & 11573,6 & 11531,2 \\
\hline \multicolumn{3}{|l|}{ Juon Jmmnll: } \\
\hline 3 an & 9329,2 & 9210,4 \\
\hline oglo & 2235,4 & 2233,0 \\
\hline 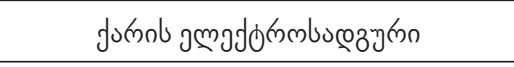 & 9,0 & 87,8 \\
\hline nдзмкnon, lym & 478,9 & 1497,2 \\
\hline aflümmon, bym & 559,0 & 685,7 \\
\hline ambasmjòs & 11493,5 & 12342,7 \\
\hline dumúblon $( \pm)$ & $+80,1$ & $-811,5$ \\
\hline
\end{tabular}

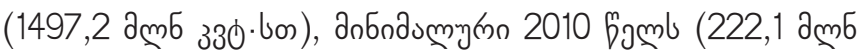

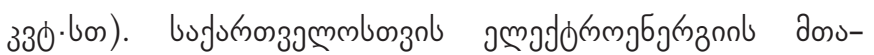

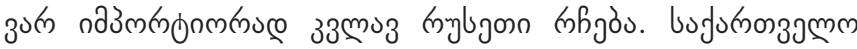

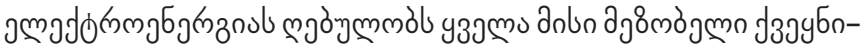

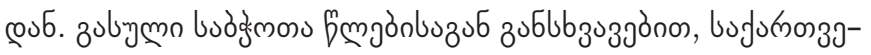

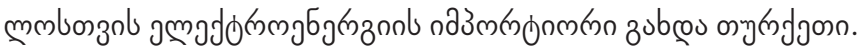

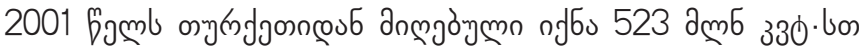

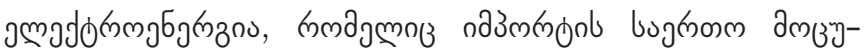

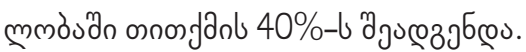

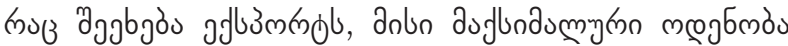

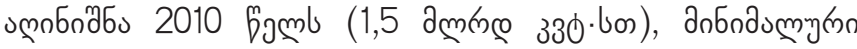

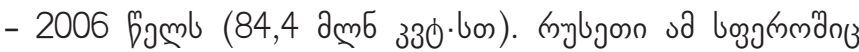

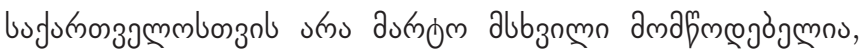

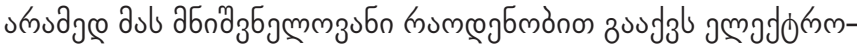

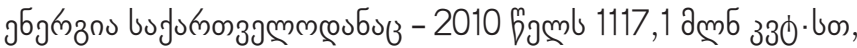

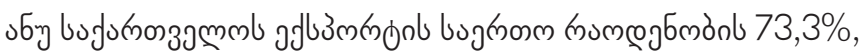
2014 Gamb - 218,6 am 330. bon, งбฑ 36,2\%.

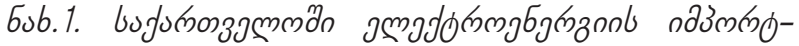
adlusmononb bonydoynd, 2017 p.

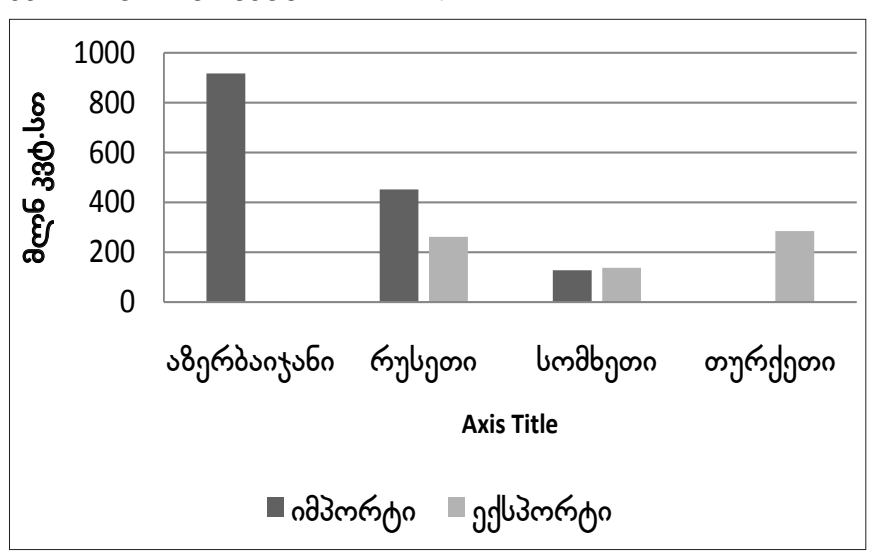

2017 famb amadd

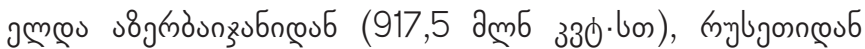

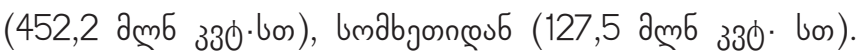

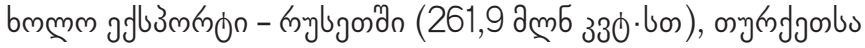

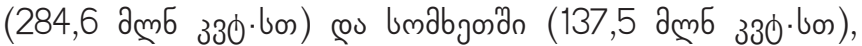

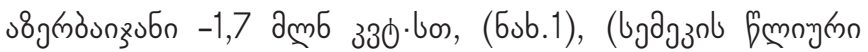

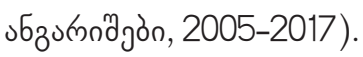

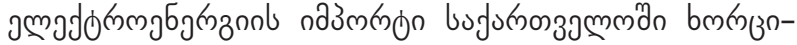

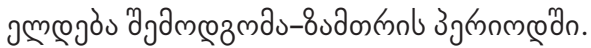

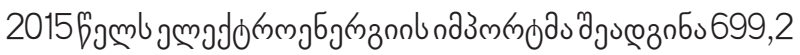
a

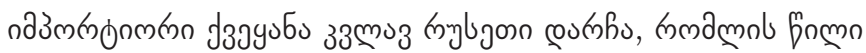

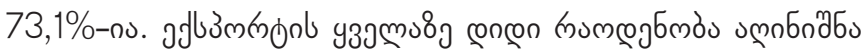

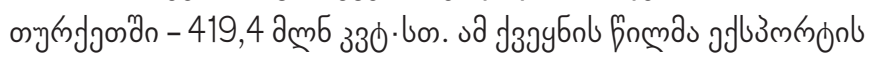

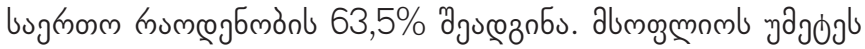

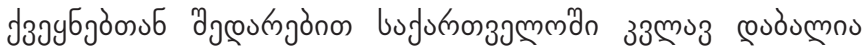

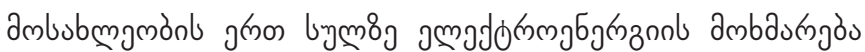
(zbrnnmn 3), (Key World Energy Statistics, 2016).

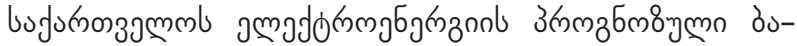

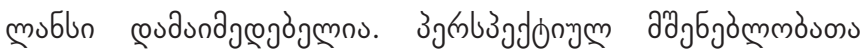

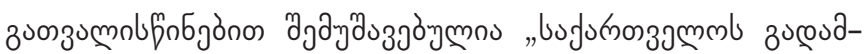

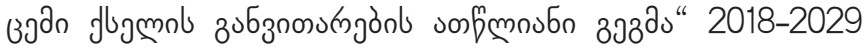

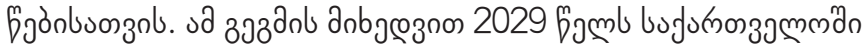

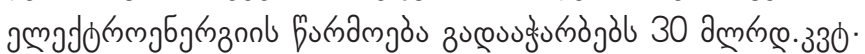

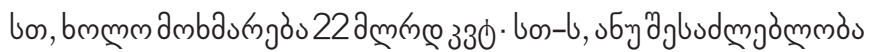

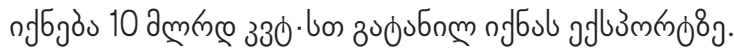

3btromano 3.

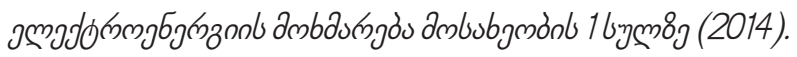

\begin{tabular}{|c|c|c|c|}
\hline dзృуงбo & $\begin{array}{c}\text { ambsbmgmò, } \\
\text { дলmb }\end{array}$ & 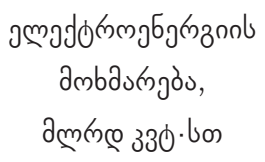 & 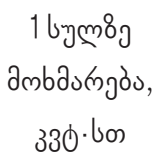 \\
\hline alımoुलnलm & 7249 & 21963 & 3029,8 \\
\hline د $2 \nexists$ & 319,17 & 4137,1 & 12962 \\
\hline (x) & 143,82 & 949,59 & 6602,6 \\
\hline 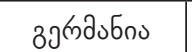 & 80,98 & 569,75 & 7035,7 \\
\hline no3̈mbno & 127,12 & 995,26 & 7829,3 \\
\hline bsogñbagyon & 66,17 & 460,2 & 6954,8 \\
\hline mod3no & 1,99 & 6,9 & 3460 \\
\hline mond & 2,93 & 11,21 & 3820 \\
\hline 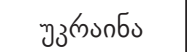 & 45,38 & 154,77 & 3410 \\
\hline bufunonsgmm & 4,5 & 10,02 & 2226,6 \\
\hline 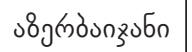 & 9,54 & 21,0 & 2201 \\
\hline lıabjon & 3,01 & 5,71 & 1897 \\
\hline
\end{tabular}




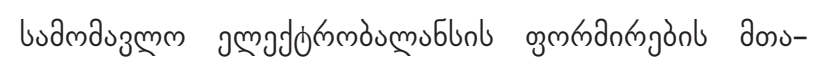

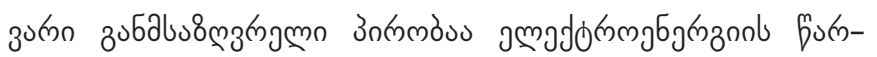

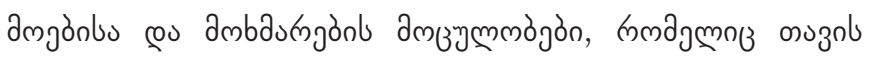

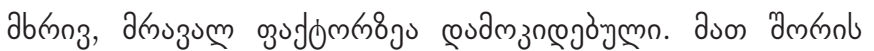

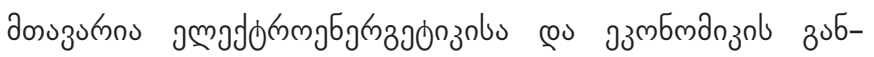

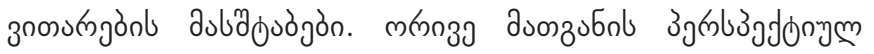

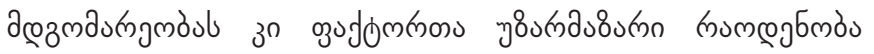
zublus 8 लुз mngòn (hydropower, thermal power, renewable power

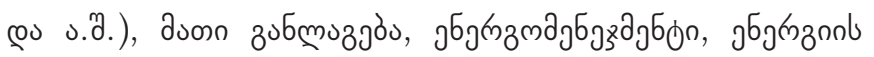

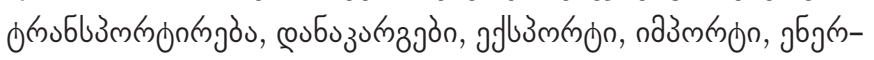

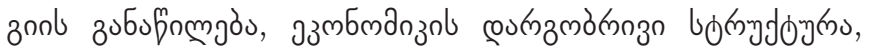

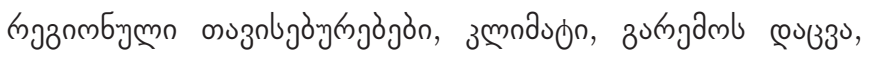

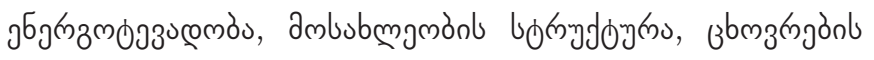

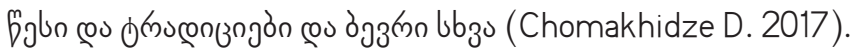

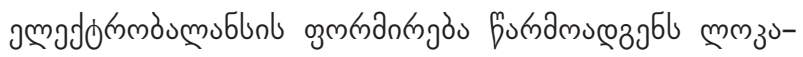

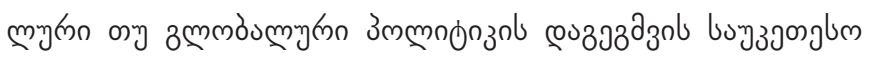

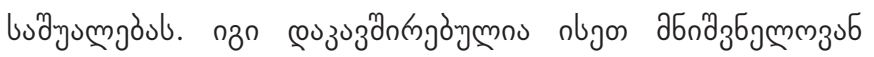

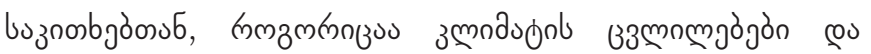

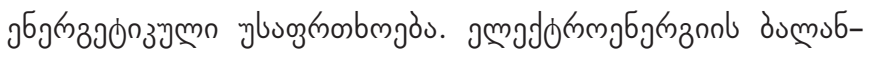

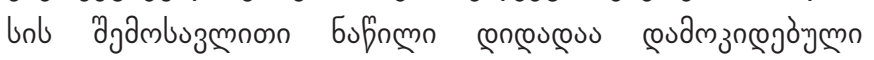

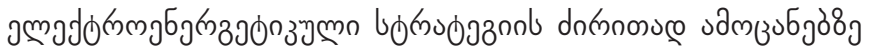

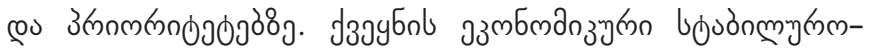

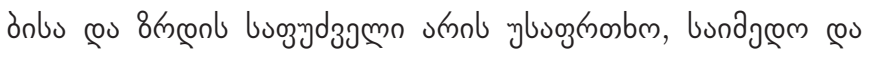

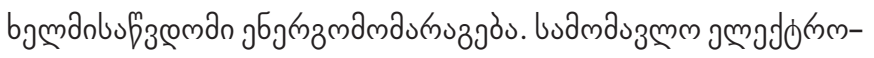

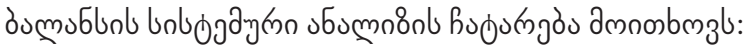

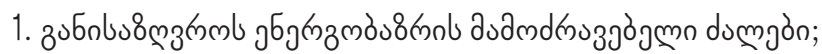

2. zus bumno

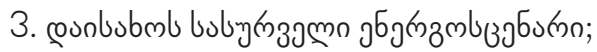

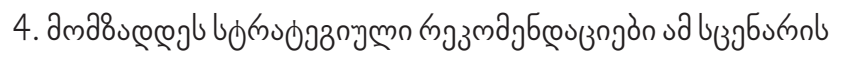
зubbminzogmajobluonzol.

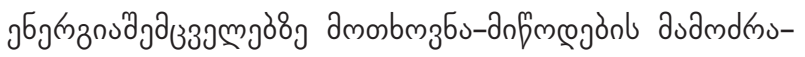

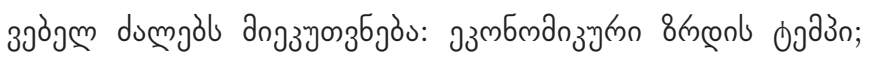

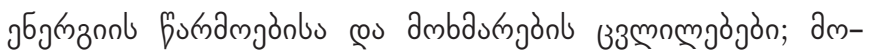
мbmzб

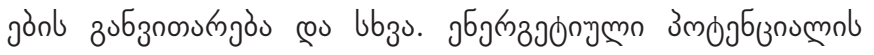
z дамy

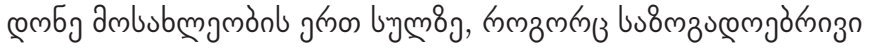
उ ऊrmz

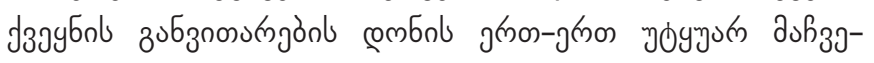

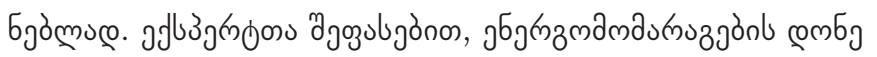

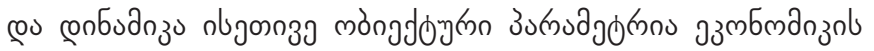

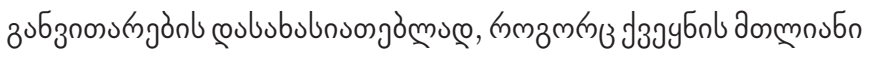

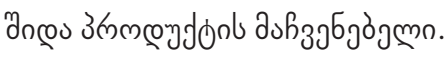

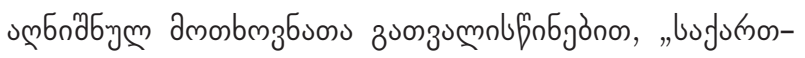

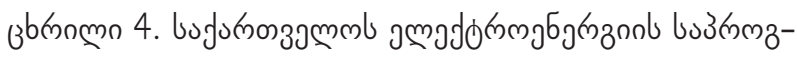

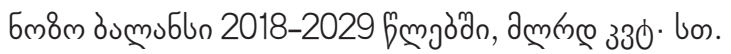

\begin{tabular}{|c|c|c|c|c|c|c|}
\hline \multirow[b]{2}{*}{ Emgòn } & \multirow[b]{2}{*}{$83^{6} g$ mogrou } & \multicolumn{3}{|c|}{ ason anmonls } & \multirow[b]{2}{*}{ ambà mjò } & \multirow[b]{2}{*}{ o adb3mmon } \\
\hline & & 3 & ol & 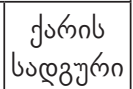 & & \\
\hline 2018 & 13,34 & 11,13 & 2,12 & 0,093 & 13,00 & 0,34 \\
\hline 2019 & 14,47 & 11,85 & 2,53 & 0,093 & 13,65 & 0,82 \\
\hline 2020 & 15,95 & 12,46 & 3,40 & 0,093 & 14,33 & 1,62 \\
\hline 2021 & 18,03 & 15,10 & 2,84 & 0,093 & 15,05 & 2,98 \\
\hline 2022 & 20,35 & 17,91 & 2,34 & 0,093 & 5,80 & 4,55 \\
\hline 2023 & 9 & 36 & 2,43 & 093 & 6,59 & 5,30 \\
\hline 2024 & 23,20 & 20,56 & 2,55 & 0,093 & 17,42 & 5,79 \\
\hline 2025 & 26,34 & 23,63 & 2,61 & 0,093 & 18,29 & 8,05 \\
\hline 2026 & 27,82 & 24,63 & 3,09 & 0,093 & 19,21 & 8,61 \\
\hline 2027 & 29,57 & 26,28 & 3,20 & 0,093 & 20,17 & 9.41 \\
\hline 2028 & 29.92 & 26.62 & 3.20 & 0.093 & 21.17 & 8,74 \\
\hline 2029 & 32.26 & 28.97 & 3.20 & 0.093 & 22.23 & 10.03 \\
\hline
\end{tabular}

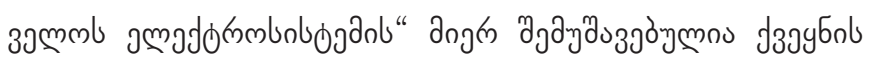

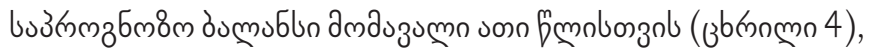

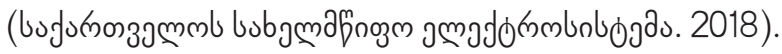

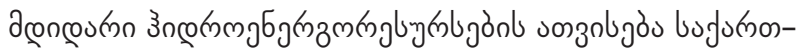

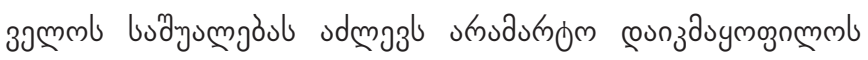

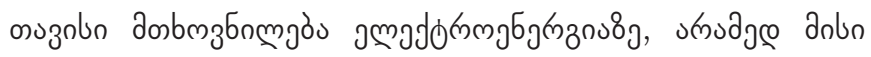
26пдз

\section{esı336s}

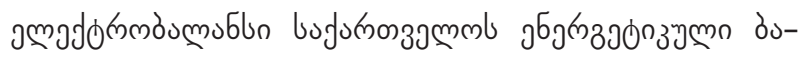
mublin dosszumn zaduœ ornn r.

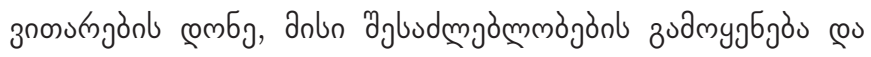

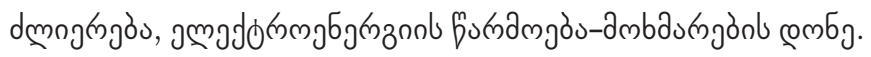

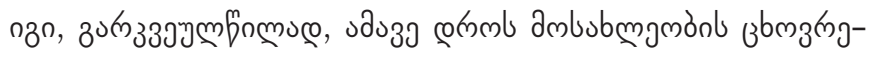

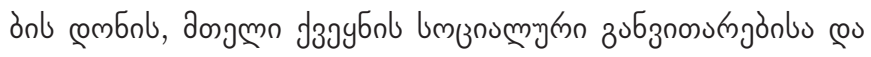

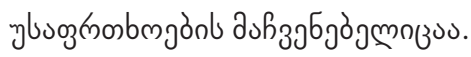

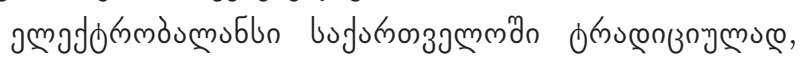

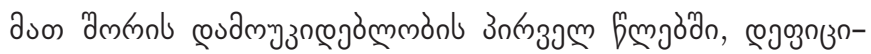

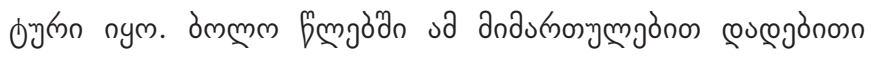

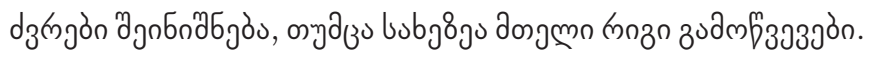

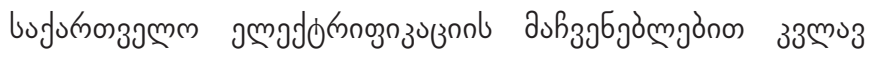

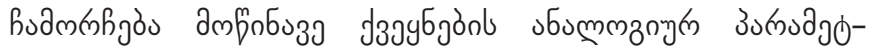

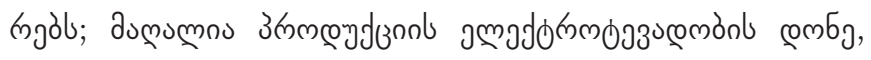

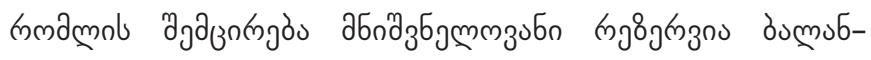

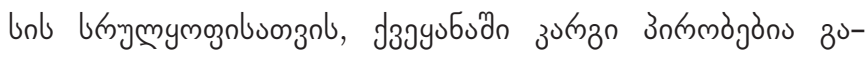




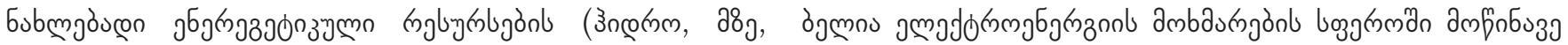

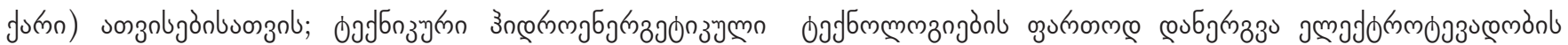

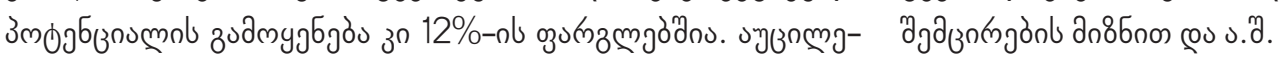

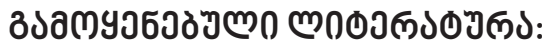

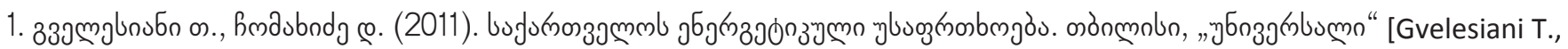
Chomakhidze D. (2011). Georgia ss energy security. Tbilisi, «Universali“. In Georgian].

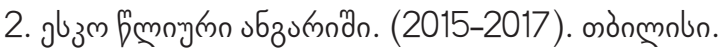

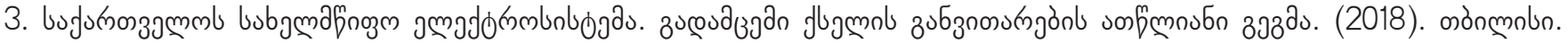
[Georgian State Electro system Transmission network development ten year plan. (2018). Tbilisi. In Georgian].

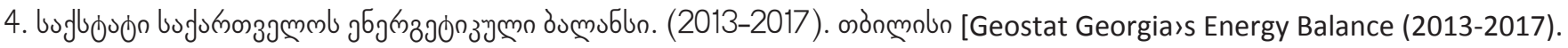
Tbilisi. In Georgia].

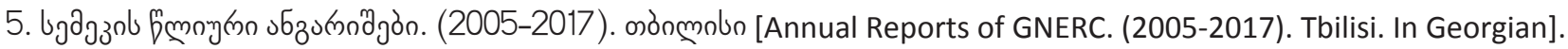

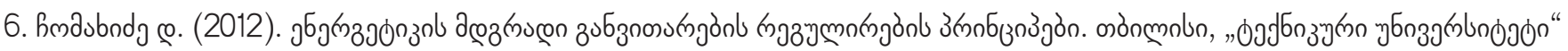
[Chomakhidze D. (2012). Principles of regulating sustainable development of energy. Tbilisi, «Technical University». In Georgian].

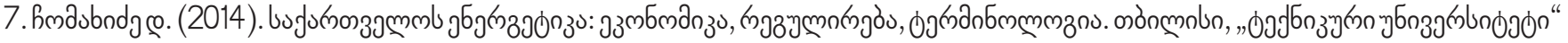
[Chomakhidze D. (2014). Georgia Energy: Economics, Regulation, Terminology. Tbilisi, «Technical University». In Georgian].

8. Chomakhidze D. Tskhakaia K., others. Electricity balance of Georgia: trends and prospects, Energy Procedia (2018) pp. 581587. DOI: 10.1016/j.egypro.2018.07.074.

9. Chomakhidze D., Shengelia G. (2017). Energy Complex of Georgia. Lap, Lambert Academic Publishing.

10. Key World Energy Statistics. (2016). 


\section{THE TRENDS OF DRAWING UP THE ELECTRICITY BALANCE OF GEORGIA}

\section{DEMUR CHOMAKHIDZE}

Doctor of Economics, Professor

Georgian Technical University

Academician of the Academy of Economic Sciences of Georgia, Georgia

demurchomakhidze@yahoo.com

\section{KETEVAN TSKHAKAIA}

Candidat of Technical Science, Associate Professor

Akaki Tsereteli State University, Georgia

ketevan.tskhakaia@gmail.com

\section{KEYWORDS: BALANCE, ELECTRICITY, EXPORT-IMPORT, POWER PLANTS, THERMAL PLANTS.}

For citation: Chomakhidze D., Tskhakaia K. (2019), The Trends Of Drawing Up The Electricity Balance Of Georgia, Globalization And Business, №7, pp. 117-122. (In Georgian). https://doi.org/10.35945/gb.2019.07.014

\section{SUMMARY}

The paper dwells on the analysis of electricity balance of Georgia in the years of 2005-2017 in accordance with the parameters such as electricity generation-consumption and exports-imports. Significant attention was paid to the structural development of electricity generation and consumption. The paper also addresses the issues of exportsimports with neighboring countries. Electricity exports and imports in Georgia is characterized by changing dynamics. Over the past 17 years, imports have grown by $39,3 \%$. It has been highlighted that the electricity balance in the years of
2016-2017 differs substantially from the electricity balance of previous years that is due to the operation of wind power plant, which just in 2017 generated 87,8 million kWh, representing $1 \%$ of generated electricity.

The paper highlights that the level of electric intensity of production in Georgia is still high, the reduction of which is considerable room for improvement in the electricity balance; there is need for development of renewable energy resources (hydro, solar, wind), placing greater reliance on advanced technologies in the field of electricity consumption and so on. 


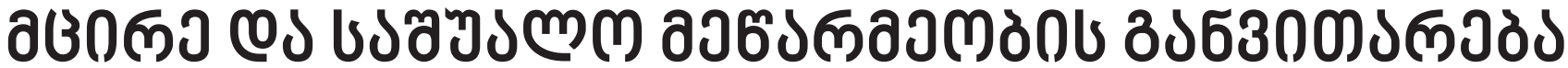

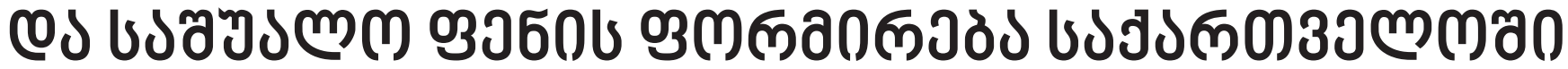

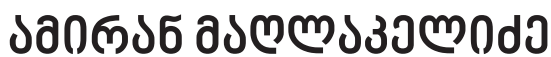

\section{एолоm}

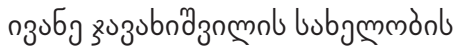

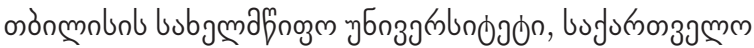

maglakelidze.amirani@gmail.com

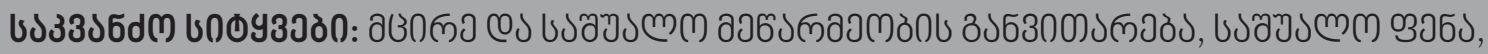

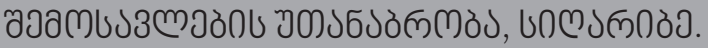

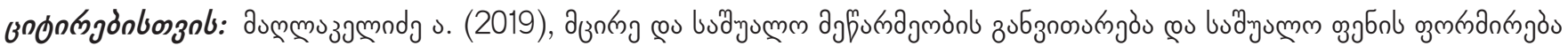

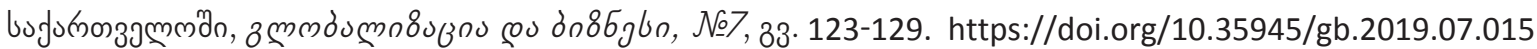

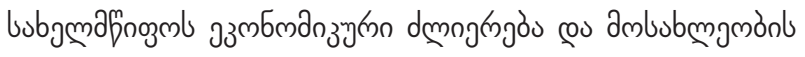

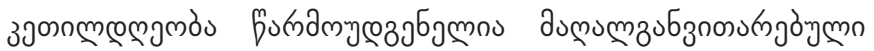

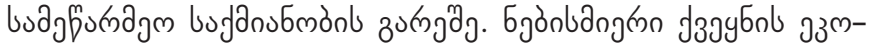

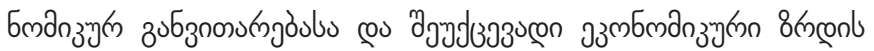

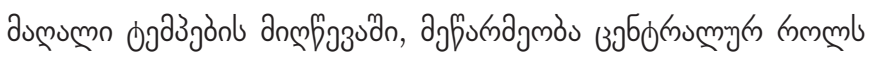

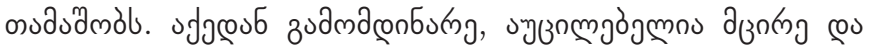

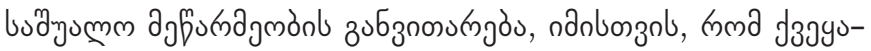

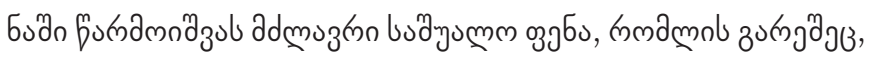

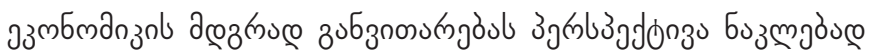

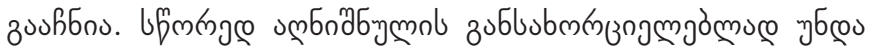

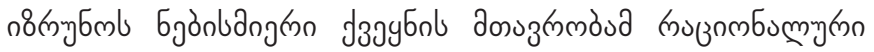

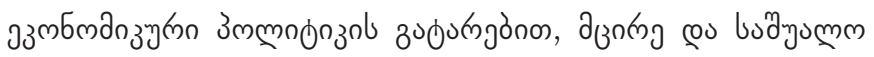

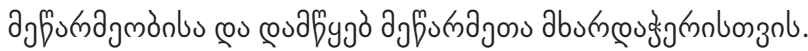

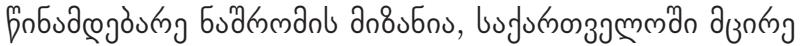

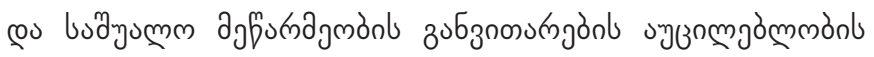

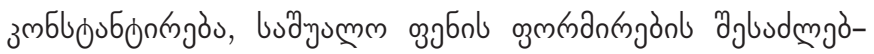

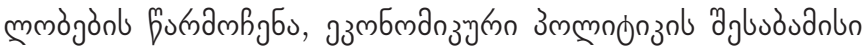

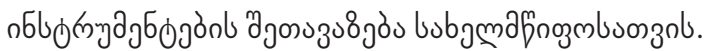

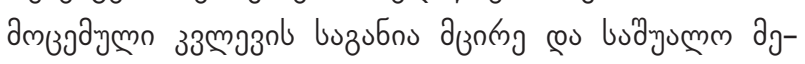

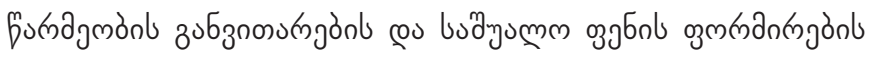

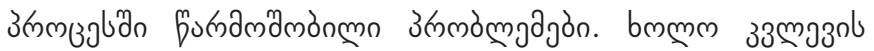

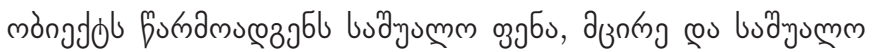

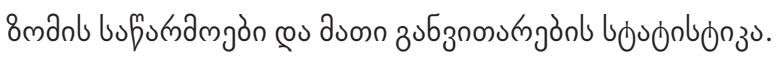

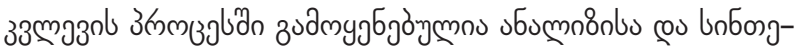

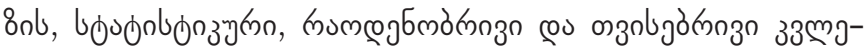

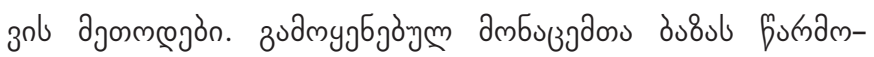

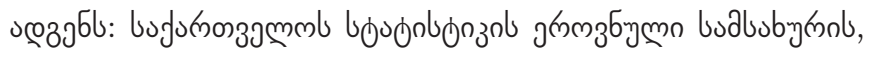

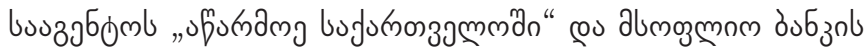

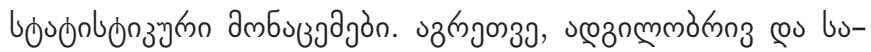

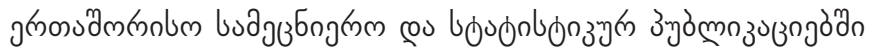

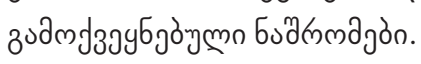

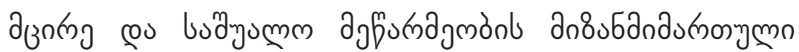

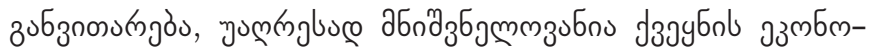

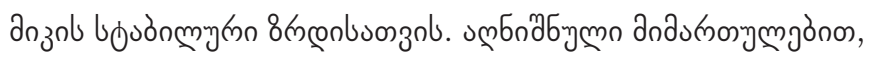

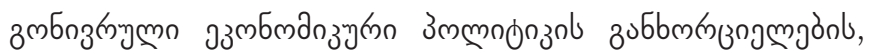

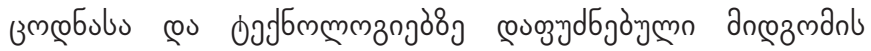

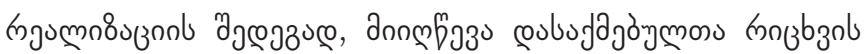

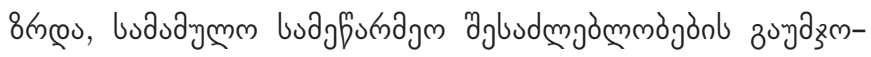

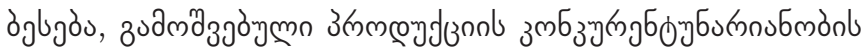

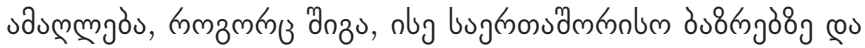

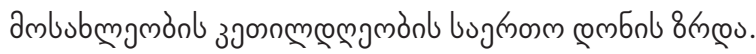

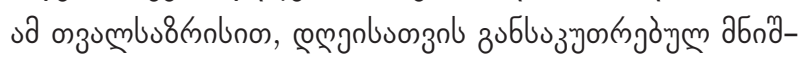

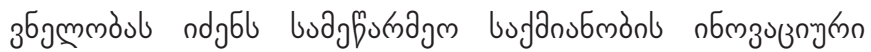

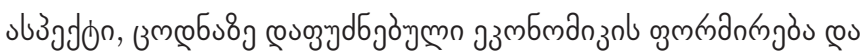

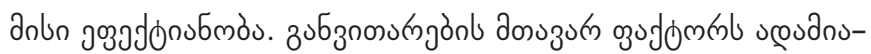

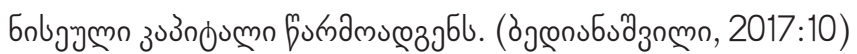

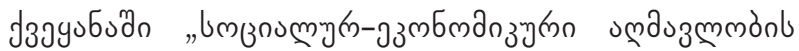

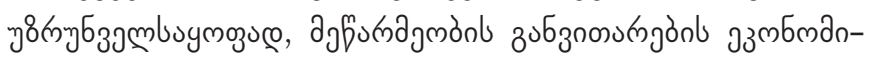

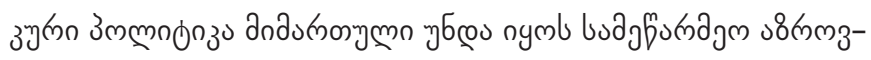

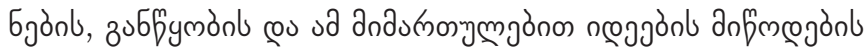

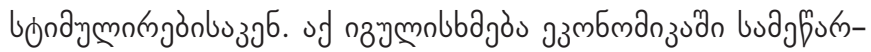

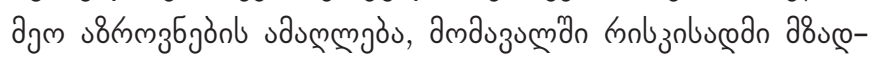




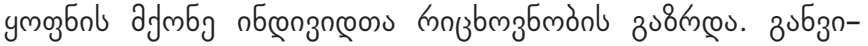

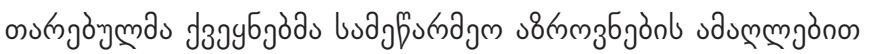

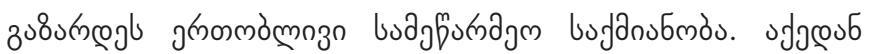

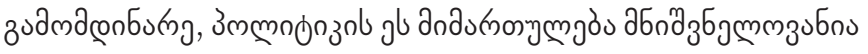

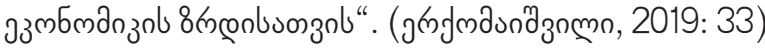

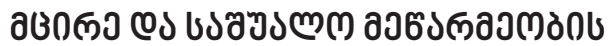

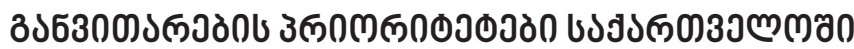

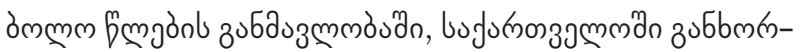

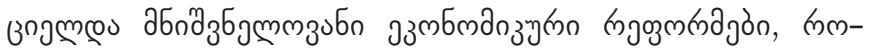

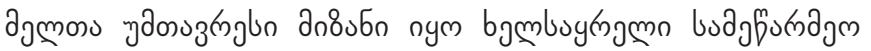

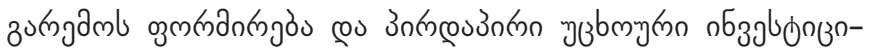

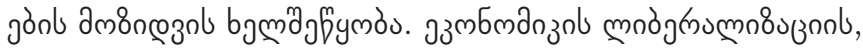

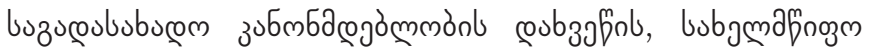

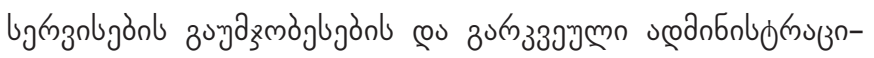

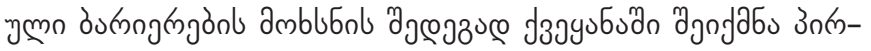

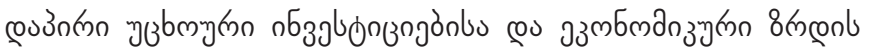

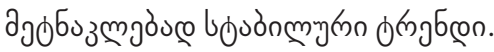

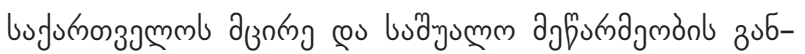

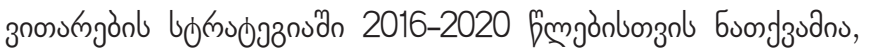

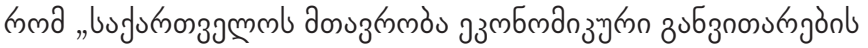

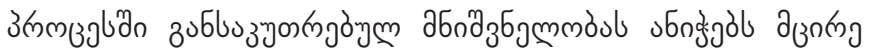

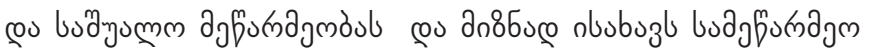

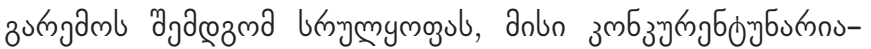

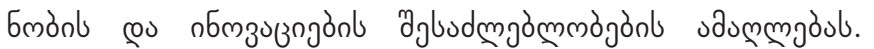

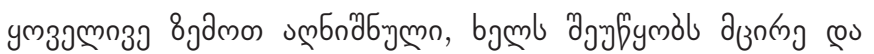

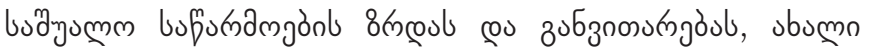

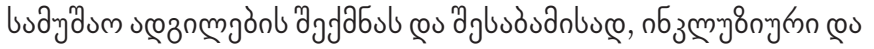

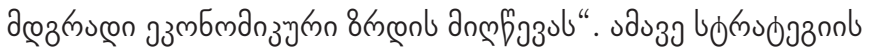

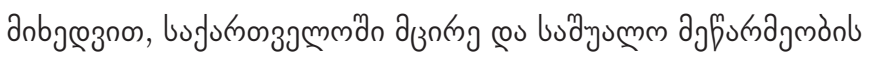

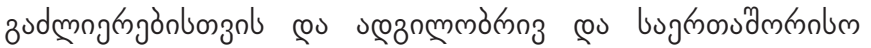

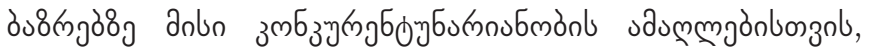

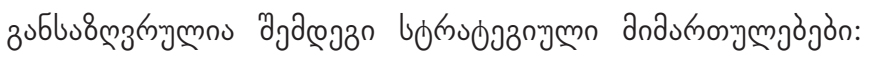

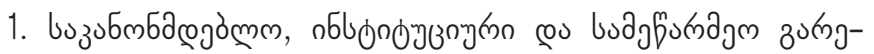

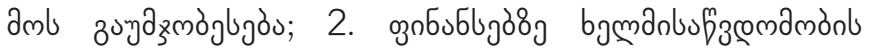

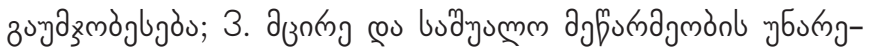

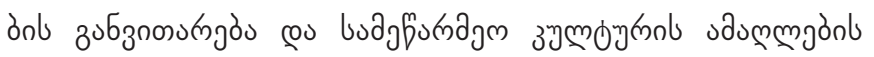

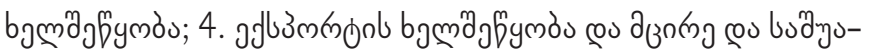

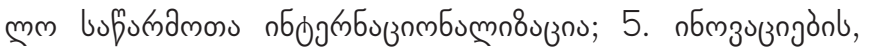

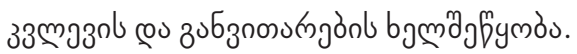

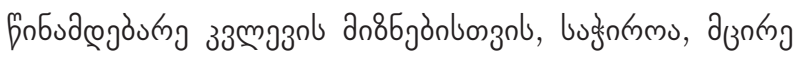

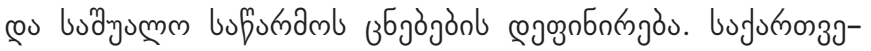

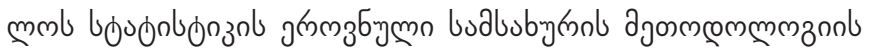

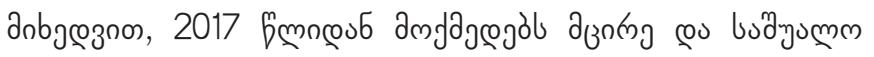

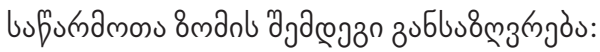

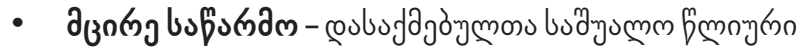

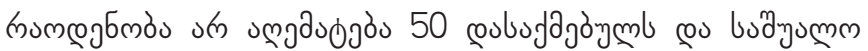

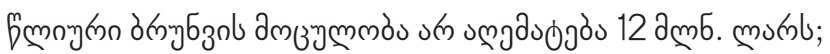

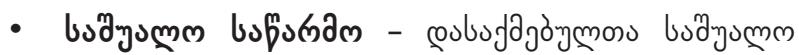

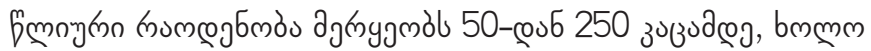

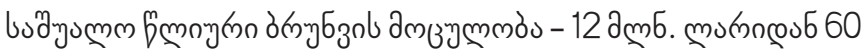

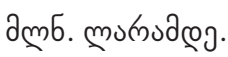

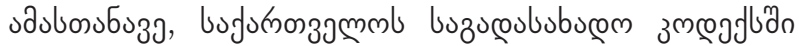

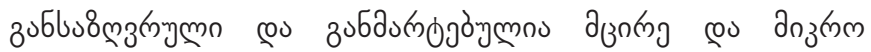

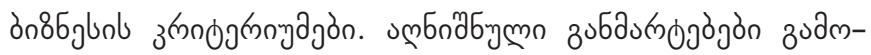

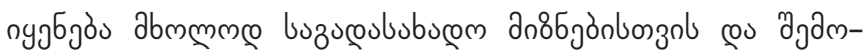

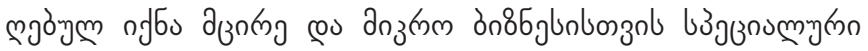

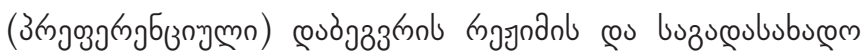

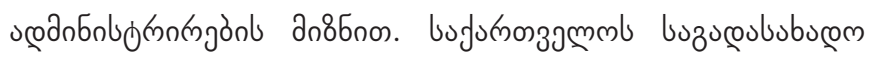

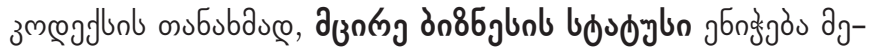

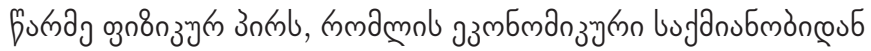

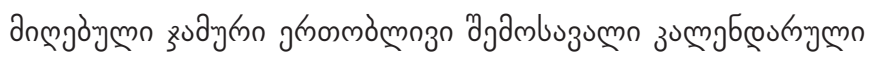

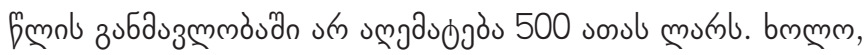

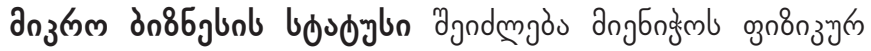

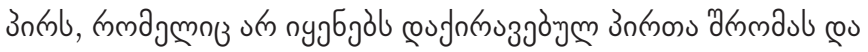

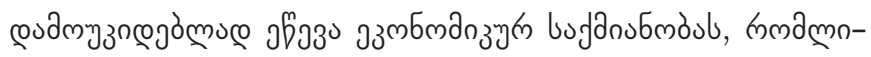

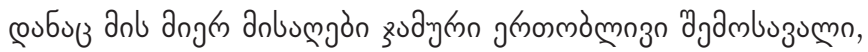

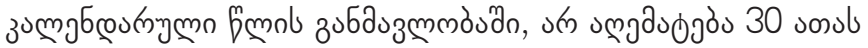
monbl.

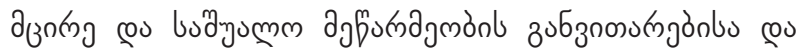

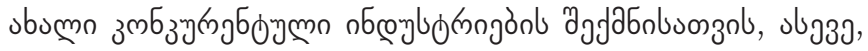

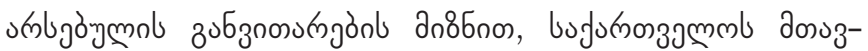

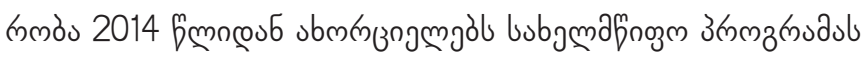

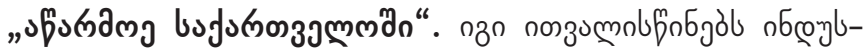

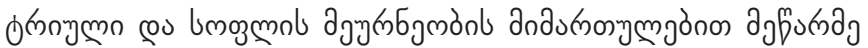

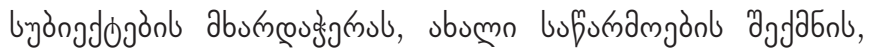

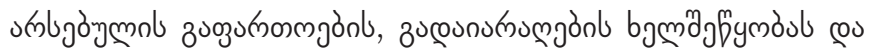
зgrndm bajom

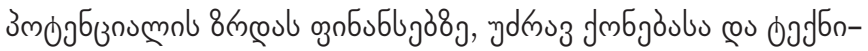

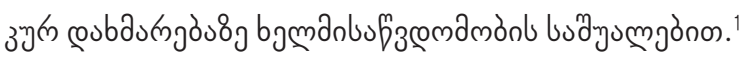

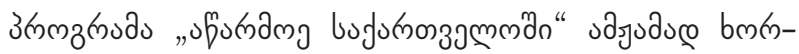

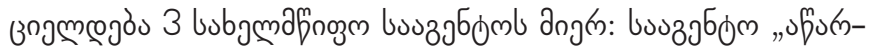

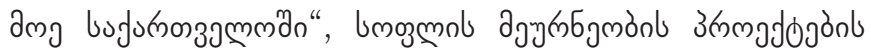

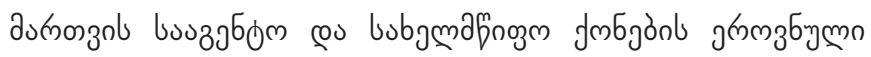
bouzjejom.

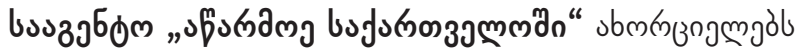

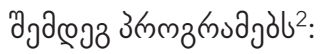

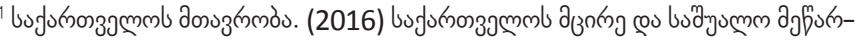

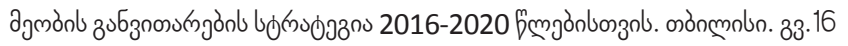

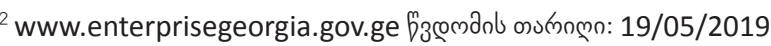




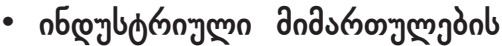

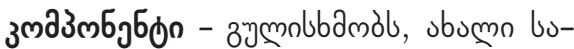

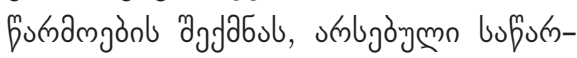

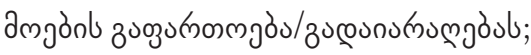

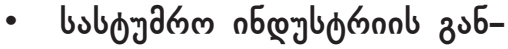

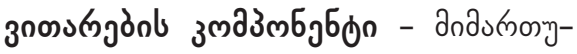
mos, sbumn buboydrnmgònl gadabnl,

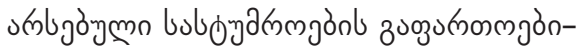
bo 396 ;

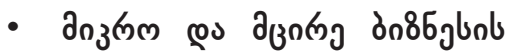

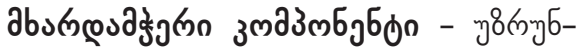

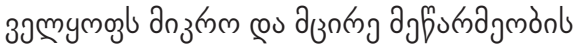

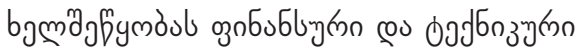

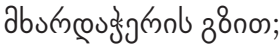

- उmmźrada "zuganga bajarm-

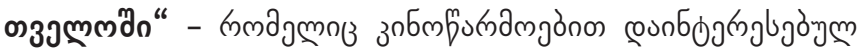

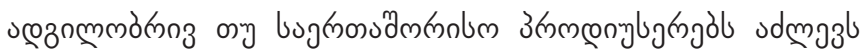

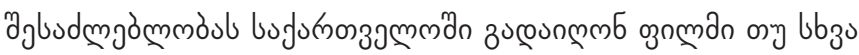

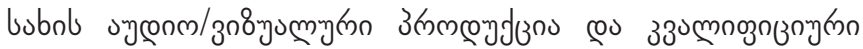

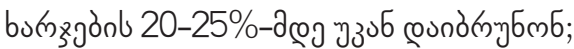

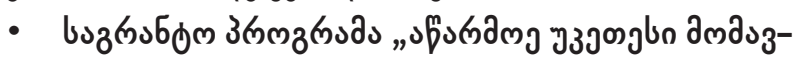

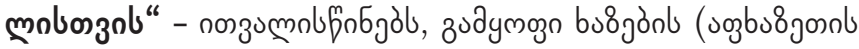

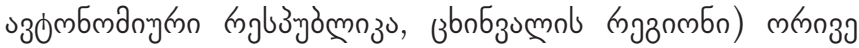

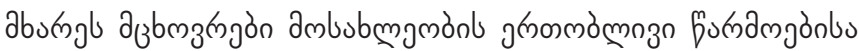

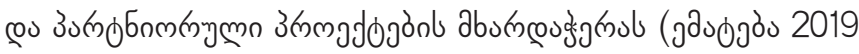
हmaños);

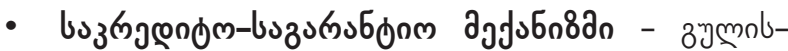

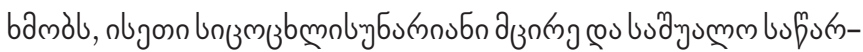

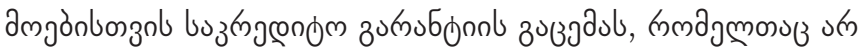

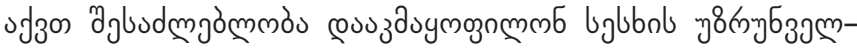

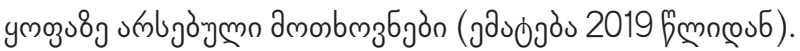

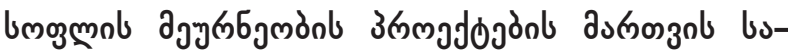

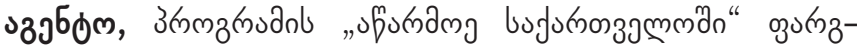

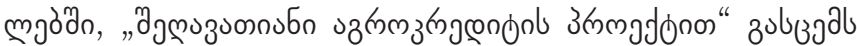

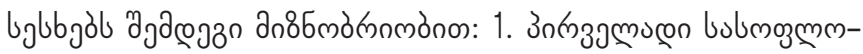

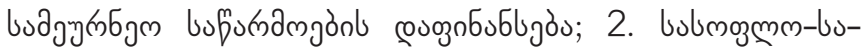

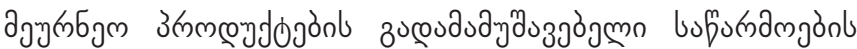

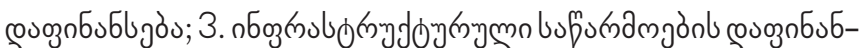

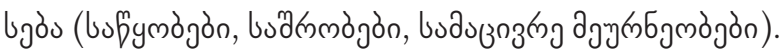

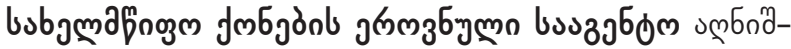

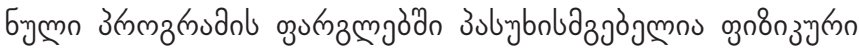
nбogm

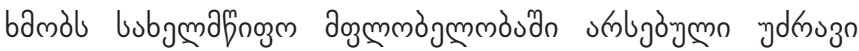

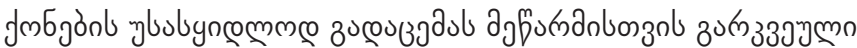

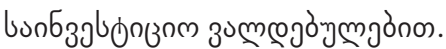

2019 fmonl aunbnl on

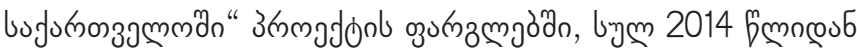

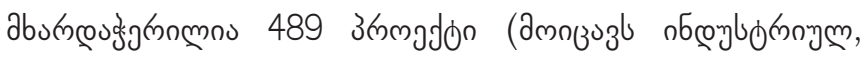

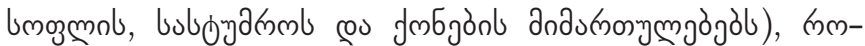

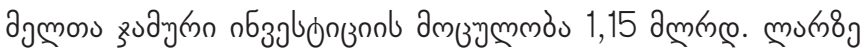

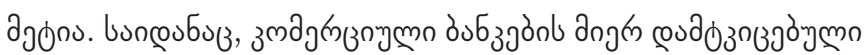

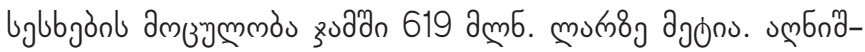

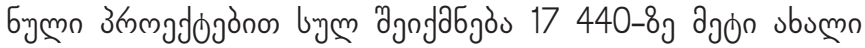

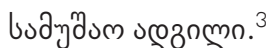

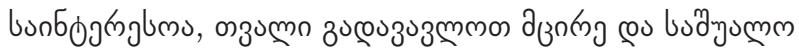

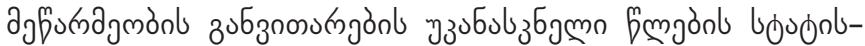

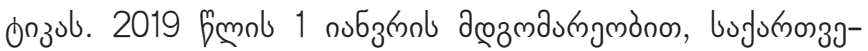

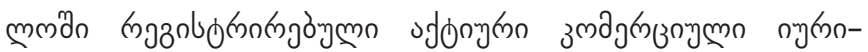

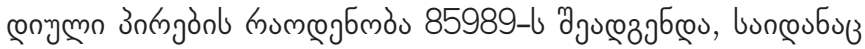

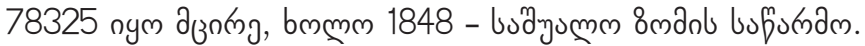

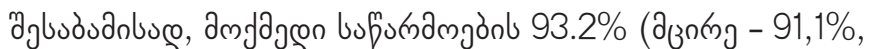

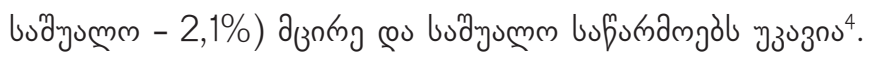

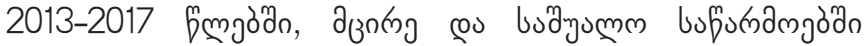

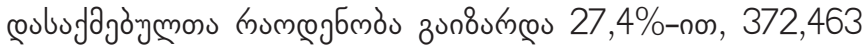

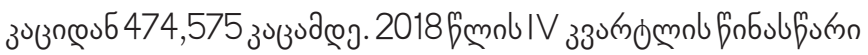

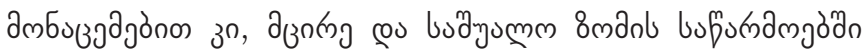

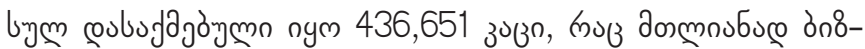

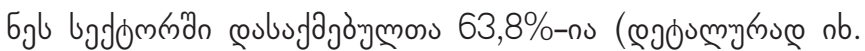
conozmoas).

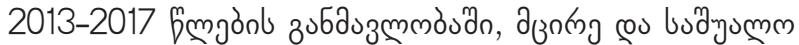

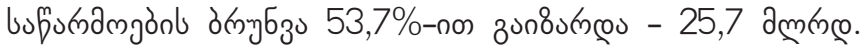

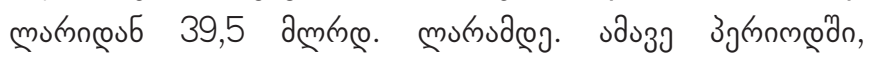

3 fyıґnn: www.enterprisegeorgia.gov.ge/ka/bnubm

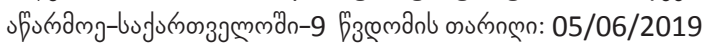

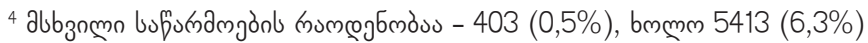

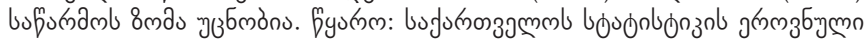
buabubingn

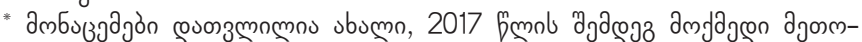

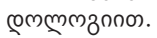

fysmm: www.geostat.ge/ka/modules/categories/326/sacarmota-statisti-

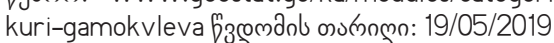




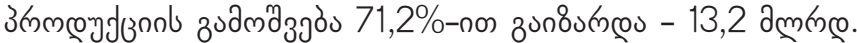

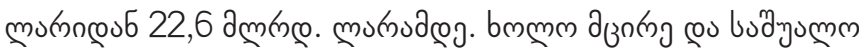

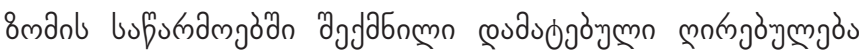

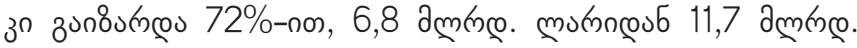

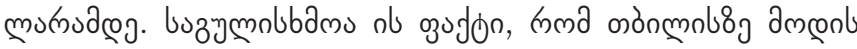

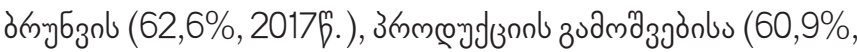

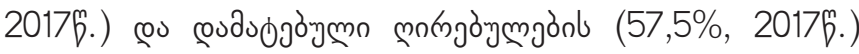

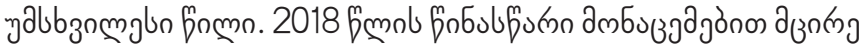

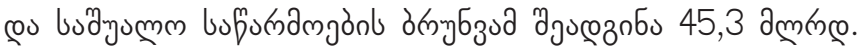

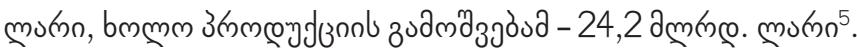

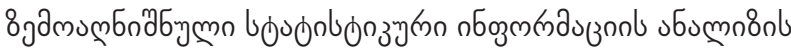

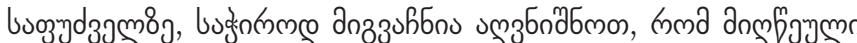

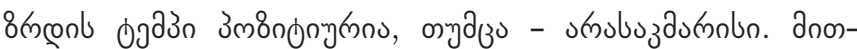

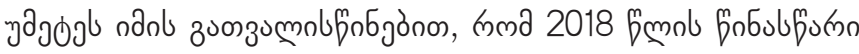

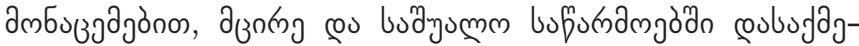

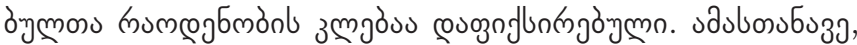

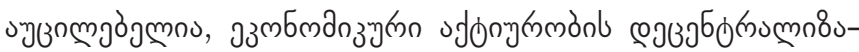

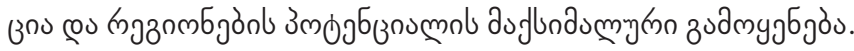

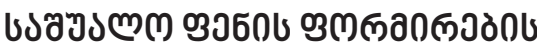

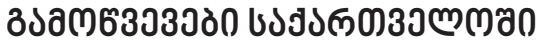

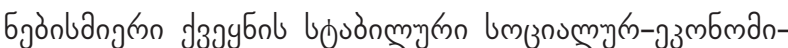

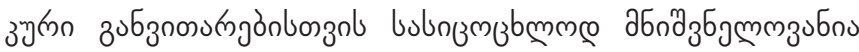

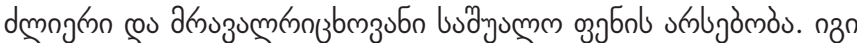

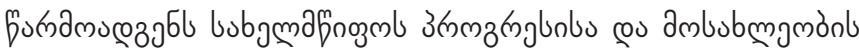

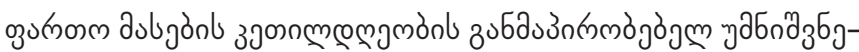

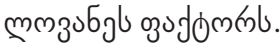

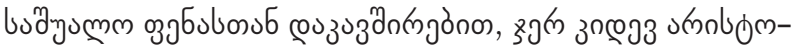

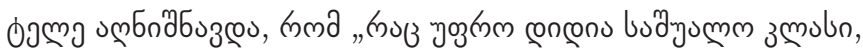

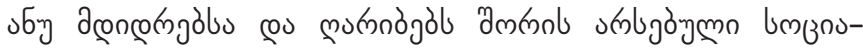

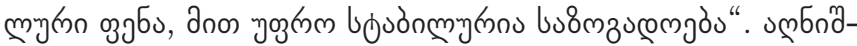

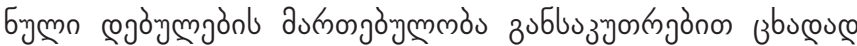

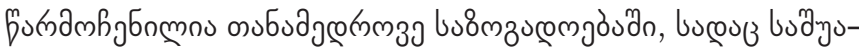

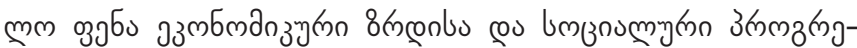

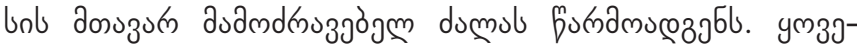
mп3э

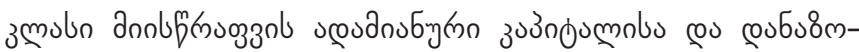

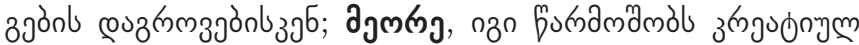

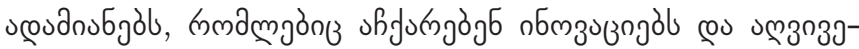

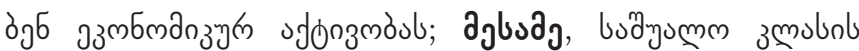

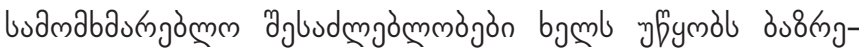

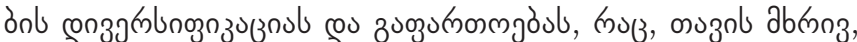

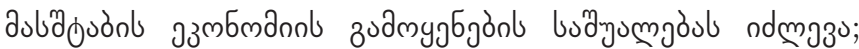

5 булюm: www.geostat.ge/ka/modules/categories/326/sacarmota-

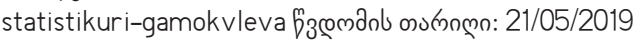

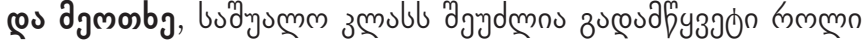

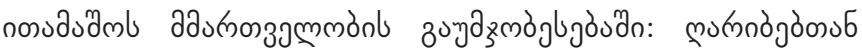

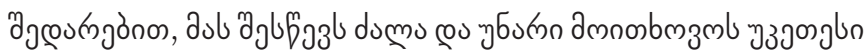

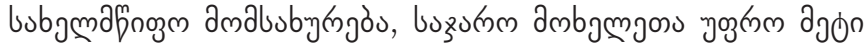

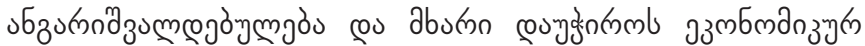

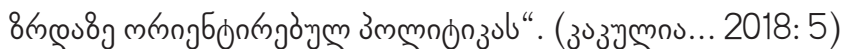

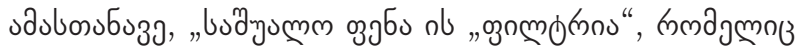

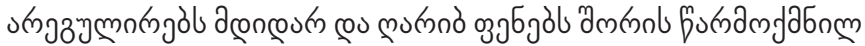

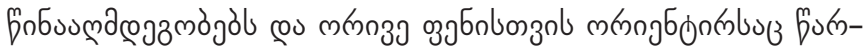

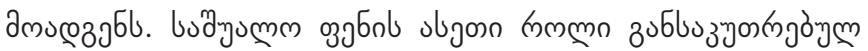

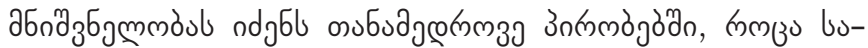

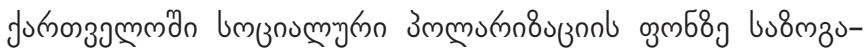

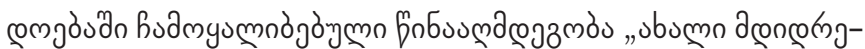

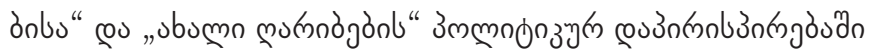
zucougno". (gुnosubuzu, 2018: 106)

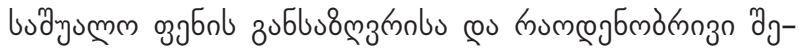

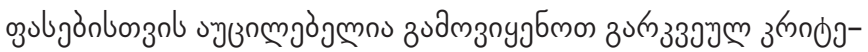

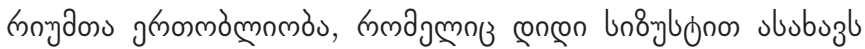

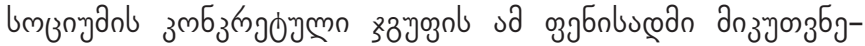

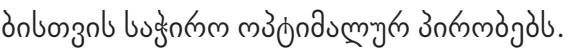

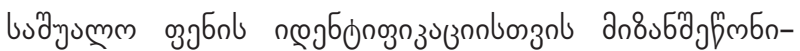

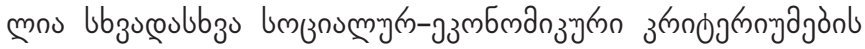

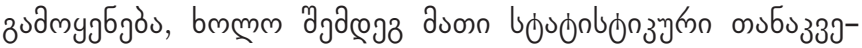
mol zublus

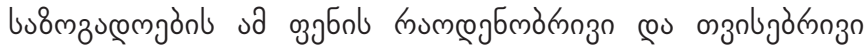

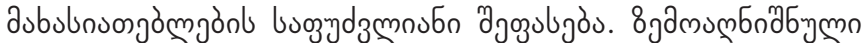

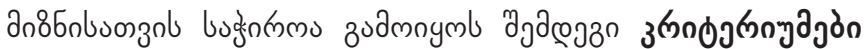

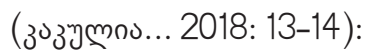

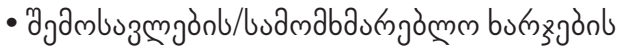

z

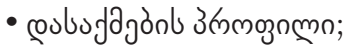

- zubuonmadol combj;

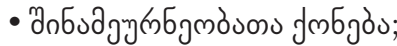

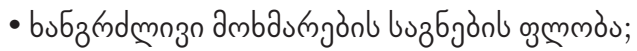

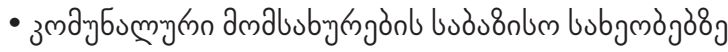
бзгода;

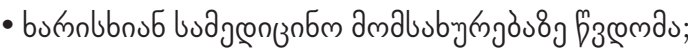

- couluzabjónl ajbudmà̃mmós;

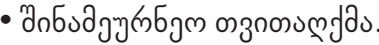

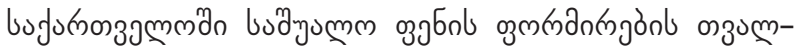

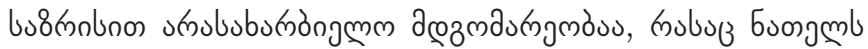

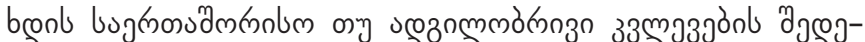

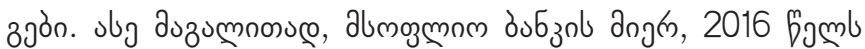

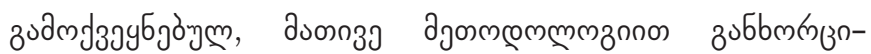

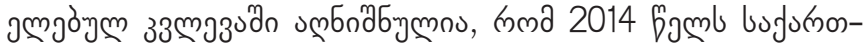




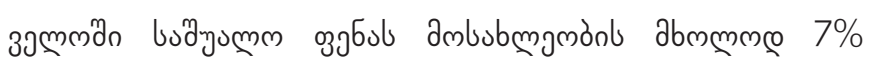

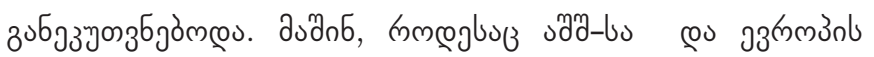

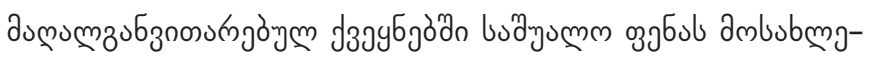

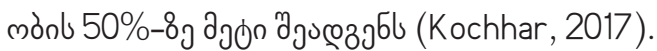

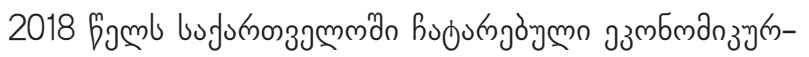

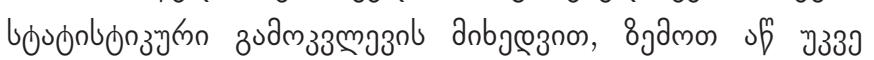

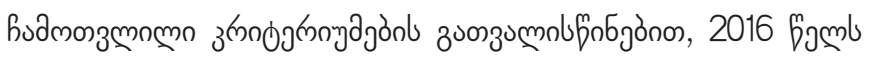

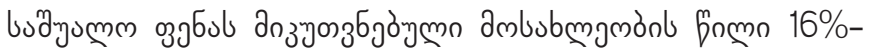

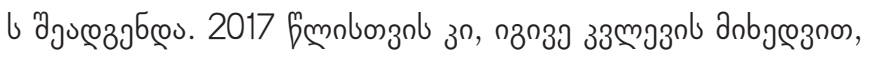

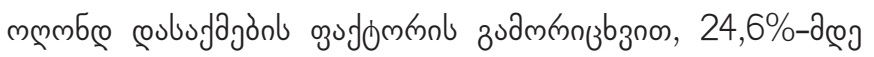

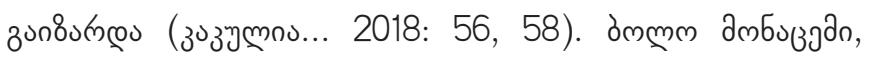

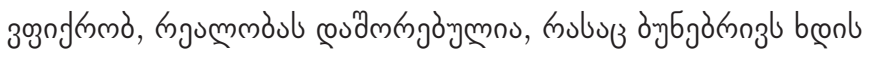

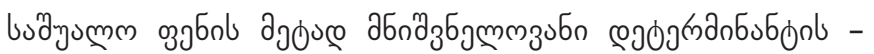

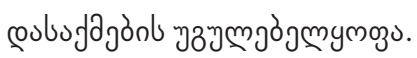

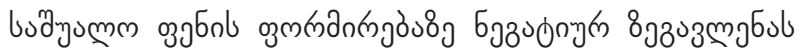

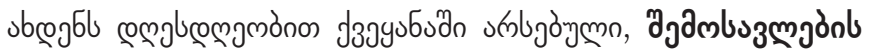

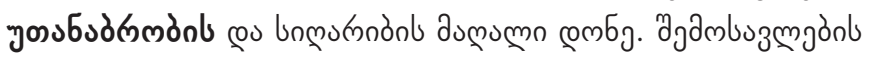

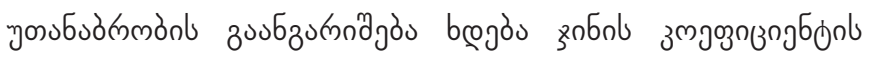

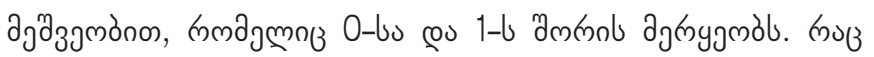

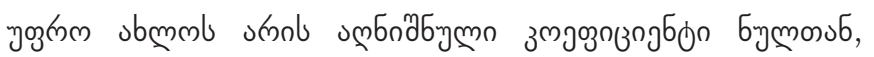

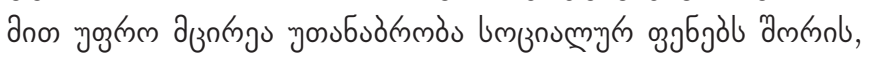

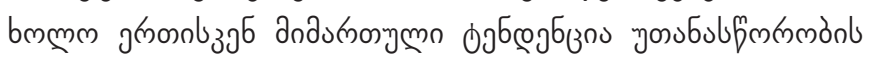

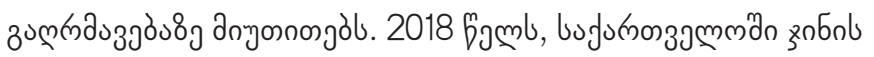

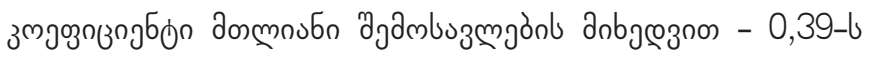

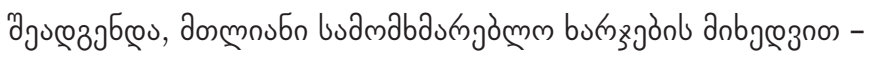

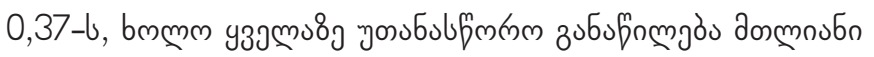

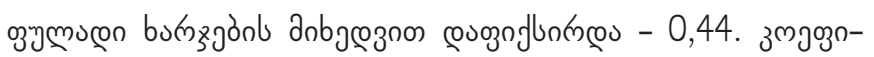

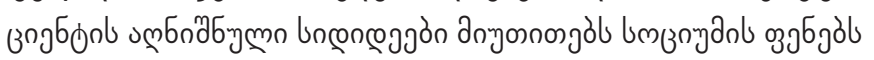

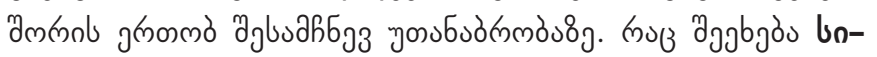

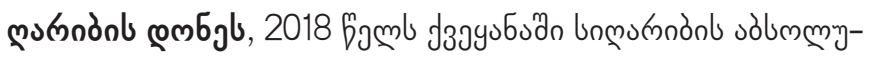

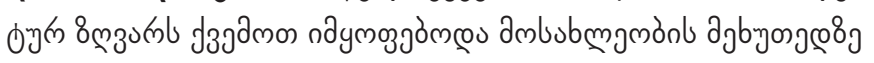
ajon - 20,1\%.

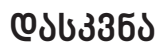

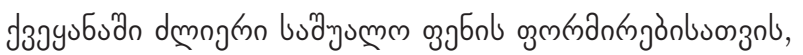

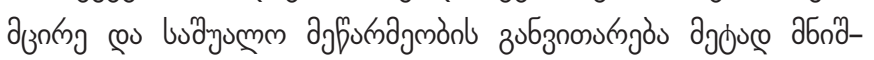

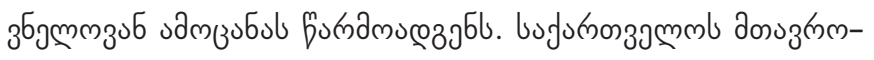

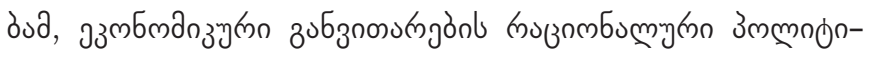

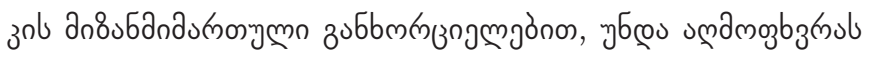

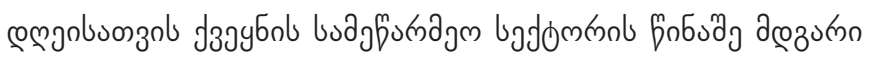

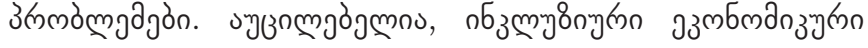

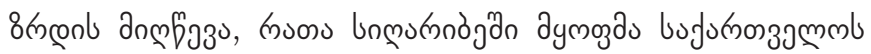

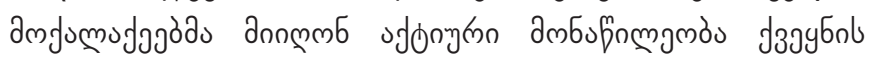

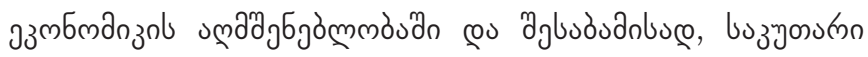

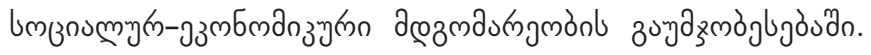

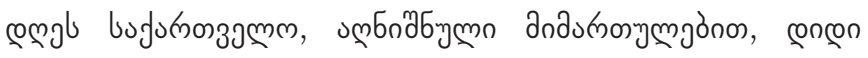

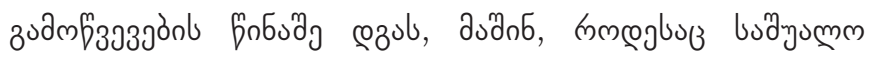

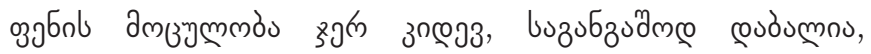

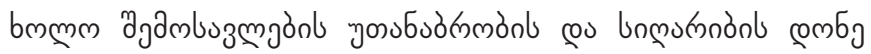

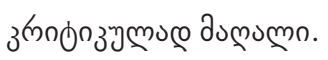

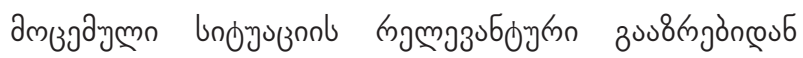

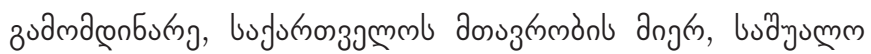

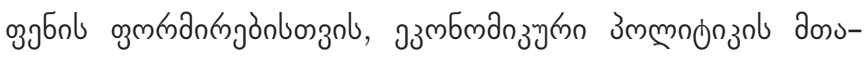

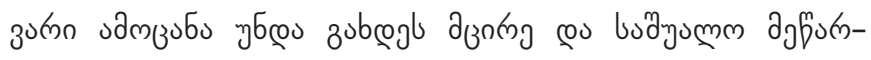

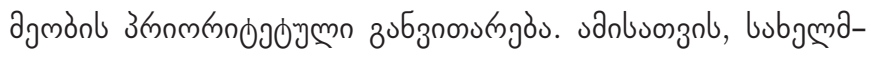

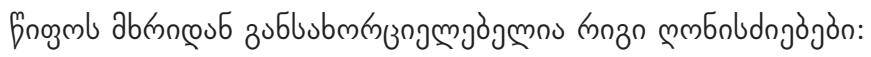

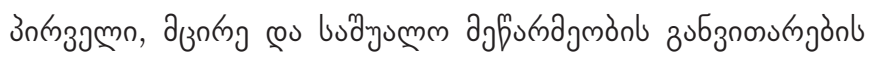

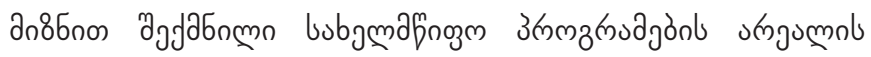

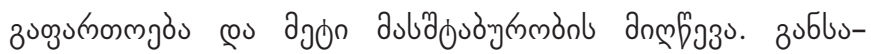

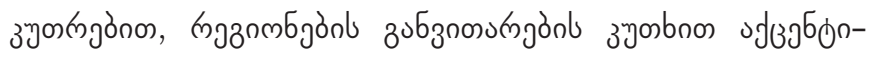

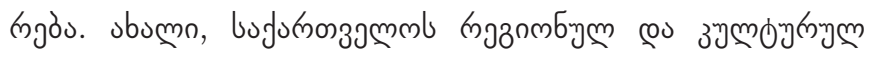
๓uznl

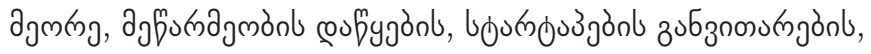

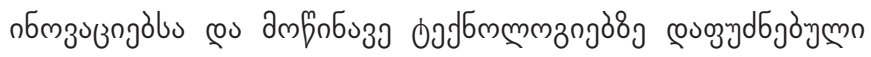

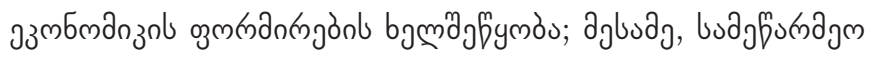

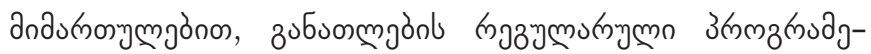

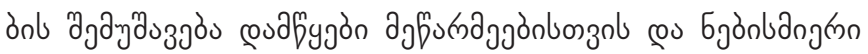

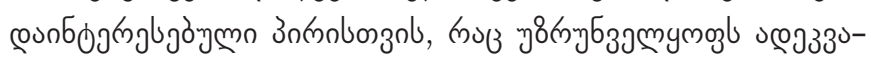

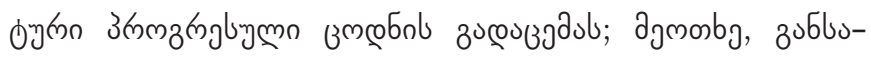

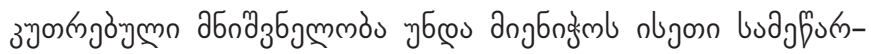

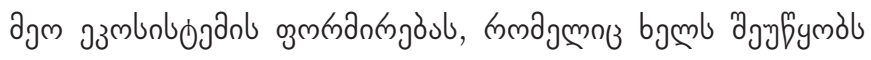

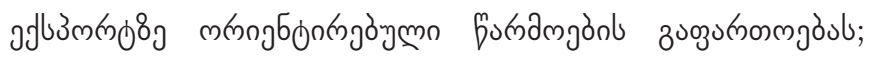

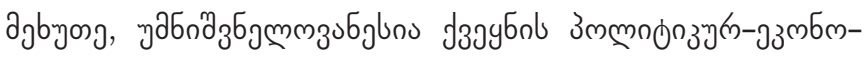

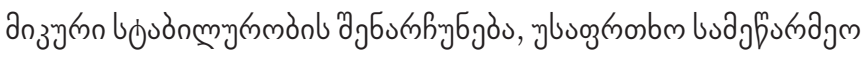

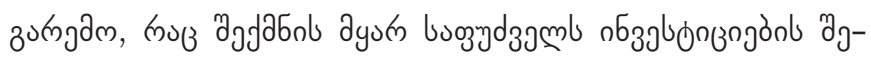

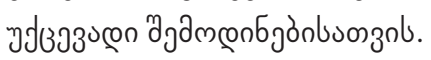

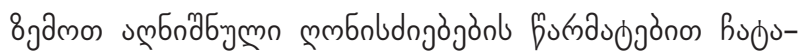

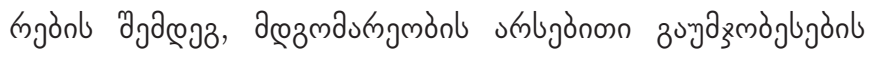

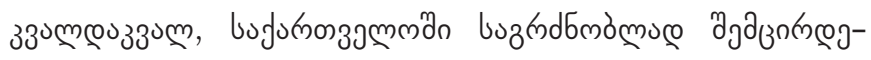

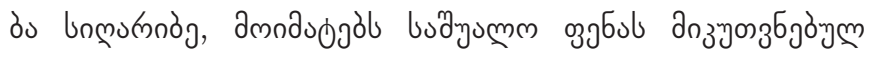

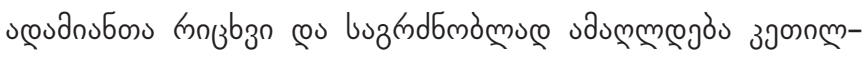
exgmòn bugrnom comby. 


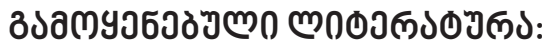

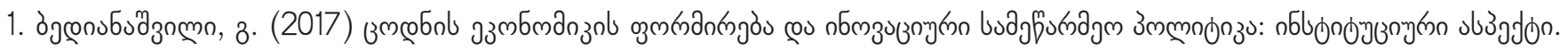
जुgु Entrepreneurial Policy: Institutional Aspects. Journal “Globalization and Business", N3 P.10-16]

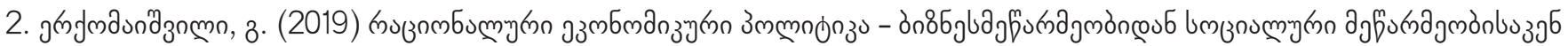

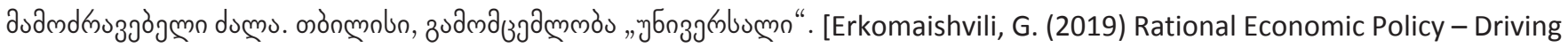
force from business to social entrepreneurship. Publishing House "UNIVERSAL", Tbilisi]

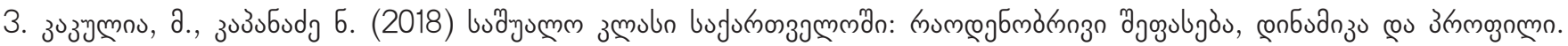

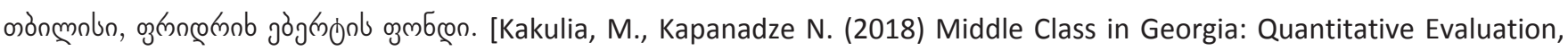
Dynamics and Profile. Friedrich Ebert Foundation, Tbilisi]

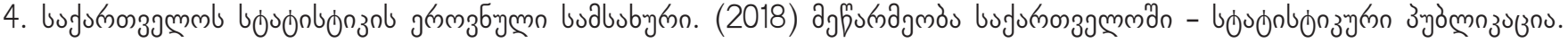
os̀nmolon. [National Statistics Office of Georgia. (2018) Entrepreneurship in Georgia - Statistical Publication. Tbilisi]

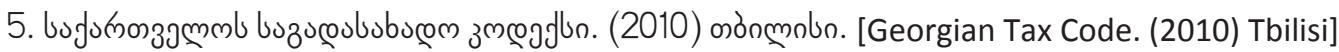

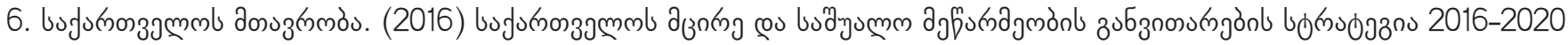
fmgònbonzol. ob̀nmonbn. [Government of Georgia. (2016) Georgia's Small and Medium Entrepreneurship Development Strategy for 2016-2020. Tbilisi]

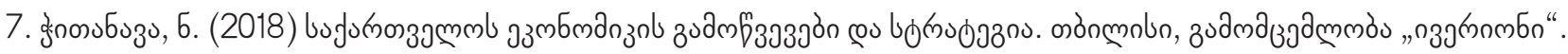

[Chitanava, N. (2018) Challenges and Strategy of the Economy of Georgia. Publishing House "IVERIONI", Tbilisi]

8. Kochhar, Rakesh (2017) Middle Class Fortunes in Western Europe. Luxembourg Income Study (LIS), asbl. https://www. econstor.eu/bitstream/10419/169262/1/702.pdf

9. Pew Research Center (2015) The American Middle Class Is Losing Ground. https://www.pewsocialtrends.org/2015/12/09/ the-american-middle-class-is-losing-ground

10. World Bank Group, GEORGIA: RECENT TRENDS AND DRIVERS OF POVERTY REDUCTION. August 17 2016. http://pubdocs. worldbank.org/en/980951472223098077/Georgia-PPA-FY16-presentation-AUG2016-final.pdf

11. www.geostat.ge

12. www.enterprisegeorgia.gov.ge

13. www.apma.ge

14. www.nasp.gov.ge 


\section{DEVELOPMENT OF SMALL AND MEDIUM ENTREPRENEURSHIP AND FORMATION OF A MIDDLE CLASS IN GEORGIA}

\section{AMIRANI MAGLAKELIDZE}

PhD Student

Ivane Javakhishvili Tbilisi State University, Georgia

maglakelidze.amirani@gmail.com

KEYWORDS: DEVELOPMENT OF SMALL AND MEDIUM ENTREPRENEURSHIP; MIDDLE CLASS; INCOME INEQUALITY; POVERTY

For citation: Maglakelidze A., (2019), Development Of Small And Medium Entrepreneurship And Formation Of A Middle Class In Georgia, Globalization And Business, №7, pp. 123-129. (In Georgian). https://doi.org/10.35945/gb.2019.07.015

\section{SUMMARY}

Formation of a strong middle class in Georgia requires a development of small and medium entrepreneurship. As a result of implementation of a rational economic policy and realization of the knowledge and technology based approach in this field, increasing the number of employment, improvement of the national enterprise possibilities, enhancing the competitiveness of the production and growth of the overall level of prosperity of population could be achieved. Small and medium enterprises have been growing during the recent years, however, the growth rate is not sufficient.

Share of the population belonging to the middle class is still low in Georgia. According to the studies conducted by the World Bank, in 2014 only 7\% of the population of Georgia belonged to the middle class. According to the economicstatistical study conducted in Georgia in 2018, share of the population belonging to the middle class in 2016 was $16 \%$. The same study, with exclusion of the employment factor, shows the growth of the above-mentioned share to $24,6 \%$. Ignoring the employment - very important determinant of the middle class - naturally puts the last figure far from reality. Current income inequality and high level of poverty negatively impact formation of the middle class in the country.

In order to achieve the growth of the small and medium entrepreneurship, and respectively, growth of the middle class share, the following actions should be taken by the Government of Georgia: Increase of the scale of small and medium entrepreneurship programs, maximally inclusion of the regions; Support of the start-ups, formation of innovative economy; Promotion of the entrepreneurial education, formation of the corresponding environmental system for the export oriented industries; Maintaining political and economic stability. 


\section{STUDY OF THE DISTRIBUTION OF WEALTH IN THE MIDDLE AND TOP SEGMENTS OF THE POPULATION OF GEORGIA}

\section{QETEVAN PIPIA}

Phd student

Samtskhe-Javakheti State University, Georgia

qetino.pipia@gmail.com

\section{KEYWORDS: PARETO DISTRIBUTION, LOGNORMAL DISTRIBUTION, MODELS OF ECONOMICAL LAYERS OF SOCIETY}

For citation: Pipia Q. (2019), Study Of The Distribution Of Wealth In The Middle And Top Segments Of The Population Of The Population Of Georgia, Globalization And Business, №7, pp. 130-142. https://doi.org/10.35945/gb.2019.07.016

Due to social, economic, political and other differences, the distribution of the population by income is a subject of different laws of distributions, for example, of a normal distribution, a log-normal distribution, Pareto distribution, and so on. In some countries the distribution of elements of society by income per unit of time in the lower and middle groups will be close to normal [10] (later it turned out that the distribution of income in the lower and middle groups better described by a log-normal distribution). The Italian economist Wilfredo Pareto remarked that in the richer segments of society, the income corresponding to the elements of society would be much higher than in the case of a normal distribution, because the large income, in contrast to the small and medium, makes it possible to invest in growth. The study of this phenomenon led to a distribution with two parameters, which is also found in statistics, sociology, etc., and is called the Pareto distribution [1].

The following distribution with two parameters $a$ and $v$ is called Pareto distribution:

$$
f=\frac{a}{x^{v}} \text {. }
$$

In [10] the structure of society consisting of three groups is considered: the bottom $10 \%$, with the average distribution of GDP I (low), the average $80 \%$ with the average distribution of GDP $\mathrm{m}$ (middle), the top $10 \%$, - with the average distribution of GDP $t$ ( top). If we denote the share of GDP per capita by $d$, then we get the balance equation

$$
0,1 l+0,8 m+0,1 t=d \text {. }
$$

Indeed,

$$
l=\frac{G D P(\text { low })}{N_{\text {low }}}=\frac{G D P(\text { low })}{0.1 N}, \quad m=\frac{G D P(\text { middle })}{N_{\text {middle }}}=\frac{G D P(\text { middle })}{0.8 N}, \quad t=\frac{G D P(\text { top })}{N_{\text {top }}}=\frac{G D P(\text { top })}{0.1 N},
$$

and it follows the equality (1):

$$
0,1 l+0,8 m+0,1 t=\frac{G D P(\text { low })}{N}+\frac{G D P(\text { middle })}{N}+\frac{G D P(\text { top })}{N}=\frac{G D P}{N}=d .
$$

The ratio of funds is defined as the ratio of incomes of the same number population, but belonging to different income groups. When $10 \%$ is selected from the upper and lower levels, this ratio is called decile ratio [8].

In the United States, the ratio of the upper $90 \%-95 \%$ layer to the lower $5 \%$ layer is often considered [4] since the incomes of the upper $5 \%$ layer are subject of other laws of distribution. This ratio is called the rank coefficient. We will consider the ratio of the incomes of the upper $10 \%$ stratum to the incomes to the lower $10 \%$ stratum, i.e. decimal coefficient D. Since the number of people in these groups is the same, this ratio is equal to the ratio of the average incomes of these groups:

$$
D=\frac{t}{l}
$$

Then from (Error! Reference source not found.) it follows 


$$
0,1(D+1) l+0.8 m=d
$$

If $N$ is a size of a population, then the GDP consumed by the lower, middle and upper groups (respectively $Q_{l}, Q_{m}, Q_{t}$ ) will be

and therefore

$$
Q_{l}=0,1 N l, \quad Q_{m}=0,8 N m, \quad Q_{t}=0,1 N t=0,1 N D l
$$

there $Q$ is the GDP.

$$
Q_{l}+Q_{m}+Q_{t}=Q=N d
$$

Let us suppose that the number $f$ of members of the top layer who has an income $q$ is expressed by the Pareto distribution

$$
f=\frac{a}{q^{v}}
$$

there $a>0, v>2$.

Proposition 1. Let $q_{t}$ be the minimal income of members of the top group, $N_{t}$ be the number of individuals who has the income more or equal to $q_{t}, q_{n} \geq q_{t}, N_{n}$ is the number of individuals who has the income more or equal to $q_{n}$. Then

Proof. It is clear, that

$$
N_{n}=N_{t}\left(\frac{q_{t}}{q_{n}}\right)^{v-1}
$$

$$
q f=\frac{a}{q^{v-1}}
$$

is the income of $f$ members of the top group. Then from (2) and (3) we get difeerential equations

$$
\frac{d N}{d q}=\frac{a}{q^{v}}, \quad q \frac{d N}{d q}=\frac{d Q}{d q}=\frac{a}{q^{v-1}} .
$$

Let $q_{\max }$ be a maximal income in the top gorup. Then

$$
N_{n}=\int_{q_{n}}^{q_{\max }} \frac{a}{q^{v}} d q, \quad Q_{n}=\int_{q_{n}}^{q_{\max }} \frac{a}{q^{v-1}} d q .
$$

Since no one has an income more than $q_{\max }$, we can assume that the upper bound in the integral is infinite:

$$
\begin{gathered}
N_{n}=\int_{q_{n}}^{\infty} \frac{a}{q^{v}} d q=\frac{a}{(v-1) q_{n}^{v-1}}, \\
Q_{n}=\int_{q_{n}}^{\infty} \frac{a}{q^{v-1}} d q=\frac{a}{(v-2) q_{n}^{v-2}} .
\end{gathered}
$$

After calculations we get

From these formulas it follows

$$
\begin{gathered}
N_{n}=\int_{q_{n}}^{\infty} \frac{a}{q^{v}} d q=\left.\frac{a q^{1-v}}{1-v}\right|_{q_{n}} ^{\infty}=\frac{a}{(v-1) q_{n}^{v-1}}, \\
Q_{n}=\int_{q_{n}}^{\infty} \frac{a}{q^{v-1}} d q=\left.\frac{a q^{2-v}}{2-v}\right|_{q_{n}} ^{\infty}=\frac{a}{(v-2) q_{n}^{v-2}}
\end{gathered}
$$

$$
Q_{n}=\frac{a}{(v-2) q_{n}^{\nu-2}}=\frac{a}{(v-1) q_{n}^{\nu-1}} \frac{(v-1) q_{n}^{\nu-1}}{(v-2) q_{n}^{\nu-2}}=\frac{v-1}{v-2} N_{n} q_{n}
$$

Now let $q_{n}=q_{t}$, where $q_{t}$ is the minimal income of the top layer (equivalently the maximal income of the middle layer). Then

$$
\begin{aligned}
N_{t} & =\frac{a}{(v-1) q_{t}^{\nu-1}} \\
Q_{t} & =\frac{a}{(v-2) q_{t}^{\nu-2}}
\end{aligned}
$$

Let us express $a$ by $N_{t}$ from (9) and substitute in (6). Then we get

We can write (6) and (8) shortly as

$$
N_{n}=\frac{a}{(v-1) q_{n}^{v-1}}=\frac{N_{t}(v-1) q_{t}^{\nu-1}}{(v-1) q_{n}^{v-1}}=N_{t}\left(\frac{q_{t}}{q_{n}}\right)^{v-1} .
$$

$$
\begin{gathered}
N_{n}=N_{t}\left(\frac{q_{t}}{q_{n}}\right)^{v-1}, \\
Q_{n}=\frac{v-1}{v-2} N_{n} q_{n} .
\end{gathered}
$$


The proposition is proved.

The formula of the sconomic structure of the upper layer of the society:

Let $q_{t}$ be a minimal income of the top layer, $N_{t}$ be the number of the individuals which has the income more or equal to $q_{t}$. Then

a) The number of individuals who have the income more or equal to $q_{n}$ and its whole income are respectively

$$
N_{n}=N_{t}\left(\frac{q_{t}}{q_{n}}\right)^{v-1}, Q_{n}=\frac{v-1}{v-2} N_{n} q_{n}
$$

b) The number of individuals whose income are between $q_{n_{1}}$ and $q_{n_{2}}$, is

and its whole income is

$$
N_{n_{2}}-N_{n_{1}}=N_{t}\left(\left(\frac{q_{t}}{q_{n_{2}}}\right)^{v-1}-\left(\frac{q_{t}}{q_{n_{1}}}\right)^{v-1}\right)=N_{t} q_{t}^{v-1}\left(\frac{1}{q_{n_{2}}^{v-1}}-\frac{1}{q_{n_{1}}^{v-1}}\right),
$$

The average income of top layer is

$$
Q_{n_{2}}-Q_{n_{1}}=\frac{v-1}{v-2}\left(N_{n_{2}} q_{n_{2}-} N_{n_{1}} q_{n_{1}}\right)
$$

$$
t=\frac{Q_{t}}{N_{t}}
$$

Corollary. Two parameters of Pareto distribution and other parameters are interlinked by formulas:

Proof. Let us write (12) for $Q_{t}$ :

$$
v=\frac{2 Q_{t}-N_{t} q_{t}}{Q_{t}-N_{t} q_{t}}=\frac{2 t-q_{t}}{t-q_{t}}=2+\frac{q_{t}}{t-q_{t}}>2, a=(v-1) q_{t}^{v-1} N_{t}
$$

$$
Q_{t}=\frac{v-1}{v-2} N_{t} q_{t}
$$

Now we must take into account that $Q_{t}=t N_{t}$. The second formula follows from (8).

Since usually $N_{t}$ and $q_{t}$ are known from practice, by these formulas we may calculate the parameters of Pareto distribution $v$ and $a$. If we substitute these expressions in (4), we get the following differential equations

$$
\frac{d N}{d q}=\frac{(v-1) N_{t}}{q_{t}}\left(\frac{q_{t}}{q}\right)^{v}, \frac{d Q}{d q}=(v-1) N_{t}\left(\frac{q_{t}}{q}\right)^{v-1} .
$$

Let us write (13) and (12) for $Q_{t}$ and $N_{t}$. Then the division of (12) on (11) give us the average income of the top layer $t$ :

$$
\frac{Q_{t}}{N_{t}}=\frac{v-1}{v-2} q_{t}=\bar{q}=t
$$

Let us suppose that the boundary values of low, middle and top layers are interlinked by each other by parameters $\alpha, \beta, \gamma$ :

$$
\text { Lower boundary of the low group } q_{l}=\frac{l}{\alpha} \text {; }
$$

Top boundary of the low group (since $l$ is small enough than $m$ ) $q_{m}=\frac{l+m}{\beta} \approx \frac{m}{\beta}=$

$$
\text { = Lower boundary of the middle group; }
$$

Top boundary of the middle group $=$ Lower boundary of the top group $q_{t}=\frac{\gamma m}{\beta}$.

The ratio of the top and lower boundaries of the middle group is

$$
\frac{\gamma m}{\beta}: \frac{l+m}{\alpha}=\frac{\alpha \gamma m}{\beta(l+m)} \approx \frac{\alpha \gamma}{\beta},
$$

since $l$ is small enough than $m$.

Let us substitute $q_{t}=\frac{\gamma m}{\beta}$ in (14):

$$
\frac{v-1}{v-2} q_{t}=\frac{v-1}{v-2} \frac{\gamma m}{\beta}=t, \quad \frac{v-1}{v-2}=\frac{\beta t}{\gamma m} ;
$$

If we suppose that $m=k l$ for some $k$, when we may estimate $D$ :

$$
\frac{v-1}{v-2}=\frac{\beta t}{\gamma m}=\frac{\beta t}{\gamma k l}, \quad D=\frac{t}{l}=\frac{\gamma k}{\beta} \frac{v-1}{v-2} .
$$

From this expression it is clear that the distribution in the top layer depends on the distribution in the low layer.

For example, let be

$$
\alpha=\beta=2, \gamma=3 \text {. }
$$

Then from (16) we get that the ratio of the top and low boundaries of the middle layer is $\gamma$. Then 
Lower boundary of the low group $q_{l}=\frac{l}{2}$;

Top boundary of the low group (since $l$ is small enough than $m$ ) $q_{m}=\frac{l+m}{2} \approx \frac{m}{2}=$

= Lower boundary of the middle group;

Top boundary of the middle group $=$ Lower boundary of the top group $q_{t}=\frac{3 m}{2}$.

Let us remark that in some countries, for example for the USA [10] such a choise of $\gamma$ is justified because a ratio of the top and low boundaries of the middle group is equal to $\gamma=3$.

The analysis of the above theoretical conclusions shows that for their application it is necessary to know the boundary between the middle and upper levels $q_{t}$ and the number of individuals in the upper layer $N_{t}$. It is not necessary that the top layer consist of 10 percent of the population.

Let us apply this results to Georgia. According to the World Bank [5], the population of Georgia in 2017 was distributed by Gross Domestic Product (GDP) as follows:

Distribution of the population (\%) according to GDP consumption in Georgia

$\begin{array}{rrrrrrrr}20176 . & 10 \% & 20 \% & 20 \% & 20 \% & 20 \% & 20 \% & 10 \% \\ & 2.6 & 6.7 & 11.5 & 16 & 22.2 & 43.6 & 28.1\end{array}$

From this list it follows that decile ratio is

$$
D=\frac{28.1}{2.6}=10.8 \approx 11
$$

The middle group consists with $80 \%$ of population and GDP consumed by him is

$$
(6.7-2.6)+11.5+16+22.2+(43.6-28.1)=69.3 \%
$$

Therefore the share of low, middle and top layers in GDP is equal respectively

$$
\frac{2.6}{10}=0.26, \quad \frac{69.3}{80}=0.86625, \quad \frac{28.1}{10}=2.81 .
$$

According to Geostat, the population of Georgia, GDP and GDP by purchasing power in 2017 were respectively

$$
N=3700000, \quad G D P=14,100,000,000 \$, G D P_{\text {purch }}=37,600,000,000 \$ \text {. }
$$

As a result, purchasing power coefficient in 2017 was

$$
\frac{37,600,000,000}{14,100,000,000}=2.67 \text {. }
$$

GDP per capita (by nominal and purchasing power) will be in Georgia

Since $10 \%$ and $80 \%$ of population are

$$
d=\frac{14,100,000,000}{3,700,000}=3810.811 \$, d_{\text {purch }}=\frac{37,600,000,000}{3,700,000}=10162.1622 \$ .
$$

\begin{tabular}{|r|r|}
\hline $0.1 \mathrm{~N}$ & $0.8 \mathrm{~N}$ \\
\hline 370000 & 2960000 \\
\hline
\end{tabular}

then for one representative of the population from the lower, middle and upper layers GDP is distributed approximately as follows (in dollars):

The same dates by purchasing power are equal to

$$
\begin{aligned}
l=\frac{14100000000 \cdot 0.26}{370000} & =990.81, \quad m=\frac{14100000000 \cdot 0.86625}{2960000}=3301.115, \\
t & =\frac{14100000000 \cdot 2.81}{370000}=10708.38 .
\end{aligned}
$$

$$
l_{\text {purch }}=2642, \quad m_{\text {purch }}=8803, \quad t_{\text {purch }}=28556 .
$$

In this example, we limit ourselves to nominal GDP.

By (17) the top bound of the consumption of GDP per capita in middle group, that is equal to the lower bound of the consumption of GDP per capita in top group is

$$
q_{t_{1}}=\frac{3 m}{2}=\frac{3 \cdot 3301}{2}=4952 \text {. }
$$

Similarly, the top bound of the consumption per capita in the middle group i.e. the lower bound of the consumption per capita in the top group (by purchasing power) is

$$
q_{t, \text { purch }_{1}}=\frac{3 m_{\text {purch }}}{2}=\frac{3 \cdot 8803}{2}=13204.46 .
$$


Now let's try to evaluate $q_{t}$ using some well-known economic indicator. According to [12] the subjective margin of wellbeing of one household in the upper middle class in 2017 was 1978 GEL per month. Since one household in Georgia consists approximately of 3.3 persons [13], then the subjective margin of well-being per capita in the upper middle class is 1978:3.3=599.394 GEL per month, and 599.394x12=7192.727 GEL per year. The dollar rate in 2017 was an average of 2.5 [11]. Therefore the subjective margin of well-being per capita in the upper middle class per year is 7192.727:2.5=2877.091\$. If we hypothesize that GDP per person is 1.5 times higher than its direct income, then we will get the maximum income of the upper middle class, that is, the lower boundary of the upper stratum

$$
q_{t_{2}}=2877.091 \times 1.5=4315.636 \$
$$

and because

$$
q_{t, \text { purch }_{2}}=11508.364 \text {. }
$$

If we substitues values of $q_{t_{1}}$ and $q_{t_{2}}$ in first formula of (14), we get two values of $v$ :

$$
v_{1}=2.860, \quad v_{2}=2.675 \text {. }
$$

The income of top groups will be

$$
\begin{gathered}
Q_{t}=0.281 \cdot 14,100,000,000=3,962,100,000, \\
Q_{t, \text { purch }}=0.281 \cdot 37,600,000,000=10,565,600,000,
\end{gathered}
$$

and number of members of top group will be

$$
N_{t}=3,700,000 \cdot 0.1=370,000 .
$$

Now we hypothesize that the rich stratum of the society in Georgia is described by the Pareto distribution - this hypothesis is widespread and is confirmed in the works of many authors, for example in [1], [9], [10]. The fact is that the exponent $e^{-\frac{x^{2}}{2}}$ decreases faster than the power function $\frac{1}{q^{v-1}}, v>2$. Therefore, Pareto distribution decreases more slowly than the normal distribution. This adequately reflects the fact that high returns allow you to invest.

As we know $q_{t_{1}}=4951.672 \$$. Let us compute the number of persons which share in GDP exceeds $10,000 \$$. This means that $q_{1}=10,000$. Formula (11) get

$$
N_{1}=N_{t}\left(\frac{q_{t_{1}}}{q_{1}}\right)^{\nu_{1}-1}=370000 *\left(\frac{4,951.672297}{10,000}\right)^{2.86-1} \approx 100090 .
$$

Also by formula (12) we have

$$
Q_{1}=\frac{v-1}{v-2} N_{1} q_{1}=\frac{2.86-1}{2.86-2} \cdot 100090 \cdot 10,000=2,164,532,303,
$$

which means that the share in GDP of 100090 persons exceeds $10,000 \$$, and they consume from GDP 2,164,532,303\$. In the lists below, the distribution of GDP (according to the nominal and the purchasing power) is calculated when the share of one person in GDP is more than $10,000 \$$, more than $100,000 \$$, more than $1,000,000 \$$, more than $10,000,000 \$$ and more than $100,000,000 \$$. From these tables, in particular, we can conclude that in Georgia's economy it is impossible to generate $10,000,000$ dollars during a year!

Now let us do the same calculations for $q_{t_{2}}=4315.636 \$$. As we know in this case $v_{2}=2.675$. We have:

$$
\begin{gathered}
N_{1}=N_{t}\left(\frac{q_{t_{2}}}{q_{1}}\right)^{v_{2}-1}=370000 *\left(\frac{4315.636}{10,000}\right)^{2.675-1} \approx 90546 ; \\
Q_{1}=\frac{v-1}{v-2} N_{1} q_{1} \frac{2.675-1}{2.675-2} \cdot 90546 \cdot 10,000=2,246,726,101 .
\end{gathered}
$$

\begin{tabular}{|r|r|r|r|r|r|r|r|r|r|r|}
\hline $\mathrm{t}$ & $\mathrm{qt}$ & $\mathrm{Nt}$ & $\mathrm{Qt}$ & $\mathrm{niu}$ & $\mathrm{q1}$ & $\mathrm{N1}$ & $\mathrm{Q1}$ & $\mathrm{q} 2$ & $\mathrm{N2}$ & Q2 \\
\hline 10708.38 & 4951.672 & 370000 & 3962100000 & 2.86015722 & 10000 & 100090.4 & 2164532303 & 100000 & 1381.131526 & 298680487.7 \\
\hline 28555.68 & 13204.46 & 370000 & 10565600000 & 2.86015722 & 20000 & 170922 & 7392643442 & 100000 & 8562.564407 & 1851721480 \\
\hline 10708.38 & 4315.636 & 370000 & 3962100000 & 2.675083767 & 10000 & 90546.42 & 2246726101 & 100000 & 1913.319504 & 474751519.4 \\
\hline 28555.68 & 11508.36 & 370000 & 10565600000 & 2.675083767 & 20000 & 146606.8 & 7275502195 & 100000 & 9892.835954 & 2454707063 \\
\hline
\end{tabular}




\begin{tabular}{|r|r|r|r|r|r|r|r|r|}
\hline \multicolumn{3}{|c|}{ domom6o } & \multicolumn{2}{|c|}{10 dom } & \multicolumn{2}{|c|}{100 dom } \\
\hline q3 & N3 & Q3 & q4 & N4 & Q4 & q5 & N5 & Q5 \\
\hline 1000000 & 19.05802178 & 41214461.72 & 10000000 & 0.262979 & 5687120 & 100000000 & 0.003629 & 784757 \\
\hline 1000000 & 118.153511 & 255516202.8 & 10000000 & 1.630382 & 35258288 & 100000000 & 0.022497 & 4865237 \\
\hline 1000000 & 40.42999906 & 100318861.8 & 10000000 & 0.854319 & 21198192 & 100000000 & 0.018052 & 4479351 \\
\hline 1000000 & 209.043679 & 518699590 & 10000000 & 4.417263 & $1.1 E+08$ & 100000000 & 0.09334 & 23160523 \\
\hline
\end{tabular}

If we compute $a$ for example, in the case $q_{t_{1}}$, we will get a huge number:

$$
a=(v-1) q_{t_{1}}^{v-1} N_{t}=5,128,692,385,795.29868 .
$$

A density of a lognormal distribution is

and a cumulative distribution function [9] is

$$
f(x)=\frac{1}{x \sigma \sqrt{2 \pi}} e^{-\frac{(\ln x-\mu)^{2}}{2 \sigma^{2}}}
$$

$$
F(x)=\frac{1}{\sigma \sqrt{2 \pi}} \int_{0}^{x} \frac{1}{x} e^{-\frac{(\ln x-\mu)^{2}}{2 \sigma^{2}}} d x .
$$

In the lognormal distribution a logarithm of a variables has a normal distribution. Indeed, in this case we must have

$$
F(x)=\frac{1}{\sigma \sqrt{2 \pi}} \int_{0}^{x} e^{-\frac{(\ln x-\mu)^{2}}{2 \sigma^{2}}} d(\ln x)=\frac{1}{\sigma \sqrt{2 \pi}} \int_{0}^{x} \frac{1}{x} e^{-\frac{(\ln x-\mu)^{2}}{2 \sigma^{2}}} d x .
$$

The log-normal distribution has two parameters: $\mu$ @s $\sigma>0$.

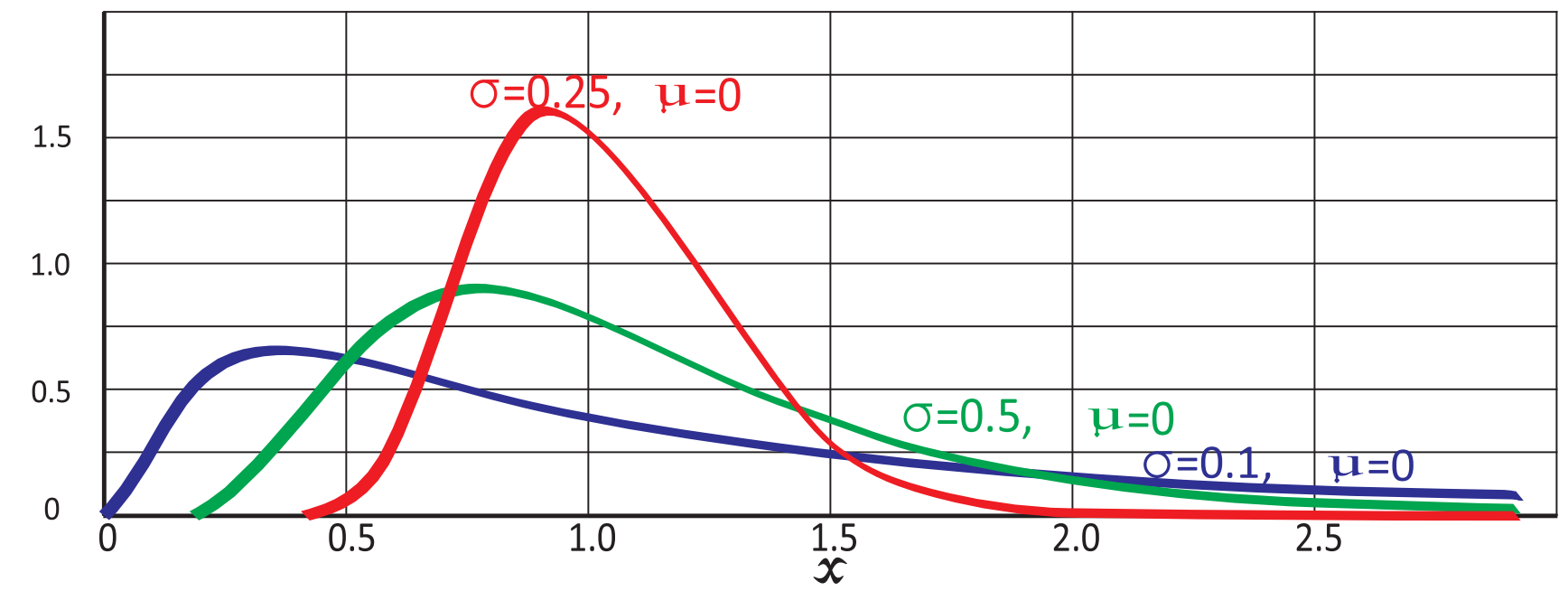

Picture 1.

The lognormal distribution is right asymmetric - it reduces slowly on the right (see Picture 1). In order to obtain the density of a distribution characterized by a left asymmetry, one must consider a distribution whose density is obtained by mirroring the density of the lognormal distribution with respect to a straight line passing through the mode parallel to the oY axis. Since the mode of the lognormal distribution is

$$
X_{\text {mod }}=e^{\mu-\sigma^{2}} \text {, and } K_{x}=X_{c} / X_{\text {mod }},
$$

then the density and the cumulative distribution function of left-asymmetric distribution will be

$$
\begin{gathered}
f(x)=\frac{1}{\left(2 e^{\mu-\sigma^{2}}-x\right) \sigma \sqrt{2 \pi}} e^{-\frac{\left(\ln \left(e^{\mu-\sigma^{2}}-x\right)-\mu\right)^{2}}{2 \sigma^{2}}}, \\
F(x)=\frac{1}{\sigma \sqrt{2 \pi}} \int_{0}^{x} \frac{1}{\left(2 e^{\mu-\sigma^{2}}-x\right)} e^{-\frac{\left(\operatorname { l n } \left(e^{\left.\left.\mu-\sigma^{2}-x\right)-\mu\right)^{2}}\right.\right.}{2 \sigma^{2}}} d x .
\end{gathered}
$$

See the plot of the density of the left-asymmetric distribution in Picture 2 below when $a=1, \sigma=1$. 


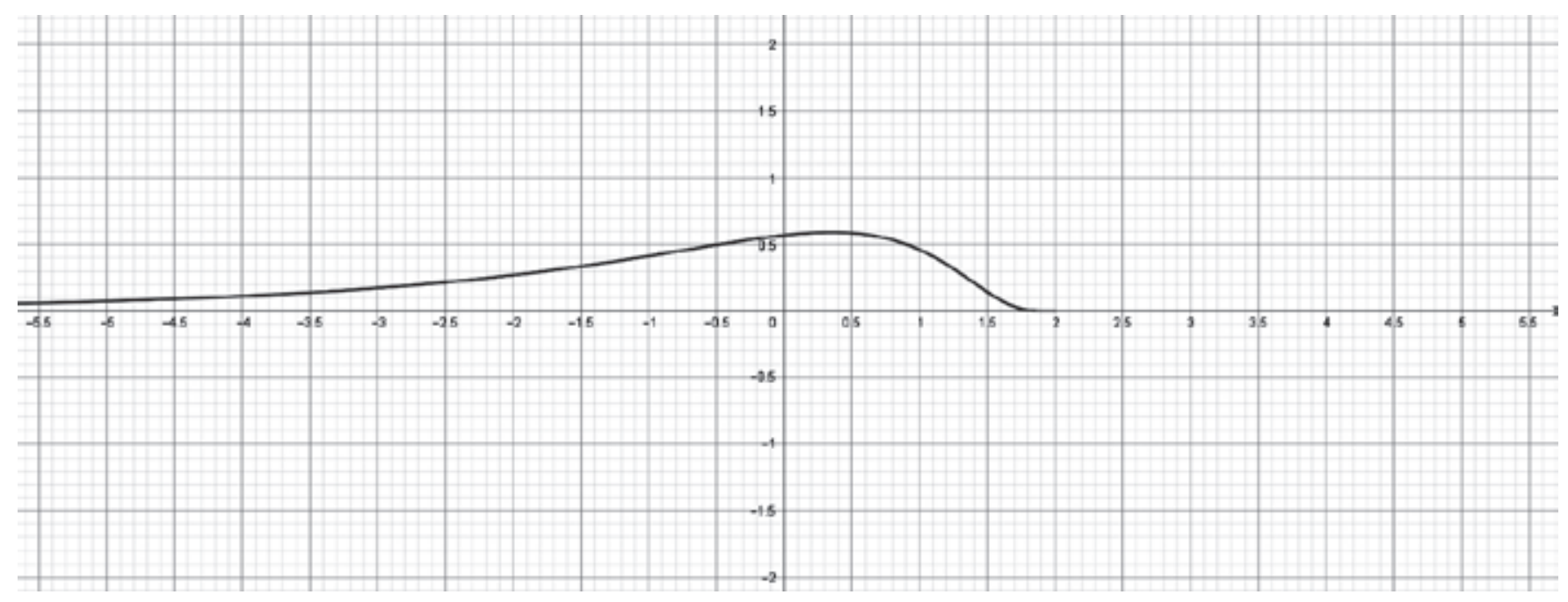

Picture 2.

Methods for computation of lognormal distribution parameters are described, for example, in [9]. Suppose we know the average monthly salary $X_{c}$ (which is calculated by counting macroparameters and size of the population) and the value of the logical function when the upper bound of the integral is $x_{i}$ :

$$
\frac{1}{\sigma \sqrt{2 \pi}} \int_{0}^{x_{i}} \frac{1}{x} e^{-\frac{\left(\ln (x-\mu)^{2}\right.}{2 \sigma^{2}}} d x=F\left(x_{i}\right)
$$

Let us try to calculate the values of $\mu$ and $\sigma$. Since a mean of the lognormal distribution is

then [9]

$$
E(X)=e^{\mu+0.5 \sigma^{2}}
$$

$$
X_{c}=e^{\mu+0.5 \sigma^{2}} .
$$

(18) and (19) give the system of two equations with two variables

$$
\left\{\begin{array}{c}
\frac{1}{\sigma \sqrt{2 \pi}} \int_{0}^{x_{i}} \frac{1}{x} e^{-\frac{(\ln (x-\mu))^{2}}{2 \sigma^{2}}} d x=F\left(x_{i}\right) \\
e^{\mu+0.5 \sigma^{2}}=X_{c}
\end{array} .\right.
$$

In the first equation of the system (20) let us apply the method of changing the variable

Since

$$
z=\frac{\ln (x-\mu)}{\sigma}
$$

$$
d z=\frac{1}{\sigma x} d x
$$

then

$$
\frac{1}{\sigma \sqrt{2 \pi}} \int_{0}^{x_{i}} \frac{1}{x} e^{-\frac{(\ln (x-\mu))^{2}}{2 \sigma^{2}}} d x=\frac{1}{\sqrt{2 \pi}} \int_{0}^{z_{i}} e^{-\frac{z^{2}}{2}} d z
$$

where

$$
z_{i}=\frac{\ln \left(x_{i}-\mu\right)}{\sigma} .
$$

We got a simpler system:

$$
\begin{gathered}
\frac{1}{\sqrt{2 \pi}} \int_{0}^{z_{i}} e^{-\frac{z^{2}}{2}} d z=F\left(x_{i}\right), \\
e^{\mu-0.5 \sigma^{2}}=X_{c} .
\end{gathered}
$$


In this system we have two variables $\mu$ and $\sigma$, because $z_{i}$ depends on $\mu$.

Consequently

$$
\frac{1}{\sqrt{2 \pi}} \int_{0}^{z_{i}} e^{-\frac{z^{2}}{2}} d z=F\left(x_{i}\right)
$$

With the standard method, for example, by Excel, we can find the value of $z_{i}$ which depends on $F\left(x_{i}\right)$. From the second equation of the system (20) it follows that

Let us substitute (22) in (21):

$$
\mu=\ln X_{c}-0.5 \sigma^{2}
$$

$$
z_{i}=\frac{\ln x_{i}-\ln X_{c}+0.5 \sigma^{2}}{\sigma} .
$$

Then because $\sigma \neq 0$, we get a quadratic equation

$$
\sigma^{2}-2 z_{i} \sigma-2 \ln \left(X_{c} / x_{i}\right)=0,
$$

which solution is

$$
\sigma=z_{i} \pm \sqrt{z_{i}^{2}+2 \ln \left(X_{c} / x_{i}\right)}
$$

Only the positive root is economically significant:

From (22) we get $\mu$ :

$$
\sigma=z_{i}+\sqrt{z_{i}^{2}+2 \ln \left(X_{c} / x_{i}\right)} .
$$

$$
\mu=\ln X_{c}-0.5 \sigma^{2} .
$$

Let us now test the hypothesis that the distribution of the declared income in Georgia is lognormal. To do this, we use the data received from Revenue Service of the Ministry of Finance of Georgia (see also [15]) about the declared incomes of the population of Georgia in 2009-2017:

\begin{tabular}{|c|c|c|c|c|c|c|c|c|}
\hline 2009 & 2010 & 2011 & 2012 & 2013 & 2014 & 2015 & 2016 & 2017 \\
\hline 762,855 & 846,045 & 987,426 & $1,118,636$ & $1,104,222$ & $1,181,484$ & $1,116,625$ & $1,185,065$ & $1,189,718$ \\
\hline
\end{tabular}

List 3. The number of people with declared income, 2009-2017.

\begin{tabular}{|c|c|c|c|c|c|c|c|c|c|}
\hline $1-100$ & $100-200$ & $200-300$ & $300-400$ & $400-500$ & $500-600$ & $600-800$ & $800-1000$ & $\begin{array}{c}1000- \\
1250\end{array}$ & $\begin{array}{c}1250- \\
1666\end{array}$ \\
\hline 205,435 & 135,886 & 93,448 & 79,784 & 47,829 & 34,594 & 51,159 & 32,846 & 25,209 & 23,315 \\
\hline
\end{tabular}

\begin{tabular}{|c|c|c|c|c|c|c|c|c|c|}
\hline $1666-2083$ & $2083-2500$ & $2500-3333$ & $\begin{array}{c}3333- \\
4166\end{array}$ & $\begin{array}{c}4166- \\
5000\end{array}$ & $\begin{array}{c}5000- \\
5833\end{array}$ & $\begin{array}{c}5833- \\
6666\end{array}$ & $\begin{array}{c}6666- \\
7500\end{array}$ & $\begin{array}{c}7500- \\
8333\end{array}$ & $>8333$ \\
\hline 11,046 & 6,518 & 7,178 & 3,230 & 1,766 & 1,126 & 703 & 472 & 331 & 1,980 \\
\hline
\end{tabular}

List 4. The distribution of the population by declared incomes, 2009

\begin{tabular}{|c|c|c|c|c|c|c|c|c|c|}
\hline $1-100$ & $100-200$ & $200-300$ & $300-400$ & $400-500$ & $500-600$ & $600-800$ & $800-1000$ & $\begin{array}{c}1000- \\
1250\end{array}$ & $\begin{array}{c}1250- \\
1666\end{array}$ \\
\hline 297469 & 134559 & 103185 & 84494 & 75525 & 60955 & 90192 & 63427 & 55643 & 56682 \\
\hline
\end{tabular}

\begin{tabular}{|c|c|c|c|c|c|c|c|c|c|}
\hline $1666-2083$ & $2083-2500$ & $2500-3333$ & $3333-4166$ & $\begin{array}{c}4166- \\
5000\end{array}$ & $\begin{array}{c}5000- \\
5833\end{array}$ & $\begin{array}{c}5833- \\
6666\end{array}$ & $\begin{array}{c}6666- \\
7500\end{array}$ & $\begin{array}{c}7500- \\
8333\end{array}$ & $>8333$ \\
\hline 25878 & 15266 & 15887 & 8093 & 4646 & 2929 & 1937 & 1339 & 1097 & 5128 \\
\hline
\end{tabular}




\begin{tabular}{|c|c|c|c|c|c|c|c|c|c|}
\hline $1-100$ & $100-200$ & $200-300$ & $300-400$ & $400-500$ & $500-600$ & $600-800$ & $800-1000$ & $\begin{array}{c}1000- \\
1250\end{array}$ & $\begin{array}{c}1250- \\
1666\end{array}$ \\
\hline 230,280 & 125,013 & 99,417 & 83,823 & 75,525 & 67,610 & 115,023 & 88,848 & 80,873 & 83,744 \\
\hline
\end{tabular}

\begin{tabular}{|c|c|c|c|c|c|c|c|c|c|}
\hline $1666-2083$ & $2083-2500$ & $2500-3333$ & $3333-4166$ & $4166-5000$ & $5000-5833$ & $5833-6666$ & $6666-7500$ & $7500-8333$ & $>8333$ \\
\hline 42,423 & 24,361 & 27,309 & 14,204 & 8,141 & 5,233 & 3,784 & 2,609 & 1,884 & 9,614 \\
\hline
\end{tabular}

List 6. The distribution of the population by declared incomes, 2017

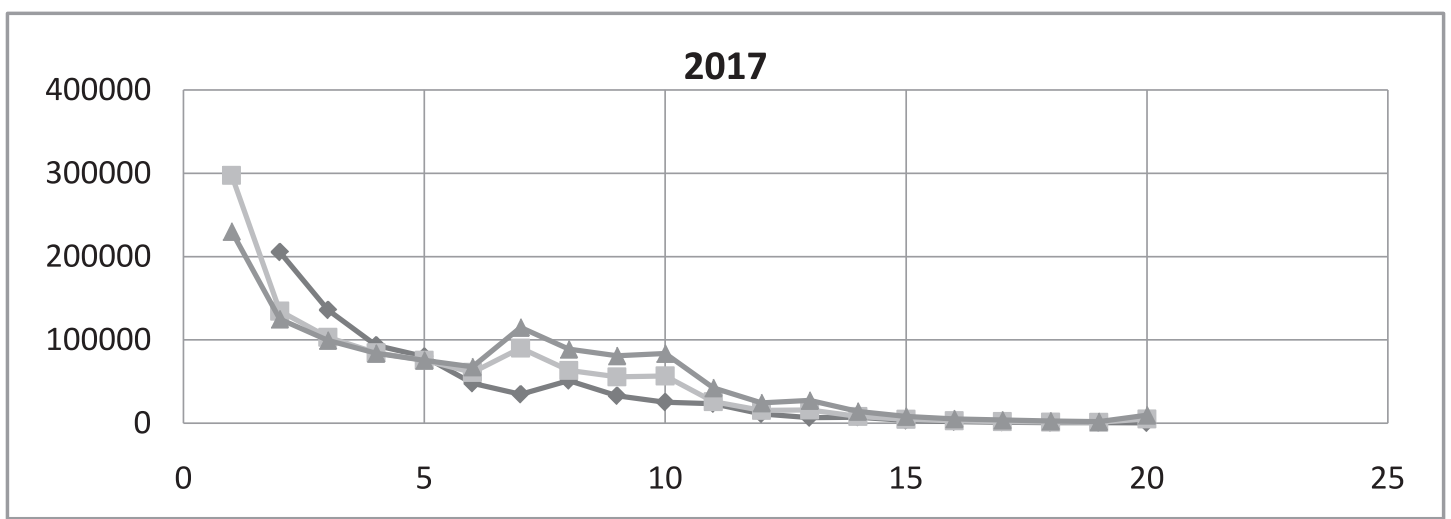

Picture 3. The distribution of the population by declared incomes:

$\checkmark$ - 2009, $\Delta-2013, \quad \square-2017$

Based on Table 6 we will create a new Table 7:

\begin{tabular}{|c|c|c|c|c|c|c|c|c|c|c|c|}
\hline 2017 & $x_{i}$ & $\mathrm{~N}$ & $\Delta F\left(x_{i}\right) \%$ & $F\left(x_{i}\right) \%$ & $F\left(x_{i}\right)$ & 2017 & $x_{i}$ & $\mathrm{~N}$ & $\Delta F\left(x_{i}\right) \%$ & $F\left(x_{i}\right) \%$ & $F\left(x_{i}\right)$ \\
\hline 1 & 0 & & 0 & 0 & 0 & 12 & 2083 & 42423 & 3.565803 & 91.83512 & 0.918351 \\
\hline 2 & 100 & 230280 & 19.35585 & 19.35585 & 0.193558 & 13 & 2500 & 24361 & 2.047628 & 93.88275 & 0.938828 \\
\hline 3 & 200 & 125013 & $\begin{array}{ll}3 & 10.50778\end{array}$ & 29.86363 & 0.298636 & 14 & 3333 & 27309 & 2.295418 & 96.17817 & 0.961782 \\
\hline 4 & 300 & 99417 & 8.35635 & 38.21998 & 0.3822 & 15 & 4166 & 14204 & 1.193896 & 97.37207 & 0.973721 \\
\hline 5 & 400 & 83823 & 37.045619 & 45.2656 & 0.452656 & 16 & 5000 & 8141 & 0.68428 & 98.05635 & 0.980563 \\
\hline 6 & 500 & 75525 & 6.348143 & 51.61374 & 0.516137 & 17 & 5833 & 5233 & 0.439852 & 98.4962 & 0.984962 \\
\hline 7 & 600 & 67610 & 5.682859 & 57.2966 & 0.572966 & 18 & 6666 & 3784 & 0.318059 & 98.81426 & 0.988143 \\
\hline 8 & 800 & 115023 & 9.668089 & 66.96469 & 0.669647 & 19 & 7500 & 2609 & 0.219296 & 99.03355 & 0.990336 \\
\hline 9 & 1000 & 88848 & 3.467988 & 74.43268 & 0.744327 & 20 & 8333 & 1884 & 0.158357 & 99.19191 & 0. \\
\hline 10 & 1250 & 80873 & 6.797661 & 81.23034 & 0.812303 & 21 & 16000 & 6014 & 0.505498 & 99.69741 & 0.996974 \\
\hline 11 & 1666 & 83744 & 7.038979 & 88.26932 & 0.882693 & $22>$ & 16000 & 3600 & 0.302593 & 100 & 1 \\
\hline
\end{tabular}

List 7.

The first column shows the numbers of the intervals, in the second - the intervals of declared income, in the third - the number of persons corresponding to the intervals. For example, this table shows that the monthly income from 0 to $100 \mathrm{GEL}$ have 230280 people, from 100 to $200 \mathrm{GEL}$ - 125013 people, etc. The fourth column shows how many percent are the third column data from the number of persons with declared income (1189718 person), in the fifth column - the accumulated value of these percent. In the sixth column, these percentages are converted to fractions of a unit.

At first, let us calculate Gini index of the declared monthly income for 2017. Let's agree that when calculating the Gini index we will not take into account incomes exceeding 16,000 GEL per month, since in this case the Gini index will be inadequate. Let us construct the Lorenc curve. In List 8 the data are translated into a convenient form for constructing Lorenz curve: 


\begin{tabular}{|c|c|c|c|c|c|}
\hline$x_{i}$ & $F\left(x_{i}\right)$ & $x_{i}$ & $x_{i} / 16000$ & $F\left(x_{i}\right)$ & $x_{i} / 16000$ \\
\hline 0 & 0 & 0 & & & \\
\hline 100 & 0.193558 & 100 & 0.00625 & 0.193558 & 0.00625 \\
\hline 200 & 0.298636 & 200 & 0.0125 & 0.298636 & 0.0125 \\
\hline 300 & 0.3822 & 300 & 0.01875 & 0.3822 & 0.01875 \\
\hline 400 & 0.452656 & 400 & 0.025 & 0.452656 & 0.025 \\
\hline 500 & 0.516137 & 500 & 0.03125 & 0.516137 & 0.03125 \\
\hline 600 & 0.572966 & 600 & 0.0375 & 0.572966 & 0.0375 \\
\hline 800 & 0.669647 & 800 & 0.05 & 0.669647 & 0.05 \\
\hline 1000 & 0.744327 & 1000 & 0.0625 & 0.744327 & 0.0625 \\
\hline 1250 & 0.812303 & 1250 & 0.078125 & 0.812303 & 0.078125 \\
\hline 1666 & 0.882693 & 1666 & 0.104125 & 0.882693 & 0.104125 \\
\hline 2083 & 0.918351 & 2083 & 0.130188 & 0.918351 & 0.130188 \\
\hline 2500 & 0.938828 & 2500 & 0.15625 & 0.938828 & 0.15625 \\
\hline 3333 & 0.961782 & 3333 & 0.208313 & 0.961782 & 0.208313 \\
\hline 4166 & 0.973721 & 4166 & 0.260375 & 0.973721 & 0.260375 \\
\hline 5000 & 0.980563 & 5000 & 0.3125 & 0.980563 & 0.3125 \\
\hline 5833 & 0.984962 & 5833 & 0.364563 & 0.984962 & 0.364563 \\
\hline 6666 & 0.988143 & 6666 & 0.416625 & 0.988143 & 0.416625 \\
\hline 7500 & 0.990336 & 7500 & 0.46875 & 0.990336 & 0.46875 \\
\hline 8333 & 0.991919 & 8333 & 0.520813 & 0.991919 & 0.520813 \\
\hline 16000 & 0.996974 & 16000 & 1 & 1 & 1 \\
\hline$>16000$ & 1 & $>16000$ & & & \\
\hline
\end{tabular}

List 8 .

Here we divide $\boldsymbol{x}_{\boldsymbol{i}}$ to 16000 , then rearrange $x_{i} / 16000$ and $F\left(x_{i}\right)$. The last two columns will give us a Lorenz curve:

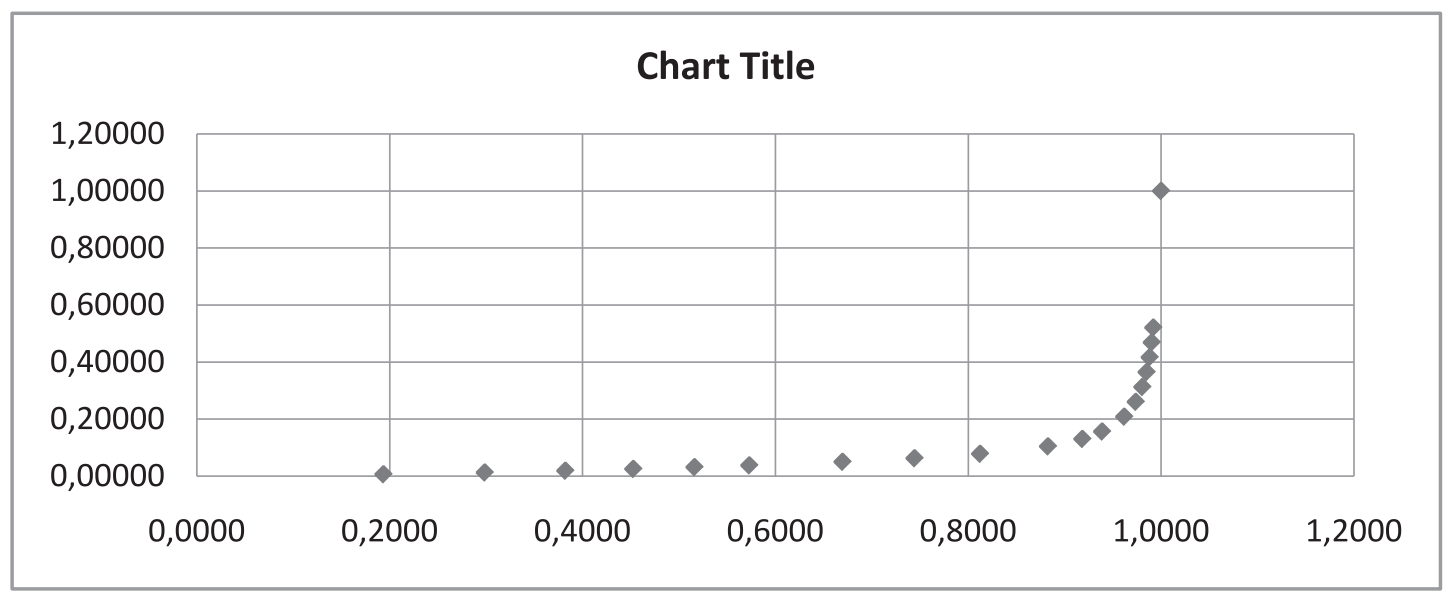

Picture 4

If $p_{i}$ part of population, $i=1,2, \ldots, n, p_{1}+p_{2}+\cdots+p_{n}=1$, owns $q_{i}$ part of a wealth, where $i=1,2, \ldots, n, q_{1}+$ $q_{2}+\cdots+q_{n}=1$, with a cumulative form $s_{i}=q_{1}+q_{2}+\cdots+q_{i}$, then Gini index can be calculated by formula [2]

$$
G=1-\sum_{i=1}^{n} p_{i}\left(s_{i}+s_{i-1}\right) \text {. }
$$

The plot in Picture 4 is almost rectangular, therefore the distribution of declared income is extremely uneven. It is easy to calculate that the Gini index corresponding to the plot on Picture 4 is equal to $\quad G=0.89$. 
According to Geostat [14] the average annual salary in the public and private sectors of Georgia in 2017 was $X_{c}=989$ GEL. For example, let us choose $\mathrm{i}=1$; According to table 3,

$$
F\left(x_{1}\right)=0.193558,
$$

i.e.

By Excel we can calculate that

$$
\frac{1}{\sqrt{2 \pi}} \int_{0}^{z_{1}} e^{-\frac{z^{2}}{2}} d z=0.193558
$$

$$
z_{1}=-0.86486
$$

Since

$$
\ln \left(X_{c} / x_{i}\right)=2.291524 \text {, }
$$

the quadratic equation (23) takes the form

$$
\sigma^{2}+1.72972 \sigma-4.583048291=0 .
$$

It has the positive solution

\begin{tabular}{|c|c|c|c|c|c|c|c|}
\hline $\mathrm{i}$ & $z_{i}$ & $X_{c} / x_{i}$ & $\ln \left(\frac{X_{c}}{c}\right)$ & $\sigma$ & $\mu$ & $X_{\text {mod }}$ & $K_{x}$ \\
\hline 1 & -0.86486 & 9.89 & 2.291524 & 1.444044 & 5.854063 & 43.32801 & 22.82587876 \\
\hline 2 & -0.52833 & 4.945 & 1.598377 & 1.336045 & 6.004186 & 67.97694 & 14.54905095 \\
\hline 3 & -0.29971 & 3.296667 & 1.192912 & 1.273711 & 6.085524 & 86.76335 & 11.39882192 \\
\hline 4 & -0.11895 & 2.4725 & 0.90523 & 1.231827 & 6.137995 & 101.5546 & 9.738605958 \\
\hline 5 & 0.040462 & 1.978 & 0.682086 & 1.20914 & 6.165684 & 110.3508 & 8.962327139 \\
\hline 6 & 0.183931 & 1.648333 & 0.499765 & 1.200474 & 6.176126 & 113.8622 & 8.68593753 \\
\hline 7 & 0.438938 & 1.23625 & 0.212083 & 1.224325 & 6.147208 & 104.4007 & 9.473114755 \\
\hline 8 & 0.656743 & 0.989 & -0.01106 & 1.296422 & 6.05634 & 79.48999 & 12.4418183 \\
\hline 9 & 0.886416 & 0.7912 & -0.2342 & 1.449733 & 5.845832 & 42.27126 & 23.39651375 \\
\hline 10 & 1.188558 & 0.593637 & -0.52149 & 1.796586 & 5.282834 & 7.807727 & 126.6693799 \\
\hline 11 & 1.394067 & 0.474796 & -0.74487 & 2.067625 & 4.759157 & 1.622688 & 609.4826824 \\
\hline 12 & 1.545005 & 0.3956 & -0.92735 & 2.274621 & 4.309745 & 0.421408 & 2346.894138 \\
\hline 13 & 1.771747 & 0.29673 & -1.21493 & 2.613898 & 3.480462 & 0.035014 & 28245.6943 \\
\hline 14 & 1.938529 & 0.237398 & -1.43802 & 2.877604 & 2.756392 & 0.003989 & 247931.6508 \\
\hline 15 & 2.065528 & 0.1978 & -1.6205 & 3.078152 & 2.159184 & 0.000665 & 1487386.178 \\
\hline 16 & 2.169088 & 0.169553 & -1.77459 & 3.244149 & 1.634444 & 0.000138 & 7179570.988 \\
\hline 17 & 2.261717 & 0.148365 & -1.90808 & 3.401544 & 1.111444 & 2.87E-05 & 34475203.52 \\
\hline 18 & 2.339125 & 0.131867 & -2.02596 & 3.530586 & 0.664175 & 7.5E-06 & 131900542 \\
\hline 19 & 2.405241 & 0.118685 & -2.13128 & 3.639183 & 0.274867 & $2.33 \mathrm{E}-06$ & 424101439.1 \\
\hline 20 & 2.744959 & 0.061813 & -2.78365 & 4.147634 & -1.70474 & $6.15 E-09$ & $1.6094 \mathrm{E}+11$ \\
\hline
\end{tabular}

$$
\sigma=1.444044073 .
$$

Then from (22) we have

$$
\mu=\ln 989-0.51 .444044073^{2}=5.85406269 .
$$

The cases $i=2,3, \ldots, 20$ are considered in List 9:

List 9.

For example let us write the lognormal distribution for data from the row 8:

$$
F\left(x_{i}\right)=\frac{1}{1.2964 \sqrt{2 \pi}} \int_{0}^{x_{i}} \frac{1}{x} e^{-\frac{(\ln (x-6.0563))^{2}}{2 * 1.2964^{2}}} d x
$$


From List 9 it is clear that with a certain accuracy it can be argued that the intervals of incomes $300-800$ GEL and 41667500 GEL is described by the log-normal distribution. Such a conclusion can be drawn because the variance and the expectation change at the same time in an insignificant way.

\section{REFERENCES:}

1. Arnold, B. C., (2015). Pareto Distributions. London: CRC Press.

2. Bellu, L. \& Liberati, P., (2006). Inequality and Axioms for its Measurement. N.-Y.: FAO.

3. Cowell, F. A., (2009). Measuring Inequality. London: Oxford University Press.

4. University of Texas., 2018. Measuring Inequality Project. https://utip.lbj.utexas.edu/tutorials.html.

5. World Bank, (2017). Percentage share of income or consumption. http://wdi.worldbank.org/table/1.3.

6. Goskomstat Rossii, (1996). Methodological provisions on statistics. Moscow (in Russian)

7. Kolmakov I. B., (2008). Methods of forecasting poverty indicators, taking into account disadvantaged groups. Problemy Prognozirovania, Issue 5, pp. 95-109 (in Russian).

8. Kolmakov I. B., (2015). Methodology of calculations and analyzes of integral estimates of the polarization of money incomes of the population. Voprosy Statistiki, pp. 23-36 (in Russian).

9. Kolmakov I. B., (2016). Conjugation of the logarithmically normal distribution of the population in terms of household income with the Pareto distribution. Audit i Finansovyj Analiz, pp. 124-131 (in Russian).

10. Rozanov V. B., (2007). Economic structure of the Russian society. In: Econophysics. Moscow, pp. 560-600 (in Russian).

11. 2018. https://m2b.ge. [Online] Available at: https://m2b.ge/post/177009-laris-kursis-dinamika-2017-wels-mizezebi-damolodinebi [Accessed 7 July 2019].

12. Kakulia M., (2018). Middle class in Georgia: quantitative assessment, dynamics And profile. Tbilisi in Georgian).

13. GeoStat, (2016). http://census.ge/. [Online] Available at: http://census.ge/files/results/Census\%20Release_GEO.pdf [Accessed 7 July 2019].

14. GeoStat, (2018). [Online] Available at: http://www.geostat.ge/?action=page\&p_id=148\&lang=geo [Accessed 27092018$].$

15. Tabula, (2018). Only $0.6 \%$ of Georgian population has more than 5,000 GEL per month [Online] Available at: http://tbl. ge/2t1g [Accessed 29. 09. 2018] (in Georgian). 


\section{STUDY OF THE DISTRIBUTION OF WEALTH IN THE MIDDLE AND TOP SEGMENTS OF THE POPULATION OF GEORGIA}

\section{QETEVAN PIPIA}

\section{Phd student}

Samtskhe-Javakheti State University, Georgia qetino.pipia@gmail.com

\section{KEYWORDS: PARETO DISTRIBUTION, LOGNORMAL DISTRIBUTION, MODELS OF ECONOMICAL LAYERS OF SOCIETY.}

For citation: Pipia Q. (2019), Study Of The Distribution Of Wealth In The Middle And Top Segments Of The Population Of The Population Of Georgia, Globalization And Business, №7, pp. 130-142. https://doi.org/10.35945/gb.2019.07.016

\section{SUMMARY}

Due to economic, social, political and other differences, different sectors of society are subject to different laws of distribution. Among these laws are Pareto distribution, the normal distribution, the lognormal distribution, and so on. It is noteworthy that the higher, richer stratum of a society more often depends on the Pareto distribution. As for the poor and middle class, there was an attempt to build their model using a normal distribution. But later it turned out that more accurate results are provided by a lognormal distribution. The article attempts to build a model of the distribution of the upper layers of the population of Georgia in terms of per capita GDP consumption (according to the World Bank) using Pareto distribution. As for the other layers, due to the lack of data in GeoStat, when trying to build a model using a lognormal distribution, data on the population's declared income are used, obtained from the Revenue Service of the Ministry of Finance of Georgia, hoping that this data correlates with the population distribution by GDP consumption. 


\section{SOCIO-ECONOMIC-GENDER DISBALANCES IN GEORGIA}

\section{NINO ABESADZE}

\section{Associate Professor}

Ivane Javakhishvili Tbilisi State University, Georgia

nino.abesadze@tsu.ge

\section{NATALIA ROBITASHVILI}

\section{Assistant Professor}

Batumi Shota Rustaveli State University, Georgia

robnato@mail.ru

\section{OTAR ABESADZE}

\section{Assistant Professor}

Caucasus University, Georgia

o.abesadze@gmail.com

\section{KEYWORDS: EMPLOYMENT, GENDER, STEREOTYPE, INDEXES, RATE}

For citation: Abesadze N., Robitashvili N., Abesadze O. (2019), Socio-Economic-Gender Disbalances In Georgia, Globalization And Business, №7, pp. 143-147. https://doi.org/10.35945/gb.2019.07.017

\section{INTRODUCTION}

The considered issue is very urgent for the Georgian reality in both, theoretical and practical respects, as the analysis and forecast of the employment dynamics in gender respect is necessary for the development and realization of the proper gender policy. The present reality of Georgia makes it necessary to significantly improve the information and methodological background of the abovedescribed problems. The work gives the modified employment indices in gender respect and major results of the economic analysis of the problem. In addition, it includes comparative analysis of unemployment in Georgia in gender respect, and the estimated figures of unemployment are gained by using modern mathematical and computing methods. (Abesadze, 2013)

A policy of gender equality made Georgia one of the most reformative countries in Caucasus. However, in spite of many positive steps, there are certain problems related to a low level of civil consciousness and strong societal stereotypes. Caucasian mentality and patriarchal environment also aggravate the problems. Probably this is one of the reasons, why according to gender index, Georgia lags behind countries, such as Tanzania, Ghana, Vietnam, Uzbekistan, Nicaragua and others. According to this classification in 2016,
Georgia occupies 90th position from 144 countries. The top three countries of global gender index are Iceland, Norway and Finland. Indicators of Montenegro, Uruguay, Greece and Indonesia are close to us, but we are ahead of Japan, Armenia, Hungary, Slovakia and others.

According to the above-mentioned report, number of women has increased at the global market since 2006 and comprises 250 million. However, inequality of wages still remains a problem. According to GIA, a gender disbalance is corrected by $4 \%$ in the fields, such as health protection, education, economy and policy. (Global-gender-gap-report-2016) We may say that political efforts to correct the gender disbalance are taking place, but in other fields, such as employment, we have many problems and some of them are quite deep.

Georgian Parliament has enacted a law against a domestic violence in 2006 and a law about a gender equality in 2010 to guarantee women's security, equality at the labor market and women's political integration. Election code has been changed in 2012 to support political parties, if they tried to protect the gender balance. However, this regulation didn't bring significant improvement. After parliamentary elections of 2012 , women occupied only $11 \%$ of parliamentary mandates, but this number was higher than in the previous parliament. 
At the local level, representation of women is even weaker. In 2016, number of women has increased compared to 2012, but level of representation of women is still low in Georgian parliament. From 150 deputies of Georgian parliament of 2017, women are only 22 and it is only $14.7 \%$. We have the same picture at the executive level. From 11 ministers only 3 are women. In parliaments of certain countries $30-40 \%$ are women. (Georgian law about gender equality, 2010). In private sector percent of employed women is higher, but majority of them occupies low-wage positions, whereas majority of men has executive positions or their wages are higher. Society still has an opinion, that it is better for women to play less active and less prestigious professional roles to combine societal activities and family business (and spend more time in caring family and children).

\section{METHODOLOGY/METHODS}

Efficient employment statistical analysis in gender respect is possible only by using relevant statistical methods. The work, through the combined application of methods of statistical observation, grouping and analysis, studies the employment dynamics in gender respect based on the method of fixed numbers. This has enabled us to identify the nature of employment changes in gender respect and impact of the factors on the changes of this phenomenon functionally linked to the resulting index. (Abesadze, 2013).

Analysis methods of the economic science were

Fig. 1.Unemployment Rate by Gender.(\%) employed, among them, statistical data processing, data grouping, inductive-deductive data analysis methods. The scientific study employs surveying, observation, as well as comparative, analytical, and graphical methods, which are used by the author to compare and analyse facts and assess solutions to specific issues. (Abesadze,2014).

\section{RESULTS}

Studying gender issues of employment and correcting the policy aiming at guaranteeing the employment gender equality on its basis is one of the priorities of the present government of Georgia. [Abesadze,2013.] The problem of unemployment is one of the acutest problems in Georgia and as the results of different statistical, conjunctive or social surveys evidence, often ranks first among the most important social-economic or political problems the country and Georgian people face. The problem of unemployment is one of the acutest problems in Georgia and as the results of different statistical, conjunctive or social surveys evidence, often ranks first among the most important social-economic or political problems the country and Georgian people face. (Abesadze, 2014).

Unfortunately, reality in our country is that abduction for marriage and premature marriages are still prevalent. In addition, women are often victims of violence from husbands or partners. Gender disbalances are significant in societal activities. We think that one clear example of stereotypical thinking is segregation upon selection of professions or

Average Monthly Earnings of Employees by Sex

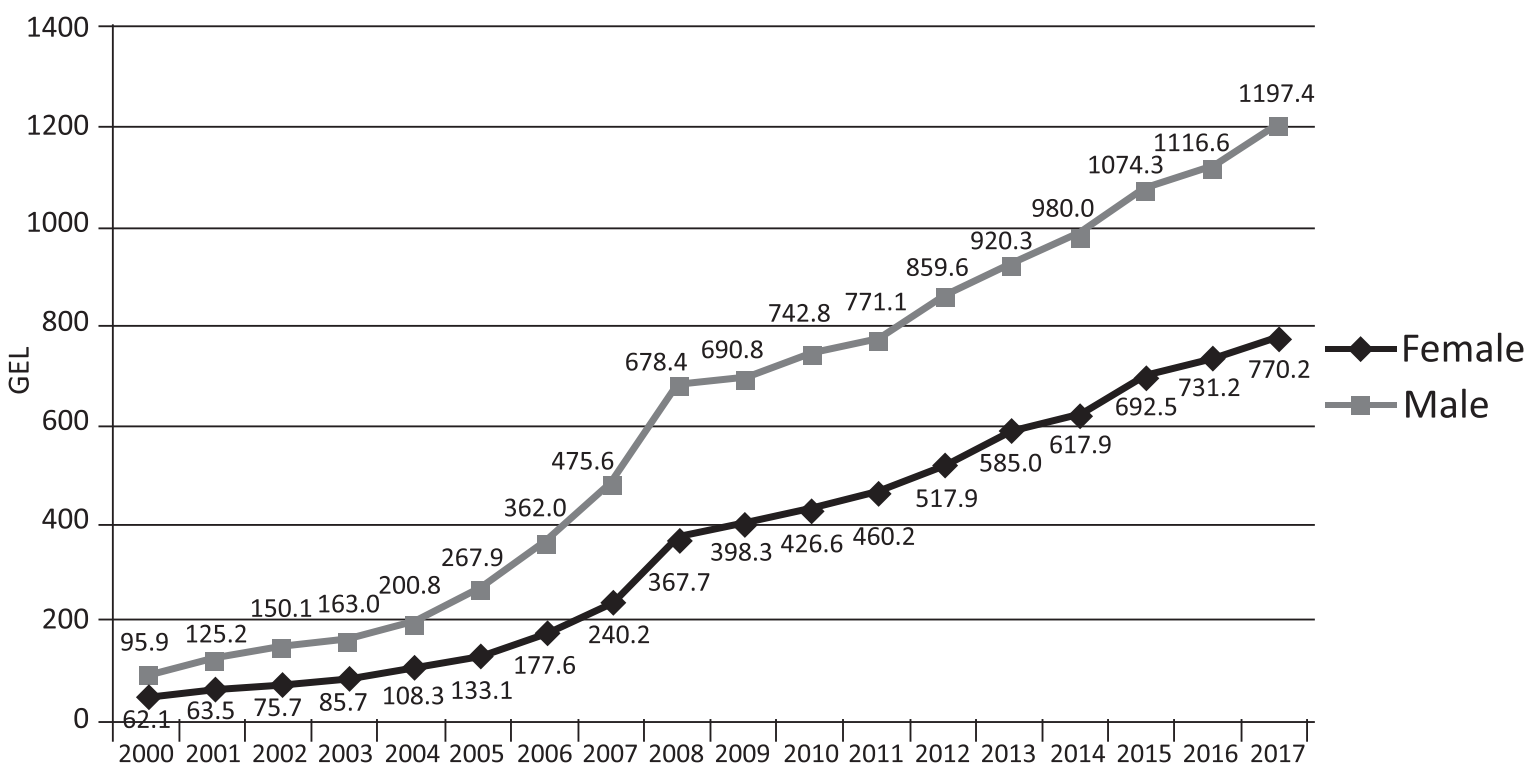

Source: Source: www. geostat.ge. Official web page of National Statistics Office of Georgia, last access 15/01/2018 
expectations of social positioning on the basis of sex. "So it is important analyze an environment, where men and women have different gender roles in political, social and societal life on the basis of sex differences». Some scientists, including Batler, [Batler, 2004] underline that sex is a complex mixture of individual activities and institutional influences, which is conditioned by power and influence of social code. It exists to form ideas about norms and deviation from norms. Sex categorizes individuals on the basis of «natural», «biological» and «bestowed by God». (Gorgadze,2015). The goal of Georgian law about gender equality is to "guarantee impermissibility of discrimination in every aspect of social life, create corresponding conditions for equal rights of men and women, as well as for realization of freedoms and opportunities; support avoidance and eradication of discrimination». However, we still meet gender disbalances in the field of employment.

It is fact that in Georgia number of employed men is more than women. A level of inactivity of women is almost two times higher than in men (correspondingly $42.0 \%$ and $21.8 \%)$. Unemployment rate of women has decreased by $1.4 \%$ in 2015 compared to 2016 and comprised $8.8 \%$. In the same period, unemployment rate of men has increased by $0.7 \%$ and comprised $14.2 \%$.

In 2017, unemployment rate for women Increased by 1.8 percentage points to 12.7 percent, and for men decreased by 1.6 percentage points and by 15 percent.

Indicators of level of inactivity also changed. The level of inactivity of women has increased by $1,8 \%$ and comprised
$58.0 \%$. The level of inactivity of men has decreased by $1,61 \%$ and comprised $78.2 \%$.

Changes for to the same period (2017/2016) are also indicators of activity level. The level of activity increased reased by 1.5 percentage points for women and decreased by 2.8 percentage points for men and 58.2 percent and 74.6 percent respectively.

We have interesting trend for indicators of employment on the basis of age. Number of employed men is especially high in the age group 45-54. Employment rate of men has been higher during the last 9 years, especially in the age group 15-24, where number of employed men is two timer higher than women.

Unemployment rate is especially high in youth. It is the main source of labor migration. Because of lack of corresponding experience, knowledge and skills, employers refuse to offer jobs to the youth and prefer more experienced personnel.

It is also fact that wage of employed men is higher than of women. Generally women earn less than men.

Growth rate of wages is also different. For example, in 2017 , average monthly wage of employed women was 770,2 Lari and of men - 1197,4 Lari (difference equals to 427,2 Lari). Wage of women is smaller in almost every field. For example, in agriculture the difference is 1.17 , in trade and repair of automobiles, consumer electronics and private usage items -1.63 , in hotels and restaurants -1.33 , in fishing -2.12 , in transport and communications - 1.45, in financial activities 2.17 , in education -1.2 , in state management -1.06 , in health protection and social assistance -1.55 etc. [11]Types of work

Fig. 2. Average monthly nominal earnings of employees by economic activity and sex, 2000-2017

Unemployment Rate by Gender, \%

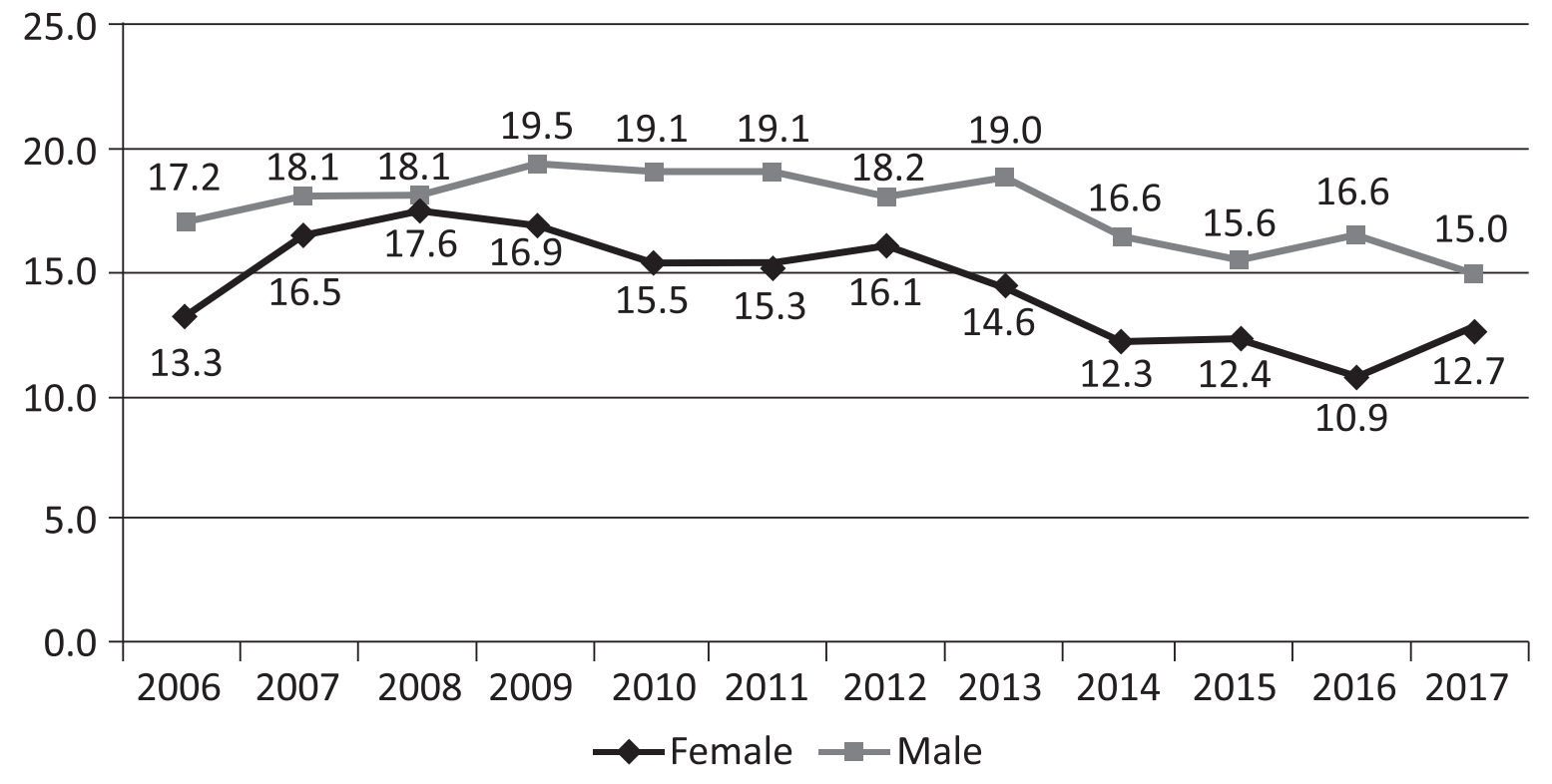

Source: www. geostat.ge. Official web page of National Statistics Office of Georgia, last access 15/01/2018 
performed by men and women are quite different, but both sex must have equal opportunity to reveal himself/herself and earn equal wage. It is also fact that in Georgia higher positions are usually occupied by men and there is a principle of socalled "glass ceiling", which implies creation invisible barriers preventing women from occupying high positions. 6h Article of 2nd chapter of Georgian law about gender equality regulates the principles of gender equality in labor relationships. It states that state supports equal availability of employment for both sexes. (Georgian law about gender equality, 2010).

What is an international practice in protection of labor relationships? For example, let us consider the experience of Iceland, as it has the best indicator in global gender index.

The law of Iceland obliges employer to take every measure to avoid segregation on the basis of sex at any job. In addition, the employer must care to distribute higher positions equally between men and women. If company has more than 25 employees, it is obliged to develop a program of gender equality and use principles of gender mainstreaming for a whole personnel. Companies must present a plan of gender equality or policy of personnel to the center of gender equality in defined terms. In other case they will pay fine, until they develop the program of gender employment (amount of fine is 50,000 Iceland Krona daily). Employer is also obliged to pay equal wage for equal work without distinction of sex. The law of Iceland states that employer must take any measure to support men and women combine their professional and family obligations. Besides, when employer establishes working hours, it is necessary to take into account employees' family conditions, as well as, demands of labor market. Employer must also help employee to return at work after maternity leave (both parents are indicated). The law of Iceland forbids publication of vacancy announcement, which prefers particular sex. Employers are forbidden to publish such announcement, which discriminates on the basis of sex. (Arjevanidze, 2012).

\section{CONCLUSIONS}

To optimize opportunities of working force on the basis of gender, it is necessary to correctly understand women's role and encourage their activities. These measures will support development of optimal employment policy.

Thus, the study has evidenced the necessity for the better availability of the information about

gender statistics for different types and sectors of business, outlined the existing gaps, priorities and major trends in gender employment, as well as specific measures to improve statistical methodology needed for statistical evaluation of employment dynamics. The gained result corresponds to the paper objective allowing concluding that designing employment gender indexes and regularly identifying the existing employment proportions based on it, predicting expected misbalance in employment, outlining the employment trends "dangerous" for the society and ultimately crushing the gender stereotypes may become the basis for the progress of the efficient employment policy.

\section{REFERENCES:}

1. Abesadze N. (2014). Statistics of unemployment in Georgia. Journal: Economy and management: Theory and practice. PP.15-20

2. Abesadze N. (2013). The methodological issues of the improvement of gender statistics of employment

- Economics and Management. PP.154-158.

3. Arjevanidze N. (2012). Gender equality - Review of Georgian and international legislation. Tbilisi. PP. 10-11. http://css.ge/files/ Papers/Nargiza_Arjevanidze,_Gender_Equality-legislation,_August_2012_Geo.pdf

4. Batler, J. (2004). Undoing Gender. New York and London. P. 22

5. Gelashvili S., Charekishvili L. (2016). The problems of gender statistics. Tbilisi, 2016. Gender discrimination at working place. Tbilisi.

6. Gender discrimination in labor relationships (2014). Tbilisi. P.51. http://article42.ge/wp-content/uploads/2016/03/.pdf

7. Gorgadze, N. Gender education at the initial stage of general educational schools (2015). Tbilisi. P. 22

8. Gochiashvili N. Analysis of working plans for support of women's employment (2014). Tbilisi.

National masterplan for gender equality in 2014-2016. Tbilisi.

9. Georgian law about gender equality (2010). 2nd chapter - guarantees for securing gender equality, 6 th article. Tbilisi.

10. Inter-parliamentary cooperation, women in national parliament (2013).1 February. http://www.ipu.org/wmn-e/classif.htm

11. Kvelashvili N., Abramia N., Liparteliani L., Tartarashvili T., Shioshvili G. (2013). The problems of youth employment in Georgia. Tbilisi.

12. Man and woman in Georgia. (2017). Geostat. Tbilisi.

13. The trends of employment and unemployment in Georgia, EPRC, Tbilisi, 2011.

14. Women's economic opportunities and challenges. Association of young economists. Tbilisi, 2014.

15. http://reports.weforum.org/global-gender-gap-report-2016/results-and-analysis/

16. \#199 decree of Georgian government about state strategy of formation of Georgian labor market (2013). Tbilisi. 


\section{SOCIO-ECONOMIC-GENDER DISBALANCES IN GEORGIA}

\section{NINO ABESADZE}

\section{Associate Professor}

Ivane Javakhishvili Tbilisi State University, Georgia

nino.abesadze@tsu.ge

\section{NATALIA ROBITASHVILI}

\section{Assistant Professor}

Batumi Shota Rustaveli State University, Georgia

robnato@mail.ru

\section{OTAR ABESADZE}

\section{Assistant Professor}

Caucasus University, Georgia

o.abesadze@gmail.com

\section{KEYWORDS: EMPLOYMENT, GENDER, STEREOTYPE, INDEXES, RATE}

For citation: Abesadze N., Robitashvili N., Abesadze O. (2019), Socio-Economic-Gender Disbalances In Georgia, Globalization And Business, №7, pp. 143-147. https://doi.org/10.35945/gb.2019.07.017

\section{SUMMARY}

Introduction and aim According to the policy towards gender equality, Georgia has become one of the most reformed countries in the Caucasus. Despite the fact that many steps have been taken in this regard, the problems are still in the society. The main objective of the study is to identify and analyze gender imbalances in the country on the background of gender stereotypes in the context of civil cognitive levels.

Research methodology: In development of the article, generally accepted qualitative and quantitative data analysis methods of the economic science were employed, among them, statistical data processing, data grouping, inductivedeductive data analysis methods. The scientific study employs surveying, observation, as well as comparative, analytical, and graphical methods, which are used by the author to compare and analyze facts and assess solutions to specific issues.

Results and implications: The paper considers the gender aspects of Discussion of inequality problems in Georgia. Besides, the trends of unemployment and their reasons are identified and the international instruments of protection of human rights regulating the equality between the men and the women in Georgia are considered. In addition, based on the statistical data, is given the analysis of employment and unemployment in a gender respect; a particular accent is made on the need of International practice of gender protection.

Conclusion: Stereotypical views on gender roles still exist in Georgia: the function of women is the care and upbringing of children, doing homework, and man-taking care of family finances.

The fact is that more men employed in Georgia than women. The level of inactivity in women is almost twice as high as in men with a similar indicator of $42.0 \%$ and $21.8 \%$ respectively. In 2017, unemployment rate for women Increased by 1.8 percentage points to 12.7 percent, and for men decreased by 1.6 percentage points and by 15 percent. Changes for to the same period are also indicators of activity level. The level of activity increased reased by 1.5 percentage points for women and decreased by 2.8 percentage points for men and 58.2 percent and 74.6 percent respectively.

Interesting trends are shown by employment indicators according to age marks. The number of men employed by the 45-54 age group is significantly higher than the number of women employed for the whole nine years, indicating the employment rate of women, especially in the 15-24 age group where the number of men employed is twice as high as the number of women employed.

It is also true that the average level of compensation for women is lower than men. Their rates of growth are also different. 


\section{THE PROBLEM OF THE POLITICAL AND FISCAL DECENTRALIZATION OF THE LOCAL GOVERNMENT IN 2012-2019}

\section{ANA TSITLIDZE}

PhD Student

Ivane Javakhishvili Tbilisi State University, Georgia

annatsitlidze@gmail.com

\section{KEYWORDS: LOCAL GOVERNMENT, FISCAL DECENTRALIZATION, EUROPEAN CHARTER, REGIONAL DEVELOPMENT,} SOCIO-ECONOMIC ASPECTS.

For citation: Tsitlidze A. (2019), The Problem Of The Political And Fiscal Decentralization Of The Local Government In 20122019, Globalization And Business, №7, pp. 148-154. https://doi.org/10.35945/gb.2019.07.018

\section{INTRODUCTION}

For every democratic state where a long-term democratic experience exists, a strong local government is fundamental. The local government or the bottom-up rule implies the political, fiscal, and administrative decentralization or devolution of authorities which are closer to people and which, unlike the center, know their needs.

The concept of "decentralization" implies a system of government where some functions of the central authority are devolved on the authorities of the local government (Sikander 2015: 172)

In decentralization, certain issues are devolved on the local population, which deals with those issues independently. This in itself does not mean that the issues of local importance are isolated from the issues belonging to state governance. The fundamental goal of the decentralization is the guarantee and encouragement of the independent initiative (Qurashvili 2003: 3-4).

For the perfect implementation of the decentralization of the local government, the combined and complex work of all types of decentralization is important.

The fiscal decentralization of the local government involves authorizing local governmental bodies to allocate budgets and introduce taxes.

The administrative decentralization of the local government involves the devolution of large powers onto local governmental bodies in the municipal territory, including powers in the management of state affairs and the sphere of public service (Oslen 2007: 5)

The actual political and fiscal decentralization of local government is an important challenge for Georgia, which is on its way to developing democracy and European integration, and which is facing the problems of territorial integrity and of developing a democratic state in the face of the danger of the monopolization of power. Its feasibility and effectiveness define the quality of democracy in the state.

The goal of the article: Study of the political and fiscal decentralization of the local government in Georgia in 20122019. Whether the tentative reformation was realized and what kind of resistance is coming in the way of implementation of the political and fiscal decentralization.

The objectives are:

- The study of the problem of the political and fiscal decentralization of the local government in 1991-2003;

- The problem of the political and fiscal decentralization of the local government in 2003-2012;

- The study of the problem of the political and fiscal decentralization of the local government in 2012-2019; whether the reformation was realized;

- Designing the outcomes and recommendations. 
In writing the article, the following research methods were employed:

Systematic method - the essence and the current process of the political and fiscal decentralization of the local government were analyzed following the approach of structural functioning.

Comparative research method - according to the political and fiscal decentralization of the local government, different authors' points of view and scientific investigations were compared.

Normative research method - this method was used for analyzing and evaluating the political occurrences of the political and fiscal decentralization of the local government, as well as defining the problem facing the process of the political and fiscal decentralization of the local government.

Qualitative research method - in-depth interview. Based on in-depth interviews, the evaluation by local governmental experts, non-governmental organizations, and local actors is especially valuable for the research because the problem of the political and fiscal decentralization of the local government in Georgia in 2012-2019 was seen by the practical outlook and first-hand. The implementation of the stages of reformation was evaluated in the contemporary period.

Sphere of outcome application - the article is the part of the research project "The problem of the Political decentralization of Georgia in 2012-2019 and Baltic Experience"

The outcome, based on the qualitative and quantitative research methods, will be an academic novelty for scholars and scientists about the model of the local government in modern-day Georgia, whether the reformation was held and what should be done for the perfect implementation of political and fiscal decentralization.

The second important period of the political and fiscal decentralization of the local government is connected to the period of 1992-2003, when the political union Citizens' Union, headed by Eduard Shevardnadze, came to the authorities. Civil confrontation, ethnic problems, wars in Abkhazia and Samachablo, Russian aggression, heavy socioeconomic issues in the country and the corruption in state bodies did not leave space for the development of political decentralization of the local government.

Despite these facts, in this period an important legal base (in 1997, the organic law "About Local rule and Government" was enacted) was more or less created for the local government in 1998. The representative bodies of the local government - assemblies elected by the municipal election - were created, and thus the mechanisms of the citizens' participation in the local governmental activity were defined.
The membership of the elected assemblies was characterized by pluralism. The entitlement of the local governmental bodies could not be implemented without their dependency on the central government. Territorial units were controlled by state representative-governors in regions first appointed by the Head of State, and then the president of Georgia; in municipalities, the units were controlled by the heads of local administration. The recognition of the local government and the importance of its decentralization was low not only for citizens but also for political parties and elected officials. Even the heavy socio-economic background prevented the implementation of fiscal decentralization. The governments had neither finances, property, nor ability.

Despite the frequent reformations held and steps taken forward, even the new authorities that came after the "Rose Revolution" cannot avoid the centralized tendency of the local governmental bodies. The political crisis in 2007 and the Russian aggression in Georgia in 2008 slowed the process of the political decentralization of the local government even more, even though in this period, in certain regions, some important socio-economic projects were initiated which were vitally important for the local government and regional economic development.

In Georgia, changing the authorities after the parliamentary election in 2012 led to a proposal for the implementation of reformation of the local government to be once more on the agenda.

The expectation was high that the coalition "Georgian Dream - Democratic Georgia", who came to the authorities in a majority in 2012, would express real political will for the implementation of the political and fiscal decentralization of the local government. The "Georgian Dream" leaders' political announcement before and after coming to authorities in the first years gave the basis for this. Besides the political announcements, from 2013, an intensive and large-scale work began on the local governmental reformation, and two stages of the implementation of reformation were announced: from 2014 to 2017 and then after 2017.

During the first stage of the reformation, the new local governmental code was enacted, the number of selfgoverned cities increased, and local governments were given a lot of authority. The announced reformation would have created a firm basis for the implementation of the political and fiscal decentralization of local governments.

Despite the fact that the reformation had a lot of opponents, the processes begun in 2013 is a step forward for the political and fiscal decentralization of the local government because new self-governing cities were created and local governmental bodies were given a lot of authority which had belonged to the central authorities. The necessity 
of assigning local property to self-government, leaving income tax to the local budget, was defined by legislation, and the code of the local government was enacted, which put together the legal norms of the local government. The code set out the direct path to election of the first person (the head of local administration/mayor) of the local government and the additional mechanisms for citizens' participation in local governmental bodies.

It was important that the local authorities could implement this authority independently in real life and how much the central authorities' will would be enough to accomplish the reformation of the local government in order to give real effective independence to the local government.

Unfortunately the political reality is totally different. Despite the increasing in the authority of local governmental bodies, even after nearly 30 years of independence, both central authorities and the political elite cannot abandon the Soviet practice of the local authority control. The local government elected directly by the people does not have enough political courage to be accountable to the electorate and not to the central authorities or their presenters - political parties. In fact, the informal control of local governmental bodies by the central authorities has not changed. Citizens' lack of participation and interest compound the situation. Oftentimes, the local authorities put the central authorities' will higher than the population's interest, which makes the process of political decentralization impossible, and which makes the population feel that nothing will change at the lower level.

The feeling that the political and fiscal decentralization of the local government was hindered by the authorities' political will become especially clear once again at the second stage of the announced reformation. In 2016, in their implemented reformation, for the purpose of finding and correcting mistakes, the authority eliminated the self-governing status of the seven self-governed cities established in 2014.

It is noteworthy that in spite of the internal resistance of majority members of the self-governed city assemblies against the elimination of self-governing status, the assemblies adopted the decision with the interference of the central authorities. The issue of property transfer to the local government has not yet begun.

The preparation of yet another stage of the selfgovernment reformation was begun by the Georgian authority in 2018. The Georgian government presented a new national vision of decentralization and local government. According to the initiative, the strategy, which should be ready at the end of the year, ensures the strengthening of local governments as well as societies' active inclusion in the process.

The new vision of the local government, or a middleterm strategy, will be scheduled for eight years.
It is known that the authority has a new vision about the local government, but nothing much was said about what this vision involves. It became known to the society that the new national vision of the decentralization and local government elaborated by Georgian parliament and government gives more possibilities to local authority both in decision-making and controlling financial resources. According to the notice by former Prime Minister of Georgia Giorgi Kvirikashvili, "The first, it is the expansion of self-governmental authority. The second is that self-government should have more financial resources for more efficiency. And the third, selfgovernment should satisfy the highest principles of clarity and accountability. It should ensure the citizens' inclusion in the decision making process, management process, and of course, the unity of all three axes will give us a totally different qualitative outcome in self-government (Kvirikashvili 2018).

Despite the last years' changes, experts focus on the current lacunae in the local government, including the financial part and the hindrance of decentralization, the problem of income taxes, ceasing the process of transferring the farming land and local property to the self-government, and the confines of independence and discretion of the local government. According to local government expert Irakli Melashvili, more authority should be given to the local authority both in the decision-making process and in controlling finances, and this vision should be reflected in the strategy announced by the government (Melashvili 2018).

The fact that transferring more authority, including financial issues, to local governments is hindered by the political will of the authorities is also discussed by the head of "International Center for Civil Culture "Kote Kandelaki". According to him, "At this stage, one of the most important points is fiscal decentralization, or more resources and finances on site. It involves both finances and drawing distinctions between the property of central authorities and local government. The local government should feel that they control the property and finances and consequently, they should be given incentives to facilitate local development. Nowadays, the self-governments do not have any incentives to work more in order to get more income, because whatever steps they make, their incomes, unfortunately, do not increase, and first and foremost, this is the error of the legislative base" (Kandelaki 2018).

It is noteworthy that according to Article 162 of "The Code of Local Government", the Ministry of Justice of Georgia, the Ministry of Regional Development and Infrastructure of Georgia, the Ministry of Economics and Sustainable Developments of Georgia and the Ministry of Finances of Georgia should have designed appropriate schedules and rules designating terms of the transfer of farming land to 
municipalities, though this obligation has not yet been carried out, which hinders the process of decentralization of the authorities (Transparency International Georgia 2018).

It has already been half a year that the Ministry of Infrastructure has been trying to decide when they should begin transferring local property to the self-governments They have no answer yet, and nor does the Ministry of Economics. According to an announcement by the Minister of Economics, "This is the current process, and we will always transfer property to the self-government when it is appropriate" (Kobulia 2019). This announcement by the Minister of Economics indicates once more that the authorities do not know yet whether they want to implement political decentralization and to strengthen local governments.

The elected placeholders of the local government talk about the necessity of political and fiscal decentralization of the local government, who think that non-implementation of the fiscal decentralization up today is directly connected to the problem of the political decentralization; it hinders the process of the decentralization and makes inefficient the activity of local governments. The chairperson of Khelvachauri assembly, Nadim Vashanidze, thinks that besides fiscal decentralization, educational decentralization is also important, and that municipalities should have the right to allocate natural resources - otherwise, elected mayors and assemblies lose their meaning (Vashanidze 2018). During the discussion of the strategy of decentralization, elected persons from different municipalities expressed the same position. According to the talk given by the mayor of Shuakhevi municipality, Pridon Putkaradze, the strategy is good but will not help us if we do not step forward effectively" (Putkaradze 2018). According to an announcement by the chairperson of Zugdidi assembly, Mamuka Tsotseria, in many cases, the municipality is encumbered because many authorities that should belong to the municipality belong to the Ministry of Economics, and communication with the Ministry is problematic. According to him, the municipality attempted to implement the registration of some property, though because of lack of finances the task could not be completed. Transferring the property was always the problem for the Ministry of Economics. What is already transferred to us in the case of an investor's appearance, we do not have a mean for its realization or privatization, in the event of the implementation of a strategic plan, only the capital city has the direct disposing for transferring in symbolic price. Local governments should have these rights. Farming lands should belong to the municipality as well. The fact that the Ministry of Economics sells lands discretely reflects on agriculture (Tsotseria 2019).

Representatives of non-governmental organizations and experts announce that the strategy should define clearly the issues of transferring property to self-governments. Besides this, it should be scheduled in the next two-year action plan the terms of which category of lands will be transferred from the National Agency of State Property of the Ministry of Economics to the property of the municipality (Shamugia 2019).

\section{CONCLUSION}

Thus, the process of the political decentralization of the local government has been proceeding inconsistently since 2012. Despite the twice announced and implemented reformation of political and fiscal decentralization of the local government, the problems are unchanged.

- Political powers at the top of the country do not concede the mechanisms of controlling the local governmental bodies;

- Municipalities do not have enough money or authority;

- On the one hand, the autonomy of the implementation of their authority by the self-government is recognized, but, on the other hand, in the realization this right, discretion is maximally restricted;

- The majority of financial resources is concentrated in the hands of the central authorities;

- Municipalities use their poor means inefficiently;

- The informal role of the central authorities is great in the activity of the elected persons and in the process of decision making; elected assembly members and officials depend on the central government.

- The informal role of law enforcement bodies - State Security Service and Ministry of Internal Affairs - is great on the local governmental bodies;

- Regional advisory councils are weak and inefficient whose members are elective persons of the municipality, whereas a person commissioned by the central authorities - the State Representative-Governor heads it. The decisions made by the council are recommendative in nature.

- The State Representative Institute commissioned by the mayor is inefficient at the lower level. This institute becomes a party's agitator in the election process;

- Public meetings and advisory councils work formally; public meetings gather because the legislation requires them. The practice shows us that the citizens faithful to the village representative-ruler's power are often asked for their participation in public meetings. The advisory council of the mayor works formally and inefficiently. The persons faithful to the ruler's power are often appointed the members of the council;

- Despite the fact that one of the authority's arguments was the decrease of bureaucratic expenses regarding 
the implemented changes in the local government and the unification of municipalities, nothing of the sort has happened. On the contrary, after the unification of two municipalities, the bureaucratic expenses stayed the same or increased. Public officers working in local government are mainly appointed according to party line and the announced completion is formal. There is an especially bad situation in Non-enterpreneurial (Non-commercial) Legal Entities of Municipalities;

- Despite the considerable controversies, transferring property to municipalities has not yet begun;

- The still weak political parties developing institutionally are problematic in view of the political decentralization of the local government.

\section{RECOMMENDATIONS}

1. The essence of the theoretical basis of the selfgovernment should be established on the elements of dualistic and state theory, according to which self-government is the unity of both general and state elements. Self-government as bottom-up management and the government closest to the population provides the democratic standard of management and is the main support of democracy;

2. In the transitional period of reformation, a mixed system can be used, but at the end, it is important to achieve the Anglo-Saxon system;

3. The basic principle of the political decentralization of self-government is discretion and independence in the process of making decisions. This is not possible by merely making a distinction between authorities and giving power.
The existence of political will and independence from the party's ambition are necessary;

4. Finishing and defining the administrative-territorial reformation are necessary;

5. Strengthening the role of the representative body the assembly - of which the executor can be a non-elective person. A Baltic model can be used;

6. Together with the elected member of the assembly, it is important that a lower level village representative should be elected, which will assist in the depoliticization of the representative institute; the Baltic model can be followed for the election of the lower level circle. For example, in Lithuania, Slautinis are elected. They do not have their own budget but have an expenditure account;

7. The implementation of fiscal decentralization and transferring of the local property to municipalities;

8. The issues of the regional arrangement - the existing regional advisory councils are inefficient. Besides its inefficiency, a person appointed by the authorities heads it. For political decentralization, it is important that the head of the regional council should be appointed out of the leading persons of the municipality by the council membership with the fixed period. It will decrease the informal control of the activity of the local government by the central authorities;

9. The depoliticization of the Ministry of Internal Affairs and the State Security Service is necessary. The practice showed us that the interference of two law enforcement bodies into the activity of the local governmental bodies has been great, especially in the last years;

10. It is important that the political parties should care about internal democratic development and the growth and develop local leaders.

\section{REFERENCES:}

1. Adalashvili, G. 2013. The reformation of the self-government which should strengthen the city and village. Tbilisi: Radio Independence. Pages 10-15 (in Georgian)

2. Alapishvili, L. 2015. The Self-governmental System in Tbilisi in 1991-2012. In The Rapportof the development of the local Democracy. Tbilisi: The International Center for the Civil Culture. Page 25 (in Georgian)

3. Bedianashvili, G. (1995). State, Power Structure and Socio-Economic Reform of Society. Tbilisi, Mecniereba

4. Chokheli, E., Narmania, D. 2017. On some issues of local self-government in Georgia. International Scientific Conference: Challenges of Globalization in Economics and Business. Conference Papers. Tbilisi

5. Georgian Young Lawyers' Association, Open Society - Georgia Foundation, International Society for Fair Elections and Democracy. 2017. Research: The reformation in the name of the self-government against the self-government. Tbilisi

6. Kandelaki, K. 2018. The Strategy of Decentralization in 2019-2025. www.droa.ge - last checked 10.03.2019

7. Kikvadze, O., Gersamia, M. 2014. The International Legislation of the Local Government, International Organizations and Self-governments. Tbilisi: The Guide of the Clarification of the Self-government. Page 7

8. Kobulia, G. 2018. The Strategy of decentralization in 2019-2025. www.droa.ge-last checked 10.03.2019 
9. Kvirikashvili, G. 2018. The Strategy of Decentralization in 2019-2025. www.droa.ge- last checked 10.03.2019

10. Losaberidze, D. 2015. The Reformation of the Local Government in Georgia in 2013-2014. In The Rapportof the development of the local Democracy. Tbilisi: The International Center for the Civil Culture. Page 20

11. Melashvili, I. 2018. The Strategy of Decentralization in 2019-2025. www.droa.ge-last checked 10.03.2019

12. Nakashidze, N. 2019. The Ruined Property and the Propertyless Self-governments left trusted to the Appropriateness of the Government. http://droa.ge/?p=42897- last checked 10.03.2019

13. Oslen, H. 2007. Decentralization and Local Government https://www.eda.admin.ch/dam/deza/en/documents/publikationen/Diverses/167288-decentralisation-local-governance_EN.pdfLast checked 03.03.2019.

14. Putkaradze, P. 2019. The Ruined Property and the Propertyless Self-governments left trusted to the Appropriateness of the Government. http://droa.ge/?p=42897- last checked 10.03.2019

15. Qurashvili, K. 2003. The Issues of the Realization of the Constitutional Principle of the Local Government in Georgia. Jurnal Almanac. N18. Tbilisi

16. Shamugia, E. 2019. The Depth interview.

17. Sikander, T. 2015. A Theoretical framework of Local Government. International Journal of Humanities and Social Sciences. Rawalpindi. Pakistan

18. Transparency International - Georgia. 2014. The new Local Self-Government Code: Overview of the main novelties. Tbilisi

19. Transparency International-Georgia. 2013. Local self-governance reform - A new Challenge for Georgia. Tbilisi

20. Tsotseria, M. 2019. The Ruined Property and the Propertyless Self-governments left trusted to the Appropriateness of the Government. http://droa.ge/?p=42897- last checked 10.03.2019 


\title{
THE PROBLEM OF THE POLITICAL AND FISCAL DECENTRALIZATION OF THE LOCAL GOVERNMENT IN 2012-2019
}

\author{
ANA TSITLIDZE \\ PhD Student \\ Ivane Javakhishvili Tbilisi State University, Georgia \\ annatsitlidze@gmail.com
}

\section{KEYWORDS: LOCAL GOVERNMENT, FISCAL DECENTRALIZATION, EUROPEAN CHARTER, REGIONAL DEVELOPMENT,} SOCIO-ECONOMIC ASPECTS.

For citation: Tsitlidze A. (2019), The Problem Of The Political And Fiscal Decentralization Of The Local Government In 20122019, Globalization And Business, №7, pp. 148-154. https://doi.org/10.35945/gb.2019.07.018

\section{SUMMARY}

The article contains a concept about Political and fiscal decentralization problem in Georgia during "2012-2019"

For each democratic state strong local government and its political and fiscal decentralization is a fundamental principle of democracy. A local government is a form of public administration which, in a majority of contexts, exists as the lowest tier of administration within a given state. It contains political, fiscal and administration decentralization which means to transfer power from central government to municipality.

In the way of Democracy and European integration as a still democracy development county, Local Government development and its Political and fiscal decentralization is an important and key issues for Georgia.

Aim of the Article: To analyze the process and the problem of Political and Fiscal decentralization of Local Government in Georgia during 2012-2019. What type of problem we have and what is the main problem for Political and Fiscal decentralization of Local Government in Georgia.

Despite all of reforms from independence to nowadays, political and fiscal decentralization of local Government is a problem for all governments in Georgia. The main reason for all of them was political control under the Local governments. After 2012, the hope that the new Government can implement real political da fiscal decentralization of local government was big. But reality is different. Despite two reforms of Local Government during 2012-2019, until now the problem of political and fiscal decentralization in Georgia is the same: no formal control of Local Governments, Most of Financial resources still have central Government, Local Governmnts have no possibility to implement their power independently, they have no own property and suffifient finansial resources.

In this article we give a recommendations how to improve political and fiscal decentralization aspects in Geogria and what the mainc problem is. 


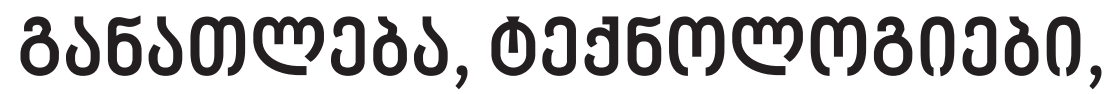

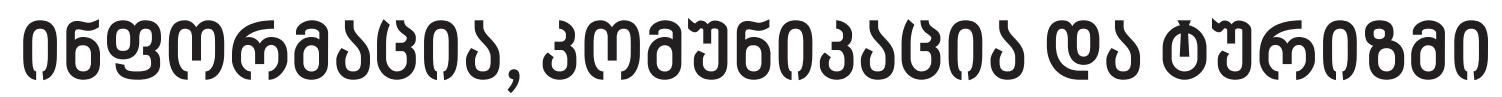

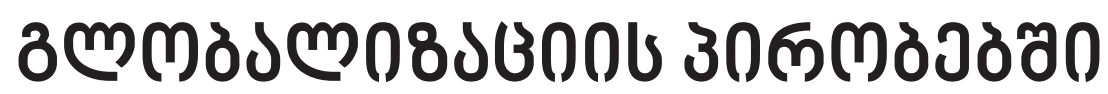

\author{
EDUCATION, TECHNOLOGIES, \\ INFORMATION, COMMUNICATION AND \\ TOURISM IN TERMS OF GLOBALIZATION
}





\title{
PROTECTED AREAS - DEVELOPMENT SUSTAINABILITY, ECONOMIC BENEFIT AND INTEGRATION OF LOCAL CULTURE IN GEORGIA
}

\section{MAIA AZMAIPARASHVILI}

\author{
Associate Professor, \\ European University, Georgia \\ maiaazmaiparashvili@gmail.com
}

\section{KEYWORDS: SUSTAINABLE TOURISM, ECOTOURISM, ECOLOGICAL SUSTAINABILITY RECREATIONAL RESOURCES, ECONOMICAL SUSTAINABILITY.}

For citation: Azmaiparashvili M. (2019), Protected Areas - Development Sustainability, Economic Benefit And Integration Of Local Culture In Georgia, Globalization And Business, №7, pp. 157-162. https://doi.org/10.35945/gb.2019.07.019

\section{INTRODUCTION}

Tourism is based on the goal-directed and clever using of tourist resources. Tourist resources are the subject of tourism, which have potential to satisfy the demands which occur in tourist process. Where there is no tourist resource, tourism cannot exist. Tourist resources are national property. Though the resources which have special meaning tourism gained worldwide significance.

Nowadays eco-tourism has become the most popular and fast developing industry. Eco-tourism means travelling to see the natural and manmade cultural variety without doing any harm to it. Eco-tourism is considered as one part of natural tourism, which joins together people, who travel with scientific - cognitive purpose. This tourists' interest is the environment, its observation, and partly its defending. In such kind of tourism the main interest is protected natural areas. According to this ecological tourism is considered as strictly specialized kind of brightly marked scientific-cognitive free time activity.

The development of tourism in Georgia underscores the existence of a network of national parks and other protected areas. With its natural landscapes it is known that protected areas now play an important role in protecting biodiversity, natural heritage of Georgia (and not only Georgia). In addition, protected areas are characterized by its unique natural-aesthetic characteristics. The purpose of the work is to study the example of protected areas, how the landscape diversity is perceived by the visitors and what role can be performed by the perceptual resources in the planning and management scheme of the Protected Areas. The paper also has an important place for visitors (statistics on the protected areas, survey of visitors s satisfaction, income from tourism and the sustainability of tourism sustainability on the quality of decadence.

The growth of demand on eco-tourism predetermined the necessity of founding protected areas and parks. Nowadays these areas represent the main objects of ecotourism. In the frame of especially defended natural area the organizers of eco-tourism prefer scientific-cognitive tourism, because they think that such eco-tours are easy to make. Some especially protected natural areas abroad which don't demand high qualification, let the participation of ecotourists. For example, a lot of eco-tourists are busy calculating the amount of biocenosis of different populations.

Ecotourism, to be successful, must promote sustainable development by establishing a durable productive base that allows local inhabitants and ecotourism service providers to enjoy rising standards of living. An ecotourism project must incorporate the social dimensions of productive organization and environmental conservation.

Tourism should develop sustainable, controlled and integrated basis. Using the principles of prudent planning. This concept provides a natural, cultural and other resources saving. Their continued use in the future so that they benefit society today. Tourism represents an important environmental protection enabled. Tourism is important to the protection of cultural heritage.

Sustainable development is concerned with acknowledging economic, social and environmental development 
aspects, catering for the current needs of society without damaging the well-being of future generations. Ecotourism is a niche market emerged because of increased market demands for sustainable tourism practices. It serves to provide tourism products and services which accommodating for the economic, social and environmental aspects of the society. However, earlier research suggests that it is difficult to handle the tree dimensions of sustainable development, indicating that trade-offs may occur. Furthermore, as tour operators are able to affect local development prospects, it is of interest to examine how they handle sustainable development, and more specifically, contribute to tradeoffs between, social and environmental pillars of sustainable development. (Eriksson, Lidstrom, 2013).

Ecotourism is a sub-component of the field of sustainable tourism. Ecotourism's perceived potential as an effective tool for sustainable development is the main reason why developing countries are now embracing it and including it in their economic development and conservation strategies. Ecotourism, as an alternative tourism, involves visiting natural areas in order to learn, to study, or to carry out activities environmentally friendly, that is, a tourism based on the nature experience, which enables the economic and social development of local communities. It focuses primarily on experiencing and learning about nature, its landscape, flora, fauna and their habitats, as well as cultural artifacts from the locality.

\section{STUDY AREA \& METHODS}

The understanding and recognition of significance of sustainable tourist achievements became the base of ecotourism. In the world of tourism the notion sustainable tourism and eco-tourism are used in the same way. Besides this close relation each of them has slight, but important special features. The conception of eco-tourism means more sympathy and duty from travelers. Sustainable tourism means the duty of the society to provide the protection and maintenance of the environment for the future generations. These conditions are not only political and economic factors and needs strengthening with the help of making decisions. We should realize what does ecologically sustainable environment means for the development of tourism and what part in this case plays eco-tourism as one of the main parts of the development of the sustainable tourism.(Charles Geldneri, JR Brent Ritchi, 2013).

The visitors opinion is very important for the protected areas and listening constructive criticism from them. We used data from visitor centers for our research. The quantitative and qualitative research methods are used. The target group and survey area were identified. Analyzed visitor statistics, visitor satisfaction survey, income and sustainability of tourism sustainability. Ensuring sustainability of sustainability, sustainable sustainability and development in the management of its management. Tourism innovative management is increasingly focused on managing local sustainability and its sustainability. Scholars do not argue that the destiny is the nucleus of tourism. Therefore, it is easy to evaluate the importance of sustainability, what practically resembles the sustainability of tourism. Nevertheless, less attention is paid to general destructions and, in particular, its sustainability problems.

In order to support safe management of tourism on the sites on the permanent bases, the monitoring is made according to the basic indicator of sustainable tourism. Here are used such indicators with the help of which the causeaffect connection between the tourism and the area is measured. The most important are two types of indicators: 1) the general indicator of sustainable tourism, which is worked out for general use in tourist sites. 2) the indicator of tourist site which is used with the particular eco-system or one of the tourist type.

In the second part of the 20th century because of the growth of tourism the mankind began to think more about difficult ecological situation in the world, in which tourism has already played its role. So in the process of developing of tourism it is important to take into consideration not only economical profit but also its ecological and social results, because the development of this branch won't be complete without its social economic and ecological.

It should be mentioned that the sustainable development of the areas which also belong to the eco-tourism sphere helps to develop tourism infrastructure, to create new working places, and to give work to the natives in the tourist service sphere, which naturally will rise people's level of ife in the region of the country.

For the sake of development of eco-tourism it has to be protected the variety of flora and fauna of the recreation regions and all natural resources. It is natural that eco-tourism won't be developed successfully if some amount of means taken from it aren't used for the protection of the recreation zones and for getting results from the recreation profit.

Unfortunately, nowadays the mankind is facing the global problem of secure of national resources, which main reason is modern way of life (principal oriented on consuming). As a result of irrational use of the national resources the natural may lose the resource of self-restoration, especially it refers to the forests, the degradation of which causes a lot of negative ecological results.

Georgia according to its geopolitical location and with its natural, historical or cultural resources has a lot of opportunities to develop tourism. Georgia represents the classical example of geographical landscape of vertical zones and has its almost complete variety from humid sub-tropics to zone. The most relevant regions to eco-tourism and health resorts are the regions, which are situated on Caucasian and South-Mtianeti's mountainous regions and on the Black Sea 
Coast. Along with aesthetic factor tourists are attracted by sea and mountainous medical features, the richest hydromineral resources.

\section{RESULTS \& DISCUSSIONS}

It must be mentioned that in the perspective of the development of eco-tourism, the international experts predict great future for Georgia. Along with other preferences they mention the geographical nearness of our country to the world's main tourist market - Europe.

Protection of nature is the strategic integral part of the development of eco-tourism. It can bring economical profit. This happens in eco-tourism when both the environment and business are healthy. Sometimes people's interests are sacrificed to the protection of the environment. For example the population maybe forbidden to perform agricultural works, to collect wood and building materials. The protection of nature is sacrificed to maximization of short -time profit, the existing eco-system is ruining. The degradation of tourist resources acts against both interests (protection of nature and eco-tourism.) It is important to understand that tourism can not develop without determined influence on the environment. It is impossible to make the main accent only on the development of tourism, without ecological care. One of the main principal of the sustainable development of eco-tourism is including the native people into it. Very often native people are excluded from this process artificially. (Gogelia, 2012).

The protection of natural, social and cultural resources and their sustainable usage is the base of long and successful activities. There are three main principles of sustainable development: ecological sustainability provides comparing of basic ecological processes with biological variety and biological resources; social and cultural sustainability predetermines such situations which provides people's control on their being along with material and cultural growth, which are concerned to this processes of development. Also helps to save local originality; economical sustainability provides effectiveness of economic development and such situation when future generations will use chosen methods of handling the operation of the resources. (Metreveli, 2012).

In tourism ecology and economy are more and more connected with the large system of causes and effects. Nowadays the mystery of popular tourist centers is based on the cleanliness of the environment. On the events of its effective defense and on the originality of the local culture in the places where there isn't any, it is noticed the decline of the level of service is noticed and also the shortage of volume of tourism. The aggravation of the environment, lots of cultural originality, the decline of economic situation. Local authorities are responsible for clever handling of the resources, so as future generation will have opportunities to use them.
So as to provide sustainable economy for tourism, tourist sustainability is needed. During the sustainable development of tourism the resources should be used so as to satisfy economic, social and esthetic needs, but at the same time maintain the originality of the country. The main biological variety of the environment and the main tourist systems, the stable development of tourism should provide social, cultural, ecological and economic criteria of harmonic mixture, which will give us not only maintenance of natural and cultural resources, but will become the predominance of their development and renovation.

The events of maintenance and protection of natural resources and cultural legacy have both global and local aspects. In order to make tourism sustainable, the character of tourist activities and capacity should foresee the existed natural and artificial resources throughput. The base of conception of tourism sustainable development is the concept of throughput potential. Throughput potential is the maximum loading which any tourist object can resist, without any serious harm to local resources, negative influence and social economic problems among the population (Birjakov, 2004).

The results of tourist statistics on protected areas are as follows: in 2018, the number of foreign visitors in the protected areas was 1108503 . As of 2018, most visitors visited Prometheus Cave - 185516 (19\%), Martvili Canyon 174,143 (136\%), Kazbegi National Park - 174,520 (17\%). Sataplia 81556 and Ottus 73 113. The number of visitors has increased to the following protected areas: Machakhela National Park - increase by $147 \%$, Martvili Canyon - increase by $136 \%$, Algeti National Park - increase by $74 \%$, Tbilisi National Park - $47 \%$ increase, Mtirala National Park - growth - $41 \%$, octane canyon - increase - $40 \%$, Prometheus cave increase - 19\%. Number of foreign visitors to Prometheus, Martvili, Chachuna, Mtirala and Tusheti Protected Areas exceeded the number of Georgian visitors. Visitor statistics by protected areas in 2019 (Source: Agency of Protected Areas. http://apa.gov.ge/en/s).

The growth of demand on eco-tourism predetermined the necessity of founding protected areas and parks. Nowadays these areas represent the main objects of ecotourism. In the frame of especially defended natural area the organizers of eco-tourism prefer scientific - cognitive tourism, because they think that such eco-tours are easy to make. Some especially protected natural areas abroad which don't demand high qualification, let the participation of ecotourists. For example, a lot of eco-tourists are busy calculating the amount of biocenosis of different populations.

Incomes received from tourism services of protected areas amounted to 4,504,473 lari, compared to the previous year $+94.7 \%$. Income of receiving income was: Prometheus Cave - GEL 1,887,064, Martvili Canyon - GEL 1,274,463, Oktes Canyon - 538,506 GEL and Sataplia - 499,019 GEL.

According to the information received from the Agency 
of Protected Areas of Georgia, $84 \%$ of visitors surveyed in the protected areas of Georgia and the service received during the summer are satisfied. Most of the visitors - $88 \%$ will definitely ask friends to visit Georgian Protected Areas. $68.8 \%$ of respondents assess the attitude of the personnel to the visitor very friendly. When traveling on protected areas, you feel protected by $88.3 \%$.

\section{CONCLUSIONS}

Sustainable development is the process of continuous changes, in the frame of which the using of resources, investment, technological progress and other innovations are developed according to the demands of future and present.

Conceptual question is, if it is reasonable to develop eco-tourism in strictly defended natural areas, the discussion topic till these days, both among scientific society and among the workers of these protected areas. The main argument is that the development of the protected areas happen the principals of the work of the preserves and nature, with the help of organized tourism and scientific expeditions, also to make excursions for servants, supporting the students' specifically, as an untouchable natural standard, on which only scientific research is made. On the territory of Georgia the main aim of preserves should be the following: to explain to the people practice and tourism.

Among the systems of the development of the preserves is such as: preserve is the protected area of natural processes and preserving the resources in genetic dynamic and untouched position and scientific research activities and monitor of the environment. This is the school of the nature protection and we shouldn't be afraid of people, but we should create such conditions that their presence in the preserves shouldn't become the violation of the conditions of being in the preserve.

The development of eco-tourism of the protected areas is the important question which influences the economic situations of the regions, it also can activate the protection of nature in the country. To our mind strictly protective natural areas can not only strengthen ecological tourism in the country, but also give a stimuli to the tourists to come to the territory of Georgia. The natural areas which are protected for this reasons have uniquely organized territory, have professional scientific and administrative person, the practice of protecting and restoration of ecological systems, close contacts with national fundamental science, the opportunity of sharing the experience of working abroad, have protected structures. The participants of eco-tourism should develop such service and offers which will correspond to the demands of tourist market, which will become a guarantee of attraction of tourists. The government should play the great part in this with its supportive policy, which involves the developing of infrastructure, the search of different educational programs and inves- tors. In this direction the last aim of the government is the development of financially profitable industry of eco-tourism in the conditions of protecting and improving the nature. It is important for tourist experts to take an active part in the planning process of natural parks and protected areas. It is important for tourist specialists and the administration of protected areas to work together in the sphere of planning the tours and administration.(Bliadze, Bordzikuli, 1998).

Georgia is rich in recreational resources. Georgia can become one of the powerful centers of the world tourism. A thorough study of the forests we see climates-recreational functions, mineral and thermal springs medicinal properties, grotto, spa resorts and places of functions, we come to the conclusion, that along with the tourism Georgia may become a health cache. Nature has richly rewarded Georgia with tourist recreational resources. The world is not found in such places, where a small area, which our country has, there are so many different landscapes. That's why Georgia for its unique natural-gives economic conditions-one of the strong possibility of becoming a tourist country. Are only a few countries in the world, which is natural - climate and recreation - spa resources, so of the God "rewarded".

It is impossible not to note, that tourism development that gives a sense of national pride, because your country, its nature and culture becomes recognizable. Tourism can be considered as the best means of communication to the world. Each of them tells a lot about his journey, thus evokes a desire for others to see it.

There is no doubt that tourism can not be developed in the country without the system of protected areas. The existence of tourism depends on the functionality of ecosystems and services offered by them. of the enormous potential of tourism development, because it has a variety of natural areas, aesthetically attractive landscapes, urbanization and gakulturebis processes intact the unique flora and fauna, and international endangered species included in the not so little amount of relict and endemic species, natural areas and ax and has developed networks. The ecotourists make the country a special attraction for the wealth of ethno-cultural, the diversity of public holidays, customs, traditional hospitality of the population.

One of the most important conditions for the ecological and tourism activities is to provide rational, sustainable nature of use in protected areas. Tourism and environmental conditions are interconnected. The environment contains a lot of elements. The most important factor in achieving sustainable development is development and management of tourism that will not harm the environment. The provision of sustainability, maintenance and development is driven by its management. The essence and sustainability of sustainable management is that it does not violate the balance of destiny, the sustainability of deserting is ensured in all three directions: environment, economy, socio-cultural heritage. 


\section{REFERENCES:}

1. Eriksson F., Lidstrom M. (2013). Sustainable development in ecotourism, Umeå Umeå School of Business and Economics, pp 34-38

2. Charles Geldneri R., JR Brent Ritchi (2013). Tourism -Basics - practice - theory, publishing “ Batumi Shota Rustaveli State University « pp 432-443;

3. Gogelia M. (2012). Ecotourism and its development prospects», Akaki Tsereteli State University, International Scientific Conference Proceedings, innovative technologies and modern materials, pp. 67-89.

4. Metreveli, M. (2012). Environment and Ecotourism Management, Publisher "forma", pp 51-62

5. Birjakov, M. (2004). Tourism Theories - 2nd Edition, "fotoni", pp 44-60

6. Bliadze - Bordzikuli M. (1998). recreational resources of Georgia. Strategic Research and Development Center of Georgia, pp 25-30

7. Qiqodze A., Goxelashvili R. (2007). Protected Areas of Georgia. Department of Protected Areas.

8. www.gccw.org

9. https://journals.gisap.eu/

10.http://drg.com.ge/team.php

11. http://apa.gov.ge/en/statistika/vizitorta-statistika/visitorOstatistics-by-protected-areas-in-2019

12. http://greenalt.org/wp-content/uploads/2018/07/biodiversity_monitoring_2018.pdf

13. www.geostat.ge 


\section{PROTECTED AREAS - DEVELOPMENT SUSTAINABILITY, ECONOMIC BENEFIT AND INTEGRATION OF LOCAL CULTURE IN GEORGIA}

\section{MAIA AZMAIPARASHVILI}

Associate Professor,

European University, Georgia

maiaazmaiparashvili@gmail.com

\section{KEYWORDS: SUSTAINABLE TOURISM, ECOTOURISM, ECOLOGICAL SUSTAINABILITY,} RECREATIONAL RESOURCES, ECONOMICAL SUSTAINABILITY.

For citation: Azmaiparashvili M. (2019), Protected Areas - Development Sustainability, Economic Benefit And Integration Of Local Culture In Georgia, Globalization And Business, №7, pp. 157-162. https://doi.org/10.35945/gb.2019.07.019

\section{SUMMARY}

Tourism is based on deliberate and reasonable consumption of tourist resources. The object of research is Ecological Tourism, Protected Areas - Development Sustainability, Economic Benefit and Integration of Local Culture in Georgia. The understanding and recognition of significance of sustainable tourist achievements became the base of eco-tourism. In the world of tourism the notion sustainable tourism and eco-tourism are used in the same way. Besides this close relation each of them has slight, but important special features. Sustainable tourism means the duty of the society to provide the protection and maintenance of the environment for the future generations. These conditions are not only political and economic factors and needs strengthening with the help of making decisions.
We should realize what does ecologically sustainable environment means for the development of tourism and what part in this case plays eco-tourism as one of the main parts of the development of the sustainable tourism.

With its natural landscapes it is known that protected areas now play an important role in protecting Georgiass biodiversity, natural heritage. In the work there is a significant place for visitors (statistics on the protected areas, survey of visitors) satisfaction, income from tourism and the sustainability of tourism sustainability. So as to provide sustainable economy for tourism, tourist sustainability is needed. During the sustainable development of tourism the resources should be used so as to satisfy economic, social and esthetic needs, but at the same time maintain the originality of the country. 


\section{SUPPORTING THE DEVELOPMENT OF BUSINESS ENVIRONMENT THROUGH ECONOMIC ORDER POLICY}

\section{IRAKLI ASLANISHVILI}

\section{PHd student}

Ivane Javakhishvili Tbilisi State University, Georgia

irakli.aslanishvili@gmail.com

KEYWORDS: BUSINESS ENVIRONMENT, ECONOMIC POLICY, GOVERNMENTAL REGULATIONS, CURRENCY EXCHANGE RATE, COURT SYSTEM, LEGISLATION ENVIRONMENT, TAX POLICY.

For citation: Aslanishvili I. (2019), Supporting The Development Of Business Environment Through Economic Order Policy, Globalization And Business, №7, pp. 163-167. https://doi.org/10.35945/gb.2019.07.020

\section{INTRODUCTION}

As a policy that determines qualitative changes in the economy, economic order aims to establish a framework for economic activities in the public and private sectors and to adapt the said activities to the social, economic and technological processes [Streit 2005, pp. 26-30; 49-53].

Within the economic order policy, there is a conflict between the social and competitive orders. The competitive order is success-oriented, while the social order is aimed at satisfying requirements, which conflicts with the principle of success at least in part (Gvelesiani, 2018: 163-166).

On the one hand, therefore, the state tries to form and expand the limits of social order within the economic order policy, while on the other hand, the market is designed to establish a viable and competitive order and enable its implementation. Establishing suitable areas for public (social order) and private (competitive order) economic activities and setting rational boundaries between them is crucially important for the entrepreneurship development strategy. In countries where such areas and boundaries are properly established, small, medium-scale and large enterprises are achieving success on domestic and international markets by 'taking over' the relevant segments and retaining leading positions in the long term (Gvelesiani and Gogorishvili 2018:12).

The issue of regulations has become increasingly topical in Georgia, be it the property rights, product quality, antidumping measures, anti-dumping legislation or labour laws. We may sometimes think that certain regulations do not affect us directly, but this is a false view - there are no regulations that are 'worthless' to the public. We all pay towards them, and we naturally wish to get a product of suitable quality in return. In order to obtain good regulations, we need to answer the following question: what should we regulate, and how?

In response to the aforementioned question, most developed countries, such as the United States and the European Union member states, turn to a rather difficult and labour-intensive process of regulatory impact assessment (RIA), which requires a systematic study of its necessity, benefit and cost. On the one hand, state regulations are a useful tool for achieving government objectives, yet on the other hand, each regulation has a broad range of impact. Identifying potential harmful effects in various areas is difficult without a RIA. Ideal regulations ought to be proportionate to their objectives and economically balanced. In order to obtain good regulations, we must assess both their effectiveness and their expediency. Effectiveness alone is not sufficient.

A regulation is not an end in itself, but a means to an end. Its objective must be clear and attainable. Last year, the Parliament of Georgia initiated legislation on labour migration which was to impose certain bureaucratic barriers and restrictions on employment of foreign nationals in Georgia. Naturally, imposing such restrictions cannot constitute an end in itself. Restricting the employment of foreigners will not automatically increase the interest of employers towards the local labour market. Most employers decide to invite foreign specialists only when they cannot source suitably qualified personnel locally (incurring additional expenses for the company). At the same time, the disbalance between supply and demand on the market cannot 
be eliminated through such restrictions. In this particular case, the legislators took the views of the stakeholders on board and did not bring this regulation into force. However, in the absence of RIA, there is no guarantee that they will always act in such a reasonable manner.

In most cases, regulations impose new obligations on certain sections of the public (individuals or businesses). Naturally, complying with these regulations requires new or higher costs that are usually born both by the supplier of the product or service and by the consumer (Hayek, 1979: 128). An overly strict and 'costly' regulation has an even further-reaching effect, as it disproportionately affects the competitive environment. Nevertheless, introduction of RIA does not in itself guarantee harmlessness of regulations. The precise integration point of this tool in the government vertical is of crucial importance. Experience shows that the higher it is integrated institutionally, the more effective and compliant it is with the government's declared priorities.

Appropriately implemented RIA procedures significantly improve the government's decision-making culture, ensure transparency of the process and involvement of all stakeholders that may be affected by the relevant regulations, be they specific state bodies or individual groups. Ensuring RIA's success requires not only coordination and clear expression of political will at top level, but also the formulation of uniform standards and criteria that must be satisfied by all regulations. It is also necessary to systematize sources of information and implement a monitoring system. Subordination of regulatory decisions to the overall state policy and coordination between various branches of government is particularly important for economic policymaking. Otherwise, the process of forming a predictable and sound business environment is doomed to failure. We simply do not have the luxury to be able to introduce ill-thought-out regulations. Establishing an attractive business environment and attracting foreign direct investment is one of Georgia's main opportunities, if not the only one.

The Georgian Business Environment through the Eyes of the Private Sector study identifies and assesses the major issues that are currently considered as most pressing by the private sector in general, and by members of the Business Association of Georgia (large companies operating in Georgia) in particular. The most serious obstacles highlighted by the private sector are as follows: fluctuating currency exchange rates; the judiciary system; unpredictable changes in the regulatory environment; and labour resources. It is also interesting to note the private sector's opinion about the effectiveness of state institutions. The study shows that the private sector sees the central government as more effective than local self-government units and municipalities. With regards to dealing with public institutions, companies are most concerned about delays and decision-making. The results of the interviews are supported by quantitative research. Private company representatives state in the interviews that communication with the government is good, but post-communication (decision-making) stages are drawn out.

The existing tax environment in Georgia is seen highly positively by the private sector. Companies provide positive assessment not only of the tax policies and rates but also the administration of taxes, noting that there has been significant improvement in the latter component over the last three years. Several aspects of the tax environment were analysed and evaluated positively.

In spite of the challenges and problems identified by the study, the private sector has positive expectations. Most companies plan to grow and expand over the next 3-5 years. Overall, the private sector views the existing business environment positively and believes it to be favourable for day-to-day activities and long-term development. Georgian Business Environment through the Eyes of the Private Sector is an important analytical study carried out by the Business Association of Georgia (BAG). Through its economic reports and analytical publications, BAG enables informed decisionmaking in the private and public sectors. This study is of particular interest to policy-makers, as it presents the private sector's views on a wide range of issues, from macroeconomics to the effectiveness of bureaucracy.

\section{METHODOLOGY}

Various research methods were used in this article - specifically, those of induction, analysis, assumption, scientific generalisation, comparative analysis, data analysis, as well as investigative evaluation methods.

The Georgian Business Environment through the Eyes of the Private Sector study was carried out by my leadership in three stages. First, surveying works were carried out to identify the elements of the business environment and its challenges in full. Qualitative research was carried out during the second stage, the results of which formed the basis for quantitative research during the third stage.

The first stage involved studying international research and publications, as well as detailed analysis of the information accumulated over many year's of BAG's activity. This information was used to plan the structure and objectives of interviews for the second stage. Owners and heads of 44 BAG member companies were surveyed for the qualitative study, which was carried out with the help of in-depth face-to-face interviews. Questionnaires for the interviews were prepared in advance, and addressed the issues that are important to the business community. The goal of the qualitative study was to identify the main concerns of the private sector and test the hypotheses prepared on the basis of prior practice or the existing literature.

The third stage of research made use of the results of the qualitative study to devise a quantitative questionnaire for 40 BAG member companies. This aim at this stage was to provide quantitative assessment of the private sector's attitudes 
towards the elements of pressing importance for the business environment and the major problems facing the sector.

\section{AREA OF RESEARCH}

BAG member companies formed the target group for the Georgian Business Environment through the Eyes of the Private Sector study. These are large Georgian and international companies operating in Georgia. As they form a significant part of the Georgian economy, by examining their positions we can get a good picture of the general mood in the private sector, learn what the sector's expectations are and where it sees threats to its development and stability.

\section{RESEARCH RESULTS}

On the basis of qualitative and quantitative research, the Business Association of Georgia identified the most topical issues for the business environment. The most pressing issues for the private sector are currently as follows: the state economic policy; the tax environment; international relations; the judicial and regulatory environment; the courts and the alternative conflict resolution mechanisms, state institutions; public procurement; finances; and the labour market.

Having identified the main areas of research, the private sector's attitude towards various sub-components was analysed. In-depth interviews revealed the most significant areas for the Georgian business community, while the quantitative survey ranked these issues based on their importance. The results are shown in detail below.

The private sector identified the fluctuating currency exchange rate as the most topical and pressing issue, followed by the judiciary and the unpredictable changes in the regulatory environment. The full list of issues in order of their importance is as follows: the fluctuating currency exchange rate; the judiciary; the unpredictable changes in the regulatory environment; regional affairs (in neighbouring countries); the labour market; infrastructure; bureaucracy in central government institutions; bureaucracy at municipal level; tax environment; access to finances.

\section{CURRENCY EXCHANGE RATE}

The fluctuating exchange rate had a certain negative effect on $95 \%$ of the surveyed companies over the last year. Respondents stated that sharp fluctuations in a short period of time were particularly damaging for their business [Business Association of Georgia, 2017 Study].

\section{JUDICIARY}

The private sector identified the judiciary as the secondmost pressing problem, with $44.7 \%$ of the respondents rating the quality of the current system as 'unacceptably poor,' $34.2 \%$ as 'poor,' $18.4 \%$ as 'satisfactory,' and only $2.6 \%$ as 'good.'

Individual components of the judiciary, such as the impartiality of courts, competence, the efficiency of conflict resolution and the effectiveness of the judicial apparatus were also assessed in the study. The efficiency of conflict resolution was identified as the gravest problem by the private sector, with $54 \%$ of the companies rating it as 'unacceptably poor.' $51 \%$ of the respondents believe that competence levels in the judiciary are low. Furthermore, $43 \%$ also rated court impartiality as 'low.' Significantly, none of the components of the judiciary were rated highly by the respondents [Business Association of Georgia, 2017 Study].

\section{LEGISLATIVE ENVIRONMENT}

As mentioned earlier, BAG members see unpredictable changes in the regulatory environment as one of the main problems. The majority of respondents rated the existing legislative/regulatory environment negatively, with $44 \%$ stating that it hindered long-term development, while $24 \%$ believed that it also restricts day-to-day activities [Business Association of Georgia, 2017 Study].

We tried to establish the specific problems most frequently encountered by businesses in the legislative/ regulatory environment, and found that ambiguity and incoherence of laws and regulations constitutes the main problem for the majority of respondents, followed by the incompatibility the laws and regulations with their needs. Notably, $20 \%$ of companies did not identify any significant problems in the legislative/regulatory environment.

\section{PROBLEMS WITH LEGISLATION/REGULATIONS}

The survey asked the respondents to name the specific laws or legislative norms, the introduction/changes to which affected their business negatively over the last three years. This was an open question, and the most frequently stated answers included changes to the Labour Code, changes to the Law on Land Ownership and the unexpected changes to the Tax Code (more specifically, making advance payments subject to VAT).

\section{BUREAUCRACY}

The qualitative study revealed that the private sector sees timely and effective decision-making as one of the main challenges in state governance. It believes that the government is open in its relationship with the private sector, is prepared to take the latter's ideas and initiatives on board and look for best ways to resolve problems. The obstacles present themselves at the following stage, when it comes to making decisions and taking specific measures to resolve the issues.

It was interesting to see the private sector's assessment 
of individual state institutions. More than 20 such institutions were evaluated in the study, with the Ministry of Finance and the Ministry of Economy and Sustainable Development receiving the highest marks. Notably, only a negligible part of the respondents (1-2\%) rated the public institutions as either 'very good' or 'unacceptably poor.' The most prevalent rating was 'satisfactory.' The private sector believes that there is a significant room and need for improvement in the public sector (Business Association of Georgia, 2017 Study).

The study aimed not only to obtain a general assessment of the state institutions, but also to learn about the problems most frequently encountered by the private sector in their relations with these institutions. 'Drawn-out processes' and 'drawn-out decisions' were identified as the most frequently encountered issues, followed by 'unpredictability of policy' and 'lack of competence.' On a few occasions, 'rudeness in communication' and 'lack of communication' were identified as problems. As we can see the findings of the qualitative study were backed up by the quantitative study - the private sector encounters obstacles when it comes to decisionmaking and enforcement of decisions.

\section{TAX ENVIRONMENT}

The tax environment is one of the most crucial components of the business environment across the world, and Georgia is no exception. Nevertheless, tax issues were not among the most pressing problems identified by the private sector, which suggests that the tax policies and administration are viewed favourably. This was confirmed by respondents during the interviews. They believe that the country has a good tax policy and the administration component is also constantly improving.

The Georgian tax environment was also positively assessed in the quantitative analysis, with $68 \%$ of the surveyed companies believing that it is good for long-term development, while $32 \%$ also think that it is favourable for day-to-day activities. $35 \%$ see the tax environment as predictable. Only $6 \%$ of the respondents see the tax environment as an obstacle to the private sector (Georgian Business Environment through the Eyes of the Private Sector, 2017).

\section{CONCLUSIONS}

The study has identified problems that can mostly be resolved through the involvement of other economic policymaking stakeholders. Results show that pressing issues highlighted by the private sector include the judiciary and the unpredictable changes in laws and regulations. Judicial reforms have long been a subject of discussion, but they are not proceeding quickly enough. This creates a serious threat to the investment climate in Georgia. Failure to resolve this problem increases the level of mistrust towards the system and exacerbates the existing problems within the system itself. Ultimately, this will have a negative impact on the country's economy.

With regards to the unpredictable changes, the private sector has been highlighting the need for a regulatory impact analysis and implementing rational regulatory procedures for numerous years. The study has once again underlined the need for such reforms.

Attention should also be paid to the fact that drawnout processes and decisions have been identified as a major problem in relation to state institutions. Significantly, the private sector encounters this problem in its dealings with both the central and the local authorities. Due to the pressing nature of the issue, we believe that we need to conduct a detailed study into the causes of these delays and learn whether this issue can be resolved by streamlining processes, increasing the levels of competence, or through other means.

\section{REFERENCES:}

1. Hayek, F.A. (1979). Law, legislation and Liberty, Volume III - The Political Order of a Free People, Chicago, London, reproduced in: Vanberg, V. Hayek Gesammelte Schriften bd. B4, Recht, Gesetzgebung und Freiheit, Tuebingen 2003.

2. Hayek, F.A. (2003), Rechtsordnung und Handelnsordnung, in: Streit, M.E. Hayek Gesammelte Schriften Bd. A4, Rechtosordnung und Haldelnsordnung, Tuebingen, p. 217.

3. Streit, M.E. (2005), Theorie der Wirtschaftspolitik, 6. Edition, Stuttgart.

4. Gvelesiani, R., Gogorishvili, I. (2012). Compatibility of Economic and Political Decisions with Core Social Values. Tbilisi.

5. Gvelesiani, R., Gogorishvili, I. (2018). Decision-Making Technology in Economic Policy. Tbilisi.

6. Gvelesiani, R. (2017). Discrepancies between the Main Social Values and the Problem with overcoming them. Economy and Business Journal, Vol. X\#1, Tbilisi.

7. Gvelesiani, R. (2018). The Problem with Creating a Specific Framework for Economic Order. In 1st International Conference on Challenges of Globalisation in Business and the Economy materials, Tbilisi.

8. Georgian Business Environment through the Eyes of the Private Sector (2017). - Business Association of Georgia, October 2017. 


\section{SUPPORTING THE DEVELOPMENT OF BUSINESS ENVIRONMENT THROUGH ECONOMIC ORDER POLICY}

\section{IRAKLI ASLANISHVILI}

\section{PHd student}

Ivane Javakhishvili Tbilisi State University, Georgia

irakli.aslanishvili@gmail.com

KEYWORDS: BUSINESS ENVIRONMENT, ECONOMIC POLICY, GOVERNMENTAL REGULATIONS, CURRENCY EXCHANGE RATE, COURT SYSTEM, LEGISLATION ENVIRONMENT, TAX POLICY.

For citation: Aslanishvili I. (2019), Supporting The Development Of Business Environment Through Economic Order Policy, Globalization And Business, №7, pp. 55-167. https://doi.org/10.35945/gb.2019.07.020

\section{SUMMARY}

The goal of this study is to analyse the impact of economic order policy on the development of the business environment, as well as to learn about the private sector's current assessment of the existing business environment, the problems and challenges encountered by the private sector, and the areas on which the efforts ought to be focused. Study team grouped and leaded by me has examined the attitudes of members of the Business Association of Georgia (BAG) towards all major elements of the business environment.

The aim of my study is to use the results to devise BAG's future advocacy campaigns. It is planned to present the results of the study to all major parties involved in devising economic and business policies to ensure that they are familiar with the private sector's vision and are able to take it into account when working on economic policies. 


\section{CAREER MANAGEMENT ANALYSIS IN TERMS OF AGE}

\section{NUGZAR PAICHADZE}

PhD in Economics, Professor

Ivane Javakhishvili Tbilisi State University, Georgia

nugzar.paitchadze@tsu.ge

\section{NATALIA KHARADZE}

PhD in Economics, Associated Professor

Ivane Javakhishvili Tbilisi State University, Georgia

Natalia.kharadze@tsu.ge

\section{NINO PARESASHVILI}

PhD in Economics, Associated Professor

Ivane Javakhishvili Tbilisi State University, Georgia

Nino.paresaSvili@tsu.ge

\section{DEA PIRTSKHALAISHVILI}

\section{PhD Student}

Ivane Javakhishvili Tbilisi State University, Georgia

pirtskhalaishvilid@gmail.com

KEYWORDS: BUSINESS CAREER, MANAGEMENT, RESEARCH, CORRELATION, HYPOTHESIS.

For citation: Paichadze N., Kharadze N., Paresashvili N., Pirtskhalaishvili D. (2019), Career Management Analysis In Terms Of Age, Globalization And Business, №7, pp. 168-175. https://doi.org/10.35945/gb.2019.07.021

\section{INTRODUCTION}

The term «business career» is perceived differently. Some identify it with the word «promotion». In fact it is determined by a person's position in the society and may be associated not only with the promotion but the relegation of a person as well. And Career management involves managing actions aimed at appointing workers in all areas of human activity according to their professional suitability and competence. In other words, we are dealing with a promotion-relegation management of people. In the past working on the same position in one organization by an employee for decades was an ordinary phenomenon. Today we are living in a different world of people, very few employers have clearly defined successive stages of possible promotion and development. At the same time, few employees remain in one organization for a long time. The process of career movement is characterized by different approaches in different age groups. Exactly the attitude of different age groups towards the career management is the subject of our research.

\section{LITERATURE REVIEW}

Special attention is paid to the attitude towards the human resource management by the scientists employed in our department. These issues are studied from different angles. General trends (Karadze, N.; Paichadze, N.; Paresashvili, N.; Pirtskhalaishvili, D., 2019); (Karadze, N.; Paichadze, N.; Paresashvili, Ni.; Pirtskhalaishvili D.;, 2019) as well as the peculiarities in the gender perspective (Paichadze, N.; Karadze, 
N.; Giorgobiani, M.; Nikvashvili, M.;, 2019) ; (Paichadze, N.; Karadze, N.; Giorgobiani, M.; Nikvashvili, M., 2019) attention was paid to career management problems in different types of organizations (Paresashvili, N.; Giorgobiani, M.; Nikvashvili, M.; Pirtskhalaishvili, D.; Kharadze, N.;, 2019) ; (Paresashvili, ; Giorgobiani, M.; Nikvashvili, M.; Pirtskhalaishvili, D.; Kharadze, N.;, 2019) Development of leadership skills is directly linked to the Human Resource Management (Amkoladze, G.; Gabrichidze, A.; Giorgobiani, M.; Zedgenidze, M.; Kharadze, N., 2014), research of success factors of Georgian business leaders (Amkoladze, G; Gabrichidze, A.; Giorgobiani, M.; Lomsadze-Kuchava, M.; Kharadze, N., 2014) , conflict situation management in an organization (Kharadze, N.; Gulua, E.;, 2018) the development of relevant organizational culture (Gulua, E.; Kharadze, N., 2018) employed students' development research (Gulua, E.; Kharadze, N., 2018) in general as well as in the context of gender (Kharadze, N; Gulua, E., 2018), the attitude of young people's attitude towards the time management influences a personal career management (Gulua, E.; Kharadze,N., 2017) that has been studied by regions (Kharadze, N.; Gulua, E., 2017); and also by the examples of various leading universities (Kharadze \& Dugladze, 2018); (Pirtskhalaishvili, D.; Dugladze, D.;, 2018) free time management issues were also analyzed (kharazde, N.; Gulua, E.; Dugladze, D.;, 2017); (Kharadze, N.; Gulua, E.; Duglaze, D., 2017). Time management problems were discussed in the public and private sectors (Kharadze, N.; Dugladze, D.; Pirtskhalaishvili, D.;, 2018) Implications of students' attitude survey amalysis of professors' evaluation criteria (Kharadze, N; Gulua, E.;, 2018); (Kharadze, N.; Gulua, E., 2018) organizational culture management ( Paichadze,N., 2010); management of personality behavior, peculiarities of human resources, managers' qualification raising system (Paichadze, N., 2013), improvement of labor performance management (Paichadze,N., 2013), performance efficiency of personnel management service (N. Paichadze, 2014), professional competence as a factor of a society progress (N. Paichadze, 2015), the problem of unemployment in Georgia (Paichadze,N., 2016), challenges of human resource management at the edge of XXI Century (Paichadze,Nugzar, 2016), labor market in Georgian state sector (Paichadze,N., 2017), human resource management issues in state service (Paichadze,N., 2017), personnel motivation management (Paichadze,N., 2018), labor sociology and human resource management (Paichadze,N., 2018), some aspects of the unemployment problem in Georgia (Paichadze,N., 2018), management of behavior of state and municipal officials. (Paichadze,N., 2018).

\section{RESEARCH METHODOLOGY}

The research was conducted in the "Human Potential Management» laboratory at Ivane Javakhishvili Tbilisi State University. 560 respondents participated in the survey. The questionnaire included 32 questions and 125 options for an answer. SPSS statistical software package was used for statistical processing and data analysis.

\section{RESEARCH ANALYSIS}

In the course of the research, we were interested in what the attitude towards career issues was in different age groups. The survey found that a large proportion of the interviewed respondents from $72.7 \%$ to $88.4 \%$ in the age group of 18 to 29 years are employed in the private sector. Most of the surveyed respondents from 45 to 54 are in the state sector and this figure is approximately $43 \%$ and most of the surveyed respondents above 65 years are employed in educational institutions and their percentage is $75 \%$.

There is an interesting and dangerous tendency connected with employment of young people in Georgia. There are frequent cases when undergraduate students have 2 years and sometimes more than 2 years of work experience. In particular, the interviewed respondents in the age group of 18 to 24 indicate that they have 1 to 5 years of work experience, $90.9 \%$ of 18-19 age group respondents have 1-2 years of work experience and $68.2 \%$ of the interviewed respondents of 20-24 age group have 1-2 years of work experience, and $28.3 \%$ - 3-5 years of work experience. One of our researches which dealt with students time management and included the state as well as the private sector in different regions of the country showed us that the condition of the employed students is desperate. They are not able to study properly. It is impossible to plan free time and manage stress, they cannot provide healthy nutrition and all these factors affect the formation of a proper human resource.

As the research analysis suggests, tendencies for hiring different age groups are different, namely in the case of young people, the majority of the interviewed respondents $82.3 \%$ from 20-24 age groups are appointed to the posts by means of an interview. The tendency is changing over the age of 50 and as it is shown from the data the number of employees employed by means of a job contest in this age group is higher, in particular, in the age group of $50-54,25 \%$ of the interviewed respondents are appointed by a job interview, 32.1\% through the job competition and $42,9 \%$ are directly appointed. The study showed that the last time a large portion of the respondents in 50-54 age group (50\%) moved on the career ladder was over 8 years ago and it happened in the last year for $7,1 \%$. In the last one year the promotion rate is high among the respondents from 30 to 44 age groups, $27.9 \%$ of the interviewed respondents of 40-44 age group were promoted one year ago; In the 35-39 age group the same indicator is $26.1 \%$ and in $30-34$ age group $31.9 \%$, in $25-29$ age group $-66.7 \%$. With the age reduction the number of the respondents who was promoted one year ago increase. After the qualification improvement, the promotion rate is high in the 18-19 age group. It is natural that often an applicant is employed after the internship. This indicator is low in pension age. In particular, from 12 respondents interviewed 
in 60-64 age group who improved their qualification only one was promoted. $60 \%$ of the interviewed respondents in the age group of 55-59 were promoted after upgrading their qualifications, $42,9 \%$ in the $50-54$ age group. The lowest rate of promotion after qualification raising is in 20-24 age group. Career movements in all age groups happen largely within the organization. Over the age of 40 the respondents are less mobile and their movement outside the organization is insignificant. The respondents interviewed in the age group of 20 to 29 often leave their jobs and continue their career in other organizations. This indicator ranges from $27.8 \%$ to $36.3 \%$. It is natural that young people are more mobile and at the same time they change their jobs in the searching process.

There is little observance of hidden career signs among the interviewed respondents who are about the pension age, in particular, 60-64 age group respondents indicate that they are frequently and never invited to meetings and this figure is $8.3 \%$. The respondents who are above 60 years have answered the same question in the following way: $25 \%$ often and $0 \%$ - almost never.

More than half of the surveyed respondents in the age group of 18 to 29 say they cannot use their skills at work. From the age of 30 this attitude changes and with the increase in age respondents say that their skills are fully utilized at the workplace. It is interesting to know what attitude towards justice in different age groups is. The respondents of 18 to 24 age group think that the promotion process is not fair in the organization. More than $50 \%$ have the same attitude. $55.8 \%$ think the same in the 25 29 age group, in the $30-34$ age group $65.9 \%$ have the feeling of injustice, in the $35-39$ age group - $71.7 \%$, in the $60-64$ age group $75 \%$ have a negative position. This tendency is deplorable and I think it should become the subject of a serious study. Despite the fact that the respondents consider the promotion process unfair in all age groups, the faith in future by the respondents seemed strange for us and that they are confident in their own success. The belief of the future is especially high in the younger generation. In particular, $72.7 \%$ of the interviewed respondents in the 18-19 age group are fully convinced that they will succeed by career growth. With the age growth the belief in future is reduced in the respondents. The fact that career advancement is done with unfair methods has been proved with one more question. Respondents point out that they have information about the intervention of an influential person in promoting their colleagues. In particular, this position was observed by: $72.8 \%$ of the interviewed respondents in the 18-19 age group and $57.6 \%$ in the $20-24$ age group. In the respondents being more than 65 years this indicator is $75 \%$. As we see every age group respondents talk about the signs of nepotism. Against this background young people's optimistic attitude to achieve success is surprising. Such a feeling can be attributable to age. It is no question of debate that there is a problem and nepotism as a «national and chronic» disease of Georgians has to be solved.

The feeling of hopelessness has been strengthened by the question: if the respondents think that they will reach the maximum of their possibilities. The negative answer was the least indicated by the respondents of $18-19$ age group and it is $18.2 \%$. And in other age groups this negative attitude ranges from $20 \%$ to $39.1 \%$.

The family has always been in the first place because of our traditions in Georgia and people often gave up their careers because of it, particularly women. The survey found that the trend is changing, in particular, if the respondents interviewed in the 18-19 and 20-24 age groups prefer the career and these indicators are $45.5 \%$ and $41.9 \%$; in 55-59 and 60-64 age groups the career is preferred by only 6.7 and $0 \%$. It is noteworthy that the majority of the interviewed respondents in all age groups consider the growth of their own material welfare as the main aspiration of career growth. In most cases this indicator is higher than $50 \%$. Exceptions are only 18-19 age group respondents, $63.6 \%$ of which pay attention to the well- being of the society. It is nice to see this kind of attitude from young people and we think that old non-professional personnel should be replaced by exactly such staff.

The interesting fact is that young generation is less employed by their profession. For example, $54.5 \%$ of the interviewed respondents in the 18-19 age group indicate that they do not work by the profession, at the age of 20-24, this indicator is $49.5 \%$ and only $20 \%$ in the $45-49$ age group. Apparently, the younger generation does not often use the chosen profession and supposedly the funds invested by them are unreasonably spent.

\section{FORMULATING HYPOTHESES AND TESTING THE RELIABILITY OF THE MODEL USING THE COMPLICATED STATISTICAL PROCEDURES}

As a result of the research we have developed the following hypotheses:

Hypothesis 1: Age Q3 affects how people are accepted at work Q5.

We used Pearson's Correlation Analysis to determine the strength of the connection between the variables of this hypothesis. We got the correlation coefficient 0.415 . By means of the correlation, it was shown that between these variables there is a moderate positive attitude. (See Table 1)

To test the reliability of hypothesis we used Chi-square test that indicates that the statistical significance level P (Sig.) does not exceed 0.001 . Accordingly, the first hypothesis is reliable. (See Table 2)

\begin{tabular}{|c|c|c|c|}
\hline $\begin{array}{c}\text { Table 1: Chi-Square } \\
\text { Tests }\end{array}$ & Value & df & $\begin{array}{c}\text { Asymp. Sig. } \\
\text { (2-sided) }\end{array}$ \\
\hline Pearson Chi-Square & $\begin{array}{c}127.86 \\
9^{a}\end{array}$ & 22 & .000 \\
\hline Likelihood Ratio & $\begin{array}{c}132.50 \\
3\end{array}$ & 22 & .000 \\
\hline $\begin{array}{c}\text { Linear-by-Linear } \\
\text { Association }\end{array}$ & 96.129 & 1 & .000 \\
\hline N of Valid Cases & 560 & & \\
\hline
\end{tabular}




\begin{tabular}{|c|c|c|c|}
\hline \multicolumn{2}{|c|}{ Table 2: Correlation } & Q3 & Q5 \\
\hline \multirow[t]{3}{*}{ Q3 } & Pearson Correlation & 1 & $.415^{* *}$ \\
\hline & Sig. (2-tailed) & & .000 \\
\hline & $\mathrm{N}$ & 560 & 560 \\
\hline \multirow[t]{3}{*}{ Q5 } & Pearson Correlation & $.415^{* *}$ & 1 \\
\hline & Sig. (2-tailed) & .000 & \\
\hline & $\mathrm{N}$ & 560 & 560 \\
\hline
\end{tabular}

According to both indicators, hypothesis 1: Age Q3 affects how people are accepted at work Q5 has been confirmed.

Hypothesis 2: Age Q3 affects working experience Q4.

We used Pearson's Correlation Analysis to determine the strength of the connection between the variables of this hypothesis. We got the correlation coefficient 0,870 . By means of the correlation, it was shown that between these variables there is a strong positive attitude (See Table 3)

From Table 4, received as a result of single factor dispersion analysis, it is shown that $\mathrm{F}=200.3, \mathrm{P}=0.000$; $\mathrm{P}<0.01$, i.e. between the groups a statistically significant link is noticed on 0.01 level. (See Table 4)

\begin{tabular}{|c|c|c|c|}
\hline \multicolumn{2}{|c|}{ Table 3: Correlation } & \multirow{2}{*}{$\begin{array}{c}\text { Q3 } \\
1\end{array}$} & \multirow{2}{*}{$\begin{array}{c}\text { Q4 } \\
.840^{* *}\end{array}$} \\
\hline Q3 & Pearson Correlation & & \\
\hline & Sig. (2-tailed) & & .000 \\
\hline & $N$ & 560 & 560 \\
\hline \multirow[t]{3}{*}{ Q4 } & Pearson Correlation & $.840^{* *}$ & 1 \\
\hline & Sig. (2-tailed) & .000 & \\
\hline & $\mathrm{N}$ & 560 & 560 \\
\hline
\end{tabular}

\begin{tabular}{|c|c|c|c|c|c|}
\hline $\begin{array}{c}\text { Table 4 : } \\
\text { ANOVA }\end{array}$ & $\begin{array}{c}\text { Sum of } \\
\text { Squares }\end{array}$ & Df & $\begin{array}{c}\text { Mean } \\
\text { Square }\end{array}$ & F & Sig. \\
\hline $\begin{array}{c}\text { Between } \\
\text { Groups }\end{array}$ & 2574.542 & 9 & 286.0 & 200.49 & .000 \\
\hline $\begin{array}{c}\text { Within } \\
\text { Groups }\end{array}$ & 784.708 & 550 & 1.427 & & \\
\hline Total & 3359.250 & 559 & & & \\
\hline
\end{tabular}

Consequently, the hypothesis 2: Age Q3 affects working experience Q4 has been confirmed.

Hypothesis 3: Age Q3 affects when was the last time you got a promotion Q6.

We used Pearson's Correlation Analysis to determine the strength of the connection between the variables of this hypothesis. We got the correlation coefficient 0.641. By means of the correlation, it was shown that between these variables there is a moderate positive attitude. (see Table 5)

The sixth table (see Table 6) shows the results of Levene's statistics, the difference of averages (the same $T$ value) equals to 11.085 , the statistical significance level $\mathrm{P}$ (Sig.) does not exceed 0.001 , i.e. between the vertical and horizontal career growth and career movement of the employed people inside and outside the organization there is a statistically high level of connection on 0.01 level.

Consequently, the hypothesis 3: Age Q3 affects when was the last time you got a promotion $Q 6$ has been confirmed.

\begin{tabular}{|c|c|c|c|}
\hline \multicolumn{2}{|c|}{ Table 5: Correlation } & Q3 & Q6 \\
\hline \multirow[t]{3}{*}{ Q3 } & Pearson Correlation & 1 & $.641^{* *}$ \\
\hline & Sig. (2-tailed) & & .000 \\
\hline & $\mathrm{N}$ & 560 & 560 \\
\hline \multirow[t]{3}{*}{ Q6 } & Pearson Correlation & $.641^{* *}$ & 1 \\
\hline & Sig. (2-tailed) & .000 & \\
\hline & $\mathrm{N}$ & 560 & 560 \\
\hline
\end{tabular}

\begin{tabular}{|c|c|c|c|}
\hline $\begin{array}{c}\text { Table 6: Test of } \\
\text { Homogeneity of } \\
\text { Variances }\end{array}$ & df1 & df2 & Sig. \\
\hline 11.085 & 2 & 556 & .000 \\
\hline
\end{tabular}

Hypothesis 4: Age Q3 affects whether or not you were promoted in a career over the last 5 years? Q12.

We used Pearson's Correlation Analysis to determine the strength of the connection between the variables of this hypothesis. We got the correlation coefficient 0.122 . By means of the correlation, it was shown that between these variables there is a weak positive attitude. (See Table 7)

To test the reliability of hypothesis we used Chi-square test that indicates that the statistical significance level P (Sig.) does not exceed 0.001 . Accordingly, the first hypothesis is reliable. (See Table 8)

\begin{tabular}{|c|c|c|c|}
\hline \multicolumn{2}{|c|}{ Table 7: Correlation } & Q3 & Q12 \\
\hline \multirow[t]{3}{*}{ Q3 } & Pearson Correlation & 1 & $.122^{* *}$ \\
\hline & Sig. (2-tailed) & & .004 \\
\hline & $\mathrm{N}$ & 560 & 560 \\
\hline \multirow[t]{3}{*}{ Q12 } & Pearson Correlation & $.122^{* *}$ & 1 \\
\hline & Sig. (2-tailed) & .004 & \\
\hline & $\mathrm{N}$ & 560 & 560 \\
\hline
\end{tabular}

\begin{tabular}{|c|c|c|c|}
\hline $\begin{array}{c}\text { Table 8: Chi-Square } \\
\text { Tests }\end{array}$ & Value & df & $\begin{array}{c}\text { Asymp. Sig. (2- } \\
\text { sided) }\end{array}$ \\
\hline $\begin{array}{c}\text { Pearson Chi-Square } \\
\text { Likelihood Ratio }\end{array}$ & $37.718^{\mathrm{a}}$ & 11 & .000 \\
\hline $\begin{array}{c}\text { Linear-by-Linear } \\
\text { Association }\end{array}$ & 8.39 .718 & 11 & .000 \\
\hline N of Valid Cases & 560 & 1 & .004 \\
\hline
\end{tabular}

Consequently, the hypothesis 4 Age Q3 affects whether you were promoted in a career over the last 5 years has been confirmed

Hypothesis 5: Age Q3 affects whether a person is searching for a job in a parallel mode Q34.

We used Pearson's Correlation Analysis to determine 
the strength of the connection between the variables of this hypothesis. We got the correlation coefficient 0.131 . By means of the correlation, it was shown that between these variables there is a weak positive attitude. (See Table 9)

To test the reliability of hypothesis we used Chi-square test that indicates that the statistical significance level $\mathrm{P}$ (Sig.) equal to 0.082 . Which means that hypothesis 5 is not reliable. (See Table 10)

\begin{tabular}{|c|c|c|c|}
\hline \multicolumn{2}{|c|}{ Table 9: Correlation } & Q3 & Q12 \\
\hline Q3 & Pearson Correlation & 1 & $.131^{* *}$ \\
\cline { 2 - 4 } & Sig. (2-tailed) & & .002 \\
\hline \multirow{2}{*}{ Q12 } & N & 560 & 560 \\
\hline & Pearson Correlation & $.131^{* *}$ & 1 \\
\cline { 2 - 4 } & Sig. (2-tailed) & .002 & \\
\hline & N & 560 & 560 \\
\hline
\end{tabular}

\begin{tabular}{|c|c|c|c|}
\hline $\begin{array}{c}\text { Table 10: Chi-Square } \\
\text { Tests }\end{array}$ & Value & df & $\begin{array}{c}\text { Asymp. Sig. (2- } \\
\text { sided) }\end{array}$ \\
\hline $\begin{array}{c}\text { Pearson Chi-Square } \\
\text { Likelihood Ratio }\end{array}$ & $17.993^{\mathrm{a}}$ & 11 & .082 \\
\hline $\begin{array}{c}\text { Linear-by-Linear } \\
\text { Association }\end{array}$ & 9.625 & 11 & .067 \\
\hline N of Valid Cases & 560 & 1 & .002 \\
\hline
\end{tabular}

Consequently, the hypothesis 5 Age Q3 affects whether a person is searching for a job in a parallel mode Q34 has not been confirmed

\section{CONCLUSIONS AND RECOMMENDATIONS}

1. The findings of the research indicate to a significant trend of human resource distribution. The aging process of human resource is noticeable in educational institutions and replacement of young people almost does not happen, which negatively impacts the education system, it is important to make appropriate state initiatives at the level of law, which will increase young people's interest;

2. The signs of nepotism have been revealed in responses to various questions that kill a feeling of justice for all generations;

3. In general, in order to increase the sense of justice, it is important to have the same attitude in any field and towards professional staff of any age group. Such an approach can make a hope and a sense of justice in our society;

4. The organizations are less likely to take into consideration the opinions of highly experienced employees, which can not be considered normal. We think that highly experienced respondents have the right experience and the management should take their proposals into account. Otherwise, this age group is better to be replaced with younger staff. And towards the people having a retirement age, the organization has to work out such policies that will make their retirement less painful. This problem is in all areas;

5. The feeling of hopelessness has a negative effect on the productivity of an employee. This attitude is especially important in the younger generation. Proper personnel policies should be introduced by joint efforts of the state and organizations not to receive disappointed generations.

\section{REFERENCES:}

1. Paichadze,N. (2013). for Improvment of Labor Effectiveness Management. ECONOMICS AND BUSINESS, 199.

2. Paichadze,N. (2010). Organizational culture as indvisible part of human resources. ECONOMICS and BUSINESS, 113-123.

3. Paichadze,N. (2017). Improvment of the Human Resources Management in State Service. ECONOMICS AND BUSINESS, $19-29$.

4. Paichadze,N. (2018). Aspects of Unemployment Problems in Georgia. BULLETIN OF THE GEORGIAN NATIONAL ACADEMY OF SCIENCES, 133-139.

5. Paichadze,N. (2018). Labor Sociology in Human Resource Management. CHALLENGES OF GLOBALIZATION IN ECONOMICS AND BUSINESS (pp. 288-291). TBILISI: Tsu.

6. Amkoladze, G.; Gabrichidze, A.; Giorgobiani, M.; Zedgenidze, M.; Kharadze, N. (2014). Characteristics of development of leadership in the environment of global economy. Modern issues of Medicine and Maagement, 141-145.

7. Amkoladze, G; Gabrichidze, A.; Giorgobiani, M.; Lomsadze-Kuchava, M.; Kharadze, N. (2014). Globalization and research of success factors of Georgian business leaders. Modern issues of Medicine and Maagement, 146-151.

8. Gulua, E.; Kharadze,N. (2017). Impact of Time Management on Personal Development of Master's Degree Students. ICSS XXIII, (pp. 110-118). Vienna.

9. Gulua, E.; Kharadze, N. (2018). Employed Studentsı Development Challenges in Georgia. European Journal of Interdisciplinary Studies, p. 188-208.

10. Gulua, E.; Kharadze, N. (2018). Organization Culture Management Challenges. European Journal of Interdisciplinary Studies, 67-79.

11. Karadze, N.; Paichadze, N.; Paresashvili, N.; Pirtskhalaishvili, D. (2019). Generan trends of business career management. 17th International Conference on Social Sciences (pp. 253-254). Murcia: EUSER. 
12. Karadze, N.; Paichadze, N.; Paresashvili, Ni.; Pirtskhalaishvili D.;. (2019). General trends of business career management. European Journal of Economics and Business Studies.

13. Kharadze, N., \& Dugladze, D. (2018). Tame management of private university students. INNOVATIVE ECONOMICS AND MANAGEMENT, 126-137.

14. Kharadze, N.; Dugladze, D.; Pirtskhalaishvili, D.; (2018). Comparative Analysis of Students Time Management at State and Private Universities. Innovative Economics and Management, 151-162.

15. Kharadze, N.; Gulua, E. (2017). Time Management Pecularities of Shota Rustaveli State University MA Students. Innovative Economics and Management, 20-25.

16. Kharadze, N.; Gulua, E. (2018, Volume 5). Analyze of Students' Attitude Survey of Professor Evaluation Criteria. Journal Innovative Economics and Management, pp. 122-131.

17. Kharadze, N.; Gulua, E.;. (2018). Organization Conflict Management Challenges. European Journal of Economics and Business Studies, 30-41.

18. Kharadze, N.; Gulua, E.; Duglaze, D. (2017). Free-Time Management among Master's Degree Students of Georgia. ICSS XXIII, (pp. 24-33). Vienna.

19. Kharadze, N; Gulua, E. (2018). Personal Development Peculiarities on Gender Perspective in Georgia. European Journal of Multidisciplinary Studies, p. 111-123.

20. Kharadze, N; Gulua, E.; (2018, Volume 5). IMPLICATIONS OF STUDENTS' ATTITUDE SURVEY ANALYSIS OF PROFESSORS' EVALUATION CRITERIA. Journal Innovative Economics and Management, pp. 122-131.

21. kharazde, N.; Gulua, E.; Dugladze, D.;. (2017). Free-Time Management among Master's Degree Students of Georgia. European Journal of Social Science Education and Research, 24-33.

22. N. Paichadze. (2014). Some issues on improvement the problems of Personnel Management Service. ECONOMICS AND BUSINESS, 27-43.

23. N. Paichadze. (2015). Professional Competence as the Decisive Factor in labour Effectiveness of Public Servants. ECONOMICS AND BUSINESS, 35-49.

24. Nugzar Paichadze. (2018). Management of Organizational Behavior of Governmental and Municipal Employees and the Ways of Perfection. ekonomisti, 37-44.

25. Paichadze, N.; Karadze, N.; Giorgobiani, M.; Nikvashvili, M. (2019). Career management trends in terms of gender. European Journal of Economics and Business Studies.

26. Paichadze, N.; Karadze, N.; Giorgobiani, M.; Nikvashvili, M.;. (2019). Career management trends in terms of gender. 17th International Conference on Social Sciences (pp. 124-143). Murcia: EUSER.

27. Paichadze,N . (2013). Coherent System for Improving Managers` Qualification- Prerequasite for Organizations to Operate succecfully. ECONOMICS and BUSINESS, 11-29.

28. Paichadze,N. (2016). Unemployment Problem in Georgia and Ways of Its Overcoming. ECONOMICS AND BUSINES, 9-27.

29. Paichadze,N. (2017). Labor Market in the State Sector. CHALLENGES OF GLOBALIZATION IN ECONOMICS AND BUSINESS (pp. 390-395). Tbilisi: Tsu.

30. Paichadze,N. (2018). STATE MOTIVATION AS AN AFFECTIVE FACTOR OF HUMAN RESOURCE USAGE. MODERN TENDENCIES OF DEVELOPMENT OF ECONOMY AND ECONOMIC SCIENCE (pp. 82-85). Tbilisi: PAATA GUGUSHVILI INSTITUTE.

31. Paichadze,Nugzar . (2016). CHALLENGES OF HUMAN RESOURCE MANAGMENT ON THE EDGE OF XXI CENTURY. INNOVATIVE ECONOMY AND PROBLEMS OF ITS FORMATION IN POST-COMMUNIST COUNTRIES (pp. 79-81). Tbilisi: PAATA GUGUSHVILI INSTITUTE.

32. Paresashvili, ; Giorgobiani, M.; Nikvashvili, M.; Pirtskhalaishvili, D.; Kharadze, N.;. (2019). Career Management Peculiarities in Educational Institutions. European Journal of Economics and Business Studies.

33. Paresashvili, N.; Giorgobiani, M.; Nikvashvili, M.; Pirtskhalaishvili, D.; Kharadze, N.;. (2019). Career Management Peculiarities in Educational Institutions. 17th International Conference on Social Sciences (pp. 212-231). Murcia: EUSER.

34. Pirtskhalaishvili, D.; Dugladze , D.;. (2018). Statistical Analysis of Students` Time Management and Private Hypotheses in Private Universities. Innovative Economics and Management, 124-130. 


\section{CAREER MANAGEMENT ANALYSIS IN TERMS OF AGE}

\section{NUGZAR PAICHADZE}

PhD in Economics, Professor

Ivane Javakhishvili Tbilisi State University, Georgia

nugzar.paitchadze@tsu.ge

\section{NATALIA KHARADZE}

PhD in Economics, Associated Professor

Ivane Javakhishvili Tbilisi State University, Georgia

Natalia.kharadze@tsu.ge

\section{NINO PARESASHVILI}

PhD in Economics, Associated Professor

Ivane Javakhishvili Tbilisi State University, Georgia

Nino.paresaSvili@tsu.ge

\section{DEA PIRTSKHALAISHVILI}

\section{PhD Student}

Ivane Javakhishvili Tbilisi State University, Georgia

pirtskhalaishvilid@gmail.com

KEYWORDS: BUSINESS CAREER, MANAGEMENT, RESEARCH, CORRELATION, HYPOTHESIS.

For citation: Paichadze N., Kharadze N., Paresashvili N., Pirtskhalaishvili D. (2019), Career Management Analysis In Terms Of Age, Globalization And Business, №7, pp. 168-175. https://doi.org/10.35945/gb.2019.07.021

\section{SUMMARY}

Business career enables human resources to increase qualification opportunities and become recognizable at the same time. In the course of the research, we got interested what kind of attitude towards career issues was in different age groups. What the manner was by which the career of different generation respondents was managed, what the respondents' attitude is to the career, how they perceive the career management policies implemented by the executives of the organization. The research was conducted in the "Human Potential Management» laboratory at Ivane Javakhishvili Tbilisi State University. 560 respondents participated in the survey. The questionnaire included 32 questions and 125 options for an answer. SPSS statistical software package was used for statistical processing and data analysis.
As a result of the research we have developed the hypotheses:

Hypothesis 1: Age Q3 affects how people are accepted at work Q5.

We used Pearson's Correlation Analysis to determine the strength of the connection between the variables of this hypothesis. We got the correlation coefficient 0.415 . By means of the correlation, it was shown that between these variables there is a moderate positive attitude.

To test the reliability of hypothesis we used Chi-square test that indicates that the statistical significance level P (Sig.) does not exceed 0.001 . Accordingly, the first hypothesis is reliable.

According to both indicators, hypothesis 1: Age Q3 affects how people are accepted at work Q5 has been confirmed.

Hypothesis 2: Age Q3 affects working experience Q4. 
We used Pearson's Correlation Analysis to determine the strength of the connection between the variables of this hypothesis. We got the correlation coefficient 0,870 . By means of the correlation, it was shown that between these variables there is a strong positive attitude received as a result of single factor dispersion analysis, it is shown that $\mathrm{F}=200.3, \mathrm{P}=0.000$; $P<0.01$, i. e. between the groups a statistically significant link is noticed on 0.01 level.

Consequently, the hypothesis 2: Age Q3 affects working experience $\mathrm{Q} 4$ has been confirmed.

Hypothesis 3: Age Q3 affects when was the last time you got a promotion Q6.

We used Pearson's Correlation Analysis to determine the strength of the connection between the variables of this hypothesis. We got the correlation coefficient 0.641 . By means of the correlation, it was shown that between these variables there is a moderate positive attitude.

The results of Levene's statistics, the difference of averages (the same $T$ value) equals to 11.085 , the statistical significance level P (Sig.) does not exceed 0.001, i.e. between the vertical and horizontal career growth and career movement of the employed people inside and outside the organization there is a statistically high level of connection on 0.01level.

Consequently, the hypothesis 3: Age Q3 affects when was the last time you got a promotion Q6 has been confirmed.

Hypothesis 4: Age Q3 affects whether you were promoted in a career over the last 5 years? Q12.
We used Pearson's Correlation Analysis to determine the strength of the connection between the variables of this hypothesis. We got the correlation coefficient 0.122 . By means of the correlation, it was shown that between these variables there is a weak positive attitude.

To test the reliability of hypothesis we used Chi-square test that indicates that the statistical significance level P (Sig.) does not exceed 0.001. Accordingly, the first hypothesis is reliable.

Consequently, the hypothesis 4 Age Q3 affects whether you were promoted in a career over the last 5 years has been confirmed

Hypothesis 5: Age Q3 affects whether a person is searching for a job in a parallel mode Q34.

We used Pearson's Correlation Analysis to determine the strength of the connection between the variables of this hypothesis. We got the correlation coefficient 0.131 . By means of the correlation, it was shown that between these variables there is a weak positive attitude.

To test the reliability of hypothesis we used Chi-square test that indicates that the statistical significance level P (Sig.) equal to 0.082 . Which means that hypothesis 5 is not reliable.

Consequently, the hypothesis 5 Q3 Age affects whether a person is searching for a job in a parallel mode Q34 has not been confirmed.

Some recommendations were worked out based on the research. 


\section{INTANGIBLE CULTURAL HERITAGE AS AN INNOVATIVE APPROACH FOR TOURISM DEVELOPMENT IN GEORGIA}

\section{LALI GIGAURI}

PhD student, International Black Sea University, Georgia

lali.gigauri@ibsu.edu.ge

\section{EKA DEVIDZE}

PhD in Business Administration, Professor, European University, Georgia edevidzeproject@gmail.com

KEYWORDS: INTANGIBLE CULTURAL HERITAGE, NICHE PRODUCTS, TOURISM, GEORGIA.

For citation: Gigauri L., Devidze E. (2019), Intangible Cultural Heritage As An Innovative Approach For Tourism Development In Georgia, Globalization And Business, №7, pp. 176-181. https://doi.org/10.35945/gb.2019.07.022

ABSTRACT

The article offers an alternative innovative approach to tourism product (Intangible Cultural Heritage-based) development in Georgia. As the trends of global travel market assume, increasingly demanded products become the ones offering unique and profound cultural experience, deep emotions and feelings that derive from a participation in the authentic cultural activity. The study suggests to explore and further promote Georgian intangible cultural heritage for the purpose of supporting better representation of regions of Georgia in tourist offers, creating "Niche” products, raising awareness and increasing self-involvement in the process.

\section{INTRODUCTION}

Georgia joined Convention for the Safeguarding of the Intangible Cultural Heritage of UNESCO in 2007. The country recognized the need for protecting intangible cultural heritage and assumed the obligations implied by the convention: identification, documentation, exploration, protection, preservation, revitalization of the intangible cultural heritage for the purpose for safeguarding its living culture; raising its awareness through formal or informal education, transferring to new generation, reviving different aspects of the intangible cultural heritage.

According to the convention, intangible cultural heritage is considered as traditions, habits, art expressions, knowledge and instruments, artefacts, cultural spaces as tangible aspects of these expressions of the specific groups or individuals of the community, such as: oral traditions and expressions (legends, fables, etc.) art and performance (singing, dancing, theater, etc.), common practices, celebrations (games, festivals, holidays, gastronomy, etc.), practices related with the environment (folk medicine, traditions related with natural forces) and crafts' directions and techniques (craftsmanship: Felt, metal, ceramics, carpets, etc. )

Intangible cultural heritage is found in the communities in a form of traditions, habits, oral traditions, songs, games, etc. They are also documented in private and state archives, museums, scientific and research institutes and similar organizations. Georgian intangible cultural heritage is represented on UNESCO's intangible cultural heritage list of the humanity (Georgian polyphonic singing (2001), traditional Kvevri wine-making (2013), the living culture of the Georgian alphabet (2016), Georgian wrestling “Chidaoba" (2018).The National Agency for Cultural Heritage Preservation Georgia has been actively implementing the activities and measures for identifying and safeguarding of the Georgian intangible 


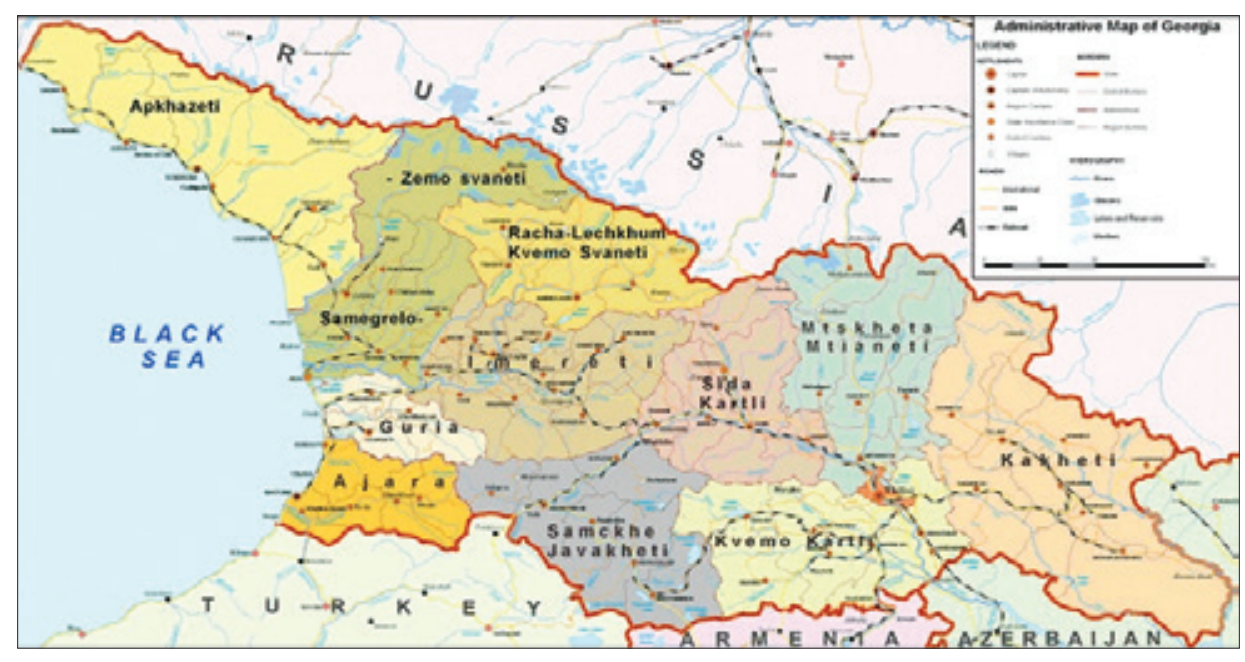

heritage. In 2012, The Minister of the Culture and Monuments Protection of Georgia issued an order \#3/28, which approved the form for identification of the cultural heritage monument of Georgia and instructions for completing the form.

\section{ABOUT OF GEORGIA}

Georgia, due to its strategic location in the Caucasus region where Europe meets Asia, is a home to biodiversity and cultural interactions. Georgia is neighbored by Russia, Azerbaijan, Armenia and Turkey. Being a part of the historic Silk Road route as well as a target of continuous invasions from various empires throughout the centuries has had deep impact on the local culture development. Nowadays, Georgia, referred to as a hidden gem of the Caucasus, attracts increasing interest of travelers from around the world. Once in Georgia, visitors discover distinct unique culture which is not comparable with others. Increasing interest of travelers has been a main driving factor for tourism development during the last 10 years in Georgia. Despite rich potential for tourism potential development in Georgia, the country still struggles with identifying and offering niche products and increasing awareness at an international level. Using intangible cultural heritage assets while developing tourism product is one of the potential tools of positioning the country as having and offering unique cultural experience, originating from traditions formed before the centuries. In addition, using intangible cultural heritage for tourism development is an effective way of showcasing and promoting regional diversity, which itself is an important supporter of regional development.

In order to refer to intangible cultural heritage as a tool for tourism product development and enrichment, measures need to be taken in order to ensure having a complete database of the explored, well-known or forgotten traditions according to regions of Georgia. As mentioned previously, The National Agency for Cultural Heritage Preservation Georgia has introduced the document, a form which is used for nominating the intangible cultural heritage asset as a monument. The document identifies the domain/category of intangible cultural heritage asset, aims to obtain photo/ video/audio material for presentation purposes; tries to identify historic and geographic area where the tradition/ practice is common; the origin and original function of it; the community or individuals bearing the tradition/cultural expression; the document also tries to identify all relevant attributes such as gender, age and social characteristics of the bearers of the tradition; its current status and any risks/ dangers it might be facing. The document is a very useful tool for identifying new monuments and further provision of valuable material for better presentation.

\section{INTANGIBLE CULTURAL HERITAGE OF GEORGIA}

Prior to the searching and identifying somehow forgotten or unexplored or less explored expressions of the intangible cultural heritage, it is important to analyze already existing list, representing districts/regions of Georgia or the country in general, as follows below:

Table 1. Intangible Cultural Heritage List Recognized by the National Agency for Cultural Heritage Preservation Georgia

\section{ICH Distribution according to Districts of Georgia}

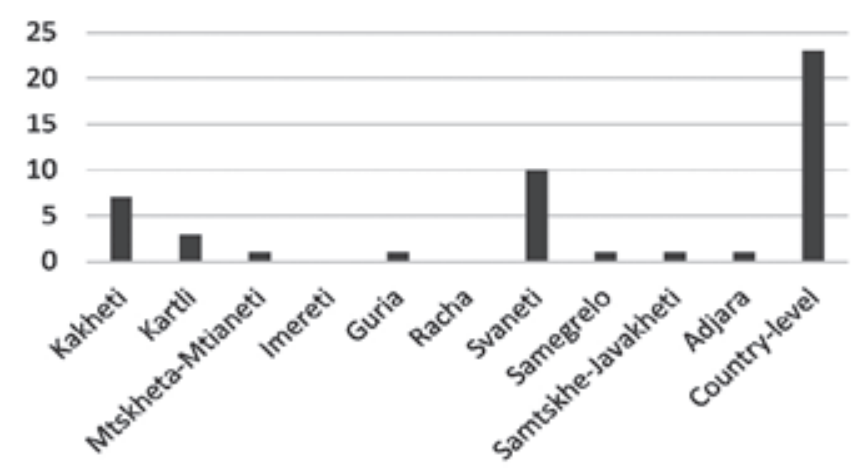

Figure 1. ICH Distribution according to Districts of Georgia 


\begin{tabular}{|c|c|c|}
\hline Districts/Regions & Name of Intangible cultural heritage & $\begin{array}{l}\text { Number } \\
\text { of ICH }\end{array}$ \\
\hline Mtskheta-Mtianeti & $\begin{array}{l}\text { Pshavian "Dambalkhacho" } \\
\text { (mold cottage cheese) producing technology and culture }\end{array}$ & 1 \\
\hline Guria & Leloburti (Georgian handball rugby) & 1 \\
\hline Imereti & & 0 \\
\hline Racha-Lechkhumi & & 0 \\
\hline Samegrelo & $\begin{array}{l}\text { Abkhazian Salt „Apirpiljika” and Megrelian ajika (Capsicum) } \\
\text { making technology }\end{array}$ & 1 \\
\hline Samtskhe-Javakheti & Traditional technology of producing Meskhian cheese "Tenili" & 1 \\
\hline Adjara & $\begin{array}{l}\text { Dance Khorumi } \\
\text { Cooking tradition on Borano }\end{array}$ & 2 \\
\hline Kartli & $\begin{array}{l}\text { Urban (Kalakuri) Mravaljamieri } \\
\text { Tskhavatian pottery } \\
\text { Ksnian Ulami - tradition of community members helping out each other with laboring works }\end{array}$ & 3 \\
\hline \multirow[t]{7}{*}{ Kakheti } & Kvevri Wine-making & \multirow[t]{7}{*}{7} \\
\hline & Kvevri & \\
\hline & Kakhetian Mravaljamieri & \\
\hline & Traditional technology of baking Kakhetian bread (Mother's bread) & \\
\hline & $\begin{array}{l}\text { Traditional technology of producing Kakhetian Churchkhela (sweets with walnuts and boiled } \\
\text { grape juice) }\end{array}$ & \\
\hline & Traditional technology of weaving Kakhetian hat & \\
\hline & Traditional pottery in Vardisubani & \\
\hline \multirow[t]{10}{*}{ Svaneti } & Svan folk instrument "Chuniri" traditional crafting technology & \multirow[t]{10}{*}{10} \\
\hline & Svan traditional hat weaving technology & \\
\hline & Svan cuisine - Millet Khachapuri (cheese pie) baking technology & \\
\hline & Svan cuisine - Kubdari (pie with spicy chopped meat) baking technology & \\
\hline & Svan cuisine - Traditional technology of Svan salt producing & \\
\hline & $\begin{array}{l}\text { Svan cuisine - Traditional technology of cooking Tashmjabi (mashed potatos with melted } \\
\text { cheese) }\end{array}$ & \\
\hline & $\begin{array}{l}\text { The tradition of using sour mineral healing waters "Mugviri", Artskheeli", "Kakhrld", "Legab", } \\
\text { "Seti", "Kvedilashi" and "Shdegi" in Upper Svaneti }\end{array}$ & \\
\hline & Traditional method of crafting Svan folk instrument "Changi" & \\
\hline & Wood carving tradition - carvings in the Svan dwelling and domestic crafts & \\
\hline & Svan traditional mourning funeral ritual & \\
\hline \multirow[t]{22}{*}{ Country Level } & Polyphonic singing & \multirow{22}{*}{22} \\
\hline & "Dedaena" - The method of alphabet teaching in primary schools by lakob Gogebashvili & \\
\hline & Perkhuli (dance) & \\
\hline & Berikaoba (performance) & \\
\hline & The tradition of issuing periodical for children "Dila" & \\
\hline & Student inauguration ceremony of the University of Theater & \\
\hline & The continuous tradition of issuing periodical "Tsiskari" (Dawn) & \\
\hline & Georgian Wrestling "Chidaoba" & \\
\hline & Living culture of three writing systems of the Georgian alphabet & \\
\hline & The tradition of learning poem "Knight in the panther's skin" by heart & \\
\hline & The continuous tradition of issuing periodical "Gantiadi" (Sunrise) & \\
\hline & Falconry hunting & \\
\hline & Culture and traditions and of the Georgian traditional feast & \\
\hline & Culture and traditional technology of producing Blue Tabla (tablecloth) & \\
\hline & Georgian folk medicine traditions and application & \\
\hline & The unique tradition of 26-century-long friendship between Georgians and Jewish & \\
\hline & Traditional technology of producing Georgian silk & \\
\hline & Traditional technology of baking Khachapuri (cheese pie) & \\
\hline & Laghidze Water Producing Technology and Culture & \\
\hline & Georgian wheat culture (Endemic types and local breeds) & \\
\hline & Georgian Folk Horse Races (Isindi, Tskhenburti -Horseball, Kabakhi, Marula ) & \\
\hline & Traditional technology of Danelia family wines & \\
\hline
\end{tabular}


The table \#1 and figure \#1 shows that intangible cultural heritage list is poorly represented on a regional basis. It is also undoubtable that remaining unexplored or less explored regions such as Racha-Lechkhumi Kvemo Svaneti, Imereti, Guria and Samegrelo have rich intangible cultural heritage with distinct traditions that can proudly be represented on the list of Georgian Intangible Cultural Heritage (National level). It is a topic of further research, based on primary and secondary sources, to identify and nominate intangible cultural heritage assets for further recognition and promotion.

\section{BENEFITS OF INTANGIBLE CULTURAL AS NICHE PRODUCTS}

Except for distinct economic benefits, which intangible cultural benefit niche products can bring, they can also deliver other equally important values for the local as well as global community:

- $\quad \mathrm{ICH}$-based tourism can revitalize intangible cultural heritage of the local community and give a new life to it;

- It can provide and strengthen a sense of identity and continuity within local communities;

- It can lead to job creation, support better state of economic and social well-being of local communities through involving them in developing, maintaining and offering authentic products to tourists;

- It can help with intercultural dialogue and encourage mutual respect among cultures;

- It can support sustainable development relying on cultural authenticity and creativity as mean resources;

- It can create a possibility to offer an innovative tourism product replying to the new demand of the travelers eager to sense unique experiences;

- It can potentially increase interest of tourists to places despite their seasonality and geographic nonattractiveness;

- It can create an opportunity of capacity building of the local community and women empowerment especially in patriarchal communities;

- It can encourage re-inhabitation of the abandoned remote places by communities to showcase their authentic culture in its original birthplace;

- As a manifestation of the freedom of expression, $\mathrm{ICH}$ based cultural tourism can be considered as a defender and promoter of human rights within communities and worldwide;

- It can, if effectively managed, significantly reduce the negative effect of globalization, which tends to erase differences among cultures;

- Revenue generated from ICH-based tourism can provide funding for initiatives aiming at revival of local cultural heritage.

\section{CONCLUSION}

The positive trend of travelers' seeking for in-depth cultural experience and emotion are driving factors of quick growth of $\mathrm{ICH}$-based tourism. ICH-based tourism brings multiple benefits for communities, among which are revitalization and strengthening its identity as well as income generation and job offers. $\mathrm{ICH}$-based tourism can successfully serve the purpose of sustainable development of the communities if risks of irresponsible use of living culture and over tourism are considered.

\section{REFERENCES:}

1. Abankina, T., (2013), Regional development models using cultural heritage resources, International Journal of Culture, Tourism and Hospitality Research, Vol. 7 Iss 1 pp. 3 - 10

2. Browse the Lists of Intangible Cultural Heritage and the Register of good safeguarding practices, UNESCO Intangible Cultural Heritage Website, 2018, ich.unesco.org/en/lists

3. Clare S. Ch. Ch., (2018) "Sustainability of indigenous folk tales, music and cultural heritage through innovation», Journal of Cultural Heritage Management and Sustainable Development, Vol. 8 Issue: 3, pp.342-361, https://doi.org/10.1108/ JCHMSD-06-2017-0044

4. Keller, K.L., (2013), Strategic Brand Management: Building, Measuring, and Managing Brand Equity, Pearson

5. Operational Directives for the implementation of the Convention for the Safeguarding of the Intangible Heritage, Intangible Cultural Heritage Website, 2011, ich.unesco.org/en/directives

6. Pietro, L. D., et al, (2015) Cultural heritage and consumer behaviour: a survey on Italian cultural visitors, Journal of Cultural Heritage Management and Sustainable Development, Vol. 5 Issue: 1, pp.61-81, https://doi.org/10.1108/JCHMSD-03-2013-0009 7. Trapani, M. S. G. D., (2010), Cultural tourism and creative regeneration: two case studies, International Journal of Culture, Tourism and Hospitality Research, Vol. 4 Iss 3 pp. $214-227$

8. Vasavada, F., \& Kour, G. (2016). Heritage Tourism: How Advertising is Branding the Intangibles? Journal of Heritage 
Management, 1(1), 22-34. https://doi.org/10.1177/2455929616640688

9. Vogt N., et al, (2013), Intangibles: enhancing access to cities) cultural heritage through interpretation, International Journal of Culture, Tourism and Hospitality Research, Vol. 7 Iss 1 pp. 68 - 77

10. What Is Intangible Cultural Heritage?, UNESCO Intangible Cultural Heritage Website, 2011, ich.unesco.org/en/what-isintangible-heritage-00003

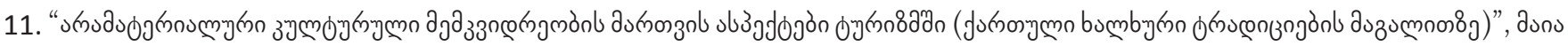
§зпбъпмпо, 2016, tafu.edu.ge/files/pdf/Disertacia/maia-gvinjilia-disertacia.pdf

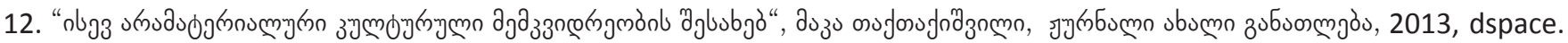
nplg.gov.ge/bitstream/1234/123906/1/Axali_Ganatleba_2013_N32.pdf

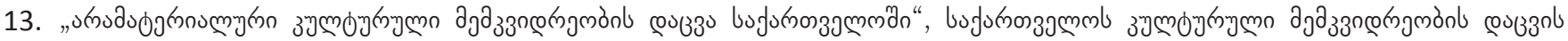

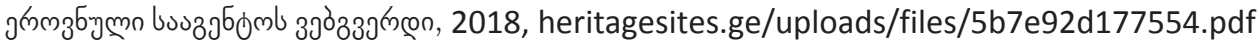

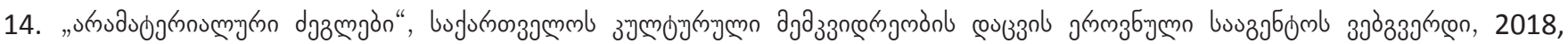
heritagesites.ge/uploads/files/5b9261cb470e4.pdf

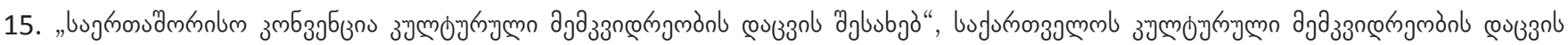

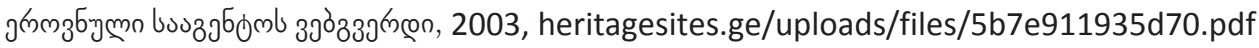




\section{INTANGIBLE CULTURAL HERITAGE AS AN INNOVATIVE APPROACH FOR TOURISM DEVELOPMENT IN GEORGIA}

\section{LALI GIGAURI}

PhD student,

International Black Sea University, Georgia

lali.gigauri@ibsu.edu.ge

\section{EKA DEVIDZE}

PhD in Business Administration,

Professor, European University, Georgia

edevidzeproject@gmail.com

KEYWORDS: INTANGIBLE CULTURAL HERITAGE, NICHE PRODUCTS, TOURISM, GEORGIA.

For citation: Gigauri L,, Devidze E. (2019), Intangible Cultural Heritage As An Innovative Approach For Tourism Development In Georgia, Globalization And Business, №7, pp. 176-181. https://doi.org/10.35945/gb.2019.07.022

\section{SUMMARY}

The article offers an alternative innovative approach to tourism product (Intangible Cultural Heritage-based) development in Georgia. As the trends of global travel market assume, increasingly demanded products become the ones offering unique and profound cultural experience, deep emotions and feelings that derive from a participation in the authentic cultural activity. The study suggests to explore and further promote Georgian intangible cultural heritage for the purpose of supporting better representation of regions of Georgia in tourist offers, creating "Niche” products, raising awareness and increasing self-involvement in the process. 


\section{MAIN CHALLENGES OF HUMAN RESOURCES MANAGEMENT IN EMPLOYEE CAREER DEVELOPMENT}

\section{KRISTINE ODISHVILI}

\section{PhD Student}

Ivane Javakhishvili Tbilisi State University, Georgia

Kristine.odishvili522@ens.tsu.edu.ge

\section{MAIA NIKVASHVILI}

\section{PhD Student}

Ivane Javakhishvili Tbilisi State University, Georgia

Maia.nikvashvili@tsu.ge

\section{KEYWORDS: MANAGEMENT, HUMAN RESOURCES MANAGEMENT, CAREER DEVELOPMENT, EMPLOYEE.}

For citation: Odishvili K., Nikvashvili M. (2019), Main Challenges Of Human Resources Management In Employee Career Development, Globalization And Business, №7, pp. 182-186. https://doi.org/10.35945/gb.2019.07.023

\section{ABSTRACT}

It is known that the creation of a perfect product / service is directly connected to the qualification of the employees.

Competition between companies has led to any organization that needs to constantly improve its product / service. The company can offer the novelty only through the employees. In addition, the process of establishing the novelty must be permanent.Thus it makes clear the need of management for the process. Obviously this is not easy because it is associated with the management of living people, with the development of creative skills, which is unpredictable. Witch is the responsibility of human resources management.

The survey examines the problems and challenges that the Human resourse management must overcome for employee career development.

In the article the discussion is about human resource management approaches to strategic management, development, motivation and corporate culture of employees.

The structure of the article: introduction, researches review of the relevant direction, career developmentresearch findings, main challenges and recommendations.

The study showed an interesting picture in terms of career development. We hope that the findings of this study will provide Human resources management specialists with a clear understanding of the challenges and will pay more attention to the career development of the employees.

\section{INTRODUCTION}

Success or failure of any organization depends on its employees.If the staff is professional, it will significantly increase the success of the company. The competition between companies has led to the fact that any organization needs to constantly improve its product. The company will be able to offer new materials only if its employees will be created. In addition, the creation / submission must be permanent, thus it makes clear the need for standardization and management of the process. Obviously, this is not easy because it is associated with the management of living people, with the development of creative skills, which is unpredictable.

As our research has shown, the following things are important for the development of creative skills in the staff: strategic management of human resources, investing in the education/development of the staff, elaborating motivation schemes, forming a healthy corporate culture.

The article describes the results and recommendations of each of the above mentioned directions.

The first issue we want to focus on is the strategic 
management of human resources in the organization, which implies the formation of a competitive workforce that allows the company to exist, develop and reach its own goals in the long run.

Strategic management of human resources is a long-term clear vision of management on what they want to achieve in human resource management. If the management issues will have a clear answer about what they want to achieve in the long term, it will make it easier for them to see the necessary steps taken in the short and medium term. Also, we will not be able to talk about the strategic management of the staff if the management does not have the companys strategy, because these two are closely linked to each other and the management is based on the companyss strategy. The main goals of the strategic management of human resources are the following:

- Company requirements for human resources should be satisfied in the longer term. The company should have longterm employees whose creative potential will be relevant to the requirements of the organization.

- Employees> pay compensation plan to help them be motivated and develop their career.

- Be able to develop leadership skills in the staff, who have the ambition, desire and qualification

- Being an effective system of teaching and professional development in the organization to enhance their creative skills.

- Set up an effective communication system between personnel, management and departments.

Itıs also important that the companyss staff should feel very strongly, taking care from the management because they do their best for the company.

\section{LITERATURE REVEW}

career management problems are actual as well as in business (Kharadze, N; Paichadze, N; Paichadze, N; Pirtskhalaishvili, D, 2019)also in educational institutions (Paresashvili, N; Giorgobiani, M; Nikvashvili, M; Pirtskhalaishvili, D; Kharadze, $\mathrm{N}$; 2019). Some peculiarities are observed in the gender aspect (Paichadze, N; Kharadze, N; Giorgobiani, M; Nikvashvili, M; 2019) management of people with modern methods affect their development (Amkoladze, G; Gabrichidze, A; Giorgobiani, M; Zedgenidze, M; Kharadze, N;, 2014) which should be taken into account when developing a labor market strategie (Paresashvili, N;, 2015) and In the process of

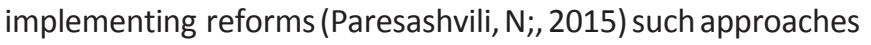
reduce the conflict situations (Nikvashvili, M., 2016) the need for reforms confarms the research that concerns the success of Georgian business leaders (Amkoladze, G; Gabrichidze, A; Giorgobiani, M; Lomsadze-Kuchava, M; Kharadze, N., 2014) during the management process it is important to take into consideration the cultural values (Kharadze, N; Chikhradze,K., 2015); (Paresashvili, N., 2016);(Nikvashvili, M., 2017) and peculiarities of the region (Korganashvili, L; Kharadze, N;; 2014) it is important for career management to include Eastern Partnership Recommendations (Paresashvili, N;, 2013), for self-development in human resource is important to conduct trainings (Kharadze, N; Dugladze, D; Pirtskhalaishvili, D; 2018); (Pirtskhalaishvili, D; Dugladze , D; 2018)

\section{RESEARCH METODOLOGY}

The research was conducted on the the example of hotel services in Tbilisi capital city of Georgia. The questionnaire according to which the survey was carried out consisted of 35 questions. The survey was attended by the respondents employed in Hotels in Tbilisi, that were randomly selected in a short period of time. The study was conducted both of them, quantitative and qualitative.

SPSS statistical software package was used for statistical processing and analysis of data.

\section{RESEARCH OBJECT}

The goal is to research the problems of career development and its impact on human resources challenges in the service sector.

The survey was carried out on the example of the service sector. Georgian brand hotels were involved and 117 people were interviewed and 35 questions were asked.

\section{RESEARCH ANALYS}

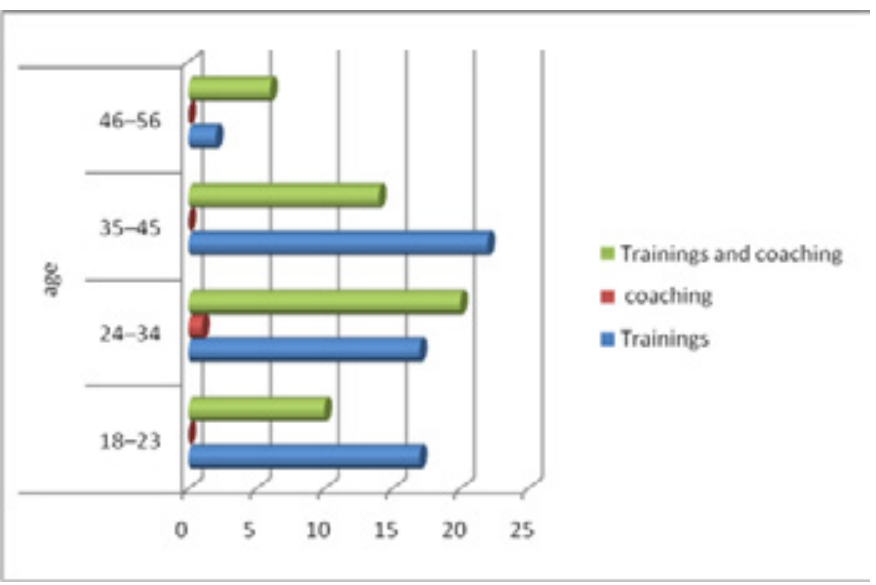

Figure 1: development

At the stage of research, the career development tools of employees were revealed

mainly training and coaching is becoming increasingly important for staff qualification. 
One of the most important functions of Human Resources Management is training and development. Training can be evaluated as a method of improvement of productivity of staff. The training means helping staff develop a proper attitude and behavior. It has played an important role in the development of human potential. Training encourages employees to be constantly productive and oriented towards advancing the knowledge of the current developments due to the selected trainings that are preceded by a number of activities: Determining the method of training and selection of content create the relevant focus groups. There are internal and external trainings, and in the first case the staff are undergoing relevant issues for the organization ss current activities, and external training is largely directed towards increasing the efficiency of the employees and acquiring their leadership skills. These kinds of trainings include lectures and conferences that help people reach a wider range and listen to ideas that will help to assess and solve a range of problems.

In todayss reality it is also important the role of coaching/»mentoring» in the development of staff. Its goal is to create collaborative, self-confidence and values that help in personal and career development. The mentor is an expert position, its function is to offer advice on solving the issue, which depends on its experience and knowledge. Many companies are actively using Mentoring today because its benefits are very large for the mentor. On the one hand, the mentor is getting more experienced, more respectful for himself, developing a better leader, and may be able to express new.

As we see, trainings and developments promote the staff more competent, and the staff are more committed to their work, as they see that they care about it, trust and do not delay - try to constantly think about the development of the organization, its advancement, protect his image and self-constantly evolving, because they are responsible for the success of the organization. Therefore, any organizationss priority is training and development, it increases the productivity and creativity of employees, which directly reflects the success of the organization.

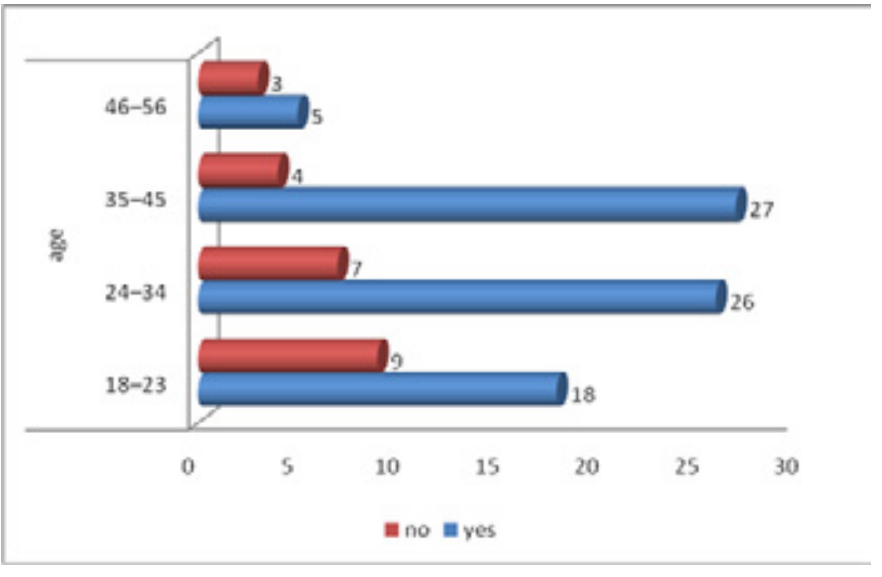

Figure 2: Employee success priority
According to the age the priority of the succes of the employee has been analyzed.The people of the age 18-45 think that the development of the employee is a priority for their organization. 45 years and older people do not feel the priority of development by their company which can be explained by the fact that they are less involved in the trainings and continue to work with accumulated experience. This attitude can be reflected on the motivation of the company and it is important to change the attitude towards them to feel like a full member of the organization.

It is interesting to evaluate the results of the survey with the priority of permanent development of employees by the age (Figure 3) and by the different level of managers (Figure 4).

The constant development of employees according to age is the following:

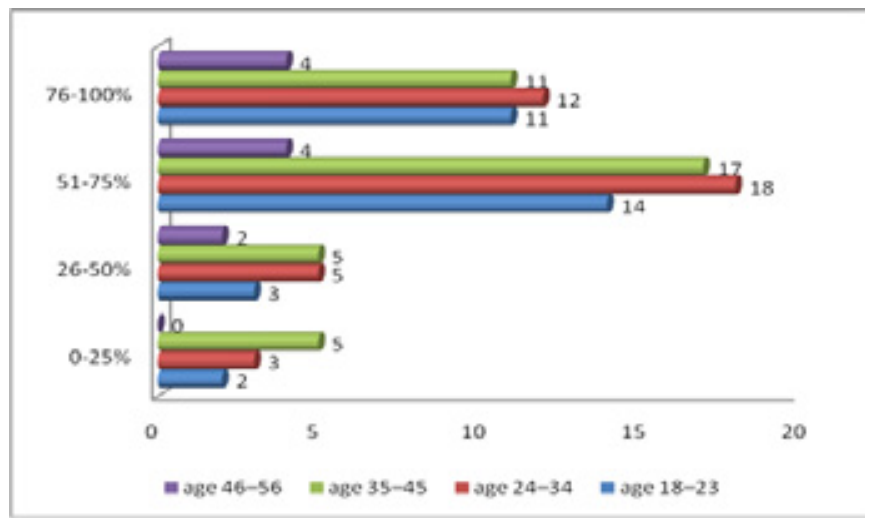

Figure 3: Priority of Constant development by the age

Career development was mostly "almost important" and "important" by age group form 18 to 45 . In edition, as we mentioned above (figure 2), the interest from 45 years is quite small in the career development.

The value of career development according to the status of employees has also been evaluated (see figure4).

It is important that middle and low HR valued the importance of career development. This is considered a positive part of organizational culture because the number indicates that they see this priority and participate in it.

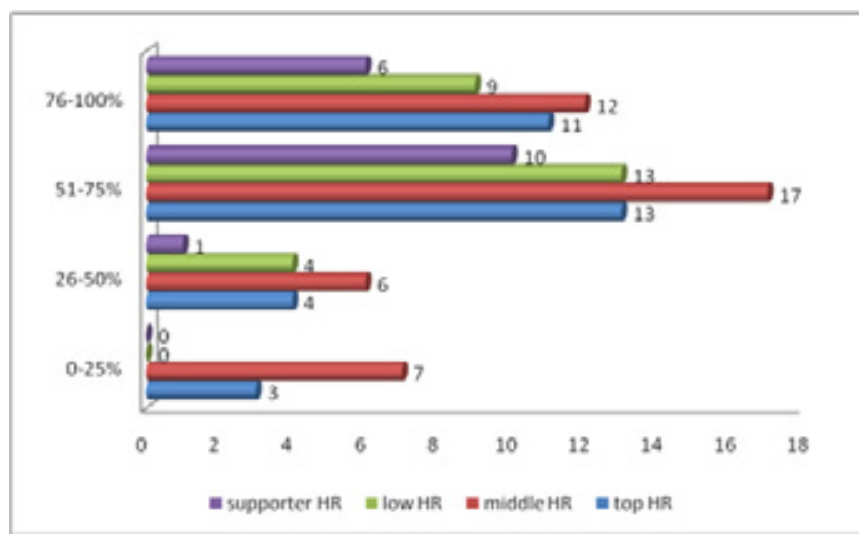

Figure 4: Priority of Constant development by different level of $H R$ 
Organizational culture is one of the key factors for promoting healthy and creative skills to achieve the success of the organization. $56 \%$ of the respondents considered organizational culture as the main factor of competitive advantage.

\section{CUNCLUSION}

As the study has shown trainings and coaching are the main factors for the development of employee qualifications.

Success of any company depends on how satisfied the customer is. Customers) satisfaction can only be achieved if the organization will offer it a constantly updated and perfect product, this requires creative skills by employees but this practice is not easy.
For the development of creative skills for employee is needed:

- Proper strategic management of human resources;

- Investing in education and development;

- Correct motivation system;

- Employee should have a clear understanding of the importance of its success for the companys success;

- Employee should have a feeling that management listens to them, provides advice and recommendations;

- Company management should teach employees to take risks;

- Healthy corporate system.

Finally, it can be said that organizational culture of promoting healthy and creative skills is one of the key factors for achieving the success of the organization.

\section{REFERENCES:}

1. Amkoladze, G; Gabrichidze, A; Giorgobiani, M; Lomsadze-Kuchava, M; Kharadze, N. (2014). Globalization and research of success factors of Georgian business leaders. Modern issues of Medicine and Maagement, 146-151.

2. Amkoladze, G; Gabrichidze, A; Giorgobiani, M; Zedgenidze, M; Kharadze, N;. (2014). Characteristics of development of leadership in the environment of global economy. Modern issues of Medicine and Maagement, 141-145.

3. Kharadze, N; Chikhradze,K. (2015). Cultural Parallels Between Russia and Georgia and Their Effect on Women Employment. CULTURE AND RUSSIAN SOCIETY. Helsinki.

4. Kharadze, N; Dugladze, D; Pirtskhalaishvili, D;. (2018). Comparative Analysis of Students Time Management at State and Private Universities. Innovative Economics and Management, 151-162.

5. Kharadze, N; Paichadze, N; Paichadze, N; Pirtskhalaishvili, D. (2019). GENERAL TRENDS OF BUSINESS CAREER MANAGEMENT. 17th International Conference on Social Sciences (pp. 253-254). Murcia: EUROPEAN CENTER FOR SCIENCE EDUCATION AND RESEARCH.

6. Korganashvili, L; Kharadze, N;. (2014). Regional Project Management of Tourism Development in Georgia. ProjectManagement-Development-Practice-and-Perspectives, 109-116.

7. Nikvashvili, M. (2016). Stimulation of Constructive Bihavior in Conflict Situations. CHALLENGES OF GLOBALIZATIN INECONOMICS ANDBUSINESS (pp. 398-402). Tbilisi: Universal.

8. Nikvashvili, M. (2017). Organizational Culture as an Integral Part of Agricultural Practices. CHALLENGES OF GLOBALIZATIONIN AND BUSINESS (pp. 363-367). Tbilisi: TSU.

9. Paichadze, N; Kharadze, N; Giorgobiani, M; Nikvashvili, M;. (2019). CAREER MANAGEMENT TRENDS IN TERMS OF GENDER. 17th International Conference on Social Sciences (pp. 124-143). Murcia: EUROPEAN CENTER FOR SCIENCE EDUCATION AND RESEARCH.

10. Paresashvili, N. (2016). CORPORATE CULTURE IN TERMS OF LABOR DIVERSITY. International Conference "Smart and Efficient Economy: Preparation for the Future Innovative Economy, (pp. 321-327 pg.). Brno, Czech Republic.

11. Paresashvili, N;. (2013). Policy Implementation of the Eastern Partnership in Georgia. Public Policy and Administration, 633-643.

12. Paresashvili, N;. (2015). Major Mechanisms to Develop the Strategies of the Labor Market in Georgia. Procedia - Social and Behavioral Sciences, 574-579.

13. Paresashvili, N;. (2015). Reforms and policies fostering Georgia as emerging market. Public Policy and Administration, 54-64.

14. Paresashvili, N; Giorgobiani, M; Nikvashvili, M; Pirtskhalaishvili, D; Kharadze, N;. (2019). Career Management Peculiarities in Educational Institutions. 17th International Conference on Social Sciences (pp. 212-231). Murcia: EUROPEAN CENTER FOR SCIENCE EDUCATION AND RESEARCH.

15. Pirtskhalaishvili, D; Dugladze , D;. (2018). Statistical Analysis of Studentsı Time Management and Private Hypotheses in Private Universities. Innovative Economics and Management, 124-130. 


\section{MAIN CHALLENGES OF HUMAN RESOURCES MANAGEMENT IN EMPLOYEE CAREER DEVELOPMENT}

\section{KRISTINE ODISHVILI}

\section{PhD Student}

Ivane Javakhishvili Tbilisi State University, Georgia

Kristine.odishvili522@ens.tsu.edu.ge

\section{MAIA NIKVASHVILI}

\section{PhD Student}

Ivane Javakhishvili Tbilisi State University, Georgia

Maia.nikvashvili@tsu.ge

\section{KEYWORDS: MANAGEMENT, HUMAN RESOURCES MANAGEMENT, CAREER DEVELOPMENT, EMPLOYEE.}

For citation: Odishvili K., Nikvashvili M. (2019), Main Challenges Of Human Resources Management In Employee Career Development, Globalization And Business, №7, pp. 182-186. https://doi.org/10.35945/gb.2019.07.023

\section{SUMMARY}

It is known that the creation of a perfect product / service is directly connected to the qualification of the employees.

Competition between companies has led to any organization that needs to constantly improve its product / service. The company can offer the novelty only through the employees. In addition, the process of establishing the novelty must be permanent.Thus it makes clear the need of management for the process. Obviously this is not easy because it is associated with the management of living people, with the development of creative skills, which is unpredictable. Witch is the responsibility of human resources management.

The survey examines the problems and challenges that the Human resourse management must overcome for employee career development.

In the article the discussion is about human resource management approaches to strategic management, development, motivation and corporate culture of employees.

The structure of the article: introduction, researches review of the relevant direction, career developmentresearch findings, main challenges and recommendations.

The study showed an interesting picture in terms of career development. We hope that the findings of this study will provide Human resources management specialists with a clear understanding of the challenges and will pay more attention to the career development of the employees. 


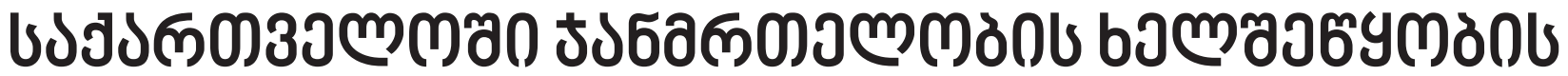

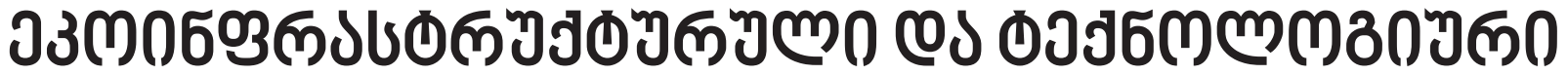

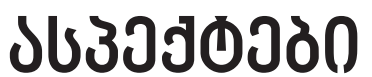

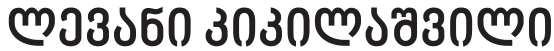

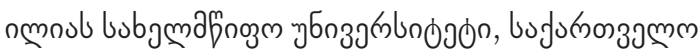

Ikikilashvili555@gmail.com

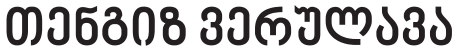

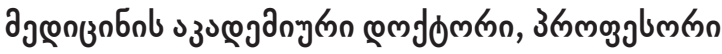

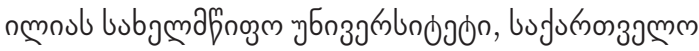

tengiz.verulava@iliauni.edu.ge

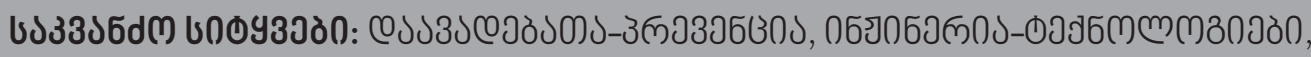

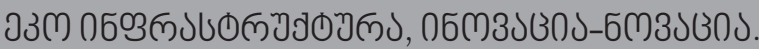

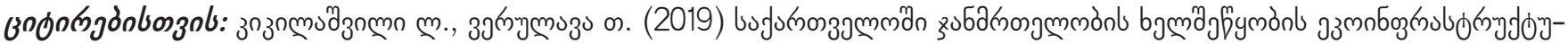

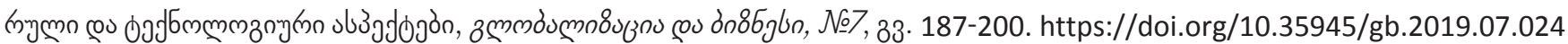

\section{ə0เ১3১ल0}

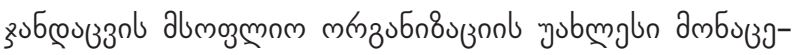

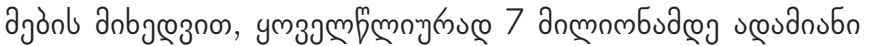

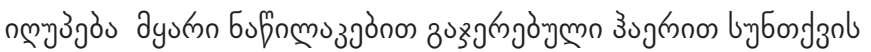

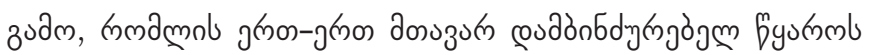

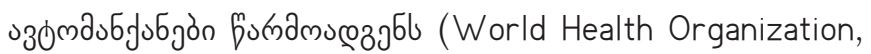

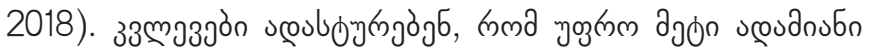

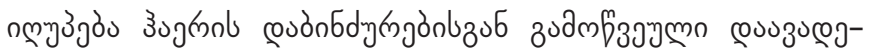

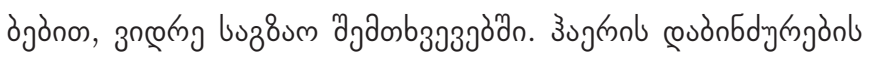

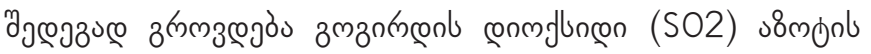

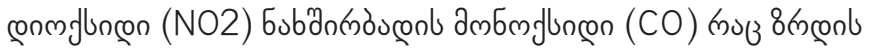

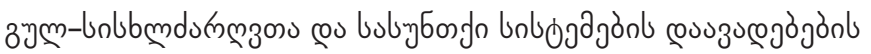

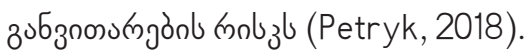

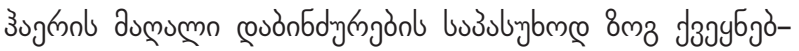

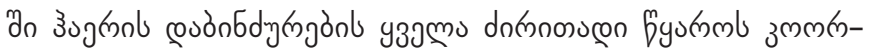

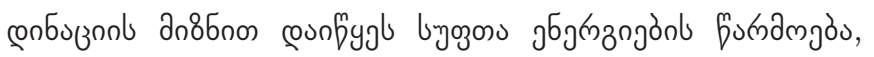

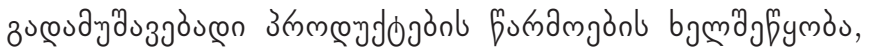

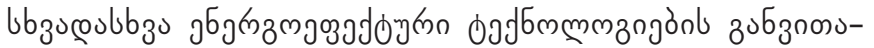

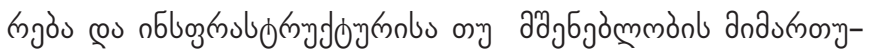

mgònon u३nmagmjöjòn (Madhipatla Kishore et al, 2018).

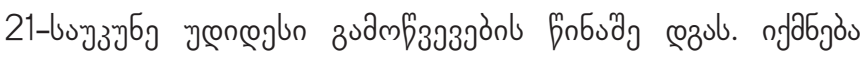

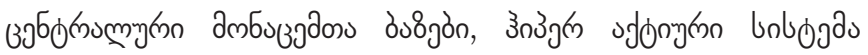

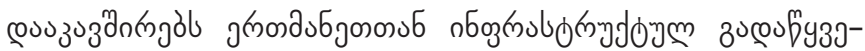

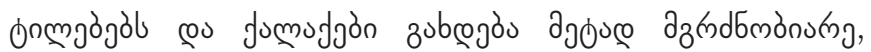

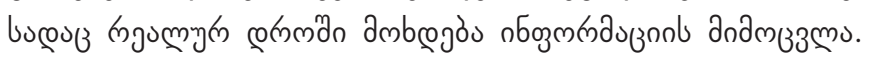

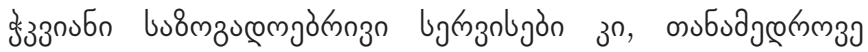

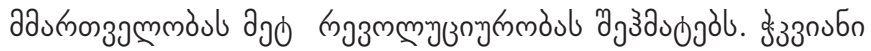

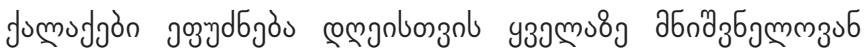

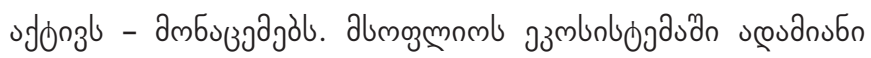

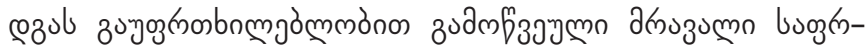

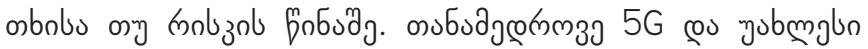

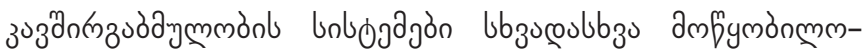

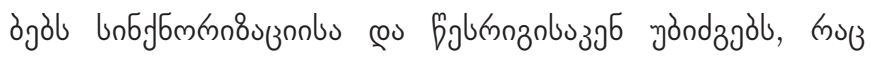

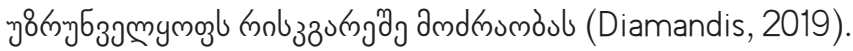

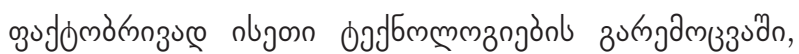

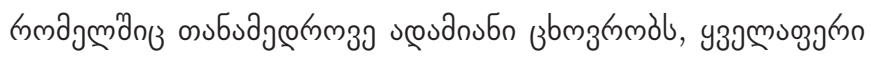

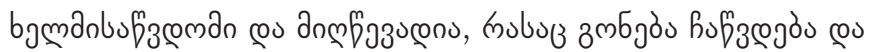

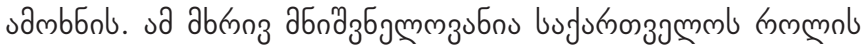

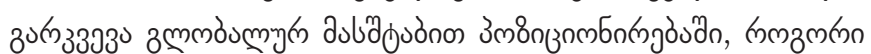




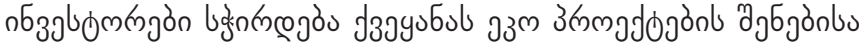

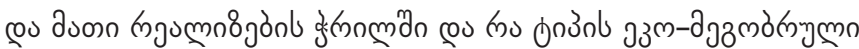

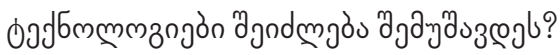

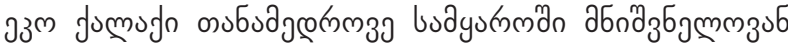

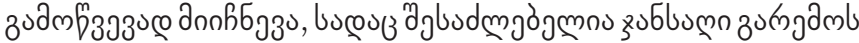

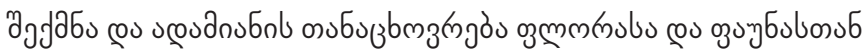

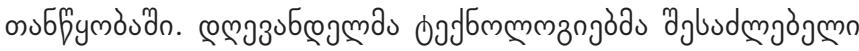

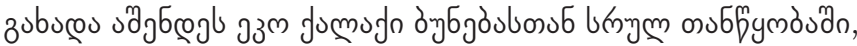

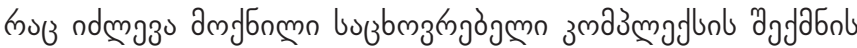

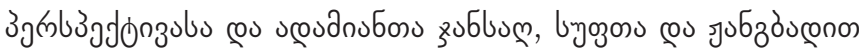

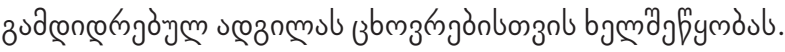

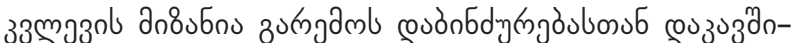

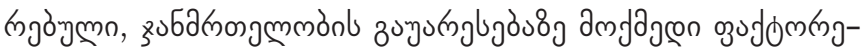

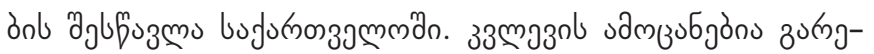

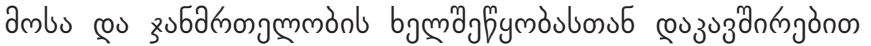

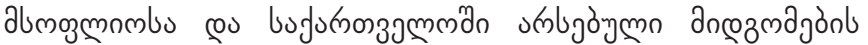

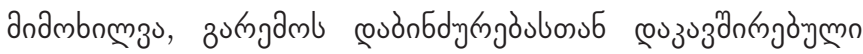

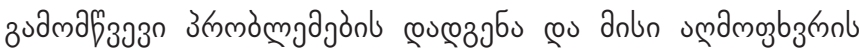
38 gòn zublus

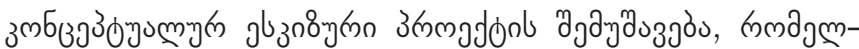

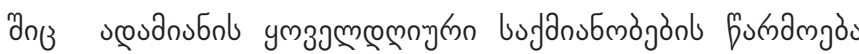

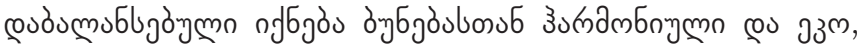

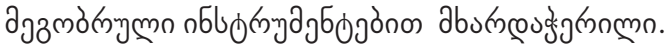

\section{аэмпюпмпвоЈ}

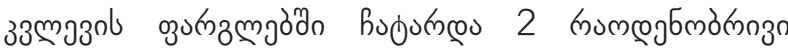

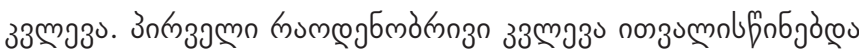

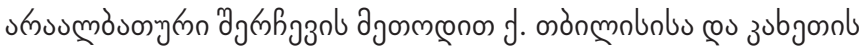

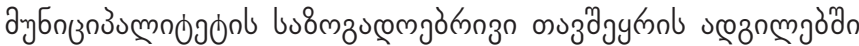

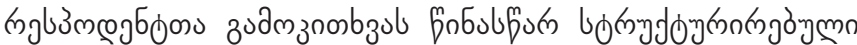

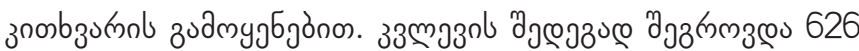

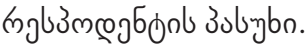

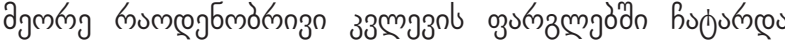

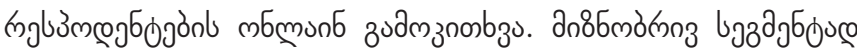

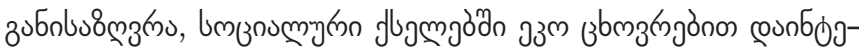

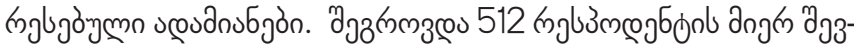

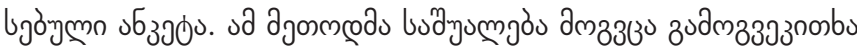

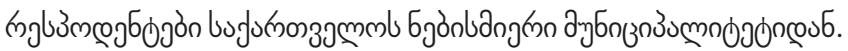

өзй

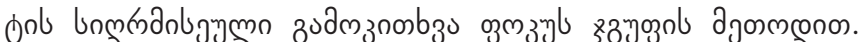

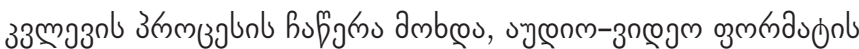

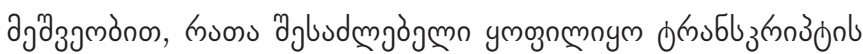

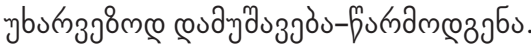

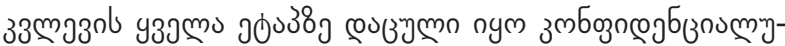

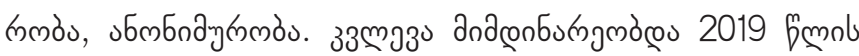
aumon-กз

\section{ซวщววอวก}

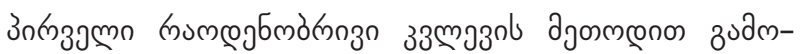

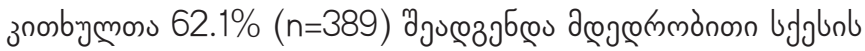

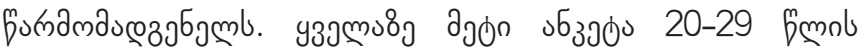

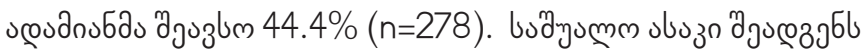

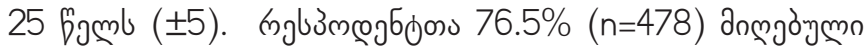

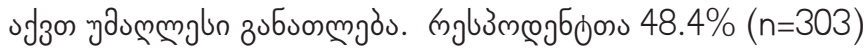

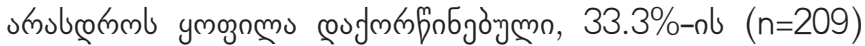

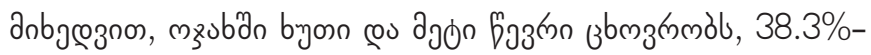

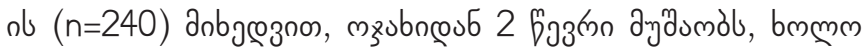

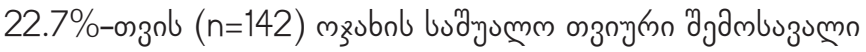
1001-2000 momos.

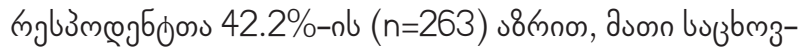

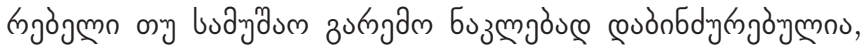

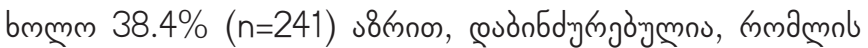

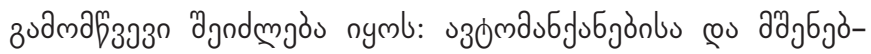

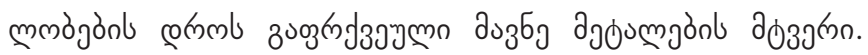

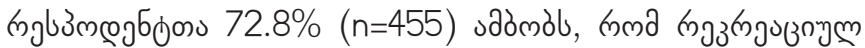

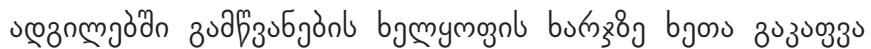

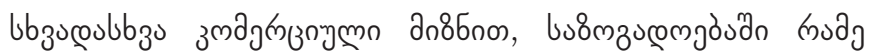

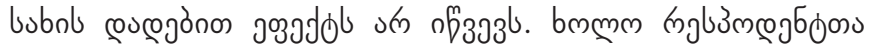

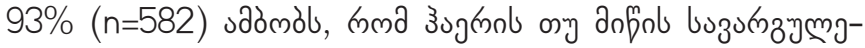

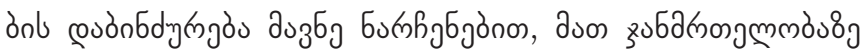

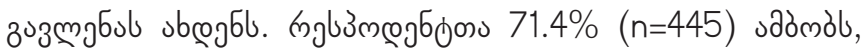

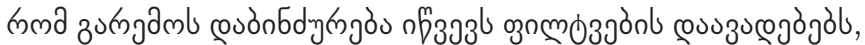

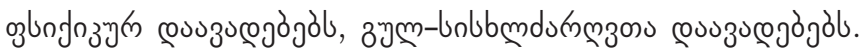

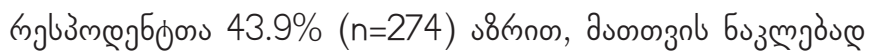

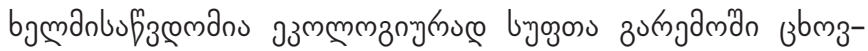

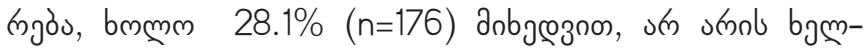

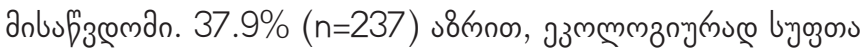

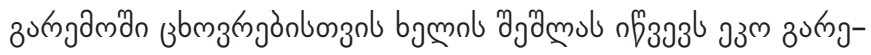

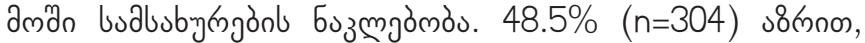

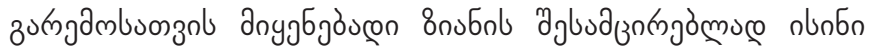

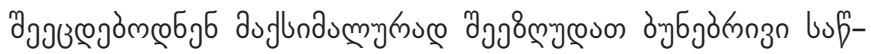

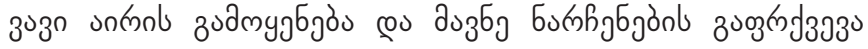

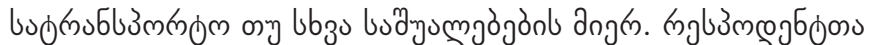

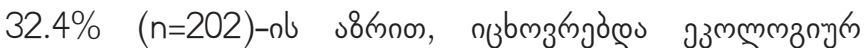

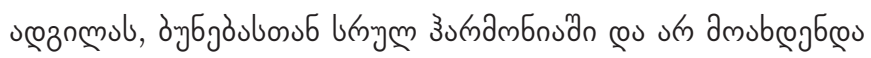

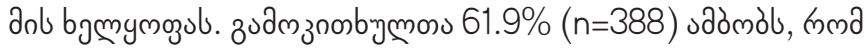

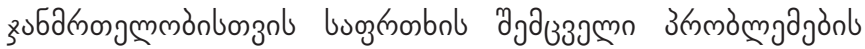

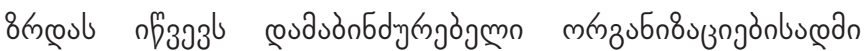

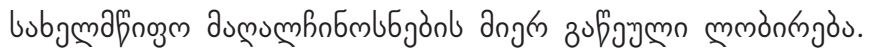

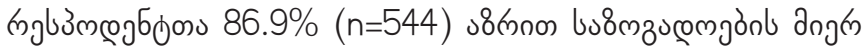

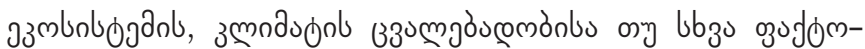

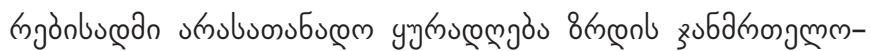

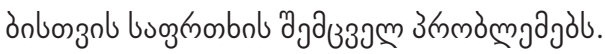




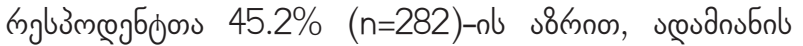

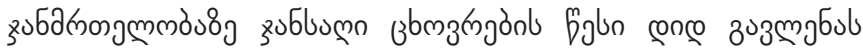

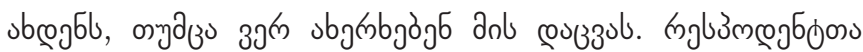

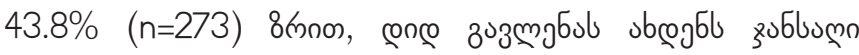

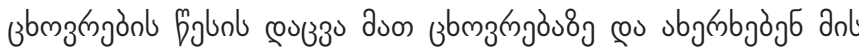

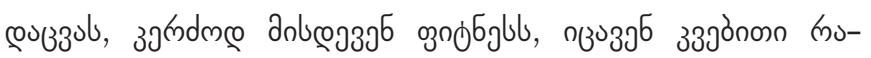

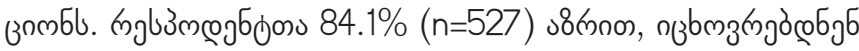

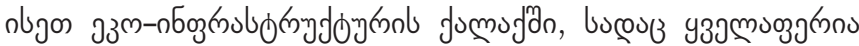

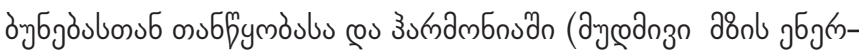

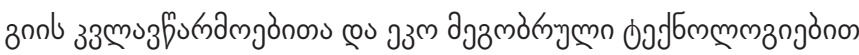

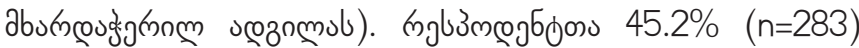

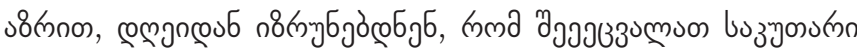

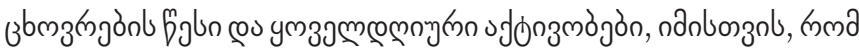

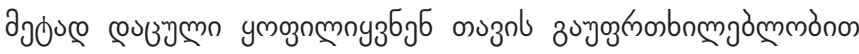

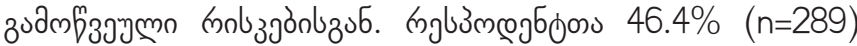

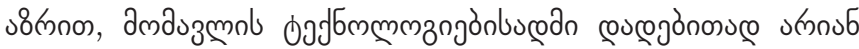

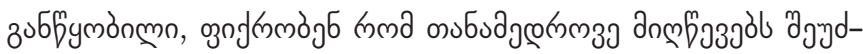

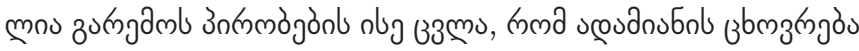

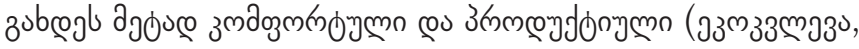
2019, 1).

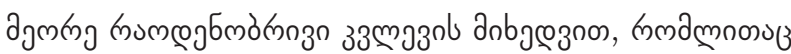

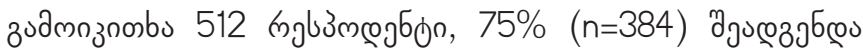

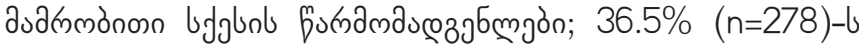

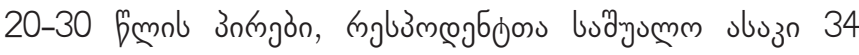
fэmos, \pm 5 .

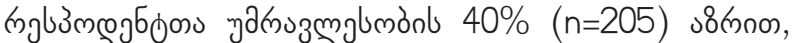

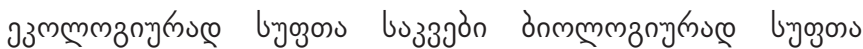

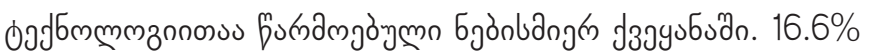

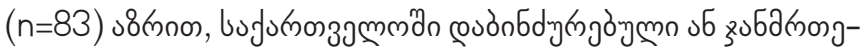

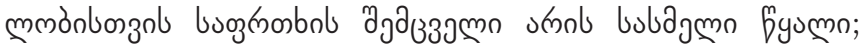

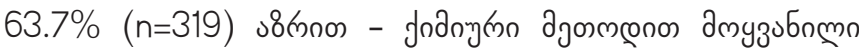

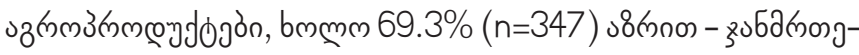

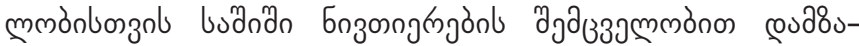

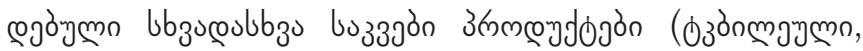
зmblg

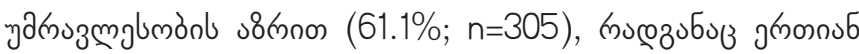

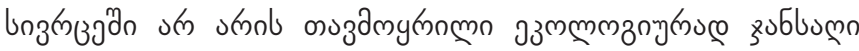

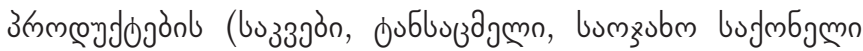

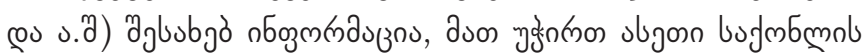

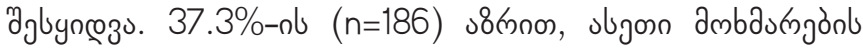

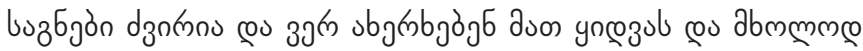

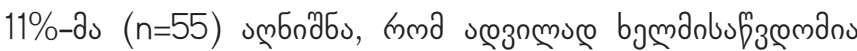

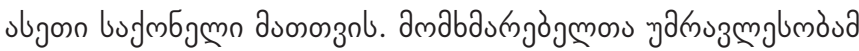

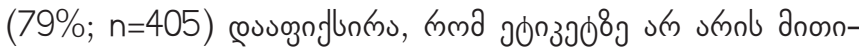

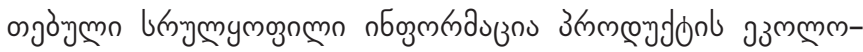

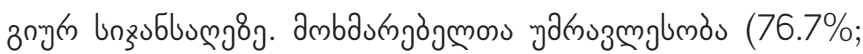

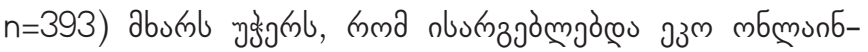

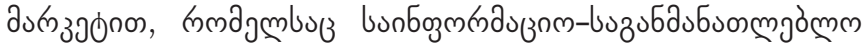

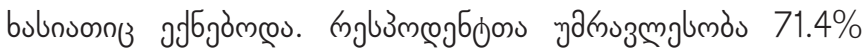

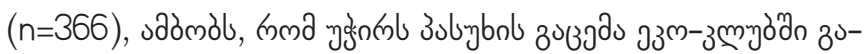

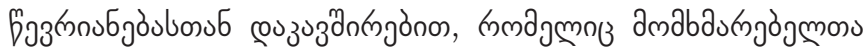
nбogm

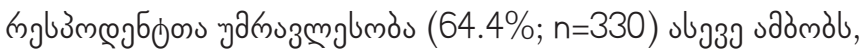

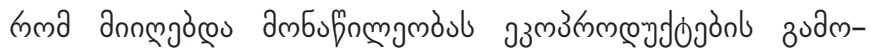

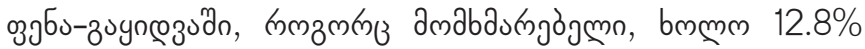

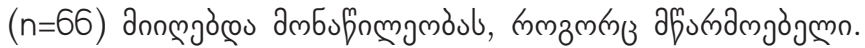

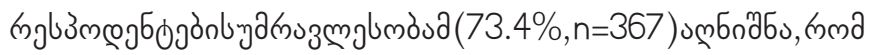

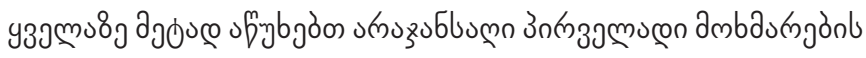

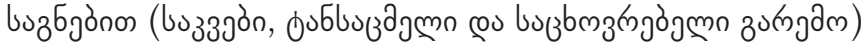

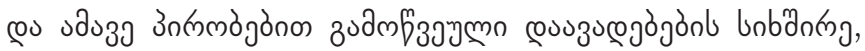

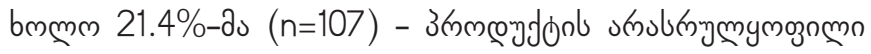

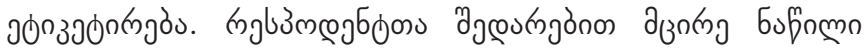

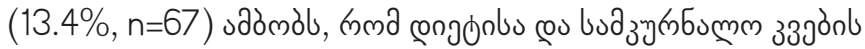

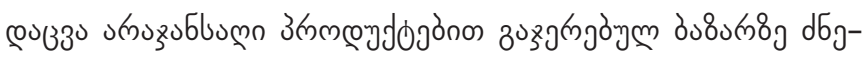
mos (эзмззмлзЈ, 2019, 2).

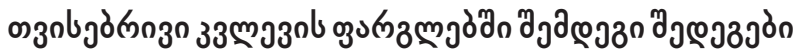 วозомуб:}

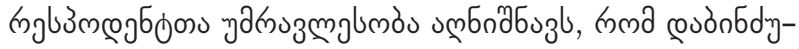

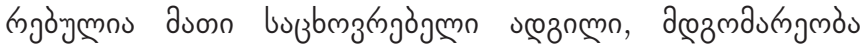

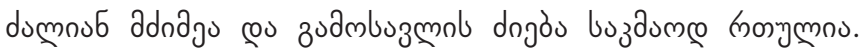

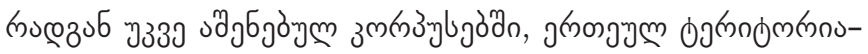

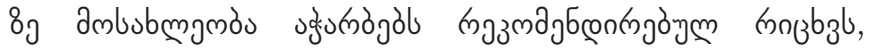

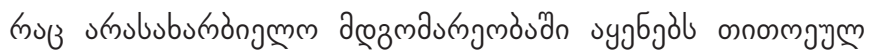

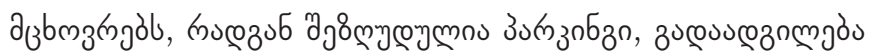
cos lbza.

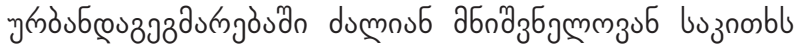

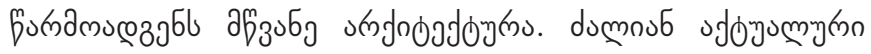

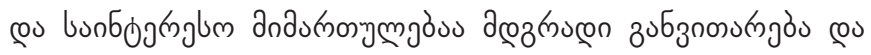

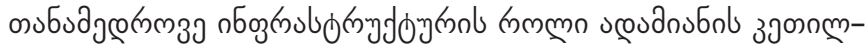

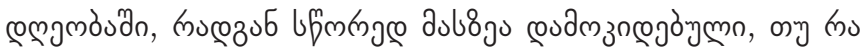

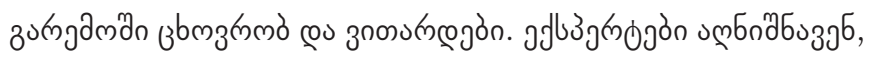

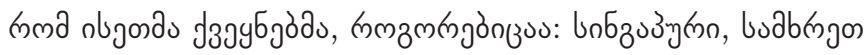

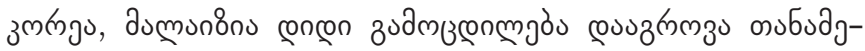

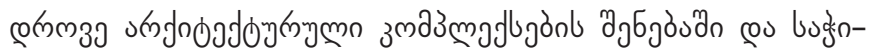

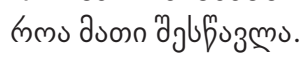

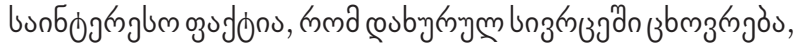

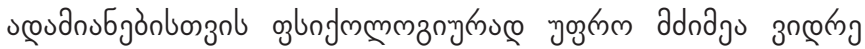

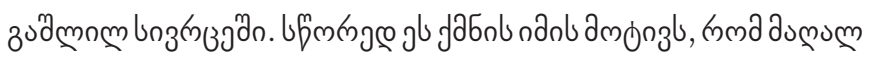

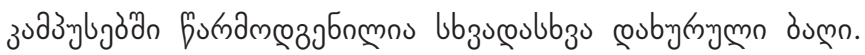

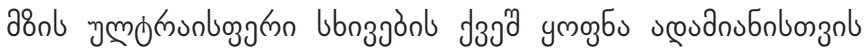

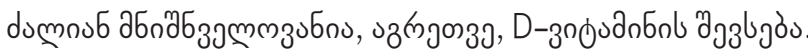

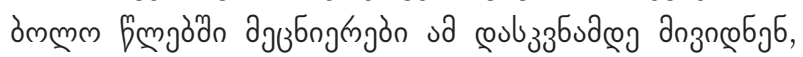

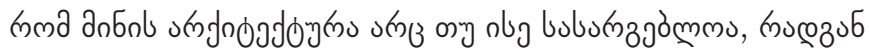




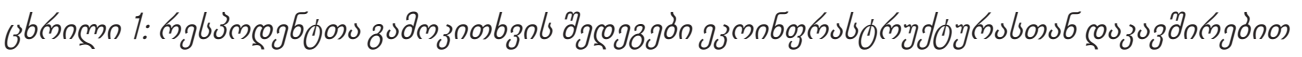

\begin{tabular}{|c|c|c|}
\hline asbsbosojరి & 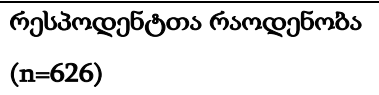 & $\%$ \\
\hline \multicolumn{3}{|l|}{ 1.bjabo } \\
\hline 1. Әсоэющмд̊поо & 389 & 62.1 \\
\hline 2. дздммдัооо & 226 & 36.1 \\
\hline 3sbybo sল essogofluoms & 11 & 1.8 \\
\hline \multicolumn{3}{|l|}{$2 . s 6 s_{30}$} \\
\hline 1. 20-дભอ & 50 & 7.9 \\
\hline 2. $20-29$ & 278 & 44.4 \\
\hline 3. $30-39$ & 110 & 17.8 \\
\hline 4. $40-49$ & 80 & 12.8 \\
\hline 5. $50-59$ & 44 & 7 \\
\hline 6. 60 cos уоз̆мио & 54 & 8.6 \\
\hline 3sbybo sm cossozofluoms & 10 & 1.5 \\
\hline bsə̃ysmm slsszo & 25 & $(\ldots \pm 5)$ \\
\hline \multicolumn{3}{|l|}{ 3. zsбsongejas: } \\
\hline 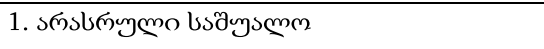 & 8 & 1.3 \\
\hline 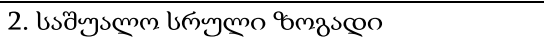 & 69 & 11 \\
\hline 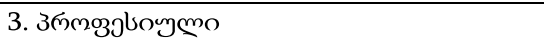 & 51 & 8.3 \\
\hline 4. удьмејыо & 479 & 76.5 \\
\hline 5. Ub3s ( (моs 3sbybo) & 6 & 0.9 \\
\hline 3sbybo sলn cossozofluoms & 13 & 2 \\
\hline \multicolumn{3}{|l|}{ 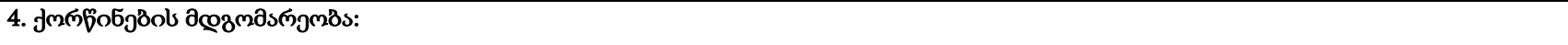 } \\
\hline 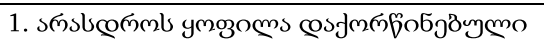 & 303 & 48.4 \\
\hline 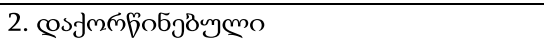 & 257 & 41.2 \\
\hline 3. d3позо & 18 & 2.9 \\
\hline 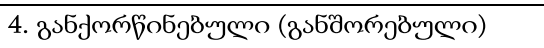 & 33 & 5.3 \\
\hline 5. Ub3s ( (mos 3sbugbo) & 1 & 0.1 \\
\hline 3sbybo sলn cossogofluoms & 14 & 2.2 \\
\hline \multicolumn{3}{|l|}{ 5. msว } \\
\hline 1. jদூо & 22 & 3.5 \\
\hline 2. mmо & 53 & 8.5 \\
\hline 3. Łsao & 135 & 21.7 \\
\hline 4. monbo & 196 & 31.3 \\
\hline 5. by๐o œ๐ วృతీం & 209 & 33.3 \\
\hline 3sbybo sল cossogofluoms & 11 & 1.7 \\
\hline \multicolumn{3}{|c|}{ 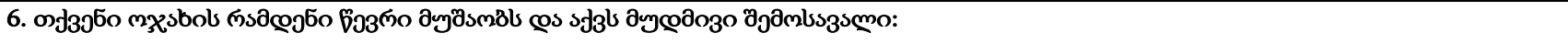 } \\
\hline 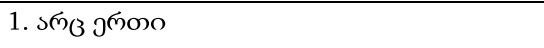 & 27 & 4.3 \\
\hline 2. jদேо & 207 & 33 \\
\hline 3. mmо & 240 & 38.3 \\
\hline 4. Łsдo & 95 & 15.2 \\
\hline 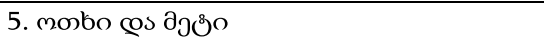 & 45 & 7.3 \\
\hline 3sbybo sলn cossozofloms & 12 & 1.9 \\
\hline
\end{tabular}




\begin{tabular}{|c|c|c|}
\hline 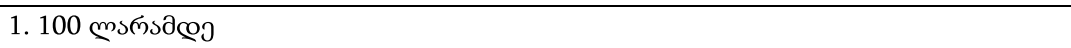 & 10 & 1.5 \\
\hline 2. $100-150$ & 10 & 1.5 \\
\hline 3. $151-500$ & 94 & 15.7 \\
\hline 4. 501-1000 & 128 & 20.9 \\
\hline 5. $1001-2000$ & 142 & 22.7 \\
\hline 6. 2001-3500 & 98 & 15.6 \\
\hline 7. 3500-\$ృ วృతిం & 81 & 12 \\
\hline 3sbybo sm cossogofluns & 63 & 10.1 \\
\hline \multicolumn{3}{|l|}{ 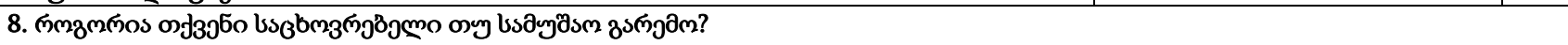 } \\
\hline 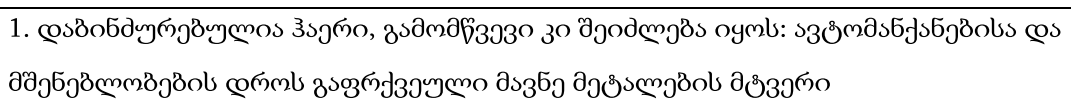 & 241 & 38.4 \\
\hline 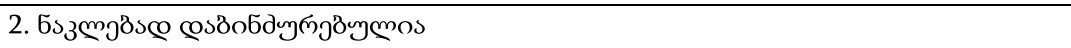 & 263 & 42.2 \\
\hline 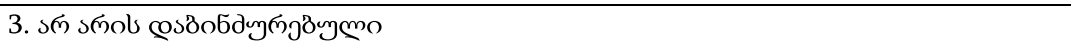 & 84 & 13.4 \\
\hline 4. lb33s (ços 3sbybo) & 12 & 2 \\
\hline 3sbrybo sলn cossogofloms & 19 & 4 \\
\hline \multirow{2}{*}{\multicolumn{3}{|c|}{ 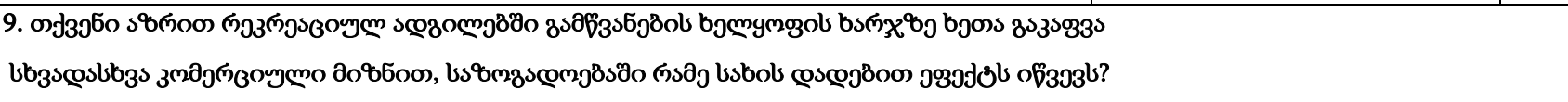 }} \\
\hline & & \\
\hline 1. $3^{\circ}$ & 141 & 22.7 \\
\hline 2. sms & 455 & 72.8 \\
\hline 3. ub3s (cos 3sbybo) & 7 & 0.9 \\
\hline 3sbyibo sল cossogofloms & 23 & 3.6 \\
\hline \multicolumn{3}{|c|}{ 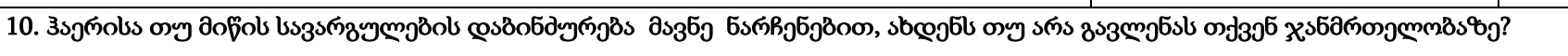 } \\
\hline 1. 30 & 582 & 93 \\
\hline 2. sms & 17 & 2.8 \\
\hline 3. lb33s (ços 3sbybo) & 8 & 1.0 \\
\hline 3sbyjbo sn cossozofloms & 19 & 3.2 \\
\hline \multicolumn{3}{|c|}{ 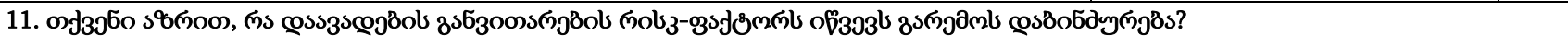 } \\
\hline 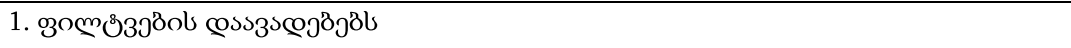 & 98 & 15.4 \\
\hline 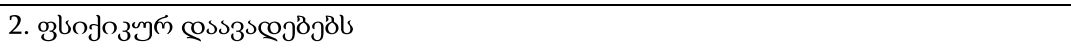 & 7 & 0.9 \\
\hline 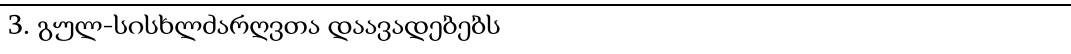 & 29 & 4.8 \\
\hline 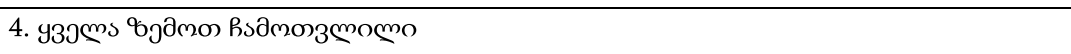 & 445 & 71.4 \\
\hline 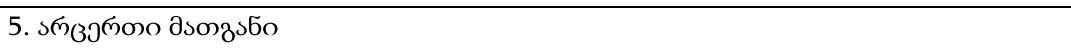 & 7 & 0.9 \\
\hline 6. Ub3s (cos 3sbybo) & 17 & 2.7 \\
\hline 3sbygbo sn cossogoflons & 23 & 3.9 \\
\hline \multicolumn{3}{|c|}{ 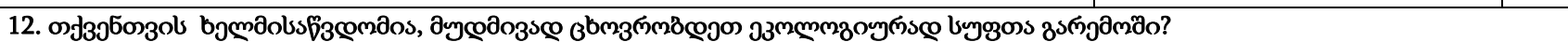 } \\
\hline 1. consb & 117 & 18.9 \\
\hline 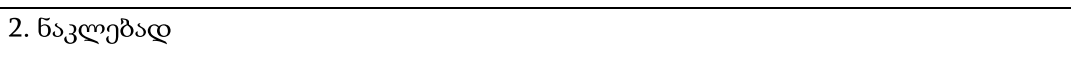 & 274 & 43.9 \\
\hline 3. sms & 176 & 28.1 \\
\hline 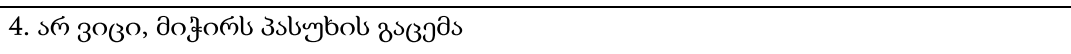 & 40 & 6.2 \\
\hline 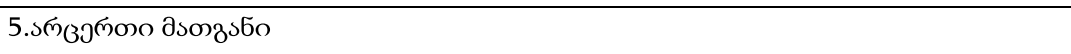 & 0 & \\
\hline 6. lb33s (ços 3sbybo) & 4 & 0.6 \\
\hline 3sbybo sm cossozofloms & 15 & 2.3 \\
\hline \multicolumn{3}{|c|}{ 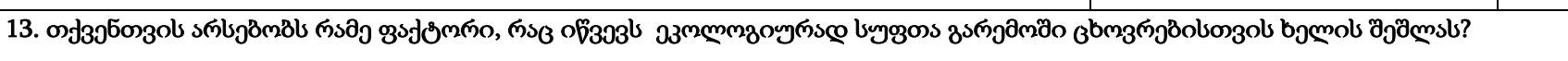 } \\
\hline 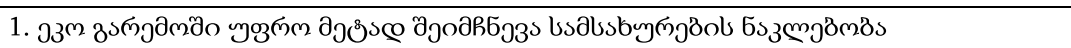 & 81 & 12.9 \\
\hline
\end{tabular}




\begin{tabular}{|c|c|c|}
\hline 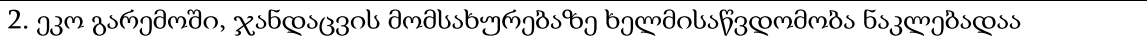 & 38 & 6 \\
\hline 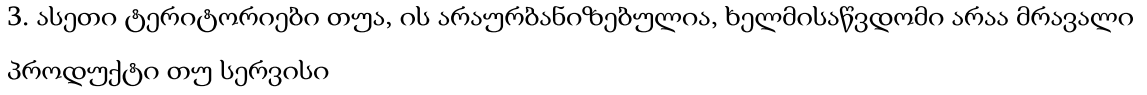 & 164 & 26.2 \\
\hline 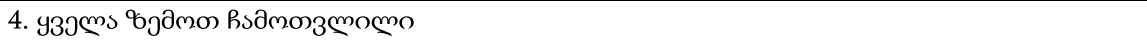 & 237 & 37.9 \\
\hline 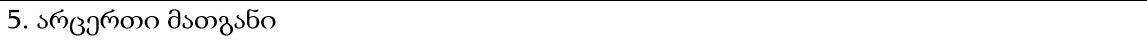 & 58 & 9.3 \\
\hline 6. Ub3s ( (mos 3sbybo) & 8 & 1.3 \\
\hline 3sbybo sm cossogofloms & 40 & 6.4 \\
\hline \multicolumn{3}{|l|}{ 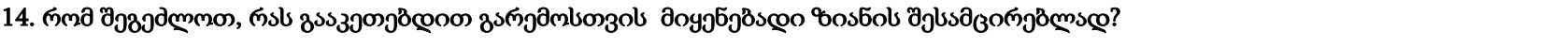 } \\
\hline 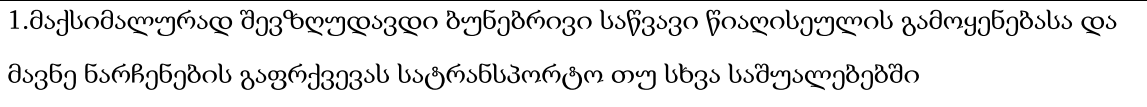 & 304 & 48.5 \\
\hline 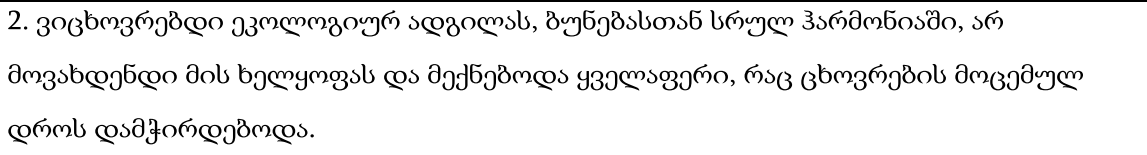 & 202 & 32.4 \\
\hline 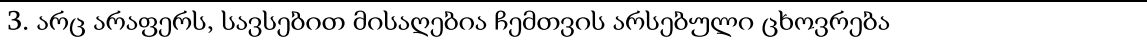 & 12 & 1.9 \\
\hline 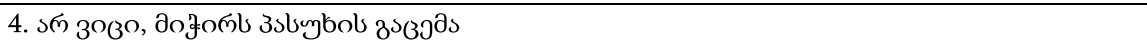 & 60 & 9.5 \\
\hline 5. Ub3s (cos 3sbybo) & 23 & 3.8 \\
\hline 3sbybo sল cossogofloms & 25 & 3.9 \\
\hline \multicolumn{3}{|c|}{ 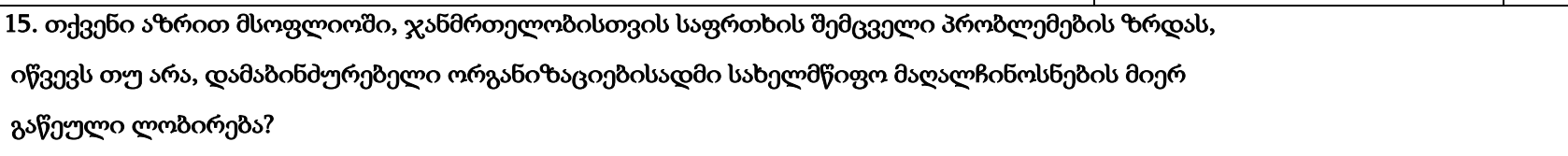 } \\
\hline 1. $3^{\circ}$ & 388 & 61.9 \\
\hline 2. sms & 21 & 3.4 \\
\hline 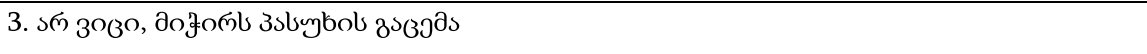 & 181 & 28.9 \\
\hline 4. $b_{3} s$ ( (mos 3sbybo) & 7 & 0.9 \\
\hline 3sbybo sm cossogofloms & 29 & 4.9 \\
\hline \multicolumn{3}{|c|}{ 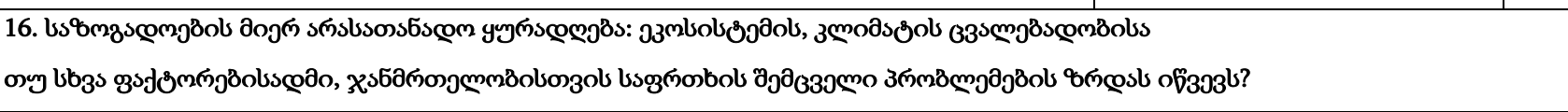 } \\
\hline $1.3^{\circ}$ & 544 & 86.9 \\
\hline 2. sms & 14 & 2.4 \\
\hline 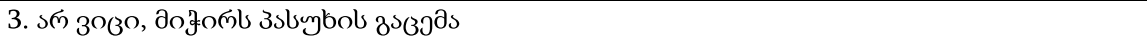 & 45 & 7.1 \\
\hline 3sbybo sm cossogofluoms & 23 & 3.6 \\
\hline \multicolumn{3}{|c|}{ 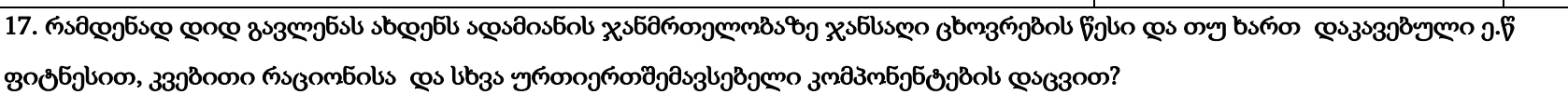 } \\
\hline 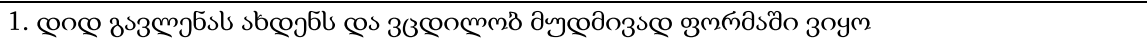 & 273 & 43.8 \\
\hline 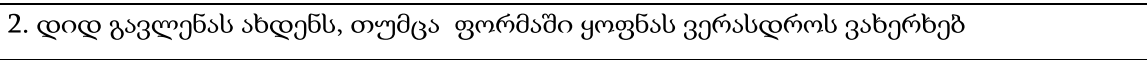 & 282 & 45.2 \\
\hline 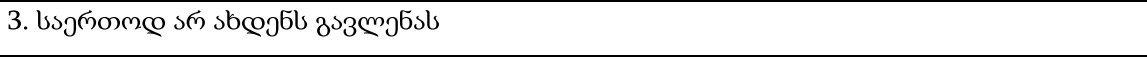 & 4 & 0.6 \\
\hline 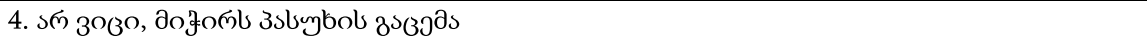 & 40 & 6.4 \\
\hline 5. ४b3s (cos 3sbybo) & 8 & 1.0 \\
\hline 3sbybo sm cossogofloms & 19 & 3.0 \\
\hline \multicolumn{3}{|c|}{ 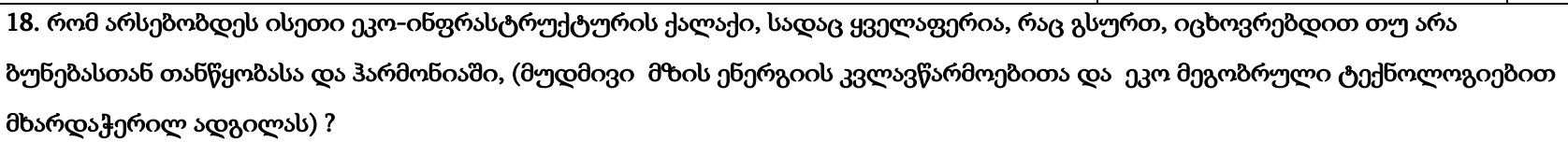 } \\
\hline 1. coosb & 527 & 84.1 \\
\hline 2. sms & 13 & 2.4 \\
\hline
\end{tabular}




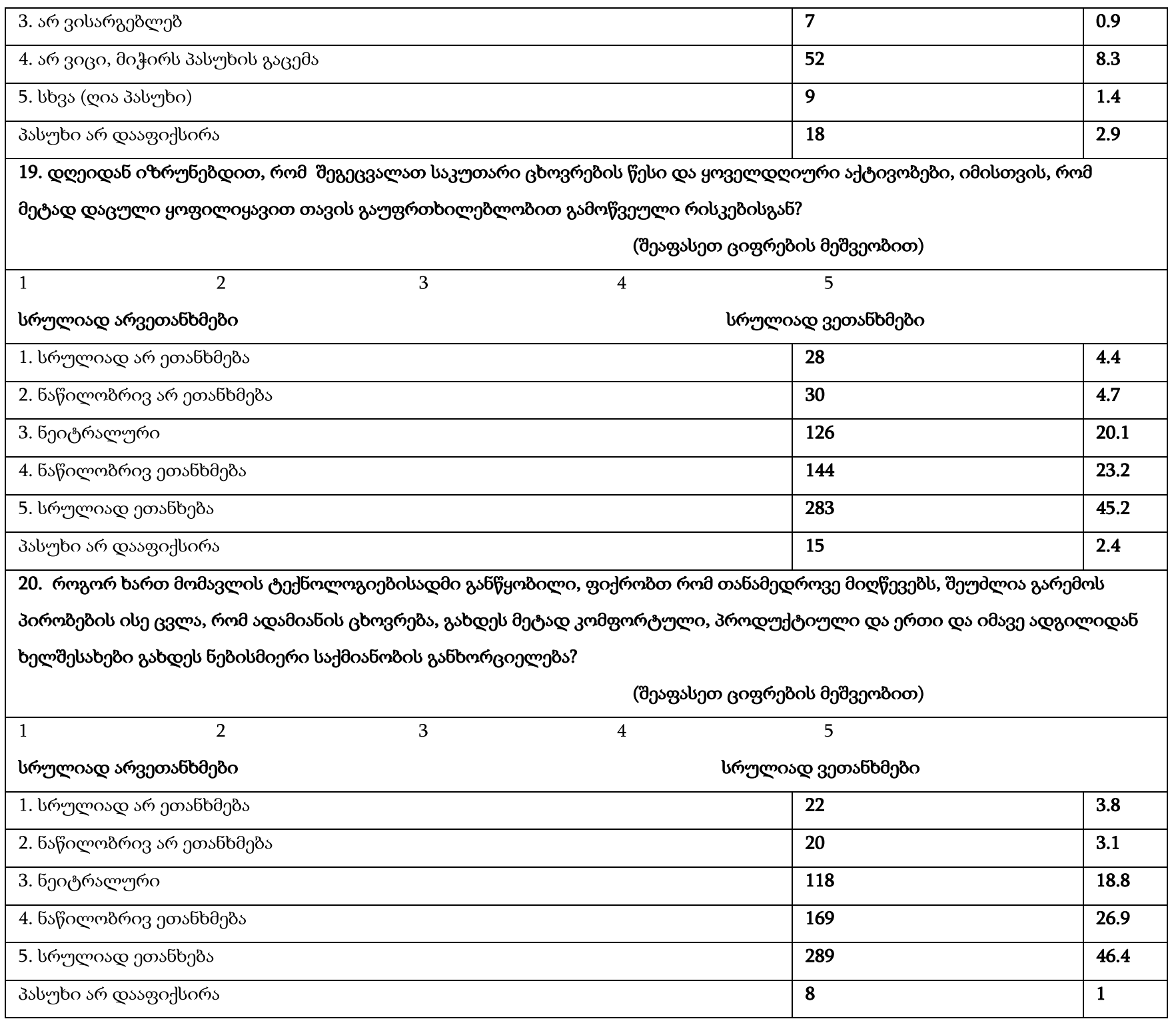

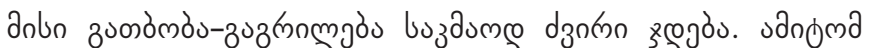

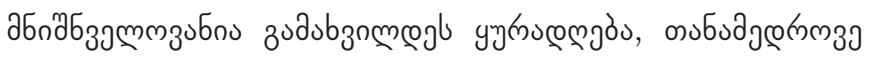

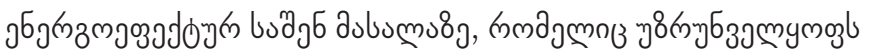

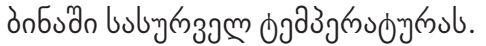

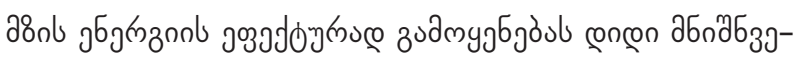

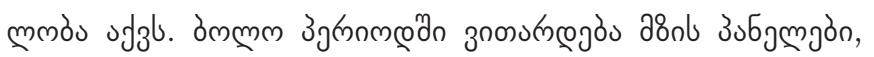

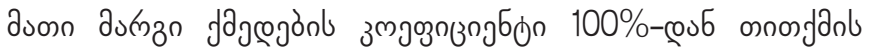

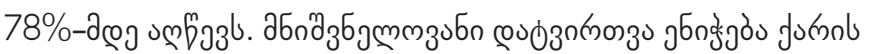

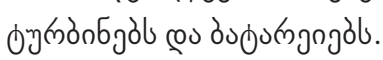

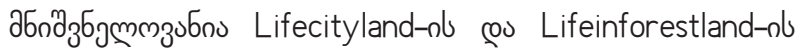

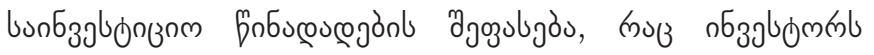

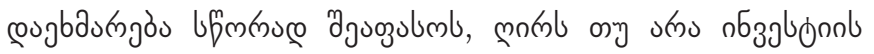

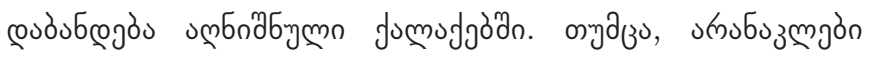

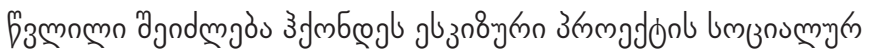

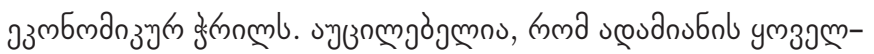

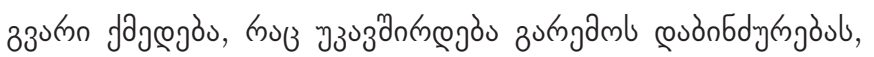

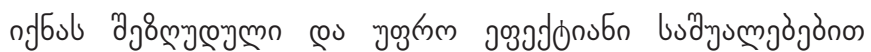

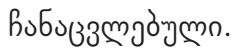

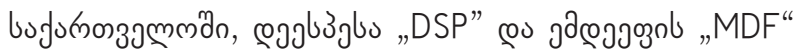

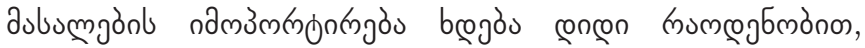

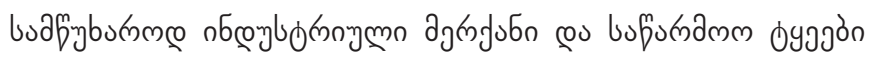

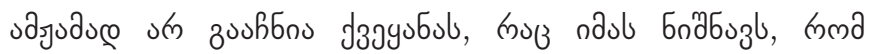

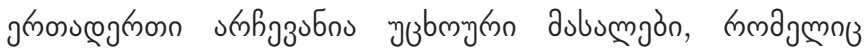

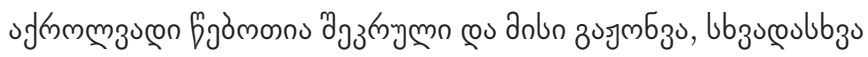

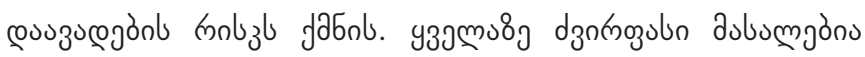

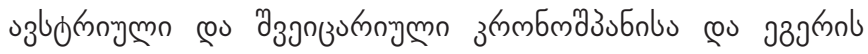

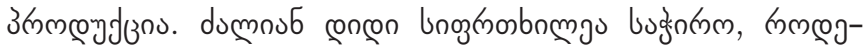

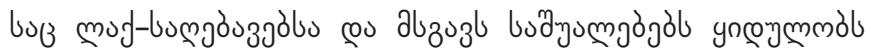

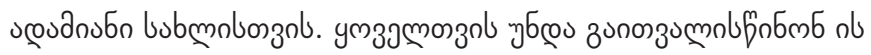

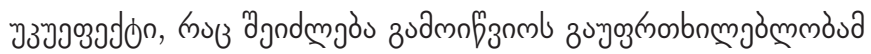

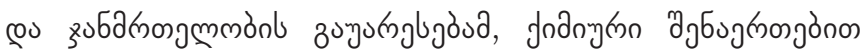

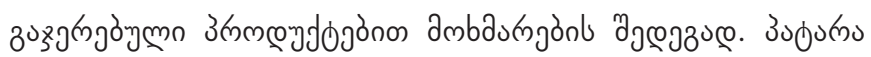




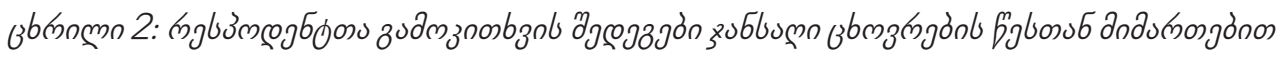

\begin{tabular}{|c|c|c|}
\hline 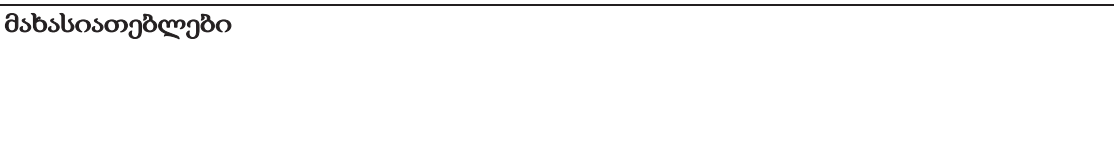 & 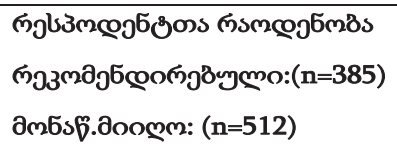 & $\%$ \\
\hline \multicolumn{3}{|l|}{ 1.bjglo } \\
\hline $1.3^{5} 3^{\circ}$ & 384 & 75 \\
\hline 2.jगलo & 115 & 23 \\
\hline 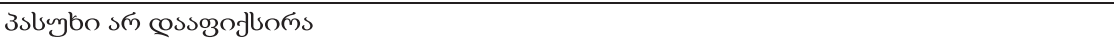 & 13 & 2 \\
\hline \multicolumn{3}{|l|}{ 2.sksзzo } \\
\hline 1.20 fmsaco & 37 & 7.2 \\
\hline $2.20-30$ & 187 & 36.5 \\
\hline $3.31-40$ & 109 & 21.2 \\
\hline $4.41-50$ & 107 & 20.8 \\
\hline $5 . \geq 50$ & 62 & 12.4 \\
\hline bsə̈ysmm slsszo & $(35 \pm 5)$ & \\
\hline 3sbybo sল cossozofloms & 10 & 1.9 \\
\hline \multicolumn{3}{|l|}{ 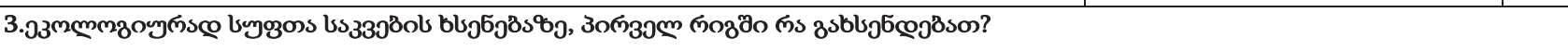 } \\
\hline 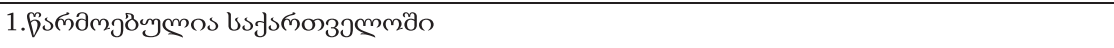 & 12 & 2.3 \\
\hline 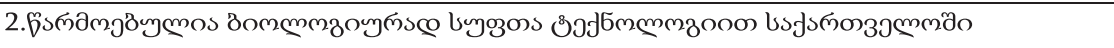 & 92 & 17.9 \\
\hline 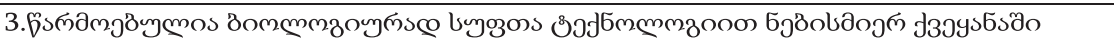 & 205 & 40 \\
\hline 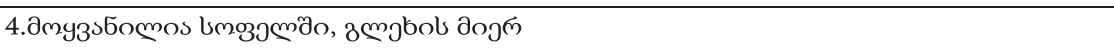 & 160 & 31.2 \\
\hline 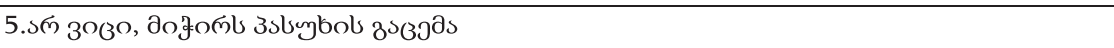 & 15 & 2.9 \\
\hline 3stuybo sm cossogoflund & 28 & 5.7 \\
\hline \multicolumn{3}{|c|}{ 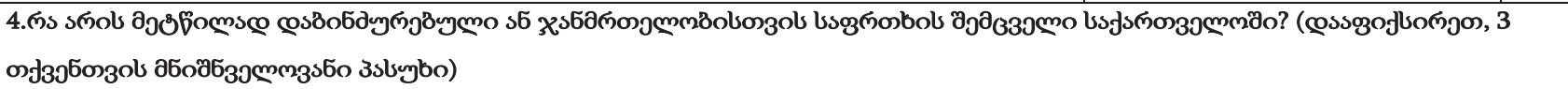 } \\
\hline 1.bsbajmo fysmo & 83 & 16.6 \\
\hline 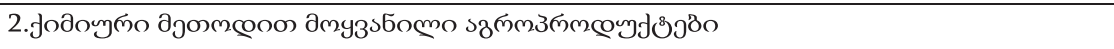 & 319 & 63.7 \\
\hline 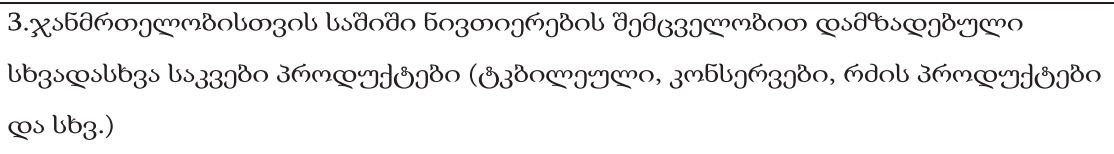 & 347 & 69.3 \\
\hline 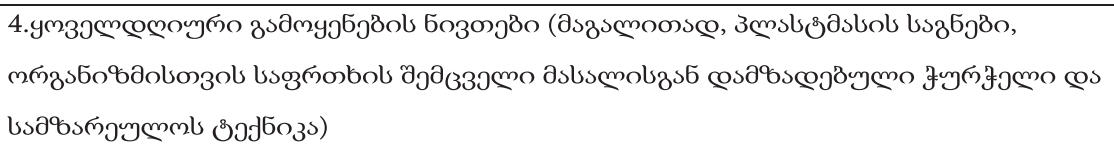 & 190 & 37.9 \\
\hline 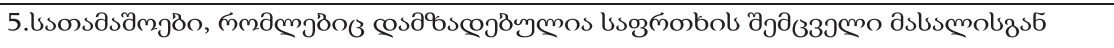 & 121 & 24.2 \\
\hline 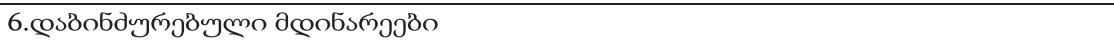 & 96 & 19.2 \\
\hline 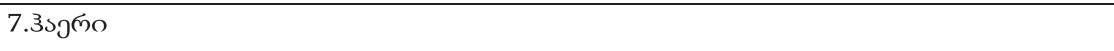 & 344 & 68.7 \\
\hline 3sbybo sm cossozofluoms & 14 & 2.7 \\
\hline \multicolumn{3}{|c|}{ 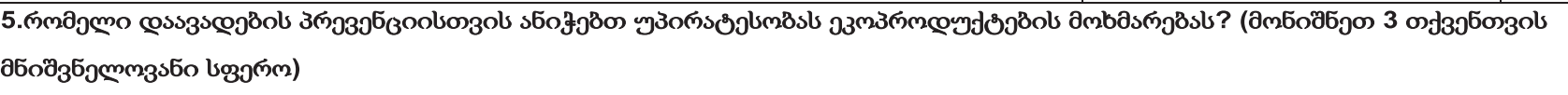 } \\
\hline 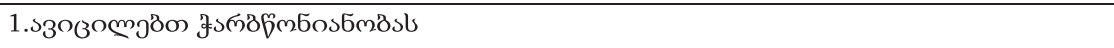 & 162 & 32.5 \\
\hline 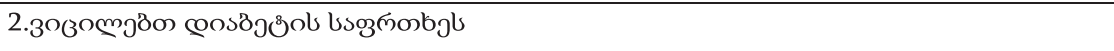 & 129 & 25.9 \\
\hline 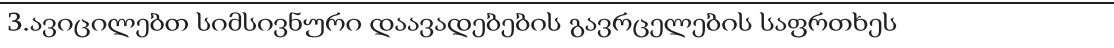 & 432 & 86.7 \\
\hline 4. & 363 & 72.9 \\
\hline 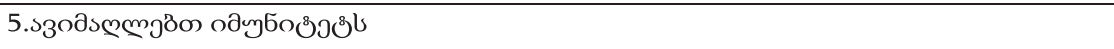 & 236 & 47.4 \\
\hline
\end{tabular}




\begin{tabular}{|c|c|c|}
\hline 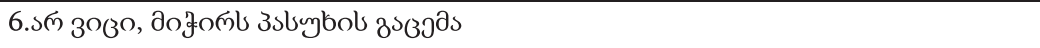 & 4 & 0.8 \\
\hline 3sbunbo sলn cossogoflunoms & 14 & 2.7 \\
\hline \multicolumn{3}{|c|}{ 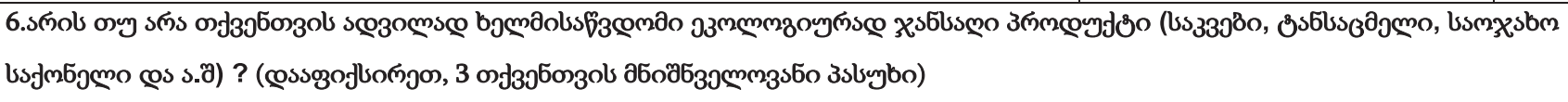 } \\
\hline 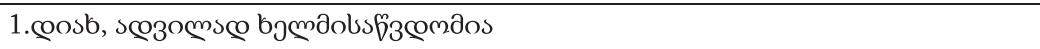 & 55 & 11 \\
\hline 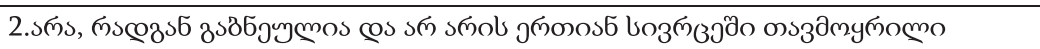 & 305 & 61.1 \\
\hline 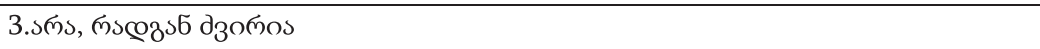 & 186 & 37.3 \\
\hline 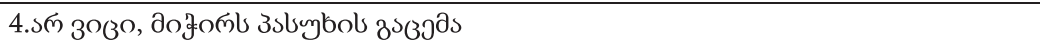 & 31 & 36.2 \\
\hline 3sbybo sm cossogofluoms & 13 & 2.5 \\
\hline \multicolumn{3}{|c|}{ 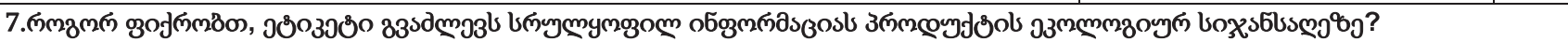 } \\
\hline 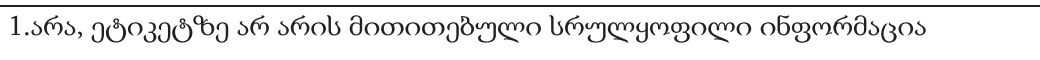 & 405 & 79 \\
\hline 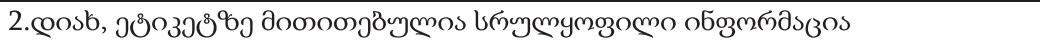 & 34 & 6.6 \\
\hline 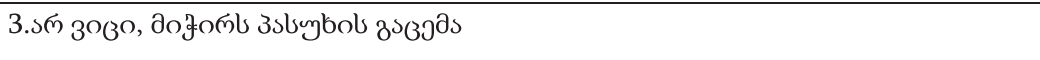 & 60 & 11.9 \\
\hline 3sbunbo sm cossozofluoms & 13 & 2.5 \\
\hline \multicolumn{3}{|c|}{ 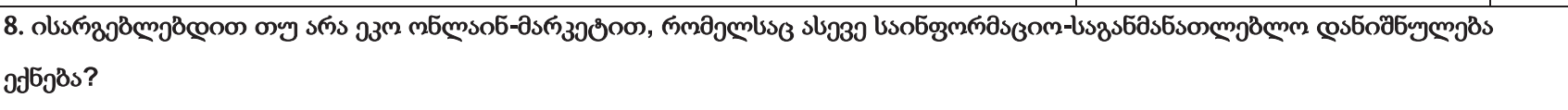 } \\
\hline 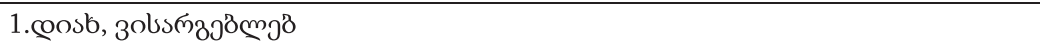 & 393 & 76.7 \\
\hline 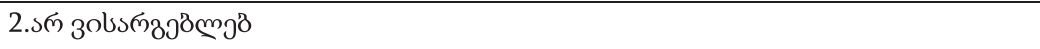 & 36 & 7.0 \\
\hline 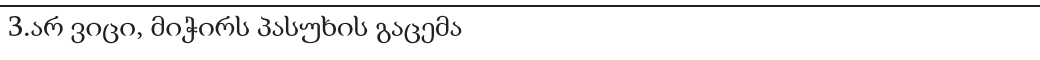 & 73 & 14.2 \\
\hline 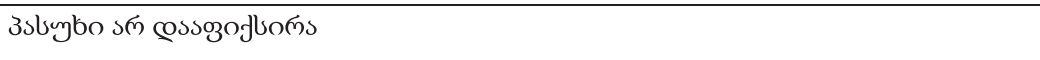 & 10 & 2.1 \\
\hline \multicolumn{3}{|c|}{ 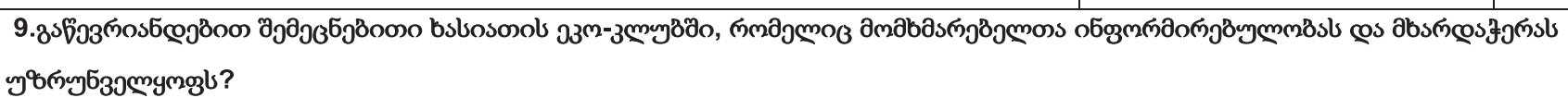 } \\
\hline 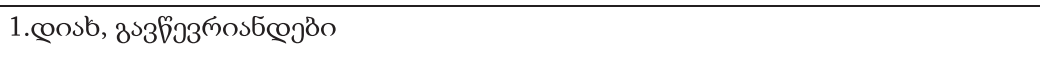 & 53 & 10.3 \\
\hline 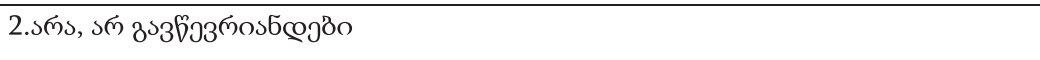 & 82 & 16.0 \\
\hline 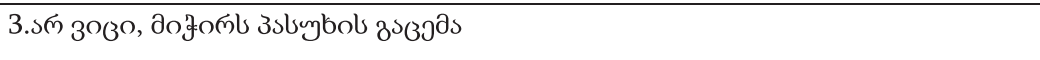 & 366 & 71.4 \\
\hline 3stuybo sm cossogofluns & 11 & 2.3 \\
\hline \multicolumn{3}{|l|}{ 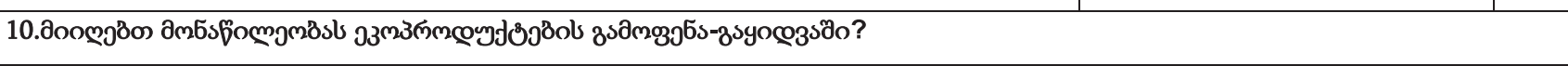 } \\
\hline 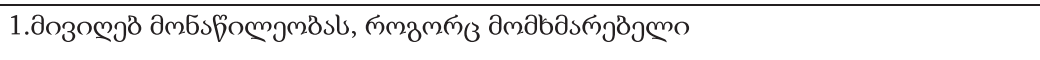 & 330 & 64.4 \\
\hline 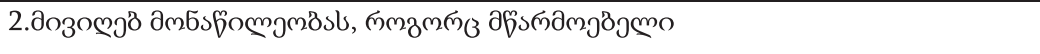 & 66 & 12.8 \\
\hline 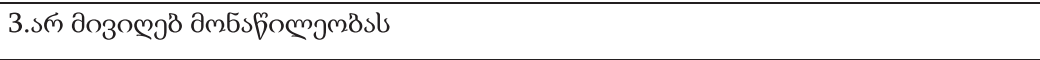 & 49 & 9.5 \\
\hline 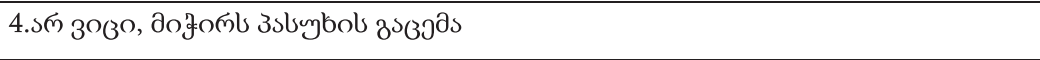 & 52 & 10.4 \\
\hline 3sbyjbo sm cossozoflunoms & 15 & 2.9 \\
\hline \multicolumn{3}{|c|}{ 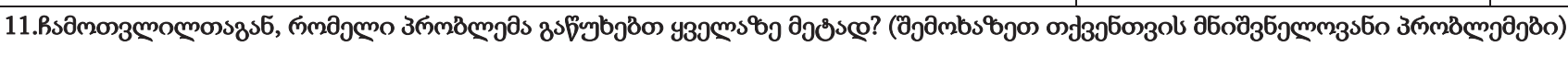 } \\
\hline 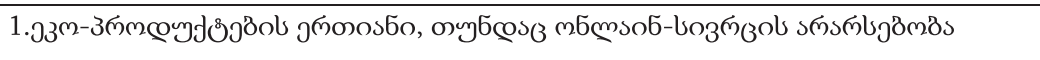 & 104 & 20.8 \\
\hline 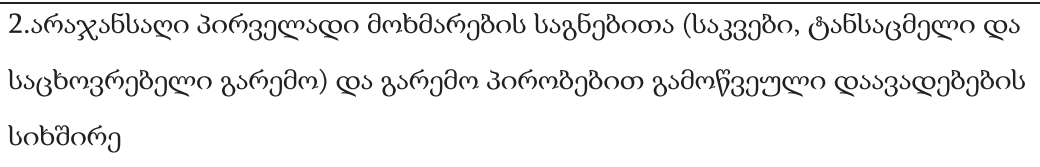 & 367 & 73.4 \\
\hline 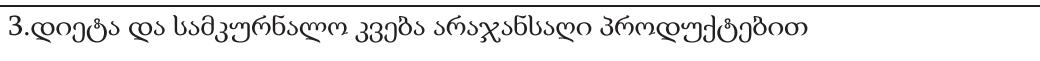 & 67 & 13.4 \\
\hline 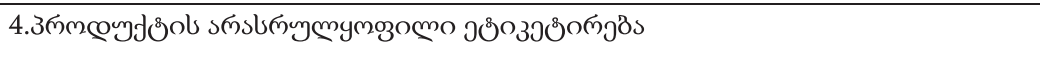 & 107 & 21.4 \\
\hline 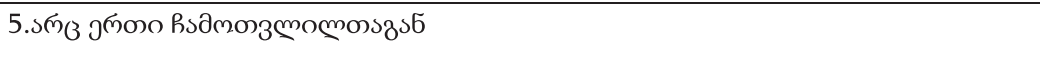 & 8 & 1.6 \\
\hline 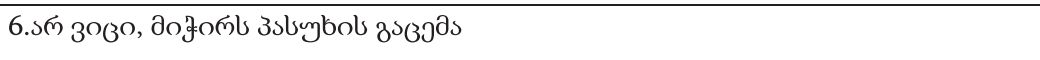 & 19 & 3.8 \\
\hline 3sbybo sলn cossogofluoms & 15 & 2.9 \\
\hline
\end{tabular}




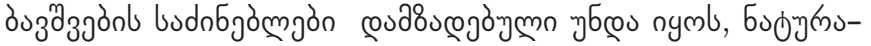

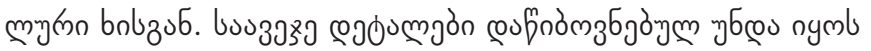

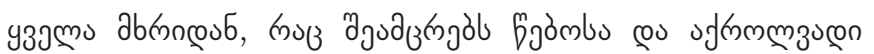

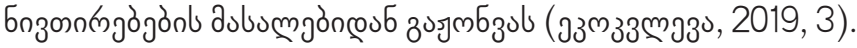

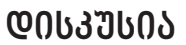

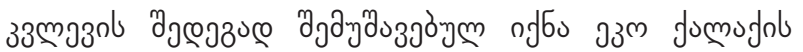

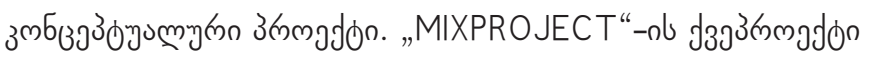

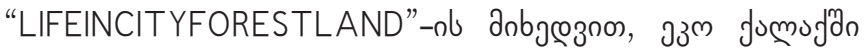

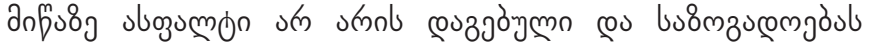

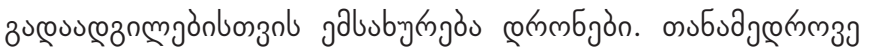

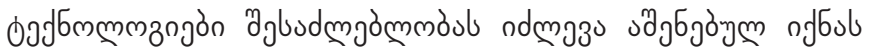

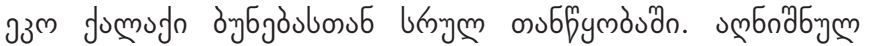

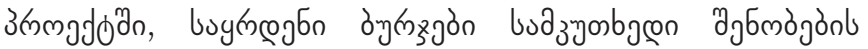

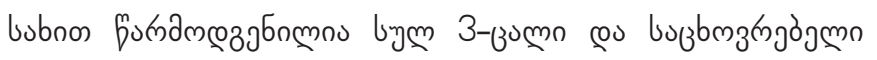
змаз

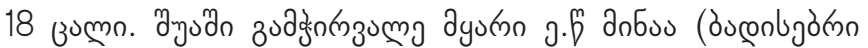

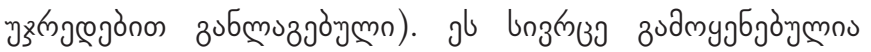

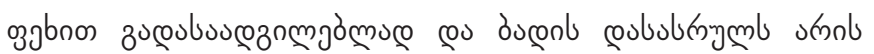

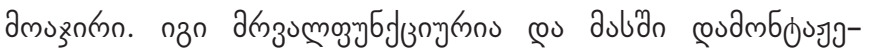

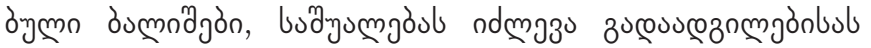

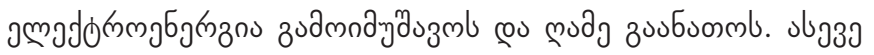

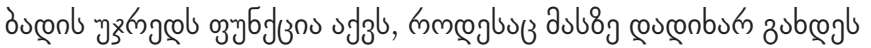

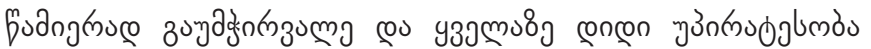

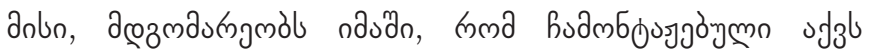

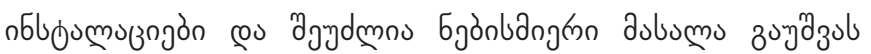

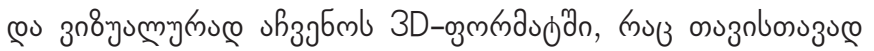

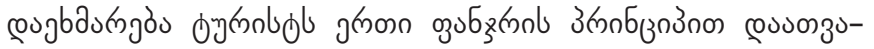

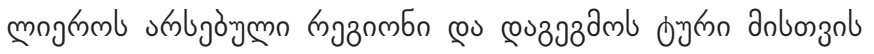

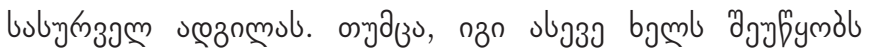

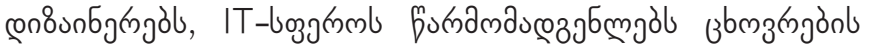

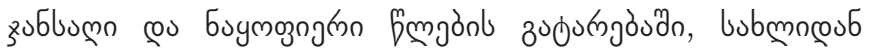

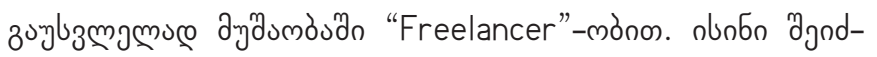

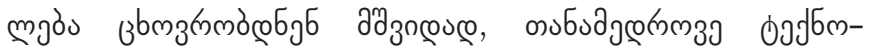

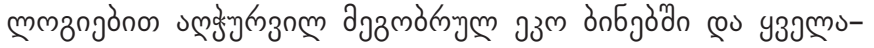

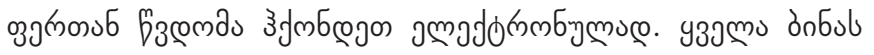

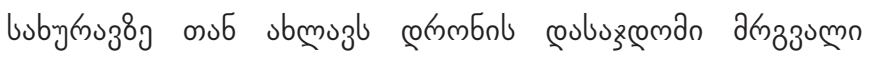

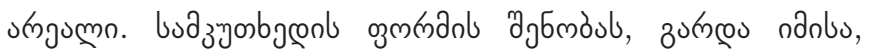
mma bugnূ

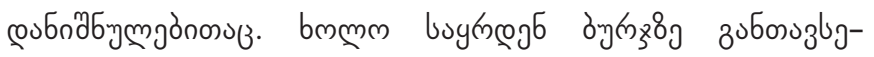

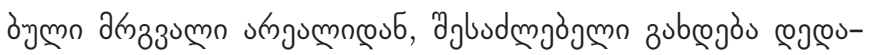

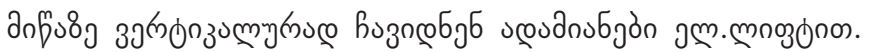

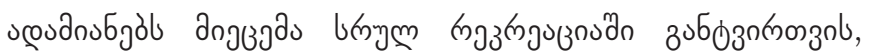

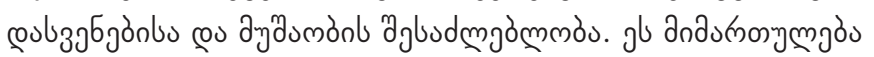

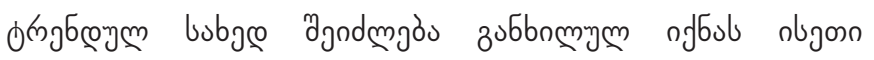

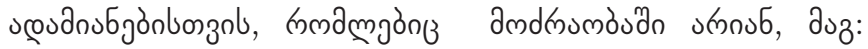

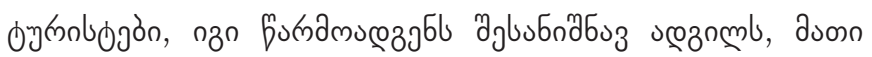

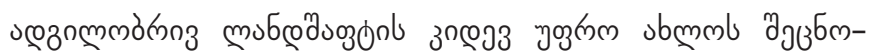

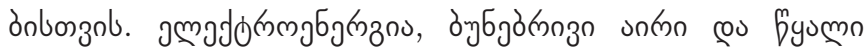

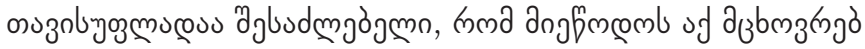

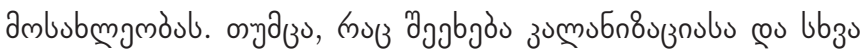

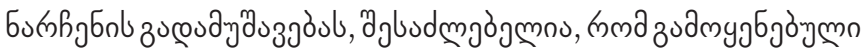

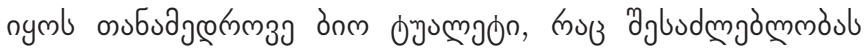

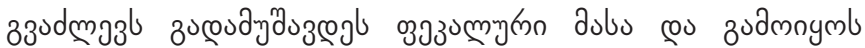

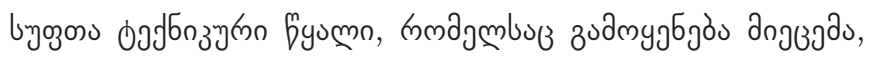
bjons cosaudjönonn am

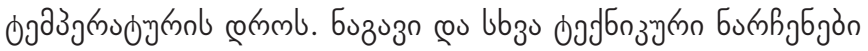

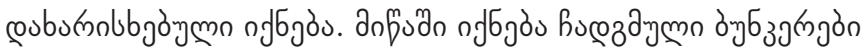

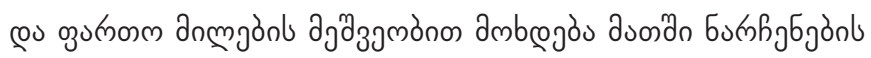

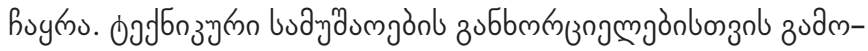

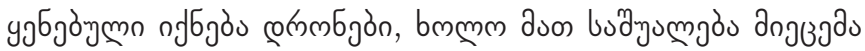

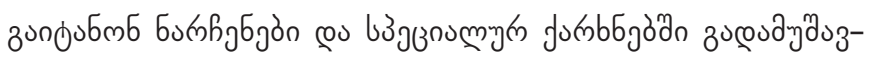

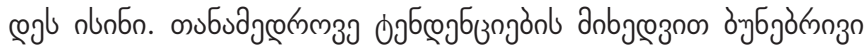

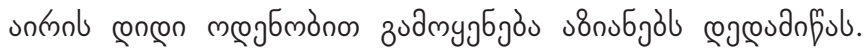

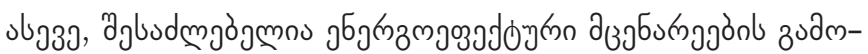

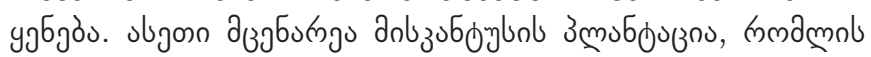

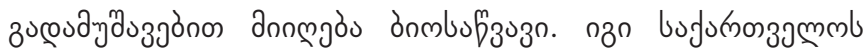

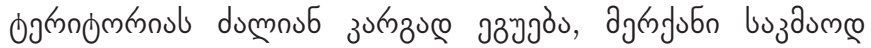

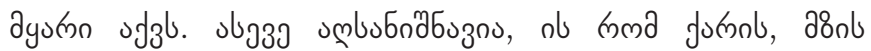

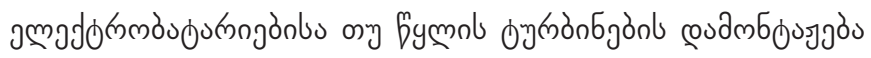

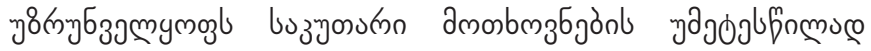

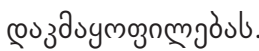

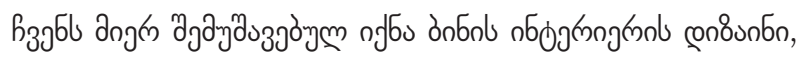

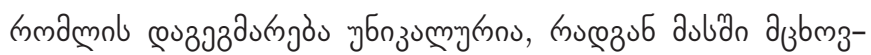

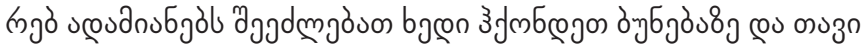

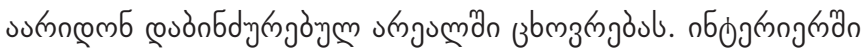

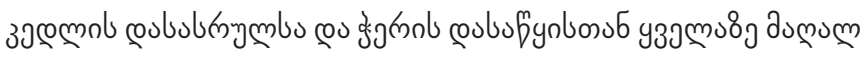

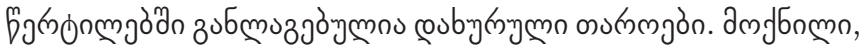

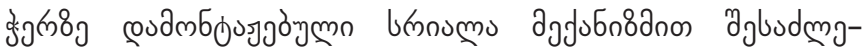

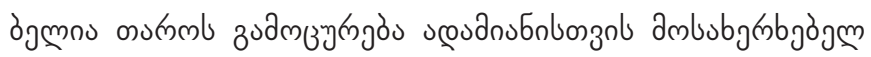

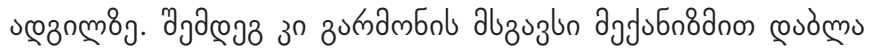

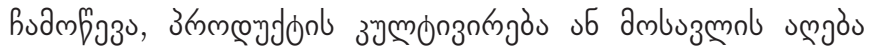

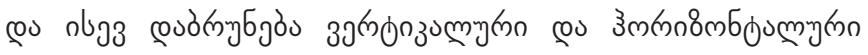

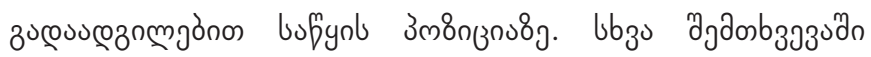

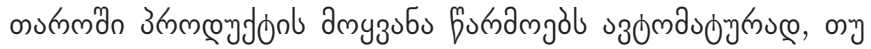

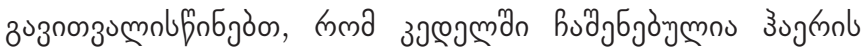

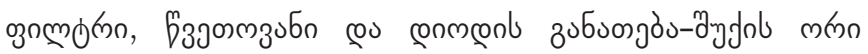

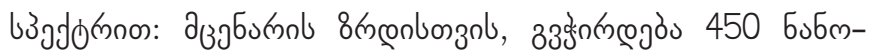

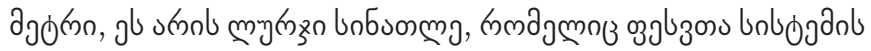

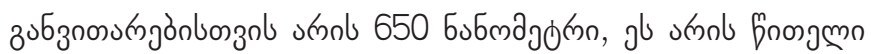

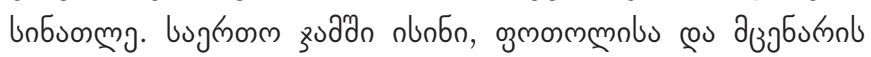

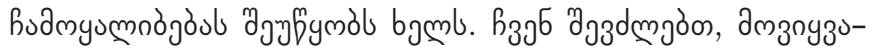




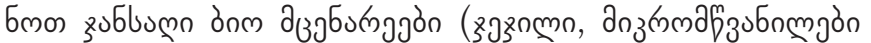

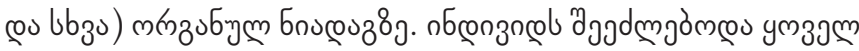

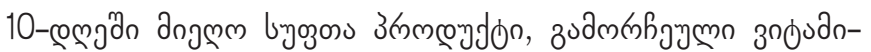

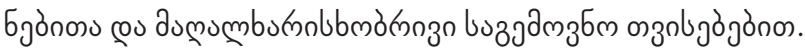

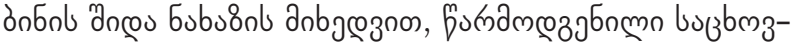

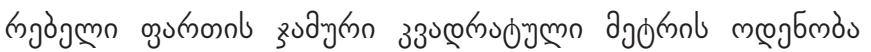

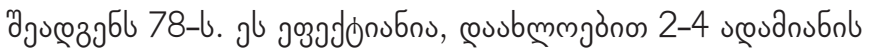

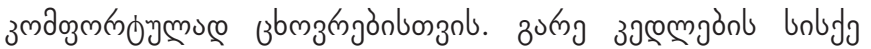

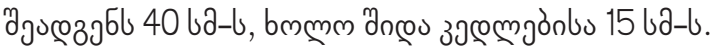

on

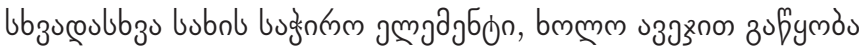

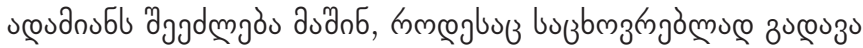

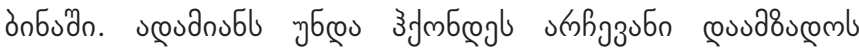

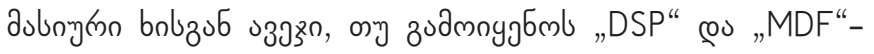

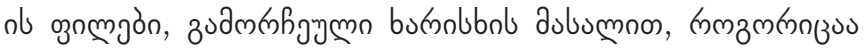

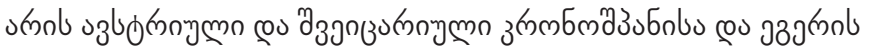

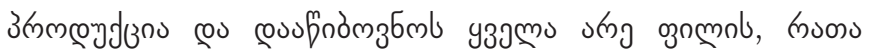

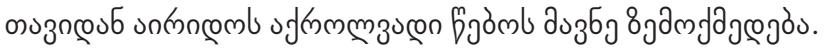

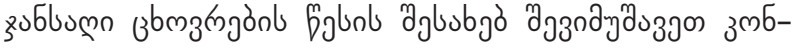

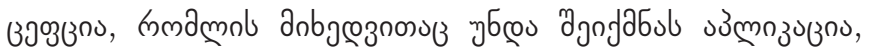

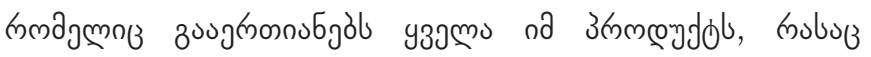

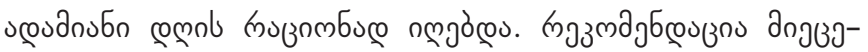

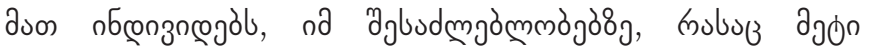

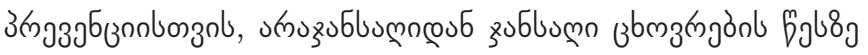

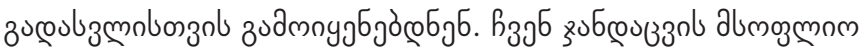

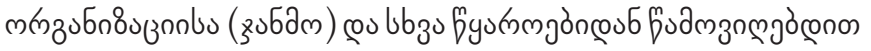

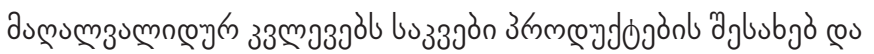

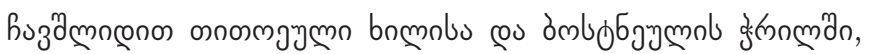

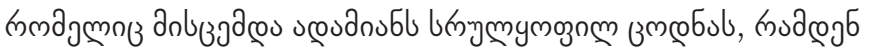

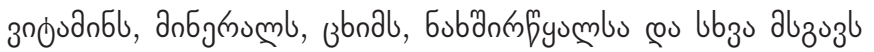

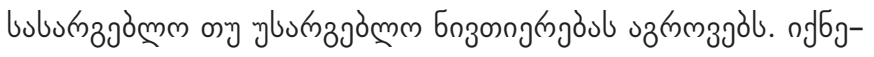

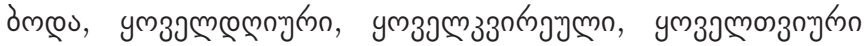

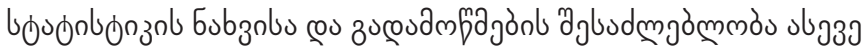

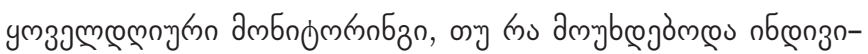

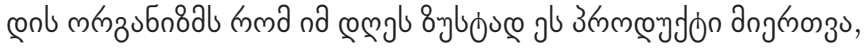

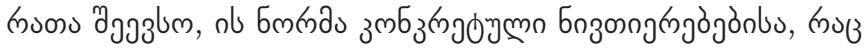

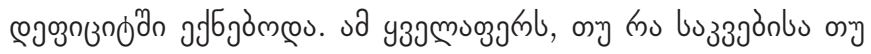

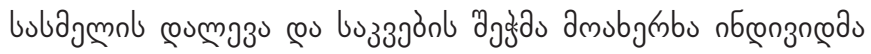

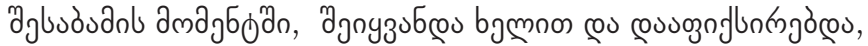

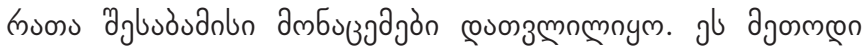

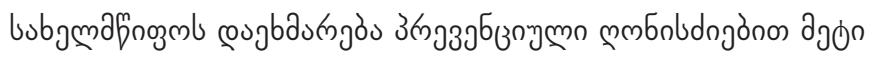

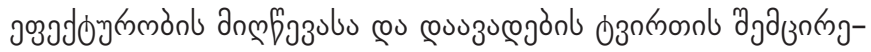

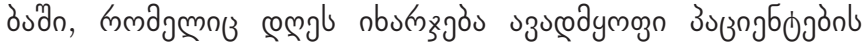

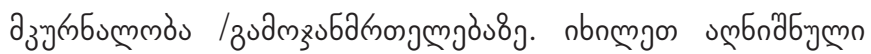

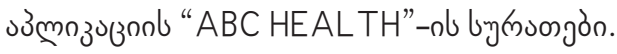

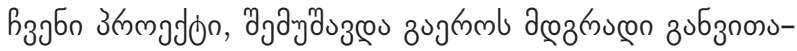

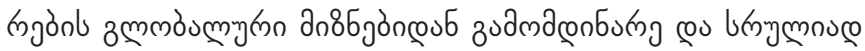

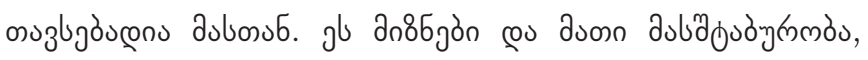

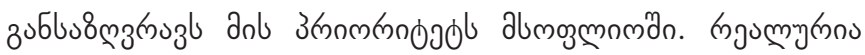

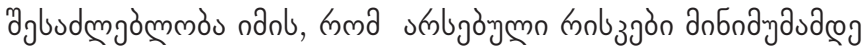
couzngzubmon eu ufmenge sanl grnon-ghon buongmn duzu-

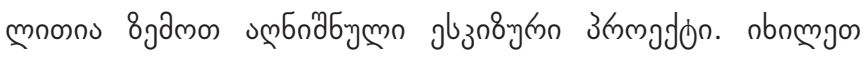

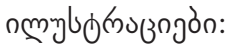

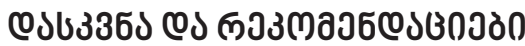

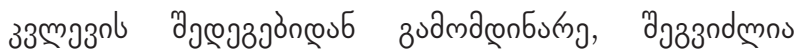

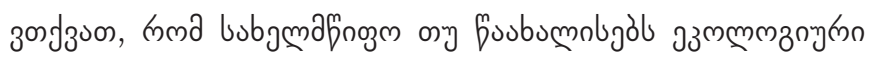

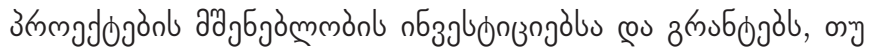

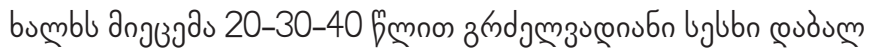

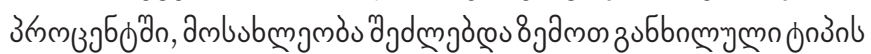

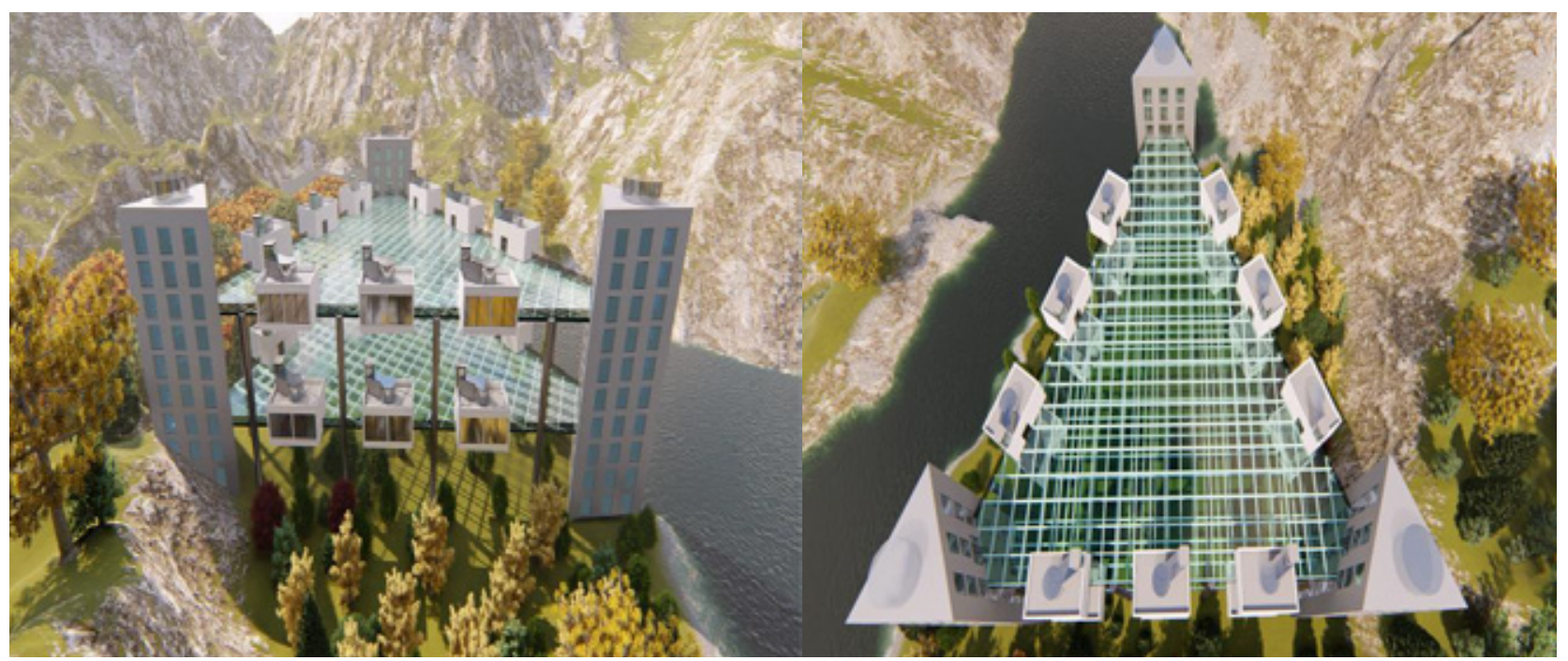




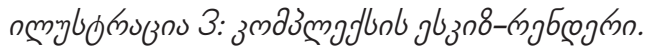

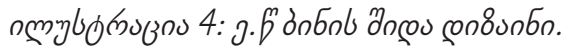

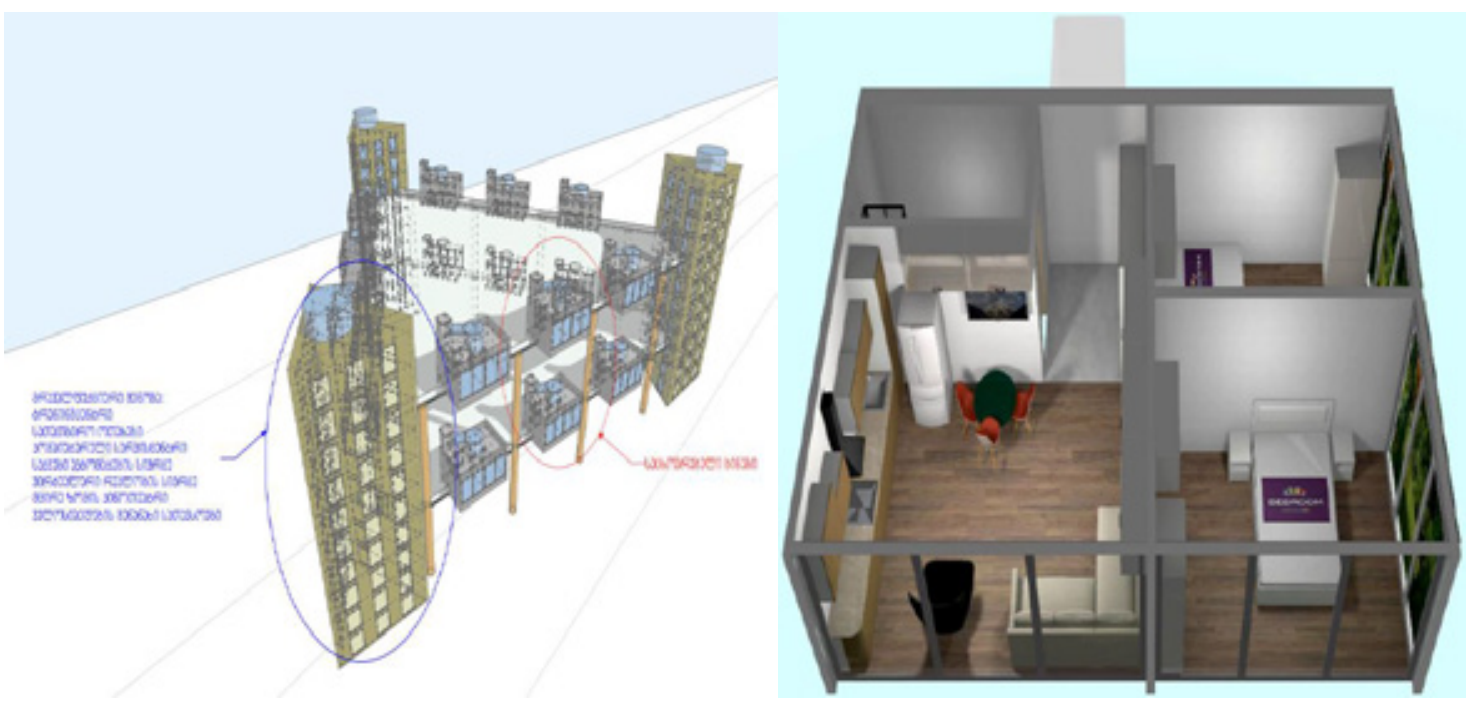

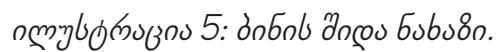

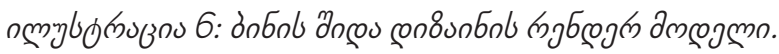
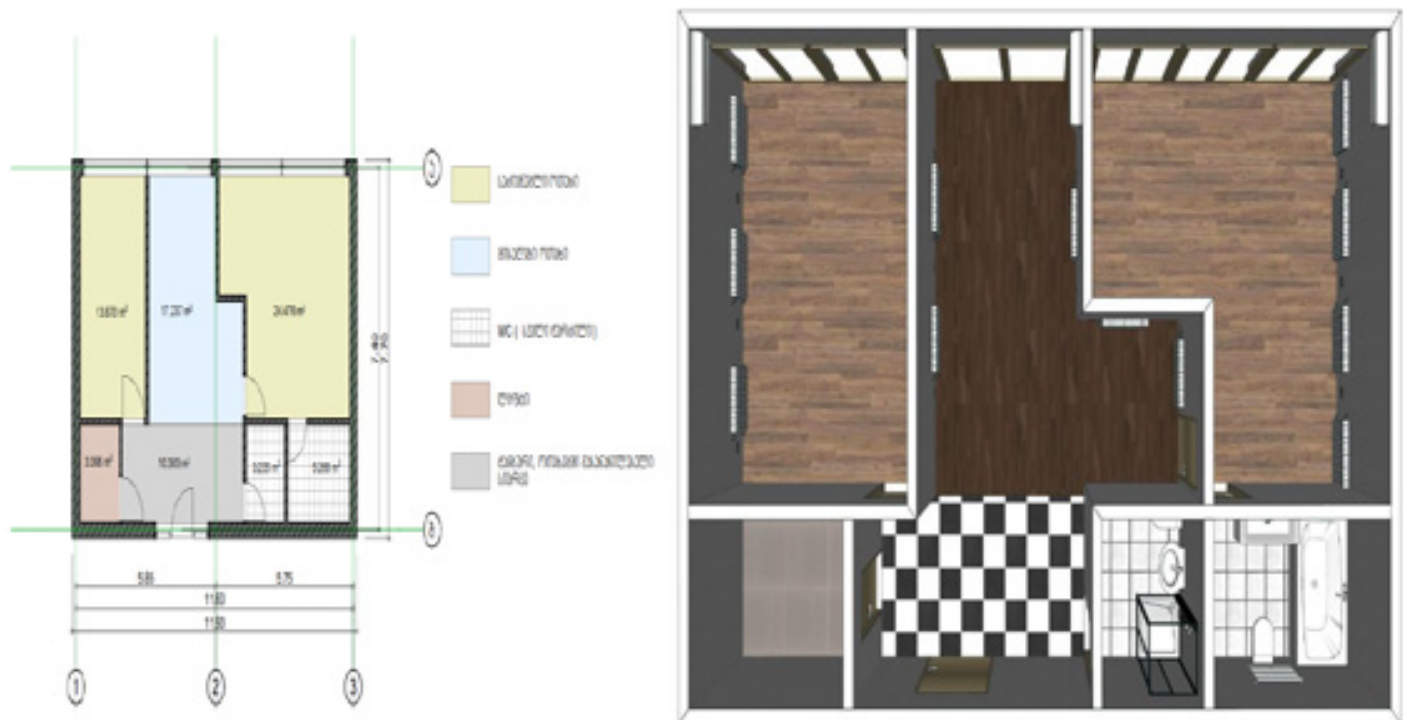

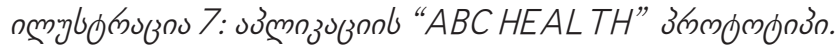
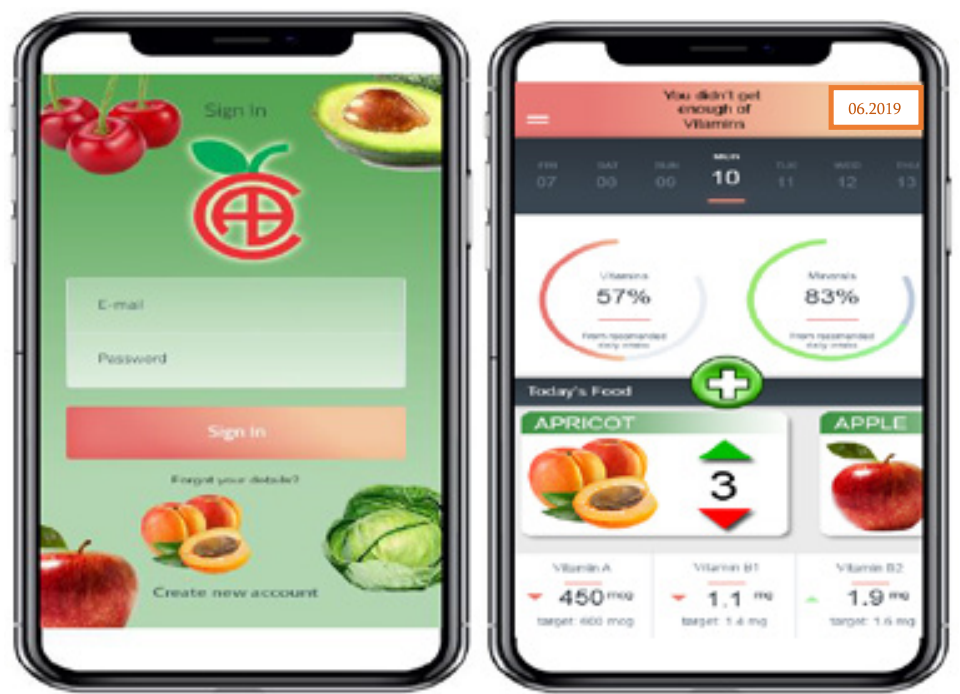


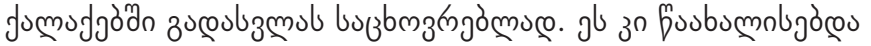

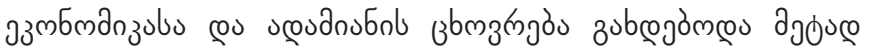

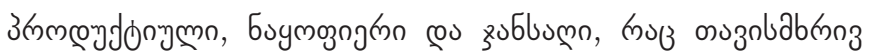

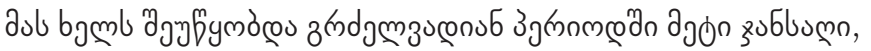

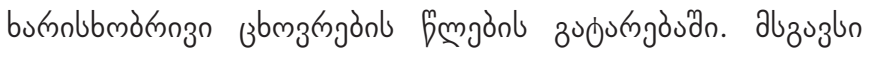

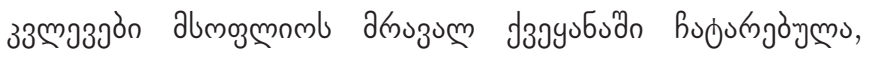

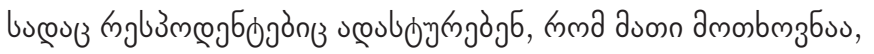

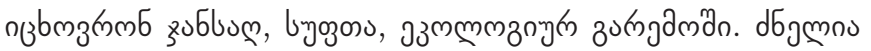

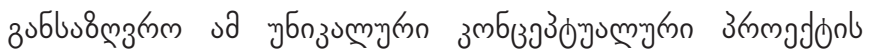

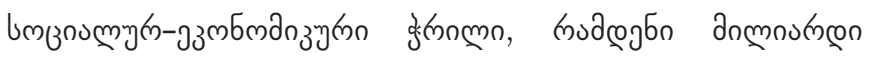

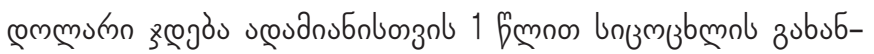

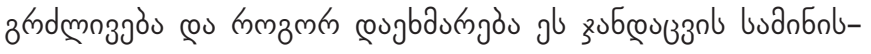

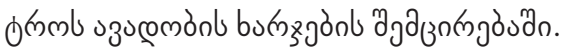

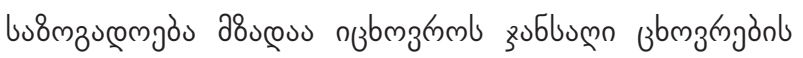

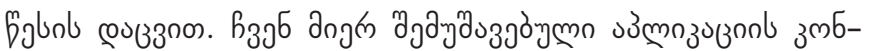

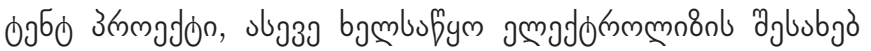

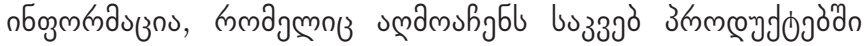

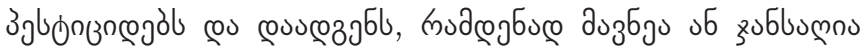

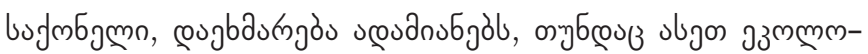

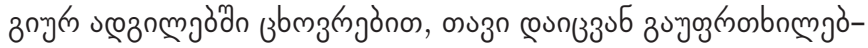

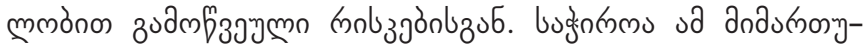

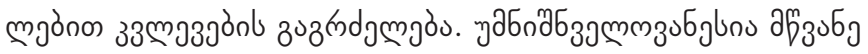

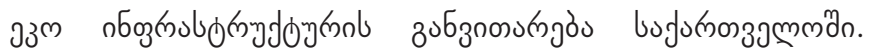

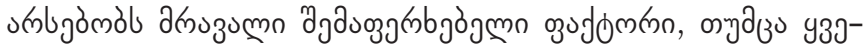

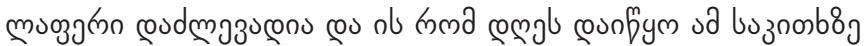

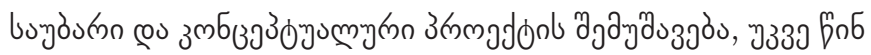
zuюszàmmo budnzno.

The research implemented by the author and was supported by the Shota Rustaveli National Science Foundation of Georgia (SRNSFG) [MR-18-093]

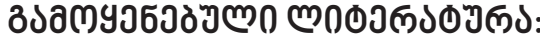

1. World Health Organization. (2018). "World Health Organization Releases New Global Air Pollution Data." CCAC Secretariat. http://ccacoalition.org/en/news/world-health-organization-releases-new-global-air-pollution-data (10.05.2019)

2. Petryk, Agnieszka. (2018). "Epidemiology of Selected Diseases Related to Air Pollution in Krakow." Journal of Ecological Engineering 19 (6): 124-31. doi.org/10.12911/22998993/92892.

3. Madhipatla Kishore, Venkatesh S, Christopher D J, Stanaway D Jeffrey, Dhaliwal R S, Dandona Lalit, Singh Virendra, et al. (2018). "The Impact of Air Pollution on Deaths, Disease Burden, and Life Expectancy across the States of India: The Global Burden of Disease Study 2017." The Lancet Planetary Health 3 (1): e26-39. doi.org/10.1016/s2542-5196(18)30261-

4. Diamandis Peter. (2019). https://www.diamandis.com/blog/future-of-cities-part-1 (5.06.2019)

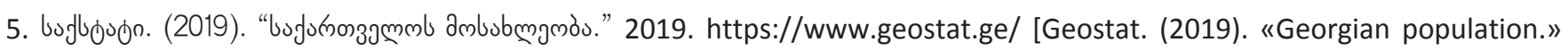
2019. https://www.geostat.ge/. In Georgian]. ka/modules/categories/41/mosakhleoba. (01.03.2019)

6. эзмз3мэз১ (2019). (1) [Eco-examination (2019). (1). In Georgian]. https://drive.google.com/open?id=1AYyimUXo-dfZQxy5N$6 \mathrm{hKn3ZCZjRm \_ IYU}$

7. эзмззмззЈ (2019). (2) [Eco-examination (2019). (2). In Georgian]. https://drive.google.com/open?id=1J-h4dn42m8q8T4ASDexgjGI1bKScEULT

8. эзмззмэзЈ (2019). (3) [Eco-examination (2019). (3). In Georgian]. https://drive.google.com/open?id=1rDavn46wuPiv8|B|J5AODOYToA11ZDk 


\section{HEALTH PROMOTION IN GEORGIA ECO INFRASTRUCTURE DEVELOPMENT AND TECHNOLOGICAL ASPECTS}

\section{LEVANI KIKILASHVILI}

Ilia State University, Georgia

Ikikilashvili555@gmail.com

\section{TENGIZ VERULAVA}

Doctor of Medicine, Professor

Ilia State University, Georgia

tengiz.verulava@iliauni.edu.ge

KEYWORDS: DISEASE-PREVENTION; ENGINEERING-TECHNOLOGY; ECO INFRASTRUCTURE; INNOVATION-NOVATION.

For citation: Kikilashvili L., Verulava T. (2019), Health Promotion In Georgia Eco Infrastructure Development And Technological Aspects, Globalization And Business, №7, pp. 187-200. (In Georgian). https://doi.org/10.35945/gb.2019.07.024

\section{SUMMARY}

At the beginning of new millennium, Georgia has faced a variety of environmental challenges. The reduction of green space and poor air quality became the major underline risk factor in the rise of many types of diseases in big cities. One of the distinct characteristics of Georgia is its unique natural resources and landscapes. That creates a possibility to design strategies for more sustainable socialeconomical development of our country. We believe the development of eco infrastructure and its appropriate support system can increase the longevity and overall life quality among the residents of Georgia. Living in the ecologically clean environment became a major challenge among many populations across the globe. The global economic development has gaps in supporting eco-friendly life standards. Therefore, it becomes critical to study environmental pollution and seek cutting-edge strategies to build a more sustainable and eco-friendly environment for the health of people and the entire planet. New, technologically advanced eco-projects have the potential to create new social and environmental climate in Georgia, thus improving health, productivity, and well-being of the entire nation. 


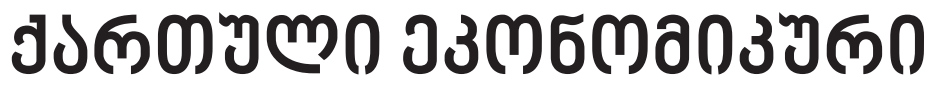

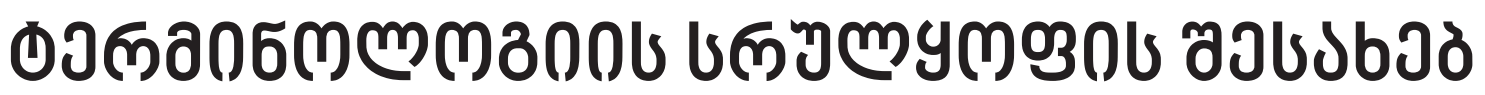

\section{ON THE IMPROVEMENT OF GEORGIAN}

ECONOMIC TERMINOLOGY 



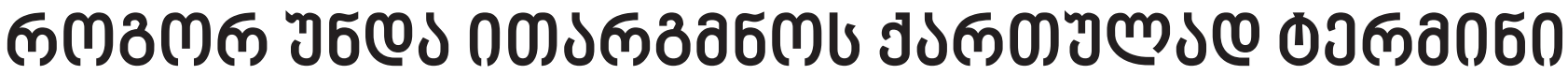 "GROSS DOMESTIC PRODUCT"1
}

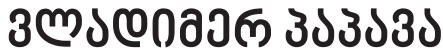

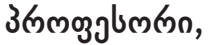

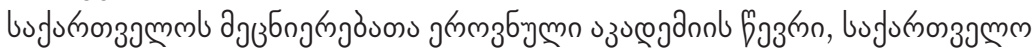

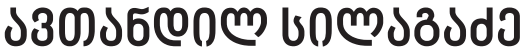

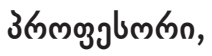

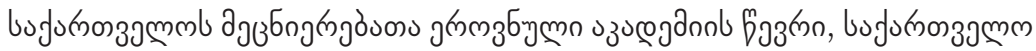

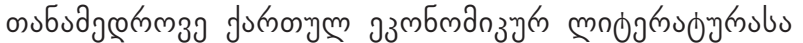

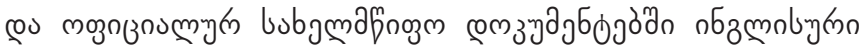

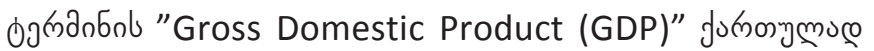

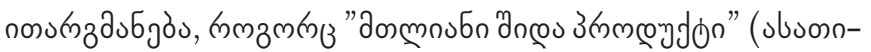

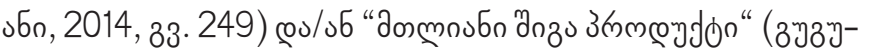

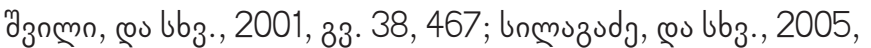
33. 408$)^{2}$.

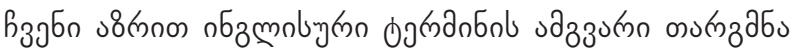

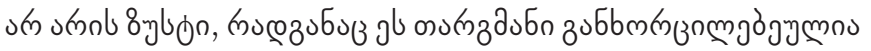

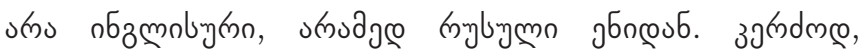

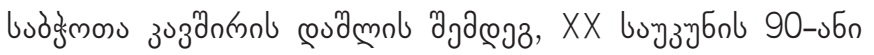

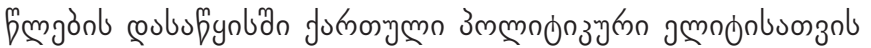

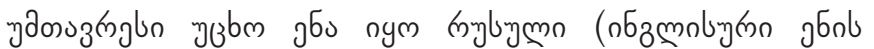

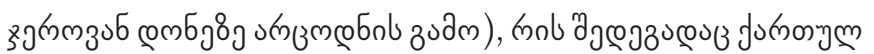
jбuдn ogr

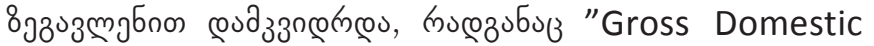

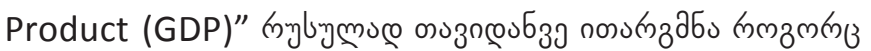
«Валовый Внутренний Продукт (ВВП)».

\footnotetext{
1. Papava V., Silagadze A., 2019. "How the Term the "Gross Domestic Product" should be Translated into Georgian." Globalization and Business, No. 7, pp. 203-204. (In Georgian.)

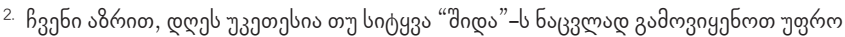

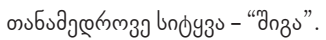

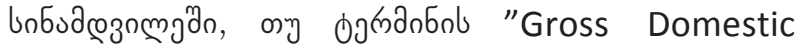

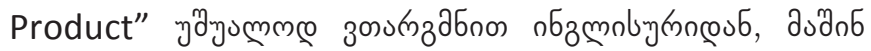

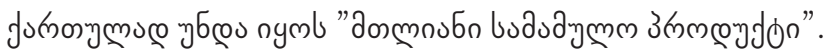

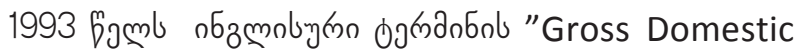

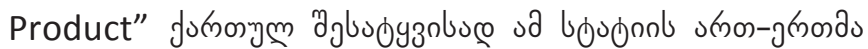

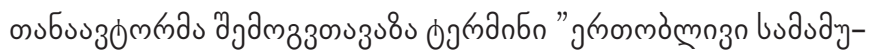

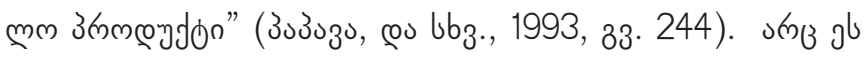

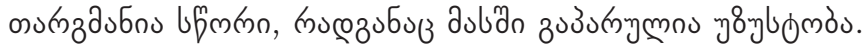

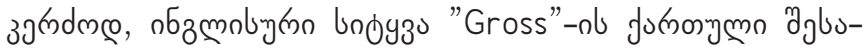

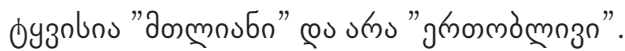

إ

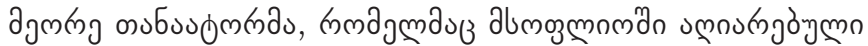

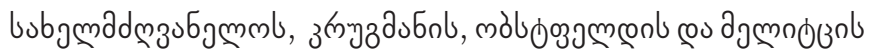
"bugrnosàmfrolum jзmbmanzol" (Krugman, Obstfeld,

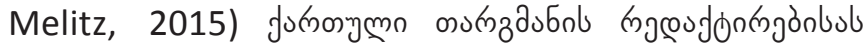

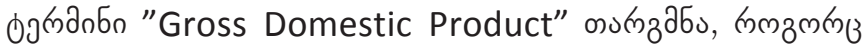

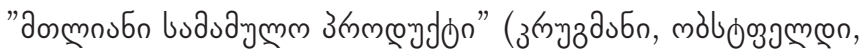
agmoo(3n, 2017, 33. 1).

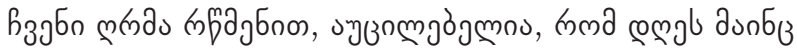

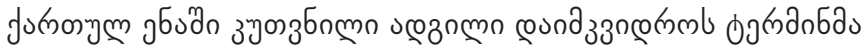

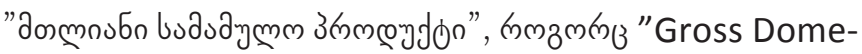

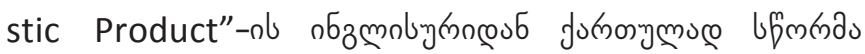
osmorasbas. 


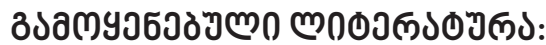

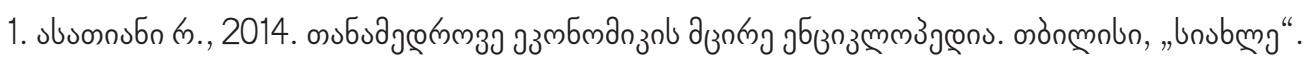

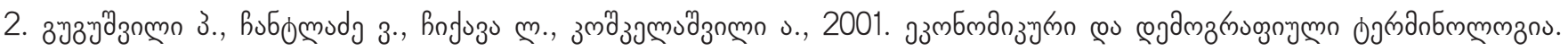
mònmolon, "lnobmg".

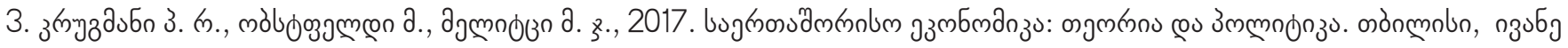

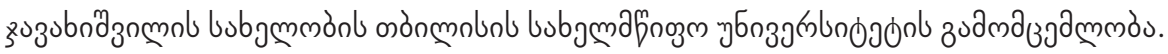

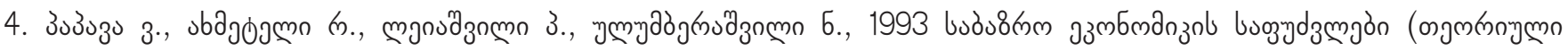

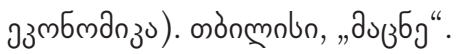

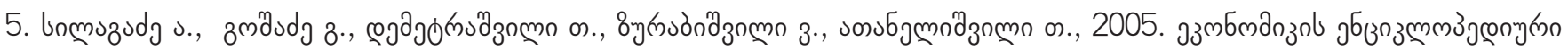

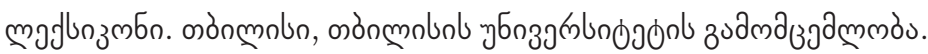

6. Krugman P. R., Obstfeld M., Melitz M. J., 2015. International Economics: Theory and Policy. Tenth Edition. Boston, Pearson Education.

\section{REFRENECES:}

1. Asatiani R., 2014. Tanamedrove ekonomikis mcire entsiklopedia [Concise Encyclopedia of Modern Economy.] Tbilisi, "Siakhle." (In. Georgian.)

2. Gugushvili P., Chantladze V., Chiqava L., Koshkelashvili A., 2001. Ekonomikuri da demographiuli terminologia [Economic and Demographic Terminology.] Tbilisi, "Siakhle." (In. Georgian.)

3. Krugmani P. R., Obstfeldi M., Melitzi M. J., 2018. Saertashoriso ekonomika: teoria da politika [International Economics: Theory and Policy.] Tbilisi, Ivane Javakhishvili Tbilisi State University Publishing House. (In. Georgian.)

4. Papava V., Akhmeteli R., Leiashvili P., Ulumberashvili N., 1993. Sabazro ekonomikis saphudzvlebi (teoriuli ekonomika) [Basis of Market Economy (Theoretical Economics.)]. Tbilisi, “Matsne." (In. Georgian.)

5. Silagadze A., Goshadze G., Demetrashvili T., Zurabishvili V., Atanelishvili T., 2005. Ekonomikis entsiklopediuri leqsikoni. [Encyclopedic Dictionary of Economics.] Tbilisi, Tbilisi University Publishing House. (In. Georgian.)

6. Krugman P. R., Obstfeld M., Melitz M. J., 2015. International Economics: Theory and Policy. Tenth Edition. Boston, Pearson Education.

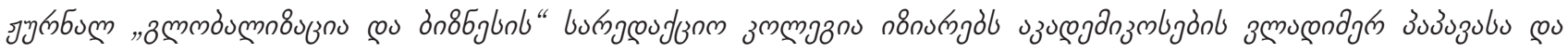

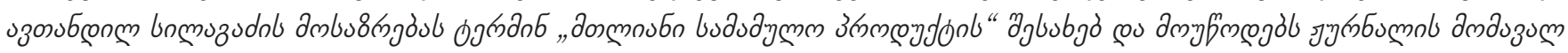

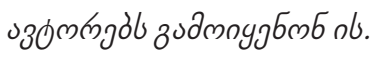




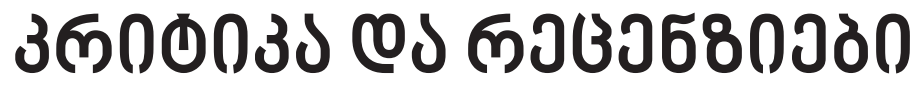

CRITICISM AND REVIEWS 



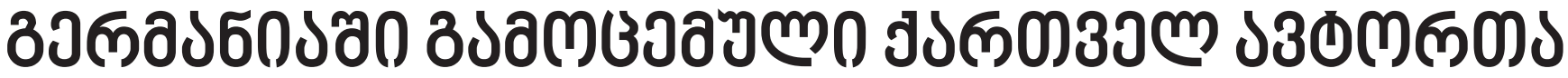

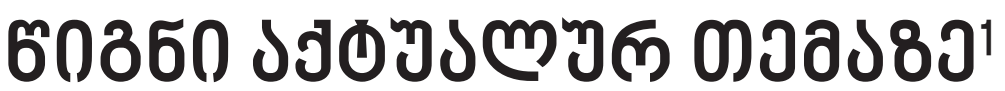

2019 G

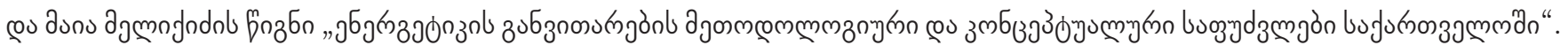

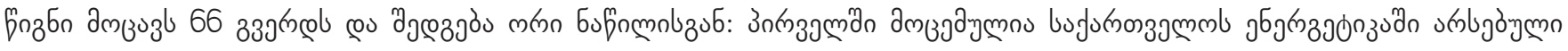

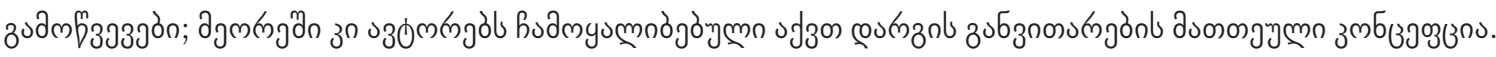

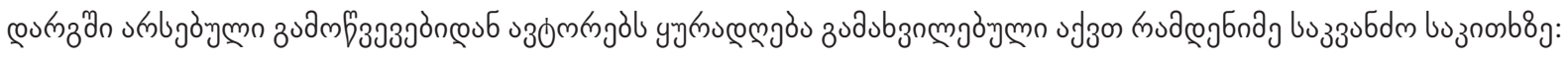

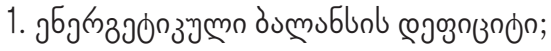

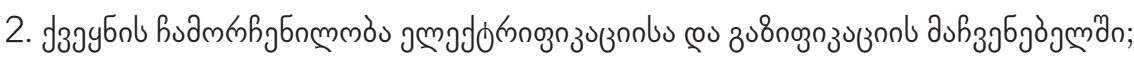

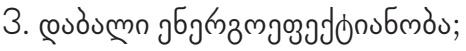

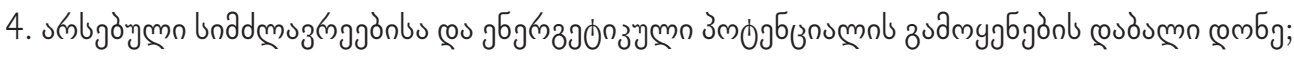

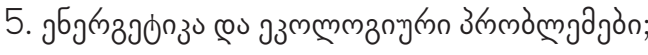

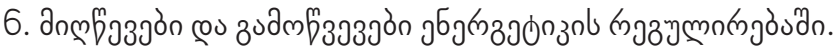

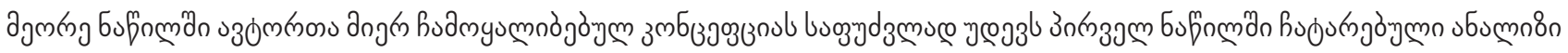

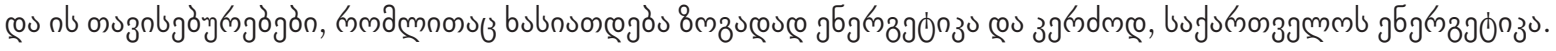

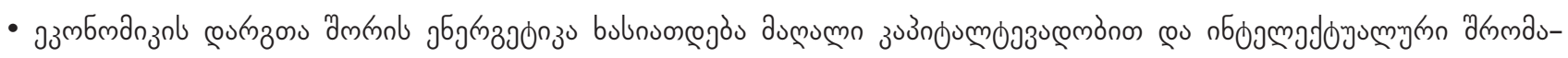

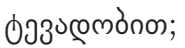

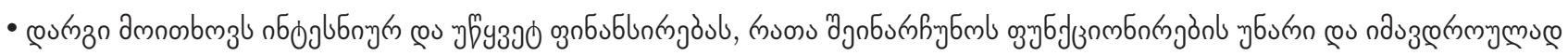

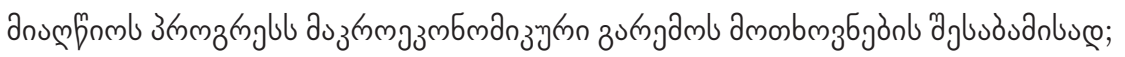

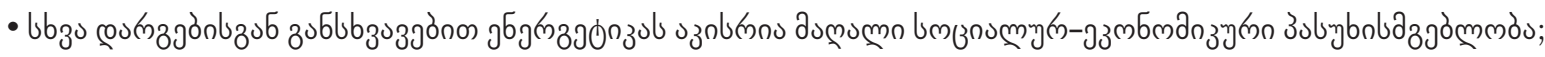

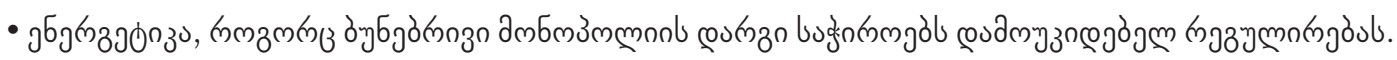

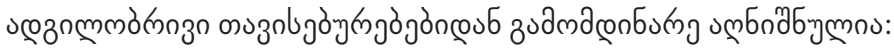

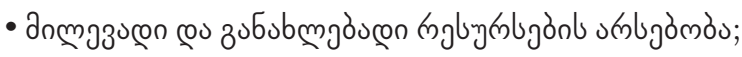

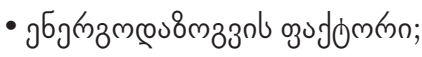

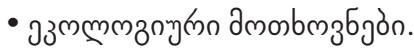

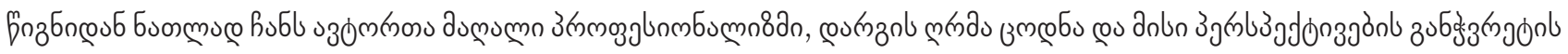

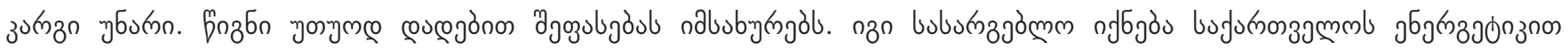

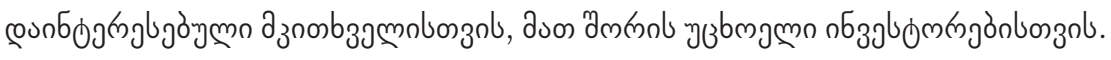

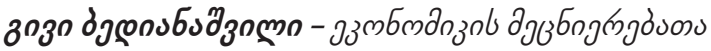

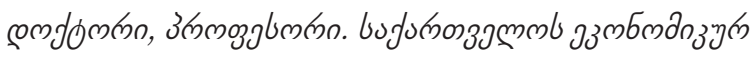
ajo 


\section{BOOK ABOUT THE KEY CHALLENGING ISSUES BY THE GEORGIAN AUTHORS THAT IS PUBLISHED IN GERMANY ${ }^{1}$}

In 2019 in Germany the Lambert Publishing has published the book - „Methodological and Conceptual Basis for the Energy Development in Georgia”. The book's authors are PhD. Mr. Demur Chomakhidze and PhD. Mrs. Maia Melikidze. The book consists of 66 pages and implies two parts: the first part refers to the existing challenges in the Georgian energy sector and the second one comprises the sector development related concepts developed by the authors.

Among existing challenges in the sector, the authors focus on the following key issues:

1. Scarcity in energy balance;

2. Low level of electrification and gasification;

3. Insufficient energy efficiency;

4. Low level of utilisation of existing capacities and energy potential;

5. Energy and environmental problems;

6. Achievements and challenges in Energy Regulation;

Concept developed by the authories presented in the second section is based on the analysis as well as on the speficities by which the energy sector (especially Georgian energy sector) is characterized. These analysis and specificities are presented in the first section of the book.

- Among other fields of economy, the energy sector is characterized by high capital intensity and requires huge intelectual resources;

- Intensive and continous financing is needed for this sector in order to ensure its sustainable development and reach the progress in accordance with the conditions of the macro-economical environment;

- High social-economical responsibilities are imposed on the energy sector unlike other fields of economy;

- Independent and seperated regulation is required for the energy as a natural monopoly.

The following factors are extremely important in terms of local specificities:

- existence of renewable and non-renewable resources;

- energy saving;

- Environmental requirements.

The book clearly shows high level of profesionalism and qualification, comprehensive knowledge of the sector and excellent ability to foresee the prospects by the authors. The book truly deserves a positive reaction and feedback. It will be useful for the readers to whom the Georgian energy sector is interesting, including foreign investors.

Givi bedianashvili - Doctor of Economic Science,

Professor, Vice-President of

the Georgian Academy of Economic Science.

1. Demur Chomakhidze, Maia Melikidze “Methodological and Conceptual Basis for the Energy Development in Georgia”. Liap. Lambert Academic publishing. 2019. 



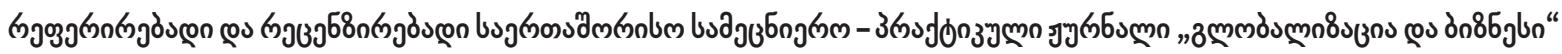

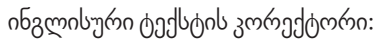

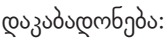

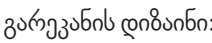

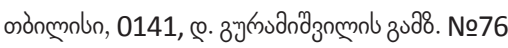

ogs

(+995 32) 2000171

(+995 599) 969459

oुoflon:

(+995 032) 2143583

jm-ogmbos:

info@eugb.ge

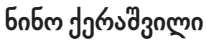

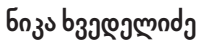

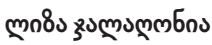

REFEREED AND PEER-REVIEWED INTERNATIONAL SCIENTIFIC-PRACTICAL JOURNAL GLOBALIZATION \& BUSINESS

Proof-reader of English text:

Page Make-up:

Cover Design:

76 D. Guramishvili ave, Tbilisi, 0141, Georgia

Phone:

(+995 32) 2000171

(+995 599) 969459

Fax:

E-mail:

(+995 032) 2143583

info@eugb.ge
Nino Kerashvili

Nika Khvedelidze

Liza Jalaghonia 


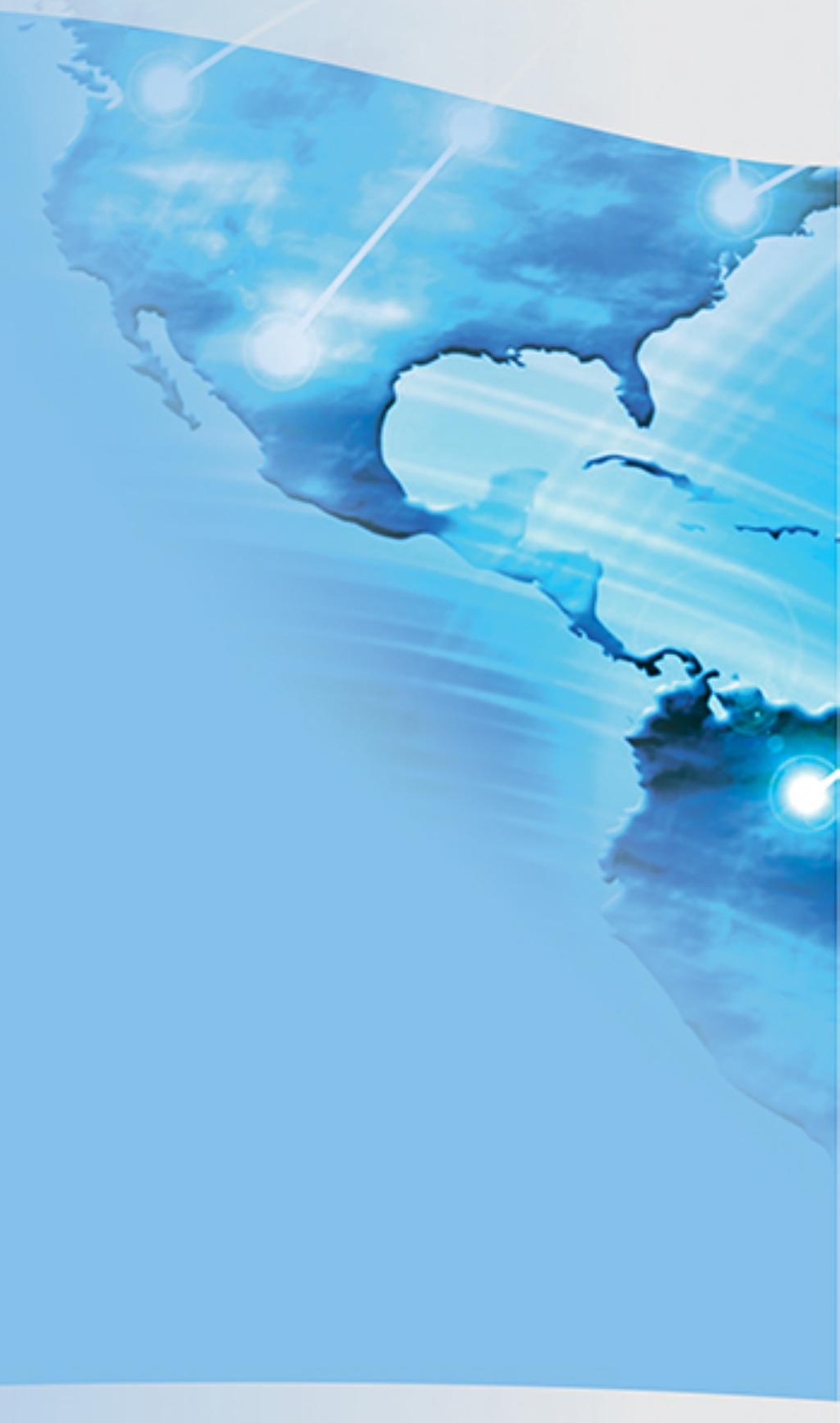

